Florida International University FIU Digital Commons

$7-3-2013$

\title{
Twelve Certain Men: The Impact of Emotional Appraisals on Juror Decision-Making
}

Stephen W. Joy

Florida International University, sjoy001@fiu.edu

DOI: $10.25148 /$ etd.FI13080705

Follow this and additional works at: https://digitalcommons.fiu.edu/etd

Part of the Other Psychology Commons

\section{Recommended Citation}

Joy, Stephen W., "Twelve Certain Men: The Impact of Emotional Appraisals on Juror Decision-Making" (2013). FIU Electronic Theses and Dissertations. 915.

https://digitalcommons.fiu.edu/etd/915

This work is brought to you for free and open access by the University Graduate School at FIU Digital Commons. It has been accepted for inclusion in FIU Electronic Theses and Dissertations by an authorized administrator of FIU Digital Commons. For more information, please contact dcc@fiu.edu. 


\section{FLORIDA INTERNATIONAL UNIVERSITY}

Miami, Florida

TWELVE CERTAIN MEN: THE IMPACT OF EMOTIONAL APPRAISALS ON JUROR DECISION-MAKING

A dissertation submitted in partial fulfillment of

the requirements for the degree of

DOCTOR OF PHILOSOPHY

in

PSYCHOLOGY

by

Stephen W. Joy

2013 
To: Dean Kenneth G. Furton

College of Arts and Sciences

This dissertation, written by Stephen W. Joy, and entitled Twelve Certain Men: The Impact of Emotional Appraisals on Juror Decision-Making, having been approved in respect to style and intellectual content, is referred to you for judgment.

We have read this dissertation and recommend that it be approved.

Steve D. Charman

Lindsay C. Malloy

Howard M. Wasserman

Ryan J. Winter, Major Professor

Date of Defense: July 3, 2013

The dissertation of Stephen W. Joy is approved.

Dean Kenneth G. Furton

College of Arts and Sciences

Dean Lakshmi N. Reddi

University Graduate School

Florida International University, 2013 


\section{DEDICATION}

This undertaking is dedicated to my infinitely patient and loving wife. Thank you for riding this out and seeing things through with me. I couldn't have done it without you. 


\section{ACKNOWLEDGMENTS}

First and foremost, I would like to thank my primary advisor, Ryan J. Winter for his invaluable guidance, advice, and tutelage throughout this entire process. I would also like to thank my committee members Steve D. Charman, Lindsay C. Malloy, and Howard M. Wasserman for their limitless patience while enduring this lengthy affair.

I must also extend my most sincere gratitude and appreciation to my two research assistants, Jenice Gonzalez and Paula Jimenez who went above and beyond to code data for me, often under time pressure and all while fielding incessant emails and worrywart text messages from me.

Finally, I would like to thank Florida International University for the very generous Presidential Fellowship. I hope I make you proud. 


\title{
ABSTRACT OF THE DISSERTATION
}

TWELVE CERTAIN MEN: THE IMPACT OF EMOTIONAL APPRAISALS ON JUROR DECISION-MAKING

\author{
by \\ Stephen W. Joy \\ Florida International University, 2013 \\ Miami, Florida \\ Professor Ryan J. Winter, Major Professor
}

Our jury system is predicated upon the expectation that jurors engage in systematic processing when considering evidence and making decisions. They are instructed to interpret facts and apply the appropriate law in a fair, dispassionate manner, free of all bias, including that of emotion. However, emotions containing an element of certainty (e.g., anger and happiness, which require little cognitive effort in determining their source) can often lead people to engage in superficial, heuristic-based processing. Compare this to uncertain emotions (e.g., hope and fear, which require people to seek out explanations for their emotional arousal), which instead has the potential to lead them to engage in deeper, more systematic processing.

The purpose of the current research is in part to confirm past research (Tiedens \& Linton, 2001; Semmler \& Brewer, 2002) that uncertain emotions (like fear) can influence decisionmaking towards a more systematic style of processing, whereas more certain emotional states (like anger) will lead to a more heuristic style of processing. Studies One, Two, and Three build upon this prior research with the goal of improving methodological rigor through the use of film clips to reliably induce emotions, with awareness of testimonial details serving as measures of processing style. 
The ultimate objective of the current research was to explore this effect in Study Four by inducing either fear, anger, or neutral emotion in mock jurors, half of whom then followed along with a trial transcript featuring eight testimonial inconsistencies, while the other participants followed along with an error-free version of the same transcript. Overall rates of detection for these inconsistencies was expected to be higher for the uncertain/fearful participants due to their more effortful processing compared to certain/angry participants. These expectations were not fulfilled, with significant main effects only for the transcript version (with or without inconsistencies) on overall inconsistency detection rates. There are a number of plausible explanations for these results, so further investigation is needed. 


\section{TABLE OF CONTENTS}

CHAPTER

PAGE

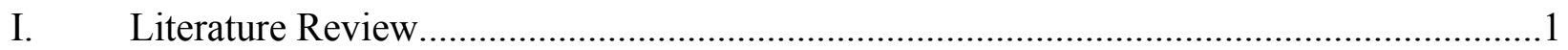

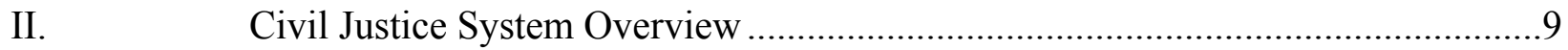

Legal decision-making ...........................................................................11

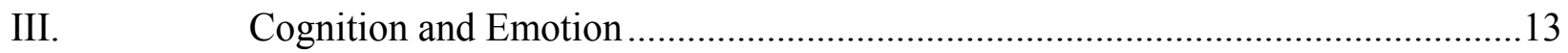

Need for cognition ................................................................................ 18

History of valence and emotion research ................................................29

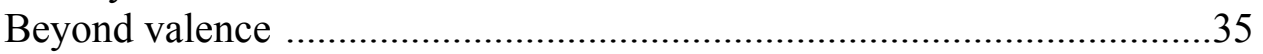

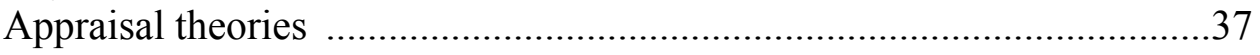

Uncertainty and certainty appraisals specifically .....................................40

Emotion induction techniques .................................................................43

Emotions dictating information processing methods and

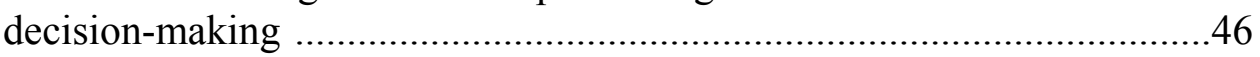

Emotional valence directing judgments.....................................................47

Emotions suggesting informational context for decision-making ..............47

How specific emotions fit in with processing and decision-making

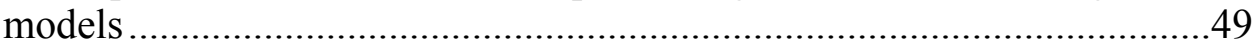

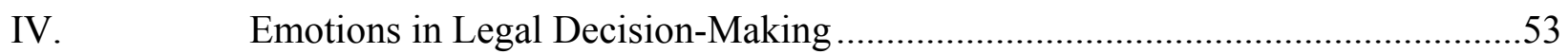

Certainty and uncertainty appraisals within the legal context ...................56

Types of inconsistencies that jurors may face …………….......................67

V. Study One: Identifying Detection Rates of Individual Inconsistencies .............................70

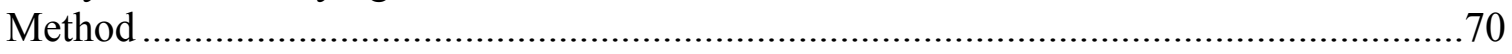

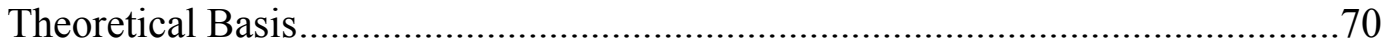

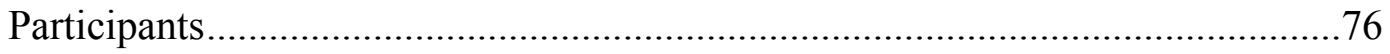

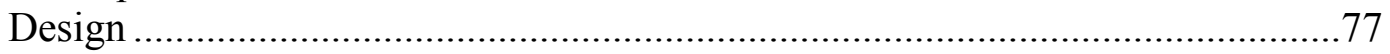

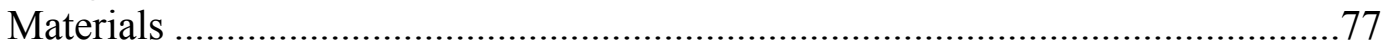

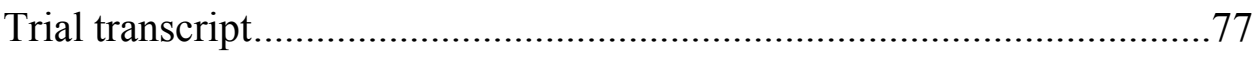

Measures to determine rate of detection for each inconsistency .................78

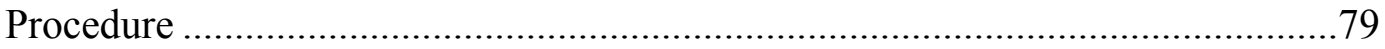

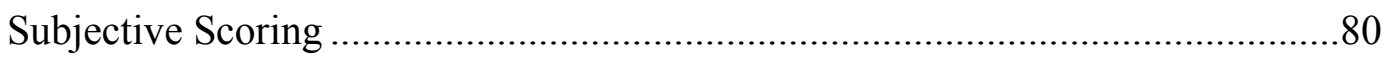

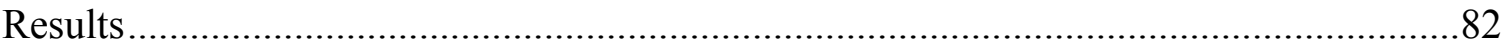

Inter-rater Reliability for Subjectively Scored Questions...................................8

Comparing Overall Detection Rates of Each Inconsistency ..................................82

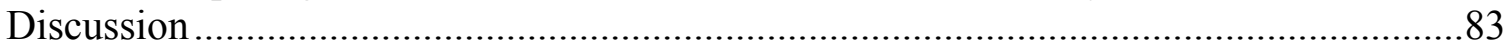

VI. Study Two: Verifying Trial Transcript Stimulus Materials ………………...................... 84

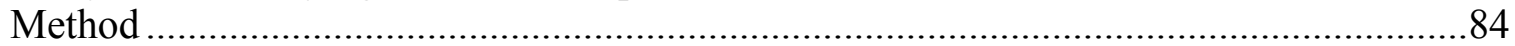

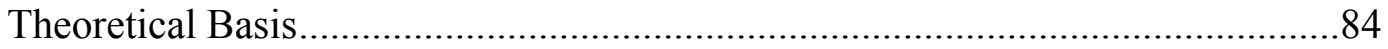

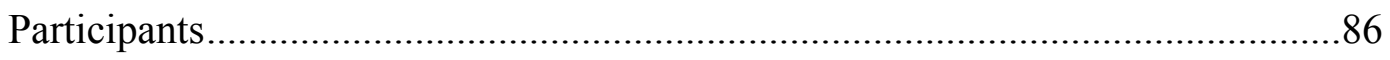




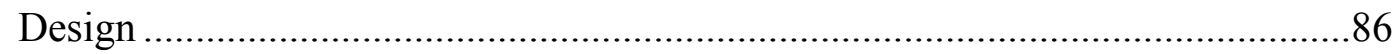

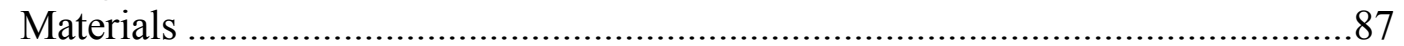

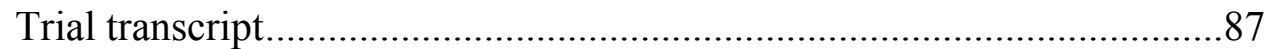

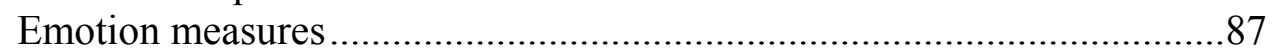

Measures to determine rate of detection for each inconsistency ................88

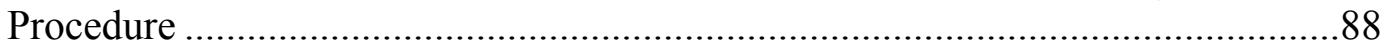

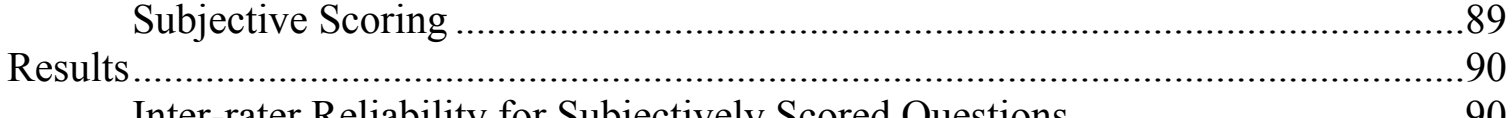

Confirming General Detection Rates of Each Inconsistency ..................................91

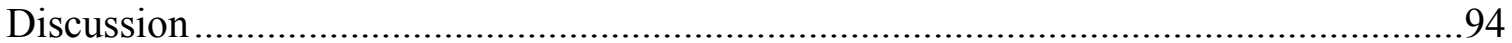

Measuring Emotional Impact of Trial Transcript ……..........................................93

VII. Study Three: Verifying Emotion Induction Manipulations ...............................................99

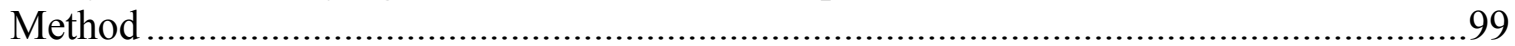

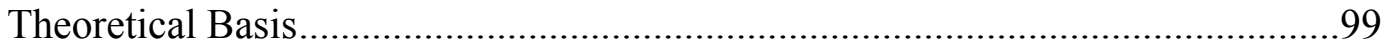

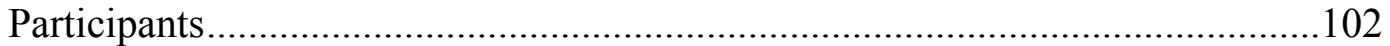

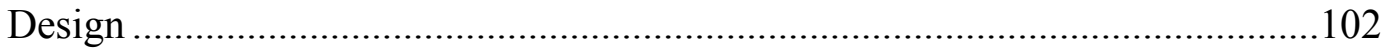

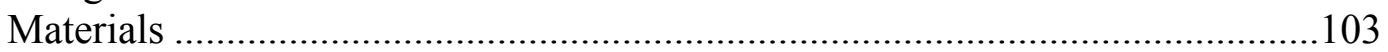

Emotion induction..........................................................................103

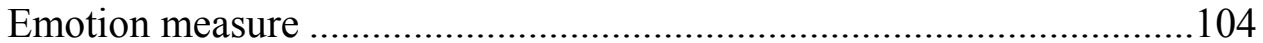

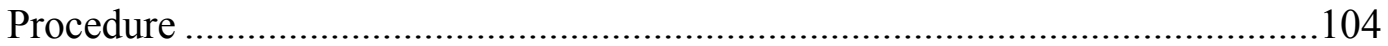

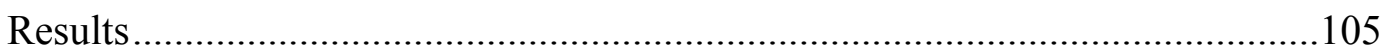

General Effectiveness of Films as Means of Induction …………….......105

Comparing Relative Effectiveness of each Film Clip and Induction

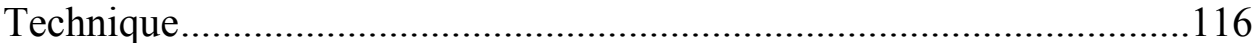

Discussion

VIII. Study Four: Effects of Emotional Appraisals of Certainty on Juror Information

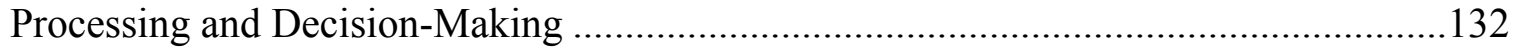

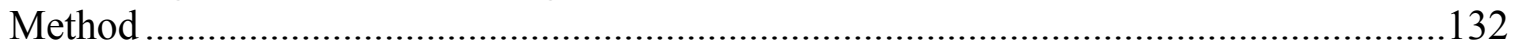

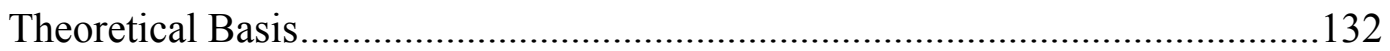

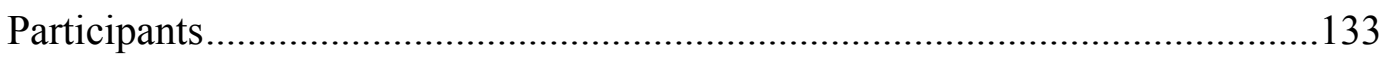

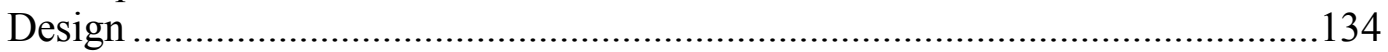

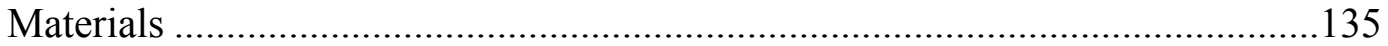

Session one: trait-based scales …………………………...................135

Session two: emotion inductions, trial transcripts, verdict, witness

ratings, inconsistency detection questions, and emotion and

film ratings

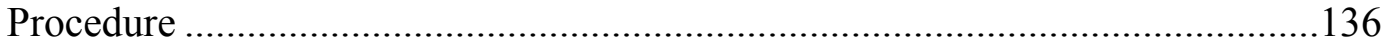

Session one: pretesting for trait-based characteristics ………………....136

Session two: emotion and decision-making ………………...................137

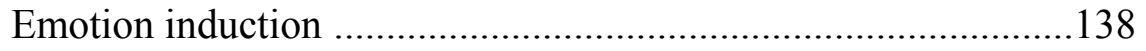

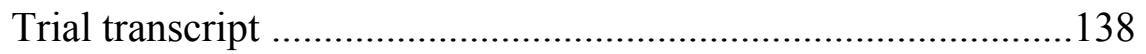

Dependent measures ...........................................................139

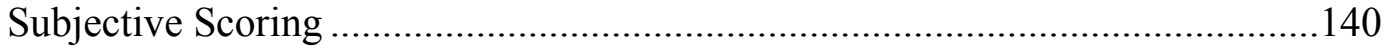




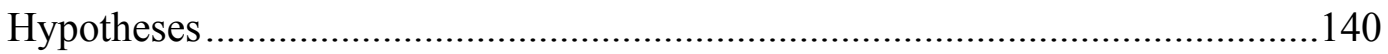

Processing style through detection of inconsistencies ..............................140

Processing style through witness credibility and persuasiveness

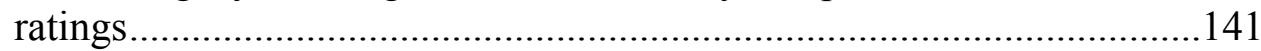

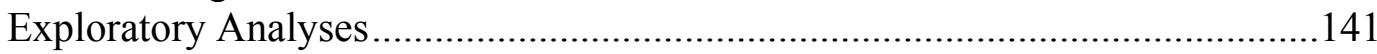

Verdict as a result of processing style...................................................142

Verdict confidence as a result of processing style ....................................143

Rational/experiential processing preference moderating verdict

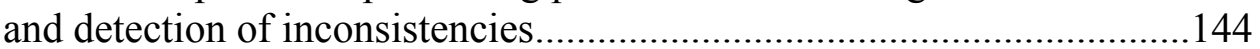

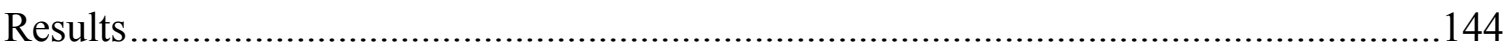

Inter-Rater Reliability for Subjectively Scored Questions ....................................144

Verification of Unequal Rates of Inconsistency Detection Based on

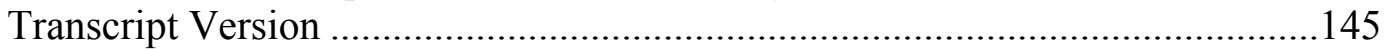

Effect of Emotion on Rate of Inconsistency Detection .......................................146

Effects of emotion on inconsistency detection according to

question type.....

Effects of emotion on inconsistency detection according to specific inconsistencies ......................................................................152

Credibility and Persuasiveness Ratings of Witnesses........................................155

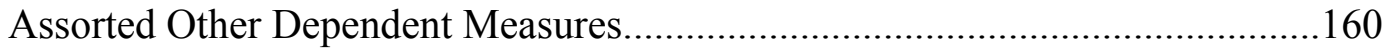

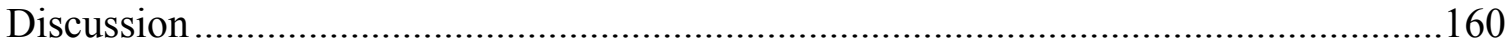

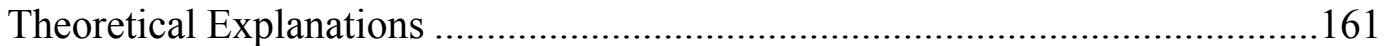

Methodological Explanations ...........................................................................163

IX. Study Five: Establishing Durability and Duration of Emotion Inductions ......................167

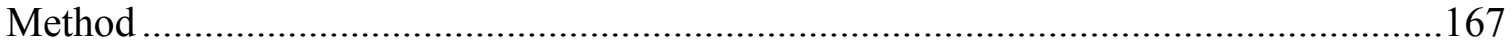

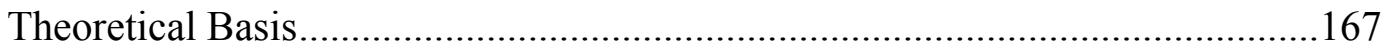

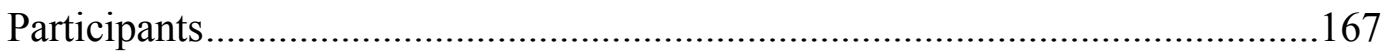

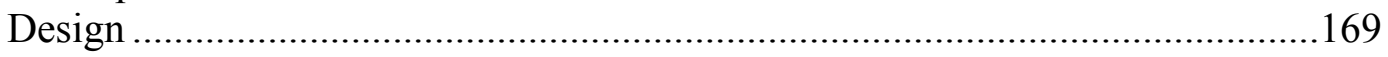

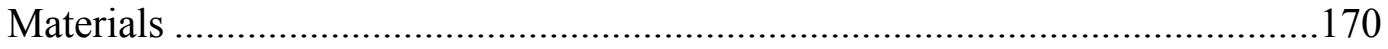

Emotion Measures ............................................................................170

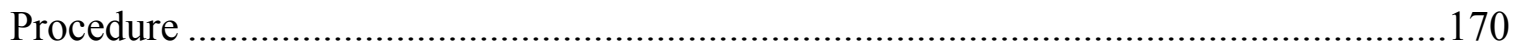

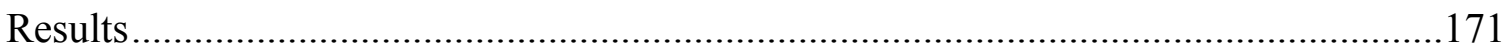

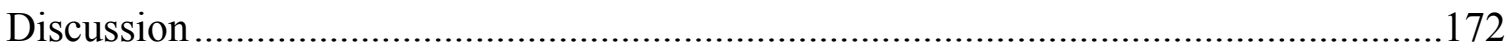

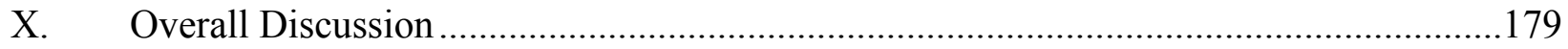

Study Limitations and Future Directions........................................................180

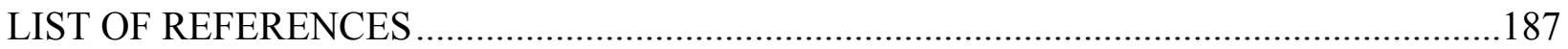

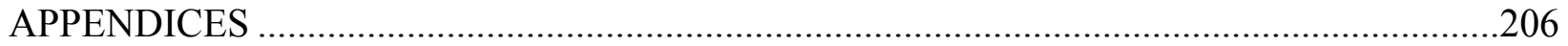

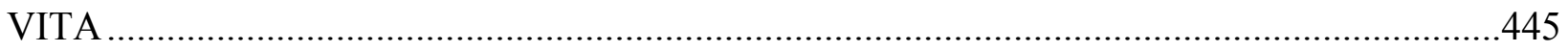




\section{LIST OF TABLES}

TABLE

PAGE

E1 Fifteen Inconsistencies and Five Dummy Questions Used in Study One Trial Transcript .....

M1 Study One: Loose Scoring Scheme Detection Rate Percentages for all 15 Inconsistencies by Question Type .....

M2 Study One: Strict Scoring Scheme Detection Rate Percentages for all 15

Inconsistencies by Question Type

R1 Eight Inconsistencies Used in Study Two Trial Transcript .368

T1 Study Two: Loose Scoring Scheme Detection Rate Percentages for all Eight Inconsistencies by Question Type .....

T2 Study Two: Strict Scoring Scheme Detection Rate Percentages for all Eight Inconsistencies by Question Type .....

1 Study Two: Kendall's tau-b Correlations Between Pre- and Post- Transcript Emotion Ratings

2 Study Two: Descriptive Statistics and Wilcoxon Signed-Test Results for Emotion Ratings Before and After Transcript

3 Study Three: Contrast of Mean Emotion Ratings Before and After The Shining Film Clip and Perspective Taking Writing Task

4 Study Three: Contrast of Mean Emotion Ratings Before and After The Silence of the Lambs Film Clip and Perspective Taking Writing Task.

5 Study Three: Contrast of Mean Emotion Ratings Before and After Cry Freedom Film Clip and Perspective Taking Writing Task

6 Study Three: Contrast of Mean Emotion Ratings Before and After My Bodyguard Film Clip and Perspective Taking Writing Task....

7 Study Three: Contrast of Mean Emotion Ratings Before and After The Shining Film Clip and No Writing Task

8 Study Three: Contrast of Mean Emotion Ratings Before and After The Silence of the Lambs Film Clip and No Writing Task.... 
9 Study Three: Contrast of Mean Emotion Ratings Before and After Cry Freedom Film Clip and No Writing Task

10 Study Three: Contrast of Mean Emotion Ratings Before and After My Bodyguard Film Clip and No Writing Task

11 Study Three: Pairwise Comparisons of Significant Differences in Postfilm Emotion Ratings

12 Emotion Ratings While Watching The Shining ..........................................................130

13 Emotion Ratings While Watching My Bodyguard ……………………......................131

AF1 Study Four: Comparison of Mean Detection Ratings by Transcript Version...................444

14 Study Four: Detection Rate Percentages for all Eight Inconsistencies by Film Clip/Induced Emotion and Question Type for Participants Exposed to Inconsistent Transcript Only

15 Study Five: Contrast of Mean Emotion Ratings Before and After The Shining Film Clip and Trial Transcript

16 Study Five: Contrast of Mean Emotion Ratings Before and After My Bodyguard Film Clip and Trial Transcript

17 Study Five: Contrast of Mean Emotion Ratings Before and After Alaska's Wild Denali Film Clip and Trial Transcript.

18 Study Five: Contrast of Mean Emotion Ratings Before and After Film Clip and Trial Transcript, Collapsing Across Conditions 


\section{Chapter I}

\section{Literature Review}

Historical belief — one shared by fairly well-respected and enlightened thinkers such as Aristotle (325 BC/1998), Sigmund Freud (1920), and Immanuel Kant (1781/1958) - holds that emotion and reason are two mutually exclusive and independently operating components of cognition. This conception is still widely endorsed today among laypersons as well as by many psychologists, psychiatrists, lawyers, legislators, and other cognoscenti who continue to believe in this sort of segregated, bilateral relationship between the emotion-oriented side of cognition and the more conscious, reason-based nature of cognition. Yet other psychologists maintain that while the two domains may seem mutually exclusive on the surface, they are in fact interrelated and interactive components within a larger scheme. This distinction fomented a decades-long debate between respected factions of psychologists with Zajonc (1980, 1984) arguing in favor of the independent, isolationist viewpoint, and Lazarus (1984, 1999) supporting the more interconnected, holistic account. While much of the disagreement can be characterized as an inability to operationalize terminology and concepts in a mutually satisfactory manner, there remains no conclusive empirically verifiable resolution to the debate. Nevertheless, in part because of research placing increased attention on the role of cognition within emotion (Schorr, 2001), a broader agreement acknowledging the permeable boundaries and overall synergy between cognition and emotion has generally prevailed, and it is under this conception that the current research shall proceed. 
The legal system of the United States is based upon a form of common law, which allows for consistency over time and across situations by deference and adherence to precedent. Our laws are designed to be predictable and consistent in their application, which leaves little room for the presumptively volatile, unpredictable emotional component in making determinations of guilt and punishment in the criminal courts, and responsibility, liability, compensation, and damage awards in civil proceedings. The contemporary legal view subscribes to the notion that reason and emotion are wholly distinct entities, and that from a practical policy-oriented, procedural, and legislative perspective, every effort should be undertaken to ensure that the subjective, unpredictable, capricious element of emotion does not invade the purview of stalwart, detached, clinical reason of law. This segregated, compartmentalized viewpoint is acknowledged by Judge Richard A. Posner of the United States Court of Appeals for the Seventh Circuit, who states that "the law itself is conventionally regarded as a bastion of 'reason' conceived of as the antithesis of emotion, as operating to rein in the emotionality of the behavior that gives rise to legal disputes" (Posner, 1999, p. 309). However, in a position not uniformly shared across the field, Posner recognizes the impossibility of creating a bright-line rule for the treatment of emotion in the legal system, an undertaking every bit as overwhelming as establishing a comprehensive method for regulating information or beliefs, and as such, the role of emotion must therefore necessarily depend upon the context of the situation and the content of the particular law in question. There are a litany of exceptions to the more commonly held isolationist rule across many domains of the law_excluding highly prejudicial evidence at trial for fear that it may unduly influence the jury by way of emotional influence (Federal Rule of Evidence 403); 
mens rea requirements in criminal law; the apprehension of fear as a constituent element for the tort of assault; pain, suffering, and mental anguish considerations in damage calculations, and more.

The legal system does not have a specific, broadly applicable policy in place for how to account for the role of emotion - nor should it, given the spectacular range of influences emotion can have on different participants at different times and under the many different circumstances within the legal process as a whole. Pattern jury instructions uniformly advise jurors to set aside their emotions when evaluating evidence and deciding upon a verdict (see Florida Standard Jury Instructions - Civil, Section 700; Florida Standard Jury Instructions - Criminal, Section 3.10), just as evidence can be excluded from admission to the jury on the grounds that it could be so emotionally charged as to unduly influence the jury's interpretation of its weight, and also because it could color perceptions of subsequent unrelated evidence. Conversely, emotion can be tolerated, scrutinized, and even emphasized in other circumstances ranging from calculating damages for emotional distress to victim impact statements during the penalty phase of capital sentencing. These contrasting and conflicting treatments of emotion are not limited to statutory construction and legislated instructions, but are even illustrated by the individual personalities and dogma of our ultimate interpreters of statutory intent, the esteemed members of our Supreme Court. During the first decade of the twentieth century, the Supreme Court included two justices of total polar contrasts in terms of personality, emotionality, and style of reasoning. Oliver Wendell Holmes, Jr. and John Marshall Harland shared the bench of our nation's highest court from 1902 through 1911, and were diametric opposites from one another in nearly every regard - upbringing, 
temperament, decision-making processes, writing style, and more—besides their mutual motivation to interpret the law as fairly and as thoroughly as possible in their own unique manner. Oliver Wendell Holmes, Jr., whose legal mind is most often criticized for being so neutrally dispassionate and coldly detached that some of his opinions seem almost amoral, especially in comparison with those of Justice Harland, whose critics assert that he twisted the law to suit his own morality above all else (Pillsbury, 1999). Despite their many contrasts, both Justices were exceedingly careful and thorough in their two different approaches to the law, and both were driven to arrive at the "right" answers in their own unique ways. Their methods of jurisprudential reasoning and the role which emotion played in their analyses were so disparate as to be complimentary in some ways, in a manner somewhat parallel to the dual-process models of cognition. For instance, Harlan's style was analogous to heuristic or experiential processing, with unequivocal, clearly defined, quick judgments motivated in a manner such that:

Harlan's faith in justice may be the simplest to explain because it comes in the most familiar form: a belief in certain substantive principles of right and wrong. A man of few doubts, his view of justice was shaped by a set of core religious, moral, and political beliefs about the nature of the American ideal. (Pillsbury, 1999, p. 349)

In the alternative, Holmes's style was more similar to systematic or rational processing, with a greater degree of attention paid to nuance, and a methodical, deliberate, careful consideration of information both on its own and within a larger context:

Holmes's faith in justice was both more procedural and more metaphysical than Harlan's. Holmes doubted the existence of clear principles of right or wrong, at 
least in the sense that Harlan believed. Holmes saw justice as a process, a struggle toward ultimate insight sometimes partially glimpsed but never fully gained. Holmes sought particular and often contingent truths, not allencompassing, ever-lasting Truth. (Pillsbury, 1999, p. 349-350)

Despite their differences, both Justices' styles of adjudication were heavily influenced by the role of emotion. Harlan's on a more visible, heart-on-a-shirt-sleeve level, but also Holmes's global, unifying sense of legal philosophizing, both of which illustrate that despite the tendency of the common law, with all of its deference to precedent and adherence to consistent predictability and a widespread insistence to the contrary, our notion of justice - and therefore the entire legal system itself — does in fact incorporate emotion all the way to its very core. However, no matter how inextricably linked emotion may be to the system as a whole, as a policy matter on a procedural level, we must take steps to ensure that jurors are processing evidence in an effortful, systematic, engaged manner, and that they refrain from determining guilt or liability based on their emotions, or that they are in any way unduly influenced by their emotions - an outcome which this research seeks to confirm can and does occur in subtle and unexpected ways.

The idiosyncracies of our foremost legal authorities and the narrow range of tacitly approved exceptions aside, the widespread and traditional "mutually exclusive" perspective does not completely block the intersection of emotion and the law. There is an effort to better understand the intricacies and nuances of their interplay, with contributions coming from multiple perspectives-lawyers (Bandes, 1999; Blumenthal, 2005a, 2005b, 2010), philosophers (Deigh, 1999), economists (Korobkin \& Ulen, 2005), 
cognitive (Forgas, 2010), social (DeWitt, Richardson, \& Warner, 1997; Inbar \& Pizarro, 2009), bio- (Goodenough \& Prehn, 2004; Salerno \& Bottoms, 2009) psychologists, and more are working towards this same interdisciplinary goal, whether through the practice of law, medicine, experimental investigation, or theoretical debate. This variegated, collective inquiry seeks to eventually create a unified, coherent, holistic, and, most importantly, interdisciplinary acknowledgement of the fact that the law does not operate in an emotional vacuum, devoid of any influence of or interference from affective states and their direct impact on cognition, experience, and behavior within the legal realm. This effort is necessary because it is clear that emotions at the time of a decision can influence outcomes independent of the basis of the emotion itself (Loewenstein, Weber, Hsee \& Welch, 2001).

Maroney (2006) set out to help distill the various ways in which the relationship between law and emotion is, can be, and should be studied. Defining both "law" and "emotion" is a thorny task, with different empirical definitions for emotions, feelings, mood, and affect, which are all subtly different depending on whose body of research one happens to be considering. Thus, simply operationalizing the relevant terms to be used can present unique compatibility issues. Nevertheless, Maroney proposed the following six methods and goals:

- Emotion-Centered Approach: Analyze how a particular emotion is, could be, or should be reflected in law

- Emotional Phenomenon Approach: Describe a mechanism by which emotion is experienced, processed, or expressed, and analyze how that emotion-driven phenomenon is, could be, or should be reflected in law 
- Emotion Theory Approach: Adopt a particular theory (or theories) of how the emotions may be approached or understood, and analyze how that theory is, could be, or should be reflected in law

- Legal Doctrine Approach: Analyze how emotion is, could be, or should be reflected in a particular area of legal doctrine or type of legal determination

- Theory of Law Approach: Analyze the theories of emotion embedded or reflected within a particular theoretical approach to the law

- Legal Actor Approach: Examine how a particular legal actor's performance of the assigned legal function is, could be, or should be influenced by emotion (Maroney, 2006, p. 126)

The current paper will address aspects of each of these six approaches. In Chapter 2, it will first examine the historical role of emotion within the legal system and how conceptions have evolved through time, trial, and error (much like our common law approach to precedent). Next, in Chapter 3, I will cover the dual-processing theories which provide the underpinnings of the proposed research. After establishing the theoretical framework, Chapter 3 will also explore how past conceptualizations of emotion, which emphasized the importance of valence, are quaintly archaic, with more contemporary theories concentrating on the subcomponents and building blocks ("appraisals") of emotion. This paper will focus on one appraisal in particular - that of certainty — which has recently emerged through empirical study as a key element in the overall experience of emotion, including its behavioral, cognitive, and decisional consequences. Chapter 3 will conclude with an exposition of the role of emotion in decision-making, culminating with the two specific emotions at the center of my studies, 
anger and fear. Chapter 4 will integrate each of the aforementioned topics, assembling them all into the basis for the proposed research. The characteristics of the emotional appraisal of certainty, including the way it has been demonstrated to supplant past theories that emotional valence determines processing style, as well as how jurors process information and make decisions will all be emphasized. Chapters 5 and 6 will explain the procedure and methodology of Studies One and Two, which will improve upon previous research by Semmler and Brewer (2002) by creating a finely tuned trial transcript for the purpose of investigating the processing style and decision-making of mock jurors. Creating a trial transcript version featuring verifiable detection rates of inconsistencies will provide for a baseline basis of comparison that will allow for a greater degree of precision in qualifying my findings. Chapter 7 will detail the methodologies of Study Three, which is focused on the selection, effectiveness, and implementation of film clips as a means of reliably eliciting emotions among participants. Chapter 8 provides an overview of how Studies One through Three integrate to inform the procedure and utility of Study Four, which will fill the void left by extant research in order to more fully explore the relationship of certainty appraisals of emotion on overall perceptiveness and processing style of mock jurors. Chapter 9 will conclude with a brief summary of potential real-world applications of these findings as well as logical extensions for future research.

There is no shortage of psychological research on emotion or legal decisionmaking, but there is a surprising dearth of research focused on both of them in tandem. More specifically, there is little research that focuses on the influence that emotions might have on legal decision-making. The purpose of the current research is in part to 
confirm past research (Tiedens \& Linton, 2001; Semmler \& Brewer, 2002) that uncertain emotions (like fear) can influence decision-making towards a more systematic style of processing, whereas more certain emotional states (like anger) will lead to a more heuristic style of processing. The current research is also designed to explore how processing style impacts overall comprehension of information and decision-making processes.

\section{Chapter II}

\section{Civil Justice System Overview}

As evidenced by the litany of legal dramas unleashed by American film and TV studios each year, the very nature of crime provides fertile soil for emotional involvement. However, our criminal justice system has a specific body of policy (no matter how inconsistent, unsupported by empirical data, or inelegant it may be to implement) that governs when, how, why, and to what extent various legal actors may acknowledge or incorporate their emotions into the legal process. Some circumstances require a blanket disregard of emotional response, such as jurors' wholesale repudiation of information learned via pretrial media publicity, whereas other determinations such as victim impact statements and other aspects of sentencing procedures hinge upon emotion. However, the civil justice system generally provides fewer opportunities for emotional engagement through news and entertainment media—one need look no further for evidence of this imbalance than to consider how few books, movies, and television programs revolve around civil law in comparison to criminal law. Torts, contractual disputes, liability determinations, damage calculations and the like rarely make for compelling theater fraught with emotional investment in the same way that procedural 
crime dramas can. Just as the entertainment industry focuses its overwhelming emphasis on the criminal justice system to the exclusion of the civil justice system, so too does the body of psychological research with respect to the impact of emotion within the legal system. Nevertheless, despite the comparative banality of much of civil law, there remains a significant role for emotion to play within the process, along with a field of inquiry ripe for study.

Within the common law system of the United States, civil justice is dedicated to issues involving disputes between private (i.e., non-governmental) parties or organizations concerning what amounts to economic interests—essentially, any noncriminal matter between two parties where there exists a potential for economic damages or recovery. Examples of civil cases include contractual disagreements, defamation, personal injury, copyright, and wrongful death claims. Civil cases may involve causes of action that are also subject to criminal charges, which are litigated in a separate and distinct criminal court system. The primary distinctions between criminal and civil cases are that in criminal cases, the defendant, who is presumed innocent until proven guilty, faces charges from the government. The government's burden of proof required for a guilty verdict is that the evidence must support the verdict beyond a reasonable doubt, and if found guilty, the defendant may face sentences ranging from probation to incarceration to a death sentence, in addition to financial penalties. In contrast, a civil case is filed by a non-governmental plaintiff, and the plaintiff must prove beyond a preponderance of the evidence that the defendant is liable for the damages alleged. If the defendant is found guilty, they can be liable for compensatory damages (designed to return the plaintiff to their original state) as well as punitive damages (designed to act as a 
deterrent to similar future behavior by the defendant or other, similar parties), but any prospect of punishment related to one's personal freedom are not applicable to the civil justice system. Because most civil cases are basically a matter of money, the broad doctrines of civil justice tend to have very economics-based, pragmatic views at their core.

\section{Legal decision-making.}

The legal realm tries to characterize human decision-making as a purely rational, calculating process vis-à-vis the rational-choice theory, which has at its core the notion that when making choices, humans always try to maximize their expected utility through rational actions and decisions with respect to anticipated costs and benefits resulting from the choice itself (Montgomery, 2006). While there is no single, unified Rational Choice Theory of human decision-making calculus (see generally Korobkin \& Ulen, 2000), critics assail the clinically sterile economist's perspective for being an incomplete and/or inaccurate summary of human decision-making because we are not always purely rational actors, and we do not always make optimal choices. This is partly because it is sometimes impossible to fully and accurately estimate the potential outcomes in a costbenefit analysis - but mainly because psychological literature is fraught with examples of how we act in counterintuitive, suboptimal, or seemingly inexplicable yet predictable ways. One empirical example of how emotions and appraisal tendencies can affect the very basis of the Rational Choice Theory — the cost/benefit analysis - was conducted by Raghunathan and Pham (1999) wherein participants were experimentally induced into either anxious or sad moods and were then offered a choice of a high risk/high reward job or a job with low risks and low rewards. Those participants who were anxious sought to 
reduce uncertainty by more frequently selecting the low-stakes job because it was a more reliable, dependable option, whereas the sad participants preferred the uncertain, highstakes job because of its possibility for greater mood improvement through the replacement of loss. These same unpredictable, inefficient and/or suboptimal decisionmaking strategies can often manifest within the legal realm or within domains that eventually are at issue in a court of law, and both by parties and by jurors.

Such a distinction in preferences between anxious and sad decision-makers suggests that to some extent, emotional interference on decision-making could influence the course of an investigation if it affects the parties involved. The examples are virtually limitless if we imagine the extent to which emotion could influence legal decisionmakers' processing styles due to reliance on heuristics as opposed to deliberate, logical, rational, careful analysis. Utilizing heuristic processing during trial proceedings could invoke the representativeness heuristic (assessments of eyewitness credibility as illustrated by the "taxicab problem" of Kahneman \& Tversky 1973; Tversky \& Kahneman, 1974), the availability heuristic (decision-makers inappropriately considering personal anecdotal experiences as dispositive of larger overall trends and tendencies; Tversky \& Kahneman, 1974), egocentric and optimistic biases (erroneously evaluating likelihood of harm, risk calculations, reasonableness and forseeability of outcomes as they relate to both oneself and others, which is of particular concern when considering negligence claims; Weinstein, 1980; Ross \& Sicoly, 1979), hindsight bias (believing the known outcome to be more likely than it really was simply based on the fact that it occurred; Kamin \& Rachlinski, 1995; Sanchirico, 2003), outcome bias (distorted evaluation of the quality of a decision given the known outcome; Baron \& Hershey, 
1988), counterfactual thinking (constructing alternate courses of action that could have had different outcomes for the events which led to the trial; Wiener et al. 1994), and other types of cognitive tendencies contrary to the economist's view of human beings as dispassionate, rational actors. Other examples include the use of technically incorrect lay definitions of legal rules when determining verdicts as well as the comparison of case facts to "crime prototypes" which are each juror's idealized, generic expectation of what the crime typically involves (V. L. Smith, 1991). Jurors are also vulnerable to less cognition-oriented heuristics such as placing undue emphasis on the complexity of the testimony given by experts or people perceived to be in positions of authority (Cooper, Bennett, \& Sukel, 1996), the physical attractiveness of the defendant (Lieberman, 2002), the sheer number of plaintiffs (Horowitz \& Bordens, 2000), and the volume of evidence entered (Weinstock \& Flaton, 2004). The more jurors rely on these sorts of heuristics, the less likely they are to be able to reason capably and thoroughly, to understand evidence both singularly and as a part of a larger picture, and to apply their conclusions towards a verdict in accordance with judicial instruction (Greene \& Ellis, 2007).

\section{Chapter III}

\section{Cognition and Emotion}

In order to examine how emotion influences jurors, it is first necessary to consider how cognition is related to emotion. Several prominent cognitive processing models are applicable to the way in which jurors process information. Specifically, the family of dual-process models lends itself particularly well to juror decision-making. There are multiple idiosyncratically distinctive theories based upon the same general framework, but the overall purview of the dual-process models is that decision-making related to 
persuasive messages can take place in one of two ways. Fundamentally, the dual-process models of decision-making posit that persuasion can occur as a result of an effortful, deliberate consideration of information, or from a less involved, approximate, and almost automatic method of processing. While the terminology used to describe the two systems may vary (for instance, E. R. Smith and DeCoster [2000] describe associative versus rulebased processing; Strack and Deutsch [2004] use impulsive versus reflective processing; Lieberman, Gaunt, Gilbert, and Trope [2002] discriminate between reflexive and reflective systems), and each distinct model is distinguished by more than mere changes in nomenclature, their general concepts are much more alike than dissimilar.

One of the most popular of these dual-process models is the elaboration likelihood model (ELM; Petty \& Cacioppo, 1986) which provides that attitude and decision-making is accomplished through one of two primary mechanisms, either the central route or the peripheral route. These two routes each depend on the same factors (the source of the information, the content of the information, the context of the information, and the decision-maker themselves) but depending on the relative strength of these characteristics along a continuum, the decision-maker will be more or less likely to either change or maintain their attitude. The most critical determinant in this process is the likelihood of elaboration, or the probability that the decision-maker will engage in complex, dedicated, meaningful cognition in order to arrive at an attitudinal outcome. The central route of persuasion prototypically involves higher-ordered thinking, careful analysis, logical, effortful evaluation, contemplation, and close scrutiny of multiple sides of the issue (dubbed "elaboration" according to the ELM), whereas the peripheral route of persuasion includes less effortful degrees and forms of cognition, often relying on stereotypes, 
mental shortcuts, and "lazier" styles of lower-level processing. The ELM is based upon the different factors that contribute to the overall likelihood of how much elaboration is expected to occur along a continuum, given the details of the source, content, context, and decision-maker.

Motivation is an important criterion in determining whether the central or peripheral route will be taken and whether an attitude change will result. The recipient of the communication must be motivated to process the information in order to process according to the central route. Without sufficient motivation, such as a high degree of self-relevance for the information, or a high need for cognition (Cacioppo \& Petty, 1982), then there is little chance that the central route to persuasion will be employed rather than the peripheral route. Similarly, if the message recipient lacks the ability to process the information, whether because of high cognitive load, insufficient knowledge, or if the subject matter is simply beyond their innate level of comprehension, then the central route is unlikely to be used above the peripheral route. If the peripheral route of persuasion is activated due to low motivation or low ability to process the information within the message, then the recipient will either engage in some sort of peripheral processing (simple heuristics, cognitive biases, adherence to balance theory [Heider, 1958]) which can result in a mild, often temporary and impermanent form of attitude change, but absent any readily employed heuristic or biases, the attitude will remain unchanged. If the recipient has sufficient motivation and ability to process the information, then the message content itself must also be sufficiently different from preexisting thoughts in order for the central route of persuasion to be used. Otherwise, lacking any informational novelty, the recipient will simply maintain their original 
attitude. The final critical determining factor of likelihood of elaboration under the ELM is whether the processing ultimately prompts some form of change to the recipient's cognitive structure, the chances of which are increased if the recipient is able to rehearse their thoughts or reflect on the issue over time. If all of these conditions (motivation, ability, novelty, and cognitive change) are met, then the attitude in question will change according to the central route, which will be a more enduring, firmly-held belief that is more likely to influence future behavior than a fleeting, peripherally accomplished attitude change.

The ELM is closely associated with another similar dual-process theory of attitude change, the heuristic-systematic processing model (HSM; Chaiken, Liberman, \& Eagly, 1989; see also Petty \& Wegener, 1999). The HSM is substantially similar to the ELM in that they both propose that the processing of information involves two mechanisms that differ in terms of the overall level of motivation and cognitive resources required. The more involved central route of ELM is roughly analogous to the systematic style of processing in HSM, with the less involved peripheral route of ELM being substantially similar to the heuristic processing style of HSM. Both theories are predicated upon the assumption that because of efficiency and attentional overload concerns, and irrespective of individual differences in preferred depth of processing, we generally tend to expend the least amount of effort necessary to arrive at the optimal, or at least a satisfactory, outcome (cf. Allport, 1979; Fiske \& Taylor, 1991; Operario \& Fiske, 1999; Simon, 1956). In addition, the primary determinants of which style of processing a perceiver will engage in are personal motivation or relevance, as well as cognitive ability (Chen \& Chaiken, 1999). The two models do differ in some ways, 
mostly with respect to their treatment of two assumptions. The ELM assumes that accuracy is a critical motivator, and that the relative levels of motivation to process information accurately and to arrive at accurate conclusions is a prime determinant of processing route according to the elaboration continuum (Petty \& Wegener, 1999), whereas the HSM does not postulate that various types of motivations have a direct impact on processing style (Chen \& Chaiken, 1999). The other main distinction between the two theories has to do with how they propose that their two systems operate in relation to each other. The ELM favors a mutually exclusive, either/or approach, wherein the levels of motivation, ability, nature of processing, and changes in cognitive structure each determine in sequence whether central or peripheral processing will occur. The ELM dictates that the threshold in each of these dimensions must be sufficiently exceeded in order for central processing to result, otherwise peripheral processing occurs, and that the two routes of processing cannot operate simultaneously in coordinated cooperation with each other concerning the same information. On the other hand, the HSM places no restrictions on how its two styles of processing can combine to work on the same task simultaneously in parallel as long as motivation and ability (which requires both aptitude and time) thresholds are sufficiently satisfied. These minute distinctions are more important to consider (and merit further exploration) in contexts with other applications than the current research. For the purposes of the experiments at hand, because the differences between exclusive versus simultaneous processing and the role of motivational biases are not fundamentally critical factors, the two models will hereinafter be viewed in rough equivalence. 
These models of cognition have been extensively used to investigate the way that jurors process information. For example, Dewitt et al. (1997) demonstrated that, consistent with predictions according to the ELM, when mock jurors lack the requisite motivation to process scientific evidence in a thorough and effortful manner, they are also less likely to pay close attention to dispositive arguments presented throughout the course of the trial. Similarly, according to the HSM's position that the utilization and degree of reliance upon heuristics is greatest when there is motivation to process systematically but the necessary background familiarity with the subject matter is lacking (Chaiken et al., 1989), Cooper et al. (1996) found that jurors relied upon source credibility heuristics (Hovland \& Weiss, 1951) to make determinations regarding extremely complex testimony beyond their ken, but when complicated testimony was within their realm of understanding, jurors engaged in systematic processing and disregarded source credibility as an indicator of accuracy. Not surprisingly, heuristic versus systematic styles of processing by jurors have been associated with differences in overall evidence recall, level of consideration given to evidence, and confidence in verdict (Honess \& Charman, 2002), and even the very verdict outcome itself (Bourgeois, Horowitz, \& ForsterLee, 1993; Horowitz, Bordens, Victor, Bourgeois, \& ForsterLee, 2001).

\section{Need for cognition.}

In addition to the unique situational variables which can contribute towards the use of a particular processing style according to these two dual-processing models, there are also fundamental, innate individual characteristics that help dictate style of processing. Chief among these is the notion of a stable, internal "need for cognition" (NFC; Cacioppo \& Petty, 1982) which is a single, unifying factor isolated through factor 
analysis of individual difference studies (Cacioppo \& Petty, 1982; Cacioppo, Petty, Kao, \& Rodriguez, 1986; Cacioppo, Petty, \& Morris, 1983) and which can be reliably measured with the Need for Cognition Scale (Cacioppo \& Petty, 1982; Cacioppo, Petty, \& Kao, 1984; in particular, see Cacioppo, Petty, Feinstein, \& Jarvis, 1996 for a detailed overview). This important factor is based upon Cohen, Stotland, and Wolfe's (1955) conception of need for cognition as an expression of the extent to which a person is motivated to reduce tension stemming from cognitive ambiguity. Their original definition was adapted by Cacioppo and Petty (1982) to become a more overarching construct where a person's relative need for cognition was represented as a point along a continuum that described the degree to which a person appreciates, enjoys, and seeks out the opportunity to engage in effortful cognition. Need for cognition should not be misunderstood as a need in the sense of a biological imperative or a fundamental drive, or as an outcome-centered means of goal attainment, but rather as the byproduct of an intrinsic satisfaction with one's own enduring, engaging cognitive efforts, the degree of which in turn determines one's preferred style of processing (Cacioppo, et al., 1996). People high in need for cognition demonstrate an active curiosity which impels them to seek out information and to thoroughly and effortfully analyze it, whereas people low in need for cognition do not derive the same enjoyment from the process of processing, and are instead cognitive misers (Fiske \& Taylor, 1991) who prefer to avoid engaging in the effortful, involved evaluation of new information. Despite their preference to avoid effortful thought, individuals low in need for cognition differ from those high in need for cognition in terms of motivational appetite, not necessarily due to less intellectual ability to process effortfully. Such a distinction is akin to how a person's motivation to engage 
in challenging, effort-intensive physical activities is associated with-but is not dispositive of - the capability of engaging in physical exertion (Cacioppo et al., 1996).

Need for cognition has been the focus of extensive research because of the robustness of the Need for Cognition Scale (NFC; 34-item version by Cacioppo \& Petty, 1982; 18-item short version, Cacioppo et al., 1984) and its core relationship with a wide range of individual characteristics, whether broad factors like age, income, and years of education, or narrowly defined personal traits such as gender role orientation, intrinsic motivation, and social anxiety (see generally Cacioppo et al., 1996). Need for cognition (via the NFC) has also been used as a dependent measure for many empirical examinations of various aspects of effortful information processing such as attitudes, attitude change, cognitive efforts, cognitive responses, recall, and perception of persuasive arguments. Commonly used indicators of NFC (several of which are incorporated into the present research) include the measurement of information recall (as a result of a greater degree of thought and extent of elaboration on relevant information, high NFC individuals will recall more information than low NFC individuals), responsiveness to persuasive messages of varying quality (high NFC individuals are more predisposed to expend the cognitive effort necessary to meaningfully distinguish between high and low quality arguments than are low NFC individuals), responsiveness to peripheral and/or heuristic cues (low NFC individuals tend to exhibit a greater degree of reliance on simple heuristic or peripheral processing cues when evaluating incoming information than are high NFC individuals), and other approaches (Cacioppo et al., 1996). Because motivation to process effortfully is one of the critical determinants of processing style according to both the ELM and HSM, individuals high in NFC who 
derive satisfaction from engaging in effortful processing styles are inherently more likely to process information according to the central or systematic approaches (Petty, Cacioppo, Sedikides, \& Strathman, 1988).

Just as motivation is necessary to process effortfully, so too is the ability to do so. A closely related but distinctly independent factor that interacts with need for cognition is the concept of cognitive load. No matter how motivated a person might be to process information in a thorough, careful manner, if they are distracted, occupied, mentally deficient, or otherwise prevented from putting forth the necessary effort, they will resort to peripheral or heuristic processing by default. Small and Lerner (2008) found that angry participants were less inclined to give further assistance to welfare recipients than were sad participants (who made a greater effort to evaluate the decision fairly), but once the sad participants were placed under additional cognitive load, their decisions were more in line with angry participants. However, when the angry participants were subjected to increased cognitive load demands, their decision-making did not differ from the no-load condition, suggesting that the addition of cognitive load inhibited the degree of effortful processing that sad participants were otherwise prone to engaging in, yet angry participants gave little effort to fully contemplate their decision with or without cognitive load demands.

Differing levels of need for cognition have been shown to influence the way in which real jurors understand, evaluate, and consider evidence proffered by expert witnesses, as well as overall verdict determinations in civil cases (McAuliff \& BullKovera, 2008). McAuliff and Bull-Kovera found that jurors higher in NFC were more cognizant of methodological strengths and weaknesses present in expert testimony than 
were their lower NFC counterparts, a distinction that was reflected in their respective verdicts. Beyond this straightforward example of NFC moderating the relationship between the degree of scrutiny given to evidence and verdict preferences, Shestowsky and Horowitz (2004) found that NFC had a more complex role within deliberating groups of mock jurors. During mock deliberations, high NFC participants were judged to have been more persuasive and more involved in proceedings than low NFC participants, yet their arguments were not rated as any more logically organized or valid. Furthermore, they also found that participants lower in NFC were more attuned to variations in argument strength than the high NFC jurors. This indicates that the way in which NFC relates to understanding, awareness, and consideration of evidence is not necessarily a binary, linear predictive variable. For example, Leippe, Eisenstadt, Rauch, and Seib (2004) found that among mock jurors in a criminal case, when considering a strong case in favor of the prosecution, those individuals with a more moderate level of NFC found the defendant guilty at a greater rate than did both low and high NFC participants, indicating that NFC can be a complex and multivariate dimension across different circumstances, particularly at both extremes of its spectrum. Such variegated findings suggest that despite the robustness of the Need for Cognition Scale itself, and general agreement over the basic principles associated with NFC, it is a dimension which requires continued investigation to more fully understand how it applies across different processing-related conditions and as an important independent trait within existing frameworks of cognitive processing.

In addition to the ELM, HSM, and other dual-process theories which differ in the way they parse the two competing modes of processing and whether (and how) the two 
systems can operate in relation to each other, the cognitive-experiential self-theory (CEST; Epstein, 1994; Epstein \& Pacini, 1999; Pacini \& Epstein, 1999) offers a dualprocess perspective that is more inclusive of the overall characteristics that define the two processes and which does not critically rely on specific, sequential, mutually-exclusive mechanisms. Furthermore, in addition to recognizing the individual differences and situational variables that influence processing style in much the same way as the ELM and HSM, CEST accounts for the role of emotion into the overall predictive model as well. CEST is based on the notion that we create our own "theory of reality" which involves a global viewpoint as well as our own self-image as it relates to our world. CEST draws on notable personality theories (those of Freud, 1920; Rogers, 1959; Adler, 1927; and Allport, 1961; among others) to establish that our primary goal is to lead an emotionally satisfying life, and it is a consequence of this marriage of emotion and cognitive processing that CEST best lends itself to the focus of the current investigation. The greater emphasis on the role of emotion within the unconscious elements of CEST provides for a more irrational unconscious than many contemporary cognitive theories include, but at the same time, the role of emotion in information processing is both adaptive in the sense that it compares incoming information to emotional reactions from prior experiences, while also being motivationally oriented, which allows for a model that is better supported from an evolutionary standpoint than other, more psychoanalyticallybased conceptions of the unconscious.

The less frequently invoked system is a more logic-based, conscious "rational" system predicated upon beliefs. The rational mode of processing "is a deliberative, effortful, abstract system" (Epstein, 1994, p. 715) which operates largely independent of 
emotional influence in an active, evidence-driven, analytical manner, similar to the central route of processing under the ELM and the systematic approach of HSM. Because of its effortful nature, and a focus geared more towards a long-term outlook, the rational system is used sparingly in daily operations compared to its complementary system. However, the two systems within CEST are capable of operating in tandem, simultaneously influencing one another through parallel engagement, unlike the more exclusive and directly competing processes in other dual-process theories.

The more dominant system that controls most of our behavior according to CEST is a quick, automatic, preconscious, affect-oriented "experiential" system where the fundamental constructs are neatly organized schemas (which can be either motivational and goal-oriented or descriptive, which involve broad generalizations about the nature of oneself and the world) that are based upon prior emotional experiences. The experiential system, which roughly corresponds to the ELM's peripheral route and the HSM's heuristic approach, is a gut-level style of processing that at its most elemental corresponds more closely with these two "default" types of processing. At a more advanced level, it is able to involve creativity, intuition, and can even include abstract and/or generalized forms of reasoning (Epstein, 1994), especially when utilized in cooperation with the rational system. Because one's affective state is often directly associated with (and incorporated within) the experiential system, without any deliberate consideration of that fact, the outcomes produced via experiential processing can often be interpreted as more "compelling" or "heart-felt" because they are aligned with one's affective state, as opposed to the relatively emotionally neutral tendencies of rational processing. Also, because this cooperative synergy between affect and experiential 
processing is rarely the focus of deliberate, intentional consideration, the rational system typically neglects to account for or control this influence since it is usually operating silently in the background of cognition.

Since the rational style of processing under CEST is so fundamentally similar to the central or systematic routes of processing according to the ELM and HSM, a modified version of the original Need for Cognition Scale (Cacioppo \& Petty, 1982) is used to identify an individual's preferences for the rational style of processing according to the Rational-Experential Inventory (REI; Epstein, Pacini, Denes-Raj, \& Heier, 1996; Pacini \& Epstein, 1999). However, since the counterpart to the rational mode of processing is rooted in emotion and drawing from past experiences, which is a separate dimension rather than simply a polar opposite along the same dimension, the REI also includes the Faith in Intuition scale (FI). The FI measures the extent to which a person relies on their hunches or gut feelings when making decisions. These two dimensions are independent, which allows for different types of decisions to be made according to either method, a duality best illustrated by what is termed the ratio-bias phenomenon (Epstein \& Pacini, 1999) which is based on the finding that when faced with low probability events, we often judge an odds ratio comprised of smaller numbers to be less likely than an identical ratio on a larger scale (such as the feeling that having 10 chances out of 100 affords a greater likelihood of selection than if one's chances were only 1 out of 10). Rationally, we understand that our odds are absolutely equivalent in both situations, but experientially, when listening to our hunches, we tend to prefer the larger ratio. Pacini and Epstein (1999) demonstrated that according to the REI, individuals more predisposed to rational processing were correspondingly associated with exhibiting greater degrees of 
logical, economically optimal choosing behaviors than individuals less prone to rational processing. This coexisting contrast between knowing one thing and feeling another illustrates how the two systems of processing according to CEST can function independently. Nevertheless, the REI (Pacini \& Epstein, 1999) has been shown to be predictive of several different personality factors.

Krauss, Lieberman and Olson (2004) found a reliable pattern when using CEST as a conceptual framework for juror evaluation of evidence. Inducing rational processing styles among mock jurors in a future dangerousness proceeding prompted those jurors to be more impressed by actuarial testimony, characterized by detailed statistical projections and data analysis, whereas participants induced to process experientially were primarily influenced by clinically oriented expert testimony that did not require the same level of logical consideration of evidence as the actuarial testimony. Lieberman (2002) found that in awarding damages in a civil suit, mock jurors who had been induced to process information in an experiential mode displayed a greater degree of leniency (expressed though lower damages awarded) for attractive defendants who had been found liable in comparison to otherwise equivalent unattractive defendants, but that mock jurors who had been primed to process information in a rational mode displayed no such extra-legal bias.

Information processing models of emotion, which present cognition as a complex and multifaceted operation of independent yet interrelated systems, emerged in the 1940s and 1950s but gained favor in the later $20^{\text {th }}$ century due in part to the broad, comprehensive nature of the theory (addressing a broad spectrum of cognitive elements such as language, vision, attention, recall, judgment, and reasoning) and an open-ended 
scope able to incorporate and accommodate additional, more specialized models and theories. Information processing models involve four primary themes as they relate to cognition and emotion (Dalgleish, 2003). First is the assumption that cognitive processing is a finite resource which must be judiciously allocated, like a computer's RAM. This limited reservoir of cognition therefore requires that we selectively allocate our attention such that the most important incoming stimuli are afforded the necessary amount of devotion. Like a computer's operating system, this automatic prioritization scheme apportions resources according to where they are needed most, in the case of emotions, emphasis is allocated particularly to those stimuli that are unique, unexpected, or changing. Second, the information processing models of emotion require an organizational scheme for cognitive endeavors, akin to a computer's process tree. Despite some past debate, the currently accepted design favors a hierarchy of distinct components, organized according to similar tasks, goals, and processes in order to facilitate quicker performance (see generally Dalgleish, 2003). Information processing models also include provisions to account for parallel processing of information by different systems independently and simultaneously to improve flexibility. Finally, information processing provides for two competing motivations- - top-down, which is generally active, deliberate, and goal-driven; and bottom-up, which is more perceptually based and reactive, a division which has been supported by functional magnetic resonance imaging research (Ochsner, et al., 2009).

One specific information processing theory of emotion is Bower's network theory (Bower, 1981) which is highly dependent on levels of association such that similarly grouped or categorized elements organize themselves as "nodes", which can be 
visualized as different electrons (including stimuli, both automatic and deliberate responses, and any other related or similarly classified concepts and constructs) orbiting around a common emotion node nucleus. Bower provided evidence for the model by demonstrating mood inductions that would lead to state-dependent performance in line with the induced moods in a variety of contexts, including social judgments, perceptual tasks, and recall efforts. However, this model proved to be too simplistic. It relied upon painting with an extremely broad, gestaltish brush to the exclusion of more nuanced, discrete components of emotion.

The overbroad clumsiness of Bower's theory (1981) was improved upon by Forgas's Affect Infusion Model (1994, 1995). It ameliorated Bower's flaws with explicit boundary conditions and ideas on how it can be applied more fluidly. The affect infusion model relies upon affective priming (Forgas \& Bower, 1987) whereupon memories for stimuli are associated with a particular affective state. When encountering new stimuli, these pre-existing pairings facilitate access to familiar experiences and previous reactions, which then shape and guide presently occurring perceptions. Once this emotional priming has taken place, affect infusion is particularly likely in situations that are complex, demanding, and which feature open, constructive, substantive processing (Fiedler \& Stroehm 1986, Forgas 1992, 1993; Forgas \& Bower 1987).

Oatley and Johnson-Laird's theory (1987) is an improvement, providing more specific accounts and greater detail within its framework, which revolved around a hierarchy of processing efforts dedicated to planning for and achieving goals. Emotions are thought to act as a conduit to help determine optimal goal outcomes through partly automatic planning and top-down prioritization of processing efforts. The theory is based 
on five primary emotions (happiness, sadness, fear, anger, and disgust, each oriented in some relation to goal satisfaction) which combine and blend with one another to create other emotions which are also goal-oriented in nature. This combinational characterization of emotional components provided the bedrock for later appraisal-based theories and models which remain today, and which form the basis for the current inquiry.

\section{History of valence and emotion research.}

The most basic, fundamental characteristic associated with the experience of emotion is valence. Some emotions are pleasant to experience, some distinctly unpleasant, while still others are neutral and depend on context, meaning, and concomitant emotions to qualify as having any sort of polar charge. Because overall valence of emotion is the broadest brush with which empirical examination is possible, doing so often only yields similarly broad conclusions, just as it did with respect to Bower's (1981) network theory. Early psychological research examining the influence of emotion on decision-making tended to focus exclusively on valence, and the opinion that valence was the foremost predictor of emotional influence on cognition and decisionmaking persisted until relatively recently (DeSteno, Petty, Wegener, Rucker, 2000; Lerner \& Keltner, 2000). Valence was thought to be the primary determinant in the emotion/decision-making relationship as recently as 1998 when Elster proclaimed that "the only relevant aspect of emotion is their valence" (p. 64) when referring to the role that valence plays in economic "cost-benefit analysis" forms of emotional decisionmaking, and some researchers (Forgas, 2010) continue to contend that valence and broad 
mood states are the most critical aspects of how emotions influence decision-making, even in light of research that suggests otherwise.

Theoretical proposals and empirical investigations began to depart from the overly simplistic valence-based accounts during the mid-1980s through the work of a small group of researchers essentially independently arriving at the same conclusions (Ellsworth, Roseman, \& C. A. Smith; Scherer; \& Frijda; see Schorr, 2001). These seminal efforts were dedicated to the notion that the experience of emotion is a more complex and nuanced affair than the prevailing view that emotion was simply a product of the interaction between one's pleasantness of feeling and one's level of arousal. Broadly speaking, this new movement suggested that emotions were comprised of several substituent, interrelated factors called appraisals. These early models emphasized different types and aspects of appraisals, with Roseman (1984) characterizing emotions according to dimensions of appetitive or aversive motivations, certainty, and agency; Scherer (1984b) proposing factors of novelty (suddenness and familiarity), intrinsic pleasantness, goal significance (concern relevance and outcome probability), agent and motive of cause, and compatibility with standards (both external and internal); and Frijda (1986) suggesting that emotion depends on characteristics related to change, familiarity, valence, focality, certainty, intent (of oneself and others) and value relevance. The most widely-accepted and enduring of the appraisal theories is that of C. A. Smith and Ellsworth (1985), which, building upon the ideas of Roseman (1984) and Scherer (1984a) through experimental investigation, was in response not only to the myopic valence/arousal overemphasis, but also to the then-prevailing notion that "opposite" emotions varied along one single, polar continuum. They also sought to remedy 
methodological blunders and to generally fill the theoretical void of existing emotion research. Through a variety of methods, they ultimately distilled emotion into 6 orthogonal dimensions: pleasantness, anticipated effort, certainty, attentional activity, self versus other responsibility/control and situational control, and mapped 15 core emotions according to each of these underlying appraisal dimensions (see Figures 1, 2, \& 3).

C. A. Smith and Ellsworth (1987) improved the reliability of their 1985 findings which had all been based upon recall, recollection, and/or imagined feelings by instead examining the emotions and appraisals associated with an upcoming event in the near future (students just prior to taking an exam) and compared their reports to subsequent responses upon receiving grades on the exam. Most participants at both times reported at least two emotions blending together to form overall affect. Their findings more or less echoed previous conclusions to confirm that their earlier (1985) assumptions and findings based upon recalled emotion were in fact valid and highly consistent. Although anger was not associated with high degrees of certainty after receiving grades in this study, there exists the possibility that anger was still present yet was subordinate to the feelings of uncertainty about one's future when associated with all of the normal consequences thought to accompany receiving a poor grade. That is, this circumstance was an atypical manifestation of anger, and more typical instances of anger do have appraisals of certainty associated with them (Ask \& Granhag, 2007a; Lerner \& Keltner, 2000; 2001; Lerner \& Tiedens, 2006; Litvak, Lerner, Tiedens, \& Schonk, 2010; Tiedens \& Linton, 2001). 


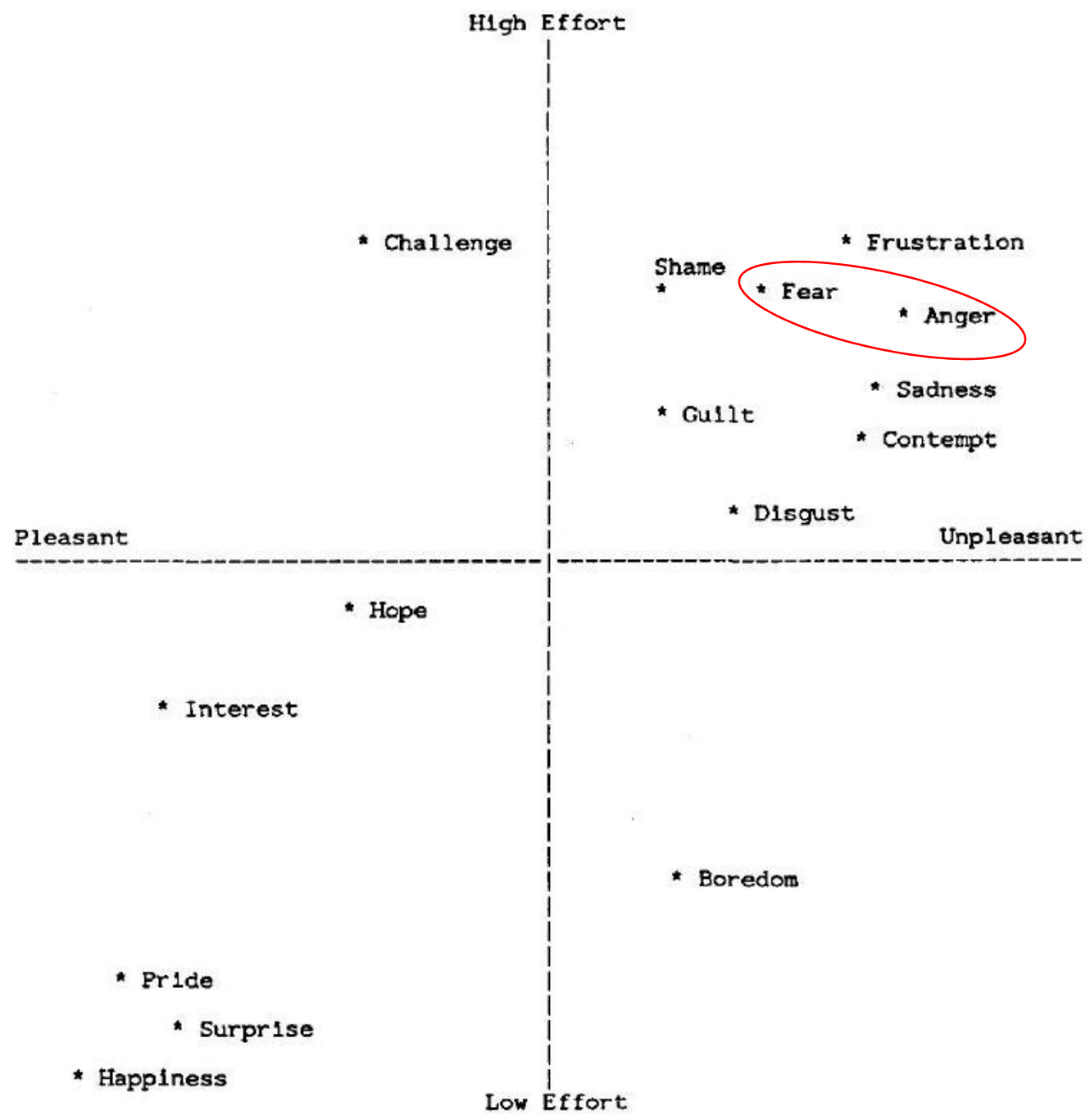

Figure 1. SINDSCAL location estimates for the 15 emotions plotted along the Pleasantness (X-axis) and Anticipated effort (Y-axis) dimensions.'

Figure 1. Smith \& Ellsworth's (1985) Symmetric Individual Differences

Multidimensional Scaling emotional dimension location plots for Pleasantness (X-axis) and Anticipate Effort (Y-axis). Principal components analysis with varimax rotation yielded similar results. 


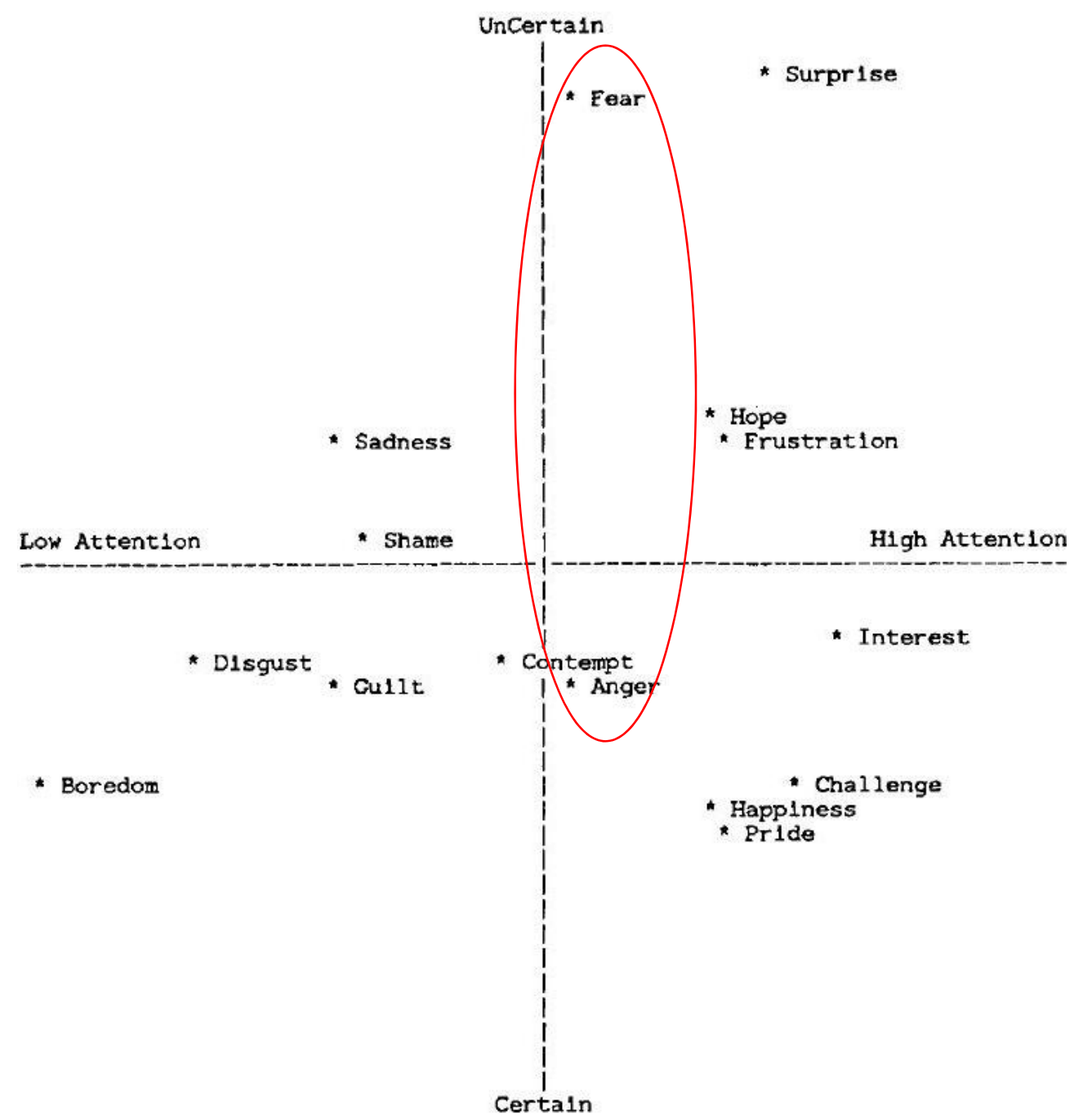

Figure 2. SINDSCAL location estimates for the 15 emotions plotted along the Certainty (X-axis) and Attentional activity (Y-axis) dimensions.

Figure 2. Smith \& Ellsworth's (1985) Symmetric Individual Differences

Multidimensional Scaling emotional dimension location plots for Certainty (X-axis) and Attentional Activity (Y-axis). Principal components analysis with varimax rotation yielded similar results. 


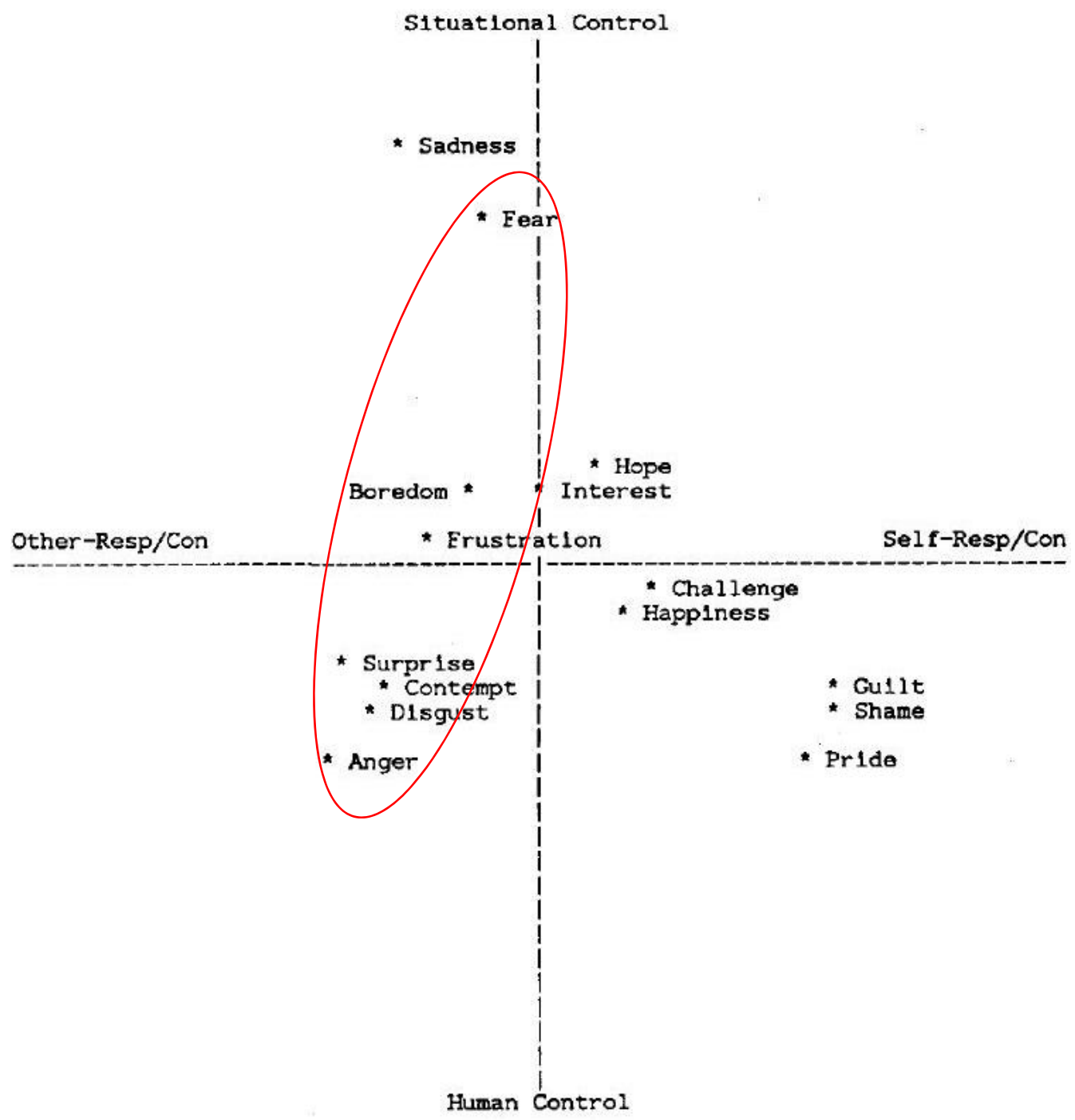

Figure 3. SINDSCAL location estimates for the 15 emotions plotted along the Self/Other-responsibility/ control (X-axis) and Situational control (Y-axis) dimensions.

Figure 3. Smith \& Ellsworth's (1985) Symmetric Individual Differences

Multidimensional Scaling emotional dimension location plots for Self/Other

Responsibility/Control (X-axis) and Situational/Human Control (Y-axis). Principal components analysis with varimax rotation yielded similar results. 


\section{Beyond valence.}

Appraisal-based efforts soon took center stage over valence in emotion research. Valence research itself yielded the basis for its ultimate disfavor, providing several converging illustrations of how different emotions of the same valence can lead to different results across a variety of dimensions — antecedent appraisals (C. A. Smith \& Ellsworth, 1985), estimations of likelihood (DeSteno et al., 2000), facial expressions (Keltner, Ekman, Gonzaga, \& Beer, 2003), autonomic responses (Levenson, Ekman, \& Friesen, 1990), police investigations (Ask \& Granhag, 2007a), tolerance for risk, optimism, self-relevant and irrelevant judgments (Lerner \& Keltner, 2001), as well as central nervous system operations (Panksepp, 1982). Lerner and Keltner (2000) continued to kick the legs out from under prior valence-based models by demonstrating that two negatively valenced emotions (anger and fear) had markedly different influences on judgment—-specifically risk perception. In line with other anger research, angry participants made optimistic assessments of risk whereas sad participants made pessimistic judgments. If mere valence was the most powerful influence of emotion on decision-making, then anger and fear should have resulted in identical (or at least similar) outcomes of decisions. Instead, the two seemingly similar (negative) emotions led to widely divergent consequences.

Lerner and Keltner (2001) provide additional basis for excluding valence as a meaningful variable of interest within decision-making contexts by comparing two emotions (happiness and anger) which are opposed in valence yet share appraisal themes of certainty and control. Using Tversky and Kahneman’s (1981) “Asian disease problem" risk preference framing paradigm, happy and angry participants both reacted to 
risk perception in similar ways, but both were different than fearful participants. Such a divergence indicates that it is more likely that the similar appraisal themes underlying happiness and anger (both being high in certainty and control) guided judgment as opposed to valence - otherwise, angry and fearful participants should have yielded more similar results. In addition to replicating their earlier findings, to address the possibility that this trend might be due to dispositional characteristics rather than experimental manipulations, Lerner and Keltner's next study pre-tested participants for their stable traits of being either angrier, more fearful, or happier by at least 1 standard deviation above the mean than average. Six to eight weeks prior to the decision-making portion of the study, participants were tested and compared according to scales measuring dispositional fear, anger, and happiness, as well as Weinstein's (1980) scale of optimism for the likelihood of various positive and negative future life events. Because past research on emotional priming indicates that priming is most effective when judgment targets are more ambiguous in relation to the primed emotion (Uleman \& Bargh, 1989), Lerner and Keltner proposed that the degree of certainty and controllability of the judged event's appraisal tendencies should moderate the effects of dispositional anger and fear on evaluations of risk when certainty and control-related appraisal tendencies are primed ahead of time. That is, ambiguous target events should be more conducive to appraisal tendencies shaping judgment than target events unequivocally perceived to be certain and controllable or uncertain and uncontrollable. For ambiguous judgments, they found that angry participants and happy participants did not differ significantly from one another, but that they both differed significantly from fearful participants in degree of optimism in risk estimates. For non-ambiguous judgments, both angry participants and fearful 
participants were equivalently pessimistic, and were both significantly less optimistic than happy participants in terms of perception of risk. To summarize, when events were ambiguous in the relevant dimensions, fear and anger had different results on judgment, which supports the appraisal-tendency model and is contrary to a valence-based approach. However, when events were unambiguous, appraisals had less of an influence on judgment and valence was a better predictor for the interaction of emotion and decision-making. Additional examples of how appraisals and attributions are more predictive of processing style include Tiedens (2001), which found that when interpreting the behavior of other people, angry participants made inferences based on scripts and schemas, (a heuristic style of processing), whereas sad participants tended to consider a wide range of possibilities when examining the motives of others, which is a more systematic approach to processing. Also, Bodenhausen, Sheppard, et al., (1994) demonstrated a greater reliance upon stereotyping for angry decision-makers than among sad decision-makers, and that angry decision-makers found peripheral, non-critical source attributes to be more persuasive than message content itself. The present dissertation will similarly look at the emotions of fear and anger, focusing more on the appraisals associated with these emotions rather than the mere valence of emotions.

\section{Appraisal theories.}

Despite coming into vogue with researchers in the late 1990s and early 2000s, the notion of appraisals has been around much longer. The term "appraisal" was first coined by Magda Arnold (1960) to describe the sort of intuitive, instinctual, instantaneous, adaptive evaluations to characterize the singularities of different emotions. She viewed emotion as the resulting experience associated with reactions to environmental changes. 
One of her contemporaries, Richard Lazarus (1966) was an early appraisal theorist whose ideas form the bedrock of most subsequent appraisal-based theories. First, he maintained that emotions have a near boundless range due to the potential for subtle gradations and distinctions in the emotional experience thanks to the unique way in which each human brain perceives its environment. Second, he argued that emotion was a fluid, ongoing event that could be recharacterized and adjusted cyclically through time such that the emotion would evolve and adapt through time and reappraisal. Frijda (1986) echoed this cyclical conceptualization of emotion by suggesting that situations guide emotional responses through appraisals, and in turn, emotional responses guide perception of situations.

Aside from a constellation of assorted variations on the theme, the fundamental underlying principle in all the various appraisal theories is the notion that emotions are a fluid, reactive, transitory/transitional process. Such a process-based account is contrary to earlier, more static, categorical positions that view emotion as a sort of ingrained, automatic response pattern of intertwined cognitive, behavioral, and biological components (Tomkins, 1962, 1963; Ekman, 1972). While different appraisal theories propose different sequences (and each model predicts different sequences for different situations - the inherent flexibility and adaptability of emotion being the very basis of this categorization/characterization of emotion), there is a sort of general sequential framework to which most emotional experiences adhere according to appraisal theories. Typically, the first appraisal is something akin to novelty stemming from a change in the environment at large, whether mental, social, or physical. An evaluation then takes place to determine whether the event is irrelevant (and can thus be ignored) or relevant (which 
requires additional attention). If the event is relevant, the next step in most models is to gauge whether the event is favorable or unfavorable to the perceiver (Zajonc, 1980). This is a largely automatic and nearly instantaneous process. Next, appraisals are formed. The specific appraisals which theoretically unfold depend on which appraisal theory is under consideration, but they generally concern issues of certainty, predictability, the ability to act in response, un/favorability with respect to one's own goals, power, agency, and accordance with social standards. This process continues in a repetitive, cyclical pattern until resolution is achieved or deemed impossible. The first emotional response to a change in the environment can provoke behavior with consequences that directly impact the trajectory or outcome of the situation itself, leading to continued reappraisals. Furthermore, there can be secondary responses to the initial response, thereby further influencing the situation, and when appraisal tendencies have been activated, they will make it more likely for future appraisals to be perceived along those same dimensions, resulting in continued emotional influences on all aspects of a situation. Whether appraisals themselves are precursors or sources of emotion (evaluation of the environment causes resultant corresponding emotion; Zajonc, 1980), or whether they are considered specific, discrete sub-components of emotion (different emotions result from different combinations of constituent appraisals; Scherer, 1984b; Kappas, 2001) is a matter of some debate, but for the most part, critics of appraisal theory tend to adopt the former viewpoint whereas appraisal theorists embrace the latter. The constituent accounting allows for an infinitely variable range of emotional experiences, shaded with nuance and subtle distinctions, which is one of the characteristics that makes the model so appealing and useful to emotion researchers. 
As previously alluded to, counterintuitive findings of seemingly similar emotions having distinctly opposed impacts on decision-making led to the formulation of the Appraisal-Tendency Framework (Lerner \& Keltner, 2000; 2001). The AppraisalTendency Framework (ATF) is founded on several assumptions. First, it assumes that emotion causes changes (whether physical, behavioral, and/or cognitive) which can linger beyond the initial emotion-provoking event and can influence subsequent decisionmaking. Second, it assumes that emotions are associated with specific appraisals which it defines as the underlying messages or meanings taken from the experience of the emotion in response to the emotion-eliciting event. Based upon these assumptions, the ATF asserts that individual emotions lead to the tendency to appraise upcoming developments according to the central appraisal dimensions which prompted the emotion in the first place. This is the appraisal tendency— the process in which "emotion activates a cognitive predisposition to appraise future events in line with the central-appraisal dimensions that triggered the emotion" (Lerner \& Keltner, 2000, p. 477). Emotions shape judgment and decision-making within situations related to the overall appraisal of the event. Emotions prompt specific cognitive, motivational, biological, and behavioral processes, which then in turn control the effect of emotion on judgment and decisionmaking.

\section{Uncertainty and certainty appraisals specifically.}

In keeping with the trend of setting aside valence in favor of constituent appraisals, the current studies will emphasize the specific appraisal dimensions of certainty and uncertainty, and how they in turn influence information processing and decision-making. Certainty, as defined by C. A. Smith and Ellsworth (1987) is "the 
extent to which the person understands or is sure of what is happening or is going to happen in the situation" (p. 475). Beyond relevance and conduciveness/motivational bases, certainty or probability is one dimension that most appraisal theorists account for. Without some measure of probabilistic likelihood of any given outcome, any fully informed emotional or behavioral response is impossible, because after all, we are primarily concerned with the outcome of the event, not necessarily the event itself. For instance, some prospective emotions like hope, anxiety, and fear are characterized by high levels of uncertainty because they are based on the future outcome of an event being unknown or unresolved. In contrast, when dealing with events that have happened or are expected to occur, responses are based upon measures higher in certainty which guide emotions like anger, disgust, and happiness accordingly (Ellsworth \& Scherer, 2003; C. A. Smith \& Ellsworth, 1985; Tiedens \& Linton, 2001). The greater degree of certainty we feel, the less motivation we have to process in an effortful, systematic manner since we are under the impression that we already have a sufficient amount of information to correctly understand and react to the situation (Feigenson \& Park, 2006).

Appraisals of certainty and uncertainty have led to a number of proposed theories to account for the mechanisms which drive the relationship between a given emotion and its cognitive consequences. Bar-Anan, Wilson, and Gilbert (2009) proposed (and confirmed) the "uncertainty intensification hypothesis" which is the notion that concomitant experience of uncertainty during emotional experiences will synergize with the predominant emotional state to intensify the relative pleasantness or unpleasantness of the event itself. Possible reasons for this effect include the notion that uncertainty increases attention and prolongs cognitive accessibility of the emotion-eliciting event 
(Wilson, Centerbar, Kermer, \& Gilbert, 2005) which might lead to intensifying reactions simply through continued consideration. In the alternative, the curiosity associated with uncertainty may increase emotional involvement and engagement moreso than a more certain event, which in turn intensifies the emotional state due to the greater levels of attention devoted to it.

Certainty appraisals are a key component within different dual-process theories of persuasion. For example, the risk-as-feelings hypothesis, (Loewenstein et al., 2001), which is similar in nature to the rational and experiential dimensions at the core of Cognitive-Experiential Self Theory (Epstein, 1994; Epstein \& Pacini 1999; Pacini \& Epstein 1999), emphasizes the role of affect at the exact moment of decision-making because emotional reactions to risk or uncertainty are often different from purely cognitive analysis, and when there is a disconnect between emotional reaction to risk (and/or uncertainty) and cognitive evaluation, emotion often trumps the more purely consequentialist, cognitive, cost-benefit analysis. Also, with respect to the HeuristicSystematic Model of persuasion (Chaiken et al., 1989), the sufficiency threshold hypothesis requires a minimum level of certainty in one's judgment of message accuracy in order to determine that enough cognitive effort has been expended on a task. If the minimum threshold of confidence is not met, additional cognitive effort is required and systematic processing results. If the threshold level of certainty has been attained, then we know that additional effort is not necessary because it will not change our decisionif we are confident and certain in our judgment, further expenditure is just gilding the lily—and heuristic processing will result. 


\section{Emotion induction techniques.}

Previous studies examining the impact of mood on decision-making typically looked at induced moods of either positive or negative valence from one context and their effects on cognition in another context characterized by dual processing models consisting of either heuristic processing or systematic processing (Chaiken et al., 1989; Petty \& Cacioppo, 1986, Schwarz, 1990). These earlier studies typically found that people who had been induced to feel traditionally negatively valenced emotions would be predisposed towards a more systematic style of processing, while those people who were induced to feel positively valenced emotions favored the use of more heuristic styles of processing (Bless, Bohner, Schwarz \& Strack, 1990; Mackie \& Worth, 1989; 1991). These valence-oriented studies proposed different mechanisms of action for their findings including capacity arguments (that a state of cognitive overload results from the fact that positive emotions are intricately intertwined with other conceptual notes; Mackie \& Worth, 1989; 1991) and motivational bases (in order to maintain or achieve positive states by avoiding or remedying negative states, we must utilize systematic thought processes; Bless et al., 1990; Wegener \& Petty, 1994; Wegener, Petty, \& S. M. Smith, 1995). Bless et al. (1996) modified the notion that happiness lessens the motivation to deeply process information. They found that while reliance on general knowledge structures was directly related to the degree of unhappiness (when tested for recognition memory by filling in details of a story about people going out to dinner, happier people substituted generic, script or schema-consistent assumptions more frequently), but that happiness did not lessen cognitive performance in all circumstances. In fact, happy participants even exhibited more accurate recall when performing two tasks 
simultaneously (listening to stories while completing a puzzle task requiring high levels of concentration), though only when the stories contained little or no script-inconsistent information. This suggests that rather than a decrease in motivation to process deeply being associated with positive moods, that a more accurate summary is that there is simply greater reliance on general knowledge structures when happy.

Another account is informational utility, which has two supporting theories, one of which being the "affect-as-information" model which proposes that mood states function as situational indicators and/or to guide our judgment such that negative states are indicative of threats to our goals and therefore careful, deliberate, systematic processing is required. Conversely, the affect-as-information model suggests that positive emotional states indicate safety and comfort with our state in relation to goals and therefore superficial, generalized, heuristic processing is all that is necessary (Bless, 2000; Bless et al., 1996; Schwarz, 1990; Schwarz \& Clore, 1983; 2003). Informational utility also forms the basis of the "mood as input" approach which is the notion that a positive affective state serves as a sort of litmus test to indicate that we have enough information to formulate a decision (Martin, Abend, Sedikides, \& Green, 1997; Martin, Ward, Achee, \& Wyer, 1993). The mood as input conception suggests that the sense of overall certainty that is associated with positive moods leads to heuristic processing instead of systematic processing (Martin et al., 1993) because it is easier for people in positive moods to meet the sufficiency threshold of certainty required for decisions than it is for people in negative moods (Chaiken et al., 1989). Each of these different models of how affect affects cognition are centered solely around the valence of the emotion, which, while obviously among the most fundamental attributes of emotion and a natural 
starting point for investigation, is far too broad of a dimension upon which to base an entire spectrum of decision-making theories. The nature of this mischaracterization was highlighted by the simple observation that two negatively valenced emotions - anger and sadness - each have dramatically different influences on processing style in that sadness facilitates deeper, effortful, systematic processing whereas anger provokes basic, unsophisticated, heuristic processing styles (Bodenhausen, Kramer, \& Süsser, 1994; Lerner, Goldberg, \& Tetlock, 1998; Tiedens, 2001).

The current research is focused on further identifying the relationship between emotional appraisals (specifically that of certainty) and its impact on processing style, which will require that I experimentally induce emotion. Previous studies have successfully induced various emotions through a variety of research designs (for comprehensive reviews and comparative effectiveness of different induction methods, see Lench, Flores, \& Bench, 2011; Martin, 1990; Philippot, 1993; Westermann, Spies, Stahl, \& Hesse, 1996), but due to the desire to maintain maximum consistency across participants, minimize the risk of ineffective inductions due to half-hearted efforts by participants (an issue which can sabotage autobiographical writing exercises as a means of induction), and on the basis of the overall relative reliability that has been established, the current research induced emotional states through the use of film clips (Gross \& Levenson, 1995; Rottenberg, Ray, \& Gross, 2007). Each of the film clips used to experimentally manipulate emotion has been extensively validated and confirmed to be a reliable means of inducing emotion (Gross \& Levenson, 1995; Rottenberg, Ray, \& Gross, 2007), though the effectiveness of each film's emotional elicitation among the current sampling frame was experimentally tested and verified prior to any theoretical reliance 
upon them. Since the focus of this effort revolves around appraisals of certainty, the emotions of fear (characterized by an appraisal of uncertainty) and anger (characterized by an appraisal of certainty) were induced. The anger-inducing clips were a bullying scene from My Bodyguard (1980) and a scene featuring South African police abusing and shooting anti-apartheid protestors from Cry Freedom (1987); the fear-inducing clips were of a boy playing in a deserted hallway from The Shining (1980) and a basement chase scene from The Silence of the Lambs (1991); neutral, non-emotional control clips of a screen-saver featuring abstract shapes and a nature scene from Alaska's Wild Denali (1997) were also used (Gross \& Levenson, 1995; Rottenberg, Ray, \& Gross, 2007; see Appendix A).

\section{Emotions dictating information processing methods and decision-making.}

In order to investigate the impact of certainty appraisals on information processing style and ability, it is important to start by considering the several ways that broader emotions have been demonstrated to influence cognition. First, emotions have been shown to dictate information processing methods. For example, Fiedler (2000) and Forgas $(1998 ; 2000)$ propose that in a broad, valence-based sense, sad/negative moods generally lead to or improve more careful, deliberate, elaborate, analytic, systematic processing. They also maintain that moderately positive moods facilitate creative thinking, forming associations, and inductive reasoning compared to neutral moods. Similarly, others argue that happy moods foster more heuristic thinking and "top-down" processing (Bless et al., 1996, Park \& Banaji, 2000), whereas negative moods lead to more "bottom-up", deliberative processing (Forgas, 2003; Park \& Banaji 2000; Petty, 
Fabrigar \& Wegener, 2003), to the exclusion of heuristics and automatic processing according to the ELM (Petty \& Cacioppo, 1986).

\section{Emotional valence directing judgments.}

Other research has shown that emotional valence can influence judgments directly and in accordance with the emotional orientation, a phenomenon generally known as mood (or affect) congruence effects. Mood congruent effects manifest when ambiguous information is interpreted in a manner consistent with whatever affective state the observer happens to be in (Bower, 1981; Forgas \& Bower, 1987; Petty et al., 2003). An example of mood congruent effects is the practice of cognitive priming, in which emotions seep into judgment and shape perceptions and decision-making accordingly (Keltner, Ellsworth, \& Edwards, 1993; Lerner \& Keltner, 2000).

\section{Emotions suggesting informational context for decision-making.}

The inferential mechanisms at the core of the affect-as-information models (Schwarz \& Clore, 1983; 2003) are another possible explanation for mood congruent effects on decision-making. Because this theory incorporates a sort of internal inventory of one's own emotional state, which is then used to inform the context for decisionmaking, any lingering emotional state we might be experiencing will be interpreted as a reaction to the target of our decision task. Affect-as-information theories for the influence of emotion on decision-making and the appraisal tendency theory are each applicable to both direct and incidental effects of emotions, though affect-as-information tends to rely more on direct effects whereas the appraisal tendency theory is generally more attuned to incidental effects (Feigenson \& Park, 2006). 
The current investigation (and nearly all citations of significant utility within) is focused on the role of incidental affect (that which is not directly relevant to or stemming from the decision task itself) on decision-making, not the more straightforward and obvious effects of integral affect which is a direct result or is directly related to the decision itself. Incidental affect influences decision-making through the reliance upon current feelings as an indicator of information — the affect-as-information model. When affect is presumed to be somehow relevant to the decision itself, it can be heuristically relied upon to help inform the decision. However, according to Lerner and Keltner's cognitive awareness hypothesis (2000), if the feelings are thought to be due to some other outside influence - especially if the decision-maker is made aware of the possibility that their affect may be due to an unrelated, external factor - then there is no heuristic use of feelings as a diagnostic indicator, and they cease to have any impact on decision-making (Keltner, Locke, \& Audrain, 1993; Schwarz, 1990; Schwarz \& Clore, 1983). For this reason, the current experiments ensured that emotional inductions were seen as separate and distinct from the case facts.

There will be no manipulation of valence because of findings that disconfirm the existence of a "cross-valence" effect—anger and sadness do not influence judgments related to circumstances that are positively valenced (Keltner, Ellsworth et al., 1993). Schwarz (1990) says that this is due to the fact that only similarly valenced events (either recalled or predicted) are relevant to the judgment tasks at hand. Because the subject matter at issue in civil trials is typically unpleasant or neutrally valenced at best, there is no need to examine the effects of valence in this circumstance. 


\section{How specific emotions fit in with processing and decision-making models.}

Depending on the individual characteristics of the trial, emotions that jurors are potentially likely to experience run the gamut of the emotional spectrum, including all 15 discrete emotional states isolated by C. A. Smith and Ellsworth (sadness, shame, fear, surprise, hope, frustration, boredom, disgust, guilt, contempt, anger, interest, happiness, challenge, and pride; 1985, 1987). Because anger and fear are highly similar to one another according to most of C. A. Smith and Ellsworth's categorical dimensions of appraisal (with the notable exception of certainty, which is addressed in the current dissertation), and because they are each directly relevant to the sorts of emotions that real-life jurors report feeling (Antonio, 2006; Landau, 2011), inducing these two specific emotions will permit us to directly investigate the role of certainty appraisals on processing style and decision-making.

C. A. Smith and Ellsworth (1985) determined that anger is best characterized as being unpleasant, involving a high degree of anticipated effort, a sense of human agency involvement as well as other-responsibility/control, and a fair amount of certainty which was associated with no clear approach or avoidance dimension. They found that the prototypical anger-inducing experience revolves around someone else doing something perceived as unfair which has negative repercussions for the individual perceiver across a broad range of circumstances. In comparison, they determined that fear is best characterized as an unpleasant condition associated with high degrees of effort, a sense of situational control as well as other-responsibility/control, and extremely high degrees of uncertainty concerning whether it will be possible to avoid or escape a highly unpleasant development for the perceiver, and that, like anger, the uncertainty was also not 
associated with any particular approach or avoidance characterization. The prototypical fearful experiences involved a wide range of scenarios, ranging from facing the possibility of arrest for possession of drugs at a rock concert, to discovering a prowler near one's home, being robbed, being chased by a vicious dog, getting lost in the wilderness, and losing control of a car or bicycle during challenging conditions. The situational versus human control dimension, on which anger was originally loaded towards situational control appraisals (compared to fear, which was loaded more towards human control appraisals), was later discovered to be less replicable across different contexts (C. A. Smith \& Ellsworth, 1987). For the current purposes, it is assumed to be essentially equivalent among jury decision-makers.

The manner in which these two distinct emotions can affect processing has been studied across multiple tasks and research paradigms, including in both dispositional and induced emotional states. In social judgment contexts as well as guilt determinations, a greater reliance upon stereotypes (a form of heuristic processing; Bodenhausen \& Wyer, 1985), heuristics, and source credibility cues over message content were utilized by angry decision-makers (Bodenhausen, Sheppard, et al., 1994; Tiedens \& Linton, 2001). Fewer informational cues were used by angry participants when determining liability in a personal injury case (along with exhibiting a greater degree of punitiveness; Lerner et al., 1998), and the rate and veridicality of detecting inconsistencies in trial testimony among angry participants (Semmler \& Brewer, 2002), was poorer than less angry participants. This final characteristic of angry jurors is a dimension which the current series of studies will investigate by improving upon and extending Semmler and Brewer's research. 
Stable, trait-based measures of dispositional anger have been shown to be negatively related to assessments of perceived risk, whereas dispositional fear has been found to be positively related to risk judgments (Lerner \& Keltner, 2000; 2001). Similarly, participants induced to feel anger consistently estimated their degree of personal risk and likelihood of suffering personal harm in a more optimistic and risktolerant manner than did participants induced to feel sad or fearful (Fischhoff, Gonzalez, Lerner, \& Small, 2005; Lerner, Gonzalez, Small, \& Fischhoff, 2003; Lerner \& Keltner, 2001). The finding that two emotions of similar valence have diametrically opposed influences on cognition and decision making serves as a perfect reference to further illustrate the possible mechanisms (Tiedens \& Linton, 2001) that drive the AppraisalTendency Framework (ATF; Lerner \& Keltner, 2000; 2001). To view these examples of the ATF through the lens of Bower's (1981) associative network theory and Forgas's (1995) Affect Infusion Model, we would conclude that fearful appraisals activate a network of response featuring low levels of coping potential and certainty, but that angry appraisals would activate a network comprised of responses involving high levels of coping ability and certainty.

The affect-as-information account (Schwarz, 1990; Schwarz \& Clore, 1983; 2003), while initially constructed to incorporate the role of valence at informing processing style and decision-making, can simply be adjusted to focus beyond the polarity of an emotion. Instead, we can expand the scope of the model to consider the types of appraisals that are activated in response to the situation, which in turn help determine how best to govern cognition. For example, instead of positively valenced emotions signaling that all is well and that nothing requires intense attention, this same 
outcome can be achieved through appraisals high in certainty. Similarly, rather than negative emotions indicating the potential for threat or unpleasantness, appraisals low in certainty communicate the message that effortful processing may be required.

The motivation-oriented hedonic contingency account (Wegener \& Petty, 1994; Wegener et al., 1995) of processing states that we will only engage in systematic processing if it is likely to result in a desirable outcome-if we are in a negative mood, we will not process systematically if we expect that doing so will worsen our mood, nor will we do so if in a positive mood and we expect it will lessen our mood. Conversely, it holds that we process systematically if we expect it will improve a negative mood or maintain a positive mood. Like the information-based accounts, the motivational basis is similarly updated by simply substituting appraisal tendencies where valence was once incorporated, and acknowledging that the mechanism proceeds according to a much more fine-tuned design taking into account the precise nature of appraisals in comparison to the broader, general character of valence. By interpreting emotional appraisals, we can compare the themes to our innate motivational drives in order to determine how to best process information and/or react to it. For instance, when we are in an uncertain mood state, the motivation to reduce uncertainty (Eagly \& Chaiken, 1993) prompts us to consider altering our circumstance in some way, which requires more careful processing than does simply maintaining the status quo, so when people are experiencing appraisals related to uncertainty, then they will likely process information in a more careful, deliberate manner. Besides influencing our style of processing, appraisal tendencies can also dictate how we respond to information, as illustrated by the increased risk tolerance in gambling decision-making tasks which sadness promotes (motivated by the interest of 
improving mood), compared to the increased risk aversion which appraisals of anxiety provoke, motivated by the interest of reducing uncertainty (Raghunathan \& Pham, 1999). In the current research, I will demonstrate the contrasting ways in which appraisals of certainty (via anger induction) and uncertainty (via fear induction) differentially dictate the way mock jurors process information. I will also explore how appraisals of certainty and uncertainty shape the way mock jurors make decisions, as well as how information processing style itself directly influences decision-making. I will show that the appraisal tendencies associated with certainty and uncertainty will guide processing and outcome effects, which they facilitate in accordance with both informational and motivational mechanisms.

\section{Chapter IV}

\section{Emotions in Legal Decision-Making}

Investigators (and other decision-makers, including jurors) are social hypothesis testers who generally practice hypothesis-consistent testing, which amounts to seeking consistent, confirmatory evidence over that which suggests that perhaps a different hypothesis is more accurate (Trope \& Liberman, 1996). In fact, when presented with ambiguous evidence, we often tend to frame it within a context complimentary to our current hypothesis, and that in order for ambiguous information to be considered in a truly objective manner, we require high motivation, ample cognitive resources, and to have already preconstructed alternative, viable hypotheses in addition to the primary hypothesis (Trope \& Lieberman, 1996). To test this relationship, Ask and Granhag (2007b) manipulated the motivation levels and cognitive ability of police investigators through varying time pressure constraints when evaluating new evidence in a homicide 
case. They found that investigators without any sort of time pressure were more sensitive to new information and were flexible in terms of altering their preconceptions of the case theory, while those investigators under time pressure did not take into account any of the new information about the case and did not change their assessment of the case. Ask and Granhag (2007b) also found that a reduction in processing capacity increases the degree to which preconceptions and assumptions are acceded to, and inversely, the degree to which subsequent evidence is carefully evaluated among police investigators. The demonstration that an external factor which increases cognitive load can dictate processing styles and overall consideration of evidence among experienced police investigators suggests that similar factors could direct the processing style of jurors at trial, who are less familiar with the practice of evaluating evidence than seasoned police.

Even during the very earliest stages of an investigation, emotions can shape cognition to such an extent that heuristic processing and cognitive biases could influence whether a case proceeds to trial. Building off Keltner, Ellsworth et al., (1993) who found that emotions can influence attributional tendencies to emphasize either personal (when angry) or situational (when sad) responsibility for events, Ask and Granhag (2007a) also incorporated research on attribution tendencies and their influence on cognition (Lerner $\&$ Keltner, 2000) to consider how criminal investigators may change the way in which they judge reliability of witness statements depending on attributional tendencies of their emotional state. They demonstrated that police investigators induced to feel sad tended to consider a wider variety of evidence (characteristics associated with both the witnessing conditions as well as the credibility of the witness themselves) and were more aware of inconsistencies within witness statements than investigators induced to feel 
angry, who tended to only consider factors associated with the credibility of the witness and who did not detect witness inconsistencies to the same degree. The sad investigators were better able to consider the consistency of witness statements with respect to the main theory of the case investigation, which is a more effortful form of processing, compared to the angry investigators who did not attend to the consistency between statements and their own hypotheses, indicating a more heuristic form of processing.

Ask and Granhag (2007a) suggested that judging reliability was akin to causal attribution, and that similar to the earlier findings of Keltner, Ellsworth et al., (1993), angry investigators would be predisposed to ascribe greater significance to personal information whereas the sad investigators would place more credence upon the diagnosticity of situational variables. The wider range of evidence types considered and the greater degree of awareness of inconsistencies among the sad investigators indicates systematic processing, whereas the angry investigators' reliance on less evidence of a more simple nature is representative of heuristic processing. Practical implications of Ask and Granhag (2007a) are easily adapted from police investigators to jurors. Just as the emotional state of investigators was shown to influence the degree of open-minded, careful, deliberate consideration a police officer gave to new evidence, so might the emotional state of a juror influence the evaluation of evidence in the same manner. Those decision-makers (whether investigator or juror) who only utilize superficial, heuristic processing due to their emotional state and its accompanying appraisals might rely too much on their preconceptions and initial judgments to the exclusion of subsequent informative evidence. Similarly, an increased reliance on stereotypeconsistent cues by angry decision-makers (Bodenhausen, Sheppard, et al., 1994) can also 
bias the way in which new information is processed and evaluated. These findings can also be troubling within the context of the confirmation bias (Nickerson, 1998) such that when police have a suspect early in the investigation, or when jurors decide their verdict prior to hearing both parties present all of their available evidence, the elevated certainty appraisals associated with anger can increase the threshold which the decision-maker requires in order to change their mind because the superficial processing of information leaves the full scope of the evidence ignored. Furthermore, all subsequent ambiguous information may also be perceived according to the preexisting expectations and conclusions.

\section{Certainty and uncertainty appraisals within the legal context.}

Uncertainty has been shown to lead to systematic thinking across a variety of contexts. For instance, Weary and Jacobson (1997) demonstrated a trait-dependent correlation among people who report being in a persistent and pervasive state of uncertainness in that they are more inclined to process information in a systematic manner than people who are chronically certain. Other studies focusing on the depressed and the pathologically dysphoric arrived at similar findings of increased levels of systematic processing compared to non-depressed populations (Edwards \& Weary, 1993; Hartlage, Alloy, Vazquez, \& Dykman, 1993; Yost \& Weary, 1996). Non-clinical illustrations of the relationship between certainty and processing elsewhere in psychology include the motivational drive of reducing dissonance stemming from personal uncertainty through the use of social comparison strategies (Festinger, 1954), and that these social comparisons involve even greater degrees of systematic processing when a person is uncertain regarding their own opinions (Pelham \& Wachsmuth, 1995). These 
diverse effects prompted Tiedens and Linton (2001) to investigate whether the certainty and uncertainty appraisals associated with specific emotions in one context could influence the style of processing used in a subsequent and unrelated information processing domain. Building off of Lerner and Keltner's (2000, 2001) AppraisalTendency Framework, they predicted that appraisals of certainty within emotions were more critical predictors of processing effects than emotional valence. Specifically, they asserted that uncertainty leads to systematic, effortful processing (the outcome or ultimate impact of the emotion eliciting event is still unresolved, therefore it is adaptively beneficial to carefully consider the situation) whereas certainty leads to more heuristic, script-based processing (the outcome and impact are seen as fixed and determined, therefore it is fruitless to devote any more cognitive effort to the issue than is strictly necessary). Tiedens and Linton proffered that emotions associated with high levels of certainty (such as happiness and anger) ought to dictate the way people feel in later situations as well as the type of subsequent processing a person will be likely to engage in.

To test their theory of certainty appraisals dictating information processing, Tiedens and Linton (2001) conducted a series of four experiments. In the first study, in order to determine whether certainty appraisals of emotions foster appraisal-congruent judgments as predicted by both the network models (Bower, 1981; Forgas, 1995) and the affect-as-information theories (Schwarz, 1990; Schwarz \& Clore, 1983), they induced participants to feel either positive or negative emotions that were either certain (positive - happy, negative — disgust) or uncertain (positive — hopeful, negative—fear) through autobiographical written narratives. Participants were asked to make predictions 
concerning eight different general knowledge issues (sports records, legislation, tuition increases, and the like) for a year into the future and to indicate the level of their certainty for each of their responses. They expected that there would be certainty appraisal congruency such that those participants induced with certain moods would give higher certainty ratings than those in uncertain moods. They found that in the induced certainty conditions, participants were more certain of their subsequent, unrelated predictions, building on Clore and Parrott's (1994) findings that emotionally induced certainty influences judgment, and like Keltner, Ellsworth et al. (1993) and Lerner and Keltner (2000, 2001) found, that congruency effects are possible in a broad range of emotional ranges and contexts. This provided them with the basis for their larger hypothesis that certainty can influence depth of processing.

Tiedens and Linton's (2001) second experiment on the influence of certainty appraisals focused on depth of processing to further inform the valence versus appraisal tendency debate. It built on existing dual process theories concerning the relationship between positive moods and heuristic processing versus negative moods and systematic processing (Bless et al., 1990; Mackie \& Worth, 1991; Schwarz et al., 1991), and also adapted Bodenhausen, Sheppard, et al. (1994) which established that both happy and angry emotion-induced participants were similarly influenced by source credibility heuristics, but that sad participants were not. This was the beginning of the end for the valence-based accounts, and Tiedens and Linton's model suggested that certainty appraisals are the deciding factor in terms of whether systematic or heuristic processing will result. 
To plumb the issue of depth of processing, they induced both positively and negatively valenced emotions that were either high in certainty (positive - contentment, negative — anger) or low in certainty (positive — surprise, negative—worry), and then presented participants with a persuasive essay concerning the issue of grade inflation. Half of the participants were told that the essay was written by a well-regarded professor (a high credibility source), while the other half—who read the exact same essay—were told that the author was a student from a local community college (a low credibility source). Following the reading of the essay, participants were asked to rate how much they agreed with the sentiments expressed in it. If certainty appraisals are the driving force behind selection of processing style rather than emotional valence, then the certain (content or angry) participants should be more influenced by the source credibility information than by the message itself, expressed by greater credibility ratings for the essay purportedly written by a professor than the essay believed to be written by a community college student. Their expectations were founded upon the fact that deference to source credibility cues above message content is a common exemplar of heuristic processing (Eagly \& Chaiken, 1993). On the other hand, if uncertain (surprised or worried) participants processed the information contained in the essay more systematically, they would provide more equivalent ratings of persuasiveness for the essays regardless of authorship. Predictably, they found that certainty and source had a main effect on persuasion but that valence did not, and Certainty X Source was the only significant two-way interaction. Certain participants saw a large difference in persuasiveness according to source credibility, whereas uncertain participants saw no difference because they were likely attending to the quality of the message rather than the 
status of the author. Their findings further support the appraisal tendency account of information processing above the purely valence-based theories.

Dual process theories of attitude change (Chaiken et al., 1989) apply across a wide range of contexts, so after considering the appraisal versus valence debate vis a vis persuasion and persuasiveness-related heuristics, they next investigated how the likelihood of relying upon stereotypes (Bodenhausen, 1993; Bodenhausen, Kramer, et al., 1994) to make inferences about another person's attributes (Bodenhausen \& Wyer, 1985; Chaiken et al., 1989) might inform the debate. There is widespread agreement across studies that positive moods facilitate heuristic processing while negative moods promote systematic processing, but again, this narrow view that valence is the key is too simplistic for something as complex as the use of stereotyping and prejudice, which involve a whole host of different negative emotions (and each in different ways) in their promulgation (E. R. Smith, 1993; Bodenhausen, Kramer, et al., 1994). To refine this issue, Tiedens and Linton (2001) considered two negatively valenced emotions differing in certainty — fear, which is high in uncertainty, and disgust, which is high in certainty. To further support the appraisal account and reject the valence model, emotional certainty would have to impact degree of stereotyping such that participants in negative certain moods (disgust) would process heuristically as shown through a greater tendency to activate and rely on stereotypes than participants in negative uncertain moods (fear). Instead of using the autobiographical writing emotion induction technique of Experiments 1 and 2 (and Bodenhausen, 1993), Tiedens and Linton (2001) used reliably pre-tested film clips (Gross \& Levenson, 1995; Rosenberg \& Ekman, 1994) to induce fear (The Shining) or disgust (a scene from the movie Maria's Lovers featuring a rat 
crawling into a man's mouth), and also measured participant certainty about the emotioneliciting event itself (the film clips) and subsequent unrelated tasks. The appraisal questionnaire had only one certainty-related question ("How certain are you about what will happen next in the film?") to prevent participant suspicion, but also asked participants to rate pleasantness of the film and how much attention they had paid to it out of concern that perhaps the content or emotional tenor of the two clips might unequally affect attention levels, which in turn could have influenced the degree to which they processed information in upcoming tasks.

The stereotyping task was presented as a second study concerning attitudes toward educational policies regarding ethical and legal infractions, and participants were told that they would later be given the opportunity to adjudicate a case to help determine whether outcomes were consistent with student perceptions of fairness. Prior to reading the case, participants were asked to indicate their certainty that they would be able to decide the resolution of the case in the optimal manner. After the pre-decisional certainty measure, participants read a case involving a student who had been accused of cheating by a professor. The professor alleged that the student added to his answers on an exam after it had been graded and returned, while the student maintained that they did not alter their answer, and that that the teacher's assistant who graded the exams must have overlooked that part of their answer and had incorrectly marked it wrong. In the stereotype-salient condition, the student was described as "a well-known athlete on the basketball team". Past research has uncovered a stereotype among students that studentathletes are more likely to engage in cheating or academic dishonesty than non-athlete students (Bodenhausen, Kramer, et al., 1994). In the other condition, no such description 
was included and the case materials were otherwise identical. Following the case, participants had to give their opinion on a 1-11 scale of how likely it was that the student was lying, that the disputed portion of the test had been added after grading, and how dishonest the student was. They also had to indicate what they thought would be an appropriate punishment, if any, and were asked to rate (1-11) their enjoyment, anger, disgust, and fear while watching the film clip earlier.

They found that their disgust and fear manipulations both held, there was no difference in anger ratings across conditions, there was no difference in attention ratings, and that the disgust emotional induction resulted in greater degrees of certainty than fear. They made sure to check for any differences in valence because if it emerged that the disgust and fear manipulations varied at all in terms of overall valence, then any results might have been due to valence and not certainty distinctions. There was no difference in pleasantness between fear and disgust clips, but there was a difference in enjoyment with the fear clip being rated as more enjoyable than the disgust clip. This finding was unexpected, though it provided for an additional element of diagnosticity through a direct comparison between the certainty account and the valence account. Their contention that certainty drives processing would predict more stereotyping/heuristic processing among the certain (disgusted) participants than the uncertain (fearful) participants, but the opposing valence-based approach would predict that the more enjoyable experience should result in greater use of stereotyping. Tiedens and Linton (2001) expected to find an interaction between emotion and athlete-ness wherein the disgust-induced (certain) participants would be more influenced by the inclusion of athlete status than those participants in the fear induction (uncertain) condition, which was exactly what they 
found. The most basic outcomes of Experiment 3 were replicating the findings of Experiment 2-that increased certainty leads to more heuristic, general knowledge-based processing than does uncertainty, and extending the certainty-appraisal-congruency findings to the domain stereotype activation. More importantly though, Experiment 3 also uncovered more of the mediation at work - that appraisals of certainty carry over to into other situations and powers the mechanism of mood effect from one domain to another, unrelated subsequent situation, and that certainty-appraisal congruence mediates processing style.

Tiedens and Linton (2001) leveled criticisms at the existing body of valenceoriented work that those studies did not control for the possibility that extraneous differences besides valence (i.e., certainty) might have been driving the findings. Nevertheless, their own Experiments 1-3 each also suffered from this uncomfortable weakness - for example, fear and disgust differ in more underlying appraisal characteristics than simply their associated degrees of certainty. To address this issue, Experiment 4 was designed to look at a single emotion under conditions of certainty versus that same emotion under conditions of uncertainty—rather than two distinct and potentially confounding emotions. They decided that rather than looking at two distinct emotions on opposite ends of the spectrum of certainty like they had done previously, that instead a single emotion from the middle portion of the certainty spectrum with a wide range of potential to vary towards both extremes would allow for the elimination of any potential confounds stemming from the use of completely separate and distinct emotions. They elected to examine sadness because it is considered to be more uncertain than happiness or neutral non-emotion, but considerably less uncertain than fear, a more 
typically uncertain emotion. C. A. Smith and Ellsworth (1985) plotted sadness as being just slightly more uncertain than certain, but still quite close to the middle of the axis (see Figure 2). They later (Ellsworth \& C. A. Smith, 1988) claimed that this central location of sadness is an indication that certainty is not typically an important component of sadness, and that the interplay between sadness and certainty appraisals is fluid and dynamic, with uncertain sad states being every bit as common as certain sad states. Tiedens and Linton asserted that in conditions of certainty-oriented sadness, there would be a greater likelihood of heuristic processing than in conditions of more uncertaintyoriented sadness which would promote more systematic processing.

Experiment 4 featured three sad emotion induction conditions varying according to certainty appraisals (certain, uncertain, and no certainty component), and a fourth, nonemotional, non-certainty inducing control condition. They used a persuasion paradigm featuring either a strong or weak argument, which discriminates between systematic processors (who are able to distinguish convincing messages from unpersuasive ones) and heuristic processors (who will not process thoroughly enough to notice any differential levels of persuasiveness). Tiedens and Linton expected to find that the sad/no certainty participants' processing styles should be nearly identical to the sad/uncertainty participants as far as being systematic because both are low on certainty, whereas the sad/certainty appraisal participants should be more heuristic in their style of processing. Experiment 4 had three objectives: to keep emotion constant while manipulating only certainty, to further explore sadness since it has been widely used in earlier research on emotions and processing, and to incorporate more meaningful measures of processing styles than had been used in the past. 
They induced emotion the using the same sort of autobiographical experience writing task from Experiments 1 and 2 (writing about an experience when they felt sad and certain vs. sad and uncertain vs. sad vs. describing minutia from the previous day's events as the control condition). The "second study" persuasive message manipulation was presented as market research for advertising a camcorder. The weak argument and strong argument conditions both included some of the same material (that it was a good product, superior to competitors' models, and one worth purchasing) but differed in terms of supplemental information. The weak argument condition emphasized information unrelated to the overall quality of the camera (the available colors for the accompanying tote bag, a lens cleaning rag, and a long extension cord) while the strong argument materials covered more meaningful characteristics directly related to the quality of the camera (color and picture quality, battery life).

They found further evidence to bolster results from Experiments 2 and 3 where emotions higher in certainty prompt less substantive processing than uncertain emotions. They improved upon those results in that very little other than degree of certainty was different between conditions - all participants felt similar sadness, and experimental conditions only varied with respect to certainty. The sad/certain participants were less able to discriminate between varied levels of argument strength than were the sad/uncertain or just the sad. Also, because the sad and the sad/uncertain conditions had such similar results, it affirms Tiedens and Linton's position that most studies which induce sadness to examine processing effects are actually inducing an emotional state that is high in uncertainty. The neutral "control" condition, while varying considerably from the three sad conditions, still allowed for meaningful conclusions. The neutral condition 
prompted less systematic processing than the sad condition, and the sad/uncertain condition also differed from the neutral condition in most respects. The sad/certain was most similar to the neutral condition, which raised the question of just how important emotion is in this situation if the sad and certain participants were the most indistinguishable from the neutral, control participants.

Overall, Tiedens and Linton (2001) established the importance of appraisals with respect to the content (through emotionally congruent judgments) and the processing style (by determining the depth of processing) for decision-making efforts. From a directly applied standpoint, it demonstrated that angry people bear a greater degree of certainty than sad or fearful people, which leads those highly certain processors to a more pronounced reliance on heuristic processing — automatically assessing higher credibility ratings for opinions proffered by "experts", having less facility at distinguishing strong from weak arguments, and overall attending more towards superficial cues than to argument quality when evaluating persuasive messages. From a more theoretical perspective, it buttresses the general finding of past research (Johnson \& Tversky, 1983; Keltner, Ellsworth, et al., 1993; Lerner et al., 1998; Lerner \& Keltner, 2000; 2001) that established the wide-ranging prevalence of emotional congruent judgments in later, unrelated tasks. More importantly, it also broadened the appraisal congruency field by confirming the extent to which certainty appraisals can influence depth of processing, effects which were mediated by certainty appraisals such that when certainty was examined independent of emotion, it was a causal determinant of the type of processing that would be used. Ultimately, it showed that greater degrees of emotional certainty—as opposed to only emotional valence - are directly related to heuristic processing, (which, 
in turn, includes being more persuaded by heuristic cues), while feelings of less certainty are associated with systematic processing and finding persuasive messages with a more rational, logical basis to be most compelling.

\section{Types of inconsistencies that jurors may face.}

As Tiedens and Linton (2001) demonstrated, appraisals of certainty can not only influence processing style, but also responses to persuasion. Jurors can be exposed to a virtually limitless array of potential evidence, bounded only by the scope of what can be at issue in a legal matter. However, research has shown that one of the most powerfully persuasive decision-making cues which jurors are faced with in their role as triers of fact is that of inconsistent testimony (Berman \& Cutler, 1996; Berman, Narby, \& Cutler, 1995; Brewer \& Hupfeld, 2004; Potter \& Brewer, 1999), though the consistency/accuracy, accuracy/credibility, and consistency/verdict relationships are themselves somewhat varied and inconsistent (Brewer \& Burke, 2002; Brewer, Potter, Fisher, Bond, \& Luszcz, 1999). For example, mock jurors found that inconsistent statements made by a 6-year old rendered that witness as seeming less credible than when the same inconsistent statements were proffered by a 10- or 30-year old witness (Leippe \& Romanczyk, 1989).

In fact, at their very most basic, all trials boil down to the issue of inconsistency in one form or another, and it is up to triers of fact to decide which account is more likely (see generally Pennington \& Hastie, 1986; 1988; \& 1992 for how jurors assemble facts and reconcile conflicting evidence and arguments). One party claims one thing, and the opposing party claims the opposite, whether the issue at odds is a contractual dispute between multinational corporations or a grisly murder. But on a narrower scale, 
inconsistent testimony can take several forms, whether through internally inconsistent statements where one witness contradicts themselves (and the conflicting information can be offered exclusively within their testimony, during pretrial statements, or between pretrial statements and testimony), or through two witnesses offering directly competing and mutually exclusive testimony. Inconsistency within testimony can also vary according to whether it concerns details that are central to the matter at hand or peripheral details which do not directly inform matters.

In order for inconsistent testimony to influence juror decision-making, it must necessarily be perceived, and in order for it to be perceived, jurors must be processing information in an effortful manner-centrally, systematically, or rationally, and not peripherally, heuristically, or experientially (according to the ELM, Petty \& Cacioppo, 1986; HSM, Chaiken et al., 1989; Petty \& Wegener, 1999; or CEST, Epstein, 1994; Epstein \& Pacini 1999; Pacini \& Epstein 1999, respectively). Past research has shown that processing style can impact mock jurors' perception of evidentiary persuasiveness such that given the same case facts, rational processors find one type of evidence (actuarial testimony) more persuasive than do experiential processors, and that experiential processors find an alternative type of evidence (clinical testimony) more persuasive than rational processors in judgments of a defendant's potential for future dangerousness in capital cases (Krauss, et al., 2004). On top of that, other research has shown that emotional appraisals of certainty can dictate information processing style (Ask \& Granhag, 2007a; Tiedens \& Linton, 2001), so it follows that emotional appraisals of certainty can influence information processing and therefore detection of inconsistencies, which should in turn guide decision-making tendencies of jurors. 
To test this hypothesis (one which the current research intends to revisit and bolster in multiple critical respects), Semmler and Brewer (2002) manipulated mock jurors' moods (neutral vs. sad) and the testimonial inconsistency of the trial transcript (consistent vs. inconsistent) with the expectation that sad jurors would process information in a more effortful manner, which could be demonstrated via a greater degree of inconsistency detection in comparison to jurors in a neutral emotional state. However, they did not find that sad participants detected inconsistencies at a statistically significant rate greater than emotionally neutral participants. Sad jurors reported inconsistencies more accurately than did neutral mood participants, though they found no main effect of mood or the Mood x Consistency interaction they expected. These null results were thought to be due to relatively low rates of inconsistency detection in all conditions. The inconsistency detection data trended toward significance, so I am optimistic that through several improvements in methodology, the expected results will emerge. Although they did not intentionally experimentally manipulate anger, they did discover that among those participants reporting a greater sense of anger, overall rate of inconsistency detection was impaired. This suggested to them that some element of anger elicits less effortful processing.

Semmler and Brewer did not incorporate the appraisal tendency framework (Lerner \& Keltner, 2000; 2001; see also Lerner \& Tiedens, 2006; and Han, Lerner, \& Keltner, 2007 for more advanced accounts of emotional appraisals influencing processing style and decision-making), which, in combination with several methodological shortcomings, compromised the overall conclusiveness of their findings. The current series of studies incorporates the appraisal tendency framework as an important 
determinant of processing style and decision-making among mock jurors, and also systematically improves upon Semmler and Brewer's various methodological weaknesses (particularly through more rigorous development and pretesting of materials; see Methods, below) in order to better establish the role of emotion, emotional appraisals of certainty (which have been effectively induced, manipulated and measured via online research in the past; see Wiener, et al., 2007), processing style, and decision-making among mock jurors. I expected to find that emotional appraisals of certainty would dictate the style of processing used by mock jurors, which would determine their degree and accuracy of detecting testimonial inconsistencies, which in turn will have influenced a number of other dimensions such as verdict, certainty, and confidence.

\section{Chapter V}

\section{Study One: Identifying Detection Rates of Individual Inconsistencies}

\section{Method}

\section{Theoretical Basis}

In order to ensure the greatest degree of reliability and validity for the materials and measures to be used in this study, as well as to improve on methodological weaknesses within existing research, several steps are necessary in order to fully reconsider Semmler and Brewer's (2002) efforts.

Because the main focus of this series of four studies is to examine the manner in which emotion influences the processing and awareness of trial evidence, I first needed to confirm that participants were noticing the various testimonial inconsistencies when reading and listening to the transcripts. If participants were unable to detect any inconsistencies (i.e., a floor effect) or they were able to detect all inconsistencies (i.e., a 
ceiling effect), it would be difficult to ascertain whether certain versus uncertain emotions lead participants to find fewer or more inconsistencies, respectively.

The inconsistencies used in prior research (Semmler \& Brewer, 2002) on the role of emotion on processing style of mock jurors involved the use of two types of inconsistencies, those that could best be described as either inferential inconsistencies (requiring some element of consideration and deduction on the part of the participant, such as an eyewitness claiming to have seen something that they later describe as having been hidden by a blind curve) or non-inferential inconsistencies (which merely required recognition and comparison of discrepant information, such as referring to the business logo on a truck as belonging to two different oil companies when describing the same truck during separate lines of questioning). For the purposes of the current efforts, I dispensed with the logical inconsistencies and instead focused exclusively on factual inconsistencies. Simplifying inconsistencies in this way will yield two main benefits. First and foremost, because the general hypothesis being tested has yet to bear statistically significant results, it seemed prudent for nascent investigations to revolve around the detection of cognitively simple, straightforward inconsistencies rather than truly tricky, multiply nuanced, complex combinations of deductive reasoning. Second, doing so will provide for more definitive analysis rather than collapsing multiple, distinct dependent variables (detection rates of both inferential and non-inferential inconsistencies, which may not necessarily be interchangeable) into a single rate of detection.

In addition to any cognitive distinctions which may exist between the processes required to identify simple versus complex inconsistencies, the materials used by 
Semmler and Brewer (2002) also included a wide range of conspicuousness, which may also have contributed to some unexpectedly low effect sizes in their study. For example, in terms of ceiling effects, one logical inconsistency involved the witness testifying about events that preceded a car accident he was involved in as a passenger. The witness initially stated that his wife, who was driving, had time to flash their headlights to warn an oncoming truck to avoid the accident. Then, when answering a follow up question, he testified that there was no time for her to react to the unfolding accident. In the text below, note how much time his wife Celia had to react to the oncoming truck (emphasis added in italics):

Prosecution: Did the truck appear to swerve or deviate from it's course at any time prior to impact, Mr White?

Witness: Yes, it did. I remember that Celia had time to flash the lights a couple of times... [emphasis added] it swerved to our left.

Prosecution: At what speed were you travelling?

Witness: I reckon it was only about $40 \mathrm{~km} / \mathrm{h}$. We had slowed down to take the corner.

Prosecution: At what speed in your estimation was the truck travelling ? Witness: It must have been over $60 \mathrm{~km} / \mathrm{h}$. We had slowed down, it came towards us very quickly. Celia didn't have time to do anything. [emphasis added] Prosecution: Did the truck appear to slow down or brake at any point? Witness: No it didn't brake at all. As I said it happened very quickly. There was this petrol truck, or Ampol truck and the trailer across the road coming straight for us and.... It was very quick. There was no way Celia could have avoided it. 
(Semmler \& Brewer, 2002, stimulus material obtained via personal communication, N. Brewer, July 2, 2010; see Appendix B.)

These directly conflicting accounts - that Celia had time to flash the lights a couple of times versus the statement indicating that she did not have time to reactrequire some consideration of context and comparison of information to inductively detect the inconsistency, but the fact that the two contrary evidentiary "units" are so proximate to one another may have mitigated the complexity of the logical reasoning necessary for detection, so that all participants detected the discrepancy. Another logical inconsistency used by Semmler and Brewer (2002) occurred when the prosecuting attorney questioned the victim, who described his view of an oncoming truck as occurring after he rounded a blind corner. After several question and answer exchanges as well as procedural interjections from the judge spanning approximately a page and a half of printed testimony, as well as cross examination by the attorney for the defense, the same victim/witness made the contradictory statement that he had seen the truck 15 meters before rounding the bend (emphasis added in italics):

Prosecution: When did you first notice the truck?

Witness: When we rounded the left hand bend just before where the accident happened. You couldn't see around the corner because at that point the road is actually bending [emphasis added] around the steep hill on the left.

[...approximately 1.5 pages of additional testimony...] 
Judge: Thank you Mr Spence. Mr Warwick, you may now cross-examine the witness.

Defence: Thank you Your Honour. Good morning Mr White. My name is Melvin Warwick and I am the attorney for the defence in this case. I have some questions to ask you about the accident which took place on Mount Barker Road in which you were involved. The justice system demands that the jury have a clear picture of these events, so please answer the questions as accurately as possible. \{Pause\} At what point did you think you saw the truck on the wrong side of the road? Witness: I'd say at least 15 meters before we rounded the bend. [emphasis added] Before the accident scene, I could see that it was on the wrong side of the road. I could see the paint of the Mobil symbol and the chrome trailer shining through the trees. (Semmler \& Brewer, 2002, stimulus material obtained via personal communication, N. Brewer, July 2, 2010; see Appendix B.)

The distant relationship between conflicting units of information may also have been rendered even more imperceptible for participants by the vagueness of language used in the two ostensibly conflicting statements, creating floor effects where none of the participants detected the inconsistency.

The varying range of conspicuousness and "ease of detection" is not limited only to the characteristics of the logical inconsistencies, but also may have complicated participants' ability to detect less cognitively demanding factual inconsistencies. For example, when the victim mentioned two separate oil companies names when describing the truck that was involved in the accident (Mobil and Ampol), this inconsistency depends on issues of brand awareness, market saturation, logo recognizability, and other 
idiosyncratic factors dependent upon the participants' familiarity with the petroleum industry in their home country of Australia. Compare this to detecting a conflicting statement by the victim regarding whether the truck was halfway or 3/4ths of the way across the middle of the road prior to the accident, which depends upon a different set of cognitive skills such as mental visualization abilities and spatial orientation acuity.

To include such a wide variety of inconsistency types is not necessarily a weakness in design, but to assume that all inconsistencies are equally likely to be detected by participants and then to analyze them en masse, particularly in the absence of any efforts to pre-test for baseline detection rates without any emotional components, seems an ineffective and incomplete basis for making any real conclusions. Because each distinct inconsistency must, by definition, involve discrete factors, information, and bases of comparisons, and because we cannot assume that every individual perceives, processes, and integrates information identically when identifying an inconsistency, it is impossible to truly distinguish and analyze every sort of inconsistency according to its constituent characteristics since this can also vary depending on the traits of each person. These differences - those specific to the ease of detecting a given inconsistency, as well as those between individual participants — will persist whether there is only one style of inconsistency to detect or multiple subclasses of different inductive/deductive reasoning techniques required, which is why at this preliminary stage, the more streamlined and cognitively similar I can make the inconsistencies, the less likely it is that multiple effects may overshadow or obscure one another.

To be clear, Semmler and Brewer's theoretical basis, expectations, and hypotheses as they relate to the impact of emotion on processing were sound - the 
execution simply left room for several confounding factors which could have been the basis for the inconclusive nature of their findings. The current investigations attempt to improve upon these various faults by first obtaining baseline detection rates for inconsistencies, which will in turn allow me to use only those inconsistencies known to be devoid of floor or ceiling effects in order to later test my main hypothesis concerning the effect of high or low certainty appraisals on cognitive processing style and the detection of inconsistent information.

\section{Participants}

One hundred sixty-one undergraduate students at Florida International University were recruited through Sona Systems, the Psychology Department Research Management system to participate in a web-based study on "Juror Decision-Making in Civil Trials" in exchange for course credit. Because this was an online study, several safeguards were used to exclude those participants who did not give the study their undivided attention (see Appendix $\mathrm{C}$ for detailed criteria and procedures undertaken for data reduction purposes), which resulted in a total of 100 participants. The sample was predominantly female (76\%; 24\% male), Hispanic (70\%; 20\% White/Caucasian; 6\% African American; $3 \%$ Native American; $1 \%$ Asian), and ranged in age from 18 to 60 years old $(M=22.41$, $S D=5.12$ ). Participants' highest level of education was well-balanced, with $26 \%$ in their freshman year, $24 \%$ in their sophomore year, $25 \%$ in their junior year, $22 \%$ in their senior year, and 3\% having completed some professional or graduate-level education. Over half the sample (54\%) was not currently employed, with $34 \%$ holding part-time employment and $12 \%$ working full-time, and $7 \%$ had served on a jury before (5\% civil; $2 \%$ criminal). 


\section{Design}

Because my objective for Study One was to simply identify which (if any) of the 15 inconsistencies from my trial transcript were detected by no more than $66 \%$ of participants and no less than $33 \%$ of participants, there was no experimental manipulation or treatment group, and therefore there was no control group and random assignment was not necessary. Study One was a one-group post-test only non-experimental design in which the overall rates of detection for each of the 15 testimonial inconsistencies were evaluated independently and in comparison to each other. Study One is essentially a pretest to validate that the inconsistencies to be included in the later experimental versions of the trial transcript are within the optimal "Goldilocks zone" of detectability (those that fall between ceiling and floor effects - that is, those that are "just right").

\section{Materials}

\section{Trial transcript.}

Since the varying degree of obviousness may have been one of the several factors contributing to Semmler and Brewer's (2002) unexpectedly sparse significant results, my research materials will involve an abbreviated transcript based on a real Florida personal injury case (Hibbard et al. v. McGraw et al., 918 So. 2d 967; 2005 Fla. App. LEXIS 18878; 30 Fla. L. Weekly D 2714). The real case involves an automobile accident with many similarities to the trial transcript used by Semmler and Brewer (see Appendix B). By merging and incorporating Semmler and Brewer's fact patterns and four noninferential inconsistencies with the facts from Hibbard v. McGraw, the resulting trial transcript will allow me to more accurately determine the strength and overall baseline detection rates of their inconsistencies. However, my transcript also includes 11 new 
inconsistencies created for the current research (see Appendix D for trial transcript and Appendix E and Table E1 for descriptions of inconsistencies and relevant testimony) in order to have an ample number of inconsistencies to select from for inclusion in the transcript to be used in Studies Two and Four. The current study thus aims to find inconsistencies that are as isolated from both floor and ceiling effects as possible.

\section{Measures to determine rate of detection for each inconsistency.}

After their participants listened to the trial transcripts, Semmler and Brewer (2002) showed the participants several examples of the types of testimonial inconsistencies that can occur in a trial prior to having them answer questions about the content of the testimony. By providing such examples, Semmler and Brewer may have potentially indicated to participants that there had been inconsistencies in the testimony which they might not have noticed otherwise. Since the detection of inconsistencies is the basis for the primary dependent measures, any potential interference with participants' recognition or awareness between exposure to the inconsistencies and measurement could distort the actual degree of detection reflected by the measures. To avoid tipping my hand or telegraphing my research objective at the outset, I expected to more definitively determine the overall degree of detection/ease of detectability for each inconsistency by first asking very broad, open-ended questions and then funneling down to more specific, narrowly-tailored, forced-choice questions (see Appendix F for the exact questions and format).

By starting off with eight open ended questions (e.g., "Were there any eyewitnesses that testified who you think might have made mistakes in terms of describing the exact nature of events related to the accident?"), I intended to elicit 
responses which would indicate what inconsistencies participants had noticed without phrasing the questions in an unduly suggestive manner. This free response style of question presented at the outset is in direct contrast to the more "point blank" questions presented at the end of the measures. These point blank questions directly asked participants about each inconsistency while still maintaining some degree of experimental control and avoiding the influence of demand characteristics. My reasoning for "funneling" the questions from broad to narrow in this manner (starting with broad, openended questions, then short answer, then multiple choice, then true/false, then the virtually point-blank questions) is that if participants were to accurately report inconsistencies with little direct questioning right from the outset of the questionnaire, it is more diagnostic of an inconsistency detection than correctly responding to a true/false or point blank question toward the end of the questionnaire.

\section{Procedure}

Participants logged on to their secure FIU Sona Systems account to receive the link to the study itself, securely hosted online at Qualtrics.com, FIU's online survey resource. After giving their consent to participate (see Appendix G for Study One Informed Consent) in a study purportedly to assist a local attorney evaluate the merits of appealing the outcome of a trial, participants were asked to listen to an audio file embedded on the website to ensure that their computer would be able to play the audio of the trial transcript. If their computer could not play the audio, participants were told to $\log$ off and access the site through a different computer, and not to proceed further until they were able to access the audio file. After verifying that their computer was properly equipped, participants were reminded that they must complete the study all at one time, 
and that they should read along with the upcoming onscreen testimony as the audio played simultaneously, which allowed participants to encode information both visually and auditorily. This should be another improvement over the prior research by Semmler and Brewer (2002), which only presented the trial transcript auditorily. Participants were required to click on a button to proceed to each subsequent page in order to keep them engaged, and the "proceed" button did not activate on any page until that page's audio concluded. Furthermore, total time spent on each page was recorded in order to later identify and exclude any participants who did not click to proceed in a reasonable length of time and who therefore likely did not give their full, undivided attention to the materials (see Appendix C for Data Reduction Techniques).

After participants finished reading along with and listening to the transcript, they were asked to rate the credibility of each witness on a seven point Likert scale ranging from 1 (Completely Lacks Credibility) to 7 (Completely Credible) and then on the next page, to rate the persuasiveness of each witness on a seven point Likert scale ranging from 1 (Not Persuasive At All) to 7 (Very Persuasive) (see Appendix H). Following the witness credibility and persuasiveness ratings, participants answered the battery of broad (i.e., open-ended) to narrow (i.e., point blank) inconsistency detection measurement questions (see Appendix F), provided demographic information (see Appendix I), and were then debriefed (see Appendix J), thanked, and instructed to log off and close their web browser.

\section{Subjective Scoring}

To avoid any potential for research bias, all responses to the open ended and short answer question types were each coded by two research assistants blind to the hypothesis 
or intent of the study (see Appendix K, Open Ended Question Coding Instructions and Appendix L, Short Answer Question Coding Instructions). Both research assistants were furnished with a copy of the trial transcript (see Appendix D) and the description of all 15 inconsistencies (see Appendix E) and instructed to familiarize themselves with the details of all testimony, particularly the information related to the inconsistencies. Next, I met with the two of them together to explain the procedures and coding criteria and to answer any questions they might have had, and I provided them with a practice file comprised of responses given by participants who did not complete the full study and were eliminated from consideration in the final data set as well as responses that I composed myself in order to illustrate the types of distinctions and decisions I expected they would have to make. After the research assistants completed the practice data set individually, we met again to discuss the reasoning for how we coded each answer. At that point, the research assistants were given the real data set and instructed to code it individually, and to save it as a separate file. After they completed individual coding, they met to discuss their coding, each having the opportunity to discuss any uncertainties or ambiguities with the other. They were instructed to discuss all discrepancies in coding with each other to the extent that they both were comfortable with the final interpretation they recorded, regardless of whether they were in full agreement. They were told to listen to the other arguments and to come to an agreement if possible, but if not, that they should not hesitate to stand by their own decisions. My goal was not to have two identical scoring files after their meeting, but rather to have two scoring files that they each stood behind. Afterwards, using a separate rubric/decision criteria for the short answer questions (see Appendix L), they followed this same multi-step process of meeting with me, scoring 
practice data individually, meeting with me to discuss the practice data, individual scoring of the real data, discussion with each other, and submitting their pre- and postdiscussion scorings to me.

\section{Results}

\section{Inter-rater Reliability for Subjectively Scored Questions}

To confirm that the research assistants were each coding the data according to the same rules of interpretation, and because I knew the distribution to be non-normally distributed (see Appendix M), I conducted a Kendall's tau-b test to measure the correlation coefficient between the two scorers. Among the open ended questions, two inconsistencies were not reported by any participants by either scorer, so for the remaining 13 inconsistencies, the average correlation of their "Yes" codings before discussion was very high $\left(\tau_{B}=.86, p<.001\right)$, as was the average correlation of their "Yes" codings following discussion $\left(\tau_{B}=.96, p<.001\right)$. Similarly, for the short answer questions, with one inconsistency not being reported by any participants by either scorer, the average correlation of their "Yes" codings for the remaining 14 inconsistencies before discussion was very high $\left(\tau_{B}=.77, p<.001\right)$, as was the average correlation of their "Yes" codings following discussion $\left(\tau_{B}=.89, p<.001\right)$.

\section{Comparing Overall Detection Rates of Each Inconsistency}

The benefits of tapering from broad styles of questions to very narrow, direct questions for the inconsistency detection rate measures outweigh the drawbacks of relying on a single type of measure, but those benefits come at the expense of having to make multiple comparisons of multiple inconsistencies using multiple measures, which required the construction of several scoring schemes to most thoroughly interpret the data 
and identify the optimal testimonial inconsistencies for later use (see Appendix $\mathrm{M}$ for scale construction background).

Upon evaluation of all 15 inconsistencies using both the Strict and the Loose scoring schemes, eight inconsistencies were detected at overall rates within the range of detectability that I sought, while seven of them did not fall within my target range according to either scoring scheme. The Loose criterion identified eight inconsistencies with detection rates between $33 \%$ and $66 \%$, while the Strict criterion identified five inconsistencies with detection rates between $33 \%$ and $66 \%$, though the three that were not identified were very close (PI 10-color: 32.4\%, PI 11-Jared: 32.4\%, and PI 9-Year: $30.8 \%$ ) and were retained because of their comfortable margin within the Loose criterion (PI 10-color: 38.0\%, PI 11-Jared: 38.4\%, and PI 9-Year: 38.4\%). In fact, the overall mean detection percentage rate for the eight inconsistencies I will retain was appreciably greater using the Strict scoring scheme $(M=36.58 \%)$ than the mean detection percentage rate using the Loose scoring scheme $(M=25.39 \%)$ for the seven inconsistencies I will eliminate from the trial transcript (see Table 1 for the overall detection rates for all 15 inconsistencies according to both scoring schemes, and Tables M1 and M2 for detection rate percentages of all 15 inconsistencies across all question types and treatments in each scoring scheme).

\section{Discussion}

In addition to identifying those inconsistencies suitable for continued use, the results from Study One illustrate that it is very difficult to create questions (or even question types) that are uniformly diagnostic for all inconsistencies. Tables M1 and M2 show that some questions or types of questions elicited greater rates of detection than 
others, and that the detection rates for each individual inconsistency does not necessarily follow the same stable, linear trajectory predicted (and intended) by the "funneling down from broad to narrow" array of questions. In fact, some rates of detection decreased through the course of questioning for some inconsistencies, which provides additional support for the methodological justification of including an array of question types to establish a mean detection rate for each inconsistency rather than simply relying on the validity of a single type of measure as Semmler and Brewer did in their 2002 study.

The eight inconsistencies found by anywhere from $31 \%$ to $52 \%$ of participants will be retained for use in the remainder of the present research. While results from Study One are promising in terms of the solidly middling rates of detection for those eight inconsistencies, to be maximally confident in their utility for Study Four, it is critical that I verify that they remain detectable at roughly the same rate in the absence of the seven inconsistencies I will be removing from the trial transcript. It is possible that a cumulative, combinative effect was driving the overall rate of detectability, and Study Two will help to determine whether that is the case.

\section{Chapter VI}

\section{Study Two: Verifying Trial Transcript Stimulus Materials}

\section{Method}

\section{Theoretical Basis}

To ensure that the eight testimonial inconsistencies identified in Study One retain the same approximate detection rates in the absence of the seven eliminated testimonial inconsistencies (see Appendix $\mathrm{N}$ for the version of Johnson v. McGraw featuring only the eight inconsistencies), I undertook essentially the same procedure in Study Two, with the 
primary difference being the number of inconsistencies in the trial transcript. However, retesting the inconsistency detection rates also afforded me the opportunity to control for another one of the confounds which potentially tainted Semmler and Brewer's (2002) results - the emotional influence of the trial transcripts themselves. All four versions of their trial transcripts (Inconsistent Testimony/Neutral Emotion, Inconsistent Testimony/Sad Emotion, Consistent Testimony/Neutral Emotion, and Consistent Testimony/Sad Emotion, see Appendix B) were found to increase both sadness and anger among their participants, which could explain their lack of significant effects. The fact that even their ostensibly emotionally neutral fact patterns had some emotional impact could very easily have blurred any distinctions between their manipulated conditions and control groups. When creating the Johnson v. McGraw trial transcript stimulus materials, my intention was to create a realistic presentation of a civil trial for maximum ecological validity, but also to create a trial transcript that would be unlikely to arouse any emotional response (in contrast to Semmler and Brewer's materials, some of which featured a widower's testimony recounting how he and his young son watched their wife and mother slowly die over the course of 20 minutes inside the mangled wreckage of their automobile which also held the family's young daughter trapped in the backseat). To determine whether my trial transcript elicited any emotional response, I measured participants' emotional state before and after listening to and reading along with the trial transcript (see Appendix O for I-PANAS-SF, Thompson, 2007 and PANAS-X, Watson \& Clark, 1994). 


\section{Participants}

Sixty-four undergraduate students at Florida International University were recruited through Sona Systems, the Psychology Department Research Management system to participate in a web-based study on "Juror Decision-Making in Civil Trials" in exchange for course credit. Because this was an online study, several safeguards were used to exclude those participants who did not give the study their undivided attention (see Appendix $\mathrm{P}$ for detailed criteria and procedures undertaken for data reduction purposes), which resulted in a total of 27 participants. The sample was predominantly female $(70 \% ; 30 \%$ male), Hispanic $(67 \% ; 11 \%$ African American; 7\% White/Caucasian; 4\% Asian, and $11 \%$ Other), and the 25 who input their age ranged in age from 19 to 44 years old $(M=23.96, S D=5.16)$. Participants were predominantly upperclassmen, with $41 \%$ in their senior year, $22 \%$ in their junior year, $15 \%$ in their sophomore year, $15 \%$ in their freshman year, and 7\% having completed some professional or graduate-level education. Over half the sample (52\%) was not currently employed, with 33\% holding part-time employment and 15\% working full-time, and one participant (4\%) having served on a civil jury.

\section{Design}

Just as in Study One, my objective for Study Two was to evaluate the suitability of stimulus materials for use in later studies (specifically, to replicate roughly equivalent rates of detection for the remaining eight inconsistencies in the absence of the seven redacted inconsistencies from the Study One trial transcript, and to determine whether the trial transcript induced any emotions), so absent any experimental manipulation, random assignment and the inclusion of a control group was not necessary. Study Two featured 
only one group of participants, but because of its dual purposes, it can be characterized from two perspectives. In terms of the detection rate confirmation aspect of Study Two, it is best described as a one-group post-test only non-experimental design, while the emotional impact aspect is best described as a one-group pre-test/post-test design. In essence, Study Two serves as the final verification and validation of the trial transcript stimulus materials.

\section{Materials}

\section{Trial transcript.}

Study Two used a modified version of the Johnson v. McGraw fictional trial transcript used in Study One. The version used in Study Two contained only those eight inconsistencies identified in Study One as having a rate of detection between $31 \%$ and $52 \%$; the seven inconsistencies having lower rates of detections were redacted from the trial transcript with consistent information replacing the inconsistent details (see Appendix $\mathrm{N}$ for the trial transcript with eight inconsistencies, and Appendix $\mathrm{R}$ for descriptions of the remaining inconsistencies and relevant testimony.)

\section{Emotion measures.}

To obtain a baseline measure of emotional state without unduly emphasizing emotional states or introspective self-evaluation, I used the 10-item International Positive and Negative Affect Schedule Short Form (I-PANAS-SF, Thompson, 2007; see Appendix O). The I-PANAS-SF does not include any emotion ratings analogous to anger, so to maximize utility for my specific purposes, I appended "Angry" and "Mad" to the scale since one of the critical manipulations in Study Four is inducing some participants to feel anger. To obtain a more thorough measurement of emotional state 
immediately after exposure to the trial transcript, participants completed the 61 -item (adding Mad) Specific Affect Scales of the Positive and Negative Affect ScheduleExpanded (PANAS-X; Watson \& Clark, 1994; see Appendix O) following the final witness's testimony.

\section{Measures to determine rate of detection for each inconsistency.}

Remaining consistent with the inconsistency detection rate measures from Study One, I used the same battery of questions in the same order of question style, starting with very broad, open-ended questions, and tapering down to more specific short-answer questions, then multiple choice, true/false, and the most direct "point blank" questions (see Appendix Q for the full list of inconsistency detection questions.) The exact order of presentation remained randomized within question style subsections, but I eliminated those questions concerning inconsistencies that failed to reach the desired threshold of detectability rates in Study One.

\section{Procedure}

Participants logged on to their secure FIU Sona Systems account to receive the link to the study itself, securely hosted online at Qualtrics.com, FIU's online survey resource. After giving their consent to participate (see Appendix S for Study Two Informed Consent) Study Two used the same cover story, computer compatibility confirmations, instructions for following along with the trial transcript, reminder to complete the study all at once, page view duration verification, and data reduction techniques (see Appendix P for Study Two Data Reduction details) as used in Study One. Participants completed the brief, 12-item I-PANAS-SF (Thompson, 2007; see Appendix 
O) before reading and listening to the trial transcript version with eight inconsistencies (see Appendix N).

Immediately after participants finished reading along with and listening to the transcript, they completed the 61-item PANAS-X (Watson \& Clark, 1994; see Appendix O) to gauge their emotional state after having been exposed to the trial transcript. They were then asked to rate the credibility of each witness on a seven point Likert scale ranging from 1 (Completely Lacks Credibility) to 7 (Completely Credible) and then on the next page, to rate the persuasiveness of each witness on a seven point Likert scale ranging from 1-Not Persuasive At All to 7-Very Persuasive (see Appendix H). Following the witness credibility and persuasiveness ratings, participants answered the battery of broad (i.e. open-ended) to narrow (i.e point blank) inconsistency detection measurement questions (see Appendix Q), provided demographic information (see Appendix I), and were then debriefed (see Appendix J), thanked, and instructed to log off and close their web browser.

\section{Subjective Scoring}

Consistent with the safeguards employed in Study One, to avoid any potential for research bias, all responses to the open ended and short answer question types were again each coded by the same two research assistants blind to the hypothesis or intent of the study using the same guidelines and scoring rubric from Study One (see Appendix K, Open Ended Question Coding Instructions and Appendix L, Short Answer Question Coding Instructions). Both research assistants were furnished with a copy of the new trial transcript featuring only eight inconsistencies (see Appendix N) as well as a new listing of the inconsistencies without the seven inconsistencies eliminated after Study 
One (see Appendix R). Next, I met with the two of them to make sure they were comfortable making the transition back to scoring the open ended questions after last having scored data according the short answer criteria. All three of us were comfortable enough that we agreed it was unnecessary to undergo the same incremental rounds of coding practice data interspersed with meetings and expositions prior to coding the actual data. They first coded the open ended data individually, then met to discuss their reasoning with each other, and made any changes they wished to make after the benefit of debate. They each returned both open ended data files to me (one from before discussion, one from after discussion), at which point I gave them the short answer data to code individually, then discuss with each other, and to once again make any necessary revisions following discussion.

\section{Results}

\section{Inter-rater Reliability for Subjectively Scored Questions}

To confirm that the research assistants were each coding the data according to the same rules of interpretation, and because I knew the distribution to be non-normally distributed (see Appendix T), I conducted a Kendall's tau-b test to measure the correlation coefficient between the two scorers. Among the open ended questions, one inconsistency was not reported by any participants by either scorer, so for the remaining seven inconsistencies, one correlation (PI 5-model) was non-significant, but the average correlation of their "Yes" codings before discussion was high for the other $\operatorname{six}\left(\tau_{B}=.83, p\right.$ $<.001)$. Following discussion, the average correlation of their "Yes" codings for all seven (including the formerly nonsignificant) inconsistencies was very high $\left(\tau_{B}=.92, p<\right.$ .001). Similarly, for the short answer questions, with one inconsistency not being 
reported by any participants by either scorer, and a different inconsistency (PI 7-hair) showing a non-significant correlation before discussion, the average correlation of their "Yes" codings for the remaining six inconsistencies before discussion was very high ( $\tau_{B}$ $=.87, p<.001)$, as was the average correlation of their "Yes" codings for all seven (including the formerly non-significant) inconsistencies following discussion $\left(\tau_{B}=.92, p\right.$ $<.001$ ). Following the submission of all four sets of scorings (open ended before and after, short answer before and after) by both research assistants, I asked them about the two separate non-significant before values, which they explained had simply stemmed from a slight difference in the criteria for distinguishing a "Yes" response from a "Partial" response, but that upon discussion for each set of data, the discrepancy was noted and rectified, which was reflected in the strong associations between all postdiscussion scores.

\section{Confirming General Detection Rates of Each Inconsistency}

Overall, using the trial transcript featuring only eight inconsistencies, rates of detection were generally lower, with PI 7-Hair and PI 9-Year being exceptions (see Tables T1 and T2 in Appendix T for exact rates), which suggests that perhaps some cumulative effect might have contributed to greater detection rates when using the transcript featuring 15 inconsistencies. The exact decrements for any particular inconsistency, question type, or scoring scheme is of less interest than the fact that mean rates of detection remained high enough to allay concerns of floor effects, leaving room for a potential decrease in detection rate under experimental conditions in Study Four. According to both the Strict scoring scheme, which ranged in overall detection rates from $19.98 \%$ for PI 11-Jared to $39.26 \%$ for PI 9-Year $(M=28.22 \%, S D=7.22 \%)$ and the 
Loose scoring scheme, which ranged in overall detection rates from $24.68 \%$ for PI 11 Jared to $54.72 \%$ for PI 7 -Hair $(M=36.90 \%, S D=10.47 \%)$, there remains a serviceable range for potentially lower rates of detection.

The rate of chance for guessing the correct answer (which would be scored as a detection) for the point blank questions provides an additional means of validating these eight inconsistencies. There is only one correct answer among three answer choice options (see Appendix Q), which means that (at least assuming an equal likelihood of each choice being selected at random) random chance would allow for a minimum "detection" rate of approximately $33 \%$. However, the point blank detection rates $(M=$ $48.61 \%, S D=9.20 \%$ ) leave a buffer of approximately $15 \%$ which can be used to demonstrate a decrease in detection rates under experimental manipulations in Study Four.

The multiple choice questions (see Appendix Q) also offer a reliable means of determining detection rates, as they are not so vaguely worded that a participant might have noticed the inconsistency at issue, and yet might not understand the intent of the question while also avoiding a level of directness that could allow for a correct answer based solely upon deductive guessing since two specific answer options must be selected in order to count as a detection. ${ }^{1}$ Overall average detection rates of each inconsistency by all 27 participants in Study Two ranged from a low of $14.81 \%$ for PI 5-Model, PI 10-

\footnotetext{
${ }^{1}$ Because there are two specific selections per correct answer, the probability of randomly choosing the right pair of options is very low. With four reasonable alternatives, plus a "none of the above" option as well as an "I don't know" option, the probability of randomly selecting the correct two options is .067 even when eliminating the "none of the above" and the "I don't know" choices from consideration. Set aside the logical absurdity of choosing any of the four reasonable options in conjunction with "none of the above" and/or "I don't know" (in other words, treating all six response options as equally viable with equal probability of being selected, and considering all combinations ranging from the choice of only one option through the choice of all six), and the probability of randomly selecting the correct response drops to .016, effectively eliminating the possibility of artificially high detection rates due to random guessing.
} 
Color, and PI 11-Jared, and a high of 37.04\% for PI 7-Hair and PI 9-Year $(M=24.07 \%$, $S D=9.70 \%$ ), which leaves a comfortable margin for Study Four even after controlling for as much as $6.7 \%$ of the detections having been due to random chance.

\section{Measuring Emotional Impact of Trial Transcript}

Participants completed the I-PANAS-SF to measure their emotions prior to reading and listening to the trial transcript, and then the PANAS-X to measure their emotions after reading and listening to the trial transcript, and by comparing the pre- and post- transcript ratings for the twelve emotions included in the I-PANAS-SF pretest, I was able to determine what emotional responses the trial transcript elicited. However, in order to establish what statistical tests to use in making that determination, I first needed to verify whether the data was normally distributed. Pre- and post- transcript ratings for Alert, Determined, Attentive, and Active all displayed values less than twice the absolute value of the standard error for skewness $(S E=.45)$ and kurtosis $(S E=.87)$ so by that measure they are considered normally distributed, while post- transcript ratings of Inspired displayed skewness of 1.32, and Upset, Hostile, Ashamed, Nervous, Afraid, and Angry each had one or both values beyond the limits of normality for both pre- and posttranscript ratings. Even though four emotions satisfied the requirements for normality according to skewness and kurtosis parameters, all eleven pre- and post- transcript emotional measures significantly deviate from a normal distribution according to the Shapiro-Wilk test $(p<.05)$, so I will use nonparametric tests to compare pre- and posttranscript emotion ratings.

I used Kendall's tau-b to examine the correlation coefficients between each emotion rating. With the exception of Hostile, pre- and post- transcript emotion ratings 
were generally at least moderately positively correlated (see Table 1), but of particular note is that both Afraid $\left(\tau_{B}=.90, p<.001\right)$ and Angry $\left(\tau_{B}=.74, p<.001\right)$ were very strongly positively correlated, indicating that because pre- and post- ratings were relatively consistent, the trial transcript did not have much effect on these two emotions of critical focus in my study.

Because of the non-normal distribution of data, I could not use a repeated measures t-test to compare the means of each emotion rating before and after exposure to the trial transcript, so I relied upon Wilcoxon signed-rank tests instead. Seven of the eleven emotions did not exhibit a statistically significant change following exposure to the trial transcript, but ratings for Inspired $(Z=-3.25, p=.001, r=.63)$, Nervous $(Z=-$ 2.54, $p=.011, r=.49)$, Determined $(Z=-3.03, p=.002, r=.58)$, and Active $(Z=-2.37$, $p=.018, r=.46$ ) each decreased following the trial transcript (see Table 2 for pre- and post- transcript descriptive statistics and Wilcoxon signed-ranks test results). These specific decreases in ratings following the trial transcript do not present any particular concern, as they are not unforeseen or unreasonable consequences to having followed along with a mundane transcript of a civil trial or to nearing the completion of what in many ways amounts to a homework assignment.

\section{Discussion}

The eight inconsistencies featured in this version of the trial transcript should provide ample opportunity for detection rates to both increase and decrease from these baseline rates I have established. The presence of experimental control conditions in Study Four will provide additional reliability for comparing detection rates. Also, the trial transcript itself does not elicit any potentially confounding emotional responses. The 
validity and effectiveness of experimental emotion induction manipulations comprises the focus of Study Three, and then Study Four will look at the emotion inductions in conjunction with the trial transcript, examining whether the inductions are substantially durable enough to remain in effect even beyond the trial transcript. 
Table 1

Study Two: Kendall's tau-b Correlations Between Pre- and Post-Transcript Emotion

Ratings

\begin{tabular}{lcc}
\hline \multicolumn{1}{c}{ Emotion } & Correlation Coefficient & Significance (2-tailed) \\
\hline Upset & $.367^{*}$ & .036 \\
Hostile & .275 & .141 \\
Alert & $.517^{* *}$ & .001 \\
Ashamed & $.866^{* *}$ & $<.001$ \\
Inspired & $.379^{*}$ & .024 \\
Nervous & $.477^{* *}$ & .006 \\
Determined & $.445^{* *}$ & .006 \\
Attentive & $.442^{* *}$ & .007 \\
Afraid & $.900^{* *}$ & $<.001$ \\
Active & $.496^{* *}$ & .002 \\
Angry & $.741^{* *}$ & $<.001$ \\
\hline
\end{tabular}

Note. $n=27$.

*. Correlation is significant at the 0.05 level (2-tailed).

**. Correlation is significant at the 0.01 level (2-tailed). 
Table 2

Study Two: Descriptive Statistics and Wilcoxon Signed-Test Results for Emotion Ratings Before and After Transcript

\begin{tabular}{|c|c|c|c|c|c|c|c|c|c|c|c|c|c|c|c|c|c|c|}
\hline \multirow[b]{3}{*}{ Emotion } & \multicolumn{5}{|c|}{ Before } & \multicolumn{5}{|c|}{ After } & \multirow[b]{3}{*}{$\begin{array}{c}\text { Neg } \\
\text { Ranks }^{\mathrm{a}}\end{array}$} & \multirow{3}{*}{$\begin{array}{c}\text { Neg } \\
\text { Rank } \\
\text { Means }\end{array}$} & \multirow[b]{3}{*}{$\begin{array}{c}\text { Pos } \\
\text { Ranks }^{\mathrm{b}}\end{array}$} & \multirow[b]{3}{*}{$\begin{array}{c}\text { Pos Rank } \\
\text { Means }\end{array}$} & \multirow[b]{3}{*}{ Ties } & \multirow[b]{3}{*}{$Z$} & \multirow[b]{3}{*}{$p$} & \multirow[b]{3}{*}{$r$} \\
\hline & & & & cent & & & & & cent & & & & & & & & & \\
\hline & Min & Max & 25 & 50 & 75 & Min & Max & 25 & 50 & 75 & & & & & & & & \\
\hline Upset & 1 & 5 & 1 & 1 & 2 & 1 & 5 & 1 & 1 & 2 & 5 & 5.20 & 5 & 5.80 & 17 & $-.155^{\mathrm{c}}$ & .877 & \\
\hline Hostile & 1 & 3 & 1 & 1 & 1 & 1 & 5 & 1 & 1 & 2 & 4 & 3.75 & 4 & 5.25 & 19 & $-.427^{\mathrm{c}}$ & .669 & \\
\hline Alert & 1 & 5 & 3 & 3 & 4 & 1 & 5 & 2 & 3 & 4 & 10 & 9.40 & 6 & 7.00 & 11 & $-1.435^{\mathrm{d}}$ & .151 & \\
\hline Ashamed & 1 & 5 & 1 & 1 & 1 & 1 & 4 & 1 & 1 & 1 & 3 & 2.50 & 1 & 2.50 & 23 & $-1.000^{\mathrm{d}}$ & .317 & \\
\hline Inspired & 1 & 5 & 1 & 3 & 4 & 1 & 4 & 1 & 1 & 2 & 14 & 9.29 & 2 & 3.00 & 11 & $-3.253^{\mathrm{d}}$ & .001 & .63 \\
\hline Nervous & 1 & 5 & 1 & 2 & 2 & 1 & 4 & 1 & 1 & 2 & 11 & 7.27 & 2 & 5.50 & 14 & $-2.543^{\mathrm{d}}$ & .011 & .49 \\
\hline Determined & 1 & 5 & 2 & 4 & 4 & 1 & 5 & 1 & 2 & 4 & 16 & 9.63 & 2 & 8.50 & 9 & $-3.035^{\mathrm{d}}$ & .002 & .58 \\
\hline Attentive & 1 & 5 & 3 & 4 & 5 & 1 & 5 & 3 & 3 & 4 & 11 & 7.41 & 4 & 9.63 & 12 & $-1.279^{\mathrm{d}}$ & .201 & \\
\hline Afraid & 1 & 5 & 1 & 1 & 1 & 1 & 4 & 1 & 1 & 1 & 4 & 2.50 & 0 & 0.00 & 23 & $-1.890^{\mathrm{d}}$ & .059 & \\
\hline Active & 1 & 5 & 2 & 3 & 4 & 1 & 5 & 1 & 2 & 3 & 15 & 9.23 & 3 & 10.83 & 9 & $-2.365^{\mathrm{d}}$ & .018 & .46 \\
\hline Angry & 1 & 5 & 1 & 1 & 1 & 1 & 4 & 1 & 1 & 2 & 2 & 2.50 & 2 & 2.50 & 23 & $.000^{\mathrm{e}}$ & 1.000 & \\
\hline
\end{tabular}


Note. $n=27 .{ }^{a}$ Negative ranks are ranks in which the pre- transcript rating was greater than the post- transcript rating, indicating an overall decrease in rating. ${ }^{b}$ Positive ranks are ranks in which the post- transcript rating was greater than the pre- transcript rating, indicating an overall increase in rating. ${ }^{\mathrm{c}} Z$ scores based on negative ranks. ${ }^{\mathrm{d}} Z$ scores based on positive ranks. ${ }^{\mathrm{e}}$ The sum of negative ranks equals the sum of positive ranks. 


\section{Chapter VII}

\section{Study Three: Verifying Emotion Induction Manipulations}

\section{Method}

\section{Theoretical Basis}

Semmler and Brewer's mixed findings (2002) should not come as any great surprise - whether due to a paucity of moderately detectable inconsistencies or the possibility that effects due to unintentionally induced emotions may have competed with, detracted from, or even overshadowed the effects of their experimentally manipulated emotional conditions. There are two additional possibilities which might also explain their results, each having to do with their experimental induction of emotions. First, and the most easily rectified, is the fact that they relied upon the content of the trial transcript itself as the means of emotional induction. This is problematic because their independent variable (inducing sadness based on details of the trial transcript) was in some ways identical to their dependent variable (the detection of inconsistencies with the testimony) since both are based on participants noticing (or not noticing) and interpreting (or not interpreting) informational details from the same root source. The low degree of experimental control afforded by this almost circular, self-contained design can be remedied by inducing emotion through a means wholly separate and independent from the trial transcript.

The second possible pitfall having to do with Semmler and Brewer's emotion induction is their specific choice of which emotion to examine. The emotion they induced (sadness) is characterized by greater appraisals of uncertainty than certainty, but it is closer to neutral in terms of levels of certainty than to either extreme end of the 
spectrum (see Figure 2). Their findings may have been inconclusive because there was not enough of a distinction between their experimental and control conditions, or due to the unusual nature of sadness itself. Sadness is a highly complex emotion in terms of constituent appraisal tendencies (C. A. Smith \& Ellsworth 1985; 1987) so it may have been a brash choice to induce as the target emotion at such a nascent stage of exploratory investigation. I have avoided both predicaments by choosing to induce two separate emotions differing in their level of certainty appraisals (fear, which is low in certainty, and anger, which is high in certainty) as well as by including a third emotionally neutral control condition. I chose to use fear and anger because they are highly similar to one another in nearly all appraisal dimensions besides certainty (C. A. Smith \& Ellsworth, 1985; see Figures 1, 2, and 3), so comparing the two will allow for a neater design with more potential for contrast than Semmler and Brewer's efforts.

There are several popular methods which researchers have used to induce emotion in participants to varying degrees of effectiveness (see generally Lench, et al., 2011; Martin, 1990; and Schaefer, Nils, Sanchez, \& Philippot, 2010), but due to concerns over highly variable participant investment using methods such as the writing of autobiographical statements and other emotion induction methods, I used film clips to induce discrete emotions. Film clips have been successfully used to induce emotions in a variety of prior research on the relationship between emotion and cognition (appraisals influencing judgments of trustworthiness, Dunn \& Schweitzer, 2005; fMRI examinations of emotional regulation, Goldin, McRae, Ramel \& Gross, 2008; timing of emotion regulation strategies, Sheppes \& Meiran, 2008), and have been found to be the most broadly effective of induction methods (Westermann et al., 1996) but concerns of 
generalizability and uniformity have prompted several efforts to identify a battery of film clips that can be used to reliably induce emotion. Philippot (1993) compiled a selection of film clips for French-speaking participants, while Gross and Levenson (1995) independently identified 16 film clips that were shown to elicit eight discrete emotions, with care taken to isolate those films which prompt only one distinct emotion (rather than a general, diffuse valence, or a constellation of concomitant emotions) and to maximal intensity. The use of these 16 clips to induce emotions was further supported by Rottenberg, Ray, and Gross (2007), which provides a primer on the use of film clips to elicit emotions in experimental settings. Despite being a relatively new research paradigm, emotion has been successfully induced in online experimental designs in a variety of ways, including the Velten procedure, autobiographical recall, moodsuggestive photographs, picture-illustrated emotive texts, and video clips (Göritz, 2007; Göritz \& Moser, 2006; Verheyen \& Göritz, 2009; Verleuer, Verhagen, \& Heuvelman, 2007; Wiener et al., 2007), so I expected that video clips shown online would be a successful means of emotion induction (such clips are agreed to be among the most robust means of emotion induction; see Gross \& Levenson, 1995; Lench, et al., 2011; Martin, 1990; Philippot, 1993; Rottenberg, Ray, \& Gross, 2007; Schaefer, et al., 2010; Westermann et al., 1996). Study Three was undertaken to confirm the effectiveness of my emotion inductions because it is a somewhat new combination of two separate, yet previously successful experimental methodologies. In order for Study Four to have meaningful results, I need to be absolutely sure that my method of inducing fear, anger, or no emotional state are each effective. Additionally, by confirming the effectiveness of 
this type of experimental manipulation, favorable results will strengthen the basis for similar emotional induction methods in future research.

\section{Participants}

Three hundred nineteen undergraduate students at Florida International University were recruited through Sona Systems, the Psychology Department Research Management system to participate in a web-based study entitled "Watching Movies" in exchange for course credit. Because this was an online study, several safeguards were used to exclude those participants who did not give the study their undivided attention (see Appendix U for detailed criteria and procedures undertaken for data reduction purposes), which resulted in a total of 249 participants. The sample was predominantly female $(75 \% ; 25 \%$ male), Hispanic (66\%; 14\% White/Caucasian; 12\% African American; 4\% Asian, and $5 \%$ Other $)$, and they ranged in age from 18 to 49 years old $(M=22.07, S D=4.64)$. Participants were predominantly upperclassmen, with $34 \%$ in their senior year, $21 \%$ in their junior year, $12 \%$ in their sophomore year, $27 \%$ in their freshman year, and 3\% having completed some professional or graduate-level education. Over half the sample (52\%) was not currently employed, with $32 \%$ holding part-time employment and $16 \%$ working full-time, and seven participants (3\%) had previously served on a civil jury while two participants (1\%) had served on a criminal jury.

\section{Design}

Study Three was essentially run twice with the findings from the first effort (identifying which emotionally neutral film clip to use as my control, as well as uncovering unexpected wrinkles in induction techniques; see Appendix W for details) informing the design and objectives for the second iteration which is the focus here. 
Study Three was a 2 (emotion induction technique: with vs. without autobiographical perspective-taking writing exercise) $\mathrm{x} 4$ (induction target emotion/level of certainty and associated film clips: fear/low certainty: boy playing in hallway from The Shining [1980] and basement chase scene from The Silence of the Lambs [1991] as well as anger/high certainty via clips featuring police abusing protestors from Cry Freedom [1987] and a bullying scene from My Bodyguard [1980]; see Appendix A) pretest-posttest mixed factorial design. In short, the goal of Study Three was to identify and confirm the most effective techniques to experimentally induce fear and anger among my sampling frame.

\section{Materials}

\section{Emotion induction.}

Rottenberg, Ray, and Gross (2007, see Appendix A) recommend two film clips that they found to reliably induce fear (a basement chase scene from The Silence of the Lambs [1991] and a boy playing in hallway from The Shining [1980]) as well as two film clips they found to reliably induce anger (police abusing protestors from Cry Freedom [1987] and a bullying scene from My Bodyguard [1980]). The neutral/no emotion inducing film clip I would use as a control in Studies Four was validated and selected during the preliminary phase of Study Three (see Appendix W).

Each participant was randomly assigned to a condition featuring only one of the four film clips. Approximately half of the participants were randomly assigned to a condition in which after they watched the clip, they were instructed to write a few sentences recounting the clip from a first-person perspective as if they had been experiencing the events depicted themselves. The other participants were not asked to engage in this perspective-taking exercise. This manipulation was conducted in order to 
test whether the writing task bolstered or weakened the effectiveness of the emotion inductions (see Appendix W for additional background on this undertaking).

\section{Emotion measures.}

To obtain a baseline measure of participant emotional state prior to them watching a film clip without unduly altering their emotional states or introspective self-evaluation, I provided participants with the 10-item International Positive and Negative Affect Schedule Short Form (I-PANAS-SF, Thompson, 2007; see Appendix O). The I-PANASSF does not include any emotion ratings analogous to anger, so to maximize utility for my specific purposes, I appended "Angry" to the scale since one of the critical manipulations in Study Four is inducing some participants to feel anger. To obtain a more thorough measurement of emotional state immediately after exposure to their randomly assigned film clip (and after those participants in the perspective-taking condition completed the writing assignment), all participants completed the 60 -item Specific Affect Scales of the Positive and Negative Affect Schedule- Expanded (PANASX; Watson \& Clark, 1994; see Appendix O). Participants also completed the Post Film Questionnaire (Rottenberg, Ray, \& Gross, 2007; see Appendix A).

\section{Procedure}

Just as in Studies One and Two, participants logged on to their secure FIU Sona Systems account to receive the link to the study itself, securely hosted online at Qualtrics.com, FIU's online survey resource. After giving their consent to participate (see Appendix V for Study Three Informed Consent) Study Three used the same computer compatibility confirmations, reminder to complete the study all at once, page view duration verification, and similar data reduction techniques (see Appendix $U$ for 
Study Three Data Reduction details) as used in prior studies. Participants were randomly assigned to all conditions.

Following their granting of consent and verifying the compatibility of their computers, participants completed the twelve item I-PANAS-SF to establish their baseline emotional ratings. Participants then watched one of the four film clips, after which those participants assigned to the writing task conditions were instructed to "please describe the film clip you just watched as if you were there to experience it in person" in a few sentences before proceeding, while those participants not assigned to the writing task continued directly to the PANAS-X. Next, participants completed Rottenberg, Ray, and Gross's (2007) Post Film Questionnaire concerning the emotions they experienced while watching the film clip. They then answered demographic questions (see Appendix I) and were thanked, debriefed (see Appendix J), and instructed to close their web browser.

\section{Results}

\section{General Effectiveness of Films as Means of Induction}

In order to accurately compare postfilm emotions of different groups, I first needed to confirm that there were no differences between groups prior to the emotional inductions. Analysis of variance with a Bonferroni correction for multiple comparisons showed no significant differences between the eight experimental groups for any of the twelve emotions rated on the I-PANAS-SF pretest: upset, $F(1,7)=1.45, p=.185, \eta^{2}=$ .04 ; hostile, $F(1,7)=1.22, p=.289, \eta^{2}=.03$; alert, $F(1,7)=1.07, p=.385, \eta^{2}=.03$;

ashamed, $F(1,7)=0.61, p=.748, \eta^{2}=.02$; angry, $F(1,7)=1.51, p=.164, \eta^{2}=.04$; nervous, $F(1,7)=0.50, p=.834, \eta^{2}=.01$; determined, $F(1,7)=0.66, p=.705, \eta^{2}=.02$; 
attentive, $F(1,7)=0.29, p=.957, \eta^{2}=.01 ;$ afraid, $F(1,7)=1.97, p=.060, \eta^{2}=.05$;

active, $F(1,7)=1.08, p=.377, \eta^{2}=.03$; inspired, $F(1,7)=1.50, p=.168, \eta^{2}=.04$; mad, $F(1,7)=1.43, p=.195, \eta^{2}=.04$. My next step was to simply check whether (and if so, which) emotion ratings changed during each film clip by conducting a repeated measures t-test for each of the twelve emotions rated both before and after the induction procedure (see Tables 3 through 10 for pre- and post- film clip means on a scale from 1-very slightly or not at all to 5-extremely). This round of testing confirmed in a very general sense that each of the four film clips seem to be inducing the correct emotions as intended, with The Shining and The Silence of the Lambs each yielding increased ratings for nervous and afraid, both with and without the writing task, while the clips from Cry Freedom and My Bodyguard are each leading to increased ratings for hostile, angry, and mad under both writing task conditions.

However, there were also several minor adverse effects, such as the $M y$ Bodyguard clip significantly increasing afraid ratings in the no writing task condition ( $M$ $=1.20, S D=0.41)$ in comparison to pretest afraid ratings $(M=1.00, S D=0.00), t(29)=$ $2.69, p=.012, d=0.49$, but this rather small difference is even smaller when considering the absolute minimum ratings given during the pretest. Of slightly greater cause for concern is that the clip from The Silence of the Lambs elicited a statistically significant increase in anger ratings from the pretest $(M=1.23, S D=0.49)$ to the post-film ratings $(M=1.46, S D=0.82), t(34)=2.26, p=.030, d=0.38$, in the no writing task condition, but even so, this is a relatively small increase from a very low baseline level. The Cry Freedom clip, however, yielded potentially confounding results in both induction conditions. Those participants who did not engage in the writing task in addition to the 
film clip emotion induction showed a statistically significant increase in afraid ratings from the pretest $(M=1.03, S D=0.16)$ to the posttest $(M=1.43, S D=0.69), t(36)=3.83$, $p<.001, d=0.63$, but this increase is also starting from a reference point just barely above the minimum possible level. The participants who undertook the writing task showed a much more dramatic and troublesome increase from pretest afraid ratings ( $M=$ $1.19, S D=0.68)$ to the levels reported following the film clip $(M=2.58, S D=1.50)$, $t(20)=3.82, p=.001, d=0.83$, which is quite a strong effect. I evaluated these and other condition-specific results more closely in my next step of analysis. 
Table 3

Study Three: Contrast of Mean Emotion Ratings Before and After The Shining Film Clip and Perspective Taking Writing Task

\begin{tabular}{|c|c|c|c|c|c|c|c|c|c|c|c|}
\hline \multirow[b]{2}{*}{ Emotion } & \multicolumn{2}{|c|}{ Before Film } & \multicolumn{2}{|c|}{ After Film } & \multicolumn{2}{|c|}{ Difference* } & \multirow[b]{2}{*}{$t(37)$} & \multirow[b]{2}{*}{$p$} & \multicolumn{2}{|c|}{$95 \% \mathrm{CI}$} & \multirow[b]{2}{*}{ Cohen's $d$} \\
\hline & $M$ & $S D$ & $M$ & $S D$ & $M$ & $S D$ & & & $L L$ & $U L$ & \\
\hline Upset & 1.48 & 0.89 & 1.53 & 0.76 & 0.05 & 0.96 & 0.34 & .737 & -0.26 & 0.37 & 0.05 \\
\hline Hostile & 1.26 & 0.60 & 1.21 & 0.53 & -0.05 & 0.57 & -0.57 & .571 & -0.24 & 0.13 & -0.09 \\
\hline Alert & 2.89 & 1.35 & 3.21 & 1.19 & 0.32 & 1.65 & 1.18 & .244 & -0.23 & 0.86 & 0.19 \\
\hline Ashamed & 1.16 & 0.49 & 1.08 & 0.27 & -0.08 & 0.27 & -1.78 & .083 & -0.17 & 0.01 & -0.29 \\
\hline Angry & 1.37 & 0.71 & 1.37 & 0.67 & 0.00 & 0.62 & 0.00 & 1.000 & -0.20 & 0.20 & 0.00 \\
\hline Nervous & 1.42 & 0.60 & 2.24 & 1.30 & 0.82 & 1.35 & 3.71 & .001 & 0.37 & 1.26 & 0.60 \\
\hline Determined & 3.32 & 1.16 & 2.32 & 1.40 & -1.00 & 1.25 & -4.92 & $<.001$ & -1.41 & -0.59 & -0.80 \\
\hline Attentive & 3.58 & 0.89 & 3.50 & 1.06 & -0.08 & 1.00 & -0.49 & .628 & -0.41 & 0.25 & -0.08 \\
\hline Afraid & 1.18 & 0.39 & 2.24 & 1.39 & 1.24 & 1.34 & 5.67 & $<.001$ & 0.79 & 1.68 & 0.92 \\
\hline Active & 3.03 & 1.30 & 2.11 & 1.20 & -0.92 & 1.15 & -4.95 & $<.001$ & -1.30 & -0.54 & -0.80 \\
\hline Inspired & 2.87 & 1.30 & 1.74 & 1.03 & -1.13 & 1.17 & -5.98 & $<.001$ & -1.51 & -0.75 & -0.97 \\
\hline Mad & 1.32 & 0.70 & 1.24 & 0.49 & -0.08 & 0.54 & -0.90 & .373 & -0.26 & 0.09 & -0.15 \\
\hline
\end{tabular}

Note. $\mathrm{CI}=$ Confidence Interval of the Difference; $L L=$ lower limit; $U L=$ upper limit. *After minus Before; positive values indicate an increased rating. 
Table 4

Study Three: Contrast of Mean Emotion Ratings Before and After The Silence of the Lambs Film Clip and Perspective Taking Writing Task

\begin{tabular}{|c|c|c|c|c|c|c|c|c|c|c|c|}
\hline \multirow[b]{2}{*}{ Emotion } & \multicolumn{2}{|c|}{ Before Film } & \multicolumn{2}{|c|}{ After Film } & \multicolumn{2}{|c|}{ Difference* } & \multirow[b]{2}{*}{$t(21)$} & \multirow[b]{2}{*}{$p$} & \multicolumn{2}{|c|}{$95 \% \mathrm{CI}$} & \multirow[b]{2}{*}{ Cohen's $d$} \\
\hline & $M$ & $S D$ & $M$ & $S D$ & $M$ & $S D$ & & & $L L$ & $U L$ & \\
\hline Upset & 1.18 & 0.39 & 1.55 & 0.67 & 0.36 & 0.66 & 2.59 & .017 & 0.07 & 0.66 & 0.55 \\
\hline Hostile & 1.14 & 0.47 & 1.27 & 0.63 & 0.14 & 0.56 & 1.14 & .266 & -0.11 & 0.38 & 0.24 \\
\hline Alert & 2.73 & 1.24 & 3.32 & 1.29 & 0.59 & 1.22 & 2.27 & .034 & 0.05 & 1.13 & 0.48 \\
\hline Ashamed & 1.18 & 0.50 & 1.14 & 0.64 & -0.05 & 0.65 & -0.33 & .747 & -0.33 & 0.24 & -0.07 \\
\hline Angry & 1.14 & 0.35 & 1.32 & 0.65 & 0.18 & 0.73 & 1.16 & .257 & -0.14 & 0.51 & 0.25 \\
\hline Nervous & 1.45 & 0.80 & 2.09 & 1.27 & 0.64 & 1.36 & 2.19 & .040 & 0.03 & 1.24 & 0.47 \\
\hline Determined & 3.09 & 1.63 & 2.00 & 1.15 & -1.09 & 1.23 & -4.16 & $<.001$ & -1.64 & -0.55 & -0.89 \\
\hline Attentive & 3.64 & 1.09 & 3.55 & 1.06 & -0.09 & 1.06 & -0.40 & .693 & -0.56 & 0.38 & -0.09 \\
\hline Afraid & 1.14 & 0.35 & 1.77 & 0.75 & 0.64 & 0.90 & 3.31 & .003 & 0.24 & 1.04 & 0.71 \\
\hline Active & 2.32 & 1.25 & 1.86 & 1.17 & -0.45 & 1.10 & -1.94 & .066 & -0.94 & 0.03 & -0.41 \\
\hline Inspired & 2.27 & 1.49 & 1.32 & 0.95 & -0.95 & 1.29 & -3.47 & .002 & -1.53 & -0.38 & -0.74 \\
\hline Mad & 1.18 & 0.39 & 1.18 & 0.39 & 0.00 & 0.53 & 0.00 & 1.000 & -0.24 & 0.24 & 0.00 \\
\hline
\end{tabular}

Note. $\mathrm{CI}=$ Confidence Interval of the Difference; $L L=$ lower limit; $U L=$ upper limit. *After minus Before; positive values indicate an increased rating. 
Table 5

Study Three: Contrast of Mean Emotion Ratings Before and After Cry Freedom Film Clip and Perspective Taking Writing Task

\begin{tabular}{|c|c|c|c|c|c|c|c|c|c|c|c|}
\hline \multirow[b]{2}{*}{ Emotion } & \multicolumn{2}{|c|}{ Before Film } & \multicolumn{2}{|c|}{ After Film } & \multicolumn{2}{|c|}{ Difference* } & \multirow[b]{2}{*}{$t(20)$} & \multirow[b]{2}{*}{$p$} & \multicolumn{2}{|c|}{$95 \% \mathrm{CI}$} & \multirow[b]{2}{*}{ Cohen's $d$} \\
\hline & $M$ & $S D$ & $M$ & $S D$ & $M$ & $S D$ & & & $L L$ & $U L$ & \\
\hline Upset & 1.29 & 0.56 & 3.90 & 1.22 & 2.62 & 1.28 & 9.35 & $<.001$ & 2.03 & 3.20 & 2.04 \\
\hline Hostile & 1.00 & 0.00 & 1.90 & 1.22 & 0.90 & 1.22 & 3.40 & .003 & 0.35 & 1.46 & 0.74 \\
\hline Alert & 3.05 & 1.28 & 3.43 & 1.43 & 0.38 & 1.53 & 1.14 & .268 & -0.32 & 1.08 & 0.25 \\
\hline Ashamed & 1.19 & 0.40 & 1.90 & 1.26 & 0.71 & 1.23 & 2.66 & .015 & 0.15 & 1.27 & 0.58 \\
\hline Angry & 1.14 & 0.36 & 3.67 & 1.46 & 2.52 & 1.50 & 7.69 & $<.001$ & 1.84 & 3.21 & 1.68 \\
\hline Nervous & 1.52 & 0.68 & 1.90 & 1.18 & 0.38 & 1.20 & 1.45 & .162 & -0.17 & 0.93 & 0.32 \\
\hline Determined & 3.67 & 1.32 & 2.24 & 1.41 & -1.43 & 1.60 & -4.09 & .001 & -2.16 & -0.70 & -0.89 \\
\hline Attentive & 3.62 & 1.16 & 3.67 & 1.28 & 0.05 & 1.72 & 0.13 & .900 & -0.73 & 0.83 & 0.03 \\
\hline Afraid & 1.19 & 0.68 & 2.58 & 1.50 & 1.38 & 1.66 & 3.82 & .001 & 0.63 & 2.14 & 0.83 \\
\hline Active & 3.05 & 1.47 & 2.05 & 1.24 & -1.00 & 1.26 & -3.62 & .002 & -1.58 & -0.42 & -0.79 \\
\hline Inspired & 3.05 & 1.63 & 1.57 & 0.98 & -1.48 & 1.91 & -3.54 & .002 & -2.35 & -0.61 & -0.77 \\
\hline Mad & 1.24 & 0.54 & 3.24 & 1.61 & 2.00 & 1.55 & 5.92 & $<.001$ & 1.29 & 2.71 & 1.29 \\
\hline
\end{tabular}

Note. $\mathrm{CI}=$ Confidence Interval of the Difference; $L L=$ lower limit; $U L=$ upper limit. *After minus Before; positive values indicate an increased rating. 
Table 6

Study Three: Contrast of Mean Emotion Ratings Before and After My Bodyguard Film Clip and Perspective Taking Writing Task

\begin{tabular}{|c|c|c|c|c|c|c|c|c|c|c|c|}
\hline \multirow[b]{2}{*}{ Emotion } & \multicolumn{2}{|c|}{ Before Film } & \multicolumn{2}{|c|}{ After Film } & \multicolumn{2}{|c|}{ Difference* } & \multirow[b]{2}{*}{$t(29)$} & \multirow[b]{2}{*}{$p$} & \multicolumn{2}{|c|}{$95 \% \mathrm{CI}$} & \multirow[b]{2}{*}{ Cohen's $d$} \\
\hline & $M$ & $S D$ & $M$ & $S D$ & $M$ & $S D$ & & & $L L$ & $U L$ & \\
\hline Upset & 1.17 & 0.38 & 2.87 & 1.48 & 1.70 & 1.60 & 5.82 & $<.001$ & 1.10 & 2.30 & 1.06 \\
\hline Hostile & 1.03 & 0.18 & 1.67 & 1.09 & 0.63 & 1.10 & 3.16 & .004 & 0.22 & 1.04 & 0.58 \\
\hline Alert & 2.93 & 1.17 & 2.90 & 1.21 & -0.03 & 1.25 & -0.15 & .884 & -0.50 & 0.43 & -0.03 \\
\hline Ashamed & 1.10 & 0.31 & 1.53 & 0.94 & 0.43 & 0.94 & 2.54 & .017 & 0.08 & 0.78 & 0.46 \\
\hline Angry & 1.07 & 0.25 & 2.47 & 1.46 & 1.40 & 1.45 & 5.28 & $<.001$ & 0.86 & 1.94 & 0.96 \\
\hline Nervous & 1.43 & 0.82 & 1.50 & 0.78 & 0.07 & 0.87 & 0.42 & .677 & -0.26 & 0.39 & 0.08 \\
\hline Determined & 3.43 & 1.28 & 2.16 & 0.99 & -1.27 & 1.46 & -4.75 & $<.001$ & -1.81 & -0.72 & -0.87 \\
\hline Attentive & 3.50 & 1.01 & 3.07 & 1.28 & -0.43 & 1.19 & -1.99 & .056 & -0.88 & 0.01 & -0.36 \\
\hline Afraid & 1.37 & 0.89 & 1.13 & 0.35 & -0.23 & 0.97 & -1.32 & .199 & -0.60 & 0.13 & -0.24 \\
\hline Active & 2.57 & 1.10 & 2.10 & 1.12 & -0.47 & 1.22 & -2.09 & .046 & -0.92 & -0.01 & -0.38 \\
\hline Inspired & 2.40 & 1.10 & 1.37 & 0.85 & -1.03 & 1.19 & -4.76 & $<.001$ & -1.48 & -0.59 & -0.87 \\
\hline Mad & 1.03 & 0.18 & 2.37 & 1.65 & 1.33 & 1.60 & 4.55 & $<.001$ & 0.73 & 1.93 & 0.83 \\
\hline
\end{tabular}

Note. $\mathrm{CI}=$ Confidence Interval of the Difference; $L L=$ lower limit; $U L=$ upper limit. *After minus Before; positive values indicate an increased rating. 
Table 7

Study Three: Contrast of Mean Emotion Ratings Before and After The Shining Film Clip and No Writing Task

\begin{tabular}{|c|c|c|c|c|c|c|c|c|c|c|c|}
\hline \multirow[b]{2}{*}{ Emotion } & \multicolumn{2}{|c|}{ Before Film } & \multicolumn{2}{|c|}{ After Film } & \multicolumn{2}{|c|}{ Difference* } & \multirow[b]{2}{*}{$t(35)$} & \multirow[b]{2}{*}{$p$} & \multicolumn{2}{|c|}{$95 \% \mathrm{CI}$} & \multirow[b]{2}{*}{ Cohen's $d$} \\
\hline & $M$ & $S D$ & $M$ & $S D$ & $M$ & $S D$ & & & $L L$ & $U L$ & \\
\hline Upset & 1.53 & 0.84 & 1.61 & 0.93 & 0.08 & 1.05 & 0.48 & .638 & -0.27 & 0.44 & 0.08 \\
\hline Hostile & 1.19 & 0.58 & 1.50 & 1.00 & 0.31 & 1.01 & 1.82 & .078 & -0.04 & 0.65 & 0.30 \\
\hline Alert & 3.03 & 1.34 & 3.19 & 1.33 & 0.17 & 1.30 & 0.77 & .446 & -0.27 & 0.61 & 0.13 \\
\hline Ashamed & 1.08 & 0.28 & 1.11 & 0.40 & 0.03 & 0.45 & 0.37 & .711 & -0.12 & 0.18 & 0.06 \\
\hline Angry & 1.25 & 0.65 & 1.22 & 0.48 & -0.03 & 0.61 & -0.27 & .786 & -0.23 & 0.18 & -0.05 \\
\hline Nervous & 1.33 & 0.68 & 2.42 & 1.36 & 1.08 & 1.30 & 5.02 & $<.001$ & 0.64 & 1.52 & 0.84 \\
\hline Determined & 3.33 & 1.53 & 2.36 & 1.38 & -0.97 & 1.48 & -3.93 & $<.001$ & -1.47 & -0.47 & -0.66 \\
\hline Attentive & 3.47 & 1.38 & 3.72 & 1.06 & 0.25 & 1.13 & 1.33 & .193 & -0.13 & 0.63 & 0.22 \\
\hline Afraid & 1.11 & 0.32 & 2.19 & 1.31 & 1.08 & 1.40 & 4.64 & $<.001$ & 0.61 & 1.56 & 0.77 \\
\hline Active & 2.67 & 1.37 & 2.03 & 1.21 & -0.64 & 1.40 & -2.74 & .010 & -1.11 & -0.17 & -0.46 \\
\hline Inspired & 2.94 & 1.55 & 2.00 & 1.20 & -0.94 & 1.37 & -4.13 & $<.001$ & -1.41 & -0.48 & -0.68 \\
\hline Mad & 1.31 & 0.62 & 1.19 & 0.58 & -0.11 & 0.57 & -1.16 & .254 & -0.31 & 0.08 & -0.19 \\
\hline
\end{tabular}

Note. CI $=$ Confidence Interval of the Difference; $L L=$ lower limit; $U L=$ upper limit. *After minus Before; positive values indicate an increased rating. 
Table 8

Study Three: Contrast of Mean Emotion Ratings Before and After The Silence of the Lambs Film Clip and No Writing Task

\begin{tabular}{|c|c|c|c|c|c|c|c|c|c|c|c|}
\hline \multirow[b]{2}{*}{ Emotion } & \multicolumn{2}{|c|}{ Before Film } & \multicolumn{2}{|c|}{ After Film } & \multicolumn{2}{|c|}{ Difference* } & \multirow[b]{2}{*}{$t(34)$} & \multirow[b]{2}{*}{$p$} & \multicolumn{2}{|c|}{$95 \% \mathrm{CI}$} & \multirow[b]{2}{*}{ Cohen's $d$} \\
\hline & $M$ & $S D$ & $M$ & $S D$ & $M$ & $S D$ & & & $L L$ & $U L$ & \\
\hline Upset & 1.34 & 0.64 & 1.77 & 1.03 & 0.43 & 1.14 & 2.21 & .034 & 0.04 & 0.82 & 0.37 \\
\hline Hostile & 1.09 & 0.28 & 1.69 & 0.93 & 0.60 & 0.88 & 4.03 & $<.001$ & 0.30 & 0.90 & 0.68 \\
\hline Alert & 2.63 & 1.33 & 3.66 & 1.08 & 1.03 & 1.62 & 3.76 & .001 & 0.47 & 1.58 & 0.64 \\
\hline Ashamed & 1.11 & 0.40 & 1.09 & 0.28 & -0.03 & 0.45 & -0.37 & .711 & -0.18 & 0.13 & -0.06 \\
\hline Angry & 1.23 & 0.49 & 1.46 & 0.82 & 0.23 & 0.60 & 2.26 & .030 & 0.02 & 0.43 & 0.38 \\
\hline Nervous & 1.60 & 0.77 & 2.20 & 1.16 & 0.60 & 1.26 & 2.81 & .008 & 0.17 & 1.03 & 0.47 \\
\hline Determined & 3.37 & 1.21 & 2.34 & 1.39 & -1.03 & 1.29 & -4.70 & $<.001$ & -1.47 & 0.58 & -0.79 \\
\hline Attentive & 3.51 & 1.07 & 3.80 & 1.05 & 0.29 & 0.96 & 1.77 & .086 & -0.04 & 0.61 & 0.30 \\
\hline Afraid & 1.11 & 0.32 & 2.00 & 0.97 & 0.89 & 0.96 & 5.44 & $<.001$ & 0.55 & 1.22 & 0.92 \\
\hline Active & 2.86 & 1.35 & 2.49 & 1.25 & -0.37 & 1.31 & -1.68 & .102 & -0.82 & 0.08 & -0.28 \\
\hline Inspired & 2.89 & 1.30 & 1.74 & 1.09 & -1.14 & 1.46 & -4.64 & $<.001$ & -1.64 & -0.64 & -0.78 \\
\hline Mad & 1.17 & 0.38 & 1.17 & 0.38 & 0.00 & 0.25 & 0.00 & 1.00 & -0.08 & 0.08 & 0.00 \\
\hline
\end{tabular}

Note. $\mathrm{CI}=$ Confidence Interval of the Difference; $L L=$ lower limit; $U L=$ upper limit. *After minus Before; positive values indicate an increased rating. 
Table 9

Study Three: Contrast of Mean Emotion Ratings Before and After Cry Freedom Film Clip and No Writing Task

\begin{tabular}{|c|c|c|c|c|c|c|c|c|c|c|c|}
\hline \multirow[b]{2}{*}{ Emotion } & \multicolumn{2}{|c|}{ Before Film } & \multicolumn{2}{|c|}{ After Film } & \multicolumn{2}{|c|}{ Difference* } & \multirow[b]{2}{*}{$t(36)$} & \multirow[b]{2}{*}{$p$} & \multicolumn{2}{|c|}{$95 \% \mathrm{CI}$} & \multirow[b]{2}{*}{ Cohen's $d$} \\
\hline & $M$ & $S D$ & $M$ & $S D$ & $M$ & $S D$ & & & $L L$ & $U L$ & \\
\hline Upset & 1.32 & 0.67 & 4.00 & 1.11 & 2.68 & 1.33 & 12.20 & $<.001$ & 2.23 & 3.12 & 2.01 \\
\hline Hostile & 1.14 & 0.35 & 1.70 & 0.97 & 0.57 & 0.93 & 3.72 & .001 & 0.26 & 0.88 & 0.61 \\
\hline Alert & 3.00 & 1.25 & 3.22 & 1.34 & 0.22 & 1.72 & 0.77 & .449 & -0.36 & 0.79 & 0.13 \\
\hline Ashamed & 1.08 & 0.28 & 1.97 & 1.24 & 0.89 & 1.26 & 4.29 & $<.001$ & 0.47 & 1.31 & 0.71 \\
\hline Angry & 1.16 & 0.37 & 3.68 & 1.36 & 2.51 & 1.48 & 10.31 & $<.001$ & 2.02 & 3.01 & 1.69 \\
\hline Nervous & 1.41 & 0.72 & 1.49 & 0.80 & 0.08 & 0.80 & 0.62 & .539 & -0.18 & 0.35 & 0.10 \\
\hline Determined & 3.00 & 1.22 & 2.03 & 1.21 & -0.97 & 1.26 & -4.71 & $<.001$ & -1.39 & -0.55 & -0.77 \\
\hline Attentive & 3.41 & 1.09 & 3.68 & 1.03 & 0.27 & 1.28 & 1.28 & .208 & -0.16 & 0.70 & 0.21 \\
\hline Afraid & 1.03 & 0.16 & 1.43 & 0.69 & 0.41 & 0.64 & 3.83 & $<.001$ & 0.19 & 0.62 & 0.63 \\
\hline Active & 2.62 & 1.26 & 2.11 & 1.29 & -0.51 & 1.28 & -2.44 & .020 & -0.94 & -0.09 & -0.40 \\
\hline Inspired & 2.32 & 1.38 & 1.46 & 1.04 & -0.86 & 1.53 & -3.44 & .001 & -1.38 & -0.35 & -0.57 \\
\hline Mad & 1.14 & 0.35 & 3.14 & 1.29 & 2.00 & 1.39 & 8.72 & $<.001$ & 1.54 & 2.46 & 1.43 \\
\hline
\end{tabular}

Note. $\mathrm{CI}=$ Confidence Interval of the Difference; $L L=$ lower limit; $U L=$ upper limit. *After minus Before; positive values indicate an increased rating. 
Table 10

Study Three: Contrast of Mean Emotion Ratings Before and After My Bodyguard Film Clip and No Writing Task

\begin{tabular}{|c|c|c|c|c|c|c|c|c|c|c|c|}
\hline \multirow[b]{2}{*}{ Emotion } & \multicolumn{2}{|c|}{ Before Film } & \multicolumn{2}{|c|}{ After Film } & \multicolumn{2}{|c|}{ Difference* } & \multirow[b]{2}{*}{$t(29)$} & \multirow[b]{2}{*}{$p$} & \multicolumn{2}{|c|}{$95 \% \mathrm{CI}$} & \multirow[b]{2}{*}{ Cohen's $d$} \\
\hline & $M$ & $S D$ & $M$ & $S D$ & $M$ & $S D$ & & & $L L$ & $U L$ & \\
\hline Upset & 1.17 & 0.38 & 2.77 & 1.48 & 1.60 & 1.43 & 6.13 & $<.001$ & 1.07 & 2.13 & 1.12 \\
\hline Hostile & 1.17 & 0.46 & 1.53 & 0.78 & 0.37 & 0.96 & 2.08 & .046 & 0.01 & 0.73 & 0.38 \\
\hline Alert & 3.43 & 1.25 & 3.53 & 1.28 & 0.10 & 1.12 & 0.49 & .630 & -0.32 & 0.52 & 0.09 \\
\hline Ashamed & 1.03 & 0.18 & 1.47 & 0.86 & 0.43 & 0.86 & 2.77 & .010 & 0.11 & 0.75 & 0.50 \\
\hline Angry & 1.07 & 0.25 & 2.37 & 1.33 & 1.30 & 1.34 & 5.30 & $<.001$ & 0.80 & 1.80 & 0.97 \\
\hline Nervous & 1.33 & 0.76 & 1.27 & 0.52 & -0.07 & 0.78 & -0.47 & .645 & -0.36 & 0.23 & -0.08 \\
\hline Determined & 3.40 & 1.30 & 2.80 & 1.45 & -0.60 & 1.16 & -2.83 & .008 & -1.03 & -0.17 & -0.52 \\
\hline Attentive & 3.73 & 0.94 & 4.00 & 0.83 & 0.27 & 0.52 & 2.80 & .009 & 0.07 & 0.46 & 0.51 \\
\hline Afraid & 1.00 & 0.00 & 1.20 & 0.41 & 0.20 & 0.41 & 2.69 & .012 & 0.05 & 0.35 & 0.49 \\
\hline Active & 3.00 & 1.34 & 2.37 & 1.50 & -0.63 & 1.03 & -3.36 & .002 & -1.02 & -0.25 & -0.61 \\
\hline Inspired & 2.90 & 1.37 & 1.90 & 1.45 & -1.00 & 1.31 & -4.17 & $<.001$ & -1.49 & -0.51 & -0.76 \\
\hline$\underline{\mathrm{Mad}}$ & 1.10 & 0.31 & 2.13 & 1.22 & 1.03 & 1.19 & 4.76 & $<.001$ & 0.59 & 1.48 & 0.87 \\
\hline
\end{tabular}

Note. $\mathrm{CI}=$ Confidence Interval of the Difference; $L L=$ lower limit; $U L=$ upper limit. *After minus Before; positive values indicate an increased rating. 


\section{Comparing Relative Effectiveness of each Film Clip and Induction Technique}

In order to evaluate the effectiveness of emotion induction for each of the four film clips, I first looked to the emotions rated during the PANAS-X posttest which bore the closest relationships to my broader constructs of fear and anger. "Mad" and "anger" ratings were used as an analogue for assessing anger, while "afraid," "nervous," "scared," and "frightened" were used as representations of fear. I compared the two fear target clips to each other for postfilm ratings of the six benchmark emotions while also comparing the presence or absence of the writing task. Separate $2 \times 2$ (fear film levels $x$ writing task) factorial analysis of variance conducted for each of the six benchmarks yielded no significant differences except for afraid, where the film clip showed a significant main effect, $F(1,127)=4.03, p=.047, \eta^{2}=.031$, with participants who watched The Shining rating afraid higher $(M=2.31)$ than participants who watched The Silence of the Lambs $(M=1.89)$ on a scale from 1-very slightly or not at all to 5extremely. Because afraid ratings are obviously such a critical dimension in my overall goal of inducing fear and high levels of uncertainty through the use of film clips, this was a very important distinction I considered when making my ultimate choice of which film clip best induces fear and uncertainty.

I conducted the same series of separate $2 \times 2$ (film $\times$ writing task) factorial analysis of variance for the postfilm ratings of each of the six benchmark emotion comparing only the clips intended to induce anger. There was a significant main effect on angry ratings for the specific film seen $F(1,114)=22.96, p<.001, \eta^{2}=.168$ such that those participants who watched the Cry Freedom clip gave much higher ratings on angry $(M=3.67)$ than did those who watched the clip from My Bodyguard $(M=2.42)$. There 
was also a significant main effect of film clip on mad ratings $F(1,114)=12.07, p=.001$, $\eta^{2}=.096$ wherein Cry Freedom resulted in higher ratings $(M=3.17)$ than My Bodyguard $(M=2.25)$.

Viewed in isolation, these two relatively strong differences would suggest that Cry Freedom might make a much better film for my purpose of inducing anger. However, that preference is based solely on effectiveness for inducing anger. It is equally imperative that the film clips I use must elicit only their intended emotion and not the opposing one as well. For this reason, the otherwise powerful emotional impact of the Cry Freedom clip dramatically loses viability for my specific needs. A 2 x 2 (film x writing task) factorial analysis for variance in postfilm ratings of frightened revealed a main effect for the film, $F(1,114)=26.52, p<.001, \eta^{2}=.189$ with Cry Freedom having a greater frightened rating $(M=2.07)$ than My Bodyguard $(M=1.20)$. The same analysis for scared showed a main effect for the film, $F(1,114)=17.14, p<.001, \eta^{2}=.131$ with Cry Freedom having a greater scared rating $(M=1.76)$ than My Bodyguard $(M=1.23)$, as well as a main effect for the writing task, $F(1,114)=13.85, p<.001, \eta^{2}=.108$ with those who completed the writing task having a greater scared rating $(M=1.85)$ than did the induction format without the writing task $(M=1.27)$. These two main effects were qualified by a significant interaction between the two factors, $F(1,114)=4.09, p=.046$, $\eta^{2}=.035$, which a simple effects analysis for the writing task indicated that the mean scared ratings given by those participants who engaged in the writing task were significantly greater $(M=1.76)$ than those who did not complete the additional task $(M=$ 1.28) which indicates that the writing task led to greater scared ratings $(F(1,114)=$ $\left.16.56, p=.004, \eta^{2}=.108\right)$. An accompanying simple effects analysis for film clip also 
indicated a significant difference in the mean scared ratings given by those participants who watched the Cry Freedom clip $(M=1.76)$ than those given by participants after watching the My Bodyguard clip ( $M=1.23)$, indicating that the Cry Freedom clip significantly increased scared ratings compared to the My Bodyguard clip $(F(1,114)=$ $\left.15.60, p=.003, \eta^{2}=.108\right)$.

Not surprisingly, given the near interchangeability of the words, afraid ratings showed similar effects to the scared ratings among those same participants who watched either of the two film clips intended to induce anger. After a $2 \times 2$ (film $x$ writing task) factorial analysis for variance in postfilm ratings of afraid, a significant main effect for the writing task emerged $\left(F(1,114)=13.16, p<.001, \eta^{2}=.103\right)$ with those participants who completed the writing task having given significantly greater afraid ratings ( $M=$ 1.73) than those who did not complete that task $(M=1.33)$. A significant main effect for film clip also emerged $\left(F(1,114)=31.94, p<.001, \eta^{2}=.219\right)$, with participants who watched the Cry Freedom clip reporting greater afraid ratings $(M=1.84)$ than those who watched the My Bodyguard clip ( $M=1.17)$. Just as with scared ratings, these two main effects showed a significant interaction between the two factors $(F(1,114)=16.64, p<$ $.001, \eta^{2}=.127$ ). A simple effects analysis for instruction type showed that those participants who engaged in the writing task reported significantly greater afraid ratings $(M=1.73)$ than those participants who did not complete the writing task $(M=1.33)$ which again indicates that the writing exercise strengthened feelings of being afraid ( $F(1$, $\left.114)=41.34, p<.001, \eta^{2}=.103\right)$. Another simple effects analysis for film clip also indicated a significant difference in the mean afraid ratings given by those participants who watched the Cry Freedom clip $(M=1.84)$ than those given by participants after 
watching the My Bodyguard clip $(M=1.17)$, indicating that the Cry Freedom clip significantly increased afraid ratings compared to the My Bodyguard clip $(F(1,114)=$ 28.12, $\left.p<.001, \eta^{2}=.127\right)$.

Finally, the last relationships of note among the six benchmark emotion ratings for those participants who watched either of the anger inducing film clips relates to the dimension of nervousness, an emotion characterized by a high degree of uncertainty. I conducted the same $2 \times 2$ (anger films x writing task) factorial analysis for variance in postfilm ratings given for nervous, which demonstrated a significant main effect for both the writing task, $F(1,114)=4.48, p=.036, \eta^{2}=.038$, and for film clip, $F(1,114)=4.12$, $p=.045, \eta^{2}=.035$. These effects showed that mean ratings for nervousness were greater among those participants who underwent the writing task in addition to the film clips ( $M$ $=1.70)$ than for those participants who simply watched the film clips $(M=1.38)$. The participants who saw the Cry Freedom film clip gave significantly higher responses $(M=$ 2.07) for nervous than participants who watched the My Bodyguard clip $(M=1.20)$. Two of my goals for Study Three were to identify which film clips to use in order to most effectively induce anger or fear, as well as to determine whether the two part induction procedure featuring the autobiographical, perspective taking writing task was more or less effective at inducing my two target emotions than just watching the film clip without the writing task. These two objectives were critical to the success of my larger goal for Study Three, which was to finalize an empirically verified means of reliably inducing and measuring not simply the broader emotions of anger and fear, but rather inducing (and determining how to reliably measure) high and low levels of the more specific appraisal of certainty. As such, discovering significant main effects for a highly resemblant 
dimension like nervousness was very diagnostic and dispositive - it was utterly contrary to my purposes to have my anger induction leading to increased uncertainty, so because both the Cry Freedom clip and the writing task each exhibited main effects leading to greater levels of nervousness, my final emotion/certainty induction techniques became more apparent.

In order to even further confirm that the clip from My Bodyguard was superior to the clip from Cry Freedom, and having known that the writing task complicated the emotion induction to such a degree that eliminating it from consideration was the most prudent course of action, using responses from only those participants who were not instructed to complete the writing task, I ran a multivariate analysis of variance with the two anger-inducing film clips (Cry Freedom and My Bodyguard) as the independent variables and postfilm ratings of afraid, shaky, nervous, jittery, scared, and frightened as the dependent variables. There was a significant effect, $F(6,60)=3.13, p=.01, \eta^{2}=$ .238. Follow-up univariate tests with Bonferroni adjustment for multiple comparisons showed significance for shaky, $F(1,65)=10.02, p=.002$, scared, $F(1,65)=7.54, p=$ .008 , and frightened, $F(1,65)=10.10, p=.002$. After watching Cry Freedom, mean ratings for shaky $(M=2.16)$, scared $(M=1.43)$, and frightened $(M=1.87)$, emotions which should not be elicited during the anger induction film clip, were each significantly greater than after watching the My Bodyguard clip, with mean ratings of 1.34 for shaky, 1.10 for scared, and 1.20 for frightened.

Taken together, this array of unique and recursive main effects and interactions suggest that with respect to the anger induction manipulations, the Cry Freedom clip and the writing task combine to cause significantly increased ratings of fear and uncertainty- 
related emotions. In essence, either the Cry Freedom film clip or the writing task could each reduce the overall range of my certainty manipulations, but the Cry Freedom clip along with the writing task in particular would be the very worst choice to use as my anger induction technique.

After having conclusively determined that the My Bodyguard clip was preferable to the Cry Freedom clip, I needed to confirm that the more effective means of induction was in fact to avoid having participants engage in the writing task. To do this, I conducted an individual analysis of variance on the postfilm ratings for each of the six benchmark emotions (angry, mad, afraid, nervous, scared, and frightened) I had selected to most accurately illustrate induction effectiveness for any differences due presence or absence of the writing task after having watched the My Bodyguard clip. No statistically significant differences emerged, however ratings for scared were close to statistical significance $F(1,58)=3.95, p=.052, \eta^{2}=.064$ (note that no Bonferroni adjustment to the $p$ value for significance was necessary since there was only a single comparison made). However, at this level of non-significance, the mean scared ratings among participants who completed the writing task $(M=1.37, S D=0.67,95 \% \mathrm{CI}[1.18,1.56])$ was greater than those reported by the participants who did not engage in the writing task ( $M=1.10, S D=0.31,95 \%$ CI $[0.91,1.29])$. Despite being just outside the limits of statistical significance, the proximity presents a potential problem should values fluctuate slightly. Because I wished to minimize any potential for fear-based or uncertain emotions to increase after having watched the anger/certainty inducing film clip, it seemed judicious to err on the side of caution and elect to eliminate the writing task component of the inductions. 
In contrast to the myriad considerations and comparisons required to sensibly evaluate the two anger induction films and the value of the writing task in this context, a $2 \times 2$ (fear films $\mathrm{x}$ writing task) factorial analysis of variance for the postfilm ratings of each of the six benchmark emotion comparing only the clips intended to induce fear, there was only one significant main effect among all six of the benchmark emotion ratings I looked at. The mean ratings for afraid were significantly greater for The Shining ( $M=2.31)$ compared to The Silence of the Lambs $(M=1.89)$, which, even though it is not an especially large difference, nevertheless presented a very compelling reason why I should use the clip from The Shining to experimentally induce fear, $F(1,127)=4.029, p$ $=.047, \eta^{2}=.031$. To confirm that there would be no drawbacks to eliminating the writing task with respect to inducing fear and uncertainty with the clip from The Shining, I conducted the same series of individual analyses of variance on the postfilm ratings for each of the six benchmark emotions with or without the writing task after having watched The Shining, and no differences even approaching statistical significance emerged.

My final step to confirm that watching film clips from The Shining and My Bodyguard without the writing task was the optimal method of inducing fear and anger, respectively, was to run a multivariate analysis of variance with The Shining, The Silence of the Lambs, and My Bodyguard film clips as the independent variables (having conclusively rejected Cry Freedom, there was no benefit to be gained from including it at the expense of statistical reliability) and all 61 postfilm emotion ratings from the PANAS-X as the dependent variables. Afraid, nervous, scared, frightened, and jittery ratings were each significantly greater for participants who watched either The Shining or The Silence of the Lambs than for those who watched My Bodyguard. Angry, mad, 
disgusted, irritable, sad, upset, and ashamed ratings were each significantly greater for participants who watched My Bodyguard than either The Shining or The Silence of the Lambs. Fearless, bold, and loathing ratings were all significantly greater for participants who watched My Bodyguard than The Shining, while alone and lonely ratings were significantly higher after having watched The Shining than My Bodyguard. Shaky and sheepish were both greater among participants who saw the clip from The Silence of the Lambs than those who saw My Bodyguard, but there was no significant difference between viewers of The Shining and either of the other two movies for these emotions. Table 12 features complete pairwise comparisons for all 19 emotions with at least one significant difference between movies.

Since I intended to use the emotion inducing film clips for the slightly novel purpose of inducing certainty, I wanted to confirm that the inductions as I had implemented them were effective to the same extent as when they were validated by Rottenberg, Ray, and Gross (2007). See Tables 13 and 14 for mean emotion ratings on the Post Film Questionnaire (see Appendix A) for both The Shining and My Bodyguard ratings from the present study in comparison to Rottenberg et al.'s ratings. My findings tracked quite well with the original data set, which lends validity and credibility to my design. 
Table 11

Study Three: Pairwise Comparisons of Significant Differences in Postfilm Emotion Ratings

\begin{tabular}{|c|c|c|c|c|c|c|c|}
\hline \multirow[b]{2}{*}{ Emotion } & \multirow[b]{2}{*}{ Film I } & \multirow[b]{2}{*}{ Film J } & \multirow{2}{*}{$\begin{array}{c}\text { Mean } \\
\text { Difference } \\
(\mathrm{I}-\mathrm{J}) \\
\end{array}$} & \multirow[b]{2}{*}{$S E$} & \multirow[b]{2}{*}{$p^{\mathrm{a}}$} & \multicolumn{2}{|c|}{$\begin{array}{l}95 \% \text { CI for } \\
\text { Difference }^{\mathrm{a}} \\
\end{array}$} \\
\hline & & & & & & $L L$ & $U L$ \\
\hline \multirow[t]{6}{*}{ Afraid } & \multirow[t]{2}{*}{ The Shining } & $\begin{array}{c}\text { The Silence of } \\
\text { the Lambs }\end{array}$ & .194 & .235 & 1.000 & -.379 & .768 \\
\hline & & My Bodyguard & $.994^{*}$ & .245 & .000 & .397 & 1.592 \\
\hline & \multirow{2}{*}{$\begin{array}{c}\text { The Silence of } \\
\text { the Lambs }\end{array}$} & The Shining & -.194 & .235 & 1.000 & -.768 & .379 \\
\hline & & My Bodyguard & $.800^{*}$ & .247 & .005 & .199 & 1.401 \\
\hline & \multirow[b]{2}{*}{ My Bodyguard } & The Shining & $-.994^{*}$ & .245 & .000 & -1.592 & -.397 \\
\hline & & $\begin{array}{c}\text { The Silence of } \\
\text { the Lambs }\end{array}$ & $-.800^{*}$ & .247 & .005 & -1.401 & -.199 \\
\hline \multirow[t]{6}{*}{ Nervous } & \multirow[t]{2}{*}{ The Shining } & $\begin{array}{c}\text { The Silence of } \\
\text { the Lambs }\end{array}$ & .217 & .261 & 1.000 & -.418 & .852 \\
\hline & & My Bodyguard & $1.150^{*}$ & .272 & .000 & .489 & 1.811 \\
\hline & \multirow{2}{*}{$\begin{array}{c}\text { The Silence of } \\
\text { the Lambs }\end{array}$} & The Shining & -.217 & .261 & 1.000 & -.852 & .418 \\
\hline & & My Bodyguard & $.933^{*}$ & .273 & .003 & .268 & 1.599 \\
\hline & \multirow[b]{2}{*}{ My Bodyguard } & The Shining & $-1.150^{*}$ & .272 & .000 & -1.811 & -.489 \\
\hline & & $\begin{array}{c}\text { The Silence of } \\
\text { the Lambs }\end{array}$ & $-.933^{*}$ & .273 & .003 & -1.599 & -.268 \\
\hline \multirow[t]{6}{*}{ Scared } & \multirow[t]{2}{*}{ The Shining } & $\begin{array}{c}\text { The Silence of } \\
\text { the Lambs }\end{array}$ & .222 & .255 & 1.000 & -.398 & .843 \\
\hline & & My Bodyguard & $1.122^{*}$ & .265 & .000 & .476 & 1.769 \\
\hline & \multirow{2}{*}{$\begin{array}{c}\text { The Silence of } \\
\text { the Lambs }\end{array}$} & The Shining & -.222 & .255 & 1.000 & -.843 & .398 \\
\hline & & My Bodyguard & $.900^{*}$ & .267 & .003 & .249 & 1.551 \\
\hline & \multirow[b]{2}{*}{ My Bodyguard } & The Shining & $-1.122^{*}$ & .265 & .000 & -1.769 & -.476 \\
\hline & & $\begin{array}{c}\text { The Silence of } \\
\text { the Lambs }\end{array}$ & $-.900^{*}$ & .267 & .003 & -1.551 & -.249 \\
\hline \multirow[t]{2}{*}{ Frightened } & \multirow[t]{2}{*}{ The Shining } & $\begin{array}{c}\text { The Silence of } \\
\text { the Lambs }\end{array}$ & .029 & .260 & 1.000 & -.605 & .662 \\
\hline & & My Bodyguard & $.800^{*}$ & .271 & .012 & .140 & 1.460 \\
\hline
\end{tabular}




\begin{tabular}{|c|c|c|c|c|c|c|}
\hline & The Silence of & The Shining & -.029 & .2601 .000 & -.662 & .605 \\
\hline & the Lambs & My Bodyguard & $.771^{*}$ & $.273 \quad .017$ & .107 & 1.436 \\
\hline & & The Shining & $-.800^{*}$ & $.271 \quad .012$ & -1.460 & -.140 \\
\hline & My Bodyguard & $\begin{array}{c}\text { The Silence of } \\
\text { the Lambs }\end{array}$ & $-.771^{*}$ & $.273 \quad .017$ & -1.436 & -.107 \\
\hline \multirow[t]{6}{*}{ Shaky } & \multirow[t]{2}{*}{ The Shining } & $\begin{array}{c}\text { The Silence of } \\
\text { the Lambs }\end{array}$ & -.336 & $.245 \quad .522$ & -.933 & .261 \\
\hline & & My Bodyguard & .383 & $.255 \quad .409$ & -.238 & 1.005 \\
\hline & \multirow{2}{*}{$\begin{array}{c}\text { The Silence of } \\
\text { the Lambs }\end{array}$} & The Shining & .336 & $.245 \quad .522$ & -.261 & .933 \\
\hline & & My Bodyguard & $.719^{*}$ & $.257 \quad .019$ & .093 & 1.345 \\
\hline & \multirow[b]{2}{*}{ My Bodyguard } & The Shining & -.383 & .255 .409 & -1.005 & .238 \\
\hline & & $\begin{array}{c}\text { The Silence of } \\
\text { the Lambs }\end{array}$ & $-.719^{*}$ & $.257 \quad .019$ & -1.345 & -.093 \\
\hline \multirow[t]{6}{*}{ Jittery } & \multirow[t]{2}{*}{ The Shining } & $\begin{array}{c}\text { The Silence of } \\
\text { the Lambs }\end{array}$ & -.113 & .2541 .000 & -.732 & .505 \\
\hline & & My Bodyguard & $.672^{*}$ & $.264 \quad .038$ & .028 & 1.316 \\
\hline & \multirow{2}{*}{$\begin{array}{c}\text { The Silence of } \\
\text { the Lambs }\end{array}$} & The Shining & .113 & .2541 .000 & -.505 & .732 \\
\hline & & My Bodyguard & $.786^{*}$ & $.266 \quad .012$ & .138 & 1.434 \\
\hline & \multirow[b]{2}{*}{ My Bodyguard } & The Shining & $-.672^{*}$ & $.264 \quad .038$ & -1.316 & -.028 \\
\hline & & $\begin{array}{c}\text { The Silence of } \\
\text { the Lambs }\end{array}$ & $-.786^{*}$ & $.266 \quad .012$ & -1.434 & -.138 \\
\hline \multirow[t]{6}{*}{ Angry } & \multirow[t]{2}{*}{ The Shining } & $\begin{array}{c}\text { The Silence of } \\
\text { the Lambs }\end{array}$ & -.235 & $.217 \quad .845$ & -.763 & .294 \\
\hline & & My Bodyguard & $-1.144^{*}$ & $.226 \quad .000$ & -1.695 & -.594 \\
\hline & \multirow{2}{*}{$\begin{array}{c}\text { The Silence of } \\
\text { the Lambs }\end{array}$} & The Shining & .235 & $.217 \quad .845$ & -.294 & .763 \\
\hline & & My Bodyguard & $-.910^{*}$ & $.227 \quad .000$ & -1.463 & -.356 \\
\hline & \multirow[b]{2}{*}{ My Bodyguard } & The Shining & $1.144^{*}$ & $.226 \quad .000$ & .594 & 1.695 \\
\hline & & $\begin{array}{c}\text { The Silence of } \\
\text { the Lambs }\end{array}$ & $.910^{*}$ & $.227 \quad .000$ & .356 & 1.463 \\
\hline \multirow[t]{3}{*}{ Mad } & \multirow[t]{2}{*}{ The Shining } & $\begin{array}{c}\text { The Silence of } \\
\text { the Lambs }\end{array}$ & .023 & .1861 .000 & -.430 & .476 \\
\hline & & My Bodyguard & $-.939^{*}$ & .194 .000 & -1.410 & -.467 \\
\hline & The Silence of & The Shining & -.023 & .1861 .000 & -.476 & .430 \\
\hline
\end{tabular}




\begin{tabular}{|c|c|c|c|c|c|c|c|}
\hline & the Lambs & My Bodyguard & $-.962^{*}$ & .195 & .000 & -1.436 & -.487 \\
\hline & \multirow[b]{2}{*}{ My Bodyguard } & The Shining & $.939^{*}$ & .194 & .000 & .467 & 1.410 \\
\hline & & $\begin{array}{c}\text { The Silence of } \\
\text { the Lambs }\end{array}$ & $.962^{*}$ & .195 & . 000 & .487 & 1.436 \\
\hline \multirow[t]{6}{*}{ Disgusted } & \multirow{2}{*}{ The Shining } & $\begin{array}{c}\text { The Silence of } \\
\text { the Lambs }\end{array}$ & $-1.662^{*}$ & .238 & . 000 & -2.242 & -1.082 \\
\hline & & My Bodyguard & $-2.500^{*}$ & .248 & .000 & -3.104 & -1.896 \\
\hline & \multirow{2}{*}{$\begin{array}{c}\text { The Silence of } \\
\text { the Lambs }\end{array}$} & The Shining & $1.662^{*}$ & .238 & .000 & 1.082 & 2.242 \\
\hline & & My Bodyguard & $-.838^{*}$ & .250 & .003 & -1.446 & -.230 \\
\hline & \multirow[b]{2}{*}{ My Bodyguard } & The Shining & $2.500^{*}$ & .248 & .000 & 1.896 & 3.104 \\
\hline & & $\begin{array}{c}\text { The Silence of } \\
\text { the Lambs }\end{array}$ & $.838^{*}$ & .250 & .003 & .230 & 1.446 \\
\hline \multirow[t]{6}{*}{ Surprised } & \multirow[t]{2}{*}{ The Shining } & $\begin{array}{c}\text { The Silence of } \\
\text { the Lambs }\end{array}$ & $-.885^{*}$ & .310 & 016 & -1.639 & -.131 \\
\hline & & My Bodyguard & $-.894^{*}$ & .322 & .020 & -1.680 & -.109 \\
\hline & \multirow{2}{*}{$\begin{array}{c}\text { The Silence of } \\
\text { the Lambs }\end{array}$} & The Shining & $.885^{*}$ & .310 & .016 & .131 & 1.639 \\
\hline & & My Bodyguard & -.010 & .325 & 1.000 & -.800 & .781 \\
\hline & \multirow[b]{2}{*}{ My Bodyguard } & The Shining & $.894^{*}$ & .322 & .020 & .109 & 1.680 \\
\hline & & $\begin{array}{c}\text { The Silence of } \\
\text { the Lambs }\end{array}$ & .010 & .325 & 1.000 & -.781 & .800 \\
\hline \multirow[t]{6}{*}{ Scornful } & \multirow[t]{2}{*}{ The Shining } & $\begin{array}{c}\text { The Silence of } \\
\text { the Lambs }\end{array}$ & $-.520^{*}$ & .205 & .038 & -1.019 & -.021 \\
\hline & & My Bodyguard & $-1.106^{*}$ & .213 & .000 & -1.626 & -.586 \\
\hline & \multirow{2}{*}{$\begin{array}{c}\text { The Silence of } \\
\text { the Lambs }\end{array}$} & The Shining & $.520^{*}$ & .205 & .038 & .021 & 1.019 \\
\hline & & My Bodyguard & $-.586^{*}$ & .215 & .023 & -1.109 & -.062 \\
\hline & \multirow[b]{2}{*}{ My Bodyguard } & The Shining & $1.106^{*}$ & .213 & .000 & .586 & 1.626 \\
\hline & & $\begin{array}{c}\text { The Silence of } \\
\text { the Lambs }\end{array}$ & $.586^{*}$ & .215 & .023 & .062 & 1.109 \\
\hline \multirow[t]{4}{*}{ Irritable } & \multirow{2}{*}{ The Shining } & $\begin{array}{c}\text { The Silence of } \\
\text { the Lambs }\end{array}$ & -.159 & .246 & 1.000 & -.757 & .439 \\
\hline & & My Bodyguard & $-1.244^{*}$ & .256 & .000 & -1.867 & -.622 \\
\hline & \multirow{2}{*}{$\begin{array}{c}\text { The Silence of } \\
\text { the Lambs }\end{array}$} & The Shining & .159 & .246 & 1.000 & -.439 & .757 \\
\hline & & My Bodyguard & $-1.086^{*}$ & .257 & .000 & -1.713 & -.459 \\
\hline
\end{tabular}




\begin{tabular}{|c|c|c|c|c|c|c|}
\hline & & The Shining & $1.244^{*}$ & .256 .000 & .622 & 1.867 \\
\hline & My Bodyguard & $\begin{array}{c}\text { The Silence of } \\
\text { the Lambs }\end{array}$ & $1.086^{*}$ & $.257 \quad .000$ & .459 & 1.713 \\
\hline \multirow[t]{6}{*}{ Fearless } & \multirow[t]{2}{*}{ The Shining } & $\begin{array}{c}\text { The Silence of } \\
\text { the Lambs }\end{array}$ & -.113 & .2921 .000 & -.823 & .598 \\
\hline & & My Bodyguard & $-.756^{*}$ & $.304 \quad .044$ & -1.496 & -.015 \\
\hline & \multirow{2}{*}{$\begin{array}{c}\text { The Silence of } \\
\text { the Lambs }\end{array}$} & The Shining & .113 & .2921 .000 & -.598 & .823 \\
\hline & & My Bodyguard & -.643 & $.306 \quad .114$ & -1.388 & .102 \\
\hline & \multirow[b]{2}{*}{ My Bodyguard } & The Shining & $.756^{*}$ & $.304 \quad .044$ & .015 & 1.496 \\
\hline & & $\begin{array}{c}\text { The Silence of } \\
\text { the Lambs }\end{array}$ & .643 & .306 .114 & -.102 & 1.388 \\
\hline \multirow[t]{6}{*}{$\mathrm{Sad}$} & \multirow[t]{2}{*}{ The Shining } & $\begin{array}{c}\text { The Silence of } \\
\text { the Lambs }\end{array}$ & -.351 & $.198 \quad .239$ & -.833 & .132 \\
\hline & & My Bodyguard & $-.922^{*}$ & $.206 \quad .000$ & -1.425 & -.420 \\
\hline & \multirow{2}{*}{$\begin{array}{c}\text { The Silence of } \\
\text { the Lambs }\end{array}$} & The Shining & .351 & $.198 \quad .239$ & -.132 & .833 \\
\hline & & My Bodyguard & $-.571^{*}$ & $.208 \quad .021$ & -1.077 & -.066 \\
\hline & \multirow[b]{2}{*}{ My Bodyguard } & The Shining & $.922^{*}$ & .206 .000 & .420 & 1.425 \\
\hline & & $\begin{array}{c}\text { The Silence of } \\
\text { the Lambs }\end{array}$ & $.571^{*}$ & $.208 \quad .021$ & .066 & 1.077 \\
\hline \multirow[t]{6}{*}{ Alone } & \multirow[t]{2}{*}{ The Shining } & $\begin{array}{c}\text { The Silence of } \\
\text { the Lambs }\end{array}$ & .391 & .246 .344 & -.207 & .990 \\
\hline & & My Bodyguard & $.972^{*}$ & $.256 \quad .001$ & .349 & 1.596 \\
\hline & \multirow{2}{*}{$\begin{array}{c}\text { The Silence of } \\
\text { the Lambs }\end{array}$} & The Shining & -.391 & $.246 \quad .344$ & -.990 & .207 \\
\hline & & My Bodyguard & .581 & $.258 \quad .079$ & -.046 & 1.208 \\
\hline & \multirow[b]{2}{*}{ My Bodyguard } & The Shining & $-.972^{*}$ & $.256 \quad .001$ & -1.596 & -.349 \\
\hline & & $\begin{array}{c}\text { The Silence of } \\
\text { the Lambs }\end{array}$ & -.581 & $.258 \quad .079$ & -1.208 & .046 \\
\hline \multirow[t]{5}{*}{ Upset } & \multirow[t]{2}{*}{ The Shining } & $\begin{array}{c}\text { The Silence of } \\
\text { the Lambs }\end{array}$ & -.160 & .2741 .000 & -.827 & .506 \\
\hline & & My Bodyguard & $-1.156^{*}$ & $.285 \quad .000$ & -1.849 & -.462 \\
\hline & \multirow{2}{*}{$\begin{array}{c}\text { The Silence of } \\
\text { the Lambs }\end{array}$} & The Shining & .160 & .2741 .000 & -.506 & .827 \\
\hline & & My Bodyguard & $-.995^{*}$ & $.287 \quad .002$ & -1.693 & -.297 \\
\hline & My Bodyguard & The Shining & $1.156^{*}$ & $.285 \quad .000$ & .462 & 1.849 \\
\hline
\end{tabular}




\begin{tabular}{|c|c|c|c|c|c|c|}
\hline & & $\begin{array}{c}\text { The Silence of } \\
\text { the Lambs }\end{array}$ & $.995^{*}$ & $.287 \quad .002$ & .297 & 1.693 \\
\hline \multirow[t]{6}{*}{ Bold } & \multirow[t]{2}{*}{ The Shining } & $\begin{array}{c}\text { The Silence of } \\
\text { the Lambs }\end{array}$ & -.166 & .2711 .000 & -.827 & .495 \\
\hline & & My Bodyguard & $-.761^{*}$ & $.282 \quad .025$ & -1.449 & -.073 \\
\hline & \multirow{2}{*}{$\begin{array}{c}\text { The Silence of } \\
\text { the Lambs }\end{array}$} & The Shining & .166 & .2711 .000 & -.495 & .827 \\
\hline & & My Bodyguard & -.595 & .284 .117 & -1.288 & .097 \\
\hline & \multirow[b]{2}{*}{ My Bodyguard } & The Shining & $.761^{*}$ & $.282 \quad .025$ & .073 & 1.449 \\
\hline & & $\begin{array}{c}\text { The Silence of } \\
\text { the Lambs }\end{array}$ & .595 & $.284 \quad .117$ & -.097 & 1.288 \\
\hline \multirow[t]{6}{*}{ Lonely } & \multirow[t]{2}{*}{ The Shining } & $\begin{array}{c}\text { The Silence of } \\
\text { the Lambs }\end{array}$ & .426 & .214 .149 & -.096 & .949 \\
\hline & & My Bodyguard & $.850^{*}$ & $.223 \quad .001$ & .306 & 1.394 \\
\hline & \multirow{2}{*}{$\begin{array}{c}\text { The Silence of } \\
\text { the Lambs }\end{array}$} & The Shining & -.426 & .214 .149 & -.949 & .096 \\
\hline & & My Bodyguard & .424 & $.225 \quad .187$ & -.124 & .971 \\
\hline & \multirow[b]{2}{*}{ My Bodyguard } & The Shining & $-.850^{*}$ & .223 .001 & -1.394 & -.306 \\
\hline & & $\begin{array}{c}\text { The Silence of } \\
\text { the Lambs }\end{array}$ & -.424 & .225 .187 & -.971 & .124 \\
\hline \multirow[t]{6}{*}{ Ashamed } & \multirow[t]{2}{*}{ The Shining } & $\begin{array}{c}\text { The Silence of } \\
\text { the Lambs }\end{array}$ & .025 & .1311 .000 & -.293 & .344 \\
\hline & & My Bodyguard & $-.356^{*}$ & $.136 \quad .031$ & -.687 & -.024 \\
\hline & \multirow{2}{*}{$\begin{array}{c}\text { The Silence of } \\
\text { the Lambs }\end{array}$} & The Shining & -.025 & .1311 .000 & -.344 & .293 \\
\hline & & My Bodyguard & $-.381^{*}$ & $.137 \quad .020$ & -.715 & -.047 \\
\hline & \multirow[b]{2}{*}{ My Bodyguard } & The Shining & $.356^{*}$ & $.136 \quad .031$ & .024 & .687 \\
\hline & & $\begin{array}{c}\text { The Silence of } \\
\text { the Lambs }\end{array}$ & $.381^{*}$ & $.137 \quad .020$ & .047 & .715 \\
\hline \multirow[t]{5}{*}{ Sheepish } & \multirow[t]{2}{*}{ The Shining } & $\begin{array}{c}\text { The Silence of } \\
\text { the Lambs }\end{array}$ & -.232 & .108 .105 & -.496 & .032 \\
\hline & & My Bodyguard & .078 & .1131 .000 & -.197 & .353 \\
\hline & \multirow{2}{*}{$\begin{array}{c}\text { The Silence of } \\
\text { the Lambs }\end{array}$} & The Shining & .232 & .108 .105 & -.032 & .496 \\
\hline & & My Bodyguard & $.310^{*}$ & $.114 \quad .023$ & .033 & .586 \\
\hline & My Bodyguard & The Shining & -.078 & .1131 .000 & -.353 & .197 \\
\hline
\end{tabular}




\begin{tabular}{|c|c|c|c|c|c|c|c|}
\hline & & $\begin{array}{c}\text { The Silence of } \\
\text { the Lambs }\end{array}$ & $-.310^{*}$ & .114 & .023 & -.586 & -.033 \\
\hline \multirow[t]{6}{*}{ Loathing } & \multirow[t]{2}{*}{ The Shining } & $\begin{array}{c}\text { The Silence of } \\
\text { the Lambs }\end{array}$ & -.175 & .180 & .994 & -.613 & .262 \\
\hline & & My Bodyguard & $-.561^{*}$ & .187 & .010 & -1.017 & -.105 \\
\hline & \multirow{2}{*}{$\begin{array}{c}\text { The Silence of } \\
\text { the Lambs }\end{array}$} & The Shining & .175 & .180 & .994 & -.262 & .613 \\
\hline & & My Bodyguard & -.386 & .188 & .130 & -.844 & .073 \\
\hline & \multirow[b]{2}{*}{ My Bodyguard } & The Shining & $.561^{*}$ & .187 & .010 & .105 & 1.017 \\
\hline & & $\begin{array}{c}\text { The Silence of } \\
\text { the Lambs }\end{array}$ & .386 & .188 & .130 & -.073 & .844 \\
\hline
\end{tabular}

Note. Based on estimated marginal means; emotion inductions featuring only film clip, no perspective-taking writing task.

${ }^{\text {a }}$ Bonferroni adjustment for multiple comparisons.

$* p<.05$. 
Table 12

Emotion Ratings While Watching The Shining

\begin{tabular}{lcccccccc}
\hline & \multicolumn{3}{c}{ Current Study } & \multicolumn{5}{c}{ Rottenberg, Ray, \& Gross } \\
\cline { 2 - 9 } \multicolumn{1}{c}{ Emotion } & \multicolumn{2}{c}{ Males $^{\mathrm{a}}$} & \multicolumn{2}{c}{ Females $^{\mathrm{b}}$} & \multicolumn{2}{c}{ Males $^{\mathrm{c}}$} & Females $^{\mathrm{d}}$ \\
\cline { 2 - 10 } & $\mathrm{M}$ & $\mathrm{SD}$ & $\mathrm{M}$ & $\mathrm{SD}$ & $\mathrm{M}$ & $\mathrm{SD}$ & $\mathrm{M}$ & $\mathrm{SD}$ \\
\hline Amusement & 2.33 & 2.35 & 1.93 & 2.30 & 1.39 & 1.37 & 0.83 & 1.23 \\
Anger & 0.44 & 0.73 & 0.63 & 1.33 & 0.65 & 1.27 & 0.17 & 0.38 \\
Confusion & 3.33 & 2.35 & 4.67 & 2.62 & 2.91 & 2.26 & 1.92 & 2.25 \\
Disgust & 0.44 & 1.33 & 0.93 & 1.86 & 0.39 & 0.78 & 0.00 & 0.00 \\
Embarrassment & 0.00 & 0.00 & 0.04 & 0.19 & 0.22 & 0.42 & 0.00 & 0.00 \\
Fear & 5.00 & 2.40 & 4.85 & 2.58 & 3.26 & 2.03 & 4.61 & 2.07 \\
Happiness & 0.67 & 1.00 & 0.15 & 0.46 & 0.96 & 1.22 & 0.19 & 0.75 \\
Interest & 5.22 & 2.77 & 4.41 & 2.59 & 4.61 & 1.27 & 3.89 & 1.72 \\
Sadness & 1.22 & 2.44 & 1.26 & 1.70 & 0.70 & 1.26 & 0.17 & 0.45 \\
Surprise & 3.11 & 3.66 & 2.11 & 2.47 & 1.74 & 2.05 & 1.08 & 1.65 \\
\hline
\end{tabular}

${ }^{a} n=9 .{ }^{b} n=27 .{ }^{c} n=23 .{ }^{d} n=36$. 
Table 13

Emotion Ratings While Watching My Bodyguard

\begin{tabular}{lcccccccc}
\hline & \multicolumn{3}{c}{ Current Study } & \multicolumn{5}{c}{ Rottenberg, Ray, \& Gross } \\
\cline { 2 - 9 } \multicolumn{1}{c}{ Emotion } & \multicolumn{2}{c}{ Males $^{\mathrm{a}}$} & \multicolumn{2}{c}{ Females $^{\mathrm{b}}$} & \multicolumn{2}{c}{ Males $^{\mathrm{c}}$} & Females $^{\mathrm{d}}$ \\
\cline { 2 - 9 } & $\mathrm{M}$ & $\mathrm{SD}$ & $\mathrm{M}$ & $\mathrm{SD}$ & $\mathrm{M}$ & $\mathrm{SD}$ & $\mathrm{M}$ & $\mathrm{SD}$ \\
\hline Amusement & 1.57 & 1.81 & 0.91 & 1.73 & 1.34 & 1.61 & 0.61 & 1.12 \\
Anger & 3.29 & 1.60 & 5.87 & 1.94 & 5.03 & 1.82 & 5.36 & 1.39 \\
Confusion & 2.43 & 3.26 & 4.39 & 2.33 & 1.21 & 1.11 & 1.82 & 2.21 \\
Disgust & 3.57 & 2.64 & 6.22 & 1.59 & 4.69 & 1.61 & 4.94 & 1.80 \\
Embarrassment & 0.29 & 0.49 & 1.52 & 2.66 & 1.10 & 1.76 & 0.61 & 1.25 \\
Fear & 0.43 & 0.79 & 1.78 & 2.43 & 1.62 & 1.57 & 2.15 & 2.00 \\
Happiness & 0.57 & 1.51 & 0.04 & 0.21 & 0.76 & 1.33 & 0.42 & 0.90 \\
Interest & 4.43 & 1.81 & 4.09 & 2.95 & 3.66 & 2.02 & 3.15 & 1.62 \\
Sadness & 2.86 & 3.08 & 4.39 & 2.64 & 3.07 & 2.12 & 4.21 & 2.13 \\
Surprise & 2.43 & 2.57 & 3.87 & 3.06 & 1.66 & 1.97 & 1.21 & 1.76 \\
\hline
\end{tabular}

${ }^{a} n=7 .{ }^{b} n=23 .{ }^{c} n=27 .{ }^{d} n=33$. 


\section{Discussion}

The goal of Study Three was to determine which combination of film clips and overall induction technique worked best for eliciting only anger and only fear among my sample. In spite of a few unexpected findings (namely, the volatility of responses elicited by the highly provocative Cry Freedom film clip and the unpredictable effects of the writing task), through a series of careful comparisons, I was able to identify the best way to induce only the emotion I wanted and (not the competing emotion) for both experimental conditions of interest. This is a fundamental cornerstone of my final study, so it was imperative to thoroughly pretest and validate the emotion induction techniques in this manner.

\section{Chapter VIII}

\section{Study Four: Effects of Emotional Appraisals of Certainty on Juror Information \\ Processing and Decision-Making}

\section{Method}

\section{Theoretical Basis}

Study Four was the final stage of my research undertaken to determine whether mock jurors' style of information processing was guided by their overall levels of certainty appraisals. After having meticulously validated both the baseline detection rates of the eight inconsistencies within my trial transcript as well as the effectiveness of my method of emotion induction, Study Four addressed my overarching research objectivewhether the rate of detection for testimonial inconsistencies was dependent in part upon emotional state and certainty. 
As a secondary research question, Study Four also allowed me to gauge whether the relationship between certainty and inconsistency detection rate could potentially carry through to influence a jurors' ultimate function of rendering a verdict. By keeping all inconsistencies loaded in one direction such that they should weaken the Plaintiff's position (or at least not substantiate it in any way) and by spreading them among multiple witnesses instead of confining them all to one single witness (the Plaintiff) as Semmler and Brewer (2002) did, Study Four had the potential to alleviate any unintended overemphasis on witness credibility or potential attitudinal backlash concerning frivolous lawsuits, legitimacy of tort claims, or the natural suspicions that can arise when faced with high-dollar damage requests. By more evenly spreading around inconsistencies among additional witnesses, this comparison allowed me to more accurately establish whether differences in comprehension and verdict emerge between the consistent and inconsistent trial summaries in the neutral emotion induction control condition.

\section{Participants}

Two hundred ninety-two undergraduate students at Florida International University were recruited through Sona Systems, the Psychology Department Research Management system to participate in a web-based study on "Juror Decision-Making in Civil Trials" in exchange for course credit. Because this was an online study, several safeguards were used to exclude those participants who did not give the study their undivided attention (see Appendix X for detailed criteria and procedures undertaken for data reduction purposes), which resulted in a total of 198 participants. The sample was predominantly female (75\%; 25\% male), Hispanic (73\%; 14\% White/Caucasian; $6 \%$ African American; 2\% Asian; 0.5\% Native American; and 4\% Other), and ranged in age 
from 18 to 47 years old $(M=22.78, S D=4.33)$. Participants' were predominantly upperclassmen, with $33 \%$ in their junior year, $24 \%$ in their senior year, $20 \%$ in their sophomore year, $19 \%$ in their freshman year, and $2 \%$ having completed some professional or graduate-level education. Just less than half the sample (48\%) was not currently employed, with 39\% holding part-time employment and 13\% working full-time, and $5 \%$ had served on a jury before ( $3 \%$ civil; $2 \%$ criminal).

\section{Design}

After creating and validating the trial transcript stimulus materials and the rates of detection for the eight inconsistencies I chose to retain in Studies One and Two, and confirming the effectiveness of emotion induction though the use of film clips in Study Three, Study Four included two different test sessions. The first measured participants' general overall emotional state (via the modified, twelve-item I-PANAS-SF used in Study Three; see Appendix O) as well as their preferred styles of cognition via the Need for Cognition Scale (NFC; see Appendix Z) and decision-making strategies via the Rational-Experiential Inventory (REI-40; see Appendix AA) in order to contrast rates of inconsistency detection with stable traits. The second test session represented the culmination of the prior three studies, which used a 2 (trial transcript version: with vs. without testimonial inconsistencies) x 3 (film clip emotion/certainty induction: The Shining [fear/uncertain] vs. My Bodyguard [anger/certain] vs. Alaska's Wild Denali [no emotion/neutral control]) between subjects factorial design. The central dependent measure was the rate of inconsistency detection, with secondary dependent measures of verdict/liability as well as credibility and persuasiveness ratings for all witnesses. 


\section{Materials}

\section{Session one: trait-based scales.}

Just as in the prior studies, this study required each participant to log on to FIU's Sona Systems website from a computer of their choosing in order to complete both sessions. During the first session, after participants consented to participate (see Appendix AB), I measured various trait-based characteristics of each participant in order to better understand my primary variables of interest to be measured during the second session. The first session included the modified 12-item I-PANAS-SF (see Appendix O), the Need for Cognition scale (NFC; 18 item version; Cacioppo et al., 1984; see Appendix Z), and the Rational-Experiential Inventory (REI-40; Pacini \& Epstein, 1999; see Appendix AA).

Session two: emotion inductions, trial transcripts, verdict, witness ratings, inconsistency detection questions, and emotion and film ratings.

The second session included the three film clips selected based on Study Three (The Shining, My Bodyguard, and Alaska's Wild Denali) as well as two versions of the trial transcript from Studies One and Two, with one version featuring the eight testimonial inconsistencies chosen during Studies One and Two (see Appendix N), and a control version without any testimonial inconsistencies (see Appendix AC). Participants also read jury instructions concerning liability and completed a verdict form (see Appendix AD), followed by a form for damage awards, if applicable (see Appendix AE). After rendering their verdict and awarding damages if they found for the plaintiff, the next page featured a list of all witnesses who testified and asked participants to rate the credibility of each witness on a seven-point scale ranging from Completely Lacks 
Credibility to Completely Credible (see Appendix H, Witness Credibility Ratings), followed by another page with a similar seven-point scale rating the persuasiveness of each witness from Not Persuasive at All to Very Persuasive (see Appendix H, Witness Persuasiveness Ratings). Next, participants completed the same series of open ended, short answer, multiple choice, true/false, and point blank inconsistency detection questions used in Study Two (see Appendix Q), followed by the 61-item version of the PANAS-X then the post-film questionnaire used by Rottenberg, Ray, and Gross (2007, see Appendix A) and were then thanked for their participation, debriefed (see Appendix J), and instructed to log off and close their browser.

\section{Procedure}

\section{Session one: pretesting for trait-based characteristics.}

A two-part design was necessary so that participants were not unduly primed by the content of the various trait measurement scales they completed during Part One, and also due to concerns that prolonged participation prior to the emotion induction and introduction of the trial transcript itself would lead to ineffective emotion induction, respondent fatigue, high dropout rates, and suboptimal attention levels among those participants who finished.

During the first session, participants accessed the secure website through FIU's Sona Systems. Participants completed their informed consent form (see Appendix AB), and then completed the modified 12-item I-PANAS-SF, followed by the Need for Cognition scale, the Rational-Experiential Inventory, and demographic information. Measuring these personal characteristics not only provided a baseline measurement for cognitive styles and emotional tendencies in an environment free of emotional content, 
but also potentially allowed for a greater understanding of how emotion influenced information processing and decision-making in the second session. After completing these surveys, participants were thanked, debriefed, and reminded to please participate in the second phase of the study for additional credit, and that they should make sure to wait until receiving a notification email before completing Part Two.

\section{Session two: emotion and decision-making.}

Study Four was conducted in two parts with a delay of no less than 48 hours between sessions. While participants were urged to complete Part Two no more than one week after completing Part One, practical realities of the participant pool and a less than breakneck pace of enrollment dictated that I relax my requirement for a narrow and uniform latency period to some extent ${ }^{2}$. I did not want participants to complete Part Two too soon with Part One still fresh in their minds, so I reminded them in several different places at the beginning and end of Part One and at the beginning of Part Two that I would email them once it was okay for them to complete Part Two a couple of days after they completed Part One. Quirks of the Sona Systems research administration website prevented me from including any sort of time-based restricted access on an individual basis, so inevitably some participants did not notice or chose not to heed this requirement. As such, I excluded from analysis any participants who began Part Two less than 48 hours after completing Part One.

\footnotetext{
${ }^{2}$ While the latency period was of no critical significance for the variables of interest, I nevertheless tried to ensure that the break between each session was relatively consistent for each participant by emailing reminders to those who I could see had signed up for Part Two prematurely or who hadn't completed Part Two after a full week of eligibility. Ultimately, the latency period for completion ranged from 2.06 days to 45.97 days $(M=8.66, S D=6.44)$, but those values are skewed by the two lengthiest gaps -45.97 and 42.08 days, and excluding those two, the longest delay was 24.81 days $(M=8.24, S D=5.18)$.
} 
During the second session, participants again accessed a secure website through FIU's Sona Systems. Upon completing the informed consent for Part Two (see Appendix Y), participants were randomly assigned to one of the six experimental conditions and were asked to confirm that their computer was capable of playing the different types of files used before proceeding to the emotion induction portion of the study.

\section{Emotion induction.}

Participants watched one of the three video clips which the results of Study Three had confirmed to induce either fear (The Shining), anger (My Bodyguard), or no emotional response (Alaska's Wild Denali). Participants were told that this portion of the study was an unrelated experiment for a different project that was appended to the design at the request of the psychology department in order to ensure that the amount of credits earned for participating in the civil trial study was not excessive. To ensure that participants effortfully attended to the emotion inducing film clip, they were instructed to pay close attention while watching the clip because they would be tested on it at the end of the study.

\section{Trial transcript.}

Participants next read along with the audio recording of the trial transcript. Approximately half of the participants were assigned to the control condition featuring the straightforward, internally consistent trial transcript with no inconsistencies (see Appendix AC), while the other half read and listened to the trial transcript version featuring the eight testimonial inconsistencies (see Appendix N) selected during Studies One and Two, all of which concern testimony proffered by witnesses that favor the plaintiff. Since the burden of proof in civil negligence cases is that a preponderance of 
the evidence must support the verdict, noticing inconsistencies within the plaintiff's case was expected to diminish the strength of the case against the defendant. In other words, to find in favor of the plaintiff and therefore hold the defendant liable, meeting the standard of proof would require the trier of fact (in this case, the mock juror participants) to conclude that the evidence presented by the plaintiff is true and accurate to a probability greater than $50 \%$. Thus, I reasoned that the more inconsistencies the participants noticed, the more likely these inconsistencies would weaken (or at the very least reflect poorly on) the plaintiff's case, and as such, the less likely they would find that the plaintiff met the burden of proof. I expected there would be a direct positive correlation between the number of inconsistencies a participant noticed and their verdict in favor of the defendant.

\section{Dependent measures.}

Upon finishing the trial transcript, participants completed a verdict form, and, if applicable, awarded punitive and/or compensatory damages (see Appendices AD and $\mathrm{AE})$. They then rated the credibility and persuasiveness of each witness on a seven-point scale ranging from Completely Lacks Credibility to Completely Credible (see Appendix H, Witness Credibility Ratings), followed by another page with a similar seven-point scale rating the persuasiveness of each witness from Not Persuasive at All to Very Persuasive (see Appendix H, Witness Persuasiveness Ratings).

After completing all of the measures based on their feelings about the case, participants answered the array of open ended, short answer, multiple choice, true/false, and point blank inconsistency detection questions used in Study Two (see Appendix Q), followed by the 61 -item version of the PANAS-X to gauge their emotional state at this 
point, then the post-film questionnaire used by Rottenberg, Ray, and Gross (2007) to confirm that the film clip had been effective. Following these measures, they were thanked, debriefed of the purpose of the study and informed that the video clip was responsible for any changes in their emotional state, and told to log off and close their browser.

\section{Subjective Scoring}

The open ended and short answer questions were scored by the same two research assistants who scored the subjective responses in Studies One and Two (see Appendix AF for how I scored inconsistency detection rates differently according to the narrower basis of Study Four). Once again, they each completed the open ended scoring individually, submitted their initial, individual scorings to me, and then met with each other to discuss any discrepancies, and to try to resolve any divergences through dialogue. After they met and made any changes to their data file based on their discussion, they each provided me with their final scorings for the open ended questions. Next, the research assistants followed the same routine of scoring all the short answer data individually, submitting it to me, discussed discrepancies and changed scores as they saw fit, and then returned each of their post-discussion score sets to me for analysis.

\section{Hypotheses}

\section{Processing style through detection of inconsistencies.}

Among participants in conditions featuring inconsistencies, uncertain emotional induction (i.e. fear) will lead to effects similar to systematic processing wherein the complexities, inconsistencies, and various nuances contained within the testimony will be more perceptible. Those participants in an uncertain emotional state will therefore have 
higher rates of inconsistencies detected, while induction of certain emotions (i.e. anger) will lead to effects similar to heuristic processing, which will be demonstrated through comparably lower rates of inconsistency detection for those participants in a certain emotional state.

\section{Processing style through witness credibility and persuasiveness ratings.}

Based on the same logic which suggests that a greater rate of inconsistency detection should occur among those participants in the uncertain (fearful) emotional state than in the neutral or certain (angry) emotional state, which will serve as a proxy for information processing style, it follows that those participants who detect the inconsistencies at a greater rate will also evaluate the credibility and persuasiveness of those inaccurate witnesses accordingly, and that credibility and persuasiveness ratings for the witnesses who made inconsistent statements will be inversely related to the extent that their inconsistent statements are detected.

\section{Exploratory Analyses}

Past research has found that individual traits such as one's need for affect (Maio \& Esses, 2001) and need for cognition (Cacioppo \& Petty, 1982) influence the way information is processed, whether cognitively oriented or emotionally-oriented arguments are found to be more persuasive, and what types of information will be recalled with greater accuracy (Haddock, Maio, Arnold, \& Huskinson, 2008). However, the interplay between these findings has rarely been applied to the triers of fact in our legal system. Due to this relative dearth of empirical research on the impact of emotional certainty on juror processing style and the corresponding effects on decision-making, the current study examined several additional areas of interest which will provide a foundation for 
future exploration. Because of their novelty, I can only offer loose predictions about what sort of outcomes may result along with possible explanatory mechanisms for each prospect.

\section{Verdict as a result of processing style.}

Participants induced to feel fear (an uncertain emotion) and who read the inconsistent testimony will exhibit the most systematic style of processing demonstrated through the highest rates of verdicts in favor of the defendant. Given the fact that the inconsistencies raise doubt as to the validity of the plaintiff's case, more awareness of these inconsistencies should weaken the plaintiff's case (and result in findings that the defendant is not liable.) I anticipate that uncertain participants, by virtue of more systematic processing, will be more aware of the inconsistencies and thus be more likely to find the defendant not liable compared to their certain counterparts.

On the other hand, participants induced to feel anger (a certain emotion) and who read the inconsistent testimony will exhibit the most heuristically-based processing, evidenced through verdicts finding the defendant liable. Because all inconsistent statements are made by plaintiff's witnesses called to testify for the purpose of assisting the plaintiff in meeting the burden of proof, the detection of the inconsistencies weakens the plaintiff's case. However, if participants experiencing a certain emotion are processing heuristically, the inconsistent statements should be less likely to be detected, making the plaintiff's case appear much stronger, which in turn should result in a greater incidence of verdicts finding liability. These certain (angry) heuristic processors will show less comprehension of trial information and less awareness of inconsistencies than those participants induced to experience uncertain emotion (fear). 


\section{Verdict confidence as a result of processing style.}

Since all inconsistencies are from plaintiff's witnesses, and since jurors tend to view inconsistency as a proxy for inaccuracy (although most research on the testimonial inconsistency/inaccuracy relationship has revolved around eyewitness identifications [Berman \& Cutler, 1996; Berman, et al., 1995; Brewer \& Burke, 2002; Wells, Olson, \& Charman, 2002]), the plaintiff's case against the defendant should be viewed as being weaker by those participants in uncertain emotional states. Because they will have an affirmative, demonstrable basis for verdicts in favor of the defendant, it is predicted that participants in uncertain emotional states who read the inconsistent transcripts will have the highest degree of confidence in their verdict. Among participants who read the consistent transcripts, regardless of verdict, the uncertain participants will have higher confidence ratings than the certain or no emotion participants simply due to having been in the most cognitively engaged mindset during the trial transcript (Honess \& Charman, 2002).

However, other research (Kuhn, Weinstock, \& Flaton, 1994; Weinstock \& Flaton, 2004) has found that those jurors who express the greatest degree of certainty in their verdicts typically consider less evidence when making their decision than do jurors who report greater uncertainty. If I find that relationship to emerge in the current study, then I should expect to find that angry participants who detect fewer inconsistencies should report the greatest degree of confidence in their verdict decisions.

Another possible finding may be that participants in uncertain emotional states may process information more deeply, render a verdict consistent with having noticed many inconsistencies in testimony, but due to emotional congruence effects, may actually 
feel less certain about their final decision than participants in certain emotional states (DeSteno, Petty, Rucker, Wegener, \& Braverman, 2004; DeSteno et al., 2000; Schwarz \& Clore, 1983). Additionally, it is also possible that participants in certain emotional states will be most confident in their verdicts because they view their verdict as a means to personally alleviate their anger (Winterich, 2011).

To further complicate the range of possibilities in this dimension, recent research (Mikels, Maglio, Reed, \& Kaplowitz, 2011) has found that under some circumstances, affect-based decision-making strategies yield greater confidence in decisions than more deliberative strategies, a factor which may be mediated by overall preferred style of processing according to the Rational-Experiential Inventory (Pacini \& Epstein, 1999).

Rational/experiential processing preference moderating verdict and detection of inconsistencies.

Participants who prefer to process information in a more rational manner will detect a greater number of inconsistencies and will return verdicts for the defense to a greater degree than participants who prefer to process information in an experiential manner. Participants who process experientially will demonstrate a stronger effect from the emotional induction than those participants who process rationally.

\section{Results}

\section{Inter-rater Reliability for Subjectively Scored Questions}

To confirm that the research assistants were each coding the data according to the same rules of interpretation, and because I knew the distribution to be non-normally distributed (see Appendix AF), I conducted a Kendall's tau-b test to measure the correlation coefficient between the two scorers. For the open ended questions, the 
average correlation of their "Yes" codings before discussion was very high $\left(\tau_{B}=.93, p<\right.$ $.001)$, as was the average correlation of their "Yes" codings following discussion $\left(\tau_{B}\right.$ $=.97, p<.001)$. However, for the short answer questions, the average correlation of their "Yes" codings before discussion was moderate at best $\left(\tau_{B}=.43, p<.003\right.$, excluding one nonsignificant pairing), which did not dramatically improve even after discussion, as the average correlation of their "Yes" codings following discussion for all eight pairs was still only $\left(\tau_{B}=.47, p<.001\right)$. Accordingly, I retained the open ended data but excluded the short answer responses from consideration for Study Four ${ }^{3}$.

\section{Verification of Unequal Rates of Inconsistency Detection Based on Transcript}

\section{Version}

In order to confirm that there was a difference in overall detection rate of inconsistencies based on the transcript version (essentially, to serve as a manipulation check that participants were in fact noticing the inconsistencies in the inconsistent trial transcript), I conducted an independent samples t-test for equality of mean detection rates. I compared the mean detection rates according to all eight individual inconsistencies, as well as collapsing the inconsistencies themselves into measures for specific question types (open ended according to both the loose and the strict criteria, multiple choice, true/false, and point blank) and then also collapsing the specific question

\footnotetext{
${ }^{3}$ Casual spot inspection of the data suggested to me that there was some confusion concerning the minimum standards for what degree of detail and accuracy was required to constitute a "Yes" answer versus a "Partial" credit for detection, indicating an overzealous criterion threshold in many cases. Unfortunately, at this point in time (again, over a year had passed since the first open ended data set they coded), one of the assistants had moved out of state to begin graduate school, and the other was taking time off from school and working full time, so rather than imposing on them to rescore the short answer data after what would have been a logistically difficult meeting in order to review the scoring criteria (which may also have caused artificially high agreement due to demand characteristics), or taking the time to train two new research associates for this one small element and in light of whatever potential confounds that may have introduced, I elected to simply drop the short answer questions from consideration, a decision which, in light of subsequent analysis, seemed unlikely to have made much possible difference either way.
} 
types into a single total detection rate (see Table AF1), consisting of the sum of detections from the strict open ended, multiple choice, true/false, and point blank question types. With eight inconsistencies and four question types, these Total Detection scores could range from 0 detections to a maximum of 32 . Mean inconsistency detection rates were higher on this global index for participants who were exposed to the trial transcript featuring inconsistent testimony $(M=13.06, S D=7.91)$ than for participants who were exposed to the trial transcript without any inconsistent testimony $(M=1.76$, $S D=1.83), t(108)=13.86, p<.001, d=2.32$. Levene's test indicated unequal variances $(F=164.42, p<.001)$, so degrees of freedom were adjusted from 196 to 108. This confirms that there is in fact a statistically significant effect stemming from the trial transcript version itself. That is, participants in the inconsistent conditions believed there were more inconsistencies than participants in the consistent conditions.

\section{Effect of Emotion on Rate of Inconsistency Detection}

The main goal of this research was to determine whether the level of certainty appraisals associated with an emotion (specifically, highly uncertain appraisals accompanying the emotion of fear, and highly certain appraisals accompanying the emotion of anger) would influence a mock juror's style of cognitive processing in a manner that could be illustrated by the overall rate of detection for minor, noninculpatory, peripheral details that are nevertheless clearly contrary to the testimony of multiple other witnesses. To address this overarching research question, I conducted a 2 (trial transcript version: with or without the eight inconsistencies) x 3 (emotion induction: fear, anger, or neutral) factorial analysis of variance with inconsistency detection rates 
(for all eight individual inconsistencies as well as collapsed across question types and total overall detections) as the dependent variable.

Despite thoroughly vetting the emotion manipulation through the course of Study Three, there was no emotion-based main effect in terms of the overall inconsistency detection rates (that is, across all eight inconsistencies and four question types), $F(2,192)$ $=0.43, p=.650$. However, in order to avoid revealing the underlying purpose of the research, emotional induction was not measured and verified immediately after the film clips as in Study Three, but rather following the trial transcript, verdict, witness credibility and persuasiveness ratings, and inconsistency detection questions. While this manipulation check of emotions at such a late stage showed no significant differences, the duration of the film clips' effectiveness was further explored in Study Five. There was a main effect for the trial transcript version, $F(1,192)=188.43, p<.001, \eta^{2}=.495$ on inconsistency detection, with those participants who were exposed to the trial transcript featuring inconsistent testimony reporting a greater total number of inconsistencies $(M=13.06, S D=7.91)$ than those participants who were exposed to the trial transcript featuring no inconsistent testimony $(M=1.76, S D=1.83)$. The interaction between the induced emotion and trial transcript version on inconsistency detection rates was also not significant $F(2,192)=0.35, p=.705$. 
Table 14

Study Four: Detection Rate Percentages for all Eight Inconsistencies by Film

Clip/Induced Emotion and Question Type for Participants Exposed to Inconsistent

Transcript Only

\begin{tabular}{|c|c|c|c|c|c|c|}
\hline Inconsistency & $\begin{array}{c}\text { Open } \\
\text { Ended } \\
\text { CombiMed }\end{array}$ & $\begin{array}{c}\text { Open Ended } \\
\text { CombiHighYes }\end{array}$ & $\begin{array}{l}\text { Multiple } \\
\text { Choice }\end{array}$ & True/False & $\begin{array}{l}\text { Point } \\
\text { Blank }\end{array}$ & $\begin{array}{l}\text { Mean } \\
\text { Score }\end{array}$ \\
\hline \multicolumn{7}{|l|}{ PI 4-Oil Co. } \\
\hline Fear/Uncertain ${ }^{\mathrm{a}}$ & 32.4 & 29.4 & 44.1 & 47.1 & 50.0 & 40.6 \\
\hline Anger/Certain ${ }^{\mathrm{b}}$ & 34.4 & 34.4 & 37.5 & 43.8 & 46.9 & 39.4 \\
\hline Neutral/Control $^{\mathrm{c}}$ & 24.2 & 24.2 & 42.4 & 45.5 & 36.4 & 34.5 \\
\hline \multicolumn{7}{|l|}{ PI 5-Model } \\
\hline Fear/Uncertain ${ }^{\mathrm{a}}$ & 44.1 & 41.2 & 32.4 & 58.8 & 52.9 & 45.9 \\
\hline Anger/Certain ${ }^{\mathrm{b}}$ & 34.4 & 34.4 & 18.8 & 59.4 & 56.3 & 40.7 \\
\hline Neutral/Control $^{\mathrm{c}}$ & 45.5 & 42.4 & 27.3 & 69.7 & 66.7 & 50.3 \\
\hline \multicolumn{7}{|l|}{ PI 7-Hair } \\
\hline Fear/Uncertain ${ }^{\mathrm{a}}$ & 55.9 & 55.9 & 50.0 & 55.9 & 47.1 & 53.0 \\
\hline Anger/Certain ${ }^{\mathrm{b}}$ & 50.0 & 50.0 & 50.0 & 59.4 & 62.5 & 54.4 \\
\hline Neutral/Control $^{\mathrm{c}}$ & 57.6 & 54.5 & 48.5 & 75.8 & 60.6 & 59.4 \\
\hline \multicolumn{7}{|l|}{ PI 9-Year } \\
\hline Fear/Uncertain ${ }^{\mathrm{a}}$ & 5.9 & 5.9 & 23.5 & 58.8 & 47.1 & 28.2 \\
\hline Anger/Certain ${ }^{\mathrm{b}}$ & 12.5 & 12.5 & 21.9 & 40.6 & 56.3 & 28.8 \\
\hline Neutral/Control $^{\mathrm{c}}$ & 18.2 & 18.2 & 36.4 & 57.6 & 39.4 & 34.0 \\
\hline \multicolumn{7}{|l|}{ PI 10-Color } \\
\hline Fear/Uncertain ${ }^{\mathrm{a}}$ & 35.3 & 32.4 & 44.1 & 55.9 & 47.1 & 43.0 \\
\hline Anger/Certain ${ }^{b}$ & 28.1 & 21.9 & 34.4 & 37.5 & 40.6 & 32.5 \\
\hline Neutral/Control $^{\mathrm{c}}$ & 21.2 & 18.2 & 39.4 & 57.6 & 45.5 & 36.4 \\
\hline \multicolumn{7}{|l|}{ PI 11-Jared } \\
\hline Fear/Uncertain $^{\mathrm{a}}$ & 29.4 & 26.5 & 38.2 & 50.0 & 35.3 & 35.9 \\
\hline Anger/Certain ${ }^{\mathrm{b}}$ & 9.4 & 9.4 & 15.6 & 31.3 & 43.8 & 21.9 \\
\hline Neutral/Control $^{\mathrm{c}}$ & 24.2 & 24.2 & 30.3 & 45.5 & 39.4 & 32.7 \\
\hline \multicolumn{7}{|l|}{ PI 12-Tree } \\
\hline Fear/Uncertain ${ }^{\mathrm{a}}$ & 23.5 & 23.5 & $41.2 *$ & 41.2 & 55.9 & 37.1 \\
\hline Anger/Certain ${ }^{b}$ & 25.0 & 25.0 & $31.3 *$ & 37.5 & 62.5 & 36.3 \\
\hline Neutral/Control ${ }^{\mathrm{c}}$ & 12.1 & 12.1 & $12.1^{*}$ & 33.3 & 57.6 & 25.4 \\
\hline \multicolumn{7}{|l|}{ PI 13-Dog } \\
\hline Fear/Uncertain ${ }^{\mathrm{a}}$ & 26.5 & 26.5 & 47.1 & 58.8 & 58.8 & 43.5 \\
\hline Anger/Certain ${ }^{\mathrm{b}}$ & 9.4 & 9.4 & 31.3 & 40.6 & 53.1 & 28.8 \\
\hline Neutral/Control $^{\mathrm{c}}$ & 12.1 & 12.1 & 39.4 & 60.6 & 48.5 & 34.5 \\
\hline \multicolumn{7}{|l|}{ Mean Rate } \\
\hline Fear/Uncertain ${ }^{\mathrm{a}}$ & 31.6 & 30.2 & 40.1 & 53.3 & 49.3 & 40.9 \\
\hline Anger/Certain ${ }^{\mathrm{b}}$ & 25.4 & 24.6 & 30.1 & 43.8 & 52.8 & 35.3 \\
\hline Neutral/Control $^{\mathrm{c}}$ & 26.9 & 25.7 & 34.5 & 55.7 & 49.3 & 38.4 \\
\hline
\end{tabular}

Note. ${ }^{\mathrm{a}} n=34 .{ }^{\mathrm{b}} n=32 .{ }^{\mathrm{c}} n=33 .{ }^{*} \chi^{2}(2, N=99)=7.16, p=.028$. All other relationships ns $(p>.05)$. 


\section{Effects of emotion on inconsistency detection according to question type.}

After finding no interaction effects or main effects for emotion on inconsistency detection rates by collapsing both inconsistencies and question types, I looked to the five different question types to see if there were any significant results at that level (see Table 14 for complete detection rates by question type and inconsistency according to induced emotion).

First, I considered the effects of scoring the open ended questions with the most accommodating scheme ("OECombiMed": counting the response as a detection if both research assistants each gave some form of credit for the answer, whether a full "Yes" score or merely a "Partial" score). There was no effect of emotion on inconsistency detection rates for the open ended questions when scored with the looser of the two criteria, $F(2,192)=0.76, p=.472$, though there was a main effect for the trial transcript version, $F(1,192)=233.96, p<.001, \eta^{2}=.400$ on inconsistency detection rates, such that the participants who were exposed to the trial transcript featuring the inconsistent testimony reported noticing a greater number of the eight inconsistencies $(M=2.24, S D=$ 1.88) than did the participants who were exposed to the trial transcript without any inconsistencies $(M=0.06, S D=0.31)$. The interaction between the induced emotion and trial transcript version on inconsistency detection rates was also not significant $F(2,192)$ $=0.53, p=.588$. Adjusting the scoring scheme used to define a detection to a more stringent criterion level ("OECombiHighYes": counting the response as a detection only if both research assistants each credited a full "Yes" score for each participants" responses) also showed no effect of emotion $F(2,192)=0.47, p=.625$, though again, there was a main effect for the trial transcript version, $F(1,192)=117.75, p<.001, \eta^{2}=$ 
.380 on inconsistency detection rates, such that the participants who were exposed to the trial transcript featuring the inconsistent testimony reported noticing a greater number of the eight inconsistencies $(M=2.15, S D=1.90)$ than did the participants who were exposed to the trial transcript without any inconsistencies $(M=0.04, S D=0.24)$. The interaction between the induced emotion and trial transcript version on inconsistency detection rates was also not significant $F(2,192)=0.53, p=.588$.

The multiple choice questions followed the same pattern. There was no significant effect of emotion on detection rates, $F(2,192)=0.89, p=.414$, though there was a main effect for the trial transcript version, $F(1,192)=125.28, p<.001, \eta^{2}=.395$ on inconsistency detection rates, such that the participants who were exposed to the trial transcript featuring the inconsistent testimony reported noticing a greater number of the eight inconsistencies $(M=2.80, S D=2.46)$ than did the participants who were exposed to the trial transcript without any inconsistencies $(M=0.01, S D=0.10)$. The interaction between the induced emotion and trial transcript version on inconsistency detection rates was also not significant $F(2,192)=0.90, p=.408$. True or false question styles did not have an effect of emotion on detection rates either, $F(2,192)=0.96, p=.384$, though again, there was a significant main effect for the trial transcript version, $F(1,192)=$ $207.25, p<.001, \eta^{2}=.519$ on inconsistency detection rates, such that the participants who were exposed to the trial transcript featuring the inconsistent testimony reported noticing a greater number of the eight inconsistencies $(M=4.08, S D=2.35)$ than did the participants who were exposed to the trial transcript without any inconsistencies $(M=$ $0.48, S D=0.83)$. The interaction between the induced emotion and trial transcript version on inconsistency detection rates remained non-significant $F(2,192)=1.854, p=$ 
.159. Finally, the point blank questions also yielded no significant effect of emotion on detection rates, $F(2,192)=0.002, p=.998$, though there was a main effect for the trial transcript version, $F(1,192)=108.55, p<.001, \eta^{2}=.36$ on inconsistency detection rates, such that the participants who were exposed to the trial transcript featuring the inconsistent testimony reported noticing a greater number of the eight inconsistencies $(M$ $=4.03, S D=2.21)$ than did the participants who were exposed to the trial transcript without any inconsistencies $(M=1.22, S D=1.48)$, and again, the interaction between the induced emotion and trial transcript version on inconsistency detection rates was also not significant $F(2,192)=0.43, p=.654$.

This pattern buttresses the fact that the lack of significant effects for an interaction between emotion/certainty and transcript version on inconsistency detection rates is not due to any kind of ceiling or floor effects similar to what may have obscured Semmler and Brewer's (2002) findings, as it is clear that the trial transcript version featuring the inconsistent testimony is leading to greater rates of inconsistency detection, just as it should if the inconsistencies are being noticed. This is exactly what I had expected would happen in terms of the transcript version (based on my validation of the transcript and the inconsistency detection rates established in Studies One and Two), but the fact that there seems to be no difference emerging between detection rates for fearful/uncertain participants in comparison to the angry/certain participants is not what I had expected. 


\section{Effects of emotion on inconsistency detection according to each specific}

\section{inconsistency.}

Since the broader measures collapsing across question types did not elicit any significant effects on inconsistency detection rate other than the logical outcome hinging upon whether the transcript featured inconsistencies, I conducted a 2 × 3 (transcript version $\mathrm{x}$ induced emotion) ANOVA testing the effects as they related to the rates of detection for all eight specific inconsistencies. There was nothing slippery about the PI 4-Oil Co. inconsistency, with no significant effect of emotion on detection rates, $F(2$, $192)=0.02, p=.978$, yet again, there was a main effect for the trial transcript version, $F(1,192)=75.00, p<.001, \eta^{2}=.281$ on inconsistency detection rates, with participants who were exposed to the trial transcript featuring the inconsistent testimony reporting having noticed the PI 4-Oil Co. inconsistency at a greater total frequency across all four question types $(M=1.61, S D=1.60)$ than those participants who were exposed to the version of the trial transcript without any inconsistent testimony $(M=0.16, S D=0.37)$. The interaction between the induced emotion and trial transcript version on inconsistency detection rates was also not significant $F(2,192)=0.46, p=.635$. For PI 5-Model, predictably, there was no significant effect of emotion either, $F(2,192)=0.24, p=.787$, though the same main effect for the trial transcript version emerged, $F(1,192)=126.39$, $p<.001, \eta^{2}=.397$ with respect to inconsistency detection rates, with participants who were exposed to the trial transcript featuring the inconsistent testimony reporting having noticed the PI 5-Model inconsistency at a greater total frequency across all four question types $(M=1.87, S D=1.41)$ than those participants who were exposed to the version of the trial transcript without any inconsistent testimony $(M=0.18, S D=0.46)$. The 
interaction between the induced emotion and trial transcript version on inconsistency detection rates was also not significant $F(2,192)=0.93, p=.396$ for PI 5-Model. For PI 7-Hair, the arrangement held firm with no effect of emotion on detection rates, $F(2,192)$ $=0.22, p=.806$, though there was the same main effect for trial transcript version, $F(1$, $192)=127.80, p<.001, \eta^{2}=.400$ on inconsistency detection rates, with participants who were exposed to the trial transcript featuring the inconsistent testimony reporting having noticed the PI 7-Hair inconsistency at a greater total frequency across all four question types $(M=2.23, S D=1.65)$ than those participants who were exposed to the version of the trial transcript without any inconsistent testimony $(M=0.24, S D=0.54)$. The interaction between the induced emotion and trial transcript version on inconsistency detection rates was also not significant $F(2,192)=0.29, p=.748$. PI 9-Year followed the same measured pace, with no main effect for emotion on detection rates, $F(2,192)=$ $0.06, p=.935$, with a main effect for the trial transcript version, $F(1,192)=62.84, p<$ $.001, \eta^{2}=.247$ on inconsistency detection rates, with participants who were exposed to the trial transcript featuring the inconsistent testimony reporting having noticed the PI 9Year inconsistency at a greater total frequency across all four question types $(M=1.39$, $S D=1.39)$ than those participants who were exposed to the version of the trial transcript without any inconsistent testimony $(M=0.22, S D=0.44)$. The interaction between the induced emotion and trial transcript version on inconsistency detection rates was once again not significant $F(2,192)=0.34, p=.712$.

PI 10-Color fell right within the very same spectrum, with no effect for emotion on detection rates, $F(2,192)=0.87, p=.421$, with a main effect for the trial transcript version, $F(1,192)=62.84, p<.001, \eta^{2}=.247$ on inconsistency detection rates, with 
participants who were exposed to the trial transcript featuring the inconsistent testimony reporting having noticed the PI 10-Color inconsistency at a greater total frequency across all four question types $(M=1.59, S D=1.53)$ than those participants who were exposed to the version of the trial transcript without any inconsistent testimony $(M=0.29, S D=$ 0.50). The interaction between the induced emotion and trial transcript version on inconsistency detection rates did not meet levels of significance $F(2,192)=0.66, p=$ .519. PI 11-Jared rang true, showing no effect of emotion on rate of detection, $F(2,192)$ $=1.70, p=.185$, a main effect for the trial transcript version, $F(1,192)=57.50, p<.001$, $\eta^{2}=.230$ on inconsistency detection rates, with participants who were exposed to the trial transcript featuring the inconsistent testimony reporting having noticed the PI 11-Jared inconsistency at a greater total frequency across all four question types $(M=1.30, S D=$ 1.51) than those participants who were exposed to the version of the trial transcript without any inconsistent testimony $(M=0.12, S D=0.33)$. The interaction between the induced emotion and trial transcript version on inconsistency detection rates was also not significant $F(2,192)=0.45, p=.638$. PI 12-Tree stood firmly rooted in line with the other inconsistencies, such that there was no effect of emotion on detection rate, $F(2$, $192)=0.81, p=.446$, though there was a main effect for the trial transcript version, $F(1$, $192)=53.45, p<.001, \eta^{2}=.218$ on inconsistency detection rates, with participants who were exposed to the trial transcript featuring the inconsistent testimony reporting having noticed the PI 12-Tree inconsistency at a greater total frequency across all four question types $(M=1.44, S D=1.41)$ than those participants who were exposed to the version of the trial transcript without any inconsistent testimony $(M=0.34, S D=0.50)$. The interaction between the induced emotion and trial transcript version on inconsistency 
detection rates was also not significant $F(2,192)=1.08, p=.343$. Finally, PI 13-Dog remained faithful to the trend as well, with no significant effect for emotion on detection rates either, $F(2,192)=1.09, p=.339$, though there was a main effect for the trial transcript version, $F(1,192)=100.31, p<.001, \eta^{2}=.343$ on inconsistency detection rates, with participants who were exposed to the trial transcript featuring the inconsistent testimony reporting having noticed the PI 13-Dog inconsistency at a greater total frequency across all four question types $(M=1.63, S D=1.35)$ than those participants who were exposed to the version of the trial transcript without any inconsistent testimony $(M=0.19, S D=0.44)$. The interaction between emotion and trial transcript version on inconsistency detection rates was not significant $F(2,192)=1.67, p=.190$.

Clearly, the only factor which significantly predicts the rate of inconsistency detection is the mere presence of the inconsistencies themselves. There is no significant effect of emotion according to any possible means of characterizing detections, nor is the interaction between emotion and transcript version significant under any arrangement or dependent measure.

\section{Credibility and Persuasiveness Ratings of Witnesses}

The final group of dependent measures which bore noteworthy results was that of participants ratings of the credibility and persuasiveness of each witness. It was my expectation that credibility and persuasiveness ratings for each witness would decrease in relation to the number of inconsistent statements participants noticed in their testimony, but similar to my other predictions, this yielded no significant effects other than those directly attributable to the exposure to the trial transcript version featuring the 
inconsistent statements. However, some unusual trends did emerge within the witness ratings.

There were two witnesses who each made two inconsistent statements, the other four inconsistent witnesses only made one inconsistent statement each (see Appendix R). The first of these two is Marc Zollinger, the translation company worker who incorrectly stated that Brock Collins drove a Corvette and not a Camry (PI 5-Model; see Appendix R), and who also described Lesley Jobin's dog as being a Dachshund when in fact it was a German Shepherd (PI 13-Dog). Credibility ratings for this witness showed a significant effect from the trial transcript version, $F(1,192)=7.32, p=.007, \eta^{2}=.037$, such that those participants who were exposed to the trial transcript featuring inconsistencies rated Marc Zollinger lower $(M=4.59, S D=1.50)$ on a seven-point scale of credibility (1Completely Lacks Credibility to 7-Completely Credible) than did those participants who were exposed to the trial transcript without inconsistencies $(M=5.12, S D=1.26)$. However, participants perception of Marc Zollinger did not show a significant effect of the emotion induced on the ratings of his credibility, $F(2,192)=0.74, p=.479$, nor was the interaction between the induced emotion and trial transcript version on credibility ratings significant, $F(2,192)=1.14, p=.323$. The second witness who made two inconsistent statements is Lesley Jobin, the tour company worker who referred to the jewelry store as a Jared when in fact it was a Zales (PI 11-Jared), and who also said that Brock Collins's car crashed into a tree, while all the other witnesses say that it crashed into a light pole (PI 12-Tree). Lesley's credibility ratings showed a significant main effect for the trial transcript version, $F(1,192)=11.55, p=.001, \eta^{2}=.057$, such that those participants who were exposed to the transcript featuring inconsistencies rated 
Lesley's credibility lower $(M=4.26, S D=1.43)$ than did those who were exposed to the transcript without any inconsistent testimony $(M=4.90, S D=1.17)$. Like Marc, participants' perceptions of Lesley did not show a significant effect of the induced emotion on the ratings of her credibility, $F(2,192)=0.19, p=.829$, nor was the interaction between the induced emotion and trial transcript version on her credibility ratings significant, $F(2,192)=0.09, p=.911$.

This next pair of witnesses also showed a main effect of transcript version on their credibility ratings, but in addition to that, they are also the only two witnesses who also showed a main effect of transcript version (or any other effect, for that matter) on the ratings of their persuasiveness as well. Barbara Feldman, the librarian who says that Michael McGraw drives a tan van when everyone else says it is white (PI 10-Color), showed a significant effect of transcript version on credibility ratings, $F(1,192)=9.79, p$ $=.002, \eta^{2}=.049$, with participants who were exposed to the inconsistent testimony rating her less credible $(M=4.32, S D=1.48)$ than those participants exposed to the consistent transcript $(M=4.92, S D=1.28)$ but no significant effect for emotion induced, $F(2,192)$ $=1.08, p=.342$, or for the interaction between emotion and transcript version on her credibility ratings, $F(2,192)=0.23, p=.798$. Barbara's persuasiveness rating also showed a significant main effect for the transcript version, $F(1,192)=4.52, p=.035, \eta^{2}$ $=.023$, with those participants who were exposed to the inconsistent transcript rating her as less persuasive $(M=4.26, S D=1.31)$ than those participants exposed to the consistent transcript $(M=4.64, S D=1.17)$, but there was no main effect of emotion, $F(2,192)=$ $0.79, p=.454$, or for the interaction between emotion and trial transcript, $F(2,192)=$ $0.49, p=.616$, on the ratings of her persuasiveness. Jill Randall, the mother on her way 
home from dropping off her child, and the person who said that Michael McGraw had red hair (everyone else states that he has black hair; PI 7-Hair) is the other witness whose credibility $\left(F[1,192]=11.59, p=.001, \eta^{2}=.057\right)$ and persuasiveness $(F[1,192]=6.00$, $\left.p=.015, \eta^{2}=.030\right)$ ratings show a significant main effect from transcript version, with the participants who were exposed to the transcript featuring inconsistencies rating Jill's credibility lower $(M=4.16, S D=1.48)$ and persuasiveness lower $(M=4.18, S D=1.40)$ than those participants who were exposed to the consistent version of the trial transcript (credibility: $M=4.82, S D=1.25$; persuasiveness: $M=4.62, S D=1.15$ ). Jill does not display a significant main effect for emotion on her credibility rating, $F(2,192)=0.25, p$ $=.781$, or for the interaction between emotion and transcript version on her credibility rating, $F(2,192)=0.54, p=.586$. Similarly, Jill also does not display a significant main effect for emotion on her persuasiveness rating, $F(2,192)=1.39, p=.251$, or for the interaction between emotion and transcript version on her persuasiveness rating, $F(2$, 192) $=0.18, p=.838$.

The next witness with an unusual expression of significance is Steve Powell, the real estate agent who was at the post office at the time of the accident. Steve Powell displays a main effect for transcript version on his credibility ratings, $F(1,192)=2.24, p$ $=.041, \eta^{2}=.022$, such that those participants who were exposed to the inconsistent transcript rated Steve's credibility lower $(M=4.32, S D=1.37)$ than did those participants exposed to the consistent transcript $(M=4.70, S D=1.22)$, but what makes Steve Powell so interesting is the fact that he doesn't make any inconsistent statements, and yet his credibility ratings shift as though he had. Though approaching significance, Steve shows no main effect for the emotion on credibility ratings, $F(2,192)=2.69, p=.071$, or for the 
interaction between emotion and transcript version on his credibility rating, $F(2,192)=$ $1.41, p=.246$

The last two witnesses whose credibility ratings merit discussion are noteworthy for the fact that they do not show any significant effects at all, and yet in the inconsistent version of the trial transcript, they gave inconsistent testimony-inconsistencies which did not go unnoticed. The first of these is Dr. Matthew Freeman, the plaintiff's accident reconstruction expert witness, who testified that Brock Collins drove a 1986 Toyota Camry (PI 9-Year) when in fact he drove a 2006 Toyota Camry. The emotion induced has no significant main effect on his credibility rating, $F(2,192)=0.59, p=.556$, nor does the trial transcript version, $F(2,192)=0.50, p=.479$, or the interaction between the two, $F(2,192)=0.80, p=.450$. While this particular inconsistency was detected at one of the lower overall rates collapsing across all three experimental conditions which were exposed to the inconsistent transcript, the overall average rate of detection for all three film/emotion conditions was $30.33 \%$, so this lack of significance is not simply due to a floor effect, but rather it seems as though some kind of conscious decision to disregard or devalue this inconsistency when judging Dr. Freeman's credibility.

The second witness who lacks significant effects regarding credibility ratings is the plaintiff, Amanda Johnson, who described having seen the Exxon tanker trailer prior to the accident (PI 4-Oil Co.), when in fact everyone else agrees that it was a Shell tanker. Amanda's nonsignificant effects for emotion induction on credibility are $F(2,192)=$ $1.09, p=.338$; for the trial transcript version on credibility, $F(2,192)=0.97, p=.327$; and for the interaction between the two, the effect was $F(2,192)=0.26, p=.774$. The average rate of detection for all three emotional induction conditions that were exposed to 
the inconsistent transcript was $38.16 \%$, so the potential for a floor effect here is even lower than for Dr. Freeman's non-significant effects. There are a number of possible explanations that come to mind - the plaintiff could have been given additional leeway in terms of inconsistent statements out of consideration for the fact that she was grievously injured moments after the time she saw the tanker which she misidentified, or a general pro-plaintiff bias, or the perception of a functional equivalence between two of the largest oil companies in the world, or simply a dismissal of the conflation because it is not an issue which should reasonably impact any findings of fact. It is unlikely that any reasonable person might conclude that because Amanda misidentified the oil company, that there may therefore be any doubt as to whether the accident actually occurred, or whether it occurred in accordance with whichever testimonial account the participant might have found most compelling at the time of the inaccurate statement.

\section{Assorted Other Dependent Measures}

Every other dependent measure collected, including the Need for Cognition and Rational-Experiential Inventory scales from Part One, categorical and continuous verdict/liability determinations, verdict confidence, damage awards (if applicable), ratings of the emotions experienced while watching the film clip, the PANAS-X measures, and any additional witness credibility and persuasiveness ratings I have not mentioned were all statistically non-significant.

\section{Discussion}

My overall view of these unexpectedly non-significant main effects and interactions for emotion/level of certainty, inconsistency detection rates, and liability determinations is that perhaps rather than a floor or ceiling effect obscuring differences in 
cognitive processing style, that the null results could be due to any number of factors individually or collectively, both theoretical and methodological — the very nature of the inconsistencies themselves; the characteristics and motivation of the participants; unfounded theoretical conclusions, assumptions, expectations, and presumptions; methodological missteps, miscalculations, over- and/or under-corrections, etc. I remain confident in the theoretical basis for this research, but am now of the impression that the exact circumstances and experimental conditions necessary to effectively capture this elusive and ephemeral effect of certainty appraisals shaping information processing styles may be much, much more practically challenging to study than I had initially believed. After all, I have conclusively established that the inconsistencies were in fact detected at the rates I had hoped for from the outset, and which I had predicted based upon Studies One and Two, with Study Four detection rates generally averaging approximately $40 \%$. Furthermore, I was confident that although overall effect sizes were on the low side (but as high as could reasonably be expected for experimental inductions), the many iterations and analyses of Study Three confirmed that the three film clips I selected would in fact induce the three intended emotional states among my research sample. However, as the results of Study Five suggest, each emotion induction was likely not intense and/or durable enough to effectively influence cognitive processing style throughout the entire trial transcript.

\section{Theoretical Explanations}

It is possible that my participants took their jobs as mock jurors (ostensibly to help a local attorney evaluate the merits of appealing the outcome of an actual personal injury case) more seriously than I had expected, and that a comparatively weak effect of 
certainty appraisals increasing or decreasing effortful processing was simply overshadowed by their motivation to do a good job. Conversely, it is possible that despite my best efforts to identify and exclude all participants who did not pay close attention or devote legitimate effort to the requirements of the study, an overwhelming sense of apathy and disinterest inhibited the manifestation of any opposing effects of high or low certainty appraisals on cognitive processing. Each of these two extreme possibilities could lead to the same end result of statistically indistinguishable inconsistency detection rates for the participants in the fearful and uncertain condition compared to those in the angry and certain experimental condition. This two-pronged possibility would mirror the findings of Leippe et al. (2004) who found a curvilinear relationship between mock jurors' overall need for cognition (NFC) and guilt determinations in light of a strong case for the prosecution where mock jurors with high or low degrees of NFC each returned convictions at a lower rate than moderate NFC mock jurors but for widely divergent reasons - a lack of understanding and elaboration on the part of the low NFC participants, versus a more thorough consideration of evidence and a greater degree of skepticism and apprehension regarding the defendant's overall guilt by those high in NFC.

The possibility of a more complex relationship between need for cognition, preferred processing style, juror decision-making, and the integrated role of emotional appraisals (particularly that of certainty) makes the task of disentangling and understanding the various dynamics at work a much more challenging proposition. Even though I expected to find that a high degree of uncertainty appraisals would lead to more effortful cognitive processing (demonstrated through a greater rate of inconsistency 
detection) of the sort typically favored by people high in NFC, and that a high degree of certainty appraisals would lead to a less effortful style of cognitive processing (demonstrated through a lower rate of inconsistency detection) of the sort typically favored by people low in NFC, and despite the larger body of extant research supporting my unfulfilled expectations, alternative accounts are not without some empirical support. For example, Shestowsky and Horowitz (2004) found that in a deliberation setting, mock jurors lower in NFC actually discriminated between strong and weak arguments more thoroughly than those higher in NFC.

\section{Methodological Explanations}

Since this body of research is still in its infancy, I deliberately created the inconsistencies to be relatively easy to notice without too much cognitive exertion and "puzzle solving" so that I would avoid floor effects in detection rates. While I made sure that all inconsistencies were made by plaintiff's witnesses so that the detected inconsistencies would all (theoretically) impact liability in the same direction, I also took care not to make them so diagnostic or of such great probative value towards a liability determination that they would become central issues of the case and cease to be the types of small, peripheral details that only a person processing information systematically rather than heuristically would pick up on. This safeguard might have had the unintended result of causing participants to almost perfunctorily dismiss any inconsistencies they detected because to them, detecting the inconsistency was so easy that they assumed it was such a simple, obvious mistake without any kind of "Eureka!" or "Gotcha!" implications that they simply disregarded it as irrelevant, an assumption which, from the perspective of a mock juror, could have been confirmed by the mere fact that there were 
no efforts by the plaintiff's attorney to clarify or correct the inconsistencies during direct examination, as well as the fact that the defendant's attorney never seized upon the inconsistencies as a means of impeaching an inconsistent witness's credibility.

In addition to the possible perceived ease of detection and the potential for the content of the trial transcript itself to have contributed to the lack of any effect of inconsistency detection on verdict, the nature of the inconsistencies themselves could have colored participants' beliefs. Prior research on the effects of inconsistent testimony by eyewitnesses has found that exposing mock jurors to different levels of inconsistency (new testimony offered during the trial which was not disclosed during any investigation, contradictions between pretrial assertions and in-court testimony, and inconsistent statements within trial testimony), no matter how diagnostic the content, any exposure to inconsistent eyewitness testimony reduced likelihood of conviction, degree of defendant guilt, and eyewitness credibility (Berman \& Cutler, 1996). Similarly, Berman, Narby, and Cutler (1995) found that with regard to eyewitness testimony, any inconsistent statements by the witness having to do with their view of the perpetrator, whether perceptually central (i.e., having to do with the perpetrator's appearance) or perceptually peripheral (i.e., having to do with information irrelevant to the accuracy of a personal identification, such as nearby objects) in nature, resulted in lower ratings of defendant culpability and lower ratings of eyewitness credibility by mock juror participants, while those participants who were exposed to eyewitnesses making inconsistent statements regarding perceptually central details convicted the defendant at a lower rate. However, these distinct effects of inconsistencies focused on central and/or peripheral details of perception and not central and/or peripheral details in terms of relevance to legal 
decision-making or any probative value, so it is possible that the overall legal relevance of the inconsistencies themselves may explain why I did not find what I had expected to find. Perhaps the inconsequential nature of each of the inconsistencies with respect to the ultimate decision of liability, or even with respect to the credibility of the witness who makes them (since for the most part, they can be characterized as a sort of "slip of the tongue" or an obvious but irrelevant oversight) rendered inconsistency detection rates impotent as my overall dependent variables representing processing style as moderated by emotional appraisals of certainty.

The heightened importance of the overall relevance of the informational cue to the decision-making task itself is supported by the findings of Moons and Mackie (2007). Their findings are at even greater odds with the theoretical basis for my research, having found results very nearly directly contrary to my expectations. They not only found that participants experimentally induced to feel angry were inclined to undergo a more thorough, rigorous, and ultimately accurate evaluation of argument strength in comparison to neutrally-induced participants, but also that individual traits such as the need for cognition and associated preferences for style of cognitive processing are less predictive of information processing style than the emotional state of the decision-maker. In other words, they found that even among participants predisposed to heuristic, loweffort cognitive processing styles, that the induction of anger was sufficient motivation to engage in systematic processing, irrespective of personal preferences. They did find that the induction of anger prompted heuristic processing, but only for specific types of accessible, task-relevant heuristics, which suggests that the nature of the decision in concert with the informational cue itself are better predictors of cognitive processing style 
rather than the emotional state of the decision-maker. The non-significant differences in verdict (both for induced emotion as well as transcript version) would suggest that there were equivalent degrees of cognitive engagement across conditions, and that regardless of induced emotion, those participants who were exposed to the inconsistent trial transcript were adept at determining the overall importance of the inconsistent information as it pertains to the fact-finding objective of a mock juror's liability determination.

If we assume that the emotion inductions held throughout the entire trial transcript, and if anger truly was influential in the way that I predicted, then angry participants should have largely overlooked the inconsistencies. Conversely, if fear was influential in the way that I predicted, then the fearful/uncertain participants should not have overlooked the inconsistencies. However, both angry/certain and fearful/uncertain participants detected the inconsistencies at roughly the same rate (see Table 14).

Participants in all conditions clearly noticed the inconsistencies, and since the rates of detection simply did not show what I thought they would, either the inconsistencies were being disregarded as inconsequential by everyone who noticed them, or else they were not the ideal dependent variable for my very narrow, specific, and delicate purpose. However, a more parsimonious explanation which still allows for the possibility that the predicted effect exists is that the emotion inductions were too weak or too fleeting to register under these experimental conditions (see Study Five and Study Limitations and Future Directions, below.) 


\section{Chapter IX}

\section{Study Five: Establishing Durability and Duration of Emotion Inductions}

\section{Method}

\section{Theoretical Basis}

In anticipation of the potential utility in better understanding the overall depth and durability of the emotion inductions during the interpretation of Study Four's results, I collected data in a separate study (running simultaneously to Study Four) endeavoring to ascertain whether the emotional inductions from the film clips would (and to what extent) endure through the presentation of the entire trial transcript.

\section{Participants}

I identified and excluded inattentive participants using the all of the same methods and criteria used in Study Three since this was essentially a replication of Study Three with the exception of having exposed participants to the trial transcript between the film clip emotional induction stage and the full post-film measure of emotion using the PANAS-X. To start, there were 560 discrete data entries, and after eliminating 141 entries from participants who accessed the study on at least one separate occasion prior to actually completing it and 111 entries from participants who did not complete the entire study, there were 308 participants remaining.

I calculated the duration of the additional time each participant spent on the web page featuring their randomly assigned film clip by taking the total time spent on the page and subtracting the duration of the film clip they watched. I summarily eliminated the very most egregious extreme outliers (who spent an additional 15,152; 18,365; 32,136 ; and 111,325 seconds) before calculating the threshold of the merely extreme 
outliers, defined as exceeding the $97.5^{\text {th }}$ percentile in total delay, which was 263.4 seconds delay, which resulted in 19 participants being identified as extreme outliers ranging from 273.9 to 2766.6 seconds spent $(M=816.05, S D=701.64)$ and eliminated from further analysis, leaving 285 participants.

Next, I calculated the duration of total time each participant spent on the entire study. I defined the most egregious outliers as anyone who spent over four hours $(14,400$ seconds) on the study, which eliminated 13 participants ranging in total times of 14,486 seconds to 256,604 seconds. I then defined the extreme outliers of the remaining participants as those who exceeded the $97.5^{\text {th }}$ percentile in total time spend, which was 6708.0 seconds (111.8 minutes), and eliminated another 7 participants as a result, ranging from 6714.1 seconds to $10,644.2$ seconds spent $(M=9072.3, S D=1607.7)$, leaving a total of 265 participants.

After eliminating those extreme outliers which would skew the overall distribution of time spent, I calculated the distribution of total time spent for the remaining 265 participants on a condition by condition basis, resulting in the elimination of thirteen participants whose total time spent was in excess of two standard deviations above the mean for their experimental conditions. There were no participants who completed the study in less time than two standard deviations below the means of their experimental conditions, leaving 252 participants remaining.

Having excluded the extreme outliers for both the duration of lag time after the film clip as well as for total time spent, and without those participants whose total time spent exceeded two standard deviations beyond the mean, I still wanted to eliminate those remaining participants who had an unusually long delay between the time their film clip 
ended and the point when they clicked the "Continue" button to load the next webpage. I calculated the mean latency for each film clip condition and eliminated those 21 participants whose times were greater than two standard deviations past the mean, leaving me with 231 participants.

Just as in Study Three, I wanted to eliminate those participants who, based on their I-PANAS-SF pretest, were already in an elevated mood state for any emotions related to fear and anger - angry, nervous, afraid, mad, and the composite Negative Affect value (consisting of the mean score of the sum of upset, hostile, ashamed, angry, nervous, afraid, and mad) so I cut 41 participants whose ratings on any one or more of those five measures exceeded two standard deviations above the mean, which left 190 participants remaining.

Finally, I eliminated 29 participants who failed the attention check question ("Please Select 2-A Little") I inserted into the PANAS-X, resulting in a total of 161 participants.

\section{Design}

The design was a 3 (film clip induction emotion/level of certainty: The Shining [1980] for fear/low certainty vs. My Bodyguard [1980] for anger/high certainty vs. Alaska's Wild Denali [1997] for neutral emotional impact/control level of certainty) x 2 (trial transcript version: with testimonial inconsistencies vs. without testimonial inconsistencies) pretest-posttest control mixed factorial design. The purpose of this undertaking was simply to learn whether the levels of induced emotion found immediately after the film clips as in Study Three would still remain even after having been exposed to the trial transcript. 


\section{Materials}

I used the same three film clips selected based on Study Three (The Shining, My Bodyguard, and Alaska's Wild Denali) as well as two versions of the trial transcript from Studies One and Two (and just as in Study Four), with one version featuring the eight testimonial inconsistencies chosen during Studies One and Two (see Appendix N), and a control version without any testimonial inconsistencies (see Appendix AC).

\section{Emotion measures.}

To obtain a baseline measure of emotional state without unduly emphasizing emotional states or introspective self-evaluation, I used the 10-item International Positive and Negative Affect Schedule Short Form (I-PANAS-SF, Thompson, 2007; see Appendix O). The I-PANAS-SF does not include any emotion ratings analogous to anger, so to maximize utility for my specific purposes, I appended "Angry" and "Mad" to the scale since one of the critical manipulations in Study Four is inducing some participants to feel anger. To obtain a more thorough measurement of emotional state immediately after exposure to the trial transcript, participants completed the 61 -item (adding Mad) Specific Affect Scales of the Positive and Negative Affect ScheduleExpanded (PANAS-X; Watson \& Clark, 1994; see Appendix O) following the final witness's testimony.

\section{Procedure}

Participants believed that they were participating in a mock juror study to assist a local attorney evaluate the merits of appealing a verdict, but that due to the duration of that study, they would also have to complete a short, unrelated study having to do with film clips to round out the total time necessary to merit the full amount of course credit. 
Participants first completed the I-PANAS-SF to measure their baseline emotions, then watched one of the three randomly assigned film clips, then were exposed to one of the two randomly selected trial transcripts (with or without testimonial inconsistencies), then completed the PANAS-X, and concluded their participation by completing the witness credibility and persuasiveness ratings.

\section{Results}

I first needed to confirm that there were no differences between groups prior to the emotional inductions. Analysis of variance with a Bonferroni correction for multiple comparisons showed no significant differences between the six experimental groups for any of the twelve emotions rated on the I-PANAS-SF pretest: upset, $F(1,5)=0.50, p=$

$.777, \eta^{2}=.02 ;$ hostile, $F(1,5)=0.61, p=.694, \eta^{2}=.02$; alert, $F(1,5)=0.61, p=.694, \eta^{2}$ $=.02$; ashamed, $F(1,5)=1.05, p=.393, \eta^{2}=.03$; angry, $F(1,5)=0.26, p=.934, \eta^{2}=$ .01 ; nervous, $F(1,5)=0.49, p=.783, \eta^{2}=.02$; determined, $F(1,5)=0.94, p=.456, \eta^{2}=$ .03 ; attentive, $F(1,5)=1.03, p=.403, \eta^{2}=.03$; afraid, $F(1,5)=0.66, p=.653, \eta^{2}=.02$; active, $F(1,5)=1.55, p=.177, \eta^{2}=.05$; inspired, $F(1,5)=1.39, p=.231, \eta^{2}=.04 ;$ mad, $F(1,5)=0.15, p=.980, \eta^{2}=.01$.

To investigate my suspicion that perhaps the null results of Study Four may have been due to the dissipation of the emotion inductions from each film clip, my next step was to check whether (and if so, which) emotional ratings were significantly different during the post-trial ratings in comparison to their initial baseline ratings. To accomplish this, I conducted a repeated measures t-test for each of the twelve emotions rated both before the film clip and after exposure to one of the trial transcripts (see Tables 15 through 17 for pre- and post- film clip means on a scale from 1-very slightly or not at all 
to 5-extremely). I included the emotion ratings from the corresponding conditions testing the effectiveness of emotion induction techniques (watching The Shining with no perspective taking writing task and watching My Bodyguard with no perspective taking writing task) from Study Three to more fully illustrate the effect of including the trial transcript on the overall ratings of each emotion. In essence, the only difference in procedure from each of the conditions in Study Three and this undertaking is that rather than providing their postfilm emotion ratings immediately following the film clip as in Study Three, they were also exposed to one of the trial transcripts between the film clip and giving their emotion ratings.

\section{Discussion}

At first blush, these effects do not appear noteworthy in any way, especially for the emotions of alert, ashamed, determined, attentive, active, and inspired, in which virtually no significant differences between conditions emerge, and for this reason in combination with their general lack of similarity to either fear, anger, or appraisals of certainty or uncertainty, they were omitted from further analysis, though they do paint a potentially bleak picture with respect to how trial testimony can impact an observer's overall levels of attention and engagement.

Next, since there were no significant interactions specifically associated with the film clips or their intended emotional inductions, I collapsed across conditions for film clip and for transcript version for the 161 participants in this phase (this does not include any participants from Study Three), and after making Bonferroni adjustments to the critical alpha levels for multiple comparisons in each set of collapsed conditions (The Shining: $n=67$, adjusted $\alpha=.05 / 67$ or $.0007 ;$ My Bodyguard: $n=44$, adjusted $\alpha=.05 / 44$ 
or .0011; Alaska's Wild Denali: $n=50$, adjusted $\alpha=.05 / 50$ or .001 ; Inconsistent Transcript: $n=94$, adjusted $\alpha=.05 / 94$ or .0005 ; Consistent Transcript: $n=67$, adjusted $\alpha$ $=.05 / 67$ or .0007$)$ no statistically significant relationships emerged according to these broader characterizations either (see Table 18). 
Table 15

Study Five: Contrast of Mean Emotion Ratings Before and After The Shining Film Clip and Trial Transcript

\begin{tabular}{|c|c|c|c|c|c|c|c|c|c|c|c|c|}
\hline \multirow[b]{2}{*}{ Emotion } & \multirow{2}{*}{$\begin{array}{l}\text { Trial } \\
\text { Transcript } \\
\text { Version }\end{array}$} & \multicolumn{2}{|c|}{$\begin{array}{l}\text { Before } \\
\text { Film }\end{array}$} & \multicolumn{2}{|c|}{$\begin{array}{l}\text { After } \\
\text { Film }\end{array}$} & \multicolumn{2}{|c|}{ Difference* } & \multirow[b]{2}{*}{$t$} & \multirow[b]{2}{*}{$p$} & \multicolumn{2}{|c|}{$95 \% \mathrm{CI}$} & \multirow{2}{*}{$\begin{array}{l}\text { Cohen's } \\
d\end{array}$} \\
\hline & & $M$ & $S D$ & $M$ & $S D$ & $M$ & $S D$ & & & $L L$ & $U L$ & \\
\hline \multirow[t]{3}{*}{ Upset } & Inconsistent $^{\mathrm{a}}$ & 1.22 & 0.48 & 1.38 & 0.64 & 0.16 & 0.73 & 1.36 & .183 & -0.08 & 0.40 & 0.22 \\
\hline & Consistent $^{\mathrm{b}}$ & 1.13 & 0.43 & 1.33 & 0.66 & 0.20 & 0.71 & 1.53 & .136 & -0.07 & 0.47 & 0.28 \\
\hline & Study $3^{c}$ & 1.53 & 0.84 & 1.61 & 0.93 & 0.08 & 1.05 & 0.48 & .638 & -0.27 & 0.44 & 0.08 \\
\hline \multirow[t]{3}{*}{ Hostile } & Inconsistent $^{\mathrm{a}}$ & 1.05 & 0.23 & 1.19 & 0.52 & 0.14 & 0.48 & 1.71 & .096 & -0.03 & 0.30 & 0.28 \\
\hline & Consistent $^{\mathrm{b}}$ & 1.07 & 0.25 & 1.13 & 0.35 & 0.07 & 0.37 & 1.00 & .326 & -0.07 & 0.20 & 0.18 \\
\hline & Study $3^{\mathrm{c}}$ & 1.19 & 0.58 & 1.50 & 1.00 & 0.31 & 1.01 & 1.82 & .078 & -0.04 & 0.65 & 0.30 \\
\hline \multirow[t]{3}{*}{$\overline{\text { Alert }}$} & Inconsistent $\mathrm{t}^{\mathrm{a}}$ & 2.89 & 1.24 & 2.73 & 1.17 & -0.16 & 1.40 & -0.70 & .487 & -0.63 & 0.31 & -0.12 \\
\hline & Consistent $^{\mathrm{b}}$ & 3.00 & 1.34 & 3.27 & 1.23 & 0.27 & 1.70 & 0.86 & .397 & -0.37 & 0.90 & 0.16 \\
\hline & Study $3^{c}$ & 3.03 & 1.34 & 3.19 & 1.33 & 0.17 & 1.30 & 0.77 & .446 & -0.27 & 0.61 & 0.13 \\
\hline \multirow[t]{3}{*}{ Ashamed } & Inconsistent $^{\mathrm{a}}$ & 1.19 & 0.62 & 1.19 & 0.57 & 0.00 & 0.53 & 0.00 & 1.00 & -0.18 & 0.18 & 0.00 \\
\hline & Consistent $^{\mathrm{b}}$ & 1.03 & 0.18 & 1.03 & 0.18 & 0.00 & 0.26 & 0.00 & 1.00 & -0.10 & 0.10 & 0.00 \\
\hline & Study $3^{\mathrm{c}}$ & 1.08 & 0.28 & 1.11 & 0.40 & 0.03 & 0.45 & 0.37 & .711 & -0.12 & 0.18 & 0.06 \\
\hline \multirow[t]{3}{*}{$\overline{\text { Angry }}$} & Inconsistent $^{\mathrm{a}}$ & 1.11 & 0.31 & 1.24 & 0.60 & 0.14 & 0.63 & 1.30 & .201 & 0.35 & 1.30 & 0.21 \\
\hline & Consistent $^{\mathrm{b}}$ & 1.10 & 0.31 & 1.13 & 0.35 & 0.03 & 0.41 & 0.44 & .662 & -0.12 & 0.19 & 0.08 \\
\hline & Study $3^{\mathrm{c}}$ & 1.25 & 0.65 & 1.22 & 0.48 & -0.03 & 0.61 & -0.27 & .786 & -0.23 & 0.18 & -0.05 \\
\hline \multirow[t]{3}{*}{ Nervous } & Inconsistent $^{\mathrm{a}}$ & 1.38 & 0.59 & 1.38 & 0.76 & 0.00 & 0.67 & 0.00 & 1.00 & -0.22 & 0.22 & 0.00 \\
\hline & Consistent $^{\mathrm{b}}$ & 1.40 & 0.56 & 1.27 & 0.45 & -0.13 & 0.68 & -1.07 & .293 & -0.39 & 0.12 & -0.20 \\
\hline & Study $3^{\mathrm{c}}$ & 1.33 & 0.68 & 2.42 & 1.36 & 1.08 & 1.30 & 5.02 & $<.001$ & 0.64 & 1.52 & 0.84 \\
\hline \multirow[t]{3}{*}{ Determined } & Inconsistent $\mathrm{t}^{\mathrm{a}}$ & 3.49 & 1.07 & 2.16 & 1.19 & -1.32 & 1.33 & -6.04 & $<.001$ & -1.77 & -0.88 & -0.99 \\
\hline & Consistent $^{\mathrm{b}}$ & 3.53 & 1.11 & 2.70 & 1.32 & -0.83 & 1.15 & -3.98 & $<.001$ & -1.26 & -0.40 & -0.73 \\
\hline & Study $3^{\mathrm{c}}$ & 3.33 & 1.53 & 2.36 & 1.38 & -0.97 & 1.48 & -3.93 & $<.001$ & -1.47 & -0.47 & -0.66 \\
\hline \multirow[t]{3}{*}{ Attentive } & Inconsistent $^{\mathrm{a}}$ & 3.49 & 1.04 & 3.14 & 1.13 & -0.35 & 1.32 & -1.62 & .113 & -0.79 & 0.09 & -0.27 \\
\hline & Consistent $^{\mathrm{b}}$ & 3.67 & 0.99 & 3.47 & 1.17 & -0.20 & 1.19 & -0.92 & .363 & -0.64 & 0.24 & -0.17 \\
\hline & Study $3^{c}$ & 3.47 & 1.38 & 3.72 & 1.06 & 0.25 & 1.13 & 1.33 & .193 & -0.13 & 0.63 & 0.22 \\
\hline \multirow[t]{3}{*}{ Afraid } & Inconsistent $\mathrm{t}^{\mathrm{a}}$ & 1.16 & 0.37 & 1.27 & 0.51 & 0.11 & 0.57 & 1.16 & .254 & -0.08 & 0.30 & 0.19 \\
\hline & Consistent $^{\mathrm{b}}$ & 1.06 & 0.25 & 1.07 & 0.25 & 0.00 & 0.26 & 0.00 & 1.00 & -0.10 & 0.10 & 0.00 \\
\hline & Study $3^{\mathrm{c}}$ & 1.11 & 0.32 & 2.19 & 1.31 & 1.08 & 1.40 & 4.64 & $<.001$ & 0.61 & 1.56 & 0.77 \\
\hline \multirow[t]{3}{*}{$\overline{\text { Active }}$} & Inconsistent $^{\mathrm{a}}$ & 2.62 & 1.19 & 2.08 & 1.14 & -0.54 & 0.90 & -3.65 & .001 & -1.14 & -0.48 & -0.81 \\
\hline & Consistent $^{\mathrm{b}}$ & 3.10 & 1.18 & 2.43 & 1.22 & -0.67 & 0.99 & -3.67 & .001 & -1.04 & -0.30 & -0.67 \\
\hline & Study $3^{c}$ & 2.67 & 1.37 & 2.03 & 1.21 & -0.64 & 1.40 & -2.74 & .010 & -1.11 & -0.17 & -0.46 \\
\hline \multirow[t]{3}{*}{ Inspired } & Inconsistent $\mathrm{t}^{\mathrm{a}}$ & 2.76 & 1.14 & 1.95 & 1.03 & -0.81 & 1.00 & -4.95 & $<.001$ & -1.14 & -0.48 & -0.81 \\
\hline & Consistent $^{\mathrm{b}}$ & 3.23 & 1.30 & 2.13 & 1.33 & -1.10 & 1.21 & -4.97 & $<.001$ & -1.55 & -0.65 & -0.91 \\
\hline & Study $3^{c}$ & 2.94 & 1.55 & 2.00 & 1.20 & -0.94 & 1.37 & -4.13 & $<.001$ & -1.41 & -0.48 & -0.68 \\
\hline \multirow[t]{3}{*}{$\overline{\mathrm{Mad}}$} & Inconsistent $\mathrm{t}^{\mathrm{a}}$ & 1.11 & 0.31 & 1.27 & 0.84 & 0.16 & 0.80 & 1.23 & .225 & -0.10 & 0.43 & -0.20 \\
\hline & Consistent & 1.10 & 0.31 & 1.17 & 0.38 & 0.07 & 0.45 & 0.81 & .423 & -0.10 & 0.23 & 0.15 \\
\hline & Study $3^{c}$ & 1.31 & 0.62 & 1.19 & 0.58 & -0.11 & 0.57 & -1.16 & .254 & -0.31 & 0.08 & -0.19 \\
\hline
\end{tabular}

Note. $\mathrm{CI}=$ Confidence Interval of the Difference; $L L=$ lower limit; $U L=$ upper limit.

*After minus Before; positive values indicate an increased rating. ${ }^{\mathrm{a}} n=37 ; d f=36$. ${ }^{\mathrm{b}} n=$ $30 ; d f=29{ }^{\mathrm{c}}$ There was no trial transcript presented in Study Three; $n=36, d f=35$. 
Table 16

Study Five: Contrast of Mean Emotion Ratings Before and After My Bodyguard Film Clip and Trial Transcript

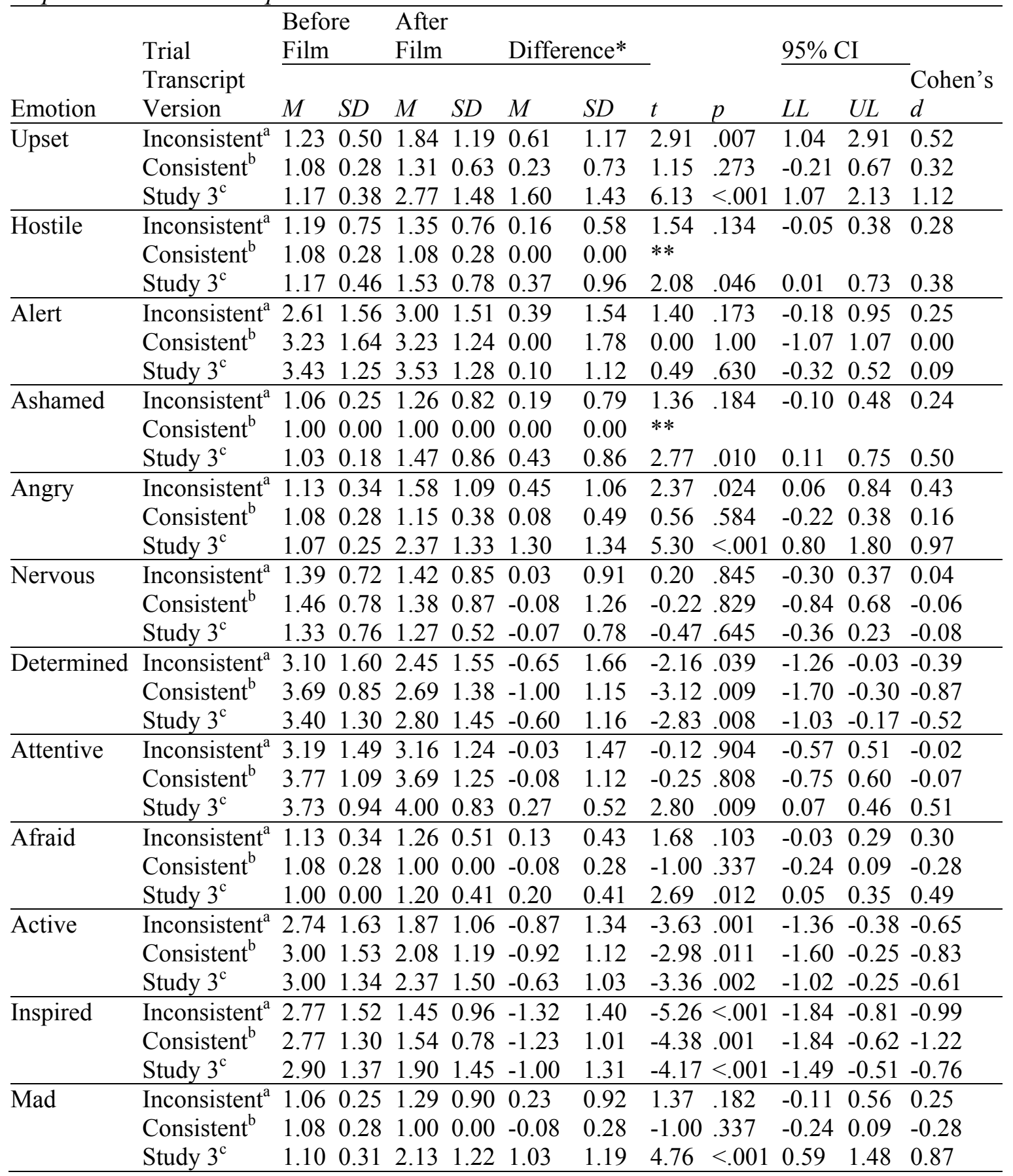

Note. $\mathrm{CI}=$ Confidence Interval of the Difference; $L L=$ lower limit; $U L=$ upper limit.

*After minus Before; positive values indicate an increased rating. $* * t$ can not be computed because the standard error of the difference is $0 .{ }^{\mathrm{a}} n=31 ; d f=30 .{ }^{\mathrm{b}} n=13 ; d f=$ 12. ${ }^{\mathrm{c}}$ There was no trial transcript presented in Study Three; $n=30, d f=29$. 
Table 17

Study Five: Contrast of Mean Emotion Ratings Before and After Alaska's Wild Denali Film Clip and Trial Transcript

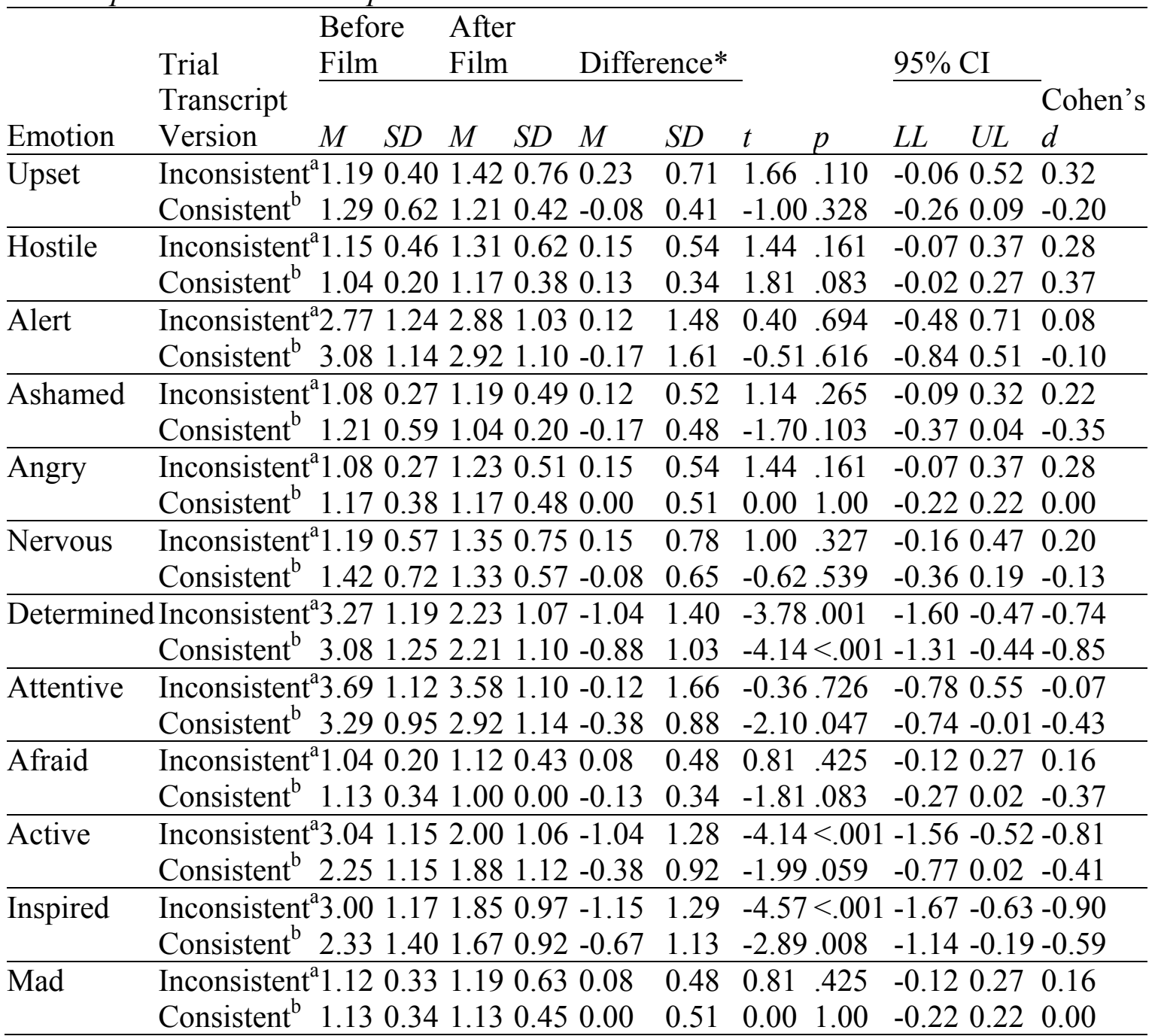

Note. $\mathrm{CI}=$ Confidence Interval of the Difference; $L L=$ lower limit; $U L=$ upper limit.

*After minus Before; positive values indicate an increased rating. **The neutral/no emotion film clip was confirmed under separate procedures; see Appendix W. ${ }^{\mathrm{a}} n=26$; $d f$ $=25{ }^{\mathrm{b}} n=24 ; d f=23$. 
Table 18

Study Five: Contrast of Mean Emotion Ratings Before and After Film and Trial

Transcript, Collapsing Across Conditions

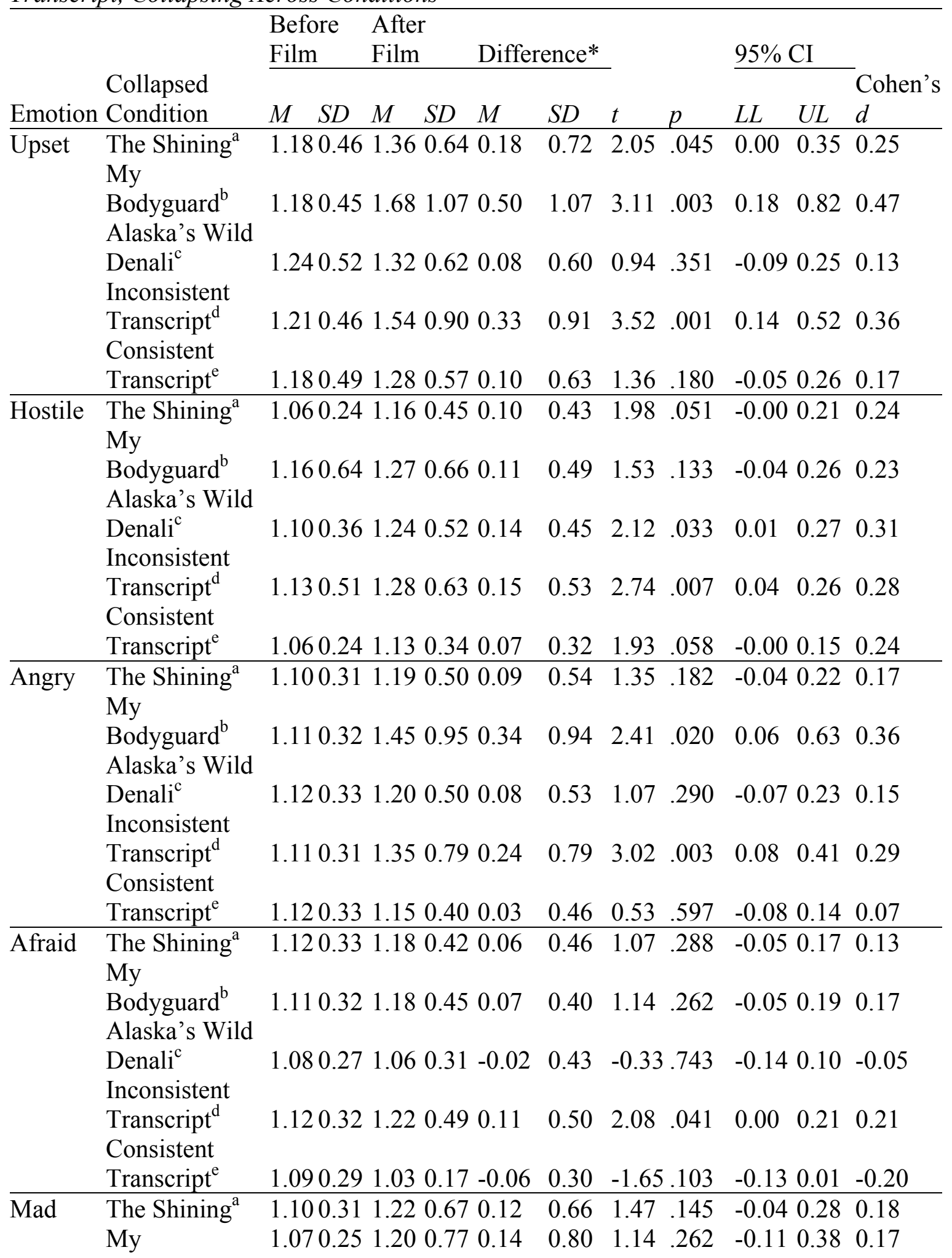


Bodyguard $^{\mathrm{b}}$

Alaska's Wild

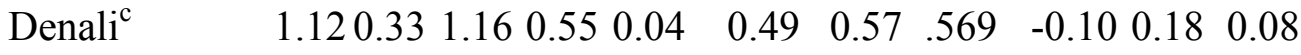

Inconsistent

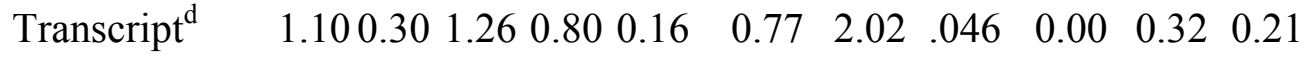

Consistent

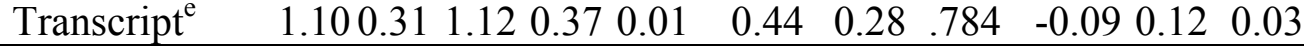

Note. $\mathrm{CI}=$ Confidence Interval of the Difference; $L L=$ lower limit; $U L=$ upper limit.

*After minus Before; positive values indicate an increased rating. ${ }^{a} n=67 ; d f=66$;

adjusted $\alpha=.0007$. ${ }^{\mathrm{b}} n=44 ; d f=43$; adjusted $\alpha=.0011$. $^{\mathrm{c}} n=50 ; d f=49$; adjusted $\alpha=$ $.001 .{ }^{\mathrm{d}} n=94 ; d f=93$; adjusted $\alpha=.0005$. ${ }^{\mathrm{e}} n=67 ; d f=66$; adjusted $\alpha=.0007$. 


\section{Chapter X}

\section{Overall Discussion}

Despite the null results, this collection of studies has yielded fruitful dividends and helped clear the way for future research. Studies One and Two finalized and streamlined a trial transcript fact pattern that is ready for use in continued efforts to isolate the relationship between information processing style and emotional appraisals, and it can be easily adapted to suit many other research applications. The detection rates of the eight inconsistencies can be used as the primary dependent variables of processing style in other, less exploratory research domains than the present undertaking turned out to be. Additionally, through the course of several unexpected challenges, Study Three rejected several unfit methods while also validating several other verifiably effective means of emotion induction - at least among the relatively unique population of Florida International University undergraduates.

The legal system of the United States is designed to be uniform and consistent, adhering to principles of common law and precedent. This leaves little room for the volatile, unpredictable influence of emotions in the law, despite the fact that we know emotions inevitably creep into jury decision-making in ways we do not yet fully understand. Inconclusive and unexpected results preclude the contribution towards a better understanding of juror decision-making I had hoped to make, so practical or policy-oriented recommendations are impossible until additional exploration is conducted. I have several suggestions that should be addressed before moving too far forward, and contingent upon eventually settling the procedural hindrances, there are several promising avenues of research that will unfold. 


\section{Study Limitations and Future Directions}

Weaknesses in the current research include the usual sorts of validity-based concerns often associated with psychological research in general, as well as limitations unique to conducting research online and difficulties associated with studying juror decision-making in particular. Using an exclusively undergraduate participant pool may have yielded results which are not perfectly generalizeable to the overall jury-eligible population since it is possible that the preferences for different types of cognitive processing and legal decision-making criteria favored by college students may not be perfectly representative of groups varying in education level. While not an unreasonable consideration, this concern has been addressed and largely withdrawn after several investigations into its merit. Bornstein (1999) considered 20 years worth of extant literature on jury simulation studies to address the general concerns of ecological validity (including concerns regarding sample populations, research paradigms, deliberation requirements, outcome variables of interest and how they were defined, and manipulation of real-world consequences) and found that much of the skepticism is unfounded. He examined twenty-six studies where sample selection (undergraduates versus community members) was a manipulated variable, and found that of those twenty-six, only five studies showed a main effect on verdict. Furthermore, only two of those twenty-six studies showed a main effect for demographic characteristics, which suggests that student and nonstudent samples are similarly affected by information at trial.

Due to the acknowledged lack of experimental control inherent in an online format, in order to best reduce the threat of participants paying less attention than they otherwise would in a more structured setting, I included multiple manipulation checks 
having to do with case familiarity and overall attention levels and excluded those participants who failed them. Objections having to do with research settings can be assuaged (though certainly not dismissed outright) as online research paradigms have been found to be largely comparable to traditional "in person" methods (Gosling, Vazire, Srivastava, \& John, 2004) and more specific to the current study, emotion has been successfully induced in past online research (Wiener et al., 2007).

Given the null results, perhaps the most noteworthy limitation to my studies can be seen as a combination of all of the aforementioned concerns - a global objection incorporating the overall induction of emotion as well as the overall emotional involvement and motivation of online mock juror participants. Breau and Brook (2007) conducted a small jury simulation experiment using law students focusing on the impact of the perceived consequences of participating in what was purported to be an honor code violation for one half of the participants, while the other half of participants were informed that they were participating in a mock jury exercise. After deliberating, each participant answered open ended questions related to their opinions on the deliberations and verdict and a multiple-choice survey addressing their perceptions of the experimental design and the degree to which they believed they were participating in a real honor code hearing. While the sample size was too small to draw any meaningful statistical conclusions, the "real" juries deliberated for a longer duration (40 and 85-90 minutes) than the known mock juries (20-25 minutes and 30 minutes), and the "real" juries either found the accused to be not guilty or had a hung jury, whereas the mock juries each delivered guilty verdicts. Furthermore, the post-deliberation questionnaires among "real" participants yielded very strong agreement with the statement "I put my heart and soul 
into resolving this issue", whereas the known mock jurors disagreed or strongly disagreed with that same statement. These findings suggest that there is a differential level of emotional and personal investment in jury simulations when the participants are unaware that they are participating in a simulation, and that to reveal the hypothetical nature of such experiments serves to dilute the motivation to reach the correct and most fair verdict, which itself indicates that the perceived level of task importance by experimental participants can influence verdict as well.

In accordance with Bornstein and McCabe's (2005) recommendation to maximize perceived real-live consequences in jury research, I expected that my cover story for the study (that participants were sought to assist a local attorney in determining the viability of appealing a past verdict) would allay this concern of participants' motivation for the most part, but the possibility remains that my results are not fully generalizeable to a real jury, since even if participants fully believed the cover story for the study, they may not have had the requisite degree of perceived task importance since the trial itself had ostensibly concluded, and their role was simply to help decide whether further action might be worthwhile.

My expectation that the detection of inconsistencies made by plaintiff's witnesses (and the plaintiff herself) should have swayed participants to find verdicts in favor of the defendant at a greater rate than those who did not notice (or were not exposed to) the inconsistent testimony is betrayed by the fact that in Study Four, 170 of 198 participants in all conditions (85.9\%) found the defendant not liable, and one would logically expect more than 28 (14.1\%) of participants to find liability when 98 participants (50\%) were exposed to the trial transcript featuring inconsistencies and 98 participants (50\%) were 
exposed to the transcript without inconsistencies--an outcome illustrating the irrelevance of trial transcript version with respect to verdict. Furthermore, the detection of inconsistent testimony showed little relation to the credibility or persuasiveness ratings for those witnesses, which also obfuscates the interpretation of the relationship between inconsistent testimony and increased durability of emotional inductions.

One way to avoid this ambiguity in undertaking future research would be to create a trial transcript that is pre-tested and confirmed to elicit a more even split of verdicts, either in the version featuring inconsistencies favoring the defendant (in which case, we would expect the transcript version without inconsistencies to lead to a greater rate of finding the defendant liable) or in the version without inconsistencies (in which case we would expect the version with the inconsistencies to find the defendant less liable). Upon determining baseline rates of liability determinations for both transcripts, this "inconsistencies lead to greater certainty, which in turn bolsters emotional induction duration" theory could be more directly tested and evaluated in relation to the more diagnostic corroborative evidence of verdict disparities for each transcript version.

In creating the trial transcript, I had intended to come up with case facts that, in the absence of testimonial inconsistencies, would lead to a roughly even percentage of verdicts in favor of the plaintiff or the defendant. It was my expectation that in so doing, the effect of inconsistency detection would translate into a greater rate of verdicts in favor of the defendant for those participants who processed information systematically due to the uncertainty appraisals inherent in their fearful emotional state, which would be evident in comparison to the higher rate of verdicts in favor of the plaintiff for those participants who detected fewer inconsistencies as a result of their more heuristic 
processing resulting from their certainty appraisals associated with their angry emotional state. However, it was beyond the scope of the current research to calibrate the trial transcript to reach a specific distribution of verdicts. Nevertheless, future investigatory efforts would be well served to at least have a baseline measure of verdict distribution, even if fine-tuning the two transcripts to reach a specific distribution of verdicts is logistically prohibitive. Future efforts might even focus on testing the overall hypothesis of certainty appraisals mediating cognitive processing styles in a much shorter trial setting, or even a less procedurally complex context than that of a jury trial in order to first find preliminary support for the underlying expectations in a more simple, direct format. Cognitive consequences of certainty appraisals could (theoretically) just as easily be demonstrated through divergences in performance on the Stroop test, brain teasers, logic puzzles and games, or simply general knowledge questions of the sort found on traditional college entrance and placement exams. These types of studies have been done in the past (Bodenhausen, Sheppard, et al. 1994; Lerner \& Tiedens, 2006; Tiedens, 2001), but they have focused more on the effect of anger and its associated certainty appraisals leading to an increased reliance on heuristic processing rather than the alternative (yet complementary) effect of increased uncertainty appraisals on an increased reliance on systematic processing as the current research attempted.

Another, more straightforward methodological explanation for my findings is that the effect of the emotional inductions dissipated too quickly to drive the mechanisms of processing style more powerfully and profoundly throughout the duration of the entire trial transcript, as Study Five suggests. Instead of measuring residual emotional induction only after the conclusion of the 45 minute trial transcript (and after full 
exposure to all elements of all eight inconsistencies), if more experimental conditions were incorporated which varied in terms of the timing of administering emotion ratings and inconsistency detection measures, then we would have a more complete picture of any sort of dynamic shift in emotion inductions in relation to exposure to (and detection of) inconsistencies, which we could compare to control conditions with identical emotion inductions measured at identical intervals but without any testimonial inconsistencies in the transcript.

Another consideration is that the experimental inductions of fear and anger might simply not have been personally relevant enough for divergent processing styles to fully manifest as a result. Working within the ethical guidelines of responsible research likely precludes the induction of fear (and perhaps even anger) through more personally relevant induction techniques, but by using more reliable physiological measures of fear such as a heart rate monitor or similar instead of less robust self-report measures, future researchers could arrive at more substantive conclusions based on physiological confirmation of emotion inductions (and give greater precision to drawing distinctions when faced with relatively small effects) which might very well shed light on the current ambiguity.

The present collection of studies has left the door open to investigate whether it is necessary to use detection rates of more cognitively complex and/or legally dispositive inconsistencies within a mock trial context in order to tease out a significant results illustrating how the overall level of certainty associated with a given emotion can influence cognitive processing style of mock jurors. Given contradictions in prior findings and the unexpected nature of the current results, this research has also raised the 
issue of whether a more prudent investigatory roadmap would instead focus on finding evidence of the "certainty appraisals on cognitive processing style" effect in more simple, straightforward, non-legal experimental formats first before rushing to apply the principles to the intricacies of legal decision-making. The current research also raises the question of whether stronger emotional inductions might be necessary in order to elicit the effects I expected to find, as well as the possibility that the detection of inconsistencies may have more control over emotional state instead of the other way around as originally expected.

Once the two overlapping foundations of determining the optimal type of inconsistency to use as the dependent measure, as well as identifying the most effective induction methods of un/certainty appraisals are each firmly established, the avenues for future research building on those core principles are manifest. For example, it would be a tremendously valuable — arguably unethically so — skill for trial attorneys, or anyone else for that matter, to be able to deliberately induce elevated or reduced levels of cognitive engagement through the activation of certain emotions. Such a discovery would in turn bring to mind the importance of studying emotion regulation efforts and how those too may have unanticipated or unchartered cognitive consequences. 


\section{REFERENCES}

Adler, A. (1927). Understanding human nature. Oxford, England: Greenberg.

Allport, G. W. (1961). Pattern and growth in personality. Oxford, England: Holt, Reinhart \& Winston.

Allport, G. W. (1979). The nature of prejudice. Reading, Mass: Addison-Wesley Pub. Co.

Antonio, M. E. (2006). Jurors' emotional reactions to serving on a capital trial. Judicature, 89(5), 282-288.

Arnold, M. B. (1960). Emotion and personality. Vol. 1. Psychological aspects. Oxford, England: Columbia Univer. Press.

Ask, K., \& Granhag, P. A. (2007a). Hot cognition in investigative judgments: The differential influence of anger and sadness. Law and Human Behavior, 31(6), 537-551. doi:10.1007/s10979-006-9075-3

Ask, K., \& Granhag, P. A. (2007b). Motivational bias in criminal investigators' judgments of witness reliability. Journal of Applied Social Psychology, 37(3), 561-591. doi:10.1111/j.1559-1816.2007.00175.x

Bandes, S. A. (Ed.), (1999). The passions of law. New York: New York University Press.

Bar-Anan, Y., Wilson, T. D., \& Gilbert, D. T. (2009). The feeling of uncertainty intensifies affective reactions. Emotion, 9(1), 123-127. doi:10.1037/a0014607

Baron, J., \& Hershey, J. C. (1988). Outcome bias in decision evaluation. Journal of Personality and Social Psychology, 54(4), 569-579. doi:10.1037/00223514.54.4.569

Berman, G. L., \& Cutler, B. L. (1996). Effects of inconsistencies in eyewitness testimony on mock-juror decision making. Journal of Applied Psychology, 81(2), 170-177. doi:10.1037/0021-9010.81.2.17

Berman, G. L., Narby, D. J., \& Cutler, B. L. (1995). Effects of inconsistent eyewitness statements on mock-juror's evaluations of the eyewitness, perceptions of defendant culpability and verdicts. Law and Human Behavior, 19(1), 79-88. doi:10.1007/BF01499074

Bless, H. (2000). The interplay of affect and cognition: The mediating role of general knowledge structures. In J. P. Forgas (Ed.), Studies in emotion and social 
interaction, second series. Feeling and thinking: The role of affect in social cognition (pp. 201-222). New York: Cambridge University Press.

Bless, H., Bohner, G., Schwarz, N., \& Strack, F. (1990). Mood and persuasion: A cognitive response analysis. Personality and Social Psychology Bulletin, 16(2), 331-345. doi:10.1177/0146167290162013

Bless, H., Clore, G. L., Schwarz, N., Golisano, V., Rabe, C., \& Wölk, M. (1996). Mood and the use of scripts: Does a happy mood really lead to mindlessness? Journal of Personality and Social Psychology, 71(4), 665-679. doi:10.1037/00223514.71.4.665

Bornstein, B. H. (1999). The ecological validity of jury simulations: Is the jury still out? Law and Human Behavior, 23(1), 75-91.

Bornstein, B. H. \& McCabe, S. G. (2005). Jurors of the absurd? The role of consequentiality in jury simulation research. Florida State University Law Review, 32, 443-467.

Blumenthal, J. A. (2005a). Does mood influence moral judgment? An empirical test with legal and policy implications. Law \& Psychology Review, 29, 1-28.

Blumenthal, J. A. (2005b). Law and the emotions: The problems of affective forecasting. Indiana Law Journal, 80, 155-238.

Blumenthal, J. A. (2010). A moody view of the law: Looking back and looking ahead at law and the emotions. In Bornstein, B. H., \& Wiener, R. L. (Eds.), Emotion and the law: psychological perspectives (pp. 185-210). New York: Springer.

Bodenhausen, G. V. (1993). Emotions, arousal, and stereotypic judgments: A heuristic model of affect and stereotyping. In D. M. Mackie \& D. L. Hamilton (Eds.), Affect, cognition, and stereotyping: Interactive processes in group perception ( $\mathrm{pp}$. 13-37). San Diego, CA: Academic Press.

Bodenhausen, G. V., Kramer, G. P., \& Süsser, K. (1994). Happiness and stereotypic thinking in social judgment. Journal of Personality and Social Psychology, 66(4), 621-632. doi:10.1037/0022-3514.66.4.621

Bodenhausen, G. V., Sheppard, L. A., \& Kramer, G. P. (1994). Negative affect and social judgment: The differential impact of anger and sadness. European Journal of Social Psychology, 24(1), 45-62. doi:10.1002/ejsp.2420240104

Bodenhausen, G. V., \& Wyer, R. S. (1985). Effects of stereotypes in decision making and information-processing strategies. Journal of Personality and Social Psychology, 48(2), 267-282. doi:10.1037/0022-3514.48.2.267 
Bourgeois, M. J., Horowitz, I. A., \& FosterLee, L. (1993). Effects of technicality and access to trial transcripts on verdicts and information processing in a civil trial. Personality and Social Psychology Bulletin, 19(2), 220-227. doi:10.1177/0146167293192012

Bower, G. H. (1981). Mood and memory. American Psychologist, 36(2), 129-148. doi:10.1037/0003-066X.36.2.129

Breau, D. L. \& Brook, B. (2007). "Mock" mock juries: A field experiment on the ecological validity of jury simulations. Law \& Psychology Review, 31, 77-92.

Brewer, N., \& Burke, A. (2002). Effects of testimonial inconsistencies and eyewitness confidence on mock-juror judgments. Law and Human Behavior, 26(3), 353-364. doi:10.1023/A:1015380522722

Brewer, N., \& Hupfeld, R. M. (2004). Effects of Testimonial Inconsistencies and Witness Group Identity on Mock-Juror Judgments. Journal of Applied Social Psychology, 34(3), 493-513. doi:10.1111/j.1559-1816.2004.tb02558.x

Brewer, N., Potter, R., Fisher, R. P., Bond, N., \& Luszcz, M. A. (1999). Beliefs and data on the relationship between consistency and accuracy of eyewitness testimony. Applied Cognitive Psychology,13(4), 297-313. doi:10.1002/(SICI)1099-0720(199908)13:4<297::AID-ACP578>3.0.CO;2-S

Cacioppo, J. T., \& Petty, R. E. (1982). The need for cognition. Journal of Personality and Social Psychology, 42, 116-131. doi:10.1037/0022-3514.42.1.116

Cacioppo, J. T., Petty, R. E., Feinstein, J., \& Jarvis, W. B. G. (1996). Dispositional differences in cognitive motivation: The life and times of individuals varying in need for cognition. Psychological Bulletin, 119, 197-253. doi:10.1037/00332909.119.2.197

Cacioppo, J. T., Petty, R. E., \& Kao, C. F. (1984). The efficient assessment of need for cognition. Journal of Personality Assessment, 48, 306-307. doi:10.1207/s15327752jpa4803_13

Cacioppo, J. T., Petty, R. E., Kao, C. F., \& Rodriguez, R. (1986). Central and peripheral routes to persuasion: An individual difference perspective. Journal of Personality and Social Psychology, 51(5), 1032-1043. doi:10.1037/0022-3514.51.5.1032

Cacioppo, J. T., Petty, R. E., \& Morris, K. J. (1983). Effects of need for cognition on message evaluation, recall, and persuasion. Journal of Personality and Social Psychology, 45, 805-818. doi:10.1037/0022-3514.45.4.805 
Chaiken, S., Liberman, A., \& Eagly, A. H. (1989). Heuristic and systematic information processing within and beyond the persuasion context. In J. S. Uleman \& J. A. Bargh (Eds.), Unintended thought (pp. 212-252). New York: Guilford Press.

Chen, S., \& Chaiken, S. (1999). The heuristic-systematic model in its broader context. In Chaiken, S., \& Trope, Y. (Eds.), Dual-process theories in social psychology, (pp. 73-96). New York, NY: Guilford Press.

Clore, G. L., \& Parrott, W. G. (1994). Cognitive feelings and metacognitive judgments. European Journal of Social Psychology, 24(1), 101-115. doi:10.1002/ejsp.2420240108

Cohen, A. R., Stotland, E., \& Wolfe, D. M. (1955). An experimental investigation of need for cognition. The Journal of Abnormal and Social Psychology, 51(2), 291294. doi:10.1037/h0042761

Cooper, J., Bennett, E. A., \& Sukel, H. L. (1996). Complex scientific testimony: How do jurors make decisions? Law and Human Behavior, 20(4), 379-394. doi:10.1007/BF01498976

Dalgleish, T. (2003). Information processing approaches to emotion. In R. J. Davidson, K. R. Scherer, \& H. H. Goldsmith (Eds.), Series in affective science. Handbook of affective sciences (pp. 661-673). New York: Oxford University Press.

Deigh, J. (1999). Emotion and the authority of law: Variation on themes in Bentham and Austin. In Bandes, S. A. (Ed.), The passions of law (pp. 285-308). New York: New York University Press.

DeSteno, D., Petty, R. E., Rucker, D. D., Wegener, D. T., \& Braverman, J. (2004). Discrete Emotions and Persuasion: The Role of Emotion-Induced Expectancies. Journal of Personality and Social Psychology, 86(1), 43-56. doi:10.1037/0022-3514.86.1.43

DeSteno, D., Petty, R. E., Wegener, D. T., \& Rucker, D. D. (2000). Beyond valence in the perception of likelihood: The role of emotion specificity. Journal of Personality and Social Psychology, 78(3), 397-416. doi:10.1037/00223514.78.3.397

Devlin, D. (Producer), \& Bill, T. (Director). (1980). My bodyguard [Motion picture]. United States: Fox Hills Video.

DeWitt, J. S., Richardson, J. T., \& Warner, L. G. (1997). Novel scientific evidence and controversial cases: A social psychological examination. Law \& Psychology Review, 21, 1-27. 
Dunn, J. R., \& Schweitzer, M. E. (2005). Feeling and Believing: The Influence of Emotion on Trust. Journal of Personality and Social Psychology, 88(5), 736-748. doi:10.1037/0022-3514.88.5.736

Eagly, A. H., \& Chaiken, S. (1993). The psychology of attitudes. Orlando, FL: Harcourt Brace Jovanovich College.

Edwards, J. A., \& Weary, G. (1993). Depression and the impression-formation continuum: Piecemeal processing despite the availability of category information. Journal of Personality and Social Psychology,64(4), 636-645. doi:10.1037/0022-3514.64.4.636

Ekman, P. (1972). Universals and cultural differences in facial expressions of emotion. In Cole, J. (Ed.), Nebraska Symposium on Motivation, 1971, 19 (pp. 207-282). Lincoln, NE: University of Nebraska Press.

Elster, J. (1998). Emotions in economic theory. Journal of Economic Literature, 36(1), 47-74.

Ellsworth, P. C., \& Scherer, K. R. (2003). Appraisal processes in emotion. In R. J. Davidson, K. R. Scherer, \& H. H. Goldsmith (Eds.), Series in affective science. Handbook of affective sciences (pp. 572-595). New York: Oxford University Press.

Ellsworth, P. C., \& Smith, C. A. (1988). From appraisal to emotion: Differences among unpleasant feelings. Motivation and Emotion, 12(3), 271-302. doi:10.1007/BF00993115

Epstein, S. (1994). Integration of the cognitive and the psychodynamic unconscious. American Psychologist, 49(8), 709-724. doi:10.1037/0003066X.49.8.709

Epstein, S., \& Pacini, R. (1999). Some basic issues regarding dual-process theories from the perspective of cognitive-experiential self-theory. In S. Chaiken \& Y. Trope (Eds.), Dual-process theories in social psychology (pp. 462-482). New York: Guilford Press.

Epstein, S., Pacini, R., Denes-Raj, V., \& Heier, H. (1996). Individual differences in intuitive-experiential and analytical-rational thinking styles. Journal of Personality and Social Psychology, 71(2), 390-405. doi:10.1037/00223514.71.2.390

Federal Rules of Evidence: FRE 403. 
Feigenson, N., \& Park, J. (2006). Emotions and attributions of legal responsibility and blame: A research review. Law and Human Behavior, 30(2), 143-161. doi:10.1007/s10979-006-9026-z

Festinger, L. (1954). A theory of social comparison processes. Human Relations, 7, 117140. doi:10.1177/001872675400700202

Fiedler, K. (2000). Toward an integrative account of affect and cognition phenomena using the BIAS computer algorithm. In J. P. Forgas (Ed.), Studies in emotion and social interaction, second series. Feeling and thinking: The role of affect in social cognition (pp. 223-252). New York: Cambridge University Press.

Fiedler, K., \& Stroehm, W. (1986). What kind of mood influences what kind of memory: The role of arousal and information structure. Memory \& Cognition, 14(2), 181188.

Fischhoff, B., Gonzalez, R. M., Lerner, J. S., \& Small, D. A. (2005). Evolving Judgments of Terror Risks: Foresight, Hindsight, and Emotion. Journal of Experimental Psychology: Applied, 11(2), 124-139. doi:10.1037/1076-898X.11.2.124

Fiske, S. T., \& Taylor, S. E. (1991). Social cognition (2nd ed.). New York, NY: McGraw-Hill Book Company.

Florida Standard Jury Instructions - Civil, $§ 700$.

Florida Standard Jury Instructions - Criminal, § 3.10.

Forgas, J. P. (1992). Affect in social judgments and decisions: A multiprocess model. In M. P. Zanna (Ed.), Advances in experimental social psychology, Vol. 25 (pp. $227-$ 275). doi:10.1016/S0065-2601(08)60285-3

Forgas, J. P. (1993). Affect, appraisal, and action: Towards a multiprocess framework. In R. S. Wyer, Jr. \& T. K. Srull (Eds.), Advances in social cognition, Vol. 6. Perspectives on anger and emotion (pp. 89-107). Hillsdale, NJ, England: Lawrence Erlbaum Associates.

Forgas, J. P. (1994). The role of emotion in social judgments: An introductory review and an Affect Infusion Model (AIM). European Journal of Social Psychology, 24(1), 1-24. doi:10.1002/ejsp.2420240102

Forgas, J. P. (1995). Mood and judgment: The affect infusion model (AIM). Psychological Bulletin, 117(1), 39-66. doi:10.1037/0033-2909.117.1.39 
Forgas, J. P. (1998). On being happy and mistaken: Mood effects on the fundamental attribution error. Journal of Personality and Social Psychology, 75(2), 318-331. doi:10.1037/0022-3514.75.2.318

Forgas, J. P. (2000). Affect and information processing strategies: An interactive relationship. In J. P. Forgas (Ed.), Studies in emotion and social interaction, second series. Feeling and thinking: The role of affect in social cognition (pp. 253-280). New York: Cambridge University Press.

Forgas, J. P. (2003). Affective influences on attitudes and judgments. In R. J. Davidson, K. R. Scherer, \& H. H. Goldsmith (Eds.), Series in affective science. Handbook of affective sciences (pp. 596-618). New York: Oxford University Press.

Forgas, J. P. (2010). Affect in legal and forensic settings: The cognitive benefits of not being too happy. In B. H. Bornstein \& R. L. Wiener (Eds.), Nebraska symposium on motivation. Emotion and the law: Psychological Perspectives (pp. 13-44). New York: Springer Science + Business Media.

Forgas, J. P., \& Bower, G. H. (1987). Mood effects on person-perception judgments. Journal of Personality and Social Psychology, 53(1), 53-60. doi:10.1037/0022-3514.53.1.53

Freud, S. (1920). A general introduction to psychoanalysis: Authorized translation with a preface. New York: Boni and Liveright. doi:10.1037/10667-000

Frijda, N. H. (1986). Studies in emotion and social interaction. The emotions. New York: Cambridge University Press.

Goldin, P. R., McRae, K., Ramel, W., \& Gross, J. J. (2008). The neural bases of emotion regulation: Reappraisal and suppression of negative emotion. Biological Psychiatry, 63(6), 577-586. doi:10.1016/j.biopsych.2007.05.031

Goodenough, O. R., \& Prehn, K. (2004). A neuroscientific approach to normative judgment in law and justice (pp. 77-109). New York, NY: Oxford University Press.

Göritz, A. S. (2007). The induction of mood via the WWW. Motivation and Emotion, 31(1), 35-47. doi:10.1007/s11031-006-9047-4

Göritz, A. S., \& Moser, K. (2006). Web-based mood induction. Cognition and Emotion, 20(6), 887-896. doi:10.1080/02699930500405386

Gosling, S. D., Vazire, S., Srivastava, S., \& John, O. P. (2004). Should we trust webbased studies? A comparative analysis of six preconceptions about internet 
questionnaires. American Psychologist, 59(2), 93-104. doi:10.1037/0003066X.59.2.93

Greene, E. \& Ellis, L. (2007). Decision making in criminal justice. In Carson, D., Milne, B., Pakes, F., Shalev, K., \& Shawyer, A. (Eds.), Applying psychology to criminal justice (pp. 183-200). West Sussex, England: John Wiley \& Sons Ltd.

Gross, J. J., \& Levenson, R. W. (1995). Emotion elicitation using films. Cognition and Emotion, 9(1), 87-108. doi:10.1080/02699939508408966

Haddock, G., Maio, G. R., Arnold, K., \& Huskinson, T. (2008). Should persuasion be affective or cognitive? The moderating effects of need for affect and need for cognition. Personality and Social Psychology Bulletin, 34(6), 769-778. doi:10.1177/0146167208314871

Han, S., Lerner, J. S., \& Keltner, D. (2007). Feelings and consumer decision making: The appraisal-tendency framework. Journal of Consumer Psychology, 17(3), 158-168. doi:10.1016/S1057-7408(07)70023-2

Hardesty, T. (Producer). (1997). Alaska's Wild Denali: Summer in Denali National Park [Motion picture]. United States: Alaska Video Postcards.

Hartlage, S., Alloy, L. B., Vázquez, C., \& Dykman, B. (1993). Automatic and effortful processing in depression. Psychological Bulletin, 113(2), 247-278. doi:10.1037/0033-2909.113.2.247

Heider, F. (1958). The psychology of interpersonal relations. Hillsdale, NJ: Lawrence Erlbaum Associates. Reprint available at http://www.questia.com/PM.qst?a=o\&d=26300808

Hibbard et al. v. McGraw et al., 918 So. 2d 967; 2005 Fla. App. LEXIS 18878; 30 Fla. L. Weekly D 2714.

Honess, T. M., \& Charman, E. A. (2002). Members of the jury--Guilty of incompetence? The Psychologist, 15(2), 72-75.

Horowitz, I. A., \& Bordens, K. S. (2000). The consolidation of plaintiffs: The effects of number of plaintiffs on jurors' liability decisions, damage awards, and cognitive processing of evidence. Journal of Applied Psychology,85(6), 909-918. doi:10.1037/0021-9010.85.6.909

Horowitz, I. A., Bordens, K. S., Victor, E., Bourgeois, M. J., \& ForsterLee, L. (2001). The effects of complexity on jurors' verdicts and construction of evidence. Journal of Applied Psychology, 86(4), 641-652. doi:10.1037/00219010.86.4.641 
Hovland, C. I., \& Weiss, W. (1951). The influence of source credibility on communication effectiveness. Public Opinion Quarterly, 15, 635-650. doi:10.1086/266350

Inbar, Y., \& Pizarro, D. (2009). Grime and punishment: How disgust influences moral, social, and legal judgments. The Jury Expert, 21(2), 11-22.

Joachim, H. H. (1998). Aristotle: The Nicomachean Ethics. Oxford University Press. Retrieved from EBSCOhost.

Johnson, E. J., \& Tversky, A. (1983). Affect, generalization, and the perception of risk. Journal of Personality and Social Psychology, 45(1), 20-31. doi:10.1037/0022-3514.45.1.20

Kahneman, D., \& Tversky, A. (1973). On the psychology of prediction. Psychological Review, 80(4), 237-251. doi:10.1037/h0034747

Kamin, K. A., \& Rachlinski, J. J. (1995). Ex post $\neq$ ex ante: Determining liability in hindsight. Law and Human Behavior, 19(1), 89-104. doi:10.1007/BF01499075

Kappas, A. (2001). A metaphor is a metaphor is a metaphor: Exorcising the homunculus from appraisal theory. In K. R. Scherer, A. Schorr, \& T. Johnstone (Eds.), Series in affective science. Appraisal processes in emotion: Theory, methods, research (pp. 157-172). New York: Oxford University Press.

Keltner, D., Ekman, P., Gonzaga, G. C., \& Beer, J. (2003). Facial expression of emotion. In R. J. Davidson, K. R. Scherer, \& H. H. Goldsmith (Eds.), Series in affective science. Handbook of affective sciences (pp. 415-432). New York: Oxford University Press.

Keltner, D., Ellsworth, P. C., \& Edwards, K. (1993). Beyond simple pessimism: Effects of sadness and anger on social perception. Journal of Personality and Social Psychology, 64(5), 740-752. doi:10.1037/0022-3514.64.5.740

Keltner, D., Locke, K. D., \& Audrain, P. C. (1993). The influence of attributions on the relevance of negative feelings to personal satisfaction. Personality and Social Psychology Bulletin, 19(1), 21-29. doi:10.1177/0146167293191003

Korobkin, R. B., \& Ulen, T. S. (2000). Law and behavioral science: Removing the rationality assumption from law and economics. California Law Review, 88, 1051-1144. 
Krauss, D. A., Lieberman, J. D., \& Olson, J. (2004). The effects of rational and experiential information processing of expert testimony in death penalty cases. Behavioral Sciences \& the Law, 22(6), 801-822. doi:10.1002/bsl.621

Kubrick, S. (Producer/Director). (1980). The shining [Motion picture]. United States: Warner Home Video.

Kuhn, D., Weinstock, M., \& Flaton, R. (1994). How well do jurors reason? Competence dimensions of individual variation in a juror reasoning task. Psychological Science, 5, 289-296. doi:10.1111/j.1467-9280.1994.tb00628.x

Landau, E. (2011, June 28). Murder trial jurors can be overwhelmed, traumatized. $C N N$ Health. Retrieved from http://www.cnn.com/2011/HEALTH/06/28/jury.stress.ptsd/index.html?hpt=hp_c2 \#

Lazarus, R.S. (1966). Psychological stress and the coping process. New York: McGrawHill.

Lazarus, R. S. (1984). On the primacy of cognition. American Psychologist, 39(2), 124129. doi:10.1037/0003-066X.39.2.124

Lazarus, R. S. (1999). The cognition-emotion debate: A bit of history. In Dalgleish, T., \& Power, M. J. (Eds.), Handbook of cognition and emotion (pp. 3-19). New York, NY: John Wiley \& Sons Ltd.

Leippe, M. R., Eisenstadt, D., Rauch, S. M., \& Seib, H. M. (2004). Timing of Eyewitness Expert Testimony, Jurors' Need for Cognition, and Case Strength as Determinants of Trial Verdicts. Journal of Applied Psychology, 89(3), 524-541. doi:10.1037/0021-9010.89.3.524

Leippe, M. R., \& Romanczyk, A. (1989). Reactions to child (versus adult) eyewitnesses: The influence of jurors' preconceptions and witness behavior. Law and Human Behavior, 13(2), 103-132. doi:10.1007/BF01055919

Lench, H. C., Flores, S. A., \& Bench, S. W. (2011). Discrete emotions predict changes in cognition, judgment, experience, behavior, and physiology: A meta-analysis of experimental emotion elicitations. Psychological Bulletin, 137, 834-855. doi:10.1037/a0024244

Lerner, J. S., Goldberg, J. H., \& Tetlock, P. E. (1998). Sober second thought: The effects of accountability, anger, and authoritarianism on attributions of responsibility. Personality and Social Psychology Bulletin, 24(6), 563-574. doi:10.1177/0146167298246001 
Lerner, J. S., Gonzalez, R. M., Small, D. A., \& Fischhoff, B. (2003). Effects of fear and anger on perceived risks of terrorism: A national field experiment. Psychological Science, 14(2), 144-150. doi:10.1111/1467-9280.01433

Lerner, J. S., \& Keltner, D. (2000). Beyond valence: Toward a model of emotion-specific influences on judgement and choice. Cognition and Emotion, 14(4), 473-493. doi:10.1080/026999300402763

Lerner, J. S., \& Keltner, D. (2001). Fear, anger, and risk. Journal of Personality and Social Psychology, 81, 146-159. doi:10.1037//0022-3514.81.1.146

Lerner, J. S., \& Tiedens, L. Z. (2006). Portrait of The Angry Decision Maker: How Appraisal Tendencies Shape Anger's Influence on Cognition. Journal of Behavioral Decision Making, 19(2), 115-137. doi:10.1002/bdm.515

Levenson, R. W., Ekman, P., \& Friesen, W. V. (1990). Voluntary facial action generates emotion-specific autonomic nervous system activity. Psychophysiology, 27(4), 363-384. doi:10.1111/j.1469-8986.1990.tb02330.x

Lieberman, J. D. (2002). Head over the heart or heart over the head? Cognitive experiential self-theory and extralegal heuristics in juror decision making. Journal of Applied Social Psychology,32(12), 2526-2553. doi:10.1111/j.15591816.2002.tb02755.x

Lieberman, M. D., Gaunt, R., Gilbert, D. T., \& Trope, Y. (2002). Reflexion and reflection: A social cognitive neuroscience approach to attributional inference. In Zanna, M. P. (Ed.), Advances in experimental social psychology, Vol. 34, (pp. 199-249). San Diego, CA, US: Academic Press.

Litvak, P. M., Lerner, J. S., Tiedens, L. Z., \& Shonk, K. (2010). Fuel in the fire: How anger impacts judgment and decision-making. In M. Potegal, G. Stemmler, \& C. Spielberger (Eds.), International handbook of anger: Constituent and concomitant biological, psychological, and social processes (pp. 287-310). New York: Springer Science + Business Media.

Loewenstein, G. F., Weber, E. U., Hsee, C. K., \& Welch, N. (2001). Risk as feelings. Psychological Bulletin, 127(2), 267-286. doi:10.1037/00332909.127.2.267

Mackie, D. M., \& Worth, L. T. (1989). Processing deficits and the mediation of positive affect in persuasion. Journal of Personality and Social Psychology, 57(1), 27-40. doi:10.1037/0022-3514.57.1.27

Mackie, D. M., \& Worth, L. T. (1991). Feeling good, but not thinking straight: The impact of positive mood on persuasion. In J. P. Forgas (Ed.), International series 
in experimental social psychology. Emotion and social judgments (pp. 201-219). Elmsford, NY: Pergamon Press.

Maio, G. R., \& Esses, V. M. (2001). The need for affect: Individual differences in the motivation to approach or avoid emotions. Journal of Personality, 69(4), 583-615. doi:10.1111/1467-6494.694156

Maroney, T. A. (2006). Law and emotion: A proposed taxonomy of an emerging field. Law and Human Behavior, 30(2), 119-142. doi:10.1007/s10979-006-9029-9

Martin, M. (1990). On the induction of mood. Clinical Psychology Review, 10(6), 669697. doi:10.1016/0272-7358(90)90075-L

Martin, L. L., Abend, T., Sedikides, C., \& Green, J. D. (1997). How would it feel if...? Mood as input to a role fulfillment evaluation process. Journal of Personality and Social Psychology, 73(2), 242-253. doi:10.1037/0022-3514.73.2.242

Martin, L. L., Ward, D. W., Achee, J. W., \& Wyer, R. S. (1993). Mood as input: People have to interpret the motivational implications of their moods. Journal of Personality and Social Psychology, 64(3), 317-326. doi:10.1037/00223514.64.3.317

McAuliff, B. D., \& Kovera, M. B. (2008). Juror need for cognition and sensitivity to methodological flaws in expert evidence. Journal of Applied Social Psychology, 38(2), 385-408.

Mikels, J. A., Maglio, S. J., Reed, A. E., \& Kaplowitz, L. J. (2011). Should I go with my gut? Investigating the benefits of emotion-focused decision making. Emotion, 11(4), 743-753. doi:10.1037/a00239

Montgomery, J. E. (2006). Cognitive biases in heuristics and tort litigation: A proposal to limit their effects without changing the world. Nebraska Law Review, 85, 15-51.

Moons, W. G., \& Mackie, D. M. (2007). Thinking straight while seeing red: The influence of anger on information processing. Personality and Social Psychology Bulletin, 33(5), 706-720. doi:10.1177/0146167206298566

Nickerson, R. S. (1998). Confirmation bias: A ubiquitous phenomenon in many guises. Review of General Psychology, 2(2), 175-220. doi:10.1037/10892680.2.2.175

Oatley, K., \& Johnson-Laird, P. N. (1987). Towards a cognitive theory of emotions. Cognition and Emotion, 1(1), 29-50. doi:10.1080/02699938708408362 
Ochsner, K. N., Ray, R. R., Hughes, B., McRae, K., Cooper, J. C., Weber, J., . . Gross, J. J. (2009). Bottom-up and top-down processes in emotion generation: Common and distinct neural mechanisms. Psychological Science, 20(11), 1322-1331. doi:10.1111/j.1467-9280.2009.02459.x

Operario, D., \& Fiske, S. T. (1999). Social cognition permeates social psychology: Motivated mental processes guide the study of human social behavior. Asian Journal of Social Psychology, 2(1), 63-78. doi:10.1111/1467-839X.00026

Pacini, R., \& Epstein, S. (1999). The relation of rational and experiential information processing styles to personality, basic beliefs, and the ratio-bias phenomenon. Journal of Personality and Social Psychology, 76(6), 972-987. doi:10.1037/00223514.76.6.972

Panksepp, J. (1982). Toward a general psychobiological theory of emotions. Behavioral and Brain Sciences, 5(3), 407-467. doi:10.1016/0166-4328(82)90044-4

Park, J., \& Banaji, M. R. (2000). Mood and heuristics: The influence of happy and sad states on sensitivity and bias in stereotyping. Journal of Personality and Social Psychology, 78(6), 1005-1023. doi:10.1037/0022-3514.78.6.1005

Pelham, B. W., \& Wachsmuth, J. O. (1995). The waxing and waning of the social self: Assimilation and contrast in social comparison. Journal of Personality and Social Psychology, 69(5), 825-838. doi:10.1037/0022-3514.69.5.825

Pennington, N., \& Hastie, R. (1986). Evidence evaluation in complex decision making. Journal of Personality and Social Psychology, 51(2), 242-258. doi:10.1037/0022-3514.51.2.242

Pennington, N., \& Hastie, R. (1988). Explanation-based decision making: Effects of memory structure on judgment. Journal of Experimental Psychology: Learning, Memory, and Cognition, 14(3), 521-533. doi:10.1037/0278-7393.14.3.521

Pennington, N., \& Hastie, R. (1992). Explaining the evidence: Tests of the Story Model for juror decision making. Journal of Personality and Social Psychology, 62(2), 189-206. doi:10.1037/0022-3514.62.2.189

Petty, R. E., \& Cacioppo, J. T. (1986). The elaboration likelihood model of persuasion. Advances in Experimental Social Psychology, 19, 123-205.

Petty, R. E., Cacioppo, J. T., Sedikides, C., \& Strathman, A. J. (1988). Affect and persuasion: A contemporary perspective. American Behavioral Scientist, 31(3), 355-371. doi:10.1177/000276488031003007 
Petty, R. E., Fabrigar, L. R., \& Wegener, D. T. (2003). Emotional factors in attitudes and persuasion. In R. J. Davidson, K. R. Scherer, \& H. H. Goldsmith (Eds.), Series in affective science. Handbook of affective sciences (pp. 752-772). New York: Oxford University Press.

Petty, R. E., \& Wegener, D. T. (1999). The elaboration likelihood model: Current status and controversies. In Chaiken, S., \& Trope, Y. (Eds.), Dual-process theories in social psychology, (pp. 37-72). New York, NY: Guilford Press.

Philippot, P. (1993). Inducing and assessing differentiated emotion-feeling states in the laboratory. Cognition and Emotion, 7(2), 171-193. doi:10.1080/02699939308409183

Pillsbury, S. H. (1999). Harlan, Holmes, and the passions of justice. In Bandes, S. A. (Ed.), The passions of law (pp. 330-362). New York: New York University Press.

Posner, R. A. (1999). Emotion vs. emotionalism in law. In Bandes, S. A. (Ed.), The passions of law (pp. 309-329). New York: New York University Press.

Potter, R., \& Brewer, N. (1999). Perceptions of witness behaviour-accuracy relationships held by police, lawyers and mock-jurors. Psychiatry, Psychology and Law, 6(1), 97-103. doi:10.1080/13218719909524952

Raghunathan, R., \& Pham, M. T. (1999). All negative moods are not equal: Motivational influences of anxiety and sadness on decision making. Organizational Behavior and Human Decision Processes, 79(1), 56-77. doi:10.1006/obhd.1999.2838

Rogers, C.R. (1959). A theory of therapy, personality and interpersonal relationships, as developed in the client-centered framework. In S. Koch (ed.), Psychology: A study of science (pp. 184-256). New York, NY: McGraw-Hill Book Company.

Roseman, I. J. (1984). Cognitive determinants of emotion: A structural theory. Review of Personality \& Social Psychology, 5, 11-36.

Rosenberg, E. L., \& Ekman, P. (2005). Coherence Between Expressive and Experiential Systems in Emotion. In P. Ekman \& E. L. Rosenberg (Eds.), Series in affective science. What the face reveals: Basic and applied studies of spontaneous expression using the facial action coding system (FACS, 2nd ed.) (pp. 63-88). New York: Oxford University Press.

Ross, M., \& Sicoly, F. (1979). Egocentric biases in availability and attribution. Journal of Personality and Social Psychology, 37(3), 322-336. doi:10.1037/00223514.37.3.322 
Rottenberg, J., \& Ray, R. D., \& Gross, J. J. (2007). Emotion elicitation using films. In Coan, J. A. \& Allen, J. J. B. (Eds.), The handbook of emotion elicitation and assessment (pp. 9-28). London: Oxford University Press.

Salerno, J. M., \& Bottoms, B. L. (2009). Emotional evidence and jurors' judgments: The promise of neuroscience for informing psychology and law. Behavioral Sciences \& the Law, 27(2), 273-296. doi:10.1002/bsl.861

Sanchirico, C. W. (2003). Finding error. Michigan State Law Review, Winter, 1189-1203.

Saxon, E., Utt, K., Bozman, R. (Producers), \& Demme, J. (Director). (1991). Silence of the lambs [Motion picture]. United States: Orion Pictures.

Schaefer, A., Nils, F., Sanchez, X., \& Philippot, P. (2010). Assessing the effectiveness of a large database of emotion-eliciting films: A new tool for emotion researchers. Cognition and Emotion, 24(7), 1153-1172. doi:10.1080/02699930903274322

Scherer, K. R. (1984a). Emotion as a multicomponent process: A model and some crosscultural data. Review of Personality \& Social Psychology, 5, 37-63.

Scherer, K. R. (1984b). On the nature and function of emotion: A component process approach. In Scherer, K. R., \& Ekman, P. (Eds.), Approaches to emotion (pp. 293317). Hillsdale, NJ: Lawrence Erlbaum.

Schorr, A. (2001). Appraisal: The evolution of an idea. In Scherer, K. R., Schorr, A., \& Johnstone, T. (Eds.), Appraisal processes in emotion: Theory, methods, research (pp. 20-34). New York, NY: Oxford University Press.

Schwarz, N. (1990). Feelings as information: Informational and motivational functions of affective states. In E. T. Higgins \& R. M. Sorrentino (Eds.), Handbook of motivation and cognition: Foundations of social behavior, Vol. 2 (pp. 527-561). New York: Guilford Press.

Schwarz, N., Bless, H., \& Bohner, G. (1991). Mood and persuasion: Affective states influence the processing of persuasive communications. In Zanna, M. (Ed.), Advances in Experimental Social Psychology, Vol. 24, (pp. 161-199).

Schwarz, N., \& Clore, G. L. (1983). Mood, misattribution, and judgments of well-being: Informative and directive functions of affective states. Journal of Personality and Social Psychology, 45(3), 513-523. doi:10.1037/0022-3514.45.3.513

Schwarz, N., \& Clore, G. L. (2003). Mood as Information: 20 Years Later. Psychological Inquiry, 14(3-4), 296-303. doi:10.1207/S15327965PLI1403\&4_20 
Semmler, C. \& Brewer, N. (2002). Effects of mood and emotion on juror processing and judgments. Behavioral Sciences and the Law, 20, 423-436. doi:10.1002/bsl.502

Sheppes, G., \& Meiran, N. (2008). Divergent cognitive costs for online forms of reappraisal and distraction. Emotion, 8(6), 870-874. doi:10.1037/a0013711

Shestowsky, D., \& Horowitz, L. M. (2004). How the need for cognition scale predicts behavior in mock jury deliberations. Law and Human Behavior, 28(3), 305-337. doi:10.1023/B:LAHU.0000029141.46850.fb

Simon, H. A. (1956). Rational choice and the structure of the environment. Psychological Review, 63(2), 129-138. doi:10.1037/h0042769

Small, D. A., \& Lerner, J. S. (2008). Emotional policy: Personal sadness and anger shape judgments about a welfare case. Political Psychology, 29(2), 149-168. doi:10.1111/j.1467-9221.2008.00621.x

Smith, C. A. \& Ellsworth, P. C. (1985). Patterns of cognitive appraisal in emotion. Journal of Personality and Social Psychology, 48(4), 813-838. doi:10.1037/00223514.48.4.813

Smith, C. A., \& Ellsworth, P. C. (1987). Patterns of appraisal and emotion related to taking an exam. Journal of Personality and Social Psychology, 52(3), 475-488. doi:10.1037/0022-3514.52.3.475

Smith, E. R. (1993). Social identity and social emotions: Toward new conceptualizations of prejudice. In D. M. Mackie \& D. L. Hamilton (Eds.), Affect, cognition, and stereotyping: Interactive processes in group perception (pp. 297-315). San Diego, CA: Academic Press.

Smith, E. R., \& DeCoster, J. (2000). Dual-process models in social and cognitive psychology: Conceptual integration and links to underlying memory systems. Personality and Social Psychology Review, 4(2), 108-131. doi:10.1207/S15327957PSPR0402_01

Smith, V. L. (1991). Prototypes in the courtroom: Lay representations of legal concepts. Journal of Personality and Social Psychology, 61(6), 857-872. doi:10.1037/0022-3514.61.6.857

Spencer, N., Briley, J. (Producers), \& Attenborough, R. (Director). (1987). Cry freedom [Motion picture]. United States: MCA/Universal Home Video.

Strack, F., \& Deutsch, R. (2004). Reflective and impulsive determinants of social behavior. Personality and Social Psychology Review, 8(3), 220-247. doi:10.1207/s15327957pspr0803_1 
Thompson, E. R. (2007). Development and Validation of an Internationally Reliable Short-Form of the Positive and Negative Affect Schedule (PANAS). Journal of Cross-Cultural Psychology, 38(2), 227-242. doi:10.1177/0022022106297301

Tiedens, L. Z. (2001). The effect of anger on the hostile inferences of aggressive and nonaggressive people: Specific emotions, cognitive processing, and chronic accessibility. Motivation and Emotion, 25(3), 233-251. doi:10.1023/A:1012224507488

Tiedens, L. Z., \& Linton, S. (2001). Judgment under emotional certainty and uncertainty: The effects of specific emotions on information processing. Journal of Personality and Social Psychology, 81(6), 973-988. doi:10.1037/00223514.81.6.973

Tomkins, S. S. (1962). Affect, imagery, consciousness: Vol. 1. The positive affects. Oxford, England: Springer.

Tomkins, S. S. (1963). Affect imagery consciousness, 2: The negative affects. New York: Tavistock/Routledge.

Trope, Y., \& Liberman, A. (1996). Social hypothesis testing: Cognitive and motivational mechanisms. In E. T. Higgins \& A. W. Kruglanski (Eds.), Social psychology: Handbook of basic principles (pp. 239-270). New York: Guilford Press.

Tversky, A., \& Kahneman, D. (1974). Judgment under uncertainty: Heuristics and biases. Science, 185(4157), 1124-1131. doi:10.1126/science.185.4157.1124

Tversky, A., \& Kahneman, D. (1981). The framing of decisions and the psychology of choice. Science, 211(4481), 453-458. doi:10.1126/science.7455683

Uleman, J. S., \& Bargh, J. A. (Eds.). (1989). Unintended thought. New York: Guilford Press.

Verheyen, C., \& Göritz, A. S. (2009). Plain texts as an online mood-induction procedure. Social Psychology, 40(1), 6-15. doi:10.1027/1864-9335.40.1.6

Verleur, R., Verhagen, P. W., \& Heuvelman, A. (2007). Can mood-inducing videos affect problem-solving activities in a web-based environment? British Journal of Educational Technology, 38(6), 1010-1019. doi:10.1111/j.14678535.2006.00688.x

Watson, D., \& Clark, L.A. (1994). The PANAS-X: Manual for the positive and negative affect schedule-expanded form. Unpublished manuscript, University of Iowa, Iowa City, IA. 
Weary, G., \& Jacobson, J. A. (1997). Causal uncertainty beliefs and diagnostic information seeking. Journal of Personality and Social Psychology, 73(4), 839848. doi:10.1037/0022-3514.73.4.839

Wegener, D. T., \& Petty, R. E. (1994). Mood management across affective states: The hedonic contingency hypothesis. Journal of Personality and Social Psychology, 66(6), 1034-1048. doi:10.1037/0022-3514.66.6.1034

Wegener, D. T., Petty, R. E., \& Smith, S. M. (1995). Positive mood can increase or decrease message scrutiny: The hedonic contingency view of mood and message processing. Journal of Personality and Social Psychology, 69(1), 5-15. doi:10.1037/0022-3514.69.1.5

Weinstein, N. D. (1980). Unrealistic optimism about future life events. Journal of Personality and Social Psychology, 39(5), 806-820. doi:10.1037/00223514.39.5.806

Weinstock, M. P., \& Flaton, R. A. (2004). Evidence coverage and argument skills: Cognitive factors in a juror's verdict choice. Journal of Behavioral Decision Making, 17(3), 191-212. doi:10.1002/bdm.470

Weldon, T. D. (1958). Kant's Critique of pure reason. Oxford: Clarendon Press.

Wells, G. L., Olson, E. A., \& Charman, S. D. (2002). The confidence of eyewitnesses in their identifications from lineups. Current Directions in Psychological Science, 11(5), 151-154. doi:10.1111/1467-8721.00189

Westermann, R., Spies, K., Stahl, G., \& Hesse, F. W. (1996). Relative effectiveness and validity of mood induction procedures: A meta-analysis. European Journal of Social Psychology, 26(4), 557-580. doi:10.1002/(SICI)10990992(199607)26:4<557::AID-EJSP769>3.0.CO;2-4

Wiener, R. L., Gaborit, M., Pritchard, C. C., McDonough, E. M., Staebler, C. R., Wiley, D. C., \& Goldkamp, K. S. (1994). Counterfactual thinking in mock juror assessments of negligence: A preliminary investigation. Behavioral Sciences \& the Law, 12(1), 89-102. doi:10.1002/bsl.2370120108

Wiener, R. L., Holtje, M., Winter, R. J., Cantone, J. A., Gross, K., \& Block-Lieb, S. (2007). Consumer credit card use: The roles of creditor disclosure and anticipated emotion. Journal of Experimental Psychology: Applied, 13(1), 32-46. doi:10.1037/1076-898X.13.1.32

Wilson, T. D., Centerbar, D. B., Kermer, D. A., \& Gilbert, D. T. (2005). The Pleasures of Uncertainty: Prolonging Positive Moods in Ways People Do Not 
Anticipate. Journal of Personality and Social Psychology, 88(1), 5-21. doi:10.1037/0022-3514.88.1.5

Winterich, K. P. (2011). Are your jurors mad or sad? Emotional blunting may influence their judgments in the courtroom. The Jury Expert, 23(3), 46-66.

Yost, J. H., \& Weary, G. (1996). Depression and the correspondent inference bias: Evidence for more effortful cognitive processing. Personality and Social Psychology Bulletin, 22(2), 192-200. doi:10.1177/0146167296222008

Zajonc, R. B. (1980). Feeling and thinking: Preferences need no inferences. American Psychologist, 35(2), 151-175. doi:10.1037/0003-066X.35.2.151

Zajonc, R. B. (1984). On the primacy of affect. American Psychologist, 39(2), 117-123. doi:10.1037/0003-066X.39.2.117 


\section{APPENDICES \\ LIST OF APPENDICES}

$\begin{array}{ll}\text { APPENDIX } & \text { PAGE }\end{array}$

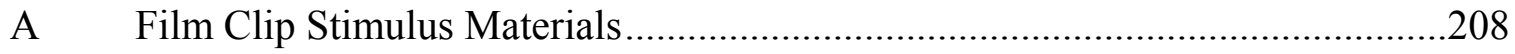

B Semmler and Brewer's (2002) Trial Transcript Materials..................................213

C Study One Data Reduction Procedures..............................................................241

D Study One Trial Transcript (15 Inconsistencies) ..............................................246

E List and Description of all 15 Inconsistencies from Study One .........................2270

F Study One Inconsistency Detection Questions ...............................................2.273

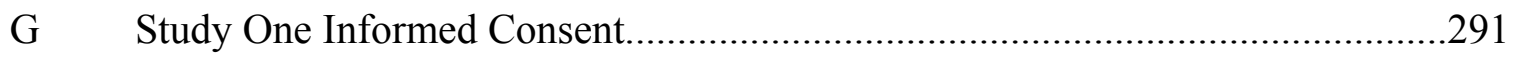

H Witness-Specific Likert Scales .....................................................................29

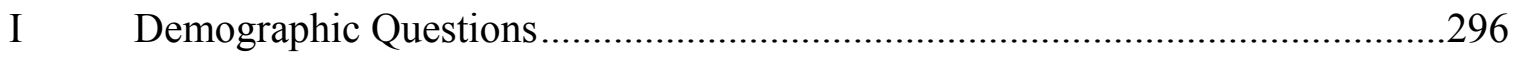

J Participant Debriefing Information ...............................................................29

K Open Ended Question Coding Instructions..........................................................300

L Short Answer Question Coding Instructions ………………..............................306

M Study One Scoring, Scale Creation, and Evaluation of Inconsistencies...............311

N Studies Two and Four Trial Transcript (Eight Inconsistencies) ............................321

O PANAS Short and Long Form Emotion Measurement Scales ..............................345

P Study Two Data Reduction Procedures ..............................................................349

Q Study Two Inconsistency Detection Questions …………………………….......354

R List and Description of all Eight Inconsistencies from Study Two …………......367

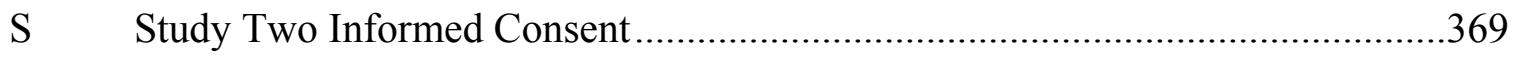

T Study Two Scoring, Scale Creation, and Evaluation of Inconsistencies ..............372 
U Study Three Data Reduction Procedures ......................................................381

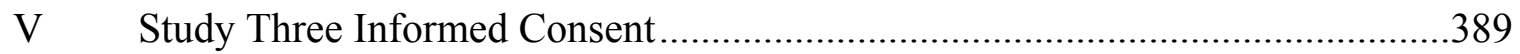

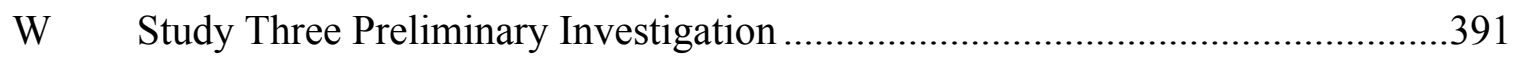

X Study Four Data Reduction Procedures ......................................................396

Y Study Four Part Two Informed Consent......................................................403

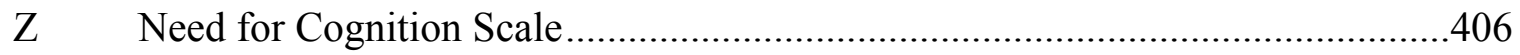

AA Rational-Experiential Inventory Scale ....................................................407

AB Study Four Part One Informed Consent......................................................408

AC Study Four Trial Transcript with No Inconsistencies ......................................411

AD Liability Jury Instructions and Verdict Form..........................................436

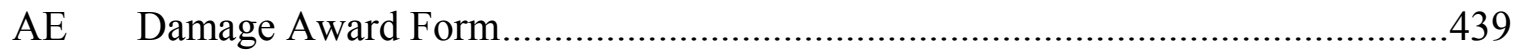

AF Study Four Scoring of Inconsistency Detection Rates ...................................440 


\section{APPENDIX A}

\section{Film Clip Stimulus Materials}

Rottenberg, J., \& Ray, R. D., \& Gross, J. J. (2007). Emotion elicitation using films. In J. A. Coan \& J. J. B. Allen (Eds.), The handbook of emotion elicitation and assessment. London: Oxford University Press.

\section{ANGER FILMS}

\section{BODYGUARD}

Film: "My Bodyguard"

Target emotion: Anger

Clip length: 4'06"'

Instructions: Advance to the first frame in which a growing circular form has the words "Magnetic Video" written under it in full. Reset the timer to 00:00:00:00 (hours:minutes:seconds:frames). Begin the clip at 01:12:23:05 (01:12:15:01). At this point, two men are visible, one wearing a red tank top and the other wearing an army jacket. A fight is about to begin. Several people are in the background, including a blondhaired boy in the lower right hand corner of the screen. Begin the clip at the first frame in which the blond-haired boy's hand covers both his mouth and nose. End the clip at 01:16:29:27 (01:16:20:20). At this point, a man dressed in a gray muscle shirt and black pants is exiting the scene, with trees, a wall, and a fence in the background. This is two frames before a shot of a man with a bloody nose on all fours. 


\section{CRY FREEDOM}

Film: "Cry Freedom”

Target emotion: Anger

Clip length: 2'36"'

Instructions: Advance to the point at which "Cry Freedom" is typed across the screen. At the first frame in which the " $\mathrm{m}$ " is visible, reset the timer to 00:00:00:00

(hours:minutes:seconds:frames). Begin the clip at 2:24:56:11 (02:25:56:07). At this point, there is a shift from a view of protesters in the distance to a close-up of a bald girl with a pink skirt and a boy crossing just in front of her. Begin the clip with the first frame of this close up. Stop recording at 2:25:16:07 (02:26:15:05). At this point, a boy in a dark gray sweater is jumping up and down. Stop recording at the last frame in which he is visible before the camera shifts to the two groups of protesters joining into one group. Begin recording at 2:25:32:06 (02:26:32:06). This is the point at which the camera switches to a view of the three groups of protesters who have just joined into one big group which is advancing straight toward the camera. End the clip at 2:27:49:10 (02:28:49:10). At this point, a man in a car has just shot a boy who was running away. Stop recording after the boy falls, at the first frame in which he is completely still.

\section{FEAR FILMS}

\section{SHINING}

Film: "The Shining"

Target emotion: Fear

Clip length: 1'22" 
Instructions: Advance to the first frame of the film, which shows a body of water surrounded by mountains. Reset the timer to 00:00:00:00 (hours:minutes:seconds:frames). Begin the clip at 00:56:51:15 (00:57:03:08). At this point, a boy's hands are visible (one flat on the floor and the other in a fist). There are toy trucks and cars on a red, brown, and orange carpet. End the clip at 00:58:12:18 (00:58:24:01). At this point, an open door with a key in the lock is visible, and one full second has passed since the boy has said "Mom, are you in there?"

\section{LAMBS}

Film: "Silence of the Lambs"

Target emotion: Fear

Clip length: 3'29”

Instructions: Advance to the first frame of the film in which the words "A STRONG HEART DEMME PRODUCTION" appear. Reset the timer to 00:00:00:00 (hours:minutes:seconds:frames). Begin the clip at 01:40:16:29 (01:40:56:01). At this point, a dirt road and trees are in the forefront and a mint green trailer is in the background. Stop recording at 01:43:44:23 (01:44:24:10). At this point, the profile of a dark-haired woman is visible. There is a metal wire hanging from the ceiling that appears to almost (but not quite) touch her nose and chin. Begin recording at 01:46:36:24 (01:47:16:01). At this point, hands holding a gun are moving rapidly into the scene from the right of the screen. In the background, there is dirty yellow wallpaper. End the clip at 01:46:38:19 (01:47:18:01). At this point, the dark-haired woman has her back to the yellow wallpaper, and has pointed her gun between the upper-middle and the upper-right 
hand portions of the screen. Her right hand obscures most of the left half of her face and we hear her exclaim as the lights go out.

\section{NEUTRAL FILMS}

\section{STICKS}

Film:"Noncommercial Screen Saver"

Target emotion: Neutral

Clip Length 3'26"

Instructions: Film available for download at

http://www.cas.usf.edu/psychology/fac_rottenbergJ.htm

\section{DENALI}

Film: "Alaska's Wild Denali"

Target emotion: Neutral

Clip Length: 5'02"

Instructions: Reset the timer to 00.00.00:00 when the credits for the Alaskan production company come up. Begin the clip at 00:33:28:00 (00:33:15:00), right after a person plays a guitar, start as the music is still playing and fading, but the visual is a silhouette of a mountain and the midnight sky; the narrator talks about the Alaskan midnight sky. End the clip at 00:38:30:00 (00:38:17:00). At this point, a buck is eating little grasses and there is a shot of a mountain stream. 


\section{POST FILM QUESTIONNAIRE}

The following questions refer to how you felt while watching the film.

\begin{tabular}{|cccccccc|}
\hline $\begin{array}{c}0 \\
\text { not at all } \\
\text { none }\end{array}$ & 1 & 2 & 3 & $\begin{array}{c}4 \\
\text { somewhat/ } \\
\text { some }\end{array}$ & 5 & 6 & $\begin{array}{c}7 \\
\text { extremely/ } \\
\text { a great deal }\end{array}$ \\
\hline
\end{tabular}

Using the scale above, please indicate the greatest amount of EACH emotion you experienced while watching the film.

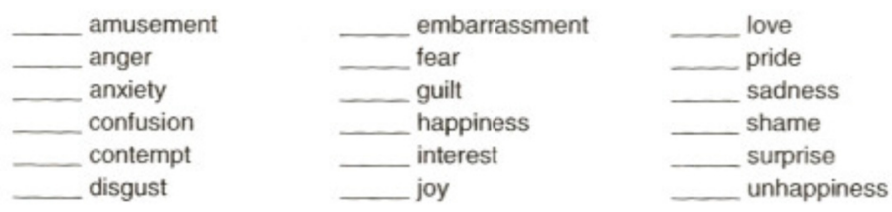

Did you feel any other emotion during the film? O No O Yes

If so, what was the emotion?

How much of this emotion did you feel?

Please use the following pleasantness scale to rate the feelings you had during the film. Circle your answer:

$\begin{array}{ccccccccc}\begin{array}{c}0 \\ \text { unpleasant }\end{array} & 1 & 2 & 3 & 4 & 5 & 6 & 7 & 8 \\ \text { pleasant }\end{array}$

Had you seen this film before? O No O Yes

Did you close your eyes or look away during any scenes? $O$ No $O$ Yes 


\section{APPENDIX B}

Semmler and Brewer's (2002) Trial Transcript Materials

\section{INCONSISTENT NEUTRAL}

Judge: Good morning Ladies and Gentlemen, my name is Judge Stevenson. Today we are to hear in the Supreme Court the case of the Crown versus Mark Alexander Fitzpatrick. Mr Fitzpatrick is charged with causing death by dangerous driving. $\mathrm{Mr}$ Spence, you are now invited to open the case for the prosecution. Prosecution : Thank you Your Honour. Ladies and Gentlemen of the jury, my name is Henry Spence, I am the prosecuting attorney in the case of the Crown vs Mark Alexander Fitzpatrick. You will hear eyewitness evidence today that proves beyond reasonable doubt that Mr Fitzpatrick is solely responsible for the death of Celia White. The accident took place at approximately 10am on the 14th of April 1998 on Mount Barker Road near Eagle on the Hill. Driving home from a visit with relatives, the family in their Corolla sedan rounded a left hand bend and were confronted with a large fuel tanker on the wrong side of the road. The driver panicked, swerved causing the trailer to jack-knife across the path of the oncoming car. The car hit the trailer on the driver's side. Unfortunately, Celia White and her daughter were trapped in the twisted wreckage. They were trapped for 20 minutes. The mother sustained only a broken and gashed leg. Celia White's husband and son were looking on. They could not help her. They had to stand back because there was fuel all over the road. The daughter was eventually rescued by the 'jaws of life'.

You will hear eyewitness evidence that proves that Mr Fitzpatrick drove his semitrailer in a manner that created potential danger to other people using the highway. This was solely and act of carelessness and inattention and an act that directly caused the death of Celia White. You, the jury, can deliver justice which by finding the defendant guilty of causing death by dangerous driving. Thank you Your Honour. Judge: Mr Warwick, you may now open the case for the defence.

Defence; Thankyou Your Honour. Ladies and Gentlemen of the Jury, My name is Melvin Warwick, I am the attorney defending my client Mr Fitzpatrick in this case. The prosecution will allege on the basis of eyewitness testimony that my client was solely responsible for the injury which occurred when his truck collided with the eyewitness' car on the morning of April 14th. Ladies and Gentlemen, I want you to first remember while listening to the eyewitness evidence that the eyewitness was actually in the car at the time of the accident which means that he may not have seen the things he alleges to have seen. Second, he and his young son had to watch his wife and daughter trapped in the wreckage of a car that was in imminent danger of bursting into flames. This is bound to cause feelings of helplessness and perhaps a strong need to blame someone. While not in any way meaning to deny the adverse nature of the events that occurred on that day, I have to emphasise that Mr White is not an impartial witness who has the benefit of objectivity. Finally, at the time of the accident he must have been under a great deal of stress and this most likely affected his ability to recall the details of an event. Ladies and Gentlemen, it is our case that it was the witness's car which crossed over the double white lines that morning causing Mr Fitzpatrick to 
swerve and his trailer to jack-knife across the road and into the witness' car. Finally, Ladies and Gentlemen, I ask you to consider the suffering of the truck's driver $\mathrm{Mr}$ Fitzpatrick. He walked away physically unarmed from the accident. However, psychologically he is distraught. He knows that he could not have avoided the accident, but he still blames himself despite the physical evidence which proves his innocence. Ladies and Gentlemen, in considering the evidence, remember that the Crown bears the burden of proof. They must prove beyond reasonable doubt that my client drove in a manner dangerous to the public, that this was the result of carelessness and further that this action was the sole cause of the death which resulted from the collision. You can deliver justice and ease Mr Fitzpatrick's suffering by delivering a not guilty verdict in accordance with the truth. Thank you Your Honour. Judge: Mr Spence, please call your first witness.

Prosecution: Thankyou Your Honour. The Crown calls Police Constable Roger McNally to the stand. \{Pause\} Mr McNally, My name is Henry Spence, I am the prosecuting Attorney. I would like to ask you some questions about the accident which took place on Mount Barker Road, near Eagle on the Hill, on the morning of April 14th 1998. We are keen to establish the truth so please answer where appropriate with either, I don't know, I 'm not sure or I am sure.

Police Constable: Yes, I will.

Prosecution: You were the investigating officer when this accident occurred?

Police Constable: Yes, that is correct.

Prosecution: Can you describe for the jury the position of the vehicles when you arrived at the scene of the accident that morning.

Police Constable: Certainly. The semi-trailer was blocking the whole road. The cabin was in the middle of the down track with its trailer perpendicular to the cabin obstructing the rest of the down track and extending all the way across the up track. The truck had been travelling down Mount Barker Road away from Eagle on the Hill and the car had been travelling up. (1)The car had impacted the trailer about half way along its right side. There wasn't much left of the car, the motor had been pushed back to the center console. The car had hit mostly on the driver's side. The car was completely caved in on the driver's side. I arrived on the scene before the ambulance. The woman was pinned in the driver's seat. She was bleeding profusely. The father and son who had managed to get out of the vehicle were yelling at me to get them out of the wreckage. I couldn't do anything because of the risk of the fuel around the car igniting and causing an explosion ...I tried to move them away from the car, they wouldn't leave the rest of their family trapped....we just had to wait...Mr Spence, I was so surprised that they all managed to make it out alive. When I got there and saw the damage to the car I was amazed that they hadn't all been killed.

Prosecution: Mr McNally......Was there any evidence that either of the vehicles had taken evasive action, such as breaking or swerving before impact?

Police Constable: (2) Yes, there were wide skid marks from the truck trailer skewing sideways which were 28 meters in length and from the truck going in a direction perpendicular to those of the truck which were a 2 meters longer. There were no skid marks from the car.

Prosecution: From the length of the skid marks, the damage caused upon impact and 
the position of the vehicles, can any estimates about the speed of the truck and car be made.

Police Constable: It is difficult to determine. However, it appears that the car may have been travelling at about $32 \mathrm{~km} /$ hour and the truck at about 50 .

Prosecution: Mr McNally, what causes a trailer to jack-knife or begin to push the cabin of a truck along ?

Police Constable; There are several different causes for a trailer to jack-knife. It can be caused by incorrect tyre pressure, insufficient or too heavy loads, under gear out of alignment or worn steering or other faults in old vehicles.

Prosecution: In your opinion was the jack-knife of the truck trailer caused by any of the problems that you just mentioned?

Police Constable; No. We checked the vehicle thoroughly. It was in good condition mechanically.

Prosecution: What in your opinion, was the cause of the collision?

Police Constable: The truck obviously swerved to it's left and braked heavily. This caused the trailer to jack-knife and the car ran into the trailer. I can not speculate on the reason why the driver of the truck had to brake.

Prosecution: Is it possible Mr McNally, that the driver swerved and braked because he suddenly realised he was on the wrong side of the road?

Police Constable: Yes it is possible.

Prosecution: Thankyou Mr McNally. No further questions Your honour.

Judge: Mr Warwick, you may now cross examine the witness.

Defence: Good morning Mr McNally. My name is Melvin Warwick, I am defending my client Mr Fitzpatrick the driver of the semi-trailer. Mr McNally, you stated to my learned friend $\mathrm{Mr}$ Spence, that there are several reasons for a trailer to jack-knife in the manner you described.

Police Constable: Yes.

Defence: Is it not equally possible that the driver of the truck, my client, may have braked and swerved to avoid a collision when he saw the car driven by Celia White on the wrong side of the road?

Police Constable: Yes it is possible. As I said before, the trailer jack-knifed because the truck braked heavily and swerved to it's left. I can only speculate on the reasons why the truck braked in the first place. I can only tell you that the trailer moved to the other side of the road because of the braking and swerving. Def: Thankyou Mr McNally. No further questions Your Honour. *

Judge: Mr Spence, you may now call your second witness to the stand.

Prosecution: Thankyou Your Honour. The Crown calls James White to the stand. Goodmorning Mr White, my name is Henry Spence, I am the prosecuting attorney, I would like to ask you some questions about the accident which took place on Mount Barker Road at Eagle on the Hill, Monday the 14th of April 1998. Mr White it is essential for the carriage of justice that the jury have a good understanding of the events leading up to the accident. We are keen to establish the truth so please answer where appropriate with either, I don't know, I'm not sure or I am sure.

Witness: Yes I will. 
Prosecution: Would you state your full name please.

Witness: James Phillip White.

Prosecution: What is your full home address.

Witness: 20 Druids Avenue, Mount Barker, South Australia.

Prosecution: At approximately 10am on Monday the 14th of April 1998 were you a passenger in the vehicle travelling along Mount Barker road, which was subsequently involved in the accident in question?

Witness: Yes, I was.

Prosecution: Why was the vehicle in which you were a passenger travelling along Mount Barker Road on the morning of the accident?

Witness: My family and I were returning from a visit with my parents at Clarence Gardens in Adelaide.

Prosecution: Who was in the car with you?

Witness: My wife Celia, who was driving, and our children Kayla and Jarrod.

Prosecution: Had Celia, your wife, had any accidents prior to the one that occurred that morning?

Witness: No. Celia had an impeccable driving record.

Prosecution: Did Celia have any impairments or disabilities which may have reduced her ability to drive ?

Witness: No, she did not.

Prosecution: Was Celia tired, stressed or distracted on the day while she was driving the vehicle?

Witness: No she was not.

Prosecution: Were the children distracting her at any point during the trip ?

Witness: No.

Prosecution: Are you sure Mr White, children can sometimes find it difficult to behave on long trips?

Witness: The children were being well behaved, talking to each other in the back.

Celia's attention was on the road the whole time.

Prosecution: Do you have any problems with your eyes Mr White?

Witness: No

Prosecution: When was the last time you had your eyes checked?

Witness: About 3 months ago. We get them checked regularly because of the type of work I do.

Prosecution: Mr White, can you tell the court in detail, what you were doing 5 minutes prior to the accident.

Witness: Well, as I said before, we were returning home from a visit to my parent's place in Adelaide. We were driving up Mount Barker Road...

Prosecution: Mr White, what exactly was going on in the car at this point?

Witness: We were talking to the kids about what they had been doing at Grandma's

place.

Prosecution: When did you first notice the truck?

Witness: (4)When we rounded the left hand bend just before where the accident happened. You couldn't see around the corner because at that point the road is actually bending around the steep hill on the left. 
Prosecution: When you first saw the truck, where in relation to your car, was it located ?

Witness: It was directly in front of us, a little to the left of the center of our car.

Prosecution: Was the truck over the double white lines on the road?

Witness: Yes.

Prosecution: In your estimation, how far over the white lines ?

Witness: At least 3 meters. About from here to the jury away. (3) It was $3 / 4$ the way over on our side of the road. So far over that we couldn't have swerved around it, there simply wasn't room.

Prosecution: Did the truck appear to swerve or deviate from it's course at any time prior to impact, Mr White?

Witness: Yes, it did. I remember that Celia had time to flash the lights a couple of times... (5)it swerved to our left (3).

Prosecution: At what speed were you travelling ?

Witness: I reckon it was only about $40 \mathrm{~km} / \mathrm{h}$. We had slowed down to take the corner.

Prosecution: At what speed in your estimation was the truck travelling?

Witness: It must have been over $60 \mathrm{~km} / \mathrm{h}$. We had slowed down, it came towards us very quickly. (5)Celia didn't have time to do anything.

Prosecution: Did the truck appear to slow down or brake at any point ?

Witness: (2) No it didn't brake at all. As I said it happened very quickly. There was this petrol truck, or Ampol truck and the trailer across the road coming straight for us and.... It was very quick. There was no way Celia could have avoided it.

Prosecution: Which side of the trailer did your car hit?

Witness: (1) We hit on the trailer's left side.

Prosecution: Is there anything else that you think is important to tell the court $\mathrm{Mr}$ White?

Witness: Yes, I'd just like the jury to know that Celia was always a good driver. Prosecution: No further questions Your Honour.

*

Judge: Thankyou Mr Spence. Mr Warwick, you may now cross-examine the witness. Defence: Thankyou Your Honour. Good morning Mr White. My name is Melvin Warwick and I am the attorney for the defence in this case. I have some questions to ask you about the accident which took place on Mount Barker Road in which you were involved. The justice system demands that the jury have a clear picture of these events, so please answer the questions as accurately as possible. \{Pause\} At what point did you think you saw the truck on the wrong side of the road ?

Witness: (4)I'd say at least 15 meters before we rounded the bend. Before the accident scene, I could see that it was on the wrong side of the road. I could see the (6) paint of the Mobil symbol and the chrome trailer shining through the trees.

Defence: And you are sure you could tell that the truck was on the wrong side of the road?

Witness: Yes. I am sure.

Defence: You were rounding the bend and travelling at a reasonable speed and yet you are sure were able to discern that the truck was on the wrong side of the road ? Witness: Yes, that's what I'm telling you. 
Defence: Mr White, if you saw the truck was on the wrong side of the road before you reached the bend, why didn't you warn your wife ?

Witness: I don't know. It happened very quickly. I wasn't $100 \%$ sure that it was on the wrong side before we rounded the bend. But I could see it definitely was once we got further the way around. (3)It was almost half way over onto our side of the road. Defence: Between the time that you saw the truck through the trees and the time that you saw it coming toward you, did your eyes remain on the road?

Witness: No.

Defence: Exactly what were you doing in between the time that you saw the truck through the trees and the time that you rounded the bend?

Witness:(7) I was getting a tissue for Kayla. She'd spilt her drink.

Defence: Mr White, your wife maintained her attention on the road the whole time while you were trying to clean up the mess on the back seat?

Witness: Yes.

Defence: Is it not possible that she too was distracted by your daughter's mishap, and that her attention left the road for a few moments also ?

W: No.

Def: Mr White, I think that perhaps she was distracted and that you needed to blame someone for your misfortune and that happens to be my client Mr Fitzpatrick.

W: No that's not true! He caused the accident, his truck was on the wrong side of the road!

Def: No further questions Your Honour.

Judge: Mr Spence, you may now address the jury with your closing statement.

Pro: Thankyou Your Honour. Ladies and Gentlemen of the jury, you have heard evidence today from two witnesses to the accident which occurred on April 14th 1999 on Mount Barker Road. Mr Fitzpatrick may well have suffered as the result of these events, however, the White family has suffered much more. The testimony of both Constable McNally and Mr White prove beyond reasonable doubt that, Mr Fitzpatrick drove in a manner that was dangerous to the public, that this was the result of carelessness due to inattention and the sum result of these actions was the death of Celia White. Celia was trapped in the wreckage for 20 minutes and could see her husband and son were out of the car and that they could not help her. Ladies and Gentlemen of the jury, Mr Fitzpatrick's suffering is insignificant by comparison to that of the White family. You have a duty to the Whites and to the public to find the defendant guilty of causing death by dangerous driving. The Whites must be given the understanding that our justice system is fair and the public must be given the understanding of how important careful attention on the road at all time really is. Thankyou for your attention Ladies and Gentlemen.

Judge: Mr Warwick you may now address the jury with your closing statement.

Def: Thank you Your Honour. Ladies and Gentlemen of the jury. The prosecution has alleged that it was Mr Fitzpatrick's truck on the wrong side of the road which caused the accident in question. We contend that the only reason the trailer ended up there was because of the driver's need to swerve sharply and to brake to avoid the White's car which had strayed on to the wrong side of the road. Ladies and Gentlemen I ask you to consider the eyewitness evidence upon which the Prosecution's allegation is 
based. Mr White had to watch his wife's life in danger right in front of him and could not do anything as they waited for help to arrive. A situation like this can be emotionally upsetting. Can you place your faith in the memory of a man who has experienced such events? Can you believe that he is able to clearly remember what he saw prior to these events? No, you cannot. The amount of stress that Mr White would have experienced is substantial, and yet the prosecution asserts that Mr White's memory would have been unaffected by this. Secondly, Ladies and Gentlemen, realise that when people see those whom they know die they often need to blame someone. Mr White cannot face the possibility that his wife's driving caused her own death. This is easy to understand. However, as the guardians of justice, you the jury have the duty to up hold the law, no matter how much people have suffered and may continue to suffer. That is why you must find my client Mr Fitzpatrick not guilty of causing grievous bodily harm by dangerous driving. That is all Your Honour.

\section{INCONSISTENT SAD}

Judge: Good morning Ladies and Gentlemen, my name is Judge Stevenson. Today we are to hear in the Supreme Court the case of the Crown versus Mark Alexander Fitzpatrick. Mr Fitzpatrick is charged with causing death by dangerous driving. $\mathrm{Mr}$ Spence, you are now invited to open the case for the prosecution.

Prosecution : Thank you Your Honour. Ladies and Gentlemen of the jury, my name is Henry Spence, I am the prosecuting attorney in the case of the Crown vs Mark Alexander Fitzpatrick. You will hear eyewitness evidence today that proves beyond reasonable doubt that Mr Fitzpatrick is solely responsible for the death of Celia White. The tragic events took place at approximately 10am on the 14th of April 1998 on Mount Barker Road near Eagle on the Hill. Driving home from a visit with relatives, the family in their Corolla sedan rounded a left hand bend and were confronted with a large fuel tanker on the wrong side of the road. The driver panicked, swerved causing the trailer to jack-knife across the path of the oncoming car. The car hit the trailer on the driver's side. Unfortunately, Celia White was crushed and trapped in the twisted wreckage with her little girl Kayla. She was trapped for 20 minutes, in agony from the steering column of the car which had crushed her chest. Her legs were crushed and she could hear her little girl trapped in the back seat crying out for her mum. But sadly she could do nothing. Celia White died that morning with her husband and son looking on, and her daughter calling for her. They could not help her. There was fuel all over the road. They had to stand back and watch two people that they dearly loved suffer. Kayla was rescued by the 'jaws of life', even more saddening was the fact that her face was severely lacerated by the glass from the passenger's side window. She also bears the psychological scars of the ordeal and these Ladies and Gentlemen will take much longer to heal.

In sum it is a very gloomy picture. You will hear eyewitness evidence that proves that Mr Fitzpatrick drove his semi-trailer in a manner that created potential danger to other people using the highway. This was solely and act of carelessness and inattention and an act that directly caused the death of Celia White, and the serious and tragic injury of her young child. Mr Fitzpatrick has destroyed the lives and happiness of many individuals who loved Celia White. Most particularly James White and his children who must live with the sad memory of the accident and face the prospect of life 
without a wife and mother. You, the jury, can deliver justice which will appease their loss by finding the defendant guilty the charge of causing death by dangerous driving. Thankyou Your Honour.

Judge: Mr Warwick, you may now open the case for the defence.

Defence; Thankyou Your Honour. Ladies and Gentlemen of the Jury, My name is

Melvin Warwick, I am the attorney defending my client Mr Fitzpatrick in this case.

The prosecution will allege on the basis of eyewitness testimony that my client was solely responsible for the death and injury which occurred when his truck collided with the eyewitness' car on the morning of April 14th. Ladies and Gentlemen, I want you to first remember while listening to the eyewitness evidence that the eyewitness was actually in the car at the time of the accident which means that he may not have seen the things he alleges to have seen. Second, he and his young son had to watch his wife bleed to death while his child was trapped in the wreckage of a car that was in imminent danger of bursting into flames. This is bound to cause feelings of helplessness and perhaps a strong need to blame someone for this tragic outcome. While not in any way meaning to deny the ad and tragic nature of the events that occurred on that day, I have to emphasise that Mr White is not an impartial witness who has the benefit of objectivity. Finally, at the time of the accident he must have been under a great deal of stress and this most likely affected his ability to recall the details of an event. Ladies and Gentlemen, it is our case that it was the witness's car which crossed over the double white lines that morning causing Mr Fitzpatrick to swerve and his trailer to jack-knife across the road and into the witness' car. Finally, Ladies and Gentlemen, I ask you to consider the suffering of the truck's driver $\mathrm{Mr}$ Fitzpatrick. He walked away physically unarmed from the accident. However, psychologically he is distraught. He knows that he could not have avoided the accident, but he still blames himself despite the physical evidence which proves his innocence. Ladies and Gentlemen, in considering the evidence, the tragedy, understand that all of those involved are victims, and sadly all will bare emotional and physical scars. Also remember that the Crown bears the burden of proof. They must prove beyond reasonable doubt that my client drove in a manner dangerous to the public, that this was the result of carelessness and further that this action was the sole cause of the death and injury which result from the collision. You can deliver justice and ease Mr Fitzpatrick's suffering by delivering a not guilty verdict in accordance with the truth. Thankyou Your Honour.

Judge: Mr Spence, please call your first witness.

Prosecution: Thankyou Your Honour. The Crown calls Police Constable Roger McNally to the stand. \{Pause\} Mr McNally, My name is Henry Spence, I am the prosecuting Attorney. I would like to ask you some questions about the accident which took place on Mount Barker Road, near Eagle on the Hill, on the morning of April 14th 1998. We are keen to establish the truth so please answer where appropriate with either, I don't know, I 'm not sure or I am sure.

Police Constable: Yes, I will.

Prosecution: You were the investigating officer when this accident occurred ?

Police Constable: Yes, that is correct.

Prosecution: Can you describe for the jury the position of the vehicles when you 
arrived at the scene of the accident that morning.

Police Constable: Certainly. The semi-trailer was blocking the whole road. The cabin was in the middle of the down track with its trailer perpendicular to the cabin obstructing the rest of the down track and extending all the way across the up track. The truck had been travelling down Mount Barker Road away from Eagle on the Hill and the car had been travelling up. (1)The car had impacted the trailer about half way along its right side. There wasn't much left of the car, the motor had been pushed back to the center console. The car had hit mostly on the driver's side. This had caused the steering column to be pushed through the chest of the driver. The car was completely caved in on the driver's side. I arrived on the scene before the ambulance. The woman was pinned in the driver's seat by the steering column and the girl in the driver's side passenger seat was trapped also. The mother was still alive when I arrived but she was in obvious pain. She was bleeding profusely. The daughter was sobbing; it was as if she could tell that her Mum wasn't going to make it. The father and son who had managed to get out of the vehicle were distraught, they were yelling at me to get the others out of the wreckage. I couldn't do anything because of the risk of the fuel around the car igniting and causing an explosion which would have killed all of us...I tried to move them away from the car, they wouldn't leave the rest of their family trapped....we just had to wait... sit there and watch her die... Mr Spence, this would have to be one of the most horrific and disturbing accidents I had ever had the misfortune to attend.

Prosecution: Mr McNally, take a moment to compose yourself. We can understand the great deal of emotional strain that recounting the accident will cause you......Was there any evidence that either of the vehicles had taken evasive action, such as breaking or swerving before impact?

Police Constable: (2) Yes, there were wide skid marks from the truck trailer skewing sideways which were 28 meters in length and from the truck going in a direction perpendicular to those of the truck which were a 2 meters longer. There were no skid marks from the car.

Prosecution: From the length of the skid marks, the damage caused upon impact and the position of the vehicles, can any estimates about the speed of the truck and car be made.

Police Constable: It is difficult to determine. However, it appears that the car may have been travelling at about $32 \mathrm{~km} /$ hour and the truck at about 50 .

Prosecution: Mr McNally, what causes a trailer to jack-knife or begin to push the cabin of a truck along?

Police Constable; There are several different causes for a trailer to jack-knife. It can be caused by incorrect tyre pressure, insufficient or too heavy loads, under gear out of alignment or worn steering or other faults in old vehicles.

Prosecution: In your opinion was the jack-knife of the truck trailer caused by any of the problems that you just mentioned?

Police Constable; No. We checked the vehicle thoroughly. It was in good condition mechanically.

Prosecution: What in your opinion, was the cause of the collision?

Police Constable: The truck obviously swerved to it's left and braked heavily. This 
caused the trailer to jack-knife and the car ran into the trailer. I can not speculate on the reason why the driver of the truck had to brake.

Prosecution: Is it possible Mr McNally, that the driver swerved and braked because he suddenly realised he was on the wrong side of the road?

Police Constable: Yes it is possible.

Prosecution: Thankyou Mr McNally. No further questions Your honour.

Judge: Mr Warwick, you may now cross examine the witness.

Defence: Good morning Mr McNally. My name is Melvin Warwick, I am defending my client Mr Fitzpatrick the driver of the semi-trailer. Mr McNally, you stated to my learned friend $\mathrm{Mr}$ Spence, that there are several reasons for a trailer to jack-knife in the manner you described.

Police Constable: Yes.

Defence: Is it not equally possible that the driver of the truck, my client, may have braked and swerved to avoid a collision when he saw the car driven by Celia White on the wrong side of the road?

Police Constable: Yes it is possible. As I said before, the trailer jack-knifed because the truck braked heavily and swerved to it's left. I can only speculate on the reasons why the truck braked in the first place. I can only tell you that the trailer moved to the other side of the road because of the braking and swerving. Def: Thankyou Mr McNally. No further questions Your Honour.

Judge: Mr Spence, you may now call your second witness to the stand.

Prosecution: Thankyou Your Honour. The Crown calls James White to the stand. Goodmorning Mr White, my name is Henry Spence, I am the prosecuting attorney, I would like to ask you some questions about the accident which took place on Mount Barker Road at Eagle on the Hill, Monday the 14th of April 1998. I realise that the events that occurred on that day are heart rending for you. We all appreciate that you and your children had to watch as someone you loved was dying and yet you were unable to do anything to ease the pain. Mr White it is essential for the carriage of justice that the jury have a good understanding of the events leading up to the accident, and so unfortunately some very sad and difficult memories will have to be experienced. Take your time with your answers and try to remain composed. We are keen to establish the truth so please answer where appropriate with either, I don't know, I'm not sure or I am sure.

Witness: Yes I will.

Prosecution: Would you state your full name please.

Witness: James Phillip White.

Prosecution: What is your full home address.

Witness: 20 Druids Avenue, Mount Barker, South Australia.

Prosecution: At approximately 10am on Monday the 14th of April 1998 were you a passenger in the vehicle travelling along Mount Barker road, which was subsequently involved in the accident in question?

Witness: Yes, I was.

Prosecution: Why was the vehicle in which you were a passenger travelling along Mount Barker Road on the morning of the accident ? 
Witness: My family and I were returning from a visit with my parents at Clarence Gardens in Adelaide.

Prosecution: Who was in the car with you?

Witness: My wife Celia, who was driving, and our children Kayla and Jarrod.

Prosecution: Had Celia, your wife, had any accidents prior to the one that occurred that morning?

Witness: No. Celia had an impeccable driving record.

Prosecution: Did Celia have any impairments or disabilities which may have reduced her ability to drive?

Witness: No, she did not.

Prosecution: Was Celia tired, stressed or distracted on the day while she was driving the vehicle?

Witness: No she was not.

Prosecution: Were the children distracting her at any point during the trip ?

Witness: No.

Prosecution: Are you sure Mr White, children can sometimes find it difficult to behave on long trips?

Witness: The children were being well behaved, talking to each other in the back.

Celia's attention was on the road the whole time.

Prosecution: Do you have any problems with your eyes Mr White?

Witness: No

Prosecution: When was the last time you had your eyes checked ?

Witness: About 3 months ago. We get them checked regularly because of the type of work I do.

Prosecution: Mr White, can you tell the court in detail, what you were doing 5 minutes prior to the accident.

Witness: Well, as I said before, we were returning home from a visit to my parent's place in Adelaide. We were driving up Mount Barker Road...

Prosecution: Mr White, what exactly was going on in the car at this point ?

Witness: We were talking to the kids about the baby rabbits Grandma had shown them. They wanted a rabbit....Celia was telling them that if they wanted a rabbit they would both have to take care of it, look after it and feed it regularly.....they promised her they would....

Judge: Take a moment, if you wish, Mr White, to compose yourself.

Witness: Thankyou.....Sorry......it's just really hard......without her....

\{Pause

Prosecution: Can we continue Mr White?

Witness: Yes.

Prosecution: When did you first notice the truck ?

Witness: (4)When we rounded the left hand bend just before where the accident happened. You couldn't see around the corner because at that point the road is actually bending around the steep hill on the left.

Prosecution: When you first saw the truck, where in relation to your car, was it located ?

Witness: It was directly in front of us, a little to the left of the center of our car. 
Prosecution: Was the truck over the double white lines on the road?

Witness: Yes.

Prosecution: In your estimation, how far over the white lines ?

Witness: At least 3 meters. About from here to the jury away. (3) It was $3 / 4$ the way over on our side of the road. So far over that we couldn't have swerved around it, there simply wasn't room.

Prosecution: Did the truck appear to swerve or deviate from it's course at any time prior to impact, Mr White?

Witness: Yes, it did. I remember that Celia had time to flash the lights a couple of times... (5)it swerved to our left (3).

Prosecution: At what speed were you travelling ?

Witness: I reckon it was only about $40 \mathrm{~km} / \mathrm{h}$. We had slowed down to take the corner. Prosecution: At what speed in your estimation was the truck travelling?

Witness: It must have been over $60 \mathrm{~km} / \mathrm{h}$. We had slowed down, it came towards us very quickly. (5)Celia didn't have time to do anything.

Prosecution: Did the truck appear to slow down or brake at any point ?

Witness: (2) No it didn't brake at all. As I said it happened very quickly. There was this petrol truck, or Ampol truck and the trailer across the road coming straight for us and.... It was very quick. There was no way Celia could have avoided it.

Prosecution: Which side of the trailer did your car hit?

Witness: (1) We hit on the trailer's left side.

Prosecution: Is there anything else that you think is important to tell the court $\mathrm{Mr}$ White?

Witness: Yes, I'd just like the jury to know that Celia was always a good driver. She was a great mother and she was my best friend. I miss her so much and our children miss her.....they keep asking when she's coming home.....

Judge: Mr White, please try to compose yourself. We understand how difficult this is for you, but please try to answer the questions.

Prosecution: No further questions Your Honour.

$*$

Judge: Thankyou Mr Spence. Mr Warwick, you may now cross-examine the witness. Defence: Thankyou Your Honour. Good morning Mr White. My name is Melvin Warwick and I am the attorney for the defence in this case. I have some questions to ask you about the accident which took place on Mount Barker Road in which you were involved. Mr White, I know that this is difficult for you , I know that you will abhor the implications of the questions that I will ask you and I too find myself feeling very sad about what took place that day. At times such as these I find it very difficult to do my job. You and your children have been through a lot. But the justice system demands that the jury have a clear picture of these events, so please answer the questions as accurately as possible. \{Pause\} At what point did you think you saw the truck on the wrong side of the road?

Witness: (4)I'd say at least 15 meters before we rounded the bend. Before the accident scene, I could see that it was on the wrong side of the road. I could see the (6) paint of the Mobil symbol and the chrome trailer shining through the trees.

Defence: And you are sure you could tell that the truck was on the wrong side of the 
road ?

Witness: Yes. I am sure.

Defence: You were rounding the bend and travelling at a reasonable speed and yet you were able to discern that the truck was on the wrong side of the road.

Witness: Yes, that's what I'm telling you.

Defence: Mr White, if you saw the truck was on the wrong side of the road before you reached the bend, why didn't you warn your wife ?

Witness: I don't know. It happened very quickly. I wasn't $100 \%$ sure that it was on the wrong side before we rounded the bend. But I could see it definitely was once we got further the way around. (3)It was almost half way over onto our side of the road.

Defence: Between the time that you saw the truck through the trees and the time that

you saw it coming toward you, did your eyes remain on the road?

Witness: No.

Defence: Exactly what were you doing in between the time that you saw the truck through the trees and the time that you rounded the bend?

Witness:(7) I was getting a tissue for Kayla. She'd spilt her drink.

Defence: Mr White, your wife maintained her attention on the road the whole time while you were trying to clean up the mess on the back seat?

Witness: Yes.

Defence: Is it not possible that she too was distracted by your daughter's mishap, and that her attention left the road for a few moments also ?

W: No.

Def: Mr White, I think that perhaps she was distracted and that out of your grief and

loss, you needed to blame someone for your misfortune and that happens to be my client Mr Fitzpatrick.

W: No that's not true ! He caused the accident, his truck was on the wrong side of the road!

Def: No further questions Your Honour.

Judge: Mr Spence, you may now address the jury with your closing statement.

Pro: Thankyou Your Honour. Ladies and Gentlemen of the jury, you have heard

evidence today from two witnesses to the tragic event which occurred on April 14th 1999 on Mount Barker Road. Mr Fitzpatrick may well have suffered as the result of these events, however, the White family has suffered much more. The testimony of both Constable McNally and $\mathrm{Mr}$ White prove beyond reasonable doubt that, $\mathrm{Mr}$

Fitzpatrick drove in a manner that was dangerous to the public, that this was the result of carelessness due to inattention and the sum result of these actions was the death of Celia White. Both of these children will have to face a life without their mother, and a life of the memories of pain and suffering as a result of the accident. Little Kayla was trapped in the wreckage with her dying mother for half an hour. She could see her father and brother were out of the car and that they could not help her or her mother. This little girl has to bear these memories for the rest of her life. Mr White and his son will never forget watching Celia die. They will never forget the monumental helplessness, the frustration, the terrible loss. Ladies and Gentlemen of the jury, $\mathrm{Mr}$ Fitzpatrick's suffering is insignificant by comparison to that of the White family. You have a duty to the Whites and to the public to find the defendant guilty of causing 
death by dangerous driving. The Whites must be given the understanding that our justice system is fair and the public must be given the understanding of how important careful attention on the road at all time really is. Thankyou for your attention Ladies and Gentlemen.

Judge: Mr Warwick you may now address the jury with your closing statement. Def: Thankyou Your Honour. Ladies and Gentlemen of the jury. The prosecution has alleged that it was Mr Fitzpatrick's truck on the wrong side of the road which caused the accident in question. We contend that the only reason the trailer ended up there was because of the driver's need to swerve sharply and to brake to avoid the White's car which had strayed on to the wrong side of the road. Ladies and Gentlemen I ask you to consider the eyewitness evidence upon which the Prosecution's allegation is based. Mr White had to watch his wife die right in front of him and could not do anything to prevent this occurrence. Similarly, he had to watch his daughter in pain and bleeding as they waited for help to arrive. A situation cannot be more emotionally upsetting than this. Can you place your faith in the memory of a man who has experienced such events ? Can you believe that he is able to clearly remember what he saw prior to these events? No, you cannot. The amount of stress that Mr White would have experienced is barely conceivable, and yet the prosecution asserts that $\mathrm{Mr}$ White's memory would have been unaffected by this. Secondly, Ladies and Gentlemen, realise that when people lose those whom they love they often go through a period of grief that involves the need to blame someone for their lose. Mr White cannot face the possibility that his wife's driving caused her own death and the injury of their children. This is easy to understand. However, as the guardians of justice, you the jury have the duty to up hold the law, no matter how much people have suffered and may continue to suffer. That is why you must find my client Mr Fitzpatrick not guilty of causing death by dangerous driving. That is all Your Honour.

\section{CONSISTENT SAD}

Judge: Good morning Ladies and Gentlemen, my name is Judge Stevenson. Today we are to hear in the Supreme Court the case of the Crown versus Mark Alexander Fitzpatrick. Mr Fitzpatrick is charged with causing death by dangerous driving. $\mathrm{Mr}$ Spence, you are now invited to open the case for the prosecution.

Prosecution : Thank you Your Honour. Ladies and Gentlemen of the jury, my name is Henry Spence, I am the prosecuting attorney in the case of the Crown vs Mark Alexander Fitzpatrick. You will hear eyewitness evidence today that proves beyond reasonable doubt that Mr Fitzpatrick is solely responsible for the death of Celia White. The tragic events took place at approximately 10am on the 14th of April 1998 on Mount Barker Road near Eagle on the Hill. Driving home from a visit with relatives, the family in their Corolla sedan rounded a left hand bend and were confronted with a large fuel tanker on the wrong side of the road. The driver panicked, swerved causing the trailer to jack-knife across the path of the oncoming car. The car hit the trailer on the driver's side. Unfortunately, Celia White was crushed and trapped in the twisted wreckage with her little girl Kayla. She was trapped for 20 minutes, in agony from the steering column of the car which had crushed her chest. Her legs were crushed and she could hear her little girl trapped in the back seat crying out for her mum. But sadly she could do nothing. Celia White died that morning with her husband and son looking 
on, and her daughter calling for her. They could not help her. There was fuel all over the road. They had to stand back and watch two people that they dearly loved suffer. Kayla was rescued by the 'jaws of life', even more saddening was the fact that her face was severely lacerated by the glass from the passenger's side window. She also bears the psychological scars of the ordeal and these Ladies and Gentlemen will take much longer to heal.

In sum it is a very gloomy picture. You will hear eyewitness evidence that proves that Mr Fitzpatrick drove his semi-trailer in a manner that created potential danger to other people using the highway. This was solely and act of carelessness and inattention and an act that directly caused the death of Celia White, and the serious and tragic injury of her young child. Mr Fitzpatrick has destroyed the lives and happiness of many individuals who loved Celia White. Most particularly James White and his children who must live with the sad memory of the accident and face the prospect of life without a wife and mother. You, the jury, can deliver justice which will appease their loss by finding the defendant guilty the charge of causing death by dangerous driving. Thankyou Your Honour.

Judge: Mr Warwick, you may now open the case for the defence.

Defence; Thankyou Your Honour. Ladies and Gentlemen of the Jury, My name is Melvin Warwick, I am the attorney defending my client Mr Fitzpatrick in this case. The prosecution will allege on the basis of eyewitness testimony that my client was solely responsible for the death and injury which occurred when his truck collided with the eyewitness' car on the morning of April 14th. Ladies and Gentlemen, I want you to first remember while listening to the eyewitness evidence that the eyewitness was actually in the car at the time of the accident which means that he may not have seen the things he alleges to have seen. Second, he and his young son had to watch his wife bleed to death while his child was trapped in the wreckage of a car that was in imminent danger of bursting into flames. This is bound to cause feelings of helplessness and perhaps a strong need to blame someone for this tragic outcome. While not in any way meaning to deny the ad and tragic nature of the events that occurred on that day, I have to emphasise that Mr White is not an impartial witness who has the benefit of objectivity. Finally, at the time of the accident he must have been under a great deal of stress and this most likely affected his ability to recall the details of an event. Ladies and Gentlemen, it is our case that it was the witness's car which crossed over the double white lines that morning causing Mr Fitzpatrick to swerve and his trailer to jack-knife across the road and into the witness' car. Finally, Ladies and Gentlemen, I ask you to consider the suffering of the truck's driver Mr Fitzpatrick. He walked away physically unarmed from the accident. However, psychologically he is distraught. He knows that he could not have avoided the accident, but he still blames himself despite the physical evidence which proves his innocence. Ladies and Gentlemen, in considering the evidence, the tragedy, understand that all of those involved are victims, and sadly all will bare emotional and physical scars. Also remember that the Crown bears the burden of proof. They must prove beyond reasonable doubt that my client drove in a manner dangerous to the public, that this was the result of carelessness and further that this action was the sole cause of the death and injury which result from the collision. You can deliver justice 
and ease Mr Fitzpatrick's suffering by delivering a not guilty verdict in accordance with the truth. Thankyou Your Honour.

Judge: Mr Spence, please call your first witness.

Prosecution: Thankyou Your Honour. The Crown calls Police Constable Roger McNally to the stand. \{Pause\} Mr McNally, My name is Henry Spence, I am the prosecuting Attorney. I would like to ask you some questions about the accident which took place on Mount Barker Road, near Eagle on the Hill, on the morning of April 14th 1998. We are keen to establish the truth so please answer where appropriate with either, I don't know, I 'm not sure or I am sure.

Police Constable: Yes, I will.

Prosecution: You were the investigating officer when this accident occurred?

Police Constable: Yes, that is correct.

Prosecution: Can you describe for the jury the position of the vehicles when you arrived at the scene of the accident that morning.

Police Constable: Certainly. The semi-trailer was blocking the whole road. The cabin was in the middle of the down track with its trailer perpendicular to the cabin obstructing the rest of the down track and extending all the way across the up track. The truck had been travelling down Mount Barker Road away from Eagle on the Hill and the car had been travelling up. (1)The car had impacted the trailer about half way along its right side. There wasn't much left of the car, the motor had been pushed back to the center console. The car had hit mostly on the driver's side. This had caused the steering column to be pushed through the chest of the driver. The car was completely caved in on the driver's side. I arrived on the scene before the ambulance. The woman was pinned in the driver's seat by the steering column and the girl in the driver's side passenger seat was trapped also. The mother was still alive when I arrived but she was in obvious pain. She was bleeding profusely. The daughter was sobbing; it was as if she could tell that her Mum wasn't going to make it. The father and son who had managed to get out of the vehicle were distraught, they were yelling at me to get the others out of the wreckage. I couldn't do anything because of the risk of the fuel around the car igniting and causing an explosion which would have killed all of us...I tried to move them away from the car, they wouldn't leave the rest of their family trapped....we just had to wait... sit there and watch her die... Mr Spence, this would have to be one of the most horrific and disturbing accidents I had ever had the misfortune to attend.

Prosecution: Mr McNally, take a moment to compose yourself. We can understand the great deal of emotional strain that recounting the accident will cause you......Was there any evidence that either of the vehicles had taken evasive action, such as breaking or swerving before impact?

Police Constable: (2) Yes, there were wide skid marks from the truck trailer skewing sideways which were 28 meters in length and from the truck going in a direction perpendicular to those of the truck which were a 2 meters longer. There were no skid marks from the car.

Prosecution: From the length of the skid marks, the damage caused upon impact and the position of the vehicles, can any estimates about the speed of the truck and car be made. 
Police Constable: It is difficult to determine. However, it appears that the car may have been travelling at about $32 \mathrm{~km} /$ hour and the truck at about 50 .

Prosecution: Mr McNally, what causes a trailer to jack-knife or begin to push the cabin of a truck along?

Police Constable; There are several different causes for a trailer to jack-knife. It can be caused by incorrect tyre pressure, insufficient or too heavy loads, under gear out of alignment or worn steering or other faults in old vehicles.

Prosecution: In your opinion was the jack-knife of the truck trailer caused by any of the problems that you just mentioned?

Police Constable; No. We checked the vehicle thoroughly. It was in good condition mechanically.

Prosecution: What in your opinion, was the cause of the collision?

Police Constable: The truck obviously swerved to it's left and braked heavily. This caused the trailer to jack-knife and the car ran into the trailer. I can not speculate on the reason why the driver of the truck had to brake.

Prosecution: Is it possible Mr McNally, that the driver swerved and braked because he suddenly realised he was on the wrong side of the road?

Police Constable: Yes it is possible.

Prosecution: Thankyou Mr McNally. No further questions Your honour.

Judge: Mr Warwick, you may now cross examine the witness.

Defence: Good morning Mr McNally. My name is Melvin Warwick, I am defending my client Mr Fitzpatrick the driver of the semi-trailer. Mr McNally, you stated to my learned friend Mr Spence, that there are several reasons for a trailer to jack-knife in the manner you described.

Police Constable: Yes.

Defence: Is it not equally possible that the driver of the truck, my client, may have braked and swerved to avoid a collision when he saw the car driven by Celia White on the wrong side of the road?

Police Constable: Yes it is possible. As I said before, the trailer jack-knifed because the truck braked heavily and swerved to it's left. I can only speculate on the reasons why the truck braked in the first place. I can only tell you that the trailer moved to the other side of the road because of the braking and swerving. Def: Thankyou Mr McNally. No further questions Your Honour.

*

Judge: Mr Spence, you may now call your second witness to the stand.

Prosecution: Thankyou Your Honour. The Crown calls James White to the stand. Good morning Mr White, my name is Henry Spence, I am the prosecuting attorney, I would like to ask you some questions about the accident which took place on Mount Barker Road at Eagle on the Hill, Monday the 14th of April 1998. I realise that the events that occurred on that day are heart rending for you. We all appreciate that you and your children had to watch as someone you loved was dying and yet you were unable to do anything to ease the pain. Mr White it is essential for the carriage of justice that the jury have a good understanding of the events leading up to the accident, and so unfortunately some very sad and difficult memories will have to be experienced. Take your time with your answers and try to remain composed. We are 
keen to establish the truth so please answer where appropriate with either, I don't know, I'm not sure or I am sure.

Witness: Yes I will.

Prosecution: Would you state your full name please.

Witness: James Phillip White.

Prosecution: What is your full home address.

Witness: 20 Druids Avenue, Mount Barker, South Australia.

Prosecution: At approximately 10am on Monday the 14th of April 1998 were you a passenger in the vehicle travelling along Mount Barker road, which was

subsequently involved in the accident in question?

Witness: Yes, I was.

Prosecution: Why was the vehicle in which you were a passenger travelling along Mount Barker Road on the morning of the accident ?

Witness: My family and I were returning from a visit with my parents at Clarence Gardens in Adelaide.

Prosecution: Who was in the car with you?

Witness: My wife Celia, who was driving, and our children Kayla and Jarrod.

Prosecution: Had Celia, your wife, had any accidents prior to the one that occurred that morning?

Witness: No. Celia had an impeccable driving record.

Prosecution: Did Celia have any impairments or disabilities which may have reduced her ability to drive?

Witness: No, she did not.

Prosecution: Was Celia tired, stressed or distracted on the day while she was driving the vehicle?

Witness: No she was not.

Prosecution: Were the children distracting her at any point during the trip ?

Witness: No.

Prosecution: Are you sure Mr White, children can sometimes find it difficult to behave on long trips?

Witness: The children were being well behaved, talking to each other in the back.

Celia's attention was on the road the whole time.

Prosecution: Do you have any problems with your eyes Mr White?

Witness: No

Prosecution: When was the last time you had your eyes checked?

Witness: About 3 months ago. We get them checked regularly because of the type of work I do.

Prosecution: Mr White, can you tell the court in detail, what you were doing 5 minutes prior to the accident.

Witness: Well, as I said before, we were returning home from a visit to my parent's place in Adelaide. We were driving up Mount Barker Road...

Prosecution: Mr White, what exactly was going on in the car at this point?

Witness: We were talking to the kids about the baby rabbits Grandma had shown them. They wanted a rabbit....Celia was telling them that if they wanted a rabbit they would both have to take care of it, look after it and feed it regularly.....they promised 
her they would....

Judge: Take a moment, if you wish, Mr White, to compose yourself.

Witness: Thankyou.....Sorry......it's just really hard.....without her....

\{Pause\}

Prosecution: Can we continue Mr White?

Witness: Yes.

Prosecution: When did you first notice the truck ?

Witness: (4)When we rounded the left hand bend just before where the accident

happened. You couldn't see around the corner because at that point the road is actually bending around the steep hill on the left.

Prosecution: When you first saw the truck, where in relation to your car, was it located ?

Witness: It was directly in front of us, a little to the left of the center of our car.

Prosecution: Was the truck over the double white lines on the road?

Witness: Yes.

Prosecution: In your estimation, how far over the white lines?

Witness: At least 3 meters. About from here to the jury away. (3) It was almost half the way over on our side of the road. So far over that we couldn't have swerved around it, there simply wasn't room.

Prosecution: Did the truck appear to swerve or deviate from it's course at any time prior to impact, Mr White ?

Witness: Yes, it did. I remember that Celia had time to flash the lights a couple of times... (5)it swerved to our right (3).

Prosecution: At what speed were you travelling?

Witness: I reckon it was only about $40 \mathrm{~km} / \mathrm{h}$. We had slowed down to take the corner.

Prosecution: At what speed in your estimation was the truck travelling ?

Witness: It must have been over $60 \mathrm{~km} / \mathrm{h}$. We had slowed down, it came towards us very quickly. (5).

Prosecution: Did the truck appear to slow down or brake at any point?

Witness: (2) It did brake. As I said it happened very quickly. There was this petrol truck, or Ampol truck and the trailer across the road coming straight for us and.... It was very quick. There was no way Celia could have avoided it.

Prosecution: Which side of the trailer did your car hit?

Witness: (1) We hit on the trailer's right side.

Prosecution: Is there anything else that you think is important to tell the court $\mathrm{Mr}$ White?

Witness: Yes, I'd just like the jury to know that Celia was always a good driver. She was a great mother and she was my best friend. I miss her so much and our children miss her.....they keep asking when she's coming home.....

Judge: Mr White, please try to compose yourself. We understand how difficult this is for you, but please try to answer the questions.

Prosecution: No further questions Your Honour.

*

Judge: Thankyou Mr Spence. Mr Warwick, you may now cross-examine the witness. Defence: Thankyou Your Honour. Good morning Mr White. My name is Melvin 
Warwick and I am the attorney for the defence in this case. I have some questions to ask you about the accident which took place on Mount Barker Road in which you were involved. Mr White, I know that this is difficult for you , I know that you will abhor the implications of the questions that I will ask you and I too find myself feeling very sad about what took place that day. At times such as these I find it very difficult to do my job. You and your children have been through a lot. But the justice system demands that the jury have a clear picture of these events, so please answer the questions as accurately as possible. \{Pause\} At what point did you think you saw the truck on the wrong side of the road?

Witness: (4)When we rounded the bend just before the accident happened, as I said before, it was a blind corner which you couldn't see around because of the hill. When we rounded the bend I could see that it was on the wrong side of the road. I could see the (6) paint of the Ampol symbol.

Defence: And you are sure you could tell that the truck was on the wrong side of the road?

Witness: Yes. I am sure.

Defence: You were rounding the bend and travelling at a reasonable speed and yet you were able to discern that the truck was on the wrong side of the road.

Witness: Yes, that's what I'm telling you.

Defence: Mr White, if you saw the truck was on the wrong side of the road, why didn't you warn your wife?

Witness: I don't know. It happened very quickly. But I could see it definitely was on the wrong side of the road. (3)It was almost half way over onto our side of the road. Defence: Did your eyes remain on the road the whole time you were rounding the corner?

Witness: Yes.

Defence: Exactly what were you doing in between the time that you rounded the bend and the time that you first saw the truck?

Witness:(7) I was watching the road.

Defence: Mr White, your wife maintained her attention on the road the whole time while you were going around the corner?

Witness: Yes.

Defence: Is it not possible that she was distracted by your children, and that her attention left the road for a few moments?

W: No.

Def: Mr White, I think that perhaps she was distracted and that out of your grief and loss, you needed to blame someone for your misfortune and that happens to be my client Mr Fitzpatrick.

W: No that's not true ! He caused the accident, his truck was on the wrong side of the road!

Def: No further questions Your Honour.

Judge: Mr Spence, you may now address the jury with your closing statement.

Pro: Thankyou Your Honour. Ladies and Gentlemen of the jury, you have heard evidence today from two witnesses to the tragic event which occurred on April 14th 1999 on Mount Barker Road. Mr Fitzpatrick may well have suffered as the result of 
these events, however, the White family has suffered much more. The testimony of both Constable McNally and Mr White prove beyond reasonable doubt that, $\mathrm{Mr}$ Fitzpatrick drove in a manner that was dangerous to the public, that this was the result of carelessness due to inattention and the sum result of these actions was the death of Celia White. Both of these children will have to face a life without their mother, and a life of the memories of pain and suffering as a result of the accident. Little Kayla was trapped in the wreckage with her dying mother for half an hour. She could see her father and brother were out of the car and that they could not help her or her mother. This little girl has to bear these memories for the rest of her life. Mr White and his son will never forget watching Celia die. They will never forget the monumental helplessness, the frustration, the terrible loss. Ladies and Gentlemen of the jury, $\mathrm{Mr}$ Fitzpatrick's suffering is insignificant by comparison to that of the White family. You have a duty to the Whites and to the public to find the defendant guilty of causing death by dangerous driving. The Whites must be given the understanding that our justice system is fair and the public must be given the understanding of how important careful attention on the road at all time really is. Thankyou for your attention Ladies and Gentlemen.

Judge: Mr Warwick you may now address the jury with your closing statement. Def: Thankyou Your Honour. Ladies and Gentlemen of the jury. The prosecution has alleged that it was Mr Fitzpatrick's truck on the wrong side of the road which caused the accident in question. We contend that the only reason the trailer ended up there was because of the driver's need to swerve sharply and to brake to avoid the White's car which had strayed on to the wrong side of the road. Ladies and Gentlemen I ask you to consider the eyewitness evidence upon which the Prosecution's allegation is based. Mr White had to watch his wife die right in front of him and could not do anything to prevent this occurrence. Similarly, he had to watch his daughter in pain and bleeding as they waited for help to arrive. A situation cannot be more emotionally upsetting than this. Can you place your faith in the memory of a man who has experienced such events ? Can you believe that he is able to clearly remember what he saw prior to these events? No, you cannot. The amount of stress that Mr White would have experienced is barely conceivable, and yet the prosecution asserts that $\mathrm{Mr}$ White's memory would have been unaffected by this. Secondly, Ladies and Gentlemen, realise that when people lose those whom they love they often go through a period of grief that involves the need to blame someone for their lose. Mr White cannot face the possibility that his wife's driving caused her own death and the injury of their children. This is easy to understand. However, as the guardians of justice, you the jury have the duty to up hold the law, no matter how much people have suffered and may continue to suffer. That is why you must find my client Mr Fitzpatrick not guilty of causing death by dangerous driving. That is all Your Honour.

\section{CONSISTENT NEUTRAL}

Judge: Good morning Ladies and Gentlemen, my name is Judge Stevenson. Today we are to hear in the Supreme Court the case of the Crown versus Mark Alexander Fitzpatrick. Mr Fitzpatrick is charged with causing grievous bodily harm by dangerous driving. Mr Spence, you are now invited to open the case for the prosecution.

Prosecution : Thank you Your Honour. Ladies and Gentlemen of the jury, my name is 
Henry Spence, I am the prosecuting attorney in the case of the Crown vs Mark Alexander Fitzpatrick. You will hear eyewitness evidence today that proves beyond reasonable doubt that Mr Fitzpatrick is solely responsible for the injury caused to Celia White. The accident took place at approximately 10am on the 14th of April 1998 on Mount Barker Road near Eagle on the Hill. Driving home from a visit with relatives, the family in their Corolla sedan rounded a left hand bend and were confronted with a large fuel tanker on the wrong side of the road. The driver panicked, swerved causing the trailer to jack-knife across the path of the oncoming car. The car hit the trailer on the driver's side. Unfortunately, Celia White and her daughter were trapped in the twisted wreckage. They were trapped for 20 minutes. The mother sustained only a broken and gashed leg. Celia White's husband and son were looking on. They could not help her. They had to stand back because there was fuel all over the road. The daughter was eventually rescued by the 'jaws of life'.

You will hear eyewitness evidence that proves that Mr Fitzpatrick drove his semitrailer in a manner that created potential danger to other people using the highway.

This was solely and act of carelessness and inattention and an act that directly caused the death of Celia White. You, the jury, can deliver justice which by finding the defendant guilty of causing death by dangerous driving. Thank you Your Honour. Judge: Mr Warwick, you may now open the case for the defence.

Defence; Thankyou Your Honour. Ladies and Gentlemen of the Jury, My name is Melvin Warwick, I am the attorney defending my client Mr Fitzpatrick in this case. The prosecution will allege on the basis of eyewitness testimony that my client was solely responsible for the injury which occurred when his truck collided with the eyewitness' car on the morning of April 14th. Ladies and Gentlemen, I want you to first remember while listening to the eyewitness evidence that the eyewitness was actually in the car at the time of the accident which means that he may not have seen the things he alleges to have seen. Second, he and his young son had to watch his wife trapped in the wreckage of a car that was in imminent danger of bursting into flames. This is bound to cause feelings of helplessness and perhaps a strong need to blame someone. While not in any way meaning to deny the adverse nature of the events that occurred on that day, I have to emphasise that Mr White is not an impartial witness who has the benefit of objectivity. Finally, at the time of the accident he must have been under a great deal of stress and this most likely affected his ability to recall the details of an event. Ladies and Gentlemen, it is our case that it was the witness's car which crossed over the double white lines that morning causing Mr Fitzpatrick to swerve and his trailer to jack-knife across the road and into the witness' car. Finally, Ladies and Gentlemen, I ask you to consider the suffering of the truck's driver $\mathrm{Mr}$ Fitzpatrick. He walked away physically unarmed from the accident. However, psychologically he is distraught. He knows that he could not have avoided the accident, but he still blames himself despite the physical evidence which proves his innocence. Ladies and Gentlemen, in considering the evidence remember that the Crown bears the burden of proof. They must prove beyond reasonable doubt that my client drove in a manner dangerous to the public, that this was the result of carelessness and further that this action was the sole cause of the death that resulted from the collision. You can deliver justice and ease Mr Fitzpatrick's suffering by 
delivering a not guilty verdict in accordance with the truth. Thankyou Your Honour. Judge: Mr Spence, please call your first witness.

Prosecution: Thankyou Your Honour. The Crown calls Police Constable Roger McNally to the stand. \{Pause\} Mr McNally, My name is Henry Spence, I am the prosecuting Attorney. I would like to ask you some questions about the accident which took place on Mount Barker Road, near Eagle on the Hill, on the morning of April 14th 1998. We are keen to establish the truth so please answer where appropriate with either, I don't know, I 'm not sure or I am sure.

Police Constable: Yes, I will.

Prosecution: You were the investigating officer when this accident occurred ?

Police Constable: Yes, that is correct.

Prosecution: Can you describe for the jury the position of the vehicles when you arrived at the scene of the accident that morning.

Police Constable: Certainly. The semi-trailer was blocking the whole road. The cabin was in the middle of the down track with its trailer perpendicular to the cabin obstructing the rest of the down track and extending all the way across the up track. The truck had been travelling down Mount Barker Road away from Eagle on the Hill and the car had been travelling up. (1)The car had impacted the trailer about half way along its right side. There wasn't much left of the car, the motor had been pushed back to the center console. The car had hit mostly on the driver's side. The car was completely caved in on the driver's side. I arrived on the scene before the ambulance. The woman was pinned in the driver's seat. The mother was in obvious pain. She was bleeding profusely. The father and son who had managed to get out of the vehicle were yelling at me to get them out of the wreckage. I couldn't do anything because of the risk of the fuel around the car igniting and causing an explosion ...I tried to move them away from the car, they wouldn't leave the rest of their family trapped....we just had to wait...Mr Spence, I was so surprised that they all managed to make it out alive. When I got there and saw the damage to the car I was amazed that they hadn't all been killed.

Prosecution: Mr McNally......Was there any evidence that either of the vehicles had taken evasive action, such as breaking or swerving before impact ?

Police Constable: (2) Yes, there were wide skid marks from the truck trailer skewing sideways which were 28 meters in length and from the truck going in a direction perpendicular to those of the truck which were a 2 meters longer. There were no skid marks from the car.

Prosecution: From the length of the skid marks, the damage caused upon impact and the position of the vehicles, can any estimates about the speed of the truck and car be made.

Police Constable: It is difficult to determine. However, it appears that the car may have been travelling at about $32 \mathrm{~km} /$ hour and the truck at about 50 .

Prosecution: Mr McNally, what causes a trailer to jack-knife or begin to push the cabin of a truck along ?

Police Constable; There are several different causes for a trailer to jack-knife. It can be caused by incorrect tyre pressure, insufficient or too heavy loads, under gear out of alignment or worn steering or other faults in old vehicles. 
Prosecution: In your opinion was the jack-knife of the truck trailer caused by any of the problems that you just mentioned ?

Police Constable; No. We checked the vehicle thoroughly. It was in good condition mechanically.

Prosecution: What in your opinion, was the cause of the collision?

Police Constable: The truck obviously swerved to it's left and braked heavily. This

caused the trailer to jack-knife and the car ran into the trailer. I can not speculate on the reason why the driver of the truck had to brake.

Prosecution: Is it possible Mr McNally, that the driver swerved and braked because he suddenly realised he was on the wrong side of the road?

Police Constable: Yes it is possible.

Prosecution: Thankyou Mr McNally. No further questions Your honour.

Judge: Mr Warwick, you may now cross examine the witness.

Defence: Good morning Mr McNally. My name is Melvin Warwick, I am defending

my client Mr Fitzpatrick the driver of the semi-trailer. Mr McNally, you stated to my learned friend Mr Spence, that there are several reasons for a trailer to jack-knife in the manner you described.

Police Constable: Yes.

Defence: Is it not equally possible that the driver of the truck, my client, may have braked and swerved to avoid a collision when he saw the car driven by Celia White on the wrong side of the road?

Police Constable: Yes it is possible. As I said before, the trailer jack-knifed because the truck braked heavily and swerved to it's left. I can only speculate on the reasons why the truck braked in the first place. I can only tell you that the trailer moved to the other side of the road because of the braking and swerving. Def: Thankyou Mr McNally. No further questions Your Honour.

Judge: Mr Spence, you may now call your second witness to the stand.

Prosecution: Thankyou Your Honour. The Crown calls James White to the stand. Goodmorning Mr White, my name is Henry Spence, I am the prosecuting attorney, I would like to ask you some questions about the accident which took place on Mount Barker Road at Eagle on the Hill, Monday the 14th of April 1998. Mr White it is essential for the carriage of justice that the jury have a good understanding of the events leading up to the accident. We are keen to establish the truth so please answer where appropriate with either, I don't know, I'm not sure or I am sure.

Witness: Yes I will.

Prosecution: Would you state your full name please.

Witness: James Phillip White.

Prosecution: What is your full home address.

Witness: 20 Druids Avenue, Mount Barker, South Australia.

Prosecution: At approximately 10am on Monday the 14th of April 1998 were you a passenger in the vehicle travelling along Mount Barker road, which was subsequently involved in the accident in question?

Witness: Yes, I was.

Prosecution: Why was the vehicle in which you were a passenger travelling along 
Mount Barker Road on the morning of the accident ?

Witness: My family and I were returning from a visit with my parents at Clarence Gardens in Adelaide.

Prosecution: Who was in the car with you?

Witness: My wife Celia, who was driving, and our children Kayla and Jarrod.

Prosecution: Had Celia, your wife, had any accidents prior to the one that occurred that morning?

Witness: No. Celia had an impeccable driving record.

Prosecution: Did Celia have any impairments or disabilities which may have reduced her ability to drive?

Witness: No, she did not.

Prosecution: Was Celia tired, stressed or distracted on the day while she was driving the vehicle?

Witness: No she was not.

Prosecution: Were the children distracting her at any point during the trip ?

Witness: No.

Prosecution: Are you sure Mr White, children can sometimes find it difficult to behave on long trips?

Witness: The children were being well behaved, talking to each other in the back.

Celia's attention was on the road the whole time.

Prosecution: Do you have any problems with your eyes Mr White ?

Witness: No

Prosecution: When was the last time you had your eyes checked?

Witness: About 3 months ago. We get them checked regularly because of the type of work I do.

Prosecution: Mr White, can you tell the court in detail, what you were doing 5 minutes prior to the accident.

Witness: Well, as I said before, we were returning home from a visit to my parent's place in Adelaide. We were driving up Mount Barker Road...

Prosecution: Mr White, what exactly was going on in the car at this point ?

Witness: We were talking to the kids about what they had been doing at Grandma's place.

Prosecution: When did you first notice the truck?

Witness: (4)When we rounded the left hand bend just before where the accident happened. You couldn't see around the corner because at that point the road is actually bending around the steep hill on the left.

Prosecution: When you first saw the truck, where in relation to your car, was it located ?

Witness: It was directly in front of us, a little to the left of the center of our car.

Prosecution: Was the truck over the double white lines on the road?

Witness: Yes.

Prosecution: In your estimation, how far over the white lines ?

Witness: At least 3 meters. About from here to the jury away. (3) It was almost half

the way over on our side of the road. So far over that we couldn't have swerved around it, there simply wasn't room. 
Prosecution: Did the truck appear to swerve or deviate from it's course at any time prior to impact, Mr White ?

Witness: Yes, it did. I remember that Celia had time to flash the lights a couple of times... (5)it swerved to our right (3).

Prosecution: At what speed were you travelling ?

Witness: I reckon it was only about $40 \mathrm{~km} / \mathrm{h}$. We had slowed down to take the corner.

Prosecution: At what speed in your estimation was the truck travelling ?

Witness: It must have been over $60 \mathrm{~km} / \mathrm{h}$. We had slowed down, it came towards us

very quickly. (5)

Prosecution: Did the truck appear to slow down or brake at any point?

Witness: (2) It did brake. As I said it happened very quickly. There was this petrol

truck, or Ampol truck and the trailer across the road coming straight for us and.... It was very quick. There was no way Celia could have avoided it.

Prosecution: Which side of the trailer did your car hit?

Witness: (1) We hit on the trailer's right side.

Prosecution: Is there anything else that you think is important to tell the court $\mathrm{Mr}$ White?

Witness: Yes, I'd just like the jury to know that Celia was always a good driver. Prosecution: No further questions Your Honour.

*

Judge: Thankyou Mr Spence. Mr Warwick, you may now cross-examine the witness. Defence: Thankyou Your Honour. Good morning Mr White. My name is Melvin Warwick and I am the attorney for the defence in this case. I have some questions to ask you about the accident which took place on Mount Barker Road in which you were involved. The justice system demands that the jury have a clear picture of these events, so please answer the questions as accurately as possible. \{Pause\} At what point did you think you saw the truck on the wrong side of the road?

Witness: (4)When we rounded the bend, just before the accident happened. As I said before, it was a blind corner which you couldn't see around because of the hill. When we rounded the bend I could see that it was on the wrong side of the road. I could see the (6) paint of the Ampol symbol.

Defence: And you are sure you could tell that the truck was on the wrong side of the road?

Witness: Yes. I am sure.

Defence: You were rounding the bend and travelling at a reasonable speed and yet you are sure were able to discern that the truck was on the wrong side of the road ?

Witness: Yes, that's what I'm telling you.

Defence: Mr White, if you saw the truck was on the wrong side of the road why didn't you warn your wife?

Witness: I don't know. It happened very quickly. But I could see it definitely was on the wrong side of the road. (3)It was almost half way over onto our side of the road. Defence: Did your eyes remain on the road the whole time you were rounding the corner?

Witness: Yes.

Defence: Exactly what were you doing in between time that you rounded the bend and 
the time that you saw the truck?

Witness:(7) I was watching the road.

Defence: Mr White, your wife maintained her attention on the road the whole time while you were going around the corner?

Witness: Yes.

Defence: Is it not possible that she was distracted by your children, and that her attention left the road for a few moments?

W: No.

Def: Mr White, I think that perhaps she was distracted and that out of your anger, you

needed to blame someone for your misfortune and that happens to be my client $\mathrm{Mr}$ Fitzpatrick.

W: No that's not true ! He caused the accident, his truck was on the wrong side of the road!

Def: No further questions Your Honour.

Judge: Mr Spence, you may now address the jury with your closing statement.

Pro: Thankyou Your Honour. Ladies and Gentlemen of the jury, you have heard evidence today from two witnesses to the accident which occurred on April 14th 1999 on Mount Barker Road. Mr Fitzpatrick may well have suffered as the result of these events, however, the White family has suffered much more. The testimony of both Constable McNally and Mr White prove beyond reasonable doubt that, Mr Fitzpatrick drove in a manner that was dangerous to the public, that this was the result of carelessness due to inattention and the sum result of these actions was the death of Celia White. Celia was trapped in the wreckage for 20 minutes and could see her husband and son were out of the car and that they could not help her. Ladies and Gentlemen of the jury, Mr Fitzpatrick's suffering is insignificant by comparison to that of the White family. You have a duty to the Whites and to the public to find the defendant guilty of causing death by dangerous driving. The Whites must be given the understanding that our justice system is fair and the public must be given the understanding of how important careful attention on the road at all time really is. Thankyou for your attention Ladies and Gentlemen.

Judge: Mr Warwick you may now address the jury with your closing statement. Def: Thankyou Your Honour. Ladies and Gentlemen of the jury. The prosecution has alleged that it was Mr Fitzpatrick's truck on the wrong side of the road which caused the accident in question. We contend that the only reason the trailer ended up there was because of the driver's need to swerve sharply and to brake to avoid the White's car which had strayed on to the wrong side of the road. Ladies and Gentlemen I ask you to consider the eyewitness evidence upon which the Prosecution's allegation is based. Mr White had to watch his wife's life in danger right in front of him and could not do anything as they waited for help to arrive. A situation like this can be emotionally upsetting. Can you place your faith in the memory of a man who has experienced such events? Can you believe that he is able to clearly remember what he saw prior to these events? No, you cannot. The amount of stress that Mr White would have experienced is substantial, and yet the prosecution asserts that Mr White's memory would have been unaffected by this. Secondly, Ladies and Gentlemen, realise that when people see those whom they know killed they often need to blame someone. 
Mr White cannot face the possibility that his wife's driving caused her own death. This is easy to understand. However, as the guardians of justice, you the jury have the duty to up hold the law, no matter how much people have suffered and may continue to suffer. That is why you must find my client Mr Fitzpatrick not guilty of causing death by dangerous driving. That is all Your Honour. 


\section{APPENDIX C \\ Study One Data Reduction Procedures}

\section{Step One: Raw Data}

At the conclusion of data collection, the final data file included 264 total entries, or one separate entry for each discrete time Study One was accessed and begun. Of these 264 entries, 147 were complete, having accessed every page of the study website. To be clear, these are not total numbers of distinct participants, but rather all separate instances of data recordings initiated each time the study was started.

\section{Step Two: Eliminating Repeat Starters}

Despite repeated admonitions and stern warnings to the contrary, some participants in online studies nevertheless begin the study and then quit partway through completion, only to return at a later time to complete the study in full. Because the objective of Study One is to determine the relative ease or rate of detecting each testimonial inconsistency in comparison to the other inconsistencies present in the trial transcript, I eliminated those participants who were exposed to the trial transcript to any extent prior to the session in which they completed the study. For example, a participant who read along through the end of all plaintiff's witnesses but quit the study before the defense presented its case, only to return two days later to restart the study (the website required participants to start over if their browser window closed at any point) and completed it during that second session would have been exposed to the inconsistent information two full times. This obviously gives that repeat participant a greater opportunity to detect the inconsistencies compared to a participant who was only exposed 
to the critical information once, so I eliminated all data associated with those participants who accessed the study on more than one occasion. However, for any participants who completed the study the first time they accessed it, but who may have started it again, I simply eliminated all data associated with any subsequent attempts since their first exposure was untainted.

After eliminating the data associated with any participants who had been preexposed to the trial transcript, 161 separate data entries remained.

\section{Step Three: Eliminating Inattentive Participants}

Because online data collection allows participants to complete the study at the time and place of their choosing, it tends to be more popular with the FIU participant pool than in-person studies which require participants to be in a specific time and place to participate. While this flexibility benefits participants and the concomitant popularity benefits researchers with faster data collection (as well as requiring less time to input and code data), the tradeoff for researchers is the lack of control over the environment in which participants complete the study. Without the presence of a supervising researcher, some participants may choose to complete online studies without actually paying attention or engaging in anything resembling legitimate, earnest effort. To combat this problem (or at least prevent it from tainting my data), I included two questions intended to confirm that the participant was in fact paying at least some minimum degree of attention to the questions they were answering. The first, presented in random order among the Multiple Choice questions, was

There are lots of witnesses and lots of evidence in trials. Sometimes testimony is consistent, sometimes it's contradictory, sometimes different witnesses disagree, and sometimes accounts vary. 
Please mark all possible answers for the following question, even those which are contradictory to each other.

These questions are designed to test your memory for the information in the trial testimony, not your personal opinion on what actually happened or what is correct. How you answer these questions should not depend on which witnesses you believe to have been accurate or those you believe to have been inaccurate-just the content of the testimony itself.

\section{Again, you may choose more than one answer.}

Thank you for participating in this study. To ensure that you are paying attention to the questions, please mark $A$ for this question.
A. He put the car into reverse
B. He stopped the car
C. He turned sharply to his right
D. He pulled the emergency brake
E. None of the above
Г F. Don't know/don't remember

The second, presented in random order among the True/False questions, was

There are lots of witnesses and lots of evidence in trials. Sometimes testimony is consistent, sometimes it's contradictory, sometimes different witnesses disagree, and sometimes accounts vary.

These True or False questions are designed to test your memory for the information in the trial testimony, not your personal opinion on what actually happened or what is correct.

How you answer these questions should not depend on which witnesses you believe to have been accurate or those you believe to have been inaccurate-just the content of the testimony itself.

Thank you for your continued attention and hard work. The study is almost over, so please mark this answer true so that we will know that you're still paying attention.
C True
False
I don't know

I eliminated all nine participants who failed to answer one or both of these questions as directed, leaving 152 remaining sets of data. 


\section{Step Four: Eliminating Partial Participants}

Steps Three and Four could have been performed in interchangeable order with no consequence, but I expressly refrained from eliminating the participants who did not complete the study at the start of the data reduction process because doing so would have prevented me from having the ability to identify those participants who began the study and were exposed to some or all of the trial transcript and quit partway through, only to restart and complete the study at a later time - and with an improper degree of exposure to the trial transcript and inconsistent testimony. It is only after this step that the remaining data entries can properly be called "participants." Of the 152 data entries remaining before this step, only 114 participants completed the entire study.

\section{Step Five: Eliminating Extreme Outliers of Total Time Spent}

Due to the fact that individual differences in reading speed, attention to detail, and overall thoughtfulness and thoroughness of answering the battery of questions designed to measure inconsistency detections (including 36 Open Ended and Short Answer questions with no restriction on response length), and I had estimated that most participants should be able to complete the entire study in approximately 90 to 120 minutes, I defined an extreme outlier as any participant who required greater than twice that duration to complete the study. Because some participants would load the first page of the study (the Informed Consent page) and then wait (sometimes minutes, hours, or even days) before actually indicating their consent and beginning the study, to prevent this practice from skewing the calculations of total time spent, I calculated the total duration by subtracting the time participants loaded the second page (immediately after 
submitting the Informed Consent page) from the time the last page of the study was submitted.

Eight participants were identified as extreme outliers ranging from 242.21

minutes spent to 1060.01 minutes spent $(M=577.04, S D=306.90)$ and eliminated from further analysis, leaving a total of 106 participants.

\section{Step Six: Eliminating Outliers of Total Time Spent}

After eliminating those extreme outliers which would skew the overall

distribution, I calculated the distribution for the remaining 106 participants, resulting in the elimination of those six participants whose total time spent was in excess of two standard deviations above the mean, or greater than 179.30 minutes $(M=113.17, S D=$ 33.06). There were no participants who completed the study in less time than two standard deviations below the mean.

The resulting data set $(n=100)$ to be used in Study One included participants who spent between 72.11 and 176.91 minutes $(M=107.36, S D=23.53)$. 


\section{APPENDIX D}

\section{Study One Trial Transcript (15 Inconsistencies)}

NOTE: Highlighted phrases (e.g. PI-Plain Inconsistency, S\&B-Inconsistency used in Semmler \& Brewer [2002], etc.) denote details of testimony relevant to the 15 inconsistencies, and are only marked here for ease of identification. This emphasis was not included in the actual materials used.

\section{Johnson v. McGraw}

The following trial transcript is an abridged version of a real trial from here in the state of Florida. The trial, which has already taken place, and for which the jury has already rendered a verdict, involved a traffic accident which resulted in injury to the plaintiff, Amanda Johnson, an injury she alleged was the result of negligence on behalf of the defendant, Michael McGraw. Although the circumstances of the accident involved multiple parties, Michael McGraw was the only defendant in this case.

While the names of all parties have been changed, one of the attorneys has asked FIU researchers to conduct this study to see how real people like you perceive the case so that they can determine whether to appeal the outcome of the trial. We will be using the data from this study to help the attorney, so please pay close attention as it will impact the final resolution of an actual civil case.

Please listen to the trial transcript and read along as if you had been chosen to serve as a juror in this case.

Judge Robert C. Underwood: Ladies and gentlemen of the jury, you are about to hear testimony concerning an automobile accident that occurred in the late afternoon of Tuesday, July $8^{\text {th }}, 2008$ in the city of Clermont, located in Lake County, Florida. The plaintiff is Amanda Johnson, and the defendant is Michael McGraw. There are no other plaintiffs or defendants in this suit, and all other disputes between these and any other parties related to the events in question have been resolved separately prior to this proceeding.

\section{Opening Statement by Seth Blum, Attorney for the Plaintiff}

Seth Blum, Attorney for the Plaintiff: Ladies and gentlemen of the jury, what we have before us today is an unfortunate story of a young lady who was simply going about her business one day, on the afternoon of Tuesday, July $8^{\text {th }}, 2008$, up in Clermont, which is in central Florida to the west of Orlando. This young lady, Amanda Johnson, was at the time a 26 year old waitress at a family restaurant. She was riding in the car with her 
boyfriend, Brock Collins, a 28 year old middle school teacher. They were in his blue Toyota Camry (PI 5-Model true)on their way to catch a movie before meeting up with Amanda's parents for dinner later that night. Unfortunately, their plans were dashed by the negligence of another driver on the road that evening, Michael McGraw.

Throughout this case, you are going to hear overwhelming evidence from a variety of witnesses-Amanda Johnson, Brock Collins, a host of different eyewitnesses to the accident, the emergency first responders on the scene, medical professionals, as well as professional scientists who specialize in the analysis of vehicles and crash scenes which they use to reconstruct accidents. The testimony of all these witnesses will show you, with no uncertainty whatsoever, that Mr. McGraw, and only Mr. McGraw—not Mr. Collins, not the driver of the semi truck, not anybody else and not anything else other than Mr. McGraw's negligent operation of his big, white (PI 10 color true) van-was the direct cause of Amanda Johnson's serious injury. I hope that you find him liable for all damages suffered by Ms. Johnson at his hand. Thank you very much for your time, service, and attention today.

\section{Opening Statement by Howard Kurtz, Attorney for the Defendant}

Howard Kurtz, Attorney for the Defendant: Esteemed members of the jury-like my colleague, I thank you in advance for your attention, effort, and diligent fulfillment of your civic duty. What we can all agree on is that you are here today to hear about a traffic accident that injured a nice young lady and her boyfriend. Everybody wishes that this accident hadn't happened, but not everybody agrees about how it happened or how Ms. Johnson ended up being hurt.

Ms. Johnson and her attorney would like for you to believe that her broken hip and other injuries are the result of negligence on the part of Mr. McGraw. Now it's certainly true that Mr. McGraw, while trying to avoid the out of control semi tractor trailer coming towards him, ended up hitting Mr. Collins's car. What we're less certain about is how Ms. Johnson was injured. You see, Ms. Johnson wasn't wearing her seat belt, and we'll be presenting evidence to you that will show that Ms. Johnson wasn't injured because Mr. McGraw hit their car, but that she was injured when the car ran into the street lamp, and that if she had been wearing a seat belt, she wouldn't have broken her hip.

Ms. Johnson and her attorney would like for you to believe that her injuries are the fault of Mr. McGraw, but through the course of this trial, you will soon see that they don't have any real evidence to support this allegation. Because they can't show that the majority of the evidence undoubtedly proves that Mr. McGraw was the cause of Ms. Johnson's injuries, you will come to the conclusion that Michael McGraw was not negligent, was not the direct cause of Amanda's broken hip, and that he should not be held liable for her injuries and you should render a verdict in favor of the defendant. 
Plaintiff's Witness Marc Zollinger, Direct Examination by Seth Blum, Attorney for the Plaintiff

Seth Blum, Attorney for the Plaintiff (Questioner): Can you please state your name and occupation for the court?

Marc Zollinger (Answerer): My name is Marc Zollinger, and I'm an administrative assistant for Universal Translation Corporation.

Q: And where is your office located?

A: It's on Foothill Drive, pretty close to the intersection of Foothill and Windsor, a little south of there, on the east side, or I guess northeast side of the road at that point, down by the bend.

Q: Tell me, Mr. Zollinger, what were you doing on the afternoon of Tuesday, July $8^{\text {th }}$, 2008 ?

A: I needed some fresh air, so I went to the little walk-up coffee window a few doors down from our office, the one on the north side of the Cuban restaurant there.

Q: Okay. And while you were there, did anything unusual happen?

A: As a matter of fact, yes. While I was waiting in line, I was just standing around watching the cars go by when there was a big traffic accident behind me up the way a little bit at the corner.

Q: Up the way a little bit? How far do you mean? Help the jury to understand how close you were.

A: Oh, not that far really. Probably a few hundred feet away, but I had a pretty clear view since it's just the parking lot for the restaurant there to the north of the coffee window up to the corner.

Q: And can you tell us what you saw?

A: I saw a car, a van, and a tanker truck get into a wreck. The tanker truck was kind of sideways, coming southbound towards the intersection and kind of weaving back and forth a little bit in the middle of the road. I saw Mr. Collins's car, a blue Corvette (PI 5model false)make a left turn to go west on Windsor to get out of the way of the truck, but then Mr. McGraw's white (PI 10 color true)Ford work van came screeching around, and then the semi locked up its brakes and the tires started squealing but the tanker trailer swung around and kind of batted Mr. McGraw's van on the passenger side (PI 1/S\&B 1 true)which then bounced into the back of Mr. Collins's car which then crashed into the 
street light (PI 12 true) on the northwest corner of the intersection. It was like one big chain reaction. They almost hit some lady walking a Dachshund. (PI 13 false)

Q: And what happened next?

A: I saw the guy with the black hair (PI 7 true), Mr. McGraw, get out of his van to make sure everyone in Mr. Collins's car was okay or something. I couldn't hear what they were saying or anything, but right away Mr. McGraw got on his phone, I guess to call 911. After a minute or two, Mr. Collins, the bald one, got out of the car and went around to the passenger side and was talking to someone in the passenger seat. He looked pretty worried based on all his movements and body language.

Q: And then what?

A: I placed my order, and by the time I got my coffee I could see that a police car was already on the scene (PI 8 true)so I grabbed my coffee and went over to tell him what I saw. I told him exactly how it happened, and he wrote it all down. The officer's name was Christopher Eaton. (PI 14 true) He was a really big guy.

Q: Thank you, Mr. Zollinger. No further questions.

Plaintiff's Witness Marc Zollinger, Cross Examination by Howard Kurtz, Attorney for the Defendant

Howard Kurtz, Attorney for the Defendant (Questioner): Could you describe for us in just a little more detail exactly what happened after all the vehicles came to a stop?

Marc Zollinger (Answerer): Sure. Mr. McGraw got out of the white (PI 10 color true)van pretty quickly and immediately ran over to Mr. Collins's blue Corvette, (PI 5model false) over to the driver's side. I couldn't hear them, of course, but it looked to me like he was making sure everyone was okay, and then shortly after talking to Mr. Collins, McGraw got his cell phone out of his pocket and made a call. A minute or two later, the police officer arrived, then I went over and told him (PI 14 true)what I saw, and then I went back to work.

Q: Thank you, Mr. Zollinger. I have nothing further.

Plaintiff's Witness Barbara Feldman, Direct Examination by Seth Blum, Attorney for the Plaintiff

Seth Blum, Attorney for the Plaintiff (Questioner): Hello there. Could you please state your name and occupation for the record? 
Barbara Feldman (Answerer): My name is Barbara Feldman, and I am a librarian.

Q: Thank you Ms. Feldman. Now you're familiar with the day in question here in this trial, right?

A: Yes I am.

Q: And did you witness the accident at issue here?

A: Yes I did. I remember it all quite vividly. I was driving southbound on Foothill Drive, north of the intersection with Windsor.

Q: And could you please describe for us what you saw?

A: Sure. As I was driving, up ahead I noticed a Shell (PI 4/S\&B 6 true) oil tanker trailer crossing over the line separating the two directions of traffic, kind of skidding its tires and fishtailing around. The stop light (PI 15 true) ahead of us was green, so I'm not sure what the deal was. It's not like he was slamming on his brakes to keep from running the light (PI 15 true) or anything. It was also driving southbound like I was, so I slowed down to stay away from the situation. It was all over the place in the left turn lane in the middle, going back and forth into the oncoming lanes of traffic and stuff.

Q: And what happened next?

A: Well, the truck's trailer sort of swung out into the intersection, where it hit Mr. McGraw's tan van, (PI 10 color False) kind of side swiping it and kind of just smacking it on the passenger side, which forced the van into the back side of Mr. Collins's blueToyota Camry (PI 5-model true), which then crashed into a light pole (PI 12 true)on the northeast corner of the intersection.

Q: Okay, and what about after the accident?

A: I just stayed around until the police officer arrived, and once she (PI 14 false) got there, I gave her (PI 14 false) my statement and my contact information, and then I left to go home.

Q: Thank you Ms. Feldman. I have no more questions.

Plaintiff's Witness Barbara Feldman, Cross Examination by Howard Kurtz, Attorney for the Defendant 
Howard Kurtz, Attorney for the Defendant: I have no questions for this witness, your Honor.

Plaintiff's Witness Jill Randall, Direct Examination by Seth Blum, Attorney for the Plaintiff

Seth Blum, Attorney for the Plaintiff (Questioner): Could you please give us your name and occupation?

Jill Randall (Answerer): My name is Jill Randall, and I sell Amway products from my home here in Clermont.

Q: Now could you please tell us about what you saw on Tuesday, July $8^{\text {th }}, 2008$ ?

A: I was waiting for my turn at the four-way stop (PI 15 false) at the intersection in order to to cross Foothill heading west, and there was a semi truck that came out of nowhere from the north, skidding and screeching and swerving all over the place out of control.

Q: That must have been pretty scary. Can you tell us more about the truck and what happened?

A: Well, I saw the truck blow the stop sign (PI 15 false) going through the intersection kind of in the middle of the southbound and northbound lanes, and he was fishtailing a little bit, you know, the cab of the truck going in one direction while the trailer was slipping and sliding around behind him in another direction. The truck swerved towards me, to the east, to the driver's left, past me as it came through the intersection. (PI 3/S\&B 3 true) As the truck was getting itself under control, I could tell that there was some sort of additional accident on the opposite side of the trailer involving some other vehicles, but since I was on the east side of the trailer and that was all happening on the west side, I really couldn't see too much about what exactly happened.

Q: Okay, so you weren't able to see much of the actual contact between the vehicles?

A: Well, no, not of the actual contact when it was happening, but after everything came to a stop, I was able to see that the white (PI 10 color true)van driven by Mr. McGraw had crashed into the back of the other car, the one driven by Mr. Collins, the blue Toyota Camry, (PI 5-model true) and Mr. Collins's car was up against the street lamp(PI 12 true). It looked like it had pretty much hit the lamppost (PI 12 true)head on, and Mr. McGraw's van was stopped behind it, and it was all banged up on the passenger side. (PI $1 / \mathrm{S} \& \mathrm{~B} 1$ true)

Q: Well it sounds like you got a good look at the aftermath of the accident. What happened after everything came to rest? 
A: The red-haired man (PI 7 false), Mr. McGraw got out of his van pretty quickly and went to go check on the people in Mr. Collins's car. He was talking to Mr. Collins for a minute, and then he got out his phone and made a call, I assume to 911. Mr. Collins got out of his car eventually, and when the police officer arrived a minute or two later, he (PI 14 true) was a real hunk, so I hung around and gave him my statement once the paramedics got there to take over (PI 8 true) making sure everyone got the medical attention they needed.

Q: We appreciate your testimony here today, Ms. Randall. No further questions.

Judge Robert C. Underwood: Your witness, Mr. Kurtz.

Plaintiff's Witness Jill Randall, Cross Examination by Howard Kurtz, Attorney for the Defendant

Howard Kurtz, Attorney for the Defendant (Questioner): So Ms. Randall, you weren't able to describe exactly what happened during the wreck itself because the trailer from the tanker truck was blocking your view, is that right?

Jill Randall (Answerer): That's right. I saw the beginning and I saw the aftermath, but I didn't see the actual accident between Mr. McGraw and Mr. Collins, no.

Q: I see. Thank you very much, Ms. Randall. I don't have any more questions.

Plaintiff's Witness Steve Powell, Direct Examination by Seth Blum, Attorney for the Plaintiff

Seth Blum, Attorney for the Plaintiff (Questioner): Could you please give your name and occupation for the record?

Steve Powell (Answerer): My name is Steve Powell, and I'm a real estate agent here in Clermont.

Q: And where were you on the afternoon of Tuesday, July $8^{\text {th }}, 2008$ ?

A: I was at the post office located there on the east side of Foothill Drive, a little ways up from, I mean north of, the intersection with Windsor Road.

Q: Okay. And did you see anything unusual at the post office?

A: Well, just as I was leaving, I was looking out the window and I saw a Shell (PI 4/S\&B 6 true) tanker truck go skidding down the street past the post office. 


\section{Q: Down Foothill you say?}

A: Yeah, it was traveling south but kind of screeching and sliding out of control.

Q: And what did you see after that?

A: Well, I didn't really see exactly what happened next. I was walking out of the building and didn't have a constant, uninterrupted view so I missed the actual crash, but after I got outside, I saw the whole accident scene with the tanker truck stopped at a funny angle over on the east side of Foothill on Windsor, and the van and the car over on the corner by the Zales(PI 11 true) jewelry store. The guy with the black hair (PI 7 true), Mr. McGraw, was standing outside the vehicles. There were a couple of people in the car, the blue Camry, (PI 5-model true) and they looked kind of hurt.

Q: How so?

A: The bald guy, Mr. Collins, had some cuts and stuff on his forehead and he was bleeding a little bit, but the lady in the passenger seat was much worse. It was kind of hard to see exactly how she was hurt, but it was clear she was pretty banged up.

Q: And can you tell us what happened next?

A: I had an important meeting I had to get to a little later on that afternoon so I didn't have time to stick around, and I don't know how I could have helped anyway, but as I was starting to walk back to my car, the bald guy, Mr. Collins, was out of his car and the ambulance showed up (PI 8 false) so I figured it would be okay now that there was an official emergency responder on the scene. Right as I got to my car, which was parked over in the lot north of the coffee shop on the southeast corner of the intersection, I saw a police officer arrive next (PI 8 false), so I figured I might as well go over to him (PI 14 true) and tell him what I had seen before I left to go to my meeting. As I was giving Officer Eaton my statement, I could see the paramedics getting Ms. Johnson out of the car and loading her into the ambulance. It was at that time that I could see that her left leg (PI 6 false) was all mangled and busted up.

Q: Thank you for your time, Mr. Powell. I don't have any more questions for you.

Plaintiff's Witness Steve Powell, Cross Examination by Howard Kurtz, Attorney for the Defendant

Howard Kurtz, Attorney for the Defendant (Questioner): Mr. Powell, you were inside the post office when the actual accident occurred, and were unable to see exactly what happened at the intersection, is that right? 
Steve Powell (Answerer): Yeah, I just saw the Shell (PI 4/S\&B 6 true) truck go past the window out of control, but no, I couldn't see the intersection or anything that far down from where I was.

Q: So you don't have any idea exactly how any of the different vehicles crashed into each other, or the exact moment in which Ms. Johnson might have been hurt, or how she was hurt, or anything specific like that, correct?

A: That's correct. I just saw the truck go by and then by the time I was outside, I saw the wreckage and stuff where it had all come to rest.

Q: So basically everything at issue here happened when you couldn't see it?

A: Well I did see two men, Mr. Collins and Mr. McGraw outside talking to each other when the ambulance arrived at the time I was walking across Windsor, and then by the time I got to my car, I saw the police had just arrived too. (PI 8 false)

Q: Thank you, Mr. Powell. No further questions.

Plaintiff's Witness Lesley Jobin, Direct Examination by Seth Blum, Attorney for the Plaintiff

Seth Blum, Attorney for the Plaintiff (Questioner): Hello there, could you please tell us your name, occupation, and city of residence for the record?

Lesley Jobin (Answerer): My name is Lesley Jobin, I'm a tour group coordinator, and I live here in Clermont.

Q: And what were you doing on the afternoon of Tuesday, July $8^{\text {th }}, 2008$ ?

A: Well, since there's really no slow days at my company because of all the visitors to the area and different tourist attractions and stuff in and around Orlando, we're open seven days a week, and I'm off on Mondays and Tuesdays each week. That was a Tuesday, and I was out walking Finnegan, my German Shepherd. (PI 13 true)

Q: And can you tell us a little more about your walk with Finnegan that day?

A: Sure. I was walking west along Windsor Road, having just crossed Foothill. I was passing in front of the Jared jewelry store (PI 11 false) there at the corner, when the crash happened.

Q: So can you tell us what you saw? 
A: Well, I saw the Shell (PI 4/S\&B 6 true) truck fishtailing out of control, well, I guess there isn't any way for a truck to fishtail but still be under control, but anyway, it was sort of sliding out toward its right side, and then it kind of smacked the side of the white (PI 10 color true)van, and that's when the van crashed into the back of the blue Toyota Camry, (PI 5-model true) which then ran straight into a tree (PI 12 false) on the corner in front of Jared(PI 11 false).

Q: Were you able to see anything that happened inside of either of the vehicles?

A: Yeah, when Mr. McGraw first hit Mr. Collins's car, I could see that both Mr. Collins and Ms. Johnson's heads went flying forward into the windshield.

Q: Thank you Ms. Jobin. I don't have any more questions for you.

Plaintiff's Witness Lesley Jobin, Cross Examination by Howard Kurtz, Attorney for the Defendant

Howard Kurtz, Attorney for the Defendant (Questioner): So Ms. Jobin, you were out walking your German Shepherd, (PI 13 true) Finnegan, that afternoon, is that correct?

Lesley Jobin (Answerer): That's right.

Q: And were you talking on the phone, or listening to music, or anything like that on your walk?

A: Well, I was listening to my iPod.

Q: Hmm. Okay. And you were walking west, passing the Zales(PI 11 true) store when you heard a crash and turned around to look, is that right?

A: Yeah, Finnegan and I were both really startled, and we ran backwards a little bit just as Mr. Collins's car hit the tree. (PI 12 false)

Q: I see. Thank you very much, Ms. Jobin.

Plaintiff's Witness Brock Collins, Direct Examination by Seth Blum, Attorney for the Plaintiff

Seth Blum, Attorney for the Plaintiff (Questioner): Hello there, could you please state your name, occupation, and city of residence for the record? 
Brock Collins (Answerer): My name is Brock Collins, I'm a teacher at Windy Hill Middle School here in Clermont where I live.

Q: And just so we're clear, what is your relationship with the plaintiff in this case, Amanda Johnson?

A: She's my girlfriend. We've been dating for a couple of years.

Q: And could you tell us a little bit about what happened on the afternoon of Tuesday, July $8^{\text {th }}, 2008$ ?

A: Well, Amanda and I were on our way to see a matinee before going out to dinner with her folks later on, when we got into a big wreck at the corner of Windsor and Foothill.

Q: Can you tell us about what you remember happening before the accident and how it all unfolded?

A: Well, I was driving my car, a blue 2006 (PI 9-year true)Toyota Camry (PI 5-model true)four door, northbound on Foothill Drive, and Amanda was in the passenger seat. We were coming around the bend, right where Foothill goes from kind of east/west to north/south, and once I got around the bend approaching the intersection, we had the green light, (PI 15 true)and I saw a semi truck skidding out of control towards us.

Q: Sounds pretty scary. Can you describe how you reacted to the situation and what happened next?

A: Yeah, it was really crazy to see a big truck barreling down on us like that. It took me a second to figure out what to do because I couldn't tell which way the truck was going to go or what was going to happen next. I kind of slowly turned to my left, to go west on Windsor because I was pretty sure that the truck was going to end up going to my right, east on Windsor. Once I could tell for sure that that's where he was headed, I was able to pull out of his way just barely fast enough to miss the end of his tanker trailer whipping around the corner behind him. It was a real relief to miss it, but the relief was very short lived.

\section{Q: How so?}

A: Well, the guy behind me in the white (PI 10 color true)Ford panel van wasn't as lucky. I guess he tried to get out of the way of the swinging trailer much the same way as I did, but he just ended up rear-ending us pretty much straight on, and our heads hit the windshield, and we then crashed into the street lamp (PI 12 true) on the northwest corner of the intersection, all pretty much at the same time as his van was getting hit by the trailer. I got dinged on the head pretty good but wasn't that messed up, no permanent scars or damage or anything, but Amanda was really hurt badly. 
Q: She certainly was. I'm really sorry you guys had to go through all of that. Thank you, Mr. Collins. I don't have any more questions for you.

Plaintiff's Witness Brock Collins, Cross Examination by Howard Kurtz, Attorney for the Defendant

Howard Kurtz, Attorney for the Defendant (Questioner): So, Mr. Collins, are you and Ms. Johnson still together?

Brock Collins (Answerer): Yes, we are. We're starting to talk about marriage, in fact.

Q: Oh, how nice! Let's talk a little more about your car that you were driving that day. It was a blue 2006 (PI 9-year true) four-door Toyota Camry (PI 5-model true), isn't that right?

A: Yes sir.

Q: And how long had you owned that car, Mr. Collins?

A: I had it for a little less than a year before the accident, but my dad bought it brand new, and when he got tired of it after a year or so (PI 9-year true), he sold it to me at a good price since I was in need of a reliable car.

Q: So you're familiar with the entire two-year (PI 9-year true) history of the vehicle. Tell me, Mr. Collins, had the car ever been in any sort of accident before?

A: Except for maybe tapping a bumper when parallel parking in a tight spot or something tiny like that, no, it hadn't.

Q: It worked okay, pretty reliable car?

A: Certainly. Wasn't too flashy, but it was definitely reliable.

Q: And did the seatbelts in the car work properly?

A: Well, they got stuck when pulling them out a lot, so it was kind of a pain to put them on all the time.

Q: And were you wearing your seatbelt on the day of the accident?

A: No, I wasn't.

Q: Why not? 
A: We weren't going very far or anything, so I guess I just figured I didn't need to mess with it.

Q: And was Ms. Johnson wearing her seat belt that day?

A: No.

Q: Nothing further, Your Honor.

Plaintiff's Witness Dr. Matthew Freeman, Direct Examination by Seth Blum, Attorney for the Plaintiff

Seth Blum, Attorney for the Plaintiff (Questioner): Hello, could you please give us your name, city of residence, and occupation?

Dr. Matthew Freeman (Answerer): My name is Matthew Freeman, I live in Jacksonville, Florida, and I am an accident reconstruction specialist.

Q: What exactly does that mean?

A: I examine vehicles, accident sites, traffic patterns, road conditions, medical records, and that sort of thing in order to figure out exactly how an accident occurred.

Q: Thank you. And, just to avoid any confusion, you are being paid to discuss your findings with us here today, isn't that right?

A: Yes, I'm being paid by the plaintiff's side to share my conclusions. What I do is complex and time consuming, and after all, this is my job. But I assure you that my determinations are in no way shaped by anything besides the evidence.

Q: I appreciate your candor and your earnestness, Dr. Freeman. So can you tell us what you found with regard to the accident involving Mr. Whitaker's Shell (PI 4/S\&B 6 true) tanker truck, Mr. Collins's Camry (PI 5-model true) and Mr. McGraw's Ford Econoline work van?

A: Given where we know the truck stopped just beyond the intersection itself, Mr. Whitaker couldn't have been going very fast at the time his trailer sideswiped Mr. McGraw's van. That means the impact from the trailer onto Mr. McGraw's van wasn't the determining factor in Ms. Johnson's injuries, but rather Mr. McGraw's driving itself was. If Mr. McGraw hadn't been driving so fast when the truck hit him, then Amanda wouldn't have been injured when he hit Mr. Collins's car. Besides, if he had been going slower, he wouldn't have been in the intersection anyway and the accident wouldn't have happened at all. 
Q: Interesting. And what about your conclusions based on inspecting Mr. Collins's vehicle?

A: Well, it was a 1986 (PI 9-year false) Toyota Camry, (PI 5-model true) four doors, blue, base model, and no aftermarket or custom modifications. It was damaged uniformly across the rear from Mr. McGraw's van hitting it, the front was damaged more deeply, but in a narrower span, consistent with hitting a tall, thin object like a street lamp pole (PI 12 true), and while the seat belts appeared to be in reasonable working order, I suspect that the airbag deployment sensors may have been faulty. For as hard as Mr. McGraw hit the car, the airbags should have inflated. But because they didn't, it is my opinion that Ms. Johnson broke her right hip as a direct result of that first impact with Mr. McGraw to their rear, not the second impact with the street lamp post. (PI 12 true) Unfortunately, due to a miscommunication between Mr. Collins's insurance company and the salvage yard where the car was being stored after the accident, shortly after I had done my preliminary inspection of the car, it was disassembled for parts before I had a chance to check the status of the airbag sensors to verify that they were faulty.

Q: Thank you for your expertise, Dr. Freeman. I have nothing further to ask.

Plaintiff's Witness Dr. Matthew Freeman, Cross Examination by Howard Kurtz, Attorney for the Defendant

Howard Kurtz, Attorney for the Defendant (Questioner): Tell me, Dr. Freeman, do you have any medical training or licensure?

Dr. Matthew Freeman (Answerer): No, I hold a doctorate degree in mechanical engineering, as well as numerous other certifications related to my field, but none in health-related areas, no.

Q: And yet you're willing to testify under oath that Amanda's broken hip was a result of the first impact, when my client hit Mr. Collins's car, and not that it was from the headon collision with the lamp pole. (PI 12 true)Very interesting conclusions, Dr. Freeman. No further questions.

Plaintiff's Witness Amanda Johnson, Direct Examination by Seth Blum, Attorney for the Plaintiff

Seth Blum, Attorney for the Plaintiff (Questioner): Hello, Ms. Johnson. How are you doing today?

Amanda Johnson (Answerer): Oh, just fine. It feels good to finally have my day in court. 
Q: I bet it does. How is your hip?

A: It's healed up pretty well so far, but it's not back to normal by any means. I'm not sure whether it will ever be like it was before the accident. Still, I'm finally able to walk without a limp or the use of a cane, but I can't run or do anything strenuous, and it really acts up during changes in barometric pressure and other weather changes. I feel like an old person, and I'm only 28.

Q: How has your injury affected your life?

A: Well, at first, it cost me my job, since I was in the hospital for a couple of weeks at first and then unable to walk for a long time after that, and it's pretty difficult to be a waitress if you can't walk.

Q: Can you tell us a little more about your injury and the treatment you received?

A: Sure. My right (PI 6 true) hip was broken just under the ball of my femur, the long bone at the top of your leg. It required immediate surgery and a metal plate and a bunch of screws to be screwed into both halves of my legbone so that the two parts of the bone would grow back together again. That surgery left me in the hospital for about a week, and I was under pretty heavy sedation for the pain, and then I had to stay in the rehabilitation center for another week before I was able to go home. I was in the wheelchair for another month before I was able to use a walker or crutches, and then it was another six weeks before I was able to walk short distances again. That whole time I was going to rehab three times a week and doing exercises every day at home too.

Q: And how are you doing today, a little over two years since the accident occurred?

A: I'm doing alright. Like I mentioned earlier, I can walk pretty much without a limp, but I still can't do anything really active or athletic that requires running around or anything like that. I'm able to sleep though the night okay now, and the pain doesn't bother me all that often except when the weather is about to change, which, unfortunately for me, seems to happen all too often here in central Florida. The doctors tell me that's never going to go away.

Q: Well, I guess at least you'll always know whether to bring an umbrella with you when you leave the house in the mornings. Now let's talk a little bit about the accident itself.

A: Okay.

Q: When you first saw the truck, where was it in relation to the car you were in?

A: It was directly in front of us, a little to the right of the center of our car. 
Q: Was the truck over the double yellow lines in the road that separate the two directions of travel?

A: Yes.

Q: In your estimation, how far over the double yellow lines?

A: It was about three-quarters of the way (PI 2/S\&B 3 true element of contradiction) over into our lanes of traffic, so far that I didn't think we'd be able to swerve around it.

Q: And did the truck appear to swerve or deviate from its course at any time before the accident?

A: Yes, it did, it swerved to our right and ended up going off to our right, to the east. (PI 3/S\&B 3 true element of contradiction)

Q: About how fast would you guess that you guys were going?

A: Not that fast. Probably 25 miles an hour or so.

Q: And at what speed would you estimate that the truck was going?

A: It had to have been over 40 miles per hour. We had slowed down, but it still came towards us very quickly and all we could see was the giant Shell (PI 4/S\&B 6 true element of contradiction) tanker trailer swerving towards us.

Q: And then what happened?

A: Brock sort of hesitated for a quick second to figure out which direction was the safest way to get out of the way of the truck since we didn't really know where it was going to end up, and after he quickly turned to the left, to go west down Windsor, we saw the tail end of the trailer go sliding past us and we thought we had managed to escape an accident.

Q: But that wasn't the case, was it?

A: No, even though we barely missed colliding with the tanker, we were then suddenly hit from behind by Mr. McGraw, who had himself been hit on his driver's (PI 1/S\&B 1 false) side by the trailer and knocked into us, and that's when I went flying forward, hitting my head on the windshield and smashing into the dashboard and breaking my right (PI 6 true) hip, and then we hit the street lamp post (PI 12 true) which caused us to finally come to a stop.

Q: So you were fine up until the point when Mr. McGraw crashed into you, is that right? 
A: Yeah, if he didn't hit us, I wouldn't have been hurt.

Q: Thank you, Ms. Johnson.

Plaintiff's Witness Amanda Johnson, Cross Examination by Howard Kurtz, Attorney for the Defendant

Howard Kurtz, Attorney for the Defendant (Questioner): Ms. Johnson, I'd like to ask you a few more questions about the events leading up to the accident and the details of the accident itself, if that's okay.

Amanda Johnson (Answerer): Of course.

Q: Ms. Johnson, could you please tell us exactly when you first noticed that the truck was on the wrong side of the road?

A: Before we got to the intersection, I could see that it was on the wrong side of the road. I could see the paint of the Exxon (PI 4/S\&B 6 false element of contradiction) logo and the chrome tank shining in the sun.

Q: And you are sure you could tell that the truck was on the wrong side of the road?

A: Yes, I'm sure.

Q: Ms. Johnson, if you saw the truck was on the wrong side of the road, why didn't you warn Mr. Collins?

A: I don't know. It happened very quickly. It was almost half way (PI 2/S\&B 3 false statement of contradiction) over onto our side of the road, swerving to our left. (PI 3/S\&B 3 false element of contradiction)

Q: Hmm. I see. Thank you, Ms. Johnson. No further questions.

Seth Blum, Attorney for the Plaintiff: Your Honor, we have no further witnesses to introduce, so the plaintiff rests.

Judge Robert C. Underwood: Thank you Mr. Blum. Mr. Kurtz, you may now present the defense's case.

Howard Kurtz, Attorney for the Defendant: Thank you, Your Honor. 
Defense Witness Tom Gorham, Direct Examination by Howard Kurtz, Attorney for the Defendant

Howard Kurtz, Attorney for the Defendant (Questioner): Hello, could you please state your name, city of residence, and occupation for the record?

Tom Gorham (Answerer): My name is Tom Gorham, I live in Orlando, and my wife and I own and operate a three-dimensional film and photography studio.

Q: How interesting! That must be a really exciting business for you two to be in. Now, could you please tell us where you were on the afternoon of Tuesday, July $8^{\text {th }}, 2008 ?$

A: I was stopped at the light (PI 15 true) at the corner of Windsor and Foothill, waiting to cross Foothill to the east, and I saw this whole big accident unfold.

Q: Can you tell us exactly what you saw?

A: Well, I first knew that something was wrong when I saw Mr. Collins's blue Toyota Camry (PI 5-model true) flashing the lights and heard him laying on the horn as he came up to the intersection from the south. Very shortly after that, I saw the Shell (PI 4/S\&B 6 true) tanker truck come skidding and fishtailing into the intersection from the north. He was pretty much swerving to his left, (PI 3/S\&B 3 true) away from me, towards the opposite side of the intersection from where I was. I was on the west side, waiting to cross Foothill and head east, and he was swerving over that way with the trailer kind of swinging out behind him.

Q: Okay, so after you saw the two vehicles both entering the intersection, what happened next?

A: I saw Mr. Collins's car skirt around the trailer pretty much right in front of my car, when the trailer kind of swung around to its right, as the cab of the truck was making a hard left turn, and the trailer sort of swiped the passenger side (PI 1/S\&B 1 true) of the white (PI 10 color true)van. Right after that, the van rear-ended the Camry, (PI 5-model true) and then the Camry (PI 5-model true) went and ran into the streetlamp (PI 12 true).

Q: And how good of a look did you get at this whole sequence of events?

A: I had a perfect view, pretty much a front-row seat to the whole thing.

Q: So did you see either Mr. Collins's or Ms. Johnson's heads strike the windshield of their car?

A: Definitely.

Q: And when did you see that happen? 
A: Right after their car crashed into the lamp post. (PI 12 true)

Q: Right. Now what happened next?

A: Well, I saw the guy with the black hair, (PI 7 true) McGraw, get out of the van right away and go over to the driver's side of Collins's car and it looked like he was checking to see if everyone was okay or to see how he could help. Collins got out of the car shortly after that, and while his head was bleeding a little bit, and he seemed a little dazed, he didn't really seem too badly hurt.

Q: Alright. Anything else?

A: Yeah, I stayed around to see if there was anything I could do, but the cops showed up and pretty much got it all under control and then the ambulance got there (PI 8 true) and I watched them take Ms. Johnson out of the car. It looked like her right leg (PI 6 true) was all messed up, and they loaded her and Mr. Collins into the ambulance and drove off.

Q: Thank you, Mr. Gorham. I have no further questions.

Defense Witness Tom Gorham, Cross Examination by Seth Blum, Attorney for the Plaintiff

Seth Blum, Attorney for the Plaintiff (Questioner): Mr. Gorham, you stated that you saw Mr. Collins's car flashing its lights and heard it honking its horn as it entered the intersection from the south, and that after that, you saw the Shell (PI 4/S\&B 6 true) truck come skidding and screeching into the intersection. Now if Mr. Collins was honking and flashing his lights and everything, obviously in response to something, doesn't it seem strange to you that you didn't notice what he was reacting to before you noticed him?

Tom Gorham (Answerer): Well, maybe, I guess. I think I probably just noticed Mr. Collins's car first because he was the one that was right in front of me, and since the truck was all the way on the other side of the intersection, further away, maybe I didn't see it first for that reason. Or maybe the angle of everything made the Zales(PI 11 true)store sort of block my line of sight. I don't know really know why I saw things when I saw them, I just know what I saw.

Q: If you say so. Nothing more, Your Honor.

Defense Witness John Kindt, Direct Examination by Howard Kurtz, Attorney for the Defendant 
Howard Kurtz, Attorney for the Defendant (Questioner): Hello there, could you please state your name and occupation for the record?

John Kindt (Answerer): My name is John Kindt, and I am the security guard for the Zales(PI 11 true) jewelry store located on the northwest corner of the intersection of Foothill and Windsor Road in Clermont.

Q: Can you tell us where you were on the afternoon of Tuesday, July $8^{\text {th }}, 2008$ ?

A: Yeah, I was at work.

Q: Tell me, did you happen to witness the events at issue in this case?

A: I saw the tail end of it. I saw Mr. Collins's car almost run over the lady out walking her German Shepherd. (PI 13 true) She was walking on the sidewalk and nearly got creamed.

Q: Lucky thing for her that she didn't. So what else did you see then?

A: I saw the Shell (PI 4/S\&B 6 true) trailer kind of smack the passenger side (PI 1/S\&B 1 true) of Mr. McGraw's van and knock it into Mr. Collins's car.

Q: And did you see the consequences of the collision between the two vehicles?

A: Well, yeah. I saw Mr. Collins's car come crashing towards the store, and that lady and her dog, and hit the street lamp (PI 12 true) in front of our store.

Q: And did you see anybody get hurt?

A: Yeah, I saw Mr. Collins's head and Ms. Johnson's head smash into their windshield when they hit the lamp post. I was kind of surprised that the airbags didn't go off, but I guess they weren't going fast enough or something.

Q: And what else did you see?

A: Not much. I got a call about security issues for an upcoming delivery so I had to go look at some paperwork, and by the time I was through with that, the show was over.

Q: Thank you, Mr. Kindt. I have no more questions.

Defense Witness John Kindt, Cross Examination by Seth Blum, Attorney for the Plaintiff Seth Blum, Attorney for the Plaintiff: I have no questions for this witness, Your Honor. 
Defense Witness Dr. Erik Dubberke, Direct Examination by Howard Kurtz, Attorney for the Defendant

Howard Kurtz, Attorney for the Defendant (Questioner): Hello there, could you please tell us your name and occupation?

Dr. Erik Dubberke (Answerer): My name is Erik Dubberke, and I am a trauma surgeon at South Lake Hospital here in Clermont.

Q: That's an emergency room doctor, right?

A: Yes, I'm one of the several surgeons on staff that are trained specifically for traumarelated procedures. Injuries, accident victims, things like that. Immediate and sometimes life-or-death repairs, as opposed to planned, preventative, or purely cosmetic procedures.

Q: Ahh. I see. Tell me, Dr. Dubberke, were you on duty on Tuesday, July $8^{\text {th }}, 2008$ ?

A: Yes, I was on call from noon until midnight that day.

Q: So, Dr. Dubberke, did you treat Ms. Amanda Johnson on that afternoon?

A: Yes I did. She was brought in by ambulance, and the paramedics had stabilized her condition, but it was pretty clear to them by the time that they arrived that she had suffered some sort of localized trauma to her right (PI 6 true) leg or hip area.

Q: And was that consistent with your observations?

A: Absolutely. We took some X-rays to see exactly what we were dealing with right after we cleaned up and stitched up her forehead. Luckily, her facial lacerations were superficial enough that I was able to use dissolvable sutures which tend to leave little or no scarring, but they don't work as well on really deep or difficult wounds.

Q: Thank heaven for small miracles. Now what did the X-rays reveal?

A: Ms. Johnson suffered a broken hip up near the ball in the joint where the right (PI 6 true) leg meets the pelvis. It's a very nasty sort of injury.

Q: So how did you treat Ms. Johnson?

A: I inserted a metal plate into her right (PI 6 true) leg that is held in place by five metal screws that are screwed directly into her bones. By holding everything in place like that, it allows for the bone to gradually fuse back together at the site of the break. 
Q: Sounds reasonable. Tell me, Dr. Dubberke, in your experience with trauma victims, are you at all able to judge the severity of accidents or make any determination about what caused or maybe even what could have prevented an injury?

A: To an extent. If what you're asking me is whether having worn her seat belt would have prevented Ms. Johnson's injury, then yes. I'm almost certain that her broken hip resulted from being thrown forward in a frontal crash.

Q: Not from having been rear-ended?

A: No, that type of impact typically results in whiplash and soft-tissue injuries, not being launched forward into the dashboard.

Q: Thank you, Dr. Dubberke. I don't have any more questions.

Defense Witness Dr. Erik Dubberke, Cross Examination by Seth Blum, Attorney for the Plaintiff

Seth Blum, Attorney for the Plaintiff (Questioner): Dr. Dubberke, how long have you been working in the emergency room?

Dr. Erik Dubberke (Answerer): In my current capacity? Almost eight years. Counting assisting, residency, interning, volunteering, and all that? Probably another four, five years total on top of that.

Q: That's quite a long time. You must have seen some pretty remarkable, unusual, and awful things in that time.

A: Yeah, but you get used to it. If you can't, you don't last long.

Q: I bet. Tell me, have you ever seen anything that just defied explanation, or didn't make any sense, or just plain freak accidents or weird flukes?

A: Sure. The human body is a very complicated, complex organism, with an almost limitless capacity for things to go wrong. And the world can be a pretty strange place too. Just when you start to think you've seen it all, something you could have never envisioned in a hundred years comes through the doors.

Q: I can only imagine. Thank you for all the good work you do, Doctor. Nothing further. 
Defense Witness Officer Christopher Eaton, Direct Examination by Howard Kurtz, Attorney for the Defendant

Howard Kurtz, Attorney for the Defendant (Questioner): Good afternoon, Officer. Could you please give us your name, rank, department, and division?

Officer Christopher Eaton (Answerer): My name is Christopher Eaton, I'm a deputy with the Clermont Police Department, and I'm primarily assigned to the Patrol Section.

Q: Okay. And is the intersection of Foothill and Windsor in Clermont part of your assigned beat?

A: Yes, sir. It's right smack dab in the middle of it, in fact.

Q: And is that where you were on the afternoon of Tuesday, July $8^{\text {th }}, 2008$ ?

A: I was patrolling not too far away when I heard the call on the radio about an automobile accident which also potentially involved a gasoline truck. Mr. McGraw had called 911 pretty quickly, and it only took me a minute or two to get there, and I was the first responder on the scene (PI 8 true).

Q: And what did you see when you arrived?

A: I observed Mr. Whitaker's Shell (PI 4/S\&B 6 true) gasoline tanker truck stopped in the rightmost lane, on the south side of Windsor Road, in the eastbound direction. To the west of the intersection, on the north side of Windsor Road, in the westbound direction, I observed Mr. Collins's blue 2006 (PI 9-year true) Toyota Camry (PI 5-model true) at rest up with the front up against the lamp post, (PI 12 true) and Mr. McGraw's white (PI 10 color true)2000 Ford Econoline work van smashed up into the rear of Mr. Collins's car. Mr. McGraw's van had sustained additional damage to the rear passenger quarter panel area (PI 1/S\&B 1 true), and Mr. Collins's car had a broken windshield in the typical pattern found when the windshield is struck by the head of the occupants of the front seat. In this case, that would be Mr. Collins and Ms. Johnson.

Q: And you've seen a lot of car accidents during your time on the force, I imagine?

A: Yeah, that's probably one of the most frequent calls we get. Hardly a day goes by that I don't deal with at least one wreck

Q: I bet. And in your experience, when you've seen patients with head trauma and broken bones, injured in cars that end up with cracked windshields like Mr. Collins's car, do those accidents typically involve a car that has been rear-ended? 
A: Well, I've never actually kept a count of something like that, but off the top of my head, I'd say that very few of them do, except for the sorts of multi-impact incidents like we had here.

Q: How do you mean?

A: Usually, passengers in cars who have cuts to their head and broken bones suffer their injuries as a result of having been forcefully thrown forward from the violence of a frontal impact. You know, running into something, not being run into from behind.

Q: Okay, well thank you very much for your assistance, Officer. I have no additional questions.

A: My pleasure, sir.

Defense Witness Officer Christopher Eaton, Cross Examination by Seth Blum, Attorney for the Plaintiff

Seth Blum, Attorney for the Plaintiff (Questioner): Officer Eaton, you've undoubtedly seen plenty of car accidents in your time patrolling, haven't you? Accidents of all kinds?

Officer Christopher Eaton (Answerer): Yes, sir. I couldn't even offer a guess as to the total number.

Q: And in your eyes, was this your typical rear-ending?

A: No, sir. Most rear-ending accidents do not involve an impact from a third vehicle like this incident.

Q: So this was a little out of the ordinary for how most rear-impact collisions occur?

A: Yes, sir. I'm not sure whether I can recall anything quite like this in all my years on the force where a semi trailer smacks into one vehicle which then crashes into another in a chain reaction like that. This was the result of some pretty unlikely combinations of forces and events.

Q: I'll say. Thank you, Officer.

A: My pleasure, sir. 


\section{APPENDIX E}

List and Description of all 15 Inconsistencies from Study One

\section{List of all Inconsistencies:}

PI 1-Side of Car: Which side of McGraw's van is damaged (passenger is correct; Amanda says driver's) (S\&B 1)

PI 2-1/2 vs 3/4: Truck was either $3 / 4$ (true) or $1 / 2$ (false-Amanda) across center line (S\&B 3)

PI 3-Swerve: Direction truck swerved-Amanda says to her Right (true) and Left (false) (S\&B 3)

PI 4-Oil Co.: Oil company name-Shell is true, Amanda says Exxon (S\&B 6)

PI 5-Model: Zollinger says Collins drives a blue Corvette (wrong), he really drives a blue Camry

PI 6-R/L leg: Powell says Amanda's left leg is hurt (correct leg is right leg)

PI 7-Hair: Jill says McGraw has red hair (false-he really has black hair)

PI 8-Arrival: Powell incorrectly says ambulance arrived before cops; reverse is correct

PI 9-Year: Freeman says Collins drives a 1986 Camry (false) when it's really a 2006

PI 10-Color: Barbara says McGraw's van is tan, it's really white

PI 11-Jared: Lesley says it's a Jared jewelry store (false); it's really a Zales

PI 12-Tree: Lesley says Collins crashes into a tree (false); it's really a light pole

PI 13-Dog: Zollinger says Lesley's dog is a dachshund (false); it's really a German

Shepherd

PI 14-Cop: Cop is a man; Barbara says it's a woman (false)

PI 15-Stop Sign: Jill says intersection has a stop sign (false); it's really a light

Key:

- PI is the number I have assigned to that particular inconsistency.

- S\&B indicates that Semmler and Brewer used this inconsistency in their trial transcripts. Note: Their \#3 inconsistency included two separate inconsistent components, how far across the road the swerving truck was prior to the accident, and which direction the truck swerved prior to the accident. I disentangled them for ease of use. 
Table E1

Fifteen Inconsistencies and Five Dummy Questions Used in Study One Trial Transcript

\begin{tabular}{|c|c|c|c|}
\hline Number & Summary & $\begin{array}{l}\text { Mistaken EW } \\
\& \text { their total \# }\end{array}$ & $\begin{array}{l}\text { Broad Type of } \\
\text { Incon }\end{array}$ \\
\hline $\begin{array}{l}\text { PI 1-Side of } \\
\text { Car: }\end{array}$ & $\begin{array}{l}\text { Which side of McGraw's van is damaged } \\
\text { (passenger is correct; Amanda says } \\
\text { driver's) }\end{array}$ & Amanda (4) & $\mathrm{L} / \mathrm{R}$ or directions \\
\hline $\begin{array}{l}\text { PI } 2-1 / 2 \text { vs } \\
3 / 4:\end{array}$ & $\begin{array}{l}\text { Truck was either } 3 / 4 \text { (true) or } 1 / 2 \text { (false- } \\
\text { Amanda) across center line }\end{array}$ & Amanda (4) & $\begin{array}{l}\text { Description of } \\
\text { events }\end{array}$ \\
\hline PI 3-Swerve: & $\begin{array}{l}\text { Direction truck swerved-Amanda says to } \\
\text { her Right (true) and Left (false) }\end{array}$ & Amanda (4) & $\begin{array}{l}\text { L/R or directions; } \\
* * * \mathbf{5 0} / \mathbf{5 0}\end{array}$ \\
\hline PI 4-Oil Co.: & $\begin{array}{l}\text { Oil company name-Shell is true, Amanda } \\
\text { says Exxon }\end{array}$ & Amanda (4) & Brand Names \\
\hline PI 5-Model: & $\begin{array}{l}\text { Zollinger says Collins drives a blue } \\
\text { Corvette (wrong), he really drives a blue } \\
\text { Camry }\end{array}$ & Zollinger (2) & Brand Names \\
\hline PI 6-R/L leg: & $\begin{array}{l}\text { Powell says Amanda's left leg is hurt } \\
\text { (correct leg is right leg) }\end{array}$ & Powell (2) & $\begin{array}{l}\text { L/R or directions; } \\
\text { Specific } \\
\text { descriptions of } \\
\text { people; } * * * \mathbf{5 0} / \mathbf{5 0}\end{array}$ \\
\hline PI 7-Hair: & $\begin{array}{l}\text { Jill says McGraw has red hair (false-he } \\
\text { really has black hair) }\end{array}$ & Jill (2) & $\begin{array}{l}\text { Color; Specific } \\
\text { descriptions of } \\
\text { people }\end{array}$ \\
\hline PI 8-Arrival: & $\begin{array}{l}\text { Powell incorrectly says ambulance } \\
\text { arrived before cops; reverse is correct }\end{array}$ & Powell (2) & $\begin{array}{l}\text { Order of events; } \\
* * * \mathbf{5 0 / 5 0}\end{array}$ \\
\hline PI 9-Year: & $\begin{array}{l}\text { Freeman says Collins drives a } 1986 \\
\text { Camry (false) when it's really a } 2006\end{array}$ & Freeman (1) & $\begin{array}{l}\text { Specific } \\
\text { descriptions of } \\
\text { objects }\end{array}$ \\
\hline PI 10-Color: & $\begin{array}{l}\text { Barbara says McGraw's van is tan, it's } \\
\text { really white }\end{array}$ & Barbara (2) & Color \\
\hline PI 11-Jared: & $\begin{array}{l}\text { Lesley says it's a Jared jewelry store } \\
\text { (false); it's really a Zales }\end{array}$ & Lesley (2) & Brand Names \\
\hline PI 12-Tree: & $\begin{array}{l}\text { Lesley says Collins crashes into a tree } \\
\text { (false); it's really a light pole }\end{array}$ & Lesley (2) & $\begin{array}{l}\text { Specific } \\
\text { descriptions of } \\
\text { objects; Physical } \\
\text { Layout }\end{array}$ \\
\hline PI 13-Dog: & $\begin{array}{l}\text { Zollinger says Lesley's dog is a } \\
\text { dachshund (false); it's really a German } \\
\text { Shepherd }\end{array}$ & Zollinger (2) & $\begin{array}{l}\text { Specific } \\
\text { descriptions of } \\
\text { objects }\end{array}$ \\
\hline PI 14-Cop: & $\begin{array}{l}\text { Cop is a man; Barbara says it's a woman } \\
\text { (false) }\end{array}$ & Barbara (2) & $\begin{array}{l}\text { Specific } \\
\text { descriptions of } \\
\text { people; } * * * \mathbf{5 0} / \mathbf{5 0}\end{array}$ \\
\hline $\begin{array}{l}\text { PI 15-Stop } \\
\text { Sign: }\end{array}$ & $\begin{array}{l}\text { Jill says intersection has a stop sign } \\
\text { (false); it's really a light }\end{array}$ & Jill (2) & $\begin{array}{l}\text { Specific } \\
\text { descriptions of } \\
\text { objects; Physical } \\
\text { Layout }\end{array}$ \\
\hline
\end{tabular}




\begin{tabular}{|l|l|l|l|}
\hline Dummy 1 & $\begin{array}{l}\text { Amanda was a waitress at the time of } \\
\text { accident }\end{array}$ & & \\
\hline Dummy 2 & McGraw called 911 & & \\
\hline Dummy 3 & $\begin{array}{l}\text { Brock \& Amanda were on their way to } \\
\text { see a movie }\end{array}$ & & \\
\hline Dummy 4 & The airbags in Brock's car didn't go off & & \\
\hline Dummy 5 & Amanda had a metal plate put in her leg & & \\
\hline
\end{tabular}




\section{APPENDIX F}

Study One Inconsistency Detection Questions

Note: The parenthetical (PI 1/S\&B 1, etc.) information at the end of each question was not present in the actual materials. It is included here for the sake of explanation and reference to assist in identifying which inconsistency or inconsistencies form the basis for the question. Please see the List and Descriptions of All 15 Inconsistencies from Study One (Appendix E) for a brief description of the nature of each inconsistency. Also, for the forced-choice questions (Multiple Choice, True/False, and Point Blank), the correct answer is included here for reference purposes.

All information which is presented here for background or clarification but which was not visible to participants is italicized.

\section{Open-Ended Questions:}

The open-ended question section featured one primary question and one followup “...if so, who/how?" elaboration per page, presented in random order. Because the open-ended questions are so general, many of them apply to more than one inconsistency.

The following instructions were listed at the top of each page:

There are lots of witnesses and lots of evidence in trials. Sometimes testimony is consistent, sometimes it's contradictory, sometimes different witnesses disagree, and sometimes accounts vary, etc. Please list all possible answers for the following question, even those which are contradictory to each other.

\section{Questions (Eight Primary and Eight Followup):}

Were there any eyewitnesses that testified who you think might have made mistakes concerning the difference between directions, or the difference left and right? (PI $1 / S \& B$ 1; PI 3/S\&B 3; PI 6)

If so, who and how? If you don't remember exactly which witness(es) might have made this type of mistake, please just describe the mistake(s) to the best of your ability.

Were there any eyewitnesses that testified who you think might have been mistaken or incorrect regarding the order of events as they occurred? (PI 8)

If so, who and how so? If you don't remember exactly which witness(es) might have made this type of mistake, please just describe the mistake(s) to the best of your ability. 
Were there any eyewitnesses that testified who you think might have made mistakes in terms of brand names? (PI 4/S\&B 6; PI 5-model; PI 11)

If so, who and how? If you don't remember exactly which witness(es) might have made this type of mistake, please just describe the mistake(s) to the best of your ability.

Were there any eyewitnesses that testified who you think might have made mistakes in terms of describing various colors? (PI 7; PI 10-color)

If so, who and how? If you don't remember exactly which witness(es) might have made this type of mistake, please just describe the mistake(s) to the best of your ability.

Were there any eyewitnesses that testified who you think might have made mistakes in terms of describing the physical layout of the intersection? (PI 12; PI 15)

If so, who and how? If you don't remember exactly which witness(es) might have made this type of mistake, please just describe the mistake(s) to the best of your ability.

Were there any eyewitnesses that testified who you think might have made mistakes in terms of describing the exact nature of events related to the accident? (PI $2 / S \& B$ 3)

If so, who and how? If you don't remember exactly which witness(es) might have made this type of mistake, please just describe the mistake(s) to the best of your ability.

Were there any eyewitnesses that testified who you think might have made mistakes in terms of specific physical descriptions of people? (PI 6; PI 7; PI 14)

If so, who and how? If you don't remember exactly which witness(es) might have made this type of mistake, please just describe the mistake(s) to the best of your ability.

Were there any eyewitnesses that testified who you think might have made mistakes in terms of specific physical descriptions of objects? (PI 9-year; PI 12; PI 13; PI 15)

If so, who and how? If you don't remember exactly which witness(es) might have made this type of mistake, please just describe the mistake(s) to the best of your ability.

\section{Short Answer Questions:}


The short answer question section featured one question per page, presented in random order.

The following instructions were listed at the top of each page:

There are lots of witnesses and lots of evidence in trials. Sometimes testimony is consistent, sometimes it's contradictory, sometimes different witnesses disagree, and sometimes accounts vary.

Please list all possible answers for the following question, even those which are contradictory to each other.

If you do not know the answer, please type "I don't know." and then click Submit to proceed to the next question.

Questions (20 total-one for each of the 15 inconsistencies, and five "dummy" questions to provide opportunities for participants to give answers that do not involve inconsistent testimony in order to camouflage the intent of the study while also acting as a manipulation check by probing their general understanding of the trial transcript):

Michael McGraw's van was damaged in the accident. Please describe the testimony concerning exactly how and where Michael McGraw's van was damaged. Make sure you talk about all of the witnesses who testified about the damage to Michael McGraw's van. (PI $1 / S \& B$ 1)

Testimony was given stating that the gasoline tanker truck had crossed over the center line of Foothill Dr. Please describe the testimony concerning where and how far the truck was across the center line. Make sure you talk about all of the witnesses who testified about the truck crossing the center line. (PI 2/S\&B 3)

Testimony was given stating that the gasoline tanker truck swerved in the moments just before the accident occurred. Please describe the testimony concerning where and in what direction the truck swerved. Make sure you talk about all of the witnesses who testified about the truck swerving. (PI $3 / S \& B$ 3)

Several witnesses mentioned the oil company logo on the gasoline tanker trailer. Please describe the testimony concerning the oil company. Make sure you talk about all of the witnesses who testified about the oil company that owned the truck. (PI 4/S\&B 6) 
Several witnesses described the make and model of the vehicles involved in the accident. Please describe the testimony concerning the kinds of vehicles. Make sure you talk about all of the witnesses who testified about the make and model of the vehicles. (PI 5-model)

Amanda Johnson broke one of her legs. Describe this injury. Make sure you talk about all of the witnesses who testified about her leg injury. (PI 6)

Several witnesses described Michael McGraw by mentioning his hair color. Please describe the testimony concerning Michael McGraw's hair color. Make sure you talk about all of the witnesses who mentioned his hair color. (PI 7)

Several witnesses testified about the order in which emergency personnel responded to the accident. Please describe the testimony concerning the order of emergency personnel arrival. Make sure you talk about all of the witnesses who mentioned the order of emergency personnel arrival. (PI 8)

Several witnesses described the year of the vehicles involved in the accident. Please describe the testimony concerning the years of the vehicles. Make sure you talk about all of the witnesses who testified about the years of the vehicles. (PI 9-year)

Several witnesses described the colors of the vehicles involved in the accident. Please describe the testimony concerning the colors of the vehicles. Make sure you talk about all of the witnesses who testified about the color of each of the vehicles. (PI 10-color)

There are several businesses located near the intersection of Foothill Dr. and Windsor Rd. Please describe the testimony regarding the businesses surrounding the intersection. Make sure you talk about all of the witnesses who testified about the nearby businesses. (PI 11)

Brock Collins's car eventually came to rest following the accident. Please describe the testimony concerning how Brock Collins's car came to a stop after the crash. Make sure you talk about all of the witnesses who testified about how his car came to a stop. (PI 12)

Lesley Jobin was walking her dog Finnegan when she witnessed the accident. Please describe the testimony regarding the breed of Lesley's dog. Make sure you talk about all of the witnesses who testified about Lesley's dog. (PI 13) 
Several witnesses referred to the physical characteristics of the police officer who responded to the accident. Please describe the testimony concerning the appearance of the police officer. Make sure you talk about all of the witnesses who testified about the police officer. (PI 14)

Several witnesses described the traffic control devices, safety measures, signage, and lane markings of the intersection where the accident occurred. Please describe the testimony concerning the traffic control devices, safety measures, signage, and lane markings. Make sure you talk about all witnesses who testified about these details. (PI 15)

Several witnesses testified regarding Amanda Johnson's employment status. Please describe the testimony concerning Amanda Johnson's employment status. Make sure you talk about all witnesses who mentioned Amanda's employment. (Dummy 1)

Several witnesses mentioned details having to do with who called 911. Please describe the testimony concerning who called 911. Make sure you talk about all witnesses who testified about calling 911. (Dummy 2)

Brock Collins and Amanda Johnson had plans for the day the accident occurred. Please describe what their plans were, and what they were on the way to do. Make sure you talk about all witnesses who testified about their plans for that day. (Dummy 3)

Multiple witnesses mentioned airbags in relation to the accident. Please describe the testimony involving airbags deploying. Make sure you talk about all witnesses who referred to airbags in any of the vehicles. (Dummy 4)

Amanda Johnson required an emergency medical procedure to help her broken leg heal. Please describe what this procedure involved. Make sure you talk about all witnesses who testified about the treatment Amanda needed on her broken leg. (Dummy 5)

\section{Multiple Choice Questions:}

The multiple choice question section featured one question per page, presented in random order.

The following instructions were listed at the top of each page: 
There are lots of witnesses and lots of evidence in trials. Sometimes testimony is consistent, sometimes it's contradictory, sometimes different witnesses disagree, and sometimes accounts vary.

Please mark all possible answers for the following question, even those which are contradictory to each other.

These questions are designed to test your memory for the information in the trial testimony, not your personal opinion on what actually happened or what is correct. How you answer these questions should not depend on which witnesses you believe to have been accurate or those you believe to have been inaccurate- just the content of the testimony itself.

\section{Again, you may choose more than one answer.}

Questions (23 total-one for each of the 15 inconsistencies, five "dummy" questions, two case theme manipulation checks, and one "attention check" question for use in identifying those participants who are not reading the questions which they are responding to):

According to the plaintiff's version of events, Amanda Johnson broke her hip when: (Manipulation Check of Plaintiff's Case-B)
A. The tanker truck hit Brock's car.
B. The van driven by the defendant, Michael McGraw, hit Brock's car.
C. Brock's car hit a pole.
D. Jill Randall ran the red light and hit Brock's car.
E. None of the above
F. Don't know/don't remember

According to the defense's version of events, Amanda Johnson broke her hip when: (Manipulation Check of Defense's Case-C)
A. The tanker truck hit Brock's car.
B. The van driven by the defendant, Michael McGraw, hit Brock's car.
C. Brock's car hit a pole.
D. Jill Randall ran the red light and hit Brock's car.
E. None of the above
F. Don't know/don't remember

Michael McGraw's van was damaged in the crash. What area(s) were described as having been damaged in the accident? (PI 1- $A \& C$ )
A. The passenger side
B. The roof
C. The driver's side 
D. The rear

E. None of the above

F. Don't know/don't remember

Testimony was given stating that the gasoline tanker truck had crossed over the center line of Foothill Drive and that it was in the oncoming lane of traffic. How far across the center line was the truck, according to all testimony? (PI 2/S\&B 3-B \& $C$ )
A. One quarter of the way
B. Halfway
C. Three quarters of the way
D. All the way
E. None of the above
F. Don't know/don't remember

There was testimony regarding the tanker truck swerving prior to the accident. From the point of view of Brock Collins's car, looking head-on at the truck, according to all testimony, in what direction(s) did the truck swerve? (PI 3/S\&B 3-A\& $B$ )
A. Left
B. Right
C. Left and into a spin
D. Right and into a spin
E. None of the above
F. Don't know/don't remember

The gasoline tanker trailer featured the logo of the oil company that owned the truck. What company (or companies) were mentioned as the owner of the truck? (PI 4/S\&B 6-A \& D)
A. Exxon
B. BP
C. Chevron
D. Shell
E. None of the above
F. Don't know/don't remember

Brock Collins's car was involved in the accident. According to the trial transcript, what make and model(s) of car was Brock said to have been driving? (PI 5-model-B \& D)
A. Chevrolet Camaro
B. Toyota Camry
C. Toyota Corolla
D. Chevrolet Corvette
E. None of the above 


\section{F. Don't know/don't remember}

Amanda Johnson was injured in the accident. According to the trial transcript, what part(s) of Amanda's body was (were) injured? (PI 6- $A \& B$ )
A. Left leg
B. Right leg
C. Left arm
D. Right arm
E. None of the above
F. Don't know/don't remember

Several witnesses mentioned Michael McGraw's hair color when describing what they had seen. What color(s) of hair did witnesses say he had? (PI 7-C\&D)
A. Blond
B. Brown
C. Black
D. Red
E. None of the above
F. Don't know/don't remember

Emergency responders arrived to help with the accident scene. Which responder(s) did witnesses say arrived first? (PI 8-A \& D)
A. Police
B. Fire department
C. Search and rescue
D. Paramedics
E. None of the above
F. Don't know/don't remember

The year of Brock Collins's car is mentioned in the testimony. What year(s) is it said to be? (PI 9-year- $B \& C$ )
A. 2002
B. 2006
C. 1986
D. 1996
E. None of the above
F. Don't know/don't remember

The color of Michael McGraw's van is mentioned in the testimony. What color(s) is it said to be? (PI 10-color- $A \& B$ ) 

A. Tan
B. White
C. Blue
D. Green
E. None of the above
F. Don't know/don't remember

There is a jewelry store on the northwest corner of the intersection. What name(s) for the store was/were mentioned? (PI 11-B \& D)
A. Kay
B. Jared
C. Tiffany \& Co.
D. Zales
E. None of the above
F. Don't know/don't remember

Brock Collins's car ran into something before coming to a complete stop. According to the transcript, what object(s)? (PI 12-A\&D)
A. A tree
B. A blue USPS mailbox
C. A bus stop
D. A street light pole
E. None of the above
F. Don't know/don't remember

There was testimony given regarding the breed of Lesley Jobin's dog Finnegan. What breed(s) were given for Finnegan? (PI 13- $A \& C$ )
A. Dachshund
B. Doberman
C. German Shepherd
D. Beagle
E. None of the above
F. Don't know/don't remember

The police officer was described as which of the following? (PI 14-B \& $C$ )
A. African-American
B. A man
C. A woman
D. Small in size
E. None of the above
F. Don't know/don't remember 
The transcript referred to how the safe flow of traffic is regulated at the intersection. What method(s) were given? (PI 15-B \& D)
A. Yield signs
B. A stoplight
C. A flashing yellow light
D. A stop sign
E. None of the above
F. Don't know/don't remember

The testimony indicated that Amanda Johnson had held what job(s) prior to the accident? (Dummy 1-C)
A. Nurse
B. Teacher
C. Waitress
D. Real estate agent
E. None of the above
F. Don't know/don't remember

According to the transcript, which person (or people) called 911 to report the accident? (Dummy 2-D)
A. Brock Collins
B. Steve Powell
C. Jill Randall
D. Michael McGraw
E. None of the above
F. Don't know/don't remember

What activity or activities did Brock and Amanda have planned for the day of the accident? (Dummy 3-A\&B)
A. Going to a movie
B. Going out to dinner
C. Going to a concert
D. Going to a party
E. None of the above
F. Don't know/don't remember

The transcript included testimony concerning airbags in Brock Collins's car. Which of the following was included? (Dummy $4-B \& C$ )

A. The airbags inflated 
B. The airbags did not inflate

C. The airbags may have been defective

D. The car did not have airbags

E. None of the above

F. Don't know/don't remember

Amanda Johnson required serious medical treatment for her broken leg when she arrived at the emergency room. What procedure(s) did she need? (Dummy 5-A \& D)
A. A metal plate to be inserted
B. The bone had to be broken further in order to heal properly
C. A bone graft
D. Metal screws to be inserted
E. None of the above
F. Don't know/don't remember

Thank you for participating in this study. To ensure that you are paying attention to the questions, please mark A for this question. (Attention check-A)
A. He put the car into reverse
B. He stopped the car
C. He turned sharply to his right
D. He pulled the emergency brake
E. None of the above
F. Don't know/don't remember

\section{True/False Questions:}

The truelfalse question section featured one question per page, presented in random order.

The following instructions were listed at the top of each page:

There are lots of witnesses and lots of evidence in trials. Sometimes testimony is consistent, sometimes it's contradictory, sometimes different witnesses disagree, and sometimes accounts vary.

Please answer the following True or False questions regarding the trial transcript.

These questions are designed to test your memory for the information in the trial testimony, not your personal opinion on what actually happened or what is correct. 
How you answer these questions should not depend on which witnesses you believe to have been accurate or those you believe to have been inaccurate - just the content of the testimony itself.

Questions (21 total-one for each of the 15 inconsistencies, five dummy questions, and one attention check; True, False, and I don't know were answer choices):

There was a discrepancy in the testimony about which side of Michael McGraw's van was hit by the tanker trailer. (T; PI $1 / S \& B$ 1)

There were different estimates given for how far across the center line of the road the truck was before the accident. (T; $P I 2 / S \& B 3$ )

One witness testified that the semi truck swerved both to the right and to the left prior to the accident. (T; PI $3 / S \& B$ 3)

Only one oil company logo was identified on the tanker trailer according to the transcript. (F; PI 4/S\&B 6)

Out of all descriptions of Brock Collins's car within the trial testimony, there was no discrepancy concerning the make and model of the car. (F; PI 5-model)

There was no testimony claiming that Amanda Johnson had hurt her left leg. (F; PI 6)

Not all witnesses agreed in their testimony about what color hair Michael McGraw had. $(T ; P I 7)$

All witnesses who described the aftermath of the accident agreed with each other about the order in which the police and ambulance arrived on the scene. (F; PI 8)

There was conflicting testimony given regarding the year of Brock Collins's car. (T; PI 9-year)

There was no conflict in testimony given regarding the color of Michael McGraw's van. (F; PI 10-color) 
There were multiple store names given for the jewelry store in the trial transcript. (T; $P I$ 11)

Brock Collins's car hit an object before finally coming to rest, and all testimony concerning that object agreed about exactly what that object was. (F; PI 12)

Different witnesses named different breeds when describing Lesley Jobin's dog. (T; PI 13)

There were no conflicting descriptions of the police officer's gender. (F; PI 14)

There were conflicting accounts of how traffic is controlled at the intersection. (T; PI 15)

There was conflicting testimony concerning Amanda Johnson's job before the accident. (F; Dummy 1)

There was no discrepancy in the transcript concerning who called 911 about the accident. (T; Dummy 2)

There was conflicting testimony concerning where Brock and Amanda were going when the accident occurred. (F; Dummy 3)

There was no testimony saying that the airbags deployed in Brock Collins's car. (T;

Dummy 4)

There was conflicting testimony given regarding the medical procedures performed on Amanda Johnson to help heal her broken bones. (F; Dummy 5)

Thank you for your continued attention and hard work. The study is almost over, so please mark this answer true so that we will know that you're still paying attention. $(T$; Attention Check)

\section{Point Blank Questions:}


These questions were designed to be as direct as possible without creating demand characteristics. There was only one set of questions per page (that is, two consecutive pages per witness - done to prevent any confusion over whether the two hypotheticals were the same, whether they should be evaluated in conjunction with each other, whether the same Juror 1 and Juror 2 are the focus each question, etc.) Order of presentation was randomized by witness, with all witnesses except Amanda Johnson featuring two consecutive pages, each page with one question apiece. Because Amanda Johnson's testimony is associated with four testimonial inconsistencies, the four pages featuring questions concerning her testimony were always presented last.

The following instructions appeared at the top of each page:

For this final section of questions, you will be asked to evaluate the way two different jurors each viewed a particular witness's testimony.

Each question should be answered independently of all others. The way you answer one question should not have any bearing on how you answer any other question.

\section{Questions (16 total-one for each of the 15 inconsistencies and one manipulation check):}

Imagine two jurors found the testimony of Marc Zollinger to be inaccurate but their opinions were for two different reasons. Which juror's opinion do you most agree with?

The juror who found Marc Zollinger's testimony to be inaccurate because he said that Brock Collins was driving a Corvette while another witness said he was driving a Camry.

The juror who found Marc Zollinger's testimony to be inaccurate because he said that Brock Collins was driving a Camaro while another witness said he was driving a Camry.

I don't agree with either of these jurors.

(Juror 1; PI 5-model)

The juror who found Marc Zollinger's testimony to be inaccurate because he said that Lesley Jobin's dog was a Doberman while another witness said it was a German Shepherd.

The juror who found Marc Zollinger's testimony to be inaccurate because he said that Lesley Jobin's dog was a Dachshund while another witness said it was a German Shepherd.

I don't agree with either of these jurors.

(Juror 2; PI 13)

Imagine two jurors found the testimony of Barbara Feldman to be inaccurate but their

opinions were for two different reasons. Which juror's opinion do you most agree with? 
The juror who found Barbara Feldman's testimony to be inaccurate because she said that Michael McGraw's van was green while another witness said it was white.

The juror who found Barbara Feldman's testimony to be inaccurate because she said that Michael McGraw's van was tan while another witness said it was white.

I don't agree with either of these jurors.

(Juror 2; PI 10-color)

The juror who found Barbara Feldman's testimony to be inaccurate because she said that the police officer who responded to the scene was a woman while another witness said it was a man.

The juror who found Barbara Feldman's testimony to be inaccurate because she said that the police officer who responded to the scene was small in size while another witness said he's large.

I don't agree with either of these jurors.

(Juror 1; PI 14)

Imagine two jurors found the testimony of Jill Randall to be inaccurate but their opinions were for two different reasons. Which juror's opinion do you most agree with?

The juror who found Jill Randall's testimony to be inaccurate because she said that the intersection of Foothill and Windsor has a stop sign while another witness said it has a stop light to control traffic.

The juror who found Jill Randall's testimony to be inaccurate because she said that the intersection of Foothill and Windsor has a yield sign while another witness said it has a stop light to control traffic.

I don't agree with either of these jurors.

(Juror 1; PI 15)

The juror who found Jill Randall's testimony to be inaccurate because she said that Michael McGraw has red hair while another witness said he has black hair.

The juror who found Jill Randall's testimony to be inaccurate because she said that Michael McGraw has blond hair while another witness said he has black hair.

I don't agree with either of these jurors.

(Juror 1; PI 7)

Imagine two jurors found the testimony of Steve Powell to be inaccurate but their opinions were for two different reasons. Which juror's opinion do you most agree with?

The juror who found Steve Powell's testimony to be inaccurate because he said that the fire department arrived at the accident scene first while another witness said the police arrived first. 
The juror who found Steve Powell's testimony to be inaccurate because he said that the ambulance arrived at the accident scene first while another witness said the police arrived first.

I don't agree with either of these jurors.

(Juror 2; PI 8)

The juror who found Steve Powell's testimony to be inaccurate because he said that Amanda Johnson's right arm was injured while another witness said her right leg was injured.

The juror who found Steve Powell's testimony to be inaccurate because he said that Amanda Johnson's left leg was injured while another witness said her right leg was injured.

I don't agree with either of these jurors.

(Juror 2; PI 6)

Imagine two jurors found the testimony of Lesley Jobin to be inaccurate but their opinions were for two different reasons. Which juror's opinion do you most agree with?

The juror who found Lesley Jobin's testimony to be inaccurate because she said that she was in front of the Kay jewelry store when she saw the accident while another witness said it was a Zales jewelry store.

The juror who found Lesley Jobin's testimony to be inaccurate because she said that she was in front of the Jared jewelry store when she saw the accident while another witness said it was a Zales jewelry store.

I don't agree with either of these jurors.

(Juror 2; PI 11)

The juror who found Lesley Jobin's testimony to be inaccurate because she said that Brock Collins's car came to a stop when it crashed into a tree while another witness said it crashed into a street light pole.

The juror who found Lesley Jobin's testimony to be inaccurate because she said that Brock Collins's car came to a stop when it crashed into a tree while another witness said it crashed into a bus stop.

I don't agree with either of these jurors.

(Juror 1; PI 12)

Imagine two jurors found the testimony of Dr. Matthew Freeman to be inaccurate but their opinions were for two different reasons. Which juror's opinion do you most agree with?

The juror who found Dr. Matthew Freeman's testimony to be inaccurate because he said that Brock Collins's car was a 1986 Toyota Camry while another witness said it was a 2006 Toyota Camry. 
The juror who found Dr. Matthew Freeman's testimony to be inaccurate because he said that Brock Collins's car was a 2002 Toyota Camry while another witness said it was a 2006 Toyota Camry.

I don't agree with either of these jurors.

(Juror 1; PI 9-year)

The juror who found Dr. Matthew Freeman's testimony to be inaccurate because he said that Brock and Amanda were on their way to a concert when the accident occurred while another witness said they were on their way to a movie and dinner.

The juror who found Dr. Matthew Freeman's testimony to be inaccurate because he said that Brock and Amanda were on their way to a party when the accident occurred while another witness said they were on their way to a movie and dinner.

I don't agree with either of these jurors.

(Neither; Dummy/Manipulation check/camouflage)

Imagine two jurors found the testimony of Amanda Johnson to be inaccurate but their opinions were for two different reasons. Which juror's opinion do you most agree with?

The juror who found Amanda Johnson's testimony to be inaccurate because she said that the rear of Michael McGraw's van was damaged by the truck while another witness said it was damaged on the passenger side.

The juror who found Amanda Johnson's testimony to be inaccurate because she said that the driver's side of Michael McGraw's van was damaged by the truck while another witness said it was damaged on the passenger side.

I don't agree with either of these jurors.

(Juror 2; PI 1/S\&B 1)

The juror who found Amanda Johnson's testimony to be inaccurate because she first said that prior to the accident, the semi truck was three-quarters of the way across the center of the road and then later said that it was only halfway across the center of the road.

The juror who found Amanda Johnson's testimony to be inaccurate because she first said that prior to the accident, the semi truck was three-quarters of the way across the center of the road and then later said that it was only a quarter of the way across the center of the road.

I don't agree with either of these jurors.

(Juror 1; PI 2/S\&B 3)

Imagine two jurors found the testimony of Amanda Johnson to be inaccurate but their opinions were for two different reasons. Which juror's opinion do you most agree with? 
The juror who found Amanda Johnson's testimony to be inaccurate because she first said that prior to the accident, the semi truck swerved to her right and then later said that it swerved to her right and went into a spin.

The juror who found Amanda Johnson's testimony to be inaccurate because she first said that prior to the accident, the semi truck swerved to her right and then later said that it swerved to her left.

I don't agree with either of these jurors.

(Juror 2; PI 3/S\&B 3)

The juror who found Amanda Johnson's testimony to be inaccurate because she first said that she saw the Shell company logo on the tanker trailer and then later said that she saw the Exxon company logo on the tanker trailer.

The juror who found Amanda Johnson's testimony to be inaccurate because she first said that she saw the Shell company logo on the tanker trailer and then later said that she saw the BP company logo on the tanker trailer.

I don't agree with either of these jurors.

(Juror 1; PI 4/S\&B 6) 


\title{
APPENDIX G
}

\author{
Study One Informed Consent
}

\section{\begin{tabular}{l|l|l|l|l|l|l|l|l|} 
FLORIDA \\
INTERNATIONAL \\
UNIVERSITY
\end{tabular}}

\section{ADULT ONLINE CONSENT TO PARTICIPATE IN A RESEARCH STUDY}

\author{
Civil Juror Decision-Making
}

IRB Approval \# 100411-01

Thank you for looking into our study. Over the next few webpages you will read a description of a civil trial and some jury instructions, and then answer some questions about the case. Before participating, please read the information in the official consent document below.

\section{**IMPORTANT: You may use any of the following web browsers AS LONG AS JAVASCRIPT IS ENABLED: Mozilla Firefox, Google Chrome, Apple Safari, and Internet Explorer. If you have JavaScript turned off, or use any add-ons, extensions, or shortcuts which block JavaScript, please either disable them, add qualtrics.com to the list of approved sites, enable JavaScript, or run your browser in Safe Mode**}

Please complete this study at one time. If for any reason you are not able to complete the study in one sitting, you will have to start over. The experiment takes roughly 60 to 90 minutes to complete.

Please note, you must have cookies and JavaScript enabled on your browser in order to participate. If you click the button below but do not leave this page, your browser is not supporting cookies. Cookies are used only so that you can have a unique identification number for this study; no personal information will be stored in cookies.

As a student in the FIU Psychology Participant Pool (enrolled in PSY 2012 or other class), you are invited to participate in a research study conducted over the Web. The following information is provided to help you make an informed decision whether or not to participate. Your participation is completely voluntary, and not participating will not affect your class grade in any way. You must be at least 18 years old to participate.

\section{PURPOSE OF THE STUDY}

You are being asked to be in a research study. The purpose of this study is to investigate how jurors make decisions. Completion of this study will take approximately one hour.

\section{NUMBER OF STUDY PARTICIPANTS}

If you decide to be in this study, you will be one of approximately 100 people in this research study.

\section{DURATION OF THE STUDY}

Your participation will require approximately 60 to 90 minutes.

\section{PROCEDURES}

If you agree to be in the study, we will ask you to do the following things:

1. You will be asked to read along with and listen to a case scenario involving a civil lawsuit in which a plaintiff is suing a defendant for negligence. You will be asked to answer questions about the events described on a Web page. You will be asked to imagine you are a juror in the case and to decide whether the defendant is liable and whether the plaintiff is entitled to any money-based damages. We will also ask how you feel and your opinion on various aspects of the case. You can refuse to answer any question and cease participation at any time. 


\section{RISKS AND/OR DISCOMFORTS}

The following risks may be associated with your participation in this study: You might become upset when you read or render a verdict about the case involving an automobile accident, especially if you or someone you know has been involved in a similar accident. This event is unlikely, but if you are concerned about it you may withdraw from the study at any time

\section{BENEFITS}

The following benefits may be associated with your participation in this study: you may find the learning experience enjoyable, you may learn a little bit about how psychological research is conducted, and the process may help you better understand the legal process. The information gained from this study will help us better understand the factors that influence juror decision-making processes and to improve trial procedures.

\section{ALTERNATIVES}

You do not need to participate in this research. Your instructor may have alternative means for you to earn class credit, though your participation here may satisfy your instructor's research requirements.

\section{CONFIDENTIALITY}

The records of this study will be kept private and will be protected to the fullest extent provided by law. In any sort of report we might publish, we will not include any information that will make it possible to identify a subject. Research records will be stored securely and only the researcher team will have access to the records.

Any information obtained during this study that could identify you will be kept strictly confidential. The study will collect information about your IP address and the browser you are using. No personal information will be placed on your browser by the use of cookies. So that participation credit may be given, you will be required to enter your Panther ID number and your name. The personal information collected will be deleted from the database once data collection is completed and credit has been given. The information gained from this study may be published in scientific journals or presented at scientific meetings, but your identity will be kept strictly confidential.

\section{COMPENSATION \& COSTS}

There is no financial compensation to you as a result of participating in this study. Completion of this study earns partial credit toward the FIU Psychology Participant Pool requirements as outlined by your professor and on http://fiu.sona-systems.com/. For participating in this study you will receive one and a half research credits. You are free to decide not to participate in this study or to withdraw at any time without adversely affecting your relationship with the investigators or Florida International University. There will be no negative consequences for deciding not to participate or for withdrawing. If you choose to not participate in this study, you may satisfy your course requirements through other studies registered with the FIU Psychology Participant Pool at http://fiu.sona-systems.com/, or you may contact your course instructor to arrange an alternative method of obtaining credit. Participation in human subjects research is not required to earn credit in any class, and your professor is required to offer an alternative method of obtaining credit. You will not be responsible for any costs to participate in this study.

\section{RIGHT TO DECLINE OR WITHDRAW}

Your participation in this study is voluntary. You are free to participate in the study or withdraw your consent at any time during the study. Your withdrawal or lack of participation will not affect any benefits to which you are otherwise entitled.

\section{RESEARCHER CONTACT INFORMATION}

If you have any questions about the purpose, procedures, or any other issues relating to this research study you may contact Stephen Joy at [PHONE NUMBER REDACTED], or at [EMAIL ADDRESS REDACTED].

\section{IRB CONTACT INFORMATION}

If you would like to talk with someone about your rights of being a subject in this research study or about ethical issues with this research study, you may contact the FIU Office of Research Integrity by phone at [PHONE NUMBER REDACTED] or by [EMAIL ADDRESS REDACTED].

\section{PARTICIPANT AGREEMENT}


I have read the information in this consent form and agree to participate in this study. I have had a chance to ask any questions I have about this study, and they have been answered for me. By clicking on the "consent to participate" button below I am providing my informed consent. 


\section{APPENDIX H}

\section{Witness-Specific Likert Scales}

\section{Witness Credibility Ratings}

\begin{tabular}{|c|c|c|c|c|c|c|c|}
\hline & $\begin{array}{l}\text { Completely } \\
\text { Lacks } \\
\text { Credibility }\end{array}$ & $\begin{array}{l}\text { Lacks a } \\
\text { Great Deal } \\
\text { of Credibility }\end{array}$ & $\begin{array}{l}\text { Somewhat } \\
\text { Lacking in } \\
\text { Credibility }\end{array}$ & Neutral & $\begin{array}{l}\text { Somewhat } \\
\text { Credible }\end{array}$ & $\begin{array}{l}\text { Has a Great } \\
\text { Deal of } \\
\text { Credibility }\end{array}$ & $\begin{array}{l}\text { Completely } \\
\text { Credible }\end{array}$ \\
\hline $\begin{array}{l}\text { Marc Zollinger, translation } \\
\text { company worker }\end{array}$ & 0 & 0 & 0 & 0 & 0 & 0 & 0 \\
\hline Barbara Feldman, librarian & 0 & 0 & 0 & 0 & 0 & 0 & 0 \\
\hline $\begin{array}{l}\text { Jill Randall, Amway } \\
\text { salesperson }\end{array}$ & 0 & 0 & 0 & 0 & 0 & 0 & 0 \\
\hline \multirow[t]{2}{*}{$\begin{array}{l}\text { Steve Powell, real estate } \\
\text { agent }\end{array}$} & 0 & 0 & 0 & 0 & 0 & 0 & 0 \\
\hline & $\begin{array}{l}\text { Completely } \\
\text { Lacks } \\
\text { Credibility }\end{array}$ & $\begin{array}{l}\text { Lacks a } \\
\text { Great Deal } \\
\text { of Credibility }\end{array}$ & $\begin{array}{l}\text { Somewhat } \\
\text { Lacking in } \\
\text { Credibility }\end{array}$ & Neutral & $\begin{array}{l}\text { Somewhat } \\
\text { Credible }\end{array}$ & $\begin{array}{l}\text { Has a Great } \\
\text { Deal of } \\
\text { Credibility }\end{array}$ & $\begin{array}{l}\text { Completely } \\
\text { Credible }\end{array}$ \\
\hline $\begin{array}{l}\text { Lesley Jobin, tour group } \\
\text { coordinator }\end{array}$ & 0 & 0 & 0 & 0 & 0 & 0 & 0 \\
\hline $\begin{array}{l}\text { Brock Collins, plaintiff's } \\
\text { boyfriend }\end{array}$ & 0 & 0 & 0 & 0 & 0 & 0 & 0 \\
\hline $\begin{array}{l}\text { Dr. Matthew Freeman, } \\
\text { plaintiff's accident } \\
\text { reconstruction specialist }\end{array}$ & 0 & 0 & 0 & 0 & 0 & 0 & 0 \\
\hline \multirow[t]{2}{*}{ Amanda Johnson, plaintiff } & 0 & 0 & 0 & 0 & 0 & 0 & 0 \\
\hline & $\begin{array}{l}\text { Completely } \\
\text { Lacks } \\
\text { Credibility }\end{array}$ & $\begin{array}{l}\text { Lacks a } \\
\text { Great Deal } \\
\text { of Credibility }\end{array}$ & $\begin{array}{l}\text { Somewhat } \\
\text { Lacking in } \\
\text { Credibility }\end{array}$ & Neutral & $\begin{array}{l}\text { Somewhat } \\
\text { Credible }\end{array}$ & $\begin{array}{l}\text { Has a Great } \\
\text { Deal of } \\
\text { Credibility }\end{array}$ & $\begin{array}{l}\text { Completely } \\
\text { Credible }\end{array}$ \\
\hline $\begin{array}{l}\text { Tom Gorham, 3D } \\
\text { photographer }\end{array}$ & 0 & 0 & 0 & 0 & 0 & 0 & 0 \\
\hline John Kindt, security guard & 0 & 0 & 0 & 0 & 0 & 0 & 0 \\
\hline $\begin{array}{l}\text { Dr. Erik Dubberke, trauma } \\
\text { surgeon }\end{array}$ & 0 & 0 & 0 & 0 & 0 & 0 & 0 \\
\hline $\begin{array}{l}\text { Christopher Eaton, police } \\
\text { officer }\end{array}$ & 0 & 0 & 0 & 0 & 0 & 0 & 0 \\
\hline
\end{tabular}




\section{Witness Persuasiveness Ratings}

\begin{tabular}{|c|c|c|c|c|c|c|c|}
\hline & $\begin{array}{l}\text { Not } \\
\text { Persuasive } \\
\text { At All }\end{array}$ & Unpersuasive & $\begin{array}{l}\text { Somewhat } \\
\text { Unpersuasive }\end{array}$ & $\begin{array}{c}\text { Neither } \\
\text { Persuasive } \\
\text { nor } \\
\text { Unpersuasive }\end{array}$ & $\begin{array}{l}\text { Somewhat } \\
\text { Persuasive }\end{array}$ & Persuasive & $\begin{array}{c}\text { Very } \\
\text { Persuasive }\end{array}$ \\
\hline $\begin{array}{l}\text { Marc Zollinger, translation } \\
\text { company worker }\end{array}$ & 0 & 0 & 0 & 0 & 0 & 0 & 0 \\
\hline $\begin{array}{l}\text { Barbara Feldman, } \\
\text { librarian }\end{array}$ & 0 & 0 & 0 & 0 & 0 & 0 & 0 \\
\hline $\begin{array}{l}\text { Jill Randall, Amway } \\
\text { salesperson }\end{array}$ & 0 & 0 & 0 & 0 & 0 & 0 & 0 \\
\hline \multirow[t]{2}{*}{$\begin{array}{l}\text { Steve Powell, real estate } \\
\text { agent }\end{array}$} & 0 & 0 & 0 & 0 & 0 & 0 & 0 \\
\hline & $\begin{array}{l}\text { Not } \\
\text { Persuasive } \\
\text { At All }\end{array}$ & Unpersuasive & $\begin{array}{l}\text { Somewhat } \\
\text { Unpersuasive }\end{array}$ & $\begin{array}{c}\text { Neither } \\
\text { Persuasive } \\
\text { nor } \\
\text { Unpersuasive }\end{array}$ & $\begin{array}{l}\text { Somewhat } \\
\text { Persuasive }\end{array}$ & Persuasive & $\begin{array}{c}\text { Very } \\
\text { Persuasive }\end{array}$ \\
\hline $\begin{array}{l}\text { Lesley Jobin, tour group } \\
\text { coordinator }\end{array}$ & 0 & 0 & 0 & 0 & 0 & 0 & 0 \\
\hline $\begin{array}{l}\text { Brock Collins, plaintiffs } \\
\text { boyfriend }\end{array}$ & 0 & 0 & 0 & 0 & 0 & 0 & 0 \\
\hline $\begin{array}{l}\text { Dr. Matthew Freeman, } \\
\text { plaintiff's accident } \\
\text { reconstruction specialist }\end{array}$ & 0 & 0 & 0 & 0 & 0 & 0 & 0 \\
\hline \multirow[t]{2}{*}{ Amanda Johnson, plaintiff } & 0 & 0 & 0 & 0 & 0 & 0 & 0 \\
\hline & $\begin{array}{l}\text { Not } \\
\text { Persuasive } \\
\text { At All }\end{array}$ & Unpersuasive & $\begin{array}{l}\text { Somewhat } \\
\text { Unpersuasive }\end{array}$ & $\begin{array}{c}\text { Neither } \\
\text { Persuasive } \\
\text { nor } \\
\text { Unpersuasive }\end{array}$ & $\begin{array}{l}\text { Somewhat } \\
\text { Persuasive }\end{array}$ & Persuasive & $\begin{array}{c}\text { Very } \\
\text { Persuasive }\end{array}$ \\
\hline $\begin{array}{l}\text { Tom Gorham, 3D } \\
\text { photographer }\end{array}$ & 0 & 0 & 0 & 0 & 0 & 0 & 0 \\
\hline John Kindt, security guard & 0 & 0 & 0 & 0 & 0 & 0 & 0 \\
\hline $\begin{array}{l}\text { Dr. Erik Dubberke, trauma } \\
\text { surgeon }\end{array}$ & 0 & 0 & 0 & 0 & 0 & $\mathrm{O}$ & 0 \\
\hline $\begin{array}{l}\text { Christopher Eaton, police } \\
\text { officer }\end{array}$ & 0 & 0 & 0 & 0 & 0 & 0 & 0 \\
\hline
\end{tabular}




\section{APPENDIX I}

\section{Demographic Questions}

1. In what year were you born (yyyy)?

2. What is your gender? Check one: Male Female

3. What is your ethnic origin/race?

White/Caucasian

American Indian

Hispanic

Asian

African American

Other

4. Indicate your highest educational training as of this date. (check one)

Freshman

Sophomore

Junior

Professional or graduate school

Senior

5. What is your current work status?

Check one:

Other

Employed full time

Employed part time

Unemployed

6 . What is your occupation?

7. What is your place of employment?

8. Is English your primary language? Ye No

How many years have you spoken English? Years

If English is not your primary language, what is your primary language?

If English is not your primary language, how fluent are you in English?

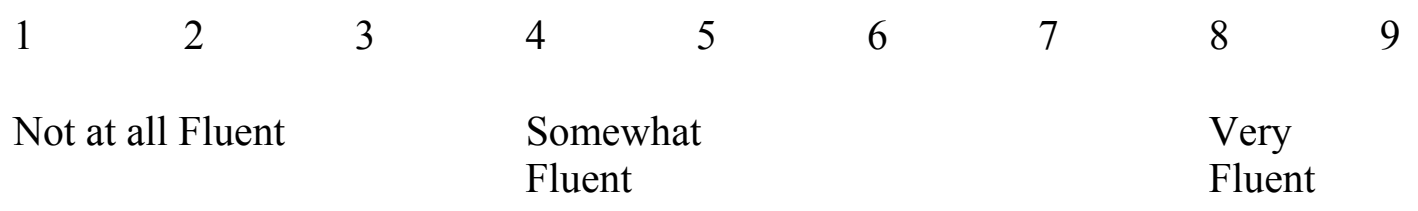

9. Do you speak Spanish?

Yes __ No 
If you speak Spanish, how many years have you spoken Spanish? Years

If you speak Spanish, how fluent are you in Spanish?

1 2 3 4 5 6 7 8 9

Not at all Fluent

Somewhat Fluent

10. Have you ever served as a juror before? Yes

If yes, was it a civil case or a criminal case?
No

Civil
Very

Fluent 


\section{APPENDIX $\mathrm{J}$}

Participant Debriefing Information

\section{FIU}

Thank you for your participation in our study. Please read the debriefing material below.

\section{Debriefing Material}

This study is investigating the impact that emotional state and emotion regulation efforts have on the way people perceive and process information in a legal context. While jurors are usually instructed by the judge to make decisions based on the law rather than emotional responses or "gut feeling" reasoning, sometimes it can be difficult to set aside emotional reactions when trying to make a decision. These experiments will allow us to gain a better understanding of how to facilitate attentive, effortful, and thorough juror decision-making.

This study exposed some participants to film clips intended to artificially manipulate their emotions. Some clips were shown in order to put participants into a more angry emotional state, some clips were show to put participants into a more fearful emotional state, and some film clips were shown for the purpose of not changing emotional state at all. This study exposed some participants to testimonial inconsistencies (witnesses confusing facts or giving testimony outside their sphere of experience) while other participants received a consistent trial transcript.

We expect to find that participants in certain moods (like anger) will process the trial transcript differently than those participants in uncertain moods (like fear). That is, prior research indicates that anger (a certain emotion) can decrease participants' ability to process information (and detect inconsistencies) while fear (an uncertain emotion) can compel deeper information processing (and may increase detection of inconsistencies). This research will further test this possibility as well as lay groundwork for future research aimed at reducing the impact of emotions on how people perceive and process information.

If you have any questions or concerns about the experiment, please contact the main 
researcher, Stephen Joy at [PHONE NUMBER REDACTED] or [EMAIL ADDRESS REDACTED]. You may also contact Patricia Price, the Institutional Review Board Chairperson at [PHONE NUMBER REDACTED] or [EMAIL ADDRESS REDACTED].

Again, thank you very much for your participation in this study. 


\section{APPENDIX K}

\section{Open Ended Question Coding Instructions}

Note: These are the exact scoring instructions furnished to the two research assistants.

\section{Purpose of the Coding:}

In a nutshell, I'm trying to determine the detection rates for each of the 15 different Plain Inconsistencies (abbreviated PIs throughout) that are present in a trial transcript.

Ultimately, I'm interested in which PIs are reported as well as how often and to what extent they're reported.

What I need from you guys is to go through the responses to determine which PIs the participants picked up on. It will be tricky, especially at the beginning, so we're going to take things slow and kind of go over everything together at first, then you'll practice a little bit on your own, and then we'll meet again to go over everything again before I give you the real data.

\section{Materials/Files:}

Johnson v McGraw (Word doc) - this is the trial transcript, complete with each PIrelevant statement marked and highlighted

List of All Incons (Excel file) - this is a list of all PIs and the individual details of each PI Open Ended Coding Instructions (Word doc) - that's this...

Open Ended Incon Detection Questions (Word doc) — this is a list of all of the Open

Ended questions

Study One OE Practice Data (Excel file; one for each of you) — this is for training purposes concerning the Open Ended questions

Study One OE Practice Answer Key (Excel file) - I'll give this to you guys after we're finished with the OE Practice Data exercise, just to be used as a reference/example later on

Study One OE Data (Excel file; one for each of you) — I'll give this to you guys after we're finished with the OE Practice Data exercise

\section{Order of Steps/Plan of Attack:}

1. First, you'll each need to familiarize yourselves with the trial transcript itself. The version you'll have to use (Johnson v McGraw 15 PIs ALL Incons Marked Word document) has each element of each PI noted and highlighted, with the number of each inconsistency given, as well as whether that element is the true/consistent portion, or whether it's the inconsistent/false portion of the testimony. Only witnesses for the plaintiff's side make any inconsistent statements, but you should still be familiar with the defense witnesses' testimony too, since noticing some of the inconsistencies depends (in whole or in part) on their testimony as well. 
While you're reading over the transcript, you can follow along with all 15 of the PIs (both the consistent and inconsistent portions) by using the List of All Incons Excel file.

2. After you've each had time to read over the trial transcript, we can meet to go over everything and I can answer any questions you might have at that point.

3. After we have a chance to go over everything in person (don't dive in on this until we have a chance to meet), you can try coding the rest of the practice data (Study One Practice OEs Only Excel file- the two files are identical except for the fact that I've made a separate one for each of you with your name on it, just to keep potential confusion to a minimum). I've done the first few participants myself so you can sort of use that as guidance. More detail on this is below...

4. Once you've worked out the practice coding and made note of any questions you have along the way and any responses you're not sure how to code, we can all meet again to go over everything together so that we're all on the same page.

5. At that point, I'll have the real data files ready for you to code. We can meet again after you've each had time to get started but before you get too far along just to make sure we're all using the same sort of criteria.

6. After confirming that we're all coding the same way, you can finish your files independently, making note of any responses that you're unsure about. After you're both finished, you two can meet without me to talk through those issues, and if there's still something you two can't figure out, or if you can't come to an agreement (which is perfectly okay - don't feel like you must agree with each other 100\%--it's far better for you both to be confident in your reasoning than to persuade the other...), then Dr. Winter and/or I can weigh in.

7. When we have all the Open Ended questions finished, we'll move on to much the same procedure for the other batch of questions but with a few minor differences that we'll worry about later on...

\section{Background Details:}

The question abbreviations are organized according to which Plain Inconsistency (PI) the question involves. See the List of All Incons Excel file for a complete description of all 15 of them.

However, the survey software only allows a certain number of characters in naming variables, which is why there are no spaces or commas between some of the numbers. They are listed in numerical order though, which should clear up some of the confusion. Also, "OEPI" stands for "Open Ended Plain Inconsistency" meaning that the broad question is intended to elicit answers including each of the inconsistencies according to the numbers listed, but they're structured to mainly elicit yes/no/I don't know sorts of 
responses. However, "OEH" stands for "Open Ended How", which is basically an elaboration on the preceding question- "If so, who and how so?" See the Open Ended Incon Detection Questions Word document for the full list of the questions.

\section{General Interpretation of Content as a Yes or a Partial Response:}

For the OE (Open Ended) questions, to count as a "Yes" response, it should be clear that they were aware of that particular inconsistency to some extent. They don't need to provide every single detail - the names of all people who testified consistently, the person who made the inconsistency, both the consistent and the inconsistent elements, etc. - just enough information that there isn't any doubt or uncertainty whether they noticed the inconsistency. Even if they get some of the details wrong, if it's undeniable that they noticed the inconsistency to some degree, it should count as a "Yes".

For the OE (Open Ended) questions, to count as a "Partial" response, there should be enough information that you're pretty sure that they noticed the inconsistency to some extent, even if they're vague and don't give you much detail. All you need to find is that the response seems to support the notion that they detected the inconsistency to some degree. A "Partial" doesn't depend on the overall percentage of accuracy or detail within their answer, but rather your certainty that they spotted it at all. If you think they probably spotted the inconsistency based on their answer but there isn't anything absolute or definite mentioned that makes it beyond a doubt that they spotted the inconsistency, that's still enough. Basically, if based on their answer you think they probably spotted something about the inconsistency, it counts as a "Partial". Even something as vague and unclear as "there was something iffy about the cop" would be enough to count as a PI 14 Partial. Since this is the most broadly open ended type of question the participants answered, we'll want to code everything with the most accommodating and flexible standards we'll be using throughout the coding process.

This kind of flexibility could be tricky if someone were to say "there's something iffy about the cars" because we wouldn't know for sure whether they're talking about PI 5 (Camry/Corvette), PI 9 (1986/2006 Camry), or even PI 10 (tan/white van). This sort of response would be perfect for you to make a note to discuss with each other later on, after you've gone through everything the first time by yourself. If you can't come to an agreement with each other, or you simply don't know how to make a ruling, then you can bring me in to help decide how to code it.

If a participant says that they noticed something inconsistent but they don't give enough information for you to tell in any way whatsoever which inconsistency they're referring to (i.e., "something didn't seem right"), or that they definitely noticed anything at all (they might just be guessing because they think they're supposed to notice something, or they might not have actually paid any attention to the trial transcript and they're just trying to BS their way through an answer because they've figured out what we're probably looking for, or if they're one of those argumentative participants who thinks it's their duty to act like the slightest degree of ambiguity — real or perceived — gives them 
license to explain why they can't possibly answer the question, etc.) or if they're just making very vague, unclear, indirect, general statements, then that shouldn't count as a detection.

\section{Specific Coding Instructions:}

- For the OEPI questions, only grade if they include actual details and content—don't bother with the "yes/no/I don't know" types of answers that most will give for these questions.

- I found it easiest to just mark an Xxx under each cell so that I would know that I had already looked at it and didn't need to revisit it when working on the Practice Excel file, but you don't have to do that if you don't think it helps you keep track of what you've already done or to keep all the different lines straight—it's totally up to you.

- For the Practice File, for the responses of any real substance (not the Yes/No/I Don't Know/Etc. sorts of things), make a little note underneath each answer explaining why you did or didn't code something as a Yes or a Partial. It will probably be helpful for you to put the actual scoring you decide to assign into bold so that you can do the final tally for each participant more easily without having to fully re-read everything you wrote.

However, when you're through with the Practice and actually coding the real data, you can put as much or as little notes or explanation in the line underneath the actual answers as you want. It's probably easiest to just put the PI \# Yes or PI \# Partial underneath unless you have questions about how to code an answer. In that case, enter whatever notes you need to make so that you'll be able to talk it over with each other once you're both done with the bulk of the coding. Otherwise, you don't need to explain the reason-I only included that in my Practice Excel file to help you to see how and why I coded things the way that I did, and I'm only asking that you do that for the Practice File so that we'll be able to discuss things when we meet again prior to working on the actual data.

- Mark the total number of times that the participant mentions each PI \# Yes and PI \# Partial, just so we can keep a tally of which inconsistencies are mentioned the most often. There is no need to tally or code any "no" or "I don't know" responses, or any "yes" answers that don't give any other information.

- The "OEPI_\#\#\#" questions are phrased in a sort of yes/no format (though obviously some participants choose to elaborate on their answers), with the following "OEH_\#\#\#" question being "If so, how?", which is set up for more detailed answers (again, see the Open Ended Incon Detection Questions Word document). If a participant gives a Yes or a Partial answer in the "OEPI_\#\#\#" question, and then again gives the same information or better in the followup "OEH_\#\#\#" question, only 
give them the credit for the strongest mention, not two credits for essentially saying the same thing twice in the two parts of the same question.

In other words, because the OEPI/OEH questions are paired up with each other, if they mention the same PI more than once within the same pairing, (basically, if they include the details in both the OEPI and the OEH answer, rather than just answering the OEPI with a "yes" and then including the details in the OEH followup question) they should only get credit for the same PI once.

- For example:

OEPI 7 10: $\quad$ Were there any eyewitnesses that testified who you think might have made mistakes in terms of describing various colors? (PI 7; PI 10color)

OEH 7 10: If so, who and how? If you don't remember exactly which witness(es) might have made this type of mistake, please just describe the mistake(s) to the best of your ability.

If they answer OEPI 710 with "Yes, someone messed up McGraw's hair color-Jill said it was red but everyone else said black." And they answer OEH 710 with "McGraw's hair color was described as red by one witness and black by the others." Then they will only receive one PI 7 Yes scoring between both of those since the OEPI and OEH questions are essentially two parts of the same question.

- However, if they refer to the same PI more than once across different sets of questions, then count each mention (still with only a maximum of one credit per question pairing) individually.

For example, if they mention Jill says McGraw has red hair in OEPI_710 and OEH_710 as in the illustration above which counts as one PI 7 Yes, and then they also mention it in OEPI_6714, and OEH_6714 like so:

OEPI 67 14: Were there any eyewitnesses that testified who you think might have made mistakes in terms of specific physical descriptions of people? (PI 6; PI 7; PI 14)

OEH 6 7 14: If so, who and how? If you don't remember exactly which witness(es) might have made this type of mistake, please just describe the mistake(s) to the best of your ability.

by answering OEPI 6714 with "Yes, McGraw's hair color was said to be both red and black, and one person said the cop was a woman." And they answer OEH 6714 with "McGraw's hair color and the cop's gender." Then they would receive one PI 7 Yes and one PI 14 Yes total for both 6714 questions to go along with the other PI 7 Yes from the 710 questions. 
Assuming they didn't mention hair color or the gender of the cop in any other responses, they'd get a total score of two PI 7 Yes responses and one PI 14 Yes response. They wouldn't get a total of four PI 7-Yes credits because they mentioned it four times total, just two, since the two mentions in both 710 questions and the two mentions in both 6714 questions are just counted as one each. I hope this makes sense - it's a pretty straightforward idea that's uncommonly difficult to succinctly describe...

- If you don't know how to code a response, make a note underneath it and you can discuss it with each other after going through the rest of the data.

- If you both are unable to come up with an agreement for how to code something tricky after discussing it with each other, then you can bring it to Dr. Winter's or my attention and we'll make a decision.

- If you have any questions about general rules or practices, please feel free to contact me via email or phone/text. If you have a more specific question like how to interpret a particular response (that doesn't have any broader, general questions associated with it), then just make a note of it and you each can discuss these with one another after you've completed the initial stage of coding. 


\section{APPENDIX L}

\section{Short Answer Question Coding Instructions}

Note: These are the exact scoring instructions furnished to the two research assistants.

\section{Purpose of the Coding:}

In a nutshell, I'm trying to determine the detection rates for each of the 15 different Plain Inconsistencies (abbreviated PIs throughout) that are present in a trial transcript.

Ultimately, I'm interested in which PIs are reported as well as how often and to what extent they're reported.

What I need from you guys is to go through the responses to determine which PIs the participants picked up on. It will be tricky, especially at the beginning, so we're going to take things slow and kind of go over everything together at first, then you'll practice a little bit on your own, and then we'll meet again to go over everything again before I give you the real data.

\section{Materials/Files:}

Johnson v McGraw (Word doc) - this is the trial transcript, complete with each PIrelevant statement marked and highlighted

List of All Incons (Excel file) — this is a list of all PIs and the individual details of each PI Short Answer Coding Instructions (Word doc)—-that's this...

Short Answer Incon Detection Questions (Word doc) — this is a list of all of the Short Answer questions

Study One SA Practice Data (Excel file; one for each of you) - this is for training purposes concerning the Short Answer questions

Study One SA Practice Answer Key (Excel file) - I'll give this to you guys after we're finished with the SA Practice Data exercise, just to be used as a reference/example later on

Study One SA Data (Excel file; one for each of you) - I'll give this to you guys after we're finished with the SA Practice Data exercise

\section{Order of Steps/Plan of Attack:}

1. You're already familiar with the trial transcript and the 15 different PIs, so really the only thing we need to do first is to meet to go over everything and I can explain the differences in how the Short Answer questions should be coded in comparison to how we coded the Open Ended questions. I'll be able to answer any questions you might have at that point.

2. After we have a chance to go over everything in person (don't dive in on this until we have a chance to meet), you can try coding the rest of the practice data (Study One Practice SAs Only Excel file - the two files are identical except for the fact that I've made a separate one for each of you with your name on it, just to keep potential 
confusion to a minimum). I've done the first few participants myself so you can sort of use that as guidance. More detail on this is below...

3. Once you've worked out the practice coding and made note of any questions you have along the way and any responses you're not sure how to code, we can all meet again to go over everything together so that we're all on the same page.

4. At that point, I'll have the real data files ready for you to code. We can meet again after you've each had time to get started but before you get too far along just to make sure we're all using the same sort of criteria.

5. After confirming that we're all coding the same way, you can finish your files independently, making note of any responses that you're unsure about. After you're both finished, you two can meet without me to talk through those issues, and if there's still something you two can't figure out, or if you can't come to an agreement (which is perfectly okay - don't feel like you must agree with each other 100\%--it's far better for you both to be confident in your reasoning than to persuade the other...), then Dr. Winter and/or I can weigh in.

6. When we have all the Short Answer questions finished, we'll use that data to help finalize the trial transcript to only include 5 or so of the original 15 PIs, and then I'll need to test that version again to make sure that people are noticing the 5 remaining PIs at the correct rate, so I'll need you both to help with another batch of OEs and SAs again soon...

\section{Background Details:}

The question abbreviations are organized according to which Plain Inconsistency (PI) the question involves. See the List of All Incons Excel file for a complete description of all 15 of them.

"SA" stands for Short Answer, and since the SA questions are less vague and more direct in scope, there's less room for overlap among the questions, so each PI has its own SA PI \# question, so there's no need to squeeze a bunch of numbers into the question/variable name.

Also, there are five Dummy questions (abbreviated SA D \#) which deal with other, fully consistent details of the transcript that are included just so that participants don't realize that each question involves a different PI. You don't need to worry about these, except to look to see if by any chance they mentioned any of the 15 PIs in their responses to the five SA D questions.

See the Short Answer Incon Detection Questions Word document for the full list of the questions. 


\section{General Interpretation of Content as a Yes or a Partial Response:}

For the SA (Short Answer) questions, to count as a "Yes" response, it should be clear that they were aware of that particular inconsistency and whatever level of detail they provide should be correct. This is different than the more forgiving way we coded "Yes" responses for the OE (Open Ended) questions. Here, they still don't have to give every single aspect and detail (such as listing every person who made the consistent statements, etc.), but what they do list must be accurate.

For the SA (Short Answer) questions, to count as a "Partial" response, it should be clear that they noticed the inconsistency, but they can have some of the details incorrect, vague, or missing. However, it should be clear that they noticed the inconsistency, not just the consistent elements (or not just the inconsistent elements).

For example,

SA PI 7: $\quad$ Several witnesses described Michael McGraw by mentioning his hair color. Please describe the testimony concerning Michael McGraw's hair color. Make sure you talk about all of the witnesses who mentioned his hair color. (PI 7)

If they only mention McGraw having black hair, that doesn't count as a partial detection, because even though it's true, and it's obviously related to PI 7, that's only the consistent portion of the testimony-it doesn't give any indication that they noticed the inconsistent part, so we wouldn't score that as a PI 7 Partial. If they said "McGraw's hair was described as different colors" that would be enough to count as a PI 7 Partial.

Also, since some of the PIs are basically a 50/50 tossup--PI 3 (direction the truck swerved), PI 6 (which leg Amanda hurt), PI 8 (order of emergency responders arrival), and PI 14 (whether the cop is a man or a woman) more or less only have two conceivable answers that can be given, and PI 2 (whether the truck was $1 / 2$ or $3 / 4$ across the center lines) is based on a contradiction by Amanda (nobody else says anything about the exact degree/extent the truck is on the wrong side of the road), simply listing one of the two possibilities is NOT enough for a Partial answer to be scored. I've marked those columns in the Practice Excel file with asterisks, and I've also bolded the columns as a reminder. I've also included $* \mathbf{5 0 / 5 0}$ for these few PIs in the "Broad Type of Incon" column on the List of All Incons Excel file.

This is different from how we coded the "Partial" responses for the OE (Open Ended) questions, where we were just looking for the very bare minimum standard of "I'm pretty sure they noticed something but I'm not absolutely positive that they did." Here, we want to know for sure that they noticed something, and the degree of flexibility only has to do with overall accuracy and correctness.

For the SA_D (Short Answer-Dummy) questions 1 thru 5, there is no inconsistency associated with these questions, they're included in order to provide a number of additional questions regarding details of the testimony but which do not feature anything 
related to inconsistent testimony. That way, participants do not think that every single question must obviously have some sort of inconsistent statement associated with it, and we won't have people guessing or trying to deduce the answers we're looking for without actually noticing the details and information themselves. Still, you should read through each of them in case there might be some additional information volunteered that has to do with one of the 15 inconsistencies, in which case you can code it accordingly.

If a participant says that they noticed something inconsistent but they don't give enough information for you to tell in any way whatsoever which inconsistency they're referring to (i.e., "something didn't seem right"), or that they definitely noticed anything at all (they might just be guessing because they think they're supposed to notice something, or they might not have actually paid any attention to the trial transcript and they're just trying to BS their way through an answer because they've figured out what we're probably looking for, or if they're one of those argumentative participants who thinks it's their duty to act like the slightest degree of ambiguity — real or perceived — gives them license to explain why they can't possibly answer the question, etc.) or if they're just making very vague, unclear, indirect, general statements, then that shouldn't count as a detection.

\section{Specific Coding Instructions:}

- I found it easiest to just mark an Xxx under each cell so that I would know that I had already looked at it and didn't need to revisit it when working on the Practice Excel file, but you don't have to do that if you don't think it helps you keep track of what you've already done or to keep all the different lines straight—it's totally up to you.

- For the Practice File, for the responses of any real substance (not the Yes/No/I Don't Know/Etc. sorts of things), make a little note underneath each answer explaining why you did or didn't code something as a Yes or a Partial. It will probably be helpful for you to put the actual scoring you decide to assign into bold so that you can do the final tally for each participant more easily without having to fully re-read everything you wrote.

However, when you're through with the Practice and actually coding the real data, you can put as much or as little notes or explanation in the line underneath the actual answers as you want. It's probably easiest to just put the PI \# Yes or PI \# Partial underneath unless you have questions about how to code an answer. In that case, enter whatever notes you need to make so that you'll be able to talk it over with each other once you're both done with the bulk of the coding. Otherwise, you don't need to explain the reason-I only included that in my Practice Excel file to help you to see how and why I coded things the way that I did, and I'm only asking that you do that for the Practice File so that we'll be able to discuss things when we meet again prior to working on the actual data. 
- Mark the total number of times that the participant mentions each PI \# Yes and PI \# Partial, just so we can keep a tally of which inconsistencies are mentioned the most often. There is no need to tally or code any "no" or "I don't know" responses, or any "yes" answers that don't give any other information.

- The Short Answer questions are pretty direct in their wording, so there shouldn't be too many cases of participants mentioning a PI other than the specific one from the question itself, but I'm sure there will be cases where someone might misunderstand the intention of the question, or where they might think that another PI is equally applicable to the way the question is phrased, so just keep your eyes open for that and make sure to record the tally accurately.

- If you don't know how to code a response, make a note underneath it and you can discuss it with each other after going through the rest of the data.

- If you both are unable to come up with an agreement for how to code something tricky after discussing it with each other, then you can bring it to Dr. Winter's or my attention and we'll make a decision.

- If you have any questions about general rules or practices, please feel free to contact me via email or phone/text. If you have a more specific question like how to interpret a particular response (that doesn't have any broader, general questions associated with it), then just make a note of it and you each can discuss these with one another after you've completed the initial stage of coding. 


\section{APPENDIX M}

Study One Scoring, Scale Creation, and Evaluation of Inconsistencies

\section{Verification of Inter-rater Reliability}

Skewness and kurtosis values for the distribution of "Yes" and "Partial" detection scores on the open ended questions exceeded twice the absolute value of their standard errors for all 15 inconsistencies calculated for two research assistants individually using their data sets from their individual ratings done before discussion with each other, after discussion with each other, and collapsing the "Yes" and "Partial" scores into a single total of scored detections for both research assistants individually before discussion and again after discussion. The short answer questions were similarly non-normally distributed based on skewness and kurtosis values predominantly exceeding twice the absolute value of their standard errors for the same treatments and tests of data.

Furthermore, because $n=100$, I used Kolmogorov-Smirnov tests of normality to confirm, which showed that for all non-constant scores (conducted on the same array of individual before and after ratings and collapses of data), inconsistency detection was not normally distributed $(p<.001)$. Because of this non-normal distribution, I relied on Kendall's taub correlation to verify inter-rater reliability rather than Pearson's or Spearman's correlations.

\section{Construction of Scales to Compare Relative Ease of Detection for Each}

\section{Inconsistency}

I used a wide range of measures to thoroughly compare the detection rates of each inconsistency to all others (see Appendix F for all questions used). Those measures included open ended questions wherein detections were coded as either a "Yes" or a 
"Partial" detection (see Appendix K for open ended scoring rubric) by two individual research assistants, short answer questions in which detections were also coded as either a "Yes" or a "Partial" detection (see Appendix L for short answer scoring rubric) by two individual research assistants, multiple choice questions which each required multiple correct answers to unequivocally count as a detection, true or false questions (which were not a typical, true 50/50 proposition because an "I don't know" option was included to deter guessing), and "point blank" questions which forced participants to identify which inconsistency was included in the testimony when presented with the details of the actual inconsistency and another similar inconsistency which was not in the transcript, as well as a third "I don't know" option to discourage guessing.

The variety of different ways to interpret and synergize the multiple disparate formats of data into a single metric for the purpose of evaluating the worthiness of each inconsistency for use in the later studies required me to come up with an overall scale to integrate the detection rate data. I was able to create several such scales, but before detailing their creation and components, I must explain how I interpreted each type of question.

\section{Open Ended and Short Answer Questions}

Although the research assistants used different thresholds of scoring for the open ended and short answer codings, the nature of the data set and the logistics of interpretation was common to both, so they will be discussed jointly for the purposes of this description.

The way I quantified the open ended data is based on the way both research assistants scored the set of data, and it takes into account the fact that they were recording 
the total number of mentions of a detection for each inconsistency (this turned out to be something I later recoded into a binary "present/absent" variable, since certain inconsistencies lended themselves to being described in response to more than one question — especially among the open ended questions — while others would only be a natural response to one question) so the number of mentions, which is reflected in all Yes and Partial values, is not a reliable means of distinguishing detection rates. However, they remained useful as an index of agreement between research assistants, particularly when comparing the Before values (each assistant coded the data independently) to the After values (after meeting to compare the way they coded everything and to discuss and justify why they coded a particular response in a particular way with the goal of coming to a consensus if/when possible.)

For a full description of coding procedures and instructions, see Appendices K and L, but the primary difference between open ended and short answer coding instructions related to the degree of flexibility allowed in defining a "Yes" or a "Partial" detection. Since open-ended questions lack specificity by nature, the degree of flexibility was greater for the open ended responses. However, since the short answer questions were far more specific in their wording, the research assistants were instructed to be much more exclusive and strict in their scoring and interpretations.

In creating my final scales to rank and evaluate the detection rates of all 15 inconsistencies in relation to each other for the open ended and short answer questions, I focused only on the data sets each research assistant submitted after their discussion. I came up with four different ways to recode or "finalize" the open ended and short answer 
data into a value corresponding to an overall detection rate for each inconsistency. In order of "loosest" level to "strictest" level, the resulting categories include:

- CombiLoose: If at least one research assistant gave any credit whatsoever (Yes or Partial) to that participant for that particular inconsistency.

- CombiMed: Both research assistants must give some sort of credit (Yes or Partial) to that participant for that particular inconsistency.

- CombiHighBoth: Both research assistants must give that participant the exact same credit (with both giving Yes or both giving Partial) for that particular inconsistency.

- CombiHighYes: Both research assistants must give that participant the exact same credit (counting only Yes scores) for that particular inconsistency.

I will return to discussion of these four scoring schemes below when outlining the process I used to aggregate all question types into metrics to assist in selecting those inconsistencies to retain for use in the remainder of the research.

\section{Multiple Choice Questions}

Out of all participants who were not excluded from analysis at the outset $(N=$ 100), 19 of them did not report a single inconsistency by selecting the two different choices corresponding to the correct consistent and correct inconsistent information presented in the trial transcript. While it is unclear whether these participants truly did not notice any of the inconsistencies, or they did notice but simply did not understand the instructions - particularly that they were told it was okay to select more than one answer, or if perhaps they were not truly engaged, paying attention, and earnestly participating, they did not meet any of the pre-established criteria for exclusion. Nevertheless, the possibility remains that they may not have been fully engaged participants (though they each did indicate having noticed inconsistencies through other question formats), so I elected to consider detection rate data for the multiple choice questions both with them $(N$ 
$=100)$, and without them $(N=81)$ to provide an upper and lower boundary to the detection rates among the multiple choice questions.

\section{True or False Questions}

Interpreting the true or false inconsistency detection rates was straightforward. Eight of the 15 non-dummy questions were phrased in a manner such that an inconsistency detection corresponded with a "True" response, and seven of 15 were

phrased in a manner where a "False" response indicated an inconsistency detection. Detection rates for each inconsistency were simply the percentage of participants who answered the non-dummy questions in a manner indicating a detection.

\section{Point Blank Questions}

The point blank questions presented participants with two jurors, both of whom found one of the witness's (whichever witness makes the inconsistent statement of interest) to be inaccurate but for two different reasons. Participants were asked which of the two hypothetical jurors they most agreed with, both of whom said there was an inconsistency. However, one provided a reason based on the exact details of the manipulated inconsistency while the other provided a reason based on details similar to but not present in the manipulated inconsistency. For example, for the point blank question associated with PI 5-Model, the choices are whether Marc Zollinger's testimony is inaccurate because he said that Brock Collins drove a Corvette (which is how the inconsistency is presented in the testimony) or because he said that Brock Collins drove a Camaro (which is not the basis of the inconsistent testimony). There is also an "I don't agree with either of these jurors." option. 
The point blank questions can actually provide two different "levels" of diagnostic information. First, those participants who correctly identify the basis of the incorrect testimony are credited with having detected the inconsistency. Second, those participants who selected the incorrect basis (essentially, those who did not get the question right, but who nevertheless indicated some belief in an inconsistency concerning the topic at issue, or who may have noticed an inconsistency but could not recalled the exact details correctly—basically, everyone but those participants who chose "I don't agree with either of these jurors.") are able to provide a very liberal definition of detection. Of course, these participants may have simply guessed or deduced the nature of the inconsistency based on exposure to prior questions, and for that reason this would make a terrible metric if it were the sole measure of inconsistency detection rate, but as the final set of questions, and as the most liberal interpretation of the most direct and "easiest" of the inconsistency detection measures, it is useful enough to simply consider these participants who did not answer the question distinctly incorrectly within the more lenient of the two detection rate scoring composites.

\section{Scales to Compare Relative Ease of Detection for Each Inconsistency}

For the open ended and short answer questions, I came up with four different schemes of standardization (in rough order of ascending criteria, CombiLoose, CombiMed, CombiHighBoth, and CombiHighYes). CombiLoose, CombiMed, and CombiHighBoth did not yield drastically different aggregate rates of detection with CombiLoose generally resulting in 1-3\% more detections of each inconsistency than CombiMed, which generally resulted in $1-3 \%$ more detections of each inconsistency than CombiHighBoth. To arrive at the upper range of detection rates, I elected to use the 
CombiMed standard because CombiLoose seemed too permissive- I did not care for the idea of counting anything as a detection where only one of the research assistants found reason to give credit. CombiHighBoth seemed like it may have been the best overall technique to use if I were constrained to only using one, but since my objective in creating these two loose and strict composite measures is to establish the most reasonable upper and lower boundaries of detection rates, the compromising, inclusive nature of the CombiHighBoth definition was not as definitive and rigorous as I wanted the components of the strict standard composite to be, while the CombiHighYes standard was exactly that.

The two criteria levels for the multiple choice questions (loose: counting only those 81 participants who got at least one multiple choice question correct by providing both the consistent and the inconsistent answers vs. strict: counting all 100 participants) are so named for their effect on overall detection rates (with loose leading to higher overall percentage of detections, and strict leading to a lower overall percentage), not the overall "standard for inclusion as an inconsistency detection", which would have resulted in the opposite naming scheme. Similarly, it is important to keep this distinction in mind for the point blank criteria naming convention (loose: counting both those participants who got the question correct as well as those who chose the incorrect option as a reason for finding the witness's testimony unconvincing — essentially everyone who did not select "I don't agree with either of these jurors" vs. strict: counting only those participants who got the question correct).

I applied the same reasoning to the names of the two composite scoring schemes, such that the Strict scheme will provide the lowest overall percentage of detections, but 
the detection rates it yields for each inconsistency will be the unequivocal minimum levels of detection. The Strict criteria level averages Open Ended CombiHighYes, Short Answer CombiHighYes, all 100 Multiple Choice responses, True/False, and Point Blank. On the other end of the spectrum, the Loose scheme will provide the highest overall percentage of detections according to the most forgiving definitions of inconsistency detection. The Loose criteria level averages Open Ended CombiMed, Short Answer CombiMed, Multiple Choice only 81 (using the Multiple Choice responses from only those 81 participants who got at least one multiple choice question correct, in percentage equivalent), True/False, and Point Blank Combined (counting detections for all participants who did not choose "I don’t know.") 
Table M1

Study One: Loose Scoring Scheme Detection Rate Percentages for all 15 Inconsistencies by Question Type

\begin{tabular}{|c|c|c|}
\hline Ope & Short & Multiple \\
\hline Ended & Answer & Choice 81 \\
\hline
\end{tabular}

\begin{tabular}{lcccccc}
\multicolumn{1}{c}{ Inconsistency } & CombiMed & CombiMed & non-0's & False & Combined & Score \\
\hline PI 7-Hair & 43.0 & 38.0 & 54.3 & 62.0 & 65.0 & $52.5^{*}$ \\
PI 4-Oil Co. & 27.0 & 33.0 & 56.8 & 54.0 & 60.0 & $46.1^{*}$ \\
PI 5-Model & 38.0 & 25.0 & 39.5 & 55.0 & 61.0 & $43.7^{*}$ \\
PI 12-Tree & 29.0 & 13.0 & 42.0 & 42.0 & 77.0 & $40.6^{*}$ \\
PI 13-Dog & 6.0 & 25.0 & 49.4 & 52.0 & 65.0 & $39.5^{*}$ \\
PI 11-Jared & 32.0 & 7.0 & 42.0 & 46.0 & 65.0 & $38.4^{*}$ \\
PI 9-Year & 16.0 & 30.0 & 30.9 & 48.0 & 67.0 & $38.4^{*}$ \\
PI 10-Color & 23.0 & 20.0 & 37.0 & 47.0 & 63.0 & $38.0^{*}$ \\
Mean Rate & 16.5 & 15.0 & 31.4 & 45.7 & 62.9 & 34.3 \\
PI 3-Swerve & 17.0 & 3.0 & 7.4 & 64.0 & 64.0 & 31.1 \\
PI 2-1/2 vs 3/4 & 0.0 & 2.0 & 12.3 & 64.0 & 53.0 & 26.3 \\
PI 15-Stop Sign & 8.0 & 4.0 & 24.7 & 33.0 & 57.0 & 25.3 \\
PI 6-R/L leg & 2.0 & 3.0 & 7.4 & 46.0 & 65.0 & 24.7 \\
PI 8-Arrival & 1.0 & 10.0 & 23.5 & 25.0 & 62.0 & 24.3 \\
PI 1-Side of Car & 3.0 & 3.0 & 27.2 & 25.0 & 60.0 & 23.6 \\
PI 14-Cop & 3.0 & 9.0 & 17.3 & 23.0 & 60.0 & 22.5
\end{tabular}

Note. *Inconsistencies 4, 5, 7, 9, 10, 11, 12, and 13 will be retained and used in Studies Two, Four, and Five. Inconsistencies 1, 2, 3, 6, 8, 14, and 15 will be redacted from the Johnson v. McGraw trial transcript to be used in Studies Two, Four, and Five. 
Table M2

Study One: Strict Scoring Scheme Detection Rate Percentages for all 15 Inconsistencies by Question Type

\begin{tabular}{|c|c|c|c|c|c|c|}
\hline Inconsistency & $\begin{array}{c}\text { Open Ended } \\
\text { CombiHighYes }\end{array}$ & $\begin{array}{l}\text { Short Answer } \\
\text { CombiHighYes }\end{array}$ & $\begin{array}{l}\text { Multiple } \\
\text { Choice }\end{array}$ & $\begin{array}{l}\text { True } \\
\text { False }\end{array}$ & $\begin{array}{l}\text { Point } \\
\text { Blank }\end{array}$ & $\begin{array}{l}\text { Strict } \\
\text { Score }\end{array}$ \\
\hline PI 7-Hair & 43.0 & 32.0 & 44.0 & 62.0 & 55.0 & $47.2^{*}$ \\
\hline PI 5-Model & 36.0 & 22.0 & 32.0 & 55.0 & 55.0 & $40.0^{*}$ \\
\hline PI 4-Oil Co. & 26.0 & 23.0 & 46.0 & 54.0 & 50.0 & $39.8^{*}$ \\
\hline PI 12-Tree & 26.0 & 12.0 & 34.0 & 42.0 & 68.0 & $36.4^{*}$ \\
\hline PI 13-Dog & 6.0 & 19.0 & 40.0 & 52.0 & 51.0 & $33.6^{*}$ \\
\hline PI 11-Jared & 31.0 & 5.0 & 34.0 & 46.0 & 46.0 & $32.4 *$ \\
\hline PI 10-Color & 22.0 & 17.0 & 30.0 & 47.0 & 46.0 & $32.4^{*}$ \\
\hline PI 9-Year & 13.0 & 20.0 & 25.0 & 48.0 & 48.0 & $30.8^{*}$ \\
\hline Mean Rate & 14.9 & 10.8 & 25.5 & 45.7 & 46.0 & 28.6 \\
\hline PI 3-Swerve & 10.0 & 1.0 & 6.0 & 64.0 & 39.0 & 24.0 \\
\hline PI 6-R/L leg & 2.0 & 1.0 & 6.0 & 46.0 & 48.0 & 20.6 \\
\hline PI 15-Stop Sign & 3.0 & 3.0 & 20.0 & 33.0 & 42.0 & 20.2 \\
\hline PI $2-1 / 2$ vs $3 / 4$ & 0.0 & 1.0 & 10.0 & 64.0 & 24.0 & 19.8 \\
\hline PI 8-Arrival & 0.0 & 3.0 & 19.0 & 25.0 & 48.0 & 19.0 \\
\hline PI 1-Side of Car & 3.0 & 2.0 & 22.0 & 25.0 & 37.0 & 17.8 \\
\hline PI 14-Cop & 3.0 & 1.0 & 14.0 & 23.0 & 33.0 & 14.8 \\
\hline
\end{tabular}

Note. *Inconsistencies 4, 5, 7, 9, 10, 11, 12, and 13 will be retained and used in Studies Two, Four, and Five. Inconsistencies 1, 2, 3, 6, 8, 14, and 15 will be redacted from the Johnson v. McGraw trial transcript to be used in Studies Two, Four, and Five. 


\section{APPENDIX N}

\section{Studies Two and Four Trial Transcript (8 Inconsistencies)}

NOTE: Highlighted phrases (e.g. PI-Plain Inconsistency, S\&B-Inconsistency used in Semmler \& Brewer [2002], etc.) denote details of testimony relevant to the eight inconsistencies, and are only marked here for ease of identification. This emphasis was not included in the actual materials used.

\section{Johnson v. McGraw}

The following trial transcript is an abridged version of a real trial from here in the state of Florida. The trial, which has already taken place, and for which the jury has already rendered a verdict, involved a traffic accident which resulted in injury to the plaintiff, Amanda Johnson, an injury she alleged was the result of negligence on behalf of the defendant, Michael McGraw. Although the circumstances of the accident involved multiple parties, Michael McGraw was the only defendant in this case.

While the names of all parties have been changed, one of the attorneys has asked FIU researchers to conduct this study to see how real people like you perceive the case so that they can determine whether to appeal the outcome of the trial. We will be using the data from this study to help the attorney, so please pay close attention as it will impact the final resolution of an actual civil case.

Please listen to the trial transcript and read along as if you had been chosen to serve as a juror in this case.

Judge Robert C. Underwood: Ladies and gentlemen of the jury, you are about to hear testimony concerning an automobile accident that occurred in the late afternoon of Tuesday, July $8^{\text {th }}, 2008$ in the city of Clermont, located in Lake County, Florida. The plaintiff is Amanda Johnson, and the defendant is Michael McGraw. There are no other plaintiffs or defendants in this suit, and all other disputes between these and any other parties related to the events in question have been resolved separately prior to this proceeding.

\section{Opening Statement by Seth Blum, Attorney for the Plaintiff}

Seth Blum, Attorney for the Plaintiff: Ladies and gentlemen of the jury, what we have before us today is an unfortunate story of a young lady who was simply going about her business one day, on the afternoon of Tuesday, July $8^{\text {th }}, 2008$, up in Clermont, which is in central Florida to the west of Orlando. This young lady, Amanda Johnson, was at the time a 26 year old waitress at a family restaurant. She was riding in the car with her 
boyfriend, Brock Collins, a 28 year old middle school teacher. They were in his blue Toyota Camry (PI 5-Model true)on their way to catch a movie before meeting up with Amanda's parents for dinner later that night. Unfortunately, their plans were dashed by the negligence of another driver on the road that evening, Michael McGraw.

Throughout this case, you are going to hear overwhelming evidence from a variety of witnesses-Amanda Johnson, Brock Collins, a host of different eyewitnesses to the accident, the emergency first responders on the scene, medical professionals, as well as professional scientists who specialize in the analysis of vehicles and crash scenes which they use to reconstruct accidents. The testimony of all these witnesses will show you, with no uncertainty whatsoever, that Mr. McGraw, and only Mr. McGraw—not Mr. Collins, not the driver of the semi truck, not anybody else and not anything else other than Mr. McGraw's negligent operation of his big, white (PI 10 color true) van-was the direct cause of Amanda Johnson's serious injury. I hope that you find him liable for all damages suffered by Ms. Johnson at his hand. Thank you very much for your time, service, and attention today.

\section{Opening Statement by Howard Kurtz, Attorney for the Defendant}

Howard Kurtz, Attorney for the Defendant: Esteemed members of the jury-like my colleague, I thank you in advance for your attention, effort, and diligent fulfillment of your civic duty. What we can all agree on is that you are here today to hear about a traffic accident that injured a nice young lady and her boyfriend. Everybody wishes that this accident hadn't happened, but not everybody agrees about how it happened or how Ms. Johnson ended up being hurt.

Ms. Johnson and her attorney would like for you to believe that her broken hip and other injuries are the result of negligence on the part of Mr. McGraw. Now it's certainly true that Mr. McGraw, while trying to avoid the out of control semi tractor trailer coming towards him, ended up hitting Mr. Collins's car. What we're less certain about is how Ms. Johnson was injured. You see, Ms. Johnson wasn't wearing her seat belt, and we'll be presenting evidence to you that will show that Ms. Johnson wasn't injured because Mr. McGraw hit their car, but that she was injured when the car ran into the street lamp, and that if she had been wearing a seat belt, she wouldn't have broken her hip.

Ms. Johnson and her attorney would like for you to believe that her injuries are the fault of Mr. McGraw, but through the course of this trial, you will soon see that they don't have any real evidence to support this allegation. Because they can't show that the majority of the evidence undoubtedly proves that Mr. McGraw was the cause of Ms. Johnson's injuries, you will come to the conclusion that Michael McGraw was not negligent, was not the direct cause of Amanda's broken hip, and that he should not be held liable for her injuries and you should render a verdict in favor of the defendant. 
Plaintiff's Witness Marc Zollinger, Direct Examination by Seth Blum, Attorney for the Plaintiff

Seth Blum, Attorney for the Plaintiff (Questioner): Can you please state your name and occupation for the court?

Marc Zollinger (Answerer): My name is Marc Zollinger, and I'm an administrative assistant for Universal Translation Corporation.

Q: And where is your office located?

A: It's on Foothill Drive, pretty close to the intersection of Foothill and Windsor, a little south of there, on the east side, or I guess northeast side of the road at that point, down by the bend.

Q: Tell me, Mr. Zollinger, what were you doing on the afternoon of Tuesday, July $8^{\text {th }}$, 2008 ?

A: I needed some fresh air, so I went to the little walk-up coffee window a few doors down from our office, the one on the north side of the Cuban restaurant there.

Q: Okay. And while you were there, did anything unusual happen?

A: As a matter of fact, yes. While I was waiting in line, I was just standing around watching the cars go by when there was a big traffic accident behind me up the way a little bit at the corner.

Q: Up the way a little bit? How far do you mean? Help the jury to understand how close you were.

A: Oh, not that far really. Probably a few hundred feet away, but I had a pretty clear view since it's just the parking lot for the restaurant there to the north of the coffee window up to the corner.

Q: And can you tell us what you saw?

A: I saw a car, a van, and a tanker truck get into a wreck. The tanker truck was kind of sideways, coming southbound towards the intersection and kind of weaving back and forth a little bit in the middle of the road. I saw Mr. Collins's car, a blue Corvette (PI 5model false)make a left turn to go west on Windsor to get out of the way of the truck, but then Mr. McGraw's white (PI 10 color true)Ford work van came screeching around, and then the semi locked up its brakes and the tires started squealing but the tanker trailer swung around and kind of batted Mr. McGraw's van on the passenger side which then bounced into the back of Mr. Collins's car which then crashed into the street light (PI 12 
true) on the northwest corner of the intersection. It was like one big chain reaction. They almost hit some lady walking a Dachshund. (PI 13 false)

Q: And what happened next?

A: I saw the guy with the black hair (PI 7 true), Mr. McGraw, get out of his van to make sure everyone in Mr. Collins's car was okay or something. I couldn't hear what they were saying or anything, but right away Mr. McGraw got on his phone, I guess to call 911. After a minute or two, Mr. Collins, the bald one, got out of the car and went around to the passenger side and was talking to someone in the passenger seat. He looked pretty worried based on all his movements and body language.

Q: And then what?

A: I placed my order, and by the time I got my coffee I could see that a police car was already on the scene so I grabbed my coffee and went over to tell him what I saw. I told him exactly how it happened, and he wrote it all down. The officer's name was Christopher Eaton. He was a really big guy.

Q: Thank you, Mr. Zollinger. No further questions.

Plaintiff's Witness Marc Zollinger, Cross Examination by Howard Kurtz, Attorney for the Defendant

Howard Kurtz, Attorney for the Defendant (Questioner): Could you describe for us in just a little more detail exactly what happened after all the vehicles came to a stop?

Marc Zollinger (Answerer): Sure. Mr. McGraw got out of the white (PI 10 color true)van pretty quickly and immediately ran over to Mr. Collins's blue Corvette, (PI 5model false) over to the driver's side. I couldn't hear them, of course, but it looked to me like he was making sure everyone was okay, and then shortly after talking to Mr. Collins, McGraw got his cell phone out of his pocket and made a call. A minute or two later, the police officer arrived, then I went over and told him what I saw, and then I went back to work.

Q: Thank you, Mr. Zollinger. I have nothing further.

Plaintiff's Witness Barbara Feldman, Direct Examination by Seth Blum, Attorney for the Plaintiff

Seth Blum, Attorney for the Plaintiff (Questioner): Hello there. Could you please state your name and occupation for the record? 
Barbara Feldman (Answerer): My name is Barbara Feldman, and I am a librarian.

Q: Thank you Ms. Feldman. Now you're familiar with the day in question here in this trial, right?

A: Yes I am.

Q: And did you witness the accident at issue here?

A: Yes I did. I remember it all quite vividly. I was driving southbound on Foothill Drive, north of the intersection with Windsor.

Q: And could you please describe for us what you saw?

A: Sure. As I was driving, up ahead I noticed a Shell (PI 4/S\&B 6 true) oil tanker trailer crossing over the line separating the two directions of traffic, kind of skidding its tires and fishtailing around. The stop light ahead of us was green, so I'm not sure what the deal was. It's not like he was slamming on his brakes to keep from running the light or anything. It was also driving southbound like I was, so I slowed down to stay away from the situation. It was all over the place in the left turn lane in the middle, going back and forth into the oncoming lanes of traffic and stuff.

Q: And what happened next?

A: Well, the truck's trailer sort of swung out into the intersection, where it hit Mr. McGraw's tan van, (PI 10 color False) kind of side swiping it and kind of just smacking it on the passenger side, which forced the van into the back side of Mr. Collins's blueToyota Camry (PI 5-model true), which then crashed into a light pole (PI 12 true)on the northeast corner of the intersection.

Q: Okay, and what about after the accident?

A: I just stayed around until the police officer arrived, and once he got there, I gave him my statement and my contact information, and then I left to go home.

Q: Thank you Ms. Feldman. I have no more questions.

Plaintiff's Witness Barbara Feldman, Cross Examination by Howard Kurtz, Attorney for the Defendant

Howard Kurtz, Attorney for the Defendant: I have no questions for this witness, your Honor. 
Plaintiff's Witness Jill Randall, Direct Examination by Seth Blum, Attorney for the Plaintiff

Seth Blum, Attorney for the Plaintiff (Questioner): Could you please give us your name and occupation?

Jill Randall (Answerer): My name is Jill Randall, and I sell Amway products from my home here in Clermont.

Q: Now could you please tell us about what you saw on Tuesday, July $8^{\text {th }}, 2008$ ?

A: I was at the intersection waiting for the light to change so that I could cross Foothill heading west, and there was a semi truck that came out of nowhere from the north, skidding and screeching and swerving all over the place out of control.

Q: That must have been pretty scary. Can you tell us more about the truck and what happened?

A: Well, I saw the truck blow the light going through the intersection kind of in the middle of the southbound and northbound lanes, and he was fishtailing a little bit, you know, the cab of the truck going in one direction while the trailer was slipping and sliding around behind him in another direction. The truck swerved towards me, to the east, to the driver's left, past me as it came through the intersection. As the truck was getting itself under control, I could tell that there was some sort of additional accident on the opposite side of the trailer involving some other vehicles, but since I was on the east side of the trailer and that was all happening on the west side, I really couldn't see too much about what exactly happened.

Q: Okay, so you weren't able to see much of the actual contact between the vehicles?

A: Well, no, not of the actual contact when it was happening, but after everything came to a stop, I was able to see that the white (PI 10 color true)van driven by Mr. McGraw had crashed into the back of the other car, the one driven by Mr. Collins, the blue Toyota Camry, (PI 5-model true) and Mr. Collins's car was up against the street lamp(PI 12 true). It looked like it had pretty much hit the lamppost (PI 12 true)head on, and Mr. McGraw's van was stopped behind it, and it was all banged up on the passenger side.

Q: Well it sounds like you got a good look at the aftermath of the accident. What happened after everything came to rest?

A: The red-haired man (PI 7 false), Mr. McGraw got out of his van pretty quickly and went to go check on the people in Mr. Collins's car. He was talking to Mr. Collins for a 
minute, and then he got out his phone and made a call, I assume to 911. Mr. Collins got out of his car eventually, and when the police officer arrived a minute or two later, he was a real hunk, so I hung around and gave him my statement once the paramedics got there to take over making sure everyone got the medical attention they needed.

Q: We appreciate your testimony here today, Ms. Randall. No further questions.

Judge Robert C. Underwood: Your witness, Mr. Kurtz.

Plaintiff's Witness Jill Randall, Cross Examination by Howard Kurtz, Attorney for the Defendant

Howard Kurtz, Attorney for the Defendant (Questioner): So Ms. Randall, you weren't able to describe exactly what happened during the wreck itself because the trailer from the tanker truck was blocking your view, is that right?

Jill Randall (Answerer): That's right. I saw the beginning and I saw the aftermath, but I didn't see the actual accident between Mr. McGraw and Mr. Collins, no.

Q: I see. Thank you very much, Ms. Randall. I don't have any more questions.

Plaintiff's Witness Steve Powell, Direct Examination by Seth Blum, Attorney for the Plaintiff

Seth Blum, Attorney for the Plaintiff (Questioner): Could you please give your name and occupation for the record?

Steve Powell (Answerer): My name is Steve Powell, and I'm a real estate agent here in Clermont.

Q: And where were you on the afternoon of Tuesday, July $8^{\text {th }}, 2008$ ?

A: I was at the post office located there on the east side of Foothill Drive, a little ways up from, I mean north of, the intersection with Windsor Road.

Q: Okay. And did you see anything unusual at the post office?

A: Well, just as I was leaving, I was looking out the window and I saw a Shell (PI 4 true/S\&B 6 true) tanker truck go skidding down the street past the post office.

Q: Down Foothill you say?

A: Yeah, it was traveling south but kind of screeching and sliding out of control. 
Q: And what did you see after that?

A: Well, I didn't really see exactly what happened next. I was walking out of the building and didn't have a constant, uninterrupted view so I missed the actual crash, but after I got outside, I saw the whole accident scene with the tanker truck stopped at a funny angle over on the east side of Foothill on Windsor, and the van and the car over on the corner by the Zales(PI 11 true) jewelry store. The guy with the black hair (PI 7 true), Mr. McGraw, was standing outside the vehicles. There were a couple of people in the car, the blue Camry, (PI 5-model true) and they looked kind of hurt.

Q: How so?

A: The bald guy, Mr. Collins, had some cuts and stuff on his forehead and he was bleeding a little bit, but the lady in the passenger seat was much worse. It was kind of hard to see exactly how she was hurt, but it was clear she was pretty banged up.

Q: And can you tell us what happened next?

A: I had an important meeting I had to get to a little later on that afternoon so I didn't have time to stick around, and I don't know how I could have helped anyway, but as I was starting to walk back to my car, the bald guy, Mr. Collins, was out of his car and the police officer had showed up so I figured it would be okay now that there was an official emergency responder on the scene. Right as I got to my car, which was parked over in the lot north of the coffee shop on the southeast corner of the intersection, I saw the ambulance arrive next, so I figured I might as well go over to the police officer and tell him what I had seen now that medical personnel were there before I left to go to my meeting. As I was giving Officer Eaton my statement, I could see the paramedics getting Ms. Johnson out of the car and loading her into the ambulance. It was at that time that I could see that her right leg was all mangled and busted up.

Q: Thank you for your time, Mr. Powell. I don't have any more questions for you.

Plaintiff's Witness Steve Powell, Cross Examination by Howard Kurtz, Attorney for the Defendant

Howard Kurtz, Attorney for the Defendant (Questioner): Mr. Powell, you were inside the post office when the actual accident occurred, and were unable to see exactly what happened at the intersection, is that right?

Steve Powell (Answerer): Yeah, I just saw the Shell (PI 4/S\&B 6 true) truck go past the window out of control, but no, I couldn't see the intersection or anything that far down from where I was. 
Q: So you don't have any idea exactly how any of the different vehicles crashed into each other, or the exact moment in which Ms. Johnson might have been hurt, or how she was hurt, or anything specific like that, correct?

A: That's correct. I just saw the truck go by and then by the time I was outside, I saw the wreckage and stuff where it had all come to rest.

Q: So basically everything at issue here happened when you couldn't see it?

A: Well I did see two men, Mr. Collins and Mr. McGraw outside talking to each other when the police officer arrived at the time I was walking across Windsor, and then by the time I got to my car, I saw the ambulance had just arrived too.

Q: Thank you, Mr. Powell. No further questions.

Plaintiff's Witness Lesley Jobin, Direct Examination by Seth Blum, Attorney for the Plaintiff

Seth Blum, Attorney for the Plaintiff (Questioner): Hello there, could you please tell us your name, occupation, and city of residence for the record?

Lesley Jobin (Answerer): My name is Lesley Jobin, I'm a tour group coordinator, and I live here in Clermont.

Q: And what were you doing on the afternoon of Tuesday, July $8^{\text {th }}, 2008$ ?

A: Well, since there's really no slow days at my company because of all the visitors to the area and different tourist attractions and stuff in and around Orlando, we're open seven days a week, and I'm off on Mondays and Tuesdays each week. That was a Tuesday, and I was out walking Finnegan, my German Shepherd. (PI 13 true)

Q: And can you tell us a little more about your walk with Finnegan that day?

A: Sure. I was walking west along Windsor Road, having just crossed Foothill. I was passing in front of the Jared jewelry store (PI 11 false) there at the corner, when the crash happened.

Q: So can you tell us what you saw?

A: Well, I saw the Shell (PI 4/S\&B 6 true) truck fishtailing out of control, well, I guess there isn't any way for a truck to fishtail but still be under control, but anyway, it was sort of sliding out toward its right side, and then it kind of smacked the side of the white (PI 10 color true)van, and that's when the van crashed into the back of the blue Toyota 
Camry, (PI 5-model true) which then ran straight into a tree (PI 12 false) on the corner in front of Jared(PI 11 false).

Q: Were you able to see anything that happened inside of either of the vehicles?

A: Yeah, when Mr. McGraw first hit Mr. Collins's car, I could see that both Mr. Collins and Ms. Johnson's heads went flying forward into the windshield.

Q: Thank you Ms. Jobin. I don't have any more questions for you.

Plaintiff's Witness Lesley Jobin, Cross Examination by Howard Kurtz, Attorney for the Defendant

Howard Kurtz, Attorney for the Defendant (Questioner): So Ms. Jobin, you were out walking your German Shepherd, (PI 13 true) Finnegan, that afternoon, is that correct?

Lesley Jobin (Answerer): That's right.

Q: And were you talking on the phone, or listening to music, or anything like that on your walk?

A: Well, I was listening to my iPod.

Q: Hmm. Okay. And you were walking west, passing the Zales(PI 11 true) store when you heard a crash and turned around to look, is that right?

A: Yeah, Finnegan and I were both really startled, and we ran backwards a little bit just as Mr. Collins's car hit the tree. (PI 12 false)

Q: I see. Thank you very much, Ms. Jobin.

Plaintiff's Witness Brock Collins, Direct Examination by Seth Blum, Attorney for the Plaintiff

Seth Blum, Attorney for the Plaintiff (Questioner): Hello there, could you please state your name, occupation, and city of residence for the record?

Brock Collins (Answerer): My name is Brock Collins, I'm a teacher at Windy Hill Middle School here in Clermont where I live.

Q: And just so we're clear, what is your relationship with the plaintiff in this case, Amanda Johnson? 
A: She's my girlfriend. We've been dating for a couple of years.

Q: And could you tell us a little bit about what happened on the afternoon of Tuesday, July $8^{\text {th }}, 2008$ ?

A: Well, Amanda and I were on our way to see a matinee before going out to dinner with her folks later on, when we got into a big wreck at the corner of Windsor and Foothill.

Q: Can you tell us about what you remember happening before the accident and how it all unfolded?

A: Well, I was driving my car, a blue 2006 (PI 9-year true)Toyota Camry (PI 5-model true)four door, northbound on Foothill Drive, and Amanda was in the passenger seat. We were coming around the bend, right where Foothill goes from kind of east/west to north/south, and once I got around the bend approaching the intersection, we had the green light, and I saw a semi truck skidding out of control towards us.

Q: Sounds pretty scary. Can you describe how you reacted to the situation and what happened next?

A: Yeah, it was really crazy to see a big truck barreling down on us like that. It took me a second to figure out what to do because I couldn't tell which way the truck was going to go or what was going to happen next. I kind of slowly turned to my left, to go west on Windsor because I was pretty sure that the truck was going to end up going to my right, east on Windsor. Once I could tell for sure that that's where he was headed, I was able to pull out of his way just barely fast enough to miss the end of his tanker trailer whipping around the corner behind him. It was a real relief to miss it, but the relief was very short lived.

\section{Q: How so?}

A: Well, the guy behind me in the white (PI 10 color true)Ford panel van wasn't as lucky. I guess he tried to get out of the way of the swinging trailer much the same way as I did, but he just ended up rear-ending us pretty much straight on, and our heads hit the windshield, and we then crashed into the street lamp (PI 12 true) on the northwest corner of the intersection, all pretty much at the same time as his van was getting hit by the trailer. I got dinged on the head pretty good but wasn't that messed up, no permanent scars or damage or anything, but Amanda was really hurt badly.

Q: She certainly was. I'm really sorry you guys had to go through all of that. Thank you, Mr. Collins. I don't have any more questions for you. 
Plaintiff's Witness Brock Collins, Cross Examination by Howard Kurtz, Attorney for the Defendant

Howard Kurtz, Attorney for the Defendant (Questioner): So, Mr. Collins, are you and Ms. Johnson still together?

Brock Collins (Answerer): Yes, we are. We're starting to talk about marriage, in fact.

Q: Oh, how nice! Let's talk a little more about your car that you were driving that day. It was a blue 2006 (PI 9-year true) four-door Toyota Camry (PI 5-model true), isn't that right?

A: Yes sir.

Q: And how long had you owned that car, Mr. Collins?

A: I had it for a little less than a year before the accident, but my dad bought it brand new, and when he got tired of it after a year or so (PI 9-year true), he sold it to me at a good price since I was in need of a reliable car.

Q: So you're familiar with the entire two-year (PI 9-year true) history of the vehicle. Tell me, Mr. Collins, had the car ever been in any sort of accident before?

A: Except for maybe tapping a bumper when parallel parking in a tight spot or something tiny like that, no, it hadn't.

Q: It worked okay, pretty reliable car?

A: Certainly. Wasn't too flashy, but it was definitely reliable.

Q: And did the seatbelts in the car work properly?

A: Well, they got stuck when pulling them out a lot, so it was kind of a pain to put them on all the time.

Q: And were you wearing your seatbelt on the day of the accident?

A: No, I wasn't.

Q: Why not?

A: We weren't going very far or anything, so I guess I just figured I didn't need to mess with it.

Q: And was Ms. Johnson wearing her seat belt that day? 
A: No.

Q: Nothing further, Your Honor.

Plaintiff's Witness Dr. Matthew Freeman, Direct Examination by Seth Blum, Attorney for the Plaintiff

Seth Blum, Attorney for the Plaintiff (Questioner): Hello, could you please give us your name, city of residence, and occupation?

Dr. Matthew Freeman (Answerer): My name is Matthew Freeman, I live in Jacksonville, Florida, and I am an accident reconstruction specialist.

Q: What exactly does that mean?

A: I examine vehicles, accident sites, traffic patterns, road conditions, medical records, and that sort of thing in order to figure out exactly how an accident occurred.

Q: Thank you. And, just to avoid any confusion, you are being paid to discuss your findings with us here today, isn't that right?

A: Yes, I'm being paid by the plaintiff's side to share my conclusions. What I do is complex and time consuming, and after all, this is my job. But I assure you that my determinations are in no way shaped by anything besides the evidence.

Q: I appreciate your candor and your earnestness, Dr. Freeman. So can you tell us what you found with regard to the accident involving Mr. Whitaker's Shell (PI 4/S\&B 6 true) tanker truck, Mr. Collins's Camry (PI 5-model true) and Mr. McGraw's Ford Econoline work van?

A: Given where we know the truck stopped just beyond the intersection itself, Mr. Whitaker couldn't have been going very fast at the time his trailer sideswiped Mr. McGraw's van. That means the impact from the trailer onto Mr. McGraw's van wasn't the determining factor in Ms. Johnson's injuries, but rather Mr. McGraw's driving itself was. If Mr. McGraw hadn't been driving so fast when the truck hit him, then Amanda wouldn't have been injured when he hit Mr. Collins's car. Besides, if he had been going slower, he wouldn't have been in the intersection anyway and the accident wouldn't have happened at all.

Q: Interesting. And what about your conclusions based on inspecting Mr. Collins's vehicle? 
A: Well, it was a 1986 (PI 9-year false) Toyota Camry, (PI 5-model true) four doors, blue, base model, and no aftermarket or custom modifications. It was damaged uniformly across the rear from Mr. McGraw's van hitting it, the front was damaged more deeply, but in a narrower span, consistent with hitting a tall, thin object like a street lamp pole (PI 12 true), and while the seat belts appeared to be in reasonable working order, I suspect that the airbag deployment sensors may have been faulty. For as hard as Mr. McGraw hit the car, the airbags should have inflated. But because they didn't, it is my opinion that Ms. Johnson broke her right hip as a direct result of that first impact with Mr. McGraw to their rear, not the second impact with the street lamp post. (PI 12 true) Unfortunately, due to a miscommunication between Mr. Collins's insurance company and the salvage yard where the car was being stored after the accident, shortly after I had done my preliminary inspection of the car, it was disassembled for parts before I had a chance to check the status of the airbag sensors to verify that they were faulty.

Q: Thank you for your expertise, Dr. Freeman. I have nothing further to ask.

\section{Plaintiff's Witness Dr. Matthew Freeman, Cross Examination by Howard Kurtz, Attorney for the Defendant}

Howard Kurtz, Attorney for the Defendant (Questioner): Tell me, Dr. Freeman, do you have any medical training or licensure?

Dr. Matthew Freeman (Answerer): No, I hold a doctorate degree in mechanical engineering, as well as numerous other certifications related to my field, but none in health-related areas, no.

Q: And yet you're willing to testify under oath that Amanda's broken hip was a result of the first impact, when my client hit Mr. Collins's car, and not that it was from the headon collision with the lamp pole. (PI 12 true)Very interesting conclusions, Dr. Freeman. No further questions.

Plaintiff's Witness Amanda Johnson, Direct Examination by Seth Blum, Attorney for the Plaintiff

Seth Blum, Attorney for the Plaintiff (Questioner): Hello, Ms. Johnson. How are you doing today?

Amanda Johnson (Answerer): Oh, just fine. It feels good to finally have my day in court.

Q: I bet it does. How is your hip? 
A: It's healed up pretty well so far, but it's not back to normal by any means. I'm not sure whether it will ever be like it was before the accident. Still, I'm finally able to walk without a limp or the use of a cane, but I can't run or do anything strenuous, and it really acts up during changes in barometric pressure and other weather changes. I feel like an old person, and I'm only 28 .

Q: How has your injury affected your life?

A: Well, at first, it cost me my job, since I was in the hospital for a couple of weeks at first and then unable to walk for a long time after that, and it's pretty difficult to be a waitress if you can't walk.

Q: Can you tell us a little more about your injury and the treatment you received?

A: Sure. My right hip was broken just under the ball of my femur, the long bone at the top of your leg. It required immediate surgery and a metal plate and a bunch of screws to be screwed into both halves of my legbone so that the two parts of the bone would grow back together again. That surgery left me in the hospital for about a week, and I was under pretty heavy sedation for the pain, and then I had to stay in the rehabilitation center for another week before I was able to go home. I was in the wheelchair for another month before I was able to use a walker or crutches, and then it was another six weeks before I was able to walk short distances again. That whole time I was going to rehab three times a week and doing exercises every day at home too.

Q: And how are you doing today, a little over two years since the accident occurred?

A: I'm doing alright. Like I mentioned earlier, I can walk pretty much without a limp, but I still can't do anything really active or athletic that requires running around or anything like that. I'm able to sleep though the night okay now, and the pain doesn't bother me all that often except when the weather is about to change, which, unfortunately for me, seems to happen all too often here in central Florida. The doctors tell me that's never going to go away.

Q: Well, I guess at least you'll always know whether to bring an umbrella with you when you leave the house in the mornings. Now let's talk a little bit about the accident itself.

A: Okay.

Q: When you first saw the truck, where was it in relation to the car you were in?

A: It was directly in front of us, a little to the right of the center of our car.

Q: Was the truck over the double yellow lines in the road that separate the two directions of travel? 
A: Yes.

Q: In your estimation, how far over the double yellow lines?

A: It was about three-quarters of the way over into our lanes of traffic, so far that I didn't think we'd be able to swerve around it.

Q: And did the truck appear to swerve or deviate from its course at any time before the accident?

A: Yes, it did, it swerved to our right and ended up going off to our right, to the east.

Q: About how fast would you guess that you guys were going?

A: Not that fast. Probably 25 miles an hour or so.

Q: And at what speed would you estimate that the truck was going?

A: It had to have been over 40 miles per hour. We had slowed down, but it still came towards us very quickly and all we could see was the giant Shell (PI 4/S\&B 6 true element of contradiction) tanker trailer swerving towards us.

Q: And then what happened?

A: Brock sort of hesitated for a quick second to figure out which direction was the safest way to get out of the way of the truck since we didn't really know where it was going to end up, and after he quickly turned to the left, to go west down Windsor, we saw the tail end of the trailer go sliding past us and we thought we had managed to escape an accident.

Q: But that wasn't the case, was it?

A: No, even though we barely missed colliding with the tanker, we were then suddenly hit from behind by Mr. McGraw, who had himself been hit on his passenger's side by the trailer and knocked into us, and that's when I went flying forward, hitting my head on the windshield and smashing into the dashboard and breaking my right hip, and then we hit the street lamp post (PI 12 true) which caused us to finally come to a stop.

Q: So you were fine up until the point when Mr. McGraw crashed into you, is that right?

A: Yeah, if he didn't hit us, I wouldn't have been hurt.

Q: Thank you, Ms. Johnson. 
Plaintiff's Witness Amanda Johnson, Cross Examination by Howard Kurtz, Attorney for the Defendant

Howard Kurtz, Attorney for the Defendant (Questioner): Ms. Johnson, I'd like to ask you a few more questions about the events leading up to the accident and the details of the accident itself, if that's okay.

Amanda Johnson (Answerer): Of course.

Q: Ms. Johnson, could you please tell us exactly when you first noticed that the truck was on the wrong side of the road?

A: Before we got to the intersection, I could see that it was on the wrong side of the road. I could see the paint of the Exxon (PI 4/S\&B 6 false element of contradiction) logo and the chrome tank shining in the sun.

Q: And you are sure you could tell that the truck was on the wrong side of the road?

A: Yes, I'm sure.

Q: Ms. Johnson, if you saw the truck was on the wrong side of the road, why didn't you warn Mr. Collins?

A: I don't know. It happened very quickly. It was almost three quarters of the way over onto our side of the road, swerving to our right.

Q: Hmm. I see. Thank you, Ms. Johnson. No further questions.

Seth Blum, Attorney for the Plaintiff: Your Honor, we have no further witnesses to introduce, so the plaintiff rests.

Judge Robert C. Underwood: Thank you Mr. Blum. Mr. Kurtz, you may now present the defense's case.

Howard Kurtz, Attorney for the Defendant: Thank you, Your Honor.

Defense Witness Tom Gorham, Direct Examination by Howard Kurtz, Attorney for the Defendant

Howard Kurtz, Attorney for the Defendant (Questioner): Hello, could you please state your name, city of residence, and occupation for the record? 
Tom Gorham (Answerer): My name is Tom Gorham, I live in Orlando, and my wife and I own and operate a three-dimensional film and photography studio.

Q: How interesting! That must be a really exciting business for you two to be in. Now, could you please tell us where you were on the afternoon of Tuesday, July $8^{\text {th }}, 2008$ ?

A: I was stopped at the light at the corner of Windsor and Foothill, waiting to cross Foothill to the east, and I saw this whole big accident unfold.

Q: Can you tell us exactly what you saw?

A: Well, I first knew that something was wrong when I saw Mr. Collins's blue Toyota Camry (PI 5-model true) flashing the lights and heard him laying on the horn as he came up to the intersection from the south. Very shortly after that, I saw the Shell (PI 4/S\&B 6 true) tanker truck come skidding and fishtailing into the intersection from the north. He was pretty much swerving to his left, away from me, towards the opposite side of the intersection from where I was. I was on the west side, waiting to cross Foothill and head east, and he was swerving over that way with the trailer kind of swinging out behind him.

Q: Okay, so after you saw the two vehicles both entering the intersection, what happened next?

A: I saw Mr. Collins's car skirt around the trailer pretty much right in front of my car, when the trailer kind of swung around to its right, as the cab of the truck was making a hard left turn, and the trailer sort of swiped the passenger side of the white (PI 10 color true)van. Right after that, the van rear-ended the Camry, (PI 5-model true) and then the Camry (PI 5-model true) went and ran into the streetlamp (PI 12 true).

Q: And how good of a look did you get at this whole sequence of events?

A: I had a perfect view, pretty much a front-row seat to the whole thing.

Q: So did you see either Mr. Collins's or Ms. Johnson's heads strike the windshield of their car?

A: Definitely.

Q: And when did you see that happen?

A: Right after their car crashed into the lamp post. (PI 12 true)

Q: Right. Now what happened next?

A: Well, I saw the guy with the black hair, (PI 7 true) McGraw, get out of the van right away and go over to the driver's side of Collins's car and it looked like he was checking 
to see if everyone was okay or to see how he could help. Collins got out of the car shortly after that, and while his head was bleeding a little bit, and he seemed a little dazed, he didn't really seem too badly hurt.

Q: Alright. Anything else?

A: Yeah, I stayed around to see if there was anything I could do, but the cops showed up and pretty much got it all under control and then the ambulance got there and I watched them take Ms. Johnson out of the car. It looked like her right leg was all messed up, and they loaded her and Mr. Collins into the ambulance and drove off.

Q: Thank you, Mr. Gorham. I have no further questions.

Defense Witness Tom Gorham, Cross Examination by Seth Blum, Attorney for the Plaintiff

Seth Blum, Attorney for the Plaintiff (Questioner): Mr. Gorham, you stated that you saw Mr. Collins's car flashing its lights and heard it honking its horn as it entered the intersection from the south, and that after that, you saw the Shell (PI 4/S\&B 6 true) truck come skidding and screeching into the intersection. Now if Mr. Collins was honking and flashing his lights and everything, obviously in response to something, doesn't it seem strange to you that you didn't notice what he was reacting to before you noticed him?

Tom Gorham (Answerer): Well, maybe, I guess. I think I probably just noticed Mr. Collins's car first because he was the one that was right in front of me, and since the truck was all the way on the other side of the intersection, further away, maybe I didn't see it first for that reason. Or maybe the angle of everything made the Zales store sort of block my line of sight. I don't know really know why I saw things when I saw them, I just know what I saw.

Q: If you say so. Nothing more, Your Honor.

Defense Witness John Kindt, Direct Examination by Howard Kurtz, Attorney for the Defendant

Howard Kurtz, Attorney for the Defendant (Questioner): Hello there, could you please state your name and occupation for the record?

John Kindt (Answerer): My name is John Kindt, and I am the security guard for the Zales jewelry store located on the northwest corner of the intersection of Foothill and Windsor Road in Clermont. 
Q: Can you tell us where you were on the afternoon of Tuesday, July $8^{\text {th }}, 2008$ ?

A: Yeah, I was at work.

Q: Tell me, did you happen to witness the events at issue in this case?

A: I saw the tail end of it. I saw Mr. Collins's car almost run over the lady out walking her German Shepherd. (PI 13 true) She was walking on the sidewalk and nearly got creamed.

Q: Lucky thing for her that she didn't. So what else did you see then?

A: I saw the Shell (PI 4/S\&B 6 true) trailer kind of smack the passenger side of Mr. McGraw's van and knock it into Mr. Collins's car.

Q: And did you see the consequences of the collision between the two vehicles?

A: Well, yeah. I saw Mr. Collins's car come crashing towards the store, and that lady and her dog, and hit the street lamp (PI 12 true) in front of our store.

Q: And did you see anybody get hurt?

A: Yeah, I saw Mr. Collins's head and Ms. Johnson's head smash into their windshield when they hit the lamp post. I was kind of surprised that the airbags didn't go off, but I guess they weren't going fast enough or something.

Q: And what else did you see?

A: Not much. I got a call about security issues for an upcoming delivery so I had to go look at some paperwork, and by the time I was through with that, the show was over.

Q: Thank you, Mr. Kindt. I have no more questions.

Defense Witness John Kindt, Cross Examination by Seth Blum, Attorney for the Plaintiff Seth Blum, Attorney for the Plaintiff: I have no questions for this witness, Your Honor.

Defense Witness Dr. Erik Dubberke, Direct Examination by Howard Kurtz, Attorney for the Defendant 
Howard Kurtz, Attorney for the Defendant (Questioner): Hello there, could you please tell us your name and occupation?

Dr. Erik Dubberke (Answerer): My name is Erik Dubberke, and I am a trauma surgeon at South Lake Hospital here in Clermont.

Q: That's an emergency room doctor, right?

A: Yes, I'm one of the several surgeons on staff that are trained specifically for traumarelated procedures. Injuries, accident victims, things like that. Immediate and sometimes life-or-death repairs, as opposed to planned, preventative, or purely cosmetic procedures.

Q: Ahh. I see. Tell me, Dr. Dubberke, were you on duty on Tuesday, July $8^{\text {th }}, 2008$ ?

A: Yes, I was on call from noon until midnight that day.

Q: So, Dr. Dubberke, did you treat Ms. Amanda Johnson on that afternoon?

A: Yes I did. She was brought in by ambulance, and the paramedics had stabilized her condition, but it was pretty clear to them by the time that they arrived that she had suffered some sort of localized trauma to her right leg or hip area.

Q: And was that consistent with your observations?

A: Absolutely. We took some X-rays to see exactly what we were dealing with right after we cleaned up and stitched up her forehead. Luckily, her facial lacerations were superficial enough that I was able to use dissolvable sutures which tend to leave little or no scarring, but they don't work as well on really deep or difficult wounds.

Q: Thank heaven for small miracles. Now what did the X-rays reveal?

A: Ms. Johnson suffered a broken hip up near the ball in the joint where the right leg meets the pelvis. It's a very nasty sort of injury.

Q: So how did you treat Ms. Johnson?

A: I inserted a metal plate into her right leg that is held in place by five metal screws that are screwed directly into her bones. By holding everything in place like that, it allows for the bone to gradually fuse back together at the site of the break.

Q: Sounds reasonable. Tell me, Dr. Dubberke, in your experience with trauma victims, are you at all able to judge the severity of accidents or make any determination about what caused or maybe even what could have prevented an injury? 
A: To an extent. If what you're asking me is whether having worn her seat belt would have prevented Ms. Johnson's injury, then yes. I'm almost certain that her broken hip resulted from being thrown forward in a frontal crash.

Q: Not from having been rear-ended?

A: No, that type of impact typically results in whiplash and soft-tissue injuries, not being launched forward into the dashboard.

Q: Thank you, Dr. Dubberke. I don't have any more questions.

Defense Witness Dr. Erik Dubberke, Cross Examination by Seth Blum, Attorney for the Plaintiff

Seth Blum, Attorney for the Plaintiff (Questioner): Dr. Dubberke, how long have you been working in the emergency room?

Dr. Erik Dubberke (Answerer): In my current capacity? Almost eight years. Counting assisting, residency, interning, volunteering, and all that? Probably another four, five years total on top of that.

Q: That's quite a long time. You must have seen some pretty remarkable, unusual, and awful things in that time.

A: Yeah, but you get used to it. If you can't, you don't last long.

Q: I bet. Tell me, have you ever seen anything that just defied explanation, or didn't make any sense, or just plain freak accidents or weird flukes?

A: Sure. The human body is a very complicated, complex organism, with an almost limitless capacity for things to go wrong. And the world can be a pretty strange place too. Just when you start to think you've seen it all, something you could have never envisioned in a hundred years comes through the doors.

Q: I can only imagine. Thank you for all the good work you do, Doctor. Nothing further.

Defense Witness Officer Christopher Eaton, Direct Examination by Howard Kurtz, Attorney for the Defendant

Howard Kurtz, Attorney for the Defendant (Questioner): Good afternoon, Officer. Could you please give us your name, rank, department, and division? 
Officer Christopher Eaton (Answerer): My name is Christopher Eaton, I'm a deputy with the Clermont Police Department, and I'm primarily assigned to the Patrol Section.

Q: Okay. And is the intersection of Foothill and Windsor in Clermont part of your assigned beat?

A: Yes, sir. It's right smack dab in the middle of it, in fact.

Q: And is that where you were on the afternoon of Tuesday, July $8^{\text {th }}, 2008$ ?

A: I was patrolling not too far away when I heard the call on the radio about an automobile accident which also potentially involved a gasoline truck. Mr. McGraw had called 911 pretty quickly, and it only took me a minute or two to get there, and I was the first responder on the scene.

Q: And what did you see when you arrived?

A: I observed Mr. Whitaker's Shell (PI 4/S\&B 6 true) gasoline tanker truck stopped in the rightmost lane, on the south side of Windsor Road, in the eastbound direction. To the west of the intersection, on the north side of Windsor Road, in the westbound direction, I observed Mr. Collins's blue 2006 (PI 9-year true) Toyota Camry (PI 5-model true) at rest up with the front up against the lamp post, (PI 12 true) and Mr. McGraw's white (PI 10 color true)2000 Ford Econoline work van smashed up into the rear of Mr. Collins's car. Mr. McGraw's van had sustained additional damage to the rear passenger quarter panel area, and Mr. Collins's car had a broken windshield in the typical pattern found when the windshield is struck by the head of the occupants of the front seat. In this case, that would be Mr. Collins and Ms. Johnson.

Q: And you've seen a lot of car accidents during your time on the force, I imagine?

A: Yeah, that's probably one of the most frequent calls we get. Hardly a day goes by that I don't deal with at least one wreck

Q: I bet. And in your experience, when you've seen patients with head trauma and broken bones, injured in cars that end up with cracked windshields like Mr. Collins's car, do those accidents typically involve a car that has been rear-ended?

A: Well, I've never actually kept a count of something like that, but off the top of my head, I'd say that very few of them do, except for the sorts of multi-impact incidents like we had here.

Q: How do you mean? 
A: Usually, passengers in cars who have cuts to their head and broken bones suffer their injuries as a result of having been forcefully thrown forward from the violence of a frontal impact. You know, running into something, not being run into from behind.

Q: Okay, well thank you very much for your assistance, Officer. I have no additional questions.

A: My pleasure, sir.

Defense Witness Officer Christopher Eaton, Cross Examination by Seth Blum, Attorney for the Plaintiff

Seth Blum, Attorney for the Plaintiff (Questioner): Officer Eaton, you've undoubtedly seen plenty of car accidents in your time patrolling, haven't you? Accidents of all kinds?

Officer Christopher Eaton (Answerer): Yes, sir. I couldn't even offer a guess as to the total number.

Q: And in your eyes, was this your typical rear-ending?

A: No, sir. Most rear-ending accidents do not involve an impact from a third vehicle like this incident.

Q: So this was a little out of the ordinary for how most rear-impact collisions occur?

A: Yes, sir. I'm not sure whether I can recall anything quite like this in all my years on the force where a semi trailer smacks into one vehicle which then crashes into another in a chain reaction like that. This was the result of some pretty unlikely combinations of forces and events.

Q: I'll say. Thank you, Officer.

A: My pleasure, sir. 


\section{APPENDIX O}

PANAS Short and Long Form Emotion Measurement Scales

International Positive and Negative Affect Schedule Short Form (I-PANAS-SF; Thompson, 2007) \& Specific Affect Scales of the Positive and Negative Affect Schedule-Expanded (PANAS-X; Watson \& Clark, 1994)

Thompson, E. R. (2007). Development and Validation of an Internationally Reliable Short-Form of the Positive and Negative Affect Schedule (PANAS). Journal of CrossCultural Psychology, 38(2), 227-242. doi:10.1177/0022022106297301

\section{The International Positive and Negative Affect Schedule Short Form (I-PANAS-SF) Question, Measure, and Item Order}

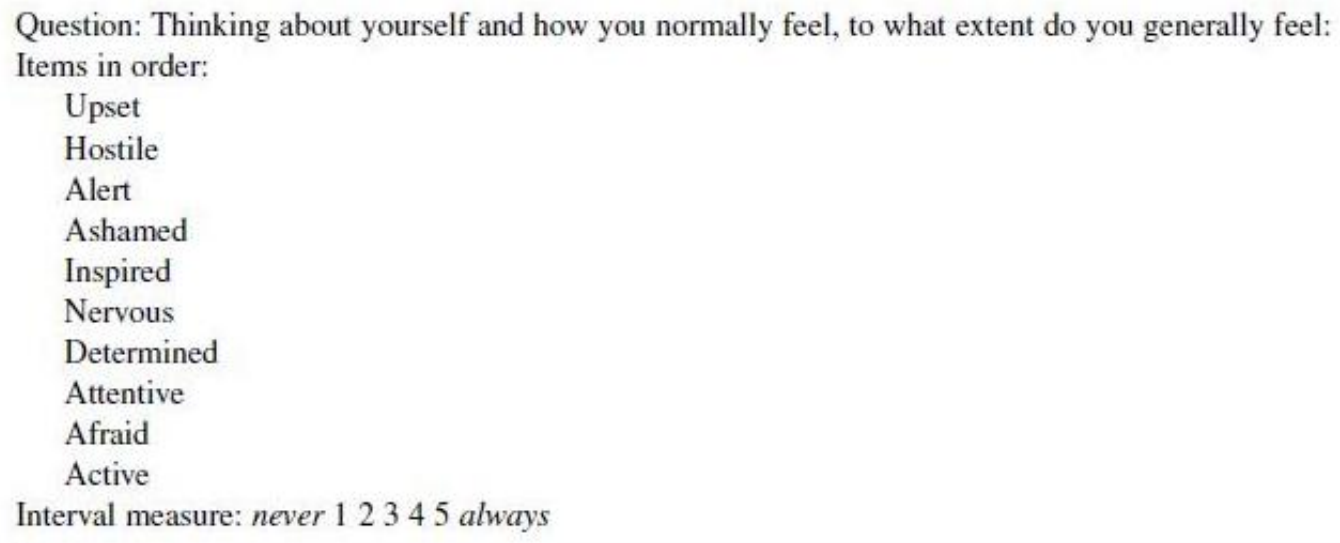

Note: I added Angry and Mad because anger will be experimentally induced in Studies Three, Four, and Five. 
Appendix O Continued: Specific Affect Scales of the Positive and Negative Affect Schedule-Expanded (PANAS-X; Watson \& Clark, 1994)

Watson, D., \& Clark, L.A. (1994). The PANAS-X: Manual for the positive and negative affect schedule-expanded form. Unpublished manuscript, University of Iowa, Iowa City, IA.

\section{PANAS-X}

This scale consists of a number of words and phrases that describe different feelings and emotions. Read each item and then mark the appropriate answer in the space next to that word. Indicate to what extent you feel this way right now. Use the following scale to record your answers:

\begin{tabular}{|ccccc|}
\hline $\begin{array}{c}1 \\
\text { very slightly } \\
\text { or not at all }\end{array}$ & $\begin{array}{c}\mathbf{3} \\
\text { a little }\end{array}$ & moderately & quite a bit & $\begin{array}{c}\mathbf{5} \\
\text { extremely }\end{array}$ \\
\hline
\end{tabular}

1.

2.

3.

4.

5 .

6.

7. surprised

8.

9.

10.

11.

12.

13.

14.

15.

16. sad

17. calm
18

19

20

21.

22.

23.

24.

25.

26.

27.

28.

29.

30.

31.

32.

33. joyful

34. nervous 


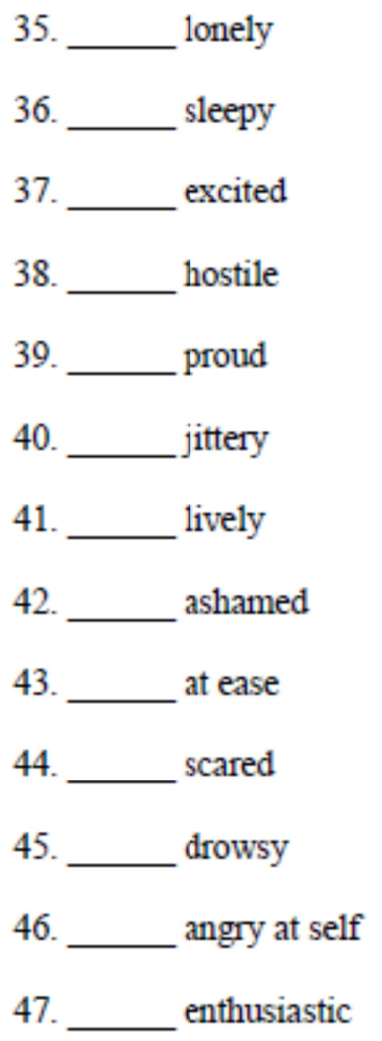

48.

49.

50.

51.

52 .

53.

54.

55.

56.

57.

58.

59.

60. dissatisfied with self

Note: I added Mad as item 61 to allow for comparisons to the modified, 12-item IPANAS-SF. 


\section{$\underline{\text { Scales }}$}

General Positive Emotion $=(p 31+p 25+p 3+p 52+p 47+p 37+p 13+p 55+p 39+p 8)$

General Negative Emotion: $=(p 18+p 44+p 34+p 40+p 11+p 38+p 32+p 42+p 26+p 50)$

fear: $=(\mathrm{p} 18+\mathrm{p} 44+\mathrm{p} 53+\mathrm{p} 34+\mathrm{p} 40+\mathrm{p} 21)$

hostility: $=(p 37+p 38+p 11+p 9+p 2+p 56)$

guilt: $=(\mathrm{p} 32+\mathrm{p} 42+\mathrm{p} 51+\mathrm{p} 46+\mathrm{p} 15+\mathrm{p} 60)$

sadness: $=(p 16+p 29+p 48+p 24+p 35)$

joviality: $=(\mathrm{p} 22+\mathrm{p} 33+\mathrm{p} 12+\mathrm{p} 1+\mathrm{p} 37+\mathrm{p} 47+\mathrm{p} 41+\mathrm{p} 58)$

self_assurance: $=(p 39+p 3+p 57+p 28+p 6+p 14)$

attentiveness: $=(\mathrm{p} 25+\mathrm{p} 3+\mathrm{p} 59+\mathrm{p} 52)$

shyness: $=(\mathrm{p} 30+\mathrm{p} 4+\mathrm{p} 49+\mathrm{p} 23)$

fatigue $=(\mathrm{p} 36+\mathrm{p} 19+\mathrm{p} 5+\mathrm{p} 45)$

serenity: $=(\mathrm{p} 17+\mathrm{p} 10+\mathrm{p} 43)$

surprise $=(\mathrm{p} 20+\mathrm{p} 7+\mathrm{p} 54)$

basic positive affect:= (joviality+self_assurance+attentiveness) $/ 3$

basic negative affect:= (sadness+guilt+hostility+fear) $/ 4$ 


\section{APPENDIX P}

Study Two Data Reduction Procedures

Note: Data reduction procedures for Study Two are virtually identical to the technique used in Study One.

\section{Step One: Raw Data}

At the conclusion of data collection, the final data file included 64 total entries, or one separate entry for each discrete time Study One was accessed and begun. Of these 64 entries, 44 were complete, having accessed every page of the study website. To be clear, these are not total numbers of distinct participants, but rather all separate instances of data recordings initiated each time the study was started.

\section{Step Two: Eliminating Repeat Starters}

Despite repeated admonitions and stern warnings to the contrary, some participants in online studies nevertheless begin the study and then quit partway through completion, only to return at a later time to complete the study in full. Because the objective of Study Two is to determine the relative ease or rate of detecting each of the eight remaining testimonial inconsistencies in comparison to their levels of detection in Study One, I eliminated those participants who were exposed to the trial transcript to any extent prior to the session in which they completed the study. For example, a participant who read along through the end of all plaintiff's witnesses but quit the study before the defense presented its case, only to return two days later to restart the study (the website required participants to start over if their browser window closed at any point) and completed it during that second session would have been exposed to the inconsistent information two full times. This obviously gives that repeat participant a greater 
opportunity to detect the inconsistencies compared to a participant who was only exposed to the critical information once, so I eliminated all data associated with those participants who accessed the study on more than one occasion. However, for any participants who completed the study the first time they accessed it, but who may have started it again, I simply eliminated all data associated with any subsequent attempts since their first exposure was untainted.

After eliminating the data associated with any participants who had been preexposed to the trial transcript, 45 separate data entries remained.

\section{Step Three: Eliminating Partial Participants}

Steps Three and Four could have been performed in interchangeable order with no consequence, but I expressly refrained from eliminating the participants who did not complete the study at the start of the data reduction process because doing so would have prevented me from having the ability to identify those participants who began the study and were exposed to some or all of the trial transcript and quit partway through, only to restart and complete the study at a later time - and with an improper degree of exposure to the trial transcript and inconsistent testimony. It is only after this step that the remaining data entries can properly be called "participants." Of the 45 data entries remaining before this step, only 40 participants completed the entire study.

\section{Step Four: Eliminating Inattentive Participants}

Because online data collection allows participants to complete the study at the time and place of their choosing, it tends to be more popular with the FIU participant pool than in-person studies which require participants to be in a specific time and place to participate. While this flexibility benefits participants and the concomitant popularity 
benefits researchers with faster data collection (as well as requiring less time to input and code data), the tradeoff for researchers is the lack of control over the environment in which participants complete the study. Without the presence of a supervising researcher, some participants may choose to complete online studies without actually paying attention or engaging in anything resembling legitimate, earnest effort. To combat this problem (or at least prevent it from tainting my data), I included two questions intended to confirm that the participant was in fact paying at least some minimum degree of attention to the questions they were answering. The first, presented in random order among the Multiple Choice questions, was

There are lots of witnesses and lots of evidence in trials. Sometimes testimony is consistent, sometimes it's contradictory, sometimes different witnesses disagree, and sometimes accounts vary.

Please mark all possible answers for the following question, even those which are contradictory to each other.

These questions are designed to test your memory for the information in the trial testimony, not your personal opinion on what actually happened or what is correct. How you answer these questions should not depend on which witnesses you believe to have been accurate or those you believe to have been inaccurate-just the content of the testimony itself.

Again, you may choose more than one answer.

Thank you for participating in this study. To ensure that you are paying attention to the questions, please mark A for this question.
A. He put the car into reverse
B. He stopped the car
C. He turned sharply to his right
ए D. He pulled the emergency brake
E. None of the above
Г F. Don't know/don't remember

The second, presented in random order among the True/False questions, was 
There are lots of witnesses and lots of evidence in trials. Sometimes testimony is consistent, sometimes it's contradictory, sometimes different witnesses disagree, and sometimes accounts vary.

These True or False questions are designed to test your memory for the information in the trial testimony, not your personal opinion on what actually happened or what is correct.

How you answer these questions should not depend on which witnesses you believe to have been accurate or those you believe to have been inaccurate-just the content of the testimony itself.

Thank you for your continued attention and hard work. The study is almost over, so please mark this answer true so that we will know that you're still paying attention.
C True
C False
C I don't know

I eliminated all seven participants who failed to answer one or both of these questions as directed, leaving 33 remaining participants.

\section{Step Five: Eliminating Extreme Outliers of Total Time Spent}

Due to the fact that individual differences in reading speed, attention to detail, and overall thoughtfulness and thoroughness of answering the battery of questions designed to measure inconsistency detections (including 23 Open Ended and Short Answer questions with no restriction on response length), and I had estimated that most participants should be able to complete the entire study in approximately 60 to 90 minutes (in contrast to the 90 to 120 minutes I estimated Study One would require because Study Two featured 36 fewer questions to answer, see Appendix Q), I defined an extreme outlier as any participant who required greater than twice that duration to complete the study. Because some participants would load the first page of the study (the Informed Consent page) and then wait (sometimes minutes, hours, or even days) before actually indicating their consent and beginning the study, to prevent this practice from 
skewing the calculations of total time spent, I calculated the total duration by subtracting the time participants loaded the second page (immediately after submitting the Informed Consent page) from the time the last page of the study was submitted.

Four participants were identified as extreme outliers ranging from 194.63 minutes spent to 869.88 minutes spent $(M=400.67, S D=314.73)$ and eliminated from further analysis, leaving a total of 29 participants.

\section{Step Six: Eliminating Outliers of Total Time Spent}

After eliminating those extreme outliers which would skew the overall

distribution, I calculated the distribution for the remaining 29 participants, resulting in the elimination of two participants whose total time spent was in excess of two standard deviations above the mean, or greater than 163.25 minutes $(M=102.90, S D=30.18)$. There were no participants who completed the study in less time than two standard deviations below the mean.

The resulting data set $(n=27)$ to be used in Study Two included participants who spent between 64.90 and 155.88 minutes $(M=98.14, S D=23.81)$. 


\section{APPENDIX Q}

\section{Study Two Inconsistency Detection Questions}

Note: The parenthetical (PI 1/S\&B 1, etc.) information at the end of each question was not present in the actual materials. It is included here for the sake of explanation and reference to assist in identifying which inconsistency or inconsistencies form the basis for the question. Please see the List and Descriptions of all Eight Inconsistencies from Study Two (Appendix R) for a brief description of the nature of each inconsistency. Also, for the forced-choice questions (Multiple Choice, True/False, and Point Blank), the correct answer is included here for reference purposes.

All information which is presented here for background or clarification but which was not visible to participants is italicized.

\section{Open-Ended Questions:}

The open-ended question section featured one primary question and one followup “...if so, who/how?" elaboration per page, presented in random order. Because the open-ended questions are so general, many of them apply to more than one inconsistency.

The following instructions were listed at the top of each page:

There are lots of witnesses and lots of evidence in trials. Sometimes testimony is consistent, sometimes it's contradictory, sometimes different witnesses disagree, and sometimes accounts vary, etc. Please list all possible answers for the following question, even those which are contradictory to each other.

\section{Questions (Five Primary and Five Followup):}

Were there any eyewitnesses that testified who you think might have made mistakes in terms of brand names? (PI 4/S\&B 6; PI 5-model; PI 11)

If so, who and how? If you don't remember exactly which witness(es) might have made this type of mistake, please just describe the mistake(s) to the best of your ability.

Were there any eyewitnesses that testified who you think might have made mistakes in terms of describing various colors? (PI 7; PI 10-color)

If so, who and how? If you don't remember exactly which witness(es) might have made this type of mistake, please just describe the mistake(s) to the best of your ability. 
Were there any eyewitnesses that testified who you think might have made mistakes in terms of describing the physical layout of the intersection? (PI 12; PI 15)

If so, who and how? If you don't remember exactly which witness(es) might have made this type of mistake, please just describe the mistake(s) to the best of your ability.

Were there any eyewitnesses that testified who you think might have made mistakes in terms of specific physical descriptions of people? (PI 6; PI 7; PI 14)

If so, who and how? If you don't remember exactly which witness(es) might have made this type of mistake, please just describe the mistake(s) to the best of your ability.

Were there any eyewitnesses that testified who you think might have made mistakes in terms of specific physical descriptions of objects? (PI 9-year; PI 12; PI 13; PI 15)

If so, who and how? If you don't remember exactly which witness(es) might have made this type of mistake, please just describe the mistake(s) to the best of your ability.

\section{Short Answer Questions:}

The short answer question section featured one question per page, presented in random order.

The following instructions were listed at the top of each page:

There are lots of witnesses and lots of evidence in trials. Sometimes testimony is consistent, sometimes it's contradictory, sometimes different witnesses disagree, and sometimes accounts vary.

Please list all possible answers for the following question, even those which are contradictory to each other.

If you do not know the answer, please type "I don't know." and then click Submit to proceed to the next question.

Questions (13 total-one for each of the eight inconsistencies, and five "dummy" questions to provide opportunities for participants to give answers that do not involve inconsistent testimony in order to camouflage the intent of the study while also acting as a manipulation check by probing their general understanding of the trial transcript): 
Several witnesses mentioned the oil company logo on the gasoline tanker trailer. Please describe the testimony concerning the oil company. Make sure you talk about all of the witnesses who testified about the oil company that owned the truck. (PI 4/S\&B 6)

Several witnesses described the make and model of the vehicles involved in the accident. Please describe the testimony concerning the kinds of vehicles. Make sure you talk about all of the witnesses who testified about the make and model of the vehicles. (PI 5-model)

Several witnesses described Michael McGraw by mentioning his hair color. Please describe the testimony concerning Michael McGraw's hair color. Make sure you talk about all of the witnesses who mentioned his hair color. (PI 7)

Several witnesses described the year of the vehicles involved in the accident. Please describe the testimony concerning the years of the vehicles. Make sure you talk about all of the witnesses who testified about the years of the vehicles. (PI 9-year)

Several witnesses described the colors of the vehicles involved in the accident. Please describe the testimony concerning the colors of the vehicles. Make sure you talk about all of the witnesses who testified about the color of each of the vehicles. (PI 10-color)

There are several businesses located near the intersection of Foothill Dr. and Windsor Rd. Please describe the testimony regarding the businesses surrounding the intersection. Make sure you talk about all of the witnesses who testified about the nearby businesses. (PI 11)

Brock Collins's car eventually came to rest following the accident. Please describe the testimony concerning how Brock Collins's car came to a stop after the crash. Make sure you talk about all of the witnesses who testified about how his car came to a stop. (PI 12)

Lesley Jobin was walking her dog Finnegan when she witnessed the accident. Please describe the testimony regarding the breed of Lesley's dog. Make sure you talk about all of the witnesses who testified about Lesley's dog. (PI 13)

Several witnesses testified regarding Amanda Johnson's employment status. Please describe the testimony concerning Amanda Johnson's employment status. Make sure you talk about all witnesses who mentioned Amanda's employment. (Dummy 1) 
Several witnesses mentioned details having to do with who called 911. Please describe the testimony concerning who called 911. Make sure you talk about all witnesses who testified about calling 911. (Dummy 2)

Brock Collins and Amanda Johnson had plans for the day the accident occurred. Please describe what their plans were, and what they were on the way to do. Make sure you talk about all witnesses who testified about their plans for that day. (Dummy 3)

Multiple witnesses mentioned airbags in relation to the accident. Please describe the testimony involving airbags deploying. Make sure you talk about all witnesses who referred to airbags in any of the vehicles. (Dummy 4)

Amanda Johnson required an emergency medical procedure to help her broken leg heal. Please describe what this procedure involved. Make sure you talk about all witnesses who testified about the treatment Amanda needed on her broken leg. (Dummy 5)

\section{Multiple Choice Questions:}

The multiple choice question section featured one question per page, presented in random order.

The following instructions were listed at the top of each page:

There are lots of witnesses and lots of evidence in trials. Sometimes testimony is consistent, sometimes it's contradictory, sometimes different witnesses disagree, and sometimes accounts vary.

Please mark all possible answers for the following question, even those which are contradictory to each other.

These questions are designed to test your memory for the information in the trial testimony, not your personal opinion on what actually happened or what is correct. How you answer these questions should not depend on which witnesses you believe to have been accurate or those you believe to have been inaccurate-just the content of the testimony itself.

\section{Again, you may choose more than one answer.}

Questions (16 total-one for each of the eight inconsistencies, five "dummy" questions, two case theme manipulation checks, and one "attention check" question 
for use in identifying those participants who are not reading the questions which they are responding to):

According to the plaintiff's version of events, Amanda Johnson broke her hip when: (Manipulation Check of Plaintiff's Case-B)
A. The tanker truck hit Brock's car.
B. The van driven by the defendant, Michael McGraw, hit Brock's car.
C. Brock's car hit a pole.
D. Jill Randall ran the red light and hit Brock's car.
E. None of the above
F. Don't know/don't remember

According to the defense's version of events, Amanda Johnson broke her hip when: (Manipulation Check of Defense's Case-C)
A. The tanker truck hit Brock's car.
B. The van driven by the defendant, Michael McGraw, hit Brock's car.
C. Brock's car hit a pole.
D. Jill Randall ran the red light and hit Brock's car.
E. None of the above
F. Don't know/don't remember

The gasoline tanker trailer featured the logo of the oil company that owned the truck. What company (or companies) were mentioned as the owner of the truck? (PI 4/S\&B 6- $A$ \& D)
A. Exxon
B. BP
C. Chevron
D. Shell
E. None of the above
F. Don't know/don't remember

Brock Collins's car was involved in the accident. According to the trial transcript, what make and model(s) of car was Brock said to have been driving? (PI 5-model-B \& D)
A. Chevrolet Camaro
B. Toyota Camry
C. Toyota Corolla
D. Chevrolet Corvette
E. None of the above
F. Don't know/don't remember 
Several witnesses mentioned Michael McGraw's hair color when describing what they had seen. What color(s) of hair did witnesses say he had? (PI 7-C\& D)
A. Blond
B. Brown
C. Black
D. Red
E. None of the above
F. Don't know/don't remember

The year of Brock Collins's car is mentioned in the testimony. What year(s) is it said to be? (PI 9-year-B \& $C$ )
A. 2002
B. 2006
C. 1986
D. 1996
E. None of the above
F. Don't know/don't remember

The color of Michael McGraw's van is mentioned in the testimony. What color(s) is it said to be? (PI 10-color- $A \& B$ )
A. Tan
B. White
C. Blue
D. Green
E. None of the above
F. Don't know/don't remember

There is a jewelry store on the northwest corner of the intersection. What name(s) for the store was/were mentioned? (PI 11-B \& D)
A. Kay
B. Jared
C. Tiffany \& Co.
D. Zales
E. None of the above
F. Don't know/don't remember

Brock Collins's car ran into something before coming to a complete stop. According to the transcript, what object(s)? (PI 12-A\&D)
A. A tree
B. A blue USPS mailbox
C. A bus stop 

D. A street light pole
E. None of the above
F. Don't know/don't remember

There was testimony given regarding the breed of Lesley Jobin's dog Finnegan. What breed(s) were given for Finnegan? (PI 13- $A$ \& $C$ )
A. Dachshund
B. Doberman
C. German Shepherd
D. Beagle
E. None of the above
F. Don't know/don't remember

The testimony indicated that Amanda Johnson had held what job(s) prior to the accident? (Dummy 1-C)
A. Nurse
B. Teacher
C. Waitress
D. Real estate agent
E. None of the above
F. Don't know/don't remember

According to the transcript, which person (or people) called 911 to report the accident? (Dummy 2-D)
A. Brock Collins
B. Steve Powell
C. Jill Randall
D. Michael McGraw
E. None of the above
F. Don't know/don't remember

What activity or activities did Brock and Amanda have planned for the day of the accident? (Dummy 3- $A \& B$ )
A. Going to a movie
B. Going out to dinner
C. Going to a concert
D. Going to a party
E. None of the above
F. Don't know/don't remember 
The transcript included testimony concerning airbags in Brock Collins's car. Which of the following was included? (Dummy 4-B \& $C$ )
A. The airbags inflated
B. The airbags did not inflate
C. The airbags may have been defective
D. The car did not have airbags
E. None of the above
F. Don't know/don't remember

Amanda Johnson required serious medical treatment for her broken leg when she arrived at the emergency room. What procedure(s) did she need? (Dummy 5-A\& D)
A. A metal plate to be inserted
B. The bone had to be broken further in order to heal properly
C. A bone graft
D. Metal screws to be inserted
E. None of the above
F. Don't know/don't remember

Thank you for participating in this study. To ensure that you are paying attention to the questions, please mark A for this question. (Attention check-A)
A. He put the car into reverse
B. He stopped the car
C. He turned sharply to his right
D. He pulled the emergency brake
E. None of the above
F. Don't know/don't remember

\section{True/False Questions:}

The true/false question section featured one question per page, presented in random order.

The following instructions were listed at the top of each page:

There are lots of witnesses and lots of evidence in trials. Sometimes testimony is consistent, sometimes it's contradictory, sometimes different witnesses disagree, and sometimes accounts vary.

Please answer the following True or False questions regarding the trial transcript. 
These questions are designed to test your memory for the information in the trial testimony, not your personal opinion on what actually happened or what is correct.

How you answer these questions should not depend on which witnesses you believe to have been accurate or those you believe to have been inaccurate - just the content of the testimony itself.

Questions (14 total - one for each of the eight inconsistencies, five dummy questions, and one attention check; True, False, and I don't know were answer choices):

Only one oil company logo was identified on the tanker trailer according to the transcript. (F; PI 4/S\&B 6)

Out of all descriptions of Brock Collins's car within the trial testimony, there was no discrepancy concerning the make and model of the car. (F; PI 5-model)

Not all witnesses agreed in their testimony about what color hair Michael McGraw had. $(T ; P I 7)$

There was conflicting testimony given regarding the year of Brock Collins's car. (T; PI 9-year)

There was no conflict in testimony given regarding the color of Michael McGraw's van. (F; PI 10-color)

There were multiple store names given for the jewelry store in the trial transcript. (T; PI 11)

Brock Collins's car hit an object before finally coming to rest, and all testimony concerning that object agreed about exactly what that object was. (F; PI 12)

Different witnesses named different breeds when describing Lesley Jobin's dog. (T; PI 13)

There was conflicting testimony concerning Amanda Johnson's job before the accident. (F; Dummy 1) 
There was no discrepancy in the transcript concerning who called 911 about the accident. (T; Dummy 2)

There was conflicting testimony concerning where Brock and Amanda were going when the accident occurred. (F; Dummy 3)

There was no testimony saying that the airbags deployed in Brock Collins's car. ( $T$; Dummy 4)

There was conflicting testimony given regarding the medical procedures performed on Amanda Johnson to help heal her broken bones. (F; Dummy 5)

Thank you for your continued attention and hard work. The study is almost over, so please mark this answer true so that we will know that you're still paying attention. ( $T$; Attention Check)

\section{Point Blank Questions:}

These questions were designed to be as direct as possible without creating demand characteristics. There was only one set of questions per page (that is, two consecutive pages per witness - done to prevent any confusion over whether the two hypotheticals were the same, whether they should be evaluated in conjunction with each other, whether the same Juror 1 and Juror 2 are the focus each question, etc.) Order of presentation was randomized by witness, with all witnesses featuring two consecutive pages, each page with one question apiece. Because Study Two features seven fewer inconsistencies than Study One, in order to maintain uniformity of presentation with two Point Blank questions for each witness, three new dummy/manipulation checks based on removed inconsistencies (PI 14, PI 15, and PI 1/S\&B 1) have been added.

The following instructions appeared at the top of each page:

For this final section of questions, you will be asked to evaluate the way two different jurors each viewed a particular witness's testimony.

Each question should be answered independently of all others. The way you answer one question should not have any bearing on how you answer any other question.

\section{Questions (12 total-one for each of the eight inconsistencies and four manipulation} checks): 
Imagine two jurors found the testimony of Marc Zollinger to be inaccurate but their opinions were for two different reasons. Which juror's opinion do you most agree with?

The juror who found Marc Zollinger's testimony to be inaccurate because he said that Brock Collins was driving a Corvette while another witness said he was driving a Camry.

The juror who found Marc Zollinger's testimony to be inaccurate because he said that Brock Collins was driving a Camaro while another witness said he was driving a Camry.

I don't agree with either of these jurors.

(Juror 1; PI 5-model)

The juror who found Marc Zollinger's testimony to be inaccurate because he said that Lesley Jobin's dog was a Doberman while another witness said it was a German Shepherd.

The juror who found Marc Zollinger's testimony to be inaccurate because he said that Lesley Jobin's dog was a Dachshund while another witness said it was a German Shepherd.

I don't agree with either of these jurors.

(Juror 2; PI 13)

Imagine two jurors found the testimony of Barbara Feldman to be inaccurate but their opinions were for two different reasons. Which juror's opinion do you most agree with?

The juror who found Barbara Feldman's testimony to be inaccurate because she said that Michael McGraw's van was green while another witness said it was white.

The juror who found Barbara Feldman's testimony to be inaccurate because she said that Michael McGraw's van was tan while another witness said it was white.

I don't agree with either of these jurors.

(Juror 2; PI 10-color)

The juror who found Barbara Feldman's testimony to be inaccurate because she said that the police officer who responded to the scene was a woman while another witness said it was a man.

The juror who found Barbara Feldman's testimony to be inaccurate because she said that the police officer who responded to the scene was small in size while another witness said he's large.

I don't agree with either of these jurors.

(Neither; Dummy/Manipulation check/camouflage based on prior PI 14)

Imagine two jurors found the testimony of Jill Randall to be inaccurate but their opinions were for two different reasons. Which juror's opinion do you most agree with? 
The juror who found Jill Randall's testimony to be inaccurate because she said that the intersection of Foothill and Windsor has a stop sign while another witness said it has a stop light to control traffic.

The juror who found Jill Randall's testimony to be inaccurate because she said that the intersection of Foothill and Windsor has a yield sign while another witness said it has a stop light to control traffic.

I don't agree with either of these jurors.

(Neither; Dummy/Manipulation check/camouflage based on prior PI 15)

The juror who found Jill Randall's testimony to be inaccurate because she said that Michael McGraw has red hair while another witness said he has black hair.

The juror who found Jill Randall's testimony to be inaccurate because she said that Michael McGraw has blond hair while another witness said he has black hair.

I don't agree with either of these jurors.

(Juror 1; PI 7)

Imagine two jurors found the testimony of Lesley Jobin to be inaccurate but their opinions were for two different reasons. Which juror's opinion do you most agree with?

The juror who found Lesley Jobin's testimony to be inaccurate because she said that she was in front of the Kay jewelry store when she saw the accident while another witness said it was a Zales jewelry store.

The juror who found Lesley Jobin's testimony to be inaccurate because she said that she was in front of the Jared jewelry store when she saw the accident while another witness said it was a Zales jewelry store.

I don't agree with either of these jurors.

(Juror 2; PI 11)

The juror who found Lesley Jobin's testimony to be inaccurate because she said that Brock Collins's car came to a stop when it crashed into a tree while another witness said it crashed into a street light pole.

The juror who found Lesley Jobin's testimony to be inaccurate because she said that Brock Collins's car came to a stop when it crashed into a tree while another witness said it crashed into a bus stop.

I don't agree with either of these jurors.

(Juror 1; PI 12)

Imagine two jurors found the testimony of Dr. Matthew Freeman to be inaccurate but their opinions were for two different reasons. Which juror's opinion do you most agree with? 
The juror who found Dr. Matthew Freeman's testimony to be inaccurate because he said that Brock Collins's car was a 1986 Toyota Camry while another witness said it was a 2006 Toyota Camry.

The juror who found Dr. Matthew Freeman's testimony to be inaccurate because he said that Brock Collins's car was a 2002 Toyota Camry while another witness said it was a 2006 Toyota Camry.

I don't agree with either of these jurors.

(Juror 1; PI 9-year)

The juror who found Dr. Matthew Freeman's testimony to be inaccurate because he said that Brock and Amanda were on their way to a concert when the accident occurred while another witness said they were on their way to a movie and dinner.

The juror who found Dr. Matthew Freeman's testimony to be inaccurate because he said that Brock and Amanda were on their way to a party when the accident occurred while another witness said they were on their way to a movie and dinner.

I don't agree with either of these jurors.

(Neither; Dummy/Manipulation check/camouflage)

Imagine two jurors found the testimony of Amanda Johnson to be inaccurate but their opinions were for two different reasons. Which juror's opinion do you most agree with?

The juror who found Amanda Johnson's testimony to be inaccurate because she said that the rear of Michael McGraw's van was damaged by the truck while another witness said it was damaged on the passenger side.

The juror who found Amanda Johnson's testimony to be inaccurate because she said that the driver's side of Michael McGraw's van was damaged by the truck while another witness said it was damaged on the passenger side.

I don't agree with either of these jurors.

(Neither; Dummy/Manipulation check/camouflage based on prior PI 1/S\&B 1)

The juror who found Amanda Johnson's testimony to be inaccurate because she first said that she saw the Shell company logo on the tanker trailer and then later said that she saw the Exxon company logo on the tanker trailer.

The juror who found Amanda Johnson's testimony to be inaccurate because she first said that she saw the Shell company logo on the tanker trailer and then later said that she saw the BP company logo on the tanker trailer.

I don't agree with either of these jurors.

(Juror 1; PI 4/S\&B 6) 


\section{APPENDIX R \\ List and Description of all Eight Inconsistencies from Study Two}

\section{List of all Inconsistencies:}

PI 4-Oil Co.: Oil company name-Shell is true, Amanda says Exxon (S\&B 6)

PI 5-Model: Zollinger says Collins drives a blue Corvette (wrong), he really drives a blue Camry

PI 7-Hair: Jill says McGraw has red hair (false-he really has black hair)

PI 9-Year: Freeman says Collins drives a 1986 Camry (false) when it's really a 2006

PI 10-Color: Barbara says McGraw's van is tan, it's really white

PI 11-Jared: Lesley says it's a Jared jewelry store (false); it's really a Zales

PI 12-Tree: Lesley says Collins crashes into a tree (false); it's really a light pole

PI 13-Dog: Zollinger says Lesley's dog is a dachshund (false); it's really a German

Shepherd

Key:

- $\mathrm{PI}$ is the number I have assigned to that particular inconsistency.

- S\&B indicates that Semmler and Brewer used this inconsistency in their trial transcripts. 


\section{Table R1}

\section{Eight Inconsistencies Used in Study Two Trial Transcript}

\begin{tabular}{|c|c|c|c|}
\hline Number & Summary & $\begin{array}{l}\text { Mistaken EW } \\
\text { \& their total \# }\end{array}$ & $\begin{array}{l}\text { Broad Type of } \\
\text { Incon }\end{array}$ \\
\hline $\begin{array}{l}\text { PI 4-Oil } \\
\text { Co.: }\end{array}$ & $\begin{array}{l}\text { Oil company name-Shell is true, } \\
\text { Amanda says Exxon }\end{array}$ & Amanda (4) & Brand Names \\
\hline $\begin{array}{l}\text { PI 5- } \\
\text { Model: }\end{array}$ & $\begin{array}{l}\text { Zollinger says Collins drives a blue } \\
\text { Corvette (wrong), he really drives a } \\
\text { blue Camry }\end{array}$ & Zollinger (2) & Brand Names \\
\hline PI 7-Hair: & $\begin{array}{l}\text { Jill says McGraw has red hair (false-he } \\
\text { really has black hair) }\end{array}$ & Jill (2) & $\begin{array}{l}\text { Color; Specific } \\
\text { descriptions of } \\
\text { people }\end{array}$ \\
\hline $\begin{array}{l}\text { PI 9- } \\
\text { Year: }\end{array}$ & $\begin{array}{l}\text { Freeman says Collins drives a } 1986 \\
\text { Camry (false) when it's really a } 2006\end{array}$ & Freeman (1) & $\begin{array}{l}\text { Specific } \\
\text { descriptions of } \\
\text { objects }\end{array}$ \\
\hline $\begin{array}{l}\text { PI 10- } \\
\text { Color: }\end{array}$ & $\begin{array}{l}\text { Barbara says McGraw's van is tan, it's } \\
\text { really white }\end{array}$ & Barbara (2) & Color \\
\hline $\begin{array}{l}\text { PI 11- } \\
\text { Jared: }\end{array}$ & $\begin{array}{l}\text { Lesley says it's a Jared jewelry store } \\
\text { (false); it's really a Zales }\end{array}$ & Lesley (2) & Brand Names \\
\hline $\begin{array}{l}\text { PI 12- } \\
\text { Tree: }\end{array}$ & $\begin{array}{l}\text { Lesley says Collins crashes into a tree } \\
\text { (false); it's really a light pole }\end{array}$ & Lesley (2) & $\begin{array}{l}\text { Specific } \\
\text { descriptions of } \\
\text { objects; Physical } \\
\text { Layout }\end{array}$ \\
\hline $\begin{array}{l}\text { PI 13- } \\
\text { Dog: }\end{array}$ & $\begin{array}{l}\text { Zollinger says Lesley's dog is a } \\
\text { dachshund (false); it's really a German } \\
\text { Shepherd }\end{array}$ & Zollinger (2) & $\begin{array}{l}\text { Specific } \\
\text { descriptions of } \\
\text { objects }\end{array}$ \\
\hline Dummy 1 & $\begin{array}{l}\text { Amanda was a waitress at the time of } \\
\text { accident }\end{array}$ & & \\
\hline Dummy 2 & McGraw called 911 & & \\
\hline Dummy 3 & $\begin{array}{l}\text { Brock \& Amanda were on their way to } \\
\text { see a movie }\end{array}$ & & \\
\hline Dummy 4 & The airbags in Brock's car didn't go off & & \\
\hline Dummy 5 & Amanda had a metal plate put in her leg & & \\
\hline
\end{tabular}




\title{
APPENDIX S
}

\author{
Study Two Informed Consent
}

\section{FIU}

\section{ADULT ONLINE CONSENT TO PARTICIPATE IN A RESEARCH STUDY}

\author{
Civil Juror Decision-Making
}

IRB Approval \# 100411-01

Thank you for looking into our study. Over the next few webpages you will read a description of a civil trial and some jury instructions, and then answer some questions about the case. Before participating, please read the information in the official consent document below.

\section{**IMPORTANT: You may use any of the following web browsers AS LONG AS JAVASCRIPT IS ENABLED: Mozilla Firefox, Google Chrome, Apple Safari, and Internet Explorer. If you have JavaScript turned off, or use any add-ons, extensions, or shortcuts which block JavaScript, please either disable them, add qualtrics.com to the list of approved sites, enable JavaScript, or run your browser in Safe Mode**}

Please complete this study at one time. If for any reason you are not able to complete the study in one sitting, you will have to start over. The experiment takes roughly between 60 and 90 minutes to complete.

Please note, you must have cookies and JavaScript enabled on your browser in order to participate. If you click the button below but do not leave this page, your browser is not supporting cookies. Cookies are used only so that you can have a unique identification number for this study; no personal information will be stored in cookies.

As a student in the FIU Psychology Participant Pool (enrolled in PSY 2012 or other class), you are invited to participate in a research study conducted over the Web. The following information is provided to help you make an informed decision whether or not to participate. Your participation is completely voluntary, and not participating will not affect your class grade in any way. You must be at least 18 years old to participate.

\section{PURPOSE OF THE STUDY}

You are being asked to be in a research study. The purpose of this study is to investigate how jurors make decisions. Completion of this study will take approximately one and a half hours.

\section{NUMBER OF STUDY PARTICIPANTS}

If you decide to be in this study, you will be one of approximately 100 people in this research study.

\section{DURATION OF THE STUDY}

Your participation will require approximately 60 to 90 minutes.

\section{PROCEDURES}

If you agree to be in the study, we will ask you to do the following things:

1. You will be asked to read along with and listen to a case scenario involving a civil lawsuit in which a plaintiff is suing a defendant for negligence. You will be asked to answer questions about the events described on a Web page. You will be asked to imagine you are a juror in the case and to decide whether the defendant is liable and whether the plaintiff is entitled to any money-based damages. We will also ask how you feel and your opinion on various aspects of the case. You can refuse to answer any question and cease participation at any time. 


\section{RISKS AND/OR DISCOMFORTS}

The following risks may be associated with your participation in this study: You might become upset when you read or render a verdict about the case involving an automobile accident, especially if you or someone you know has been involved in a similar accident. This event is unlikely, but if you are concerned about it you may withdraw from the study at any time

\section{BENEFITS}

The following benefits may be associated with your participation in this study: you may find the learning experience enjoyable, you may learn a little bit about how psychological research is conducted, and the process may help you better understand the legal process. The information gained from this study will help us better understand the factors that influence juror decision-making processes and to improve trial procedures.

\section{ALTERNATIVES}

You do not need to participate in this research. Your instructor may have alternative means for you to earn class credit, though your participation here may satisfy your instructor's research requirements.

\section{CONFIDENTIALITY}

The records of this study will be kept private and will be protected to the fullest extent provided by law. In any sort of report we might publish, we will not include any information that will make it possible to identify a subject. Research records will be stored securely and only the researcher team will have access to the records.

Any information obtained during this study that could identify you will be kept strictly confidential. The study will collect information about your IP address and the browser you are using. No personal information will be placed on your browser by the use of cookies. So that participation credit may be given, you will be required to enter your Panther ID number and your name. The personal information collected will be deleted from the database once data collection is completed and credit has been given. The information gained from this study may be published in scientific journals or presented at scientific meetings, but your identity will be kept strictly confidential.

\section{COMPENSATION \& COSTS}

There is no financial compensation to you as a result of participating in this study. Completion of this study earns partial credit toward the FIU Psychology Participant Pool requirements as outlined by your professor and on http://fiu.sona-systems.com/. For participating in this study you will receive one and a half research credits. You are free to decide not to participate in this study or to withdraw at any time without adversely affecting your relationship with the investigators or Florida International University. There will be no negative consequences for deciding not to participate or for withdrawing. If you choose to not participate in this study, you may satisfy your course requirements through other studies registered with the FIU Psychology Participant Pool at http://fiu.sona-systems.com/, or you may contact your course instructor to arrange an alternative method of obtaining credit. Participation in human subjects research is not required to earn credit in any class, and your professor is required to offer an alternative method of obtaining credit. You will not be responsible for any costs to participate in this study.

\section{RIGHT TO DECLINE OR WITHDRAW}

Your participation in this study is voluntary. You are free to participate in the study or withdraw your consent at any time during the study. Your withdrawal or lack of participation will not affect any benefits to which you are otherwise entitled.

\section{RESEARCHER CONTACT INFORMATION}

If you have any questions about the purpose, procedures, or any other issues relating to this research study you may contact Stephen Joy at [PHONE NUMBER REDACTED] or [EMAIL ADDRESS REDACTED].

\section{IRB CONTACT INFORMATION}

If you would like to talk with someone about your rights of being a subject in this research study or about ethical issues with this research study, you may contact the FIU Office of Research Integrity by phone at [PHONE NUMBER REDACTED] or [EMAIL ADDRESS REDACTED].

\section{PARTICIPANT AGREEMENT}


I have read the information in this consent form and agree to participate in this study. I have had a chance to ask any questions I have about this study, and they have been answered for me. By clicking on the "consent to participate" button below I am providing my informed consent. 


\section{APPENDIX T}

Study Two Scoring, Scale Creation, and Evaluation of Inconsistencies

\section{Verification of Inter-rater Reliability}

Skewness and kurtosis values for the distribution of "Yes" and "Partial" detection scores on the open ended questions exceeded twice the absolute value of their standard errors for all eight inconsistencies calculated for two research assistants individually using their data sets from their individual ratings done before discussion with each other, after discussion with each other, and collapsing the "Yes" and "Partial" scores into a single total of scored detections for both research assistants individually before discussion and again after discussion except for four of the 15 total distributions for PI 5Model, all of which had skewness of $.75(S E=.45)$ and kurtosis of $-1.56(S E=.87)$. The short answer questions were similarly non-normally distributed based on skewness and kurtosis values predominantly exceeding twice the absolute value of their standard errors for the same treatments and tests of data. For the short answer distributions, three of the 15 total distributions for PI 9-Year and one of the 15 total distributions for PI 7-Hair had skewness of $.75(S E=.45)$ and kurtosis of $-1.56(S E=.87)$. However, because $n=27$, I used Shapiro-Wilk tests of normality to confirm, which showed that for all non-constant scores (conducted on the same array of individual before and after ratings and collapses

of data), inconsistency detection was not normally distributed $(p<.001)$. Because of this non-normal distribution, I relied on Kendall's tau-b correlation to verify inter-rater reliability rather than Pearson's or Spearman's correlations. 


\section{Construction of Scales to Compare Relative Ease of Detection for Each}

\section{Inconsistency}

I employed the same wide range of measures used in Study One to confirm the detection rates of the remaining eight inconsistencies, retaining only those questions related to each of the eight remaining inconsistencies (see Appendix Q for all questions used). The open ended and short answer questions were independently scored by the same two research assistants blind to the hypothesis of my study using the same scoring instructions and criteria from Study One (see Appendix K for the open ended scoring rubric and Appendix L for the short answer scoring rubric). Measures also included the same multiple choice, true or false, and point blank questions used in Study One.

The variety of different ways to interpret and synergize the multiple disparate formats of data into a single metric for the purpose of evaluating the worthiness of each inconsistency for use in the later studies required me to come up with an overall scale to integrate the detection rate data. I was able to create several such scales, but before detailing their creation and components, I must explain how I interpreted each type of question.

\section{Open Ended and Short Answer Questions}

Although the research assistants used different thresholds of scoring for the open ended and short answer codings, the nature of the data set and the logistics of interpretation was common to both, so they will be discussed jointly for the purposes of this description.

The way I quantified the open ended data is based on the way both research assistants scored the set of data, and it takes into account the fact that they were recording 
the total number of mentions of a detection for each inconsistency (this turned out to be something I later recoded into a binary "present/absent" variable, since certain inconsistencies lended themselves to being described in response to more than one question — especially among the open ended questions — while others would only be a natural response to one question) so the number of mentions, which is reflected in all Yes and Partial values, is not a reliable means of distinguishing detection rates. However, they remained useful as an index of agreement between research assistants, particularly when comparing the Before values (each assistant coded the data independently) to the After values (after meeting to compare the way they coded everything and to discuss and justify why they coded a particular response in a particular way with the goal of coming to a consensus if/when possible.)

For a full description of coding procedures and instructions, see Appendices K and L, but the primary difference between open ended and short answer coding instructions related to the degree of flexibility allowed in defining a "Yes" or a "Partial" detection. Since open-ended questions lack specificity by nature, the degree of flexibility was greater for the open ended responses. However, since the short answer questions were far more specific in their wording, the research assistants were instructed to be much more exclusive and strict in their scoring and interpretations.

In creating my final scales to confirm the detection rates of all eight inconsistencies in relation to each other for the open ended and short answer questions, I focused only on the data sets each research assistant submitted after their discussion. In Study One, I came up with four different ways to recode or "finalize" the open ended and short answer data into a value corresponding to an overall detection rate for each 
inconsistency, though I ultimately used only two of them, and so I relied on these same two scoring schemes again in Study Two. In order of "loosest" level to "strictest" level, the two scoring schemes I used were:

- CombiMed: Both research assistants must give some sort of credit (Yes or Partial) to that participant for that particular inconsistency.

- CombiHighYes: Both research assistants must give that participant the exact same credit (counting only Yes scores) for that particular inconsistency.

I will return to discussion of these scoring schemes below when outlining the process I used to aggregate all question types into metrics to assist in confirming whether to retain all eight inconsistencies for use in the remainder of the research.

\section{Multiple Choice Questions}

Out of all participants who were not excluded from analysis at the outset $(N=27)$, 10 of them did not report a single inconsistency by selecting the two different choices corresponding to the correct consistent and correct inconsistent information presented in the trial transcript. While it is unclear whether these participants truly did not notice any of the inconsistencies, or they did notice but simply did not understand the instructionsparticularly that they were told it was okay to select more than one answer, or if perhaps they were not truly engaged, paying attention, and earnestly participating, they did not meet any of the pre-established criteria for exclusion. Nevertheless, the possibility remains that they may not have been fully engaged participants (though they each did indicate having noticed inconsistencies through other question formats), so I elected to consider detection rate data for the multiple choice questions both with them $(N=27)$, and without them $(N=17)$ to provide an upper and lower boundary to the detection rates among the multiple choice questions. 


\section{True or False Questions}

Interpreting the true or false inconsistency detection rates was straightforward.

Four of the eight non-dummy questions were phrased in a manner such that an inconsistency detection corresponded with a "True" response, and four of eight were phrased in a manner where a "False" response indicated an inconsistency detection. Detection rates for each inconsistency were simply the percentage of participants who answered the non-dummy questions in a manner indicating a detection.

\section{Point Blank Questions}

The point blank questions presented participants with two jurors, both of whom found one of the witness's (whichever witness makes the inconsistent statement of interest) to be inaccurate but for two different reasons. Participants were asked which of the two hypothetical jurors they most agreed with, both of whom said there was an inconsistency. However, one provided a reason based on the exact details of the manipulated inconsistency while the other provided a reason based on details similar to but not present in the manipulated inconsistency. For example, for the point blank question associated with PI 5-Model, the choices are whether Marc Zollinger's testimony is inaccurate because he said that Brock Collins drove a Corvette (which is how the inconsistency is presented in the testimony) or because he said that Brock Collins drove a Camaro (which is not the basis of the inconsistent testimony). There is also an "I don't agree with either of these jurors." option.

The point blank questions can actually provide two different "levels" of diagnostic information. First, those participants who correctly identify the basis of the incorrect testimony are credited with having detected the inconsistency. Second, those 
participants who selected the incorrect basis (essentially, those who did not get the question right, but who nevertheless indicated some belief in an inconsistency concerning the topic at issue, or who may have noticed an inconsistency but could not recalled the exact details correctly—basically, everyone but those participants who chose “I don't agree with either of these jurors.") are able to provide a very liberal definition of detection. Of course, these participants may have simply guessed or deduced the nature of the inconsistency based on exposure to prior questions, and for that reason this would make a terrible metric if it were the sole measure of inconsistency detection rate, but as the final set of questions, and as the most liberal interpretation of the most direct and "easiest" of the inconsistency detection measures, it is useful enough to simply consider these participants who did not answer the question distinctly incorrectly within the more lenient of the two detection rate scoring composites.

\section{Scales to Summarize Relative Ease of Detection for Each Inconsistency}

For the open ended and short answer questions, I employed the CombiMed as the "loose" or more liberal scale and CombiHighYes as the more discriminating "strict" scoring schemes. The two criteria levels for the multiple choice questions (loose: counting only those 17 participants who got at least one multiple choice question correct by providing both the consistent and the inconsistent answers vs. strict: counting all 27 participants) are so named for their effect on overall detection rates (with loose leading to higher overall percentage of detections, and strict leading to a lower overall percentage), not the overall "standard for inclusion as an inconsistency detection", which would have resulted in the opposite naming scheme. Similarly, it is important to keep this distinction in mind for the point blank criteria naming convention (loose: counting both those 
participants who got the question correct as well as those who chose the incorrect option as a reason for finding the witness's testimony unconvincing — essentially everyone who didn't select "I don't agree with either of these jurors" vs. strict: counting only those participants who got the question correct).

I applied the same reasoning to the names of the two composite scoring schemes, such that the Strict scheme will provide the lowest overall percentage of detections, but the detection rates it yields for each inconsistency will be the unequivocal minimum levels of detection. The Strict criteria level averages Open Ended CombiHighYes, Short Answer CombiHighYes, all 27 Multiple Choice responses, True/False, and Point Blank. On the other end of the spectrum, the Loose scheme will provide the highest overall percentage of detections according to the most forgiving definitions of inconsistency detection. The Loose criteria level averages Open Ended CombiMed, Short Answer CombiMed, Multiple Choice only 17 (using the Multiple Choice responses from only those 17 participants who got at least one multiple choice question correct, in percentage equivalent), True/False, and Point Blank Combined (counting detections for all participants who did not choose “I don't know.") 
Table T1

Study Two: Loose Scoring Scheme Detection Rate Percentages for all Eight

Inconsistencies by Question Type

\begin{tabular}{|c|c|c|c|c|c|c|}
\hline Inconsistency & $\begin{array}{c}\text { Open } \\
\text { Ended } \\
\text { CombiMed }\end{array}$ & $\begin{array}{c}\text { Short } \\
\text { Answer } \\
\text { CombiMed }\end{array}$ & $\begin{array}{l}\text { Multiple } \\
\text { Choice } \\
17 \text { non- } \\
\text { 0's }\end{array}$ & $\begin{array}{l}\text { True } \\
\text { False }\end{array}$ & $\begin{array}{c}\text { Point } \\
\text { Blank } \\
\text { Combined }\end{array}$ & $\begin{array}{l}\text { Loose } \\
\text { Score }\end{array}$ \\
\hline PI 7-Hair & 40.7 & 44.4 & 58.8 & 66.7 & 63.0 & 54.7 \\
\hline PI 9-Year & 25.9 & 29.6 & 58.8 & 63.0 & 70.4 & 49.5 \\
\hline PI 4-Oil Co. & 22.2 & 22.2 & 47.1 & 37.0 & 63.0 & 38.3 \\
\hline PI 5-Model & 33.3 & 14.8 & 23.5 & 48.1 & 59.3 & 35.8 \\
\hline PI 13-Dog & 0.0 & 18.5 & 41.2 & 37.0 & 70.4 & 33.4 \\
\hline PI 12-Tree & 7.4 & 11.1 & 29.4 & 33.3 & 77.8 & 31.8 \\
\hline PI 10-Color & 11.1 & 3.7 & 23.5 & 29.6 & 66.7 & 26.9 \\
\hline PI 11-Jared & 25.9 & 3.7 & 23.5 & 33.3 & 37.0 & 24.7 \\
\hline Mean Rate & 20.8 & 18.5 & 38.2 & 43.5 & 63.5 & 36.9 \\
\hline
\end{tabular}


Table T2

Study Two: Strict Scoring Scheme Detection Rate Percentages for all Eight

Inconsistencies by Question Type

\begin{tabular}{lcccccc}
\hline Inconsistency & $\begin{array}{c}\text { Open Ended } \\
\text { CombiHighYes }\end{array}$ & $\begin{array}{c}\text { Short Answer } \\
\text { CombiHighYes }\end{array}$ & $\begin{array}{c}\text { Multiple } \\
\text { Choice }\end{array}$ & $\begin{array}{c}\text { True } \\
\text { False }\end{array}$ & $\begin{array}{c}\text { Point } \\
\text { Blank }\end{array}$ & $\begin{array}{c}\text { Strict } \\
\text { Score }\end{array}$ \\
\hline PI 9-Year & 22.2 & 22.2 & 37.0 & 63.0 & 51.9 & 39.3 \\
PI 7-Hair & 25.9 & 11.1 & 37.0 & 66.7 & 51.9 & 38.5 \\
PI 4-Oil Co. & 14.8 & 18.5 & 29.6 & 37.0 & 40.7 & 28.1 \\
PI 5-Model & 18.5 & 11.1 & 14.8 & 48.1 & 44.4 & 27.4 \\
PI 12-Tree & 7.4 & 7.4 & 18.5 & 33.3 & 66.7 & 26.7 \\
PI 13-Dog & 0.0 & 11.1 & 25.9 & 37.0 & 51.9 & 25.1 \\
PI 10-Color & 11.1 & 3.7 & 14.8 & 29.6 & 44.4 & 20.7 \\
PI 11-Jared & 14.8 & 0.0 & 14.8 & 33.3 & 37.0 & 20.0 \\
Mean Rate & 14.3 & 10.6 & 24.1 & 43.5 & 48.6 & 28.2 \\
\hline
\end{tabular}




\section{APPENDIX U}

Study Three Data Reduction Procedures

\section{Step One: Raw Data}

At the conclusion of data collection, the final data file included 389 total entries, or one separate entry for each discrete time Study Three was accessed and begun. Of these 389 entries, 319 were complete, having accessed every page of the study website. To be clear, these are not total numbers of distinct participants, but rather all separate instances of data recordings initiated each time the study was started.

\section{Step Two: Eliminating Repeat Starters}

Despite repeated admonitions and stern warnings to the contrary, some participants in online studies nevertheless begin the study and then quit partway through completion, only to return at a later time to complete the study in full. Because the objective of Study Three is to determine the effectiveness of inducing fear, anger, or no emotional response through the use of different film clips, I wanted to use data from only those participants who were exposed to the stimulus materials a single time. Not only would participants with prior exposure have an advance familiarity with the study which could influence the way they respond to the pre-test of emotional state, but repeated viewings of the same film clip may alter its emotional impact, either intensifying or reducing its effect, neither of which I wanted distorting the results of these baseline confirmations, as participants in Studies Four and Five will not have repeated viewings. As such, I eliminated all data associated with those participants who accessed the study on more than one occasion. However, for any participants who completed the study the 
first time they accessed it, but who may have started it again, I simply eliminated all data associated with any subsequent attempts since their first exposure was untainted.

After eliminating the data associated with any participants who had been preexposed to the study, 329 separate data entries remained.

\section{Step Three: Eliminating Non-Consenting Participants}

Two participants did not agree to participate in the study after having read the Informed Consent document (see Appendix V). I eliminated those two participants who did not grant consent to participate, leaving 327 separate data entries remaining.

\section{Step Four: Eliminating Partial Participants}

Steps Two, Three, and Four could have been performed in interchangeable order with no consequence, but I expressly refrained from eliminating the participants who did not complete the study at the start of the data reduction process because doing so would have prevented me from having the ability to identify those participants who began the study and were exposed to some or all of the study materials and quit partway through, only to restart and complete the study at a later time — and with an improper degree of exposure to the measures and film clips. It is only after this step that the remaining data entries can properly be called "participants." Of the 327 data entries remaining before this step, only 319 participants completed the entire study.

\section{Step Five: Eliminating Extreme Outliers of Time Spent on Film Clips}

The goal of Study Three is to measure and confirm the effectiveness of the three different film clips as means to induce emotions, so I wanted to eliminate from consideration those participants who spent too long on the webpage featuring the film clip before continuing on to the post-film measures. Regardless of the basis for any delay 
(and a lengthy period beyond the known duration of the film clip is not necessarily an indicator of inattention, but it is obviously an indicator of atypical rate of completion), I did not want these inexplicably dilatory participants to compromise the accuracy of my assessments. To identify these extreme outliers, I created a "ClipLag" variable by subtracting the known duration of whichever film clip each participant viewed from the total time spent on the webpage featuring their randomly assigned film clip. Even though the different film clips vary in duration, by creating the ClipLag variable this way, I was able to standardize the length of time spent after each participant's film clip had ended and before they clicked "Continue" to go on to the next page.

I defined an extreme outlier as a participant at or above the $97.5^{\text {th }}$ percentile in terms of their ClipLag value. The $97.5^{\text {th }}$ percentile was 650.8 seconds delay, which resulted in six participants being identified as extreme outliers ranging from 857.1 seconds to 3332.6 seconds spent $(M=1391.1, S D=957.2)$ and eliminated from further analysis, leaving a total of 313 participants.

\section{Step Six: Eliminating Extreme Outliers of Total Time Spent on Entire Study}

In addition to using the delay between watching the film clip and proceeding to the next page as a criteria for exclusion, I also used the total time spent on the study as a way to identify atypical participants to exclude from analysis. To standardize the values for total time spent on Study Three, I subtracted the duration of each participant's randomly assigned film clip from the total time they spent on the study in order to eliminate the natural variances due to film clips of unequal length. Defining extreme outliers as those participants in the $97.5^{\text {th }}$ percentile of total time spent, which was 2486.9 seconds (or 41.4 minutes), I eliminated three participants whose total times were 2730.3 
seconds (45.5 minutes), 3279.5 (54.7 minutes), and 8059.9 (134.3 minutes), leaving 310 participants who were not extreme outliers according to either timing criteria.

\section{Step Seven: Eliminating Outliers of Total Time Spent}

After eliminating those extreme outliers which would skew the overall distribution of time spent, I calculated the distribution of total time spent for the remaining 310 participants on a condition by condition basis, resulting in the elimination of nine participants whose total time spent was in excess of two standard deviations above the mean for their experimental conditions. There were no participants who completed the study in less time than two standard deviations below the means of their experimental conditions, leaving 301 participants remaining.

\section{Step Eight: Eliminating Outliers of Duration Between Clip Conclusion and Continuing}

Having excluded the extreme outliers for the ClipLag duration and total time spent, and without those participants whose total time spent exceeded two standard deviations beyond the mean, I still wanted to eliminate those remaining participants who had an unusually long delay between the time their film clip ended and the point when they clicked the "Continue" button to load the next webpage. I calculated the mean latency for each film clip condition and eliminated those thirteen participants whose times were greater than two standard deviations past the mean, leaving me with 288 participants.

\section{Step Nine: Eliminating Participants in Highly Emotional States Prior to Induction}

In order to determine the overall effectiveness of the various film clips at inducing the target emotions, I administered the I-PANAS-SF (Thompson, 2007) prior to the film 
clips and the PANAS-X (Watson \& Clark, 1994, see Appendix O) afterwards. Gauging the effectiveness of the emotion inductions required participants who were not already in an elevated emotional state prior to receiving the film clip manipulation, so I calculated the mean ratings for each of the eleven emotions represented on the I-PANAS-SF pretest (plus a total of all eleven ratings), and then calculated the exact value of two standard deviations above and below the mean for each emotion and the combined total. Because the I-PANAS-SF uses ratings on a scale from 1 (Very Slightly or Not At All) to 5 (Extremely), two standard deviations above or below the mean was beyond the limits of the scale, but responding with a score of 1 in terms of attentiveness was two standard deviations below the mean $(M=3.51, S D=1.13)$, as was a total score of all emotion ratings of 11 or less $(M=24.72, S D=6.68)$. There were more emotions where it was possible to give a high rating greater than two standard deviations beyond the mean. Those emotions where a rating of 3 or more was greater than two standard deviations above the mean were hostile $(M=1.25, S D=0.64)$, ashamed $(M=1.23, S D=0.68)$, angry $(M=1.31, S D=0.69)$, afraid $(M=1.27, S D=0.74)$, and $\operatorname{mad}(M=1.33, S D=$ 0.75). Those emotions where a rating of 4 or more was greater than two standard deviations above the mean were upset $(M=1.46, S D=1.13)$ and nervous $(M=1.56, S D$ $=0.90$ ), while a total of 39 or more was greater than two standard deviations above the mean for a combined value of all eleven ratings $(M=24.72, S D=6.68)$.

Because I am experimentally inducing fear or anger among some participants, I eliminated six participants who exceeded two standard deviations above the mean on either afraid, angry, or mad (four of those six participants exceeded the upper limit on at least two of the three critical emotions). Of the remaining participants, I eliminated eight 
who were beyond the upper or lower boundaries on five of the nine possible ratings, two who were beyond the boundaries on six ratings, three who were beyond the boundaries on seven ratings, and one participant who was beyond the established limits on eight of the possible nine ratings where exclusion was possible. This left me with 268 remaining participants.

\section{Step Ten: Eliminating Highly Emotionally Volatile Participants}

One benefit of measuring participants' real-time emotion using the PANAS-X (which specifically instructs participants to "Indicate to what extent you feel this way right now." for each emotion) immediately following the film clip and then to follow with the Post-Film Questionnaire (Rottenberg, Ray, \& Gross, 2007, see Appendix A), which specifically instructs participants that "The following questions refer to how you felt while watching the film." which, while certainly not constituting an exact replication of the PANAS-X, still allowed me to isolate those participants who exhibited an extreme variation in emotion ratings from the time of the film clip to immediately after watching

it. Whether these highly volatile, vacillating participants were simply clicking responses haphazardly without reading the actual questions on one or both emotional rating scales, or if they are legitimately mercurial and emotionally capricious is immaterial—either way, I do not want to include their responses in my analysis.

To contrast ratings on the PANAS-X with those from the Post-Film Questionnaire, which asks for a rating on a scale from 0 (Not At All/None) to 8 (Extremely/A Great Deal), I had to convert the Post-Film Questionnaire responses into an equivalent scale, which I did by changing a rating of 0 to a 1 , a 1 to a 1.5 , a 2 remained a 2, a 3 to a $2.5,4$ to 3,5 to $3.5,6$ to 4,7 to 4.5 , and an 8 to a 5 , which aligns perfectly with 
the 1 to 5 scale used by the PANAS-X. I then calculated the absolute value of the difference between responses on the PANAS-X and the scaled Post-Film Questionnaire responses for anger, disgust, guilt, happiness, interest, joy, sadness, and surprise since those eight emotions are listed identically on both measures. I did not compare afraid (PANAS-X) to fear (Post-Film Questionnaire), proud (PANAS-X) to pride (Post-Film Questionnaire), or ashamed (PANAS-X) to shame (Post-Film Questionnaire) due to the remote possibility that these minor variations in presentation might yield unexpected differences.

I calculated the sum of the absolute values of the differences in ratings for those eight emotions as well as the mean difference across all eight emotions for each participant. I then calculated the means for both of those values from all 268 participants. The average sum of the absolute values of the difference in ratings ranged from 0.00 to $25.00(M=4.83, S D=3.28)$ while the individual average differences in ratings ranged from 0.0 to $3.57(M=0.69, S D=0.47)$. I calculated the $97.5^{\text {th }}$ percentile for each of these values, which was 13.00 for the individual sum of differences and 1.86 for the individual mean differences, and I eliminated the nine participants whose values exceeded both thresholds, leaving me with 259 participants.

\section{Step Eleven: Eliminating Internally Inconsistent Participants}

The final step of my data reduction procedure involves identifying those participants who may have been "Christmas treeing" their responses (that is, indiscriminately choosing answers based on nothing more than achieving an aesthetically balanced array of responses, the way one might approach decorating a Christmas tree) or otherwise not carefully considering their emotions while completing the measures. Part 
of what makes the PANAS-X such a reliable measure is its thoroughness at probing highly similar, interrelated traits. Three such examples are attentiveness, which is measured not only by requiring participants to rate how attentive they are, but also by including alert and concentrating. Similarly, fatigue as a broader construct is measured by tired, sleepy, and drowsy, while serenity is measured by relaxed, calm, and at ease response ratings. Because all nine of these different subcomponents are presented on the same webpage which instructs participants to rate the extent 'to which they feel that way right now,' it is unlikely that attentive, thoughtful, engaged participants will give highly disparate responses to each of these three sets of similar traits. However, since these nine emotion ratings are interspersed throughout the larger PANAS-X featuring 52 other additional emotions to rate, a participant who is not truly engaged in the study is unlikely to notice relatively redundant questions or give internally consistent responses purely by random chance.

I calculated the variance within the three attentiveness-related emotions (attentive, alert, and concentrating) for each participant, and then I calculated the $97.5^{\text {th }}$ percentile of variance for the remaining 259 participants, which was 3.56. I then did the same for the three fatigue-related emotions (tired, sleepy, and drowsy), calculating the variance between the three responses given by each participant and then the $97.5^{\text {th }}$ percentile (2.89), followed by the variance between the three serenity-related emotions (relaxed, calm, and at ease), for each participant, and the $97.5^{\text {th }}$ percentile (2.00). I eliminated the ten participants who had variances at or beyond two of the three $97.5^{\text {th }}$ percentile calculations, leaving me with a final total of 249 participants for Study Three. 


\title{
APPENDIX V
}

\author{
Study Three Informed Consent
}

\section{FIU}

\section{ADULT ONLINE CONSENT TO PARTICIPATE IN A RESEARCH STUDY Civil Juror Decision-Making}

Thank you for looking into our study. Over the next few webpages you will answer some questions and view a short film clip. Before participating, please read the information in the official consent document below.

\begin{abstract}
**IMPORTANT: You may use any of the following web browsers AS LONG AS JAVASCRIPT AND ADOBE/SHOCKWAVE FLASH ARE ENABLED: Mozilla Firefox, Google Chrome, Apple Safari, and Internet Explorer. If you have JavaScript or Flash turned off, or use any add-ons, extensions, or shortcuts which block JavaScript or Flash, please either disable them, add qualtrics.com to the list of approved sites, enable JavaScript, enable Flash, or run your browser in Safe Mode**
\end{abstract}

Please note, you must have cookies, JavaScript, and Flash enabled on your browser in order to participate. If you click the button below but do not leave this page, your browser is not supporting cookies. Cookies are used only so that you can have a unique identification number for this study; no personal information will be stored in cookies.

You must also have the capability to view Flash video clips on your computer (the same sorts of clips which are used on YouTube), and you must be able to listen to the video clips, either through the use of speakers or headphones.

Please complete this study at one time. If for any reason you are not able to complete the study in one sitting, you will have to start over. The experiment takes roughly one hour to complete.

As a student in the FIU Psychology Participant Pool (enrolled in PSY 2012 or other class), you are invited to participate in a research study conducted over the Web. The following information is provided to help you make an informed decision whether or not to participate. Your participation is completely voluntary, and not participating will not affect your class grade in any way. You must be at least 18 years old to participate.

\section{PURPOSE OF THE STUDY}

You are being asked to be in a research study. The purpose of this study is to investigate how jurors make decisions. Completion of this study will take approximately 30 minutes.

\section{NUMBER OF STUDY PARTICIPANTS}

If you decide to be in this study, you will be one of approximately 180 people in this research study.

\section{DURATION OF THE STUDY}

Your participation will require approximately 30 minutes.

\section{PROCEDURES}

If you agree to be in the study, we will ask you to do the following things:

You will be asked to watch a short film clip as well as answer some questions about how you feel. You can refuse to answer any question and cease participation at any time.

\section{RISKS AND/OR DISCOMFORTS}

The following risks may be associated with your participation in this study: You might become upset while viewing the short film clip from a popular movie. This event is unlikely, but if you are concerned about it you 
may withdraw from the study at any time.

\section{BENEFITS}

The following benefits may be associated with your participation in this study:you may find the learning experience enjoyable, you may learn a little bit about how psychological research is conducted, and the process may help you better understand the legal process. The information gained from this study will help us better understand the factors that influence juror decision-making processes and to improve trial procedures.

\section{ALTERNATIVES}

You do not need to participate in this research. Your instructor may have alternative means for you to earn class credit, though your participation here may satisfy your instructor's research requirements.

\section{CONFIDENTIALITY}

The records of this study will be kept private and will be protected to the fullest extent provided by law. In any sort of report we might publish, we will not include any information that will make it possible to identify a subject. Research records will be stored securely and only the researcher team will have access to the records.

Any information obtained during this study that could identify you will be kept strictly confidential. The study will collect information about your IP address and the browser you are using. No personal information will be placed on your browser by the use of cookies. So that participation credit may be given, you will be required to enter your Panther ID number and your name. The personal information collected will be deleted from the database once data collection is completed and credit has been given. The information gained from this study may be published in scientific journals or presented at scientific meetings, but your identity will be kept strictly confidential.

\section{COMPENSATION \& COSTS}

There is no financial compensation to you as a result of participating in this study. Completion of this study earns partial credit toward the FIU Psychology Participant Pool requirements as outlined by your professor and on http://fiu.sona-systems.com/. For participating in this study you will receive one half research credit. You are free to decide not to participate in this study or to withdraw at any time without adversely affecting your relationship with the investigators or Florida International University. There will be no negative consequences for deciding not to participate or for withdrawing. If you choose to not participate in this study, you may satisfy your course requirements through other studies registered with the FIU Psychology Participant Pool at http://fiu.sona-systems.com/, or you may contact your course instructor to arrange an alternative method of obtaining credit. Participation in human subjects research is not required to earn credit in any class, and your professor is required to offer an alternative method of obtaining credit. You will not be responsible for any costs to participate in this study.

\section{RIGHT TO DECLINE OR WITHDRAW}

Your participation in this study is voluntary. You are free to participate in the study or withdraw your consent at any time during the study. Your withdrawal or lack of participation will not affect any benefits to which you are otherwise entitled.

\section{RESEARCHER CONTACT INFORMATION}

If you have any questions about the purpose, procedures, or any other issues relating to this research study you may contact Stephen Joy at [PHONE NUMBER REDACTED] or [EMAIL ADDRESS REDACTED].

\section{IRB CONTACT INFORMATION}

If you would like to talk with someone about your rights of being a subject in this research study or about ethical issues with this research study, you may contact the FIU Office of Research Integrity by phone at [PHONE NUMBER REDACTED] or [EMAIL ADDRESS REDACTED].

\section{PARTICIPANT AGREEMENT}

I have read the information in this consent form and agree to participate in this study. I have had a chance to ask any questions I have about this study, and they have been answered for me. By clicking on the "consent to participate" button below I am providing my informed consent. 


\section{APPENDIX W}

Study Three Preliminary Investigation

My original plans did not call for Study Three to be broken up into two separate efforts, though some unexpected results required that I re-run Study Three with a few adjustments. I am appending this appendix in the interest of completeness and to provide the frame of reference necessary to support the purpose and design of the main Study Three materials featured in the body of this dissertation. However, due to nature of my findings from this effort, I will simply provide a summary rather than the full degree of elaboration appropriate for a main chapter.

My first (and what was intended to be the only) attempt at Study Three was a 3 (induction target emotion/level of certainty: fear/low certainty vs. anger/high certainty vs. neutral emotional impact/control level of certainty) x 2 (film clip; two for each target emotion - fear: boy playing in hallway from The Shining [1980] vs. basement chase scene from Silence of the Lambs [1991]; anger: police abusing protestors from Cry Freedom [1987] vs. bullying scene from My Bodyguard [1980]; neutral/no emotion: a screen-saver featuring abstract shapes [Gross \& Levenson, 1995; Rottenberg, Ray, \& Gross, 2007] versus a nature scene from Alaska's Wild Denali [1997]; see Appendix A) pretest-posttest control mixed factorial design.

The purpose was to confirm that these specific six film clips, which had been rigorously tested and validated by prior research (Gross and Levenson, 1995; Rottenberg, Ray, \& Gross, 2007) would each elicit their respective target emotions and no other emotions within my pool of participants at FIU. It had been my intention to use all six film clips so that having two distinct clips to elicit each emotional response could be 
more generalizeable. It was not my intention at this stage to pit the two film clips for each emotion against one another in order to select the more effective of the two. I had no reason to expect that prior results would not generalize to FIU students, I simply wanted to be sure in order to maintain maximum experimental control.

Participants completed the brief, 11-item I-PANAS-SF (Thompson, 2007; see Appendix O) before watching one of the six randomly assigned film clips to measure their baseline, pre-existing emotions. In the interest of maximizing the effectiveness of the emotion inductions, I included an autobiographical writing and perspective-taking exercise following the film clip in which participants were instructed to "please describe the film clip you just watched as if you were there to experience it in person" (see Lerner \& Keltner, 2001; Martin, 1990; and Westermann et al., 1996 for more on emotion induction techniques featuring autobiographical reflection and perspective taking writing tasks). Immediately after participants finished writing about how they would have felt if they were directly involved in the film clip, they completed the 60-item PANAS-X (Watson \& Clark, 1994; see Appendix O) to gauge their final emotional state after the emotion inductions.

This effort yielded three distinct discoveries, one having to do with each of the three target emotions — one troublesome, one complicated, and one straightforward. First, it became clear that experimentally inducing fear was very difficult. I had known as much at the outset of my research, and the vast collection of resources I had consulted concerning the experimental induction of emotions (through film clips and otherwise) had made it clear that due to the unavoidable artificiality of all but the most ecologically valid experimental settings (and the ethical responsibilities of researchers), fear was 
unlikely to be induced to a high degree relative to the magnitude of effectiveness found with other experimentally induced emotions. Neither of the film clips intended to induce fear (The Shining and Silence of the Lambs) produced a statistically significant increase in fear ratings in comparison to the four other film clips.

I suspected that perhaps the autobiographical writing exercise may have been compromising the effectiveness of the fear induction because of what Lerner and Keltner (2000) dubbed the "cognitive-awareness hypothesis" which suggests that upon close, deliberate awareness or consideration of their effects, appraisal tendencies have less effect because they are more readily discounted as incidental or disregarded as a simply a byproduct of circumstance. While perspective taking is not strictly analogous to being held accountable for one's decision-making process, past research has demonstrated very broadly that awareness of an unrelated influence will reduce the scope of that influence on a separate task or decision-making process (see Lerner \& Tetlock, 1999, for a wideranging summary of these types of effects). In the event that the lack of fear induction from the film clips may have been due to a sort of "disregard for emotional effects known to be from an incidental source" phenomena when faced with the perspective taking writing task, in my "second" Study Three, I added the presence or absence of the autobiographical writing task as an independent variable such that approximately half of the participants would engage in the writing task after the film clip and before the PANAS-X, and approximately half would not, instead going straight from watching the film clip to completing the PANAS-X.

Second, it was clear that of the two film clips intended to induce anger (Cry Freedom and My Bodyguard), the clip from Cry Freedom featuring white South African 
soldiers opening fire on a crowd of peaceful black anti-apartheid protestors (including a soldier taking careful aim with a pistol in order to shoot a fleeing young boy in the back) elicited a greater degree of anger than did the clip from My Bodyguard, which featured a boy getting beaten up by a bully who also damaged the victim's motorcycle before pushing it into a lake. Unfortunately, the Cry Freedom clip also elicited increased ratings of several other emotions, including afraid, frightened, and nervous. It was my suspicion that perhaps the large subset of Cuban-American students at FIU might have been more sensitive and viscerally reactive to a scene of violent political oppression than those samples selected from less atypical populations used by Gross and Levenson (1995) and later by Rottenberg, Ray, and Gross (2007), though since I was going to be rerunning Study Three with the autobiographical writing component manipulation anyway, it made good sense to reexamine both anger film clips under the new design.

The third discovery from this initial effort was that the neutral, non-emotioninducing screen saver/abstract shapes clip used by Gross and Levenson (1995) and Rottenberg, Ray, and Gross (2007) seemed to be eliciting general confusion and even annoyance among some participants. The clip was almost four minutes long, so confusion and annoyance are not totally unreasonable responses, especially when considering the relative incongruity of the autobiographical writing task. However, the Alaska's Wild Denali clip did not induce (or reduce) any emotions, so I decided to use that clip as my neutral, non-emotion-inducing control group film clip in Studies Four and Five, and to instead focus exclusively on the two fear induction and two anger induction clips for the "main" Study Three. In addition to these changes, I also implemented a much more rigorous and multi-faceted approach to data reduction for the main Study 
Three (see Appendix U) than I undertook during this initial phase in case these findings may have been due in part to some inattentive or insincere participants obscuring more robust effects among attentive, earnest, dedicated participants. 


\section{APPENDIX X \\ Study Four Data Reduction Procedures}

\section{Step One: Raw Data}

At the conclusion of data collection, the final data file included 579 total entries, or one separate entry for each discrete time Study Four was accessed and begun. Of these 579 entries, 357 were complete, having accessed every page of the study website. To be clear, these are not total numbers of distinct participants, but rather all separate instances of data recordings initiated each time the study was started.

\section{Step Two: Eliminating Repeat Starters}

Just as in the prior three studies, despite repeated reminders and unequivocal directions to the contrary, some participants in online studies nevertheless begin the study and then quit partway through completion, only to return at a later time to complete the study in full. Because Study Four requires the effective induction of fear, anger, or no emotional response through the use of different film clips, I wanted to use data from only those participants who were exposed to the stimulus materials a single time. Repeated viewings of the same film clip may alter its emotional impact, either intensifying or reducing its effect, and viewing multiple film clips would signal the nature of one of my independent variables, while multiple exposures to the trial transcript could artificially boost inconsistency detection rates. As such, I eliminated all data associated with those participants who accessed the study on more than one occasion. However, for any participants who completed the study the first time they accessed it, but who may have started it again, I simply eliminated all data associated with any subsequent attempts since their first exposure was untainted. 
After eliminating the data associated with any participants who had been preexposed to the study, 363 separate data entries remained.

\section{Step Three: Eliminating Partial Participants}

Steps Two, Three, and Four could have been performed in interchangeable order with no consequence, but I expressly refrained from eliminating the participants who did not complete the study at the start of the data reduction process because doing so would have prevented me from having the ability to identify those participants who began the study and were exposed to some or all of the study materials and quit partway through, only to restart and complete the study at a later time - and with an improper degree of exposure to the measures and film clips. It is only after this step that the remaining data entries can properly be called "participants." Of the 363 data entries remaining before this step, only 294 participants completed the entire study.

\section{Step Four: Eliminating Non-Consenting Participants}

Two participants did not agree to participate in the study after having read the Informed Consent document for Part Two of Study Four (see Appendix Y). I eliminated those participants who did not grant consent to participate, leaving 292 separate data entries remaining.

\section{Step Five: Eliminating Extreme Outliers of Time Spent on Film Clips}

Study Four depends on the effective and consistent induction of emotion through the use of film clips, so I wanted to eliminate from consideration those participants who spent too long on the webpage featuring the film clip before continuing on to the trial transcript. Just as in Study Three, I did not want these laggardly participants to compromise the accuracy of my assessments. To identify these extreme outliers, I again 
created a "ClipLag" variable by subtracting the known duration of whichever film clip each participant viewed from the total time spent on the webpage featuring their randomly assigned film clip.

I defined an extreme outlier as a participant at or above the $97.5^{\text {th }}$ percentile in terms of their ClipLag value. Before calculating the extreme outlying $97.5^{\text {th }}$ percentile, I eliminated a participant who waited 82,998 seconds (over 23 hours) to proceed to the next page. Barring that individual, the $97.5^{\text {th }}$ percentile for those participants who watched The Shining was 246.1 seconds delay, which resulted in two participants being identified as extreme outliers (259.3 and 585.1 seconds), while the $97.5^{\text {th }}$ percentile for those participants who watched My Bodyguard was 1014.7 seconds, which also resulted in two exclusions (1136.1 and 1648.1 seconds), and the $97.5^{\text {th }}$ percentile of submission time for those participants who watched Alaska's Wild Denali was 790.9 seconds, which led to the elimination of two additional participants (842.2 and 2688.6 seconds), leaving a total of 285 participants at this stage.

\section{Step Six: Eliminating Extreme Outliers of Total Time Spent on Entire Study}

In addition to using the delay between watching the film clip and proceeding to the next page as a criteria for exclusion, I also used the total time spent on the study as a way to identify atypical participants to exclude from analysis. To standardize the values for total time spent on Study Four, I subtracted the duration of each participant's randomly assigned film clip from the total time they spent on the study in order to eliminate the natural variances due to film clips of unequal length. Defining extreme outliers as those participants in the $97.5^{\text {th }}$ percentile of total time spent, I calculated separate values for each of the six experimental conditions since those participants who 
were exposed to the transcript version featuring inconsistencies (and who noticed any of them) would presumably take longer to complete the open ended and short answer questions than would those participants who were exposed to the transcript without any inconsistencies and who therefore would not have any reason to give lengthy answers to those questions.

The $97.5^{\text {th }}$ percentile for those participants who watched The Shining and who were exposed to the trial transcript featuring inconsistent testimony was $10,477.2$ seconds, eliminating two participants who spent 13,363.2 and 14,135.6 seconds. The cutoff for those participants who watched My Bodyguard and who were exposed to the trial transcript with inconsistencies had a $97.5^{\text {th }}$ percentile of $13,802.1$ seconds, excluding three participants $(M=46,620.7, S D=34,612.1)$. Those participants who watched Alaska's Wild Denali and were exposed to the inconsistent version of the transcript had a $97.5^{\text {th }}$ percentile of $12,394.6$ seconds, which eliminated six participants $(M=98,262.9$, $S D=53,413.1)$. For those who watched The Shining but did not have the inconsistent transcript, the $97.5^{\text {th }}$ percentile was $13,635.7$ seconds, which resulted in the elimination of three participants $(M=17,475.7, S D=1,719.4)$. Watching My Bodyguard in combination with the inconsistency-free transcript yielded a $97.5^{\text {th }}$ percentile of $12,218.2$ seconds, eliminating six participants $(M=20,403.3, S D=5,556.0)$. Finally, the Alaska's Wild Denali clip in conjunction with the inconsistency-free transcript had a $97.5^{\text {th }}$ percentile of $11,958.3$ seconds, which led to the elimination of five participants $(M=$ $59,975.0, S D=30,045.7)$. After this round of exclusions, 260 participants remained. 


\section{Step Seven: Eliminating Outliers of Total Time Spent}

After eliminating those extreme outliers which would skew the overall distribution of time spent, I calculated the distribution of total time spent for the remaining 260 participants on a condition by condition basis, resulting in the elimination of ten participants whose total time spent was in excess of two standard deviations above the mean for their experimental conditions. This resulted in the elimination of two participants with times of 12,257.0 and 13,802.1 from the My Bodyguard and inconsistent transcript condition; three participants from the Alaska's Wild Denali and inconsistent transcript condition $(M=11,842.5, S D=582.9)$, one participant each who watched $T h e$ Shining (13,635.7 seconds) and My Bodyguard (12,218.2 seconds) with the inconsistency-free transcript, and three participants who watched Alaska's Wild Denali and were exposed to the inconsistency-free transcript $(M=11,520.5, S D=380.3)$. There were no participants who completed the study in less time than two standard deviations below the means of their experimental conditions, leaving 250 participants remaining.

\section{Step Eight: Eliminating Outliers of Duration Between Clip Conclusion and}

\section{Continuing}

Having excluded the extreme outliers for the ClipLag duration and total time spent, and without those participants whose total time spent exceeded two standard deviations beyond the mean, I still wanted to eliminate those remaining participants who had an unusually long delay between the time their film clip ended and the point when they clicked the "Continue" button to load the next webpage. I calculated the mean latency for each film clip (The Shining: $M=22.0, S D=41.1 ;$ My Bodyguard: $M=41.1$, $S D=61.1$; Alaska's Wild Denali: $M=44.7, S D=68.5)$ and eliminated 26 participants 
whose times were greater than two standard deviations past the mean, leaving me with 224 participants.

\section{Step Nine: Eliminating Participants with Elevated Baseline Emotion Levels}

Since validation of the film clips in Study Three required that I eliminate participants showing high levels of emotion during the pre-film report, and because my inductions of emotion would have less impact on participants who are already in a highly emotional state, I calculated the mean ratings on a scale of 1 (very slightly or not at all) to 5 (extremely) as well as the standard deviation for each of the twelve emotions listed on the I-PANAS-SF which was administered during Part One (upset: $M=1.38, S D=.74$; hostile: $M=1.21, S D=.66$; alert: $M=3.00, S D=1.21$; ashamed: $M=1.21, S D=.68$; angry: $M=1.29, S D=.72$, nervous: $M=1.55, S D=.96$; determined: $M=3.39, S D=$ 1.27; attentive: $M=3.64, S D=1.04$; afraid: $M=1.29, S D=.71$; active: $M=2.67, S D=$ 1.30; inspired: $M=2.73, S D=1.33$; mad: $M=1.33, S D=.77)$. I then calculated the value of the mean plus and minus twice the standard deviation, and created a twelve sorting variables to indicate whether each participant's rating for each emotion was above or below two standard deviations from the mean. Since the scale used only ranged from 1 to 5 , it was impossible for five highly rated emotions (alert, determined, attentive, active, and inspired) to have a value exceeding two standard deviations, while only one emotion (attentive) had values which would allow for a participant's rating to be greater than two standard deviations below the mean.

I eliminated twelve participants who were beyond the boundaries on four or more of the eight possible ratings, which left $96.4 \%$ of the existing sample intact, or 212 participants. 


\section{Step Ten: Eliminating Participants who Failed Attention Checks}

I included several "attention check" questions throughout the inconsistency detection questions within Part Two of Study Four identical to the ones used in Studies One and Two. I eliminated twelve remaining participants who failed to answer the multiple choice attention check question correctly as well as two participants who failed to answer the true/false attention check question correctly.

In all, these data reduction efforts left a total of 198 participants remaining. 


\title{
APPENDIX Y
}

\author{
Study Four Part Two Informed Consent
}

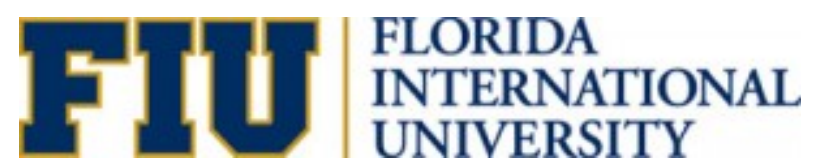

\section{ADULT ONLINE CONSENT TO PARTICIPATE IN A RESEARCH STUDY \\ Civil Juror Decision-Making}

\begin{abstract}
Thank you for looking into our study. This study is split into two separate online sessions. This is the second portion, and you must have completed the first session prior to completing the second session. Please do NOT complete Part Two until you have received an email instructing you to do so.
\end{abstract}

This session will take about an hour and a half to two hours to complete and it is worth two research credits, making a total of three research credits for your full participation.

During this session, you will view a short film clip, read a description of a civil trial and some jury instructions, and then answer some questions about the case. Before participating, please read the information in the official consent document below.

**IMPORTANT: It is strongly recommended that you use any of the following web browsers AS LONG AS JAVASCRIPT IS ENABLED: Internet Explorer, Google Chrome, or Apple Safari.

It is recommended that you DO NOT USE Mozilla Firefox.

If you have JavaScript turned off, or use any add-ons, extensions, or shortcuts which block JavaScript, please either disable them, add qualtrics.com to the list of approved sites, enable JavaScript, or run your browser in Safe Mode**

Please note, you must have cookies and JavaScript enabled on your browser in order to participate. If you click the button below but do not leave this page, your browser is not supporting cookies. Cookies are used only so that you can have a unique identification number for this study; no personal information will be stored in cookies.

You must also have the capability to view Flash video clips on your computer (the same sorts of clips which are used on YouTube), and you must be able to listen to the video clips, either through the use of speakers or headphones.

Please complete this study at one time. If for any reason you are not able to complete the study in one sitting, you will have to start over. The experiment takes roughly an hour and a half to two hours to complete.

As a student in the FIU Psychology Participant Pool (enrolled in PSY 2012 or other class), you are invited to participate in a research study conducted over the Web. The following information is provided to help you make an informed decision whether or not to participate. Your participation is completely voluntary, and not participating will not affect your class grade in any way. You must be at least 18 years old to participate.

\section{PURPOSE OF THE STUDY}

You are being asked to be in a research study. The purpose of this study is to investigate how jurors make decisions. Completion of this study will take approximately 90 to 120 minutes for this session. 


\section{NUMBER OF STUDY PARTICIPANTS}

If you decide to be in this study, you will be one of approximately 360 people in this research study.

\section{DURATION OF THE STUDY}

Your participation will require approximately one and a half to two hours.

\section{PROCEDURES}

If you agree to be in the study, we will ask you to do the following things:

You will be asked to watch a short film clip as well as read along with and listen to a case scenario involving a civil lawsuit in which a plaintiff is suing a defendant for negligence. You will be asked to answer questions about the events described on a Web page. You will be asked to imagine you are a juror in the case and to decide whether the defendant is liable and whether the plaintiff is entitled to any money-based damages. We will also ask how you feel and your opinion on various aspects of the case. You can refuse to answer any question and cease participation at any time.

\section{RISKS AND/OR DISCOMFORTS}

The following risks may be associated with your participation in this study: First, you might become upset while viewing the short film clip from a popular movie. Second, the only other risk to participating in this study is that you might become upset when you read or render a verdict about the case involving an automobile accident, especially if you or someone you know has been involved in a similar accident. This event is unlikely, but if you are concerned about it you may withdraw from the study at any time.

\section{BENEFITS}

The following benefits may be associated with your participation in this study: you may find the learning experience enjoyable, you may learn a little bit about how psychological research is conducted, and the process may help you better understand the legal process. The information gained from this study will help us better understand the factors that influence juror decision-making processes and to improve trial procedures.

\section{ALTERNATIVES}

You do not need to participate in this research. Your instructor may have alternative means for you to earn class credit, though your participation here may satisfy your instructor's research requirements.

\section{CONFIDENTIALITY}

The records of this study will be kept private and will be protected to the fullest extent provided by law. In any sort of report we might publish, we will not include any information that will make it possible to identify a subject. Research records will be stored securely and only the researcher team will have access to the records.

Any information obtained during this study that could identify you will be kept strictly confidential. The study will collect information about your IP address and the browser you are using. No personal information will be placed on your browser by the use of cookies. So that participation credit may be given, you will be required to enter your Panther ID number and your name. The personal information collected will be deleted from the database once data collection is completed and credit has been given. The information gained from this study may be published in scientific journals or presented at scientific meetings, but your identity will be kept strictly confidential.

\section{COMPENSATION \& COSTS}

There is no financial compensation to you as a result of participating in this study. Completion of this study earns partial credit toward the FIU Psychology Participant Pool requirements as outlined by your professor and on http://fiu.sona-systems.com/. For participating in the first session of this study you will have received one research credit, and for the second session you will receive two additional research credits for a total of three credits. You are free to decide not to participate in this study or to withdraw at any time without adversely affecting your relationship with the investigators or Florida International University. There will be no negative consequences for deciding not to participate or for withdrawing. If 
you choose to not participate in this study, you may satisfy your course requirements through other studies registered with the FIU Psychology Participant Pool at http://fiu.sona-systems.com/, or you may contact your course instructor to arrange an alternative method of obtaining credit. Participation in human subjects research is not required to earn credit in any class, and your professor is required to offer an alternative method of obtaining credit. You will not be responsible for any costs to participate in this study.

\section{RIGHT TO DECLINE OR WITHDRAW}

Your participation in this study is voluntary. You are free to participate in the study or withdraw your consent at any time during the study. Your withdrawal or lack of participation will not affect any benefits to which you are otherwise entitled.

\section{RESEARCHER CONTACT INFORMATION}

If you have any questions about the purpose, procedures, or any other issues relating to this research study you may contact Stephen Joy at [PHONE NUMBER REDACTED] or [EMAIL ADDRESS REDACTED].

\section{IRB CONTACT INFORMATION}

If you would like to talk with someone about your rights of being a subject in this research study or about ethical issues with this research study, you may contact the FIU Office of Research Integrity by phone at [PHONE NUMBER REDACTED] or [EMAIL ADDRESS REDACTED].

\section{PARTICIPANT AGREEMENT}

I have read the information in this consent form and agree to participate in this study. I have had a chance to ask any questions I have about this study, and they have been answered for me. By clicking on the "consent to participate" button below I am providing my informed consent. 


\section{APPENDIX Z}

\section{Need for Cognition Scale}

Cacioppo, J. T., Petty, R. E., \& Kao, C. F. (1984). The efficient assessment of need for cognition. Journal of Personality Assessment, 48, 306-307.

\section{Need for Cognition (NFC):}

Table 1

18-Item Need for Cognition Scale

\begin{tabular}{|c|c|}
\hline $\begin{array}{l}\text { Item } \\
\text { Number }\end{array}$ & Item Wording \\
\hline 1 & I would prefer complex to simple problems. \\
\hline 2 & I like to have the responsibility of handling a situation that requires a lot of thinking. \\
\hline 3 & Thinking is not my idea of fun* \\
\hline 4 & $\begin{array}{l}\text { I would rather do something that requires little thought than something that is sure to challenge } \\
\text { my thinking abilities.* }\end{array}$ \\
\hline 5 & $\begin{array}{l}\text { I try to anticipate and avoid situations where there is likely chance I will have to think in depth } \\
\text { about something.* }\end{array}$ \\
\hline 61 & I find satisfaction in deliberating hard and for long hours. \\
\hline 71 & I only think as hard as I have to. * \\
\hline 81 & I prefer to think about small, daily projects to long-term ones.* \\
\hline 9 & I like tasks that require little thought once I've learned them.* \\
\hline 10 & The idea of relying on thought to make my way to the top appeals to me. \\
\hline 111 & I really enjoy a task that involves coming up with new solutions to problems. \\
\hline 121 & Learning new ways to think doesn't excite me very much.* \\
\hline 131 & I prefer my life to be filled with puzzles that I must solve. \\
\hline 14 & The notion of thinking abstractly is appealing to me. \\
\hline 15 & $\begin{array}{l}\text { I would prefer a task that is intellectual, difficult. and important to one that is somewhat impor- } \\
\text { tant but does not require much thought. }\end{array}$ \\
\hline 16 & I feel relief rather than satisfaction after completing a task that required a lot of mental effort.* \\
\hline 17 & It's enough for me that something gets the job done; I don't care how or why it works.* \\
\hline 18 & I usually end up deliberating about issues even when they do not affect me personally. \\
\hline
\end{tabular}

* Reverse scoring is used on this item. 


\section{APPENDIX AA \\ Rational-Experiential Inventory Scale}

\section{Pacini, R., \& Epstein, S. (1999). The relation of rational and experiential information processing styles to personality, basic beliefs, and the ratio-bias phenomenon. Journal of Personality and Social Psychology, 76, 972-987. \\ Rational-Experiential Inventory (REI-40):}

\section{PACINI AND EPSTEIN}

Table 1

Study 1: Factor Analysis of Rational-Experiential Inventory Item.s

\begin{tabular}{|c|c|c|}
\hline Items & Factor 1 & Factor 2 \\
\hline \multicolumn{3}{|l|}{ Rationality scale } \\
\hline I try to avoid situations that require thinking in depth about something. (re - ) & .75 & .07 \\
\hline I'm not that good at figuring out complicated problems. ( $\mathrm{ra}-$ ) & .74 & .10 \\
\hline I enjoy intellectual challenges. (re) & .72 & -.02 \\
\hline I am not very good at solving problems that require careful logical analysis. (ra-) & .71 & -.04 \\
\hline I don't like to have to do a lot of thinking. (re-) & .70 & .09 \\
\hline I enjoy solving problems that require hard thinking. (re) & .68 & -.05 \\
\hline Thinking is not my idea of an enjoyable activity. (re-) & 65 & .10 \\
\hline I am not a very analytical thinker. $(\mathrm{ra}-)$ & 64 & -.09 \\
\hline Reasoning things out carefully is not one of my strong points. ( $\mathrm{ra}-)$ & .61 & -.01 \\
\hline I prefer complex problems to simple problems. (re) & 61 & -.05 \\
\hline \multicolumn{3}{|l|}{$\begin{array}{l}\text { Thinking bard and for a long time about something gives me little satisfaction. } \\
(\text { re-) }\end{array}$} \\
\hline I don't reason well under pressure. (ra-) & .57 & .04 \\
\hline I am much better at figuring things out logically than most people. (ra) & .56 & -.19 \\
\hline I have a logical mind. (ra) & .56 & -.19 \\
\hline I enjoy thinking in abstract terms. (re) & .49 & -.01 \\
\hline I have no problem thinking things through carefully, (ra) & .47 & .12 \\
\hline Using logic usually works well for me in figuring out problems in my life. (ra) & .46 & -.22 \\
\hline \multicolumn{3}{|l|}{$\begin{array}{l}\text { Knowing the answer without having to understand the reasoning behind it is good } \\
\text { enough for me. (re-) }\end{array}$} \\
\hline I usually have clear, explainable reasons for my decisions. (ra) & .40 & -.11 \\
\hline Learning new ways to think would be very appealing to me. (re) & .30 & .00 \\
\hline \multicolumn{3}{|l|}{ Experientiality scale } \\
\hline I like to rely on my intuitive impressions. (ee) & -.16 & 66 \\
\hline I don't have a very good sense of intuition. (ea-) & .17 & .65 \\
\hline \multicolumn{3}{|l|}{ Using my gut feelings usually works well for me in figuring out problems in my } \\
\hline & -.11 & .65 \\
\hline I believe in trusting my hunches. (ea) & -.07 & 64 \\
\hline Intuition can be a very useful way to solve problems. (ee) & .00 & .59 \\
\hline I often go by my instincts when deciding on a course of action. (ce) & -.25 & .56 \\
\hline I trust my initial feelings about people. (ea) & -.04 & .55 \\
\hline When it comes to trusting people, I can usually rely on my gut feelings. (ea) & .00 & .55 \\
\hline If I were to rely on my gut feelings, I would often make mistakes. (ea-) & .08 & .54 \\
\hline I don't like situations in which I have to rely on intuition. (ee-) & .04 & .54 \\
\hline I think there are times when one should rely on one's intuition. (ce) & -.02 & .53 \\
\hline I think it is foolish to make important decisions based on feelings. (ee-) & -.13 & .53 \\
\hline \multicolumn{3}{|l|}{ I don't think it is a good idea to rely on one's intuition for important decisions. } \\
\hline I generally don't depend on my feelings to help me make decisions. (ee-) & -.15 & .51 \\
\hline \multicolumn{3}{|l|}{ I hardly ever go wrong when I listen to my deepest gut feelings to find an answer. } \\
\hline \multicolumn{3}{|l|}{ I would not want to depend on anyone who described himself or herself as intuitive. } \\
\hline My snap judgments are probably not as good as most people's. (ea-) & .37 & .46 \\
\hline I tend to use my heart as a guide for my actions. (ee) & -.39 & .43 \\
\hline \multicolumn{3}{|l|}{ I can usually feel when a person is right or wrong, even if I can't explain how I } \\
\hline know. (ea) & -.08 & .40 \\
\hline I suspect my hunches are inaccurate as often as they are accurate. (ea-) & .15 & .35 \\
\hline
\end{tabular}

Note, $N=398$. The name of the subscale to which each item belongs appears in parentheses. ee $=$ Experiential Engagement; $\mathrm{ea}=$ Experiential Ability; re $=$ Rational Engagement; $\mathrm{ra}=$ Rational Ability. $\mathrm{A}$ minus sign $(-)$ with a scale name denotes reverse scoring 


\title{
APPENDIX AB
}

\section{Study Four Part One Informed Consent}

\section{FIU}

\section{ADULT ONLINE CONSENT TO PARTICIPATE IN A RESEARCH STUDY Civil Juror Decision-Making}

\begin{abstract}
Thank you for looking into our study. This study is split into two separate online sessions. You will receive one research credit for the first session, which should take approximately 30 minutes to complete. After completing this first session, you will receive an email several days later with instructions for how to sign up and complete the second online session. The second session must be completed no less than two days later, and no more than nine days later. The second session will take about an hour and a half to two hours to complete and it will be worth two research credits, making a total of three research credits for your full participation.
\end{abstract}

The first session involves filling out some surveys about yourself. During the second session, you will log on to the website, view a short film clip, read a description of a civil trial and some jury instructions, and then answer some questions about the case. Before participating, please read the information in the official consent document below.

\section{**IMPORTANT: You may use any of the following web browsers AS LONG AS JAVASCRIPT IS ENABLED: Mozilla Firefox, Google Chrome, Apple Safari, and Internet Explorer. If you have JavaScript turned off, or use any add-ons, extensions, or shortcuts which block JavaScript, please either disable them, add qualtrics.com to the list of approved sites, enable JavaScript, or run your browser in Safe Mode ${ }^{\star *}$}

Please note, you must have cookies and JavaScript enabled on your browser in order to participate. If you click the button below but do not leave this page, your browser is not supporting cookies. Cookies are used only so that you can have a unique identification number for this study; no personal information will be stored in cookies.

You must also have the capability to view Flash video clips on your computer (the same sorts of clips which are used on YouTube), and you must be able to listen to the video clips, either through the use of speakers or headphones.

Please complete this study at one time. If for any reason you are not able to complete the study in one sitting, you will have to start over. The experiment takes roughly thirty minutes to complete.

As a student in the FIU Psychology Participant Pool (enrolled in PSY 2012 or other class), you are invited to participate in a research study conducted over the Web. The following information is provided to help you make an informed decision whether or not to participate. Your participation is completely voluntary, and not participating will not affect your class grade in any way. You must be at least 18 years old to participate.

\section{PURPOSE OF THE STUDY}

You are being asked to be in a research study. The purpose of this study is to investigate how jurors make decisions. Completion of this study will take approximately 30 minutes for the first session and 90 to 120 minutes for the second session.

\section{NUMBER OF STUDY PARTICIPANTS}

If you decide to be in this study, you will be one of approximately 360 people in this research study. 


\section{DURATION OF THE STUDY}

Your participation will require approximately a half hour for the first session, and one and a half to two hours for the second portion.

\section{PROCEDURES}

If you agree to be in the study, we will ask you to do the following things:

You will complete some surveys about yourself during the first session.

During the second session, you will be asked to watch a short film clip as well as read along with and listen to a case scenario involving a civil lawsuit in which a plaintiff is suing a defendant for negligence. You will be asked to answer questions about the events described on a Web page. You will be asked to imagine you are a juror in the case and to decide whether the defendant is liable and whether the plaintiff is entitled to any money-based damages. We will also ask how you feel and your opinion on various aspects of the case. You can refuse to answer any question and cease participation at any time.

\section{RISKS AND/OR DISCOMFORTS}

The following risks may be associated with your participation in this study: First, you might become upset while viewing the short film clip from a popular movie. Second, the only other risk to participating in this study is that you might become upset when you read or render a verdict about the case involving an automobile accident, especially if you or someone you know has been involved in a similar accident. This event is unlikely, but if you are concerned about it you may withdraw from the study at any time.

\section{BENEFITS}

The following benefits may be associated with your participation in this study: you may find the learning experience enjoyable, you may learn a little bit about how psychological research is conducted, and the process may help you better understand the legal process. The information gained from this study will help us better understand the factors that influence juror decision-making processes and to improve trial procedures.

\section{ALTERNATIVES}

You do not need to participate in this research. Your instructor may have alternative means for you to earn class credit, though your participation here may satisfy your instructor's research requirements.

\section{CONFIDENTIALITY}

The records of this study will be kept private and will be protected to the fullest extent provided by law. In any sort of report we might publish, we will not include any information that will make it possible to identify a subject. Research records will be stored securely and only the researcher team will have access to the records.

Any information obtained during this study that could identify you will be kept strictly confidential. The study will collect information about your IP address and the browser you are using. No personal information will be placed on your browser by the use of cookies. So that participation credit may be given, you will be required to enter your Panther ID number and your name. The personal information collected will be deleted from the database once data collection is completed and credit has been given. The information gained from this study may be published in scientific journals or presented at scientific meetings, but your identity will be kept strictly confidential.

\section{COMPENSATION \& COSTS}

There is no financial compensation to you as a result of participating in this study. Completion of this study earns partial credit toward the FIU Psychology Participant Pool requirements as outlined by your professor and on http://fiu.sona-systems.com/. For participating in the first session of this study you will receive one research credit, and for the second session you will receive two additional research credits for a total of three credits. You are free to decide not to participate in this study or to withdraw at any time without adversely affecting your relationship with the investigators or Florida International University. There will be no negative consequences for deciding not to participate or for withdrawing. If you choose to not participate in this study, you may satisfy your course requirements through other studies registered with the FIU Psychology Participant Pool at http://fiu.sona-systems.com/, or you may contact your course instructor to arrange an alternative method of obtaining credit. Participation in human subjects research is not required to earn credit in any class, and your professor is required to offer an alternative method of obtaining credit. You will not be responsible for any costs to participate in this study. 
RIGHT TO DECLINE OR WITHDRAW

Your participation in this study is voluntary. You are free to participate in the study or withdraw your consent at any time during the study. Your withdrawal or lack of participation will not affect any benefits to which you are otherwise entitled.

\section{RESEARCHER CONTACT INFORMATION}

If you have any questions about the purpose, procedures, or any other issues relating to this research study you may contact Stephen Joy at [PHONE NUMBER REDACTED] or [EMAIL ADDRESS REDACTED].

\section{IRB CONTACT INFORMATION}

If you would like to talk with someone about your rights of being a subject in this research study or about ethical issues with this research study, you may contact the FIU Office of Research Integrity by phone at [PHONE NUMBER REDACTED] or [EMAIL ADDRESS REDACTED].

\section{PARTICIPANT AGREEMENT}

I have read the information in this consent form and agree to participate in this study. I have had a chance to ask any questions I have about this study, and they have been answered for me. By clicking on the "consent to participate" button below I am providing my informed consent. 


\title{
APPENDIX AC
}

\author{
Study Four Trial Transcript with No Inconsistencies
}

\section{Johnson v. McGraw}

The following trial transcript is an abridged version of a real trial from here in the state of Florida. The trial, which has already taken place, and for which the jury has already rendered a verdict, involved a traffic accident which resulted in injury to the plaintiff, Amanda Johnson, an injury she alleged was the result of negligence on behalf of the defendant, Michael McGraw. Although the circumstances of the accident involved multiple parties, Michael McGraw was the only defendant in this case.

While the names of all parties have been changed, one of the attorneys has asked FIU researchers to conduct this study to see how real people like you perceive the case so that they can determine whether to appeal the outcome of the trial. We will be using the data from this study to help the attorney, so please pay close attention as it will impact the final resolution of an actual civil case.

Please listen to the trial transcript and read along as if you had been chosen to serve as a juror in this case.

Judge Robert C. Underwood: Ladies and gentlemen of the jury, you are about to hear testimony concerning an automobile accident that occurred in the late afternoon of Tuesday, July $8^{\text {th }}, 2008$ in the city of Clermont, located in Lake County, Florida. The plaintiff is Amanda Johnson, and the defendant is Michael McGraw. There are no other plaintiffs or defendants in this suit, and all other disputes between these and any other parties related to the events in question have been resolved separately prior to this proceeding.

\section{Opening Statement by Seth Blum, Attorney for the Plaintiff}

Seth Blum, Attorney for the Plaintiff: Ladies and gentlemen of the jury, what we have before us today is an unfortunate story of a young lady who was simply going about her business one day, on the afternoon of Tuesday, July $8^{\text {th }}, 2008$, up in Clermont, which is in central Florida to the west of Orlando. This young lady, Amanda Johnson, was at the time a 26 year old waitress at a family restaurant. She was riding in the car with her boyfriend, Brock Collins, a 28 year old middle school teacher. They were in his blue Toyota Camry on their way to catch a movie before meeting up with Amanda's parents for dinner later that night. 
Unfortunately, their plans were dashed by the negligence of another driver on the road that evening, Michael McGraw.

Throughout this case, you are going to hear overwhelming evidence from a variety of witnesses-Amanda Johnson, Brock Collins, a host of different eyewitnesses to the accident, the emergency first responders on the scene, medical professionals, as well as professional scientists who specialize in the analysis of vehicles and crash scenes which they use to reconstruct accidents. The testimony of all these witnesses will show you, with no uncertainty whatsoever, that Mr. McGraw, and only Mr. McGraw-not Mr. Collins, not the driver of the semi truck, not anybody else and not anything else other than Mr. McGraw's negligent operation of his big, white van - was the direct cause of Amanda Johnson's serious injury. I hope that you find him liable for all damages suffered by Ms. Johnson at his hand. Thank you very much for your time, service, and attention today.

\section{Opening Statement by Howard Kurtz, Attorney for the Defendant}

Howard Kurtz, Attorney for the Defendant: Esteemed members of the jury-like my colleague, I thank you in advance for your attention, effort, and diligent fulfillment of your civic duty. What we can all agree on is that you are here today to hear about a traffic accident that injured a nice young lady and her boyfriend. Everybody wishes that this accident hadn't happened, but not everybody agrees about how it happened or how Ms. Johnson ended up being hurt.

Ms. Johnson and her attorney would like for you to believe that her broken hip and other injuries are the result of negligence on the part of Mr. McGraw. Now it's certainly true that Mr. McGraw, while trying to avoid the out of control semi tractor trailer coming towards him, ended up hitting Mr. Collins's car. What we're less certain about is how Ms. Johnson was injured. You see, Ms. Johnson wasn't wearing her seat belt, and we'll be presenting evidence to you that will show that Ms. Johnson wasn't injured because Mr. McGraw hit their car, but that she was injured when the car ran into the street lamp, and that if she had been wearing a seat belt, she wouldn't have broken her hip.

Ms. Johnson and her attorney would like for you to believe that her injuries are the fault of Mr. McGraw, but through the course of this trial, you will soon see that they don't have any real evidence to support this allegation. Because they can't show that the majority of the evidence undoubtedly proves that Mr. McGraw was the cause of Ms. Johnson's injuries, you will come to the conclusion that Michael McGraw was not negligent, was not the direct cause of Amanda's broken hip, and that he should not be held liable for her injuries and you should render a verdict in favor of the defendant. 
Plaintiff's Witness Marc Zollinger, Direct Examination by Seth Blum, Attorney for the Plaintiff

Seth Blum, Attorney for the Plaintiff (Questioner): Can you please state your name and occupation for the court?

Marc Zollinger (Answerer): My name is Marc Zollinger, and I'm an administrative assistant for Universal Translation Corporation.

Q: And where is your office located?

A: It's on Foothill Drive, pretty close to the intersection of Foothill and Windsor, a little south of there, on the east side, or I guess northeast side of the road at that point, down by the bend.

Q: Tell me, Mr. Zollinger, what were you doing on the afternoon of Tuesday, July $8^{\text {th }}$, $2008 ?$

A: I needed some fresh air, so I went to the little walk-up coffee window a few doors down from our office, the one on the north side of the Cuban restaurant there.

Q: Okay. And while you were there, did anything unusual happen?

A: As a matter of fact, yes. While I was waiting in line, I was just standing around watching the cars go by when there was a big traffic accident behind me up the way a little bit at the corner.

Q: Up the way a little bit? How far do you mean? Help the jury to understand how close you were.

A: Oh, not that far really. Probably a few hundred feet away, but I had a pretty clear view since it's just the parking lot for the restaurant there to the north of the coffee window up to the corner.

Q: And can you tell us what you saw?

A: I saw a car, a van, and a tanker truck get into a wreck. The tanker truck was kind of sideways, coming southbound towards the intersection and kind of weaving back and forth a little bit in the middle of the road. I saw Mr. Collins's car, a blue Toyota Camry make a left turn to go west on Windsor to get out of the way of the truck, but then Mr. McGraw's white Ford work van came screeching around, and then the semi locked up its brakes and the tires started squealing but the tanker trailer swung around and kind of batted Mr. McGraw's van on the passenger side 
which then bounced into the back of Mr. Collins's car which then crashed into the street light on the northwest corner of the intersection. It was like one big chain reaction. They almost hit some lady walking a German Shepherd.

Q: And what happened next?

A: I saw the guy with the black hair, Mr. McGraw, get out of his van to make sure everyone in Mr. Collins's car was okay or something. I couldn't hear what they were saying or anything, but right away Mr. McGraw got on his phone, I guess to call 911. After a minute or two, Mr. Collins, the bald one, got out of the car and went around to the passenger side and was talking to someone in the passenger seat. He looked pretty worried based on all his movements and body language.

Q: And then what?

A: I placed my order, and by the time I got my coffee I could see that a police car was already on the scene so I grabbed my coffee and went over to tell him what I saw. I told him exactly how it happened, and he wrote it all down. The officer's name was Christopher Eaton. He was a really big guy.

Q: Thank you, Mr. Zollinger. No further questions.

Plaintiff's Witness Marc Zollinger, Cross Examination by Howard Kurtz, Attorney for the Defendant

Howard Kurtz, Attorney for the Defendant (Questioner): Could you describe for us in just a little more detail exactly what happened after all the vehicles came to a stop?

Marc Zollinger (Answerer): Sure. Mr. McGraw got out of the white van pretty quickly and immediately ran over to Mr. Collins's blue Camry, over to the driver's side. I couldn't hear them, of course, but it looked to me like he was making sure everyone was okay, and then shortly after talking to Mr. Collins, McGraw got his cell phone out of his pocket and made a call. A minute or two later, the police officer arrived, then I went over and told him what I saw, and then I went back to work.

Q: Thank you, Mr. Zollinger. I have nothing further.

Plaintiff's Witness Barbara Feldman, Direct Examination by Seth Blum, Attorney for the Plaintiff 
Seth Blum, Attorney for the Plaintiff (Questioner): Hello there. Could you please state your name and occupation for the record?

Barbara Feldman (Answerer): My name is Barbara Feldman, and I am a librarian.

Q: Thank you Ms. Feldman. Now you're familiar with the day in question here in this trial, right?

A: Yes I am.

Q: And did you witness the accident at issue here?

A: Yes I did. I remember it all quite vividly. I was driving southbound on Foothill Drive, north of the intersection with Windsor.

Q: And could you please describe for us what you saw?

A: Sure. As I was driving, up ahead I noticed a Shell oil tanker trailer crossing over the line separating the two directions of traffic, kind of skidding its tires and fishtailing around. The stop light ahead of us was green, so I'm not sure what the deal was. It's not like he was slamming on his brakes to keep from running the light or anything. It was also driving southbound like I was, so I slowed down to stay away from the situation. It was all over the place in the left turn lane in the middle, going back and forth into the oncoming lanes of traffic and stuff.

Q: And what happened next?

A: Well, the truck's trailer sort of swung out into the intersection, where it hit Mr. McGraw's white van, kind of side swiping it and kind of just smacking it on the passenger side, which forced the van into the back side of Mr. Collins's blue Toyota Camry, which then crashed into a light pole on the northeast corner of the intersection.

Q: Okay, and what about after the accident?

A: I just stayed around until the police officer arrived, and once he got there, I gave him my statement and my contact information, and then I left to go home.

Q: Thank you Ms. Feldman. I have no more questions.

Plaintiff's Witness Barbara Feldman, Cross Examination by Howard Kurtz, Attorney for the Defendant 
Howard Kurtz, Attorney for the Defendant: I have no questions for this witness, your Honor.

Plaintiff's Witness Jill Randall, Direct Examination by Seth Blum, Attorney for the Plaintiff

Seth Blum, Attorney for the Plaintiff (Questioner): Could you please give us your name and occupation?

Jill Randall (Answerer): My name is Jill Randall, and I sell Amway products from my home here in Clermont.

Q: Now could you please tell us about what you saw on Tuesday, July $8^{\text {th }}, 2008$ ?

A: I was at the intersection waiting for the light to change so that I could cross Foothill heading west, and there was a semi truck that came out of nowhere from the north, skidding and screeching and swerving all over the place out of control.

Q: That must have been pretty scary. Can you tell us more about the truck and what happened?

A: Well, I saw the truck blow the light going through the intersection kind of in the middle of the southbound and northbound lanes, and he was fishtailing a little bit, you know, the cab of the truck going in one direction while the trailer was slipping and sliding around behind him in another direction. The truck swerved towards me, to the east, to the driver's left, past me as it came through the intersection. As the truck was getting itself under control, I could tell that there was some sort of additional accident on the opposite side of the trailer involving some other vehicles, but since I was on the east side of the trailer and that was all happening on the west side, I really couldn't see too much about what exactly happened.

Q: Okay, so you weren't able to see much of the actual contact between the vehicles?

A: Well, no, not of the actual contact when it was happening, but after everything came to a stop, I was able to see that the white van driven by Mr. McGraw had crashed into the back of the other car, the one driven by Mr. Collins, the blue Toyota Camry, and Mr. Collins's car was up against the street lamp. It looked like it had pretty much hit the lamppost head on, and Mr. McGraw's van was stopped behind it, and it was all banged up on the passenger side.

Q: Well it sounds like you got a good look at the aftermath of the accident. What happened after everything came to rest? 
A: The black-haired man, Mr. McGraw got out of his van pretty quickly and went to go check on the people in Mr. Collins's car. He was talking to Mr. Collins for a minute, and then he got out his phone and made a call, I assume to 911. Mr. Collins got out of his car eventually, and when the police officer arrived a minute or two later, he was a real hunk, so I hung around and gave him my statement once the paramedics got there to take over making sure everyone got the medical attention they needed.

Q: We appreciate your testimony here today, Ms. Randall. No further questions.

Judge Robert C. Underwood: Your witness, Mr. Kurtz.

Plaintiff's Witness Jill Randall, Cross Examination by Howard Kurtz, Attorney for the Defendant

Howard Kurtz, Attorney for the Defendant (Questioner): So Ms. Randall, you weren't able to describe exactly what happened during the wreck itself because the trailer from the tanker truck was blocking your view, is that right?

Jill Randall (Answerer): That's right. I saw the beginning and I saw the aftermath, but I didn't see the actual accident between Mr. McGraw and Mr. Collins, no.

Q: I see. Thank you very much, Ms. Randall. I don't have any more questions.

Plaintiff's Witness Steve Powell, Direct Examination by Seth Blum, Attorney for the Plaintiff

Seth Blum, Attorney for the Plaintiff (Questioner): Could you please give your name and occupation for the record?

Steve Powell (Answerer): My name is Steve Powell, and I'm a real estate agent here in Clermont.

Q: And where were you on the afternoon of Tuesday, July $8^{\text {th }}, 2008$ ?

A: I was at the post office located there on the east side of Foothill Drive, a little ways up from, I mean north of, the intersection with Windsor Road.

Q: Okay. And did you see anything unusual at the post office?

A: Well, just as I was leaving, I was looking out the window and I saw a Shell tanker truck go skidding down the street past the post office. 
Q: Down Foothill you say?

A: Yeah, it was traveling south but kind of screeching and sliding out of control.

Q: And what did you see after that?

A: Well, I didn't really see exactly what happened next. I was walking out of the building and didn't have a constant, uninterrupted view so I missed the actual crash, but after I got outside, I saw the whole accident scene with the tanker truck stopped at a funny angle over on the east side of Foothill on Windsor, and the van and the car over on the corner by the Zales jewelry store. The guy with the black hair, Mr. McGraw, was standing outside the vehicles. There were a couple of people in the car, the blue Camry, and they looked kind of hurt.

Q: How so?

A: The bald guy, Mr. Collins, had some cuts and stuff on his forehead and he was bleeding a little bit, but the lady in the passenger seat was much worse. It was kind of hard to see exactly how she was hurt, but it was clear she was pretty banged up.

Q: And can you tell us what happened next?

A: I had an important meeting I had to get to a little later on that afternoon so I didn't have time to stick around, and I don't know how I could have helped anyway, but as I was starting to walk back to my car, the bald guy, Mr. Collins, was out of his car and the police officer had showed up so I figured it would be okay now that there was an official emergency responder on the scene. Right as I got to my car, which was parked over in the lot north of the coffee shop on the southeast corner of the intersection, I saw the ambulance arrive next, so I figured I might as well go over to the police officer and tell him what I had seen now that medical personnel were there before I left to go to my meeting. As I was giving Officer Eaton my statement, I could see the paramedics getting Ms. Johnson out of the car and loading her into the ambulance. It was at that time that I could see that her right leg was all mangled and busted up.

Q: Thank you for your time, Mr. Powell. I don't have any more questions for you.

Plaintiff's Witness Steve Powell, Cross Examination by Howard Kurtz, Attorney for the Defendant 
Howard Kurtz, Attorney for the Defendant (Questioner): Mr. Powell, you were inside the post office when the actual accident occurred, and were unable to see exactly what happened at the intersection, is that right?

Steve Powell (Answerer): Yeah, I just saw the Shell truck go past the window out of control, but no, I couldn't see the intersection or anything that far down from where I was.

Q: So you don't have any idea exactly how any of the different vehicles crashed into each other, or the exact moment in which Ms. Johnson might have been hurt, or how she was hurt, or anything specific like that, correct?

A: That's correct. I just saw the truck go by and then by the time I was outside, I saw the wreckage and stuff where it had all come to rest.

Q: So basically everything at issue here happened when you couldn't see it?

A: Well I did see two men, Mr. Collins and Mr. McGraw outside talking to each other when the police officer arrived at the time I was walking across Windsor, and then by the time I got to my car, I saw the ambulance had just arrived too.

Q: Thank you, Mr. Powell. No further questions.

Plaintiff's Witness Lesley Jobin, Direct Examination by Seth Blum, Attorney for the Plaintiff

Seth Blum, Attorney for the Plaintiff (Questioner): Hello there, could you please tell us your name, occupation, and city of residence for the record?

Lesley Jobin (Answerer): My name is Lesley Jobin, I'm a tour group coordinator, and I live here in Clermont.

Q: And what were you doing on the afternoon of Tuesday, July $8^{\text {th }}, 2008$ ?

A: Well, since there's really no slow days at my company because of all the visitors to the area and different tourist attractions and stuff in and around Orlando, we're open seven days a week, and I'm off on Mondays and Tuesdays each week. That was a Tuesday, and I was out walking Finnegan, my German Shepherd.

Q: And can you tell us a little more about your walk with Finnegan that day? 
A: Sure. I was walking west along Windsor Road, having just crossed Foothill. I was passing in front of the Zales jewelry store there at the corner, when the crash happened.

Q: So can you tell us what you saw?

A: Well, I saw the Shell truck fishtailing out of control, well, I guess there isn't any way for a truck to fishtail but still be under control, but anyway, it was sort of sliding out toward its right side, and then it kind of smacked the side of the white van, and that's when the van crashed into the back of the blue Toyota Camry, which then ran straight into a light post on the corner in front of Zales.

Q: Were you able to see anything that happened inside of either of the vehicles?

A: Yeah, when Mr. McGraw first hit Mr. Collins's car, I could see that both Mr. Collins and Ms. Johnson's heads went flying forward into the windshield.

Q: Thank you Ms. Jobin. I don't have any more questions for you.

Plaintiff's Witness Lesley Jobin, Cross Examination by Howard Kurtz, Attorney for the Defendant

Howard Kurtz, Attorney for the Defendant (Questioner): So Ms. Jobin, you were out walking your German Shepherd, Finnegan, that afternoon, is that correct?

Lesley Jobin (Answerer): That's right.

Q: And were you talking on the phone, or listening to music, or anything like that on your walk?

A: Well, I was listening to my iPod.

Q: Hmm. Okay. And you were walking west, passing the Zales store when you heard a crash and turned around to look, is that right?

A: Yeah, Finnegan and I were both really startled, and we ran backwards a little bit just as Mr. Collins's car hit the light post.

Q: I see. Thank you very much, Ms. Jobin.

Plaintiff's Witness Brock Collins, Direct Examination by Seth Blum, Attorney for the Plaintiff 
Seth Blum, Attorney for the Plaintiff (Questioner): Hello there, could you please state your name, occupation, and city of residence for the record?

Brock Collins (Answerer): My name is Brock Collins, I'm a teacher at Windy Hill Middle School here in Clermont where I live.

Q: And just so we're clear, what is your relationship with the plaintiff in this case, Amanda Johnson?

A: She's my girlfriend. We've been dating for a couple of years.

Q: And could you tell us a little bit about what happened on the afternoon of Tuesday, July $8^{\text {th }}, 2008 ?$

A: Well, Amanda and I were on our way to see a matinee before going out to dinner with her folks later on, when we got into a big wreck at the corner of Windsor and Foothill.

Q: Can you tell us about what you remember happening before the accident and how it all unfolded?

A: Well, I was driving my car, a blue 2006 Toyota Camry four door, northbound on Foothill Drive, and Amanda was in the passenger seat. We were coming around the bend, right where Foothill goes from kind of east/west to north/south, and once I got around the bend approaching the intersection, we had the green light, and I saw a semi truck skidding out of control towards us.

Q: Sounds pretty scary. Can you describe how you reacted to the situation and what happened next?

A: Yeah, it was really crazy to see a big truck barreling down on us like that. It took me a second to figure out what to do because I couldn't tell which way the truck was going to go or what was going to happen next. I kind of slowly turned to my left, to go west on Windsor because I was pretty sure that the truck was going to end up going to my right, east on Windsor. Once I could tell for sure that that's where he was headed, I was able to pull out of his way just barely fast enough to miss the end of his tanker trailer whipping around the corner behind him. It was a real relief to miss it, but the relief was very short lived.

Q: How so?

A: Well, the guy behind me in the white Ford panel van wasn't as lucky. I guess he tried to get out of the way of the swinging trailer much the same way as I did, but he just ended up rear-ending us pretty much straight on, and our heads hit the 
windshield, and we then crashed into the street lamp on the northwest corner of the intersection, all pretty much at the same time as his van was getting hit by the trailer. I got dinged on the head pretty good but wasn't that messed up, no permanent scars or damage or anything, but Amanda was really hurt badly.

Q: She certainly was. I'm really sorry you guys had to go through all of that. Thank you, Mr. Collins. I don't have any more questions for you.

Plaintiff's Witness Brock Collins, Cross Examination by Howard Kurtz, Attorney for the Defendant

Howard Kurtz, Attorney for the Defendant (Questioner): So, Mr. Collins, are you and Ms. Johnson still together?

Brock Collins (Answerer): Yes, we are. We're starting to talk about marriage, in fact.

Q: Oh, how nice! Let's talk a little more about your car that you were driving that day. It was a blue 2006 four-door Toyota Camry, isn't that right?

A: Yes sir.

Q: And how long had you owned that car, Mr. Collins?

A: I had it for a little less than a year before the accident, but my dad bought it brand new, and when he got tired of it after a year or so, he sold it to me at a good price since I was in need of a reliable car.

Q: So you're familiar with the entire two-year history of the vehicle. Tell me, Mr. Collins, had the car ever been in any sort of accident before?

A: Except for maybe tapping a bumper when parallel parking in a tight spot or something tiny like that, no, it hadn't.

Q: It worked okay, pretty reliable car?

A: Certainly. Wasn't too flashy, but it was definitely reliable.

Q: And did the seatbelts in the car work properly?

A: Well, they got stuck when pulling them out a lot, so it was kind of a pain to put them on all the time.

Q: And were you wearing your seatbelt on the day of the accident? 
A: No, I wasn't.

Q: Why not?

A: We weren't going very far or anything, so I guess I just figured I didn't need to mess with it.

Q: And was Ms. Johnson wearing her seat belt that day?

A: No.

Q: Nothing further, Your Honor.

Plaintiff's Witness Dr. Matthew Freeman, Direct Examination by Seth Blum, Attorney for the Plaintiff

Seth Blum, Attorney for the Plaintiff (Questioner): Hello, could you please give us your name, city of residence, and occupation?

Dr. Matthew Freeman (Answerer): My name is Matthew Freeman, I live in Jacksonville, Florida, and I am an accident reconstruction specialist.

Q: What exactly does that mean?

A: I examine vehicles, accident sites, traffic patterns, road conditions, medical records, and that sort of thing in order to figure out exactly how an accident occurred.

Q: Thank you. And, just to avoid any confusion, you are being paid to discuss your findings with us here today, isn't that right?

A: Yes, I'm being paid by the plaintiff's side to share my conclusions. What I do is complex and time consuming, and after all, this is my job. But I assure you that my determinations are in no way shaped by anything besides the evidence.

Q: I appreciate your candor and your earnestness, Dr. Freeman. So can you tell us what you found with regard to the accident involving Mr. Whitaker's Shell tanker truck, Mr. Collins's Camry and Mr. McGraw's Ford Econoline work van?

A: Given where we know the truck stopped just beyond the intersection itself, Mr. Whitaker couldn't have been going very fast at the time his trailer sideswiped $\mathrm{Mr}$. McGraw's van. That means the impact from the trailer onto Mr. McGraw's van wasn't the determining factor in Ms. Johnson's injuries, but rather Mr. McGraw's driving itself was. If Mr. McGraw hadn't been driving so fast when the truck hit 
him, then Amanda wouldn't have been injured when he hit Mr. Collins's car. Besides, if he had been going slower, he wouldn't have been in the intersection anyway and the accident wouldn't have happened at all.

Q: Interesting. And what about your conclusions based on inspecting Mr. Collins's vehicle?

A: Well, it was a 2006 Toyota Camry, four doors, blue, base model, and no aftermarket or custom modifications. It was damaged uniformly across the rear from $\mathrm{Mr}$. McGraw's van hitting it, the front was damaged more deeply, but in a narrower span, consistent with hitting a tall, thin object like a street lamp pole, and while the seat belts appeared to be in reasonable working order, I suspect that the airbag deployment sensors may have been faulty. For as hard as Mr. McGraw hit the car, the airbags should have inflated. But because they didn't, it is my opinion that Ms. Johnson broke her right hip as a direct result of that first impact with Mr. McGraw to their rear, not the second impact with the street lamp post. Unfortunately, due to a miscommunication between Mr. Collins's insurance company and the salvage yard where the car was being stored after the accident, shortly after I had done my preliminary inspection of the car, it was disassembled for parts before I had a chance to check the status of the airbag sensors to verify that they were faulty.

Q: Thank you for your expertise, Dr. Freeman. I have nothing further to ask.

Plaintiff's Witness Dr. Matthew Freeman, Cross Examination by Howard Kurtz, Attorney for the Defendant

Howard Kurtz, Attorney for the Defendant (Questioner): Tell me, Dr. Freeman, do you have any medical training or licensure?

Dr. Matthew Freeman (Answerer): No, I hold a doctorate degree in mechanical engineering, as well as numerous other certifications related to my field, but none in health-related areas, no.

Q: And yet you're willing to testify under oath that Amanda's broken hip was a result of the first impact, when my client hit Mr. Collins's car, and not that it was from the head-on collision with the lamp pole. Very interesting conclusions, Dr. Freeman. No further questions.

Plaintiff's Witness Amanda Johnson, Direct Examination by Seth Blum, Attorney for the Plaintiff 
Seth Blum, Attorney for the Plaintiff (Questioner): Hello, Ms. Johnson. How are you doing today?

Amanda Johnson (Answerer): Oh, just fine. It feels good to finally have my day in court.

Q: I bet it does. How is your hip?

A: It's healed up pretty well so far, but it's not back to normal by any means. I'm not sure whether it will ever be like it was before the accident. Still, I'm finally able to walk without a limp or the use of a cane, but I can't run or do anything strenuous, and it really acts up during changes in barometric pressure and other weather changes. I feel like an old person, and I'm only 28.

Q: How has your injury affected your life?

A: Well, at first, it cost me my job, since I was in the hospital for a couple of weeks at first and then unable to walk for a long time after that, and it's pretty difficult to be a waitress if you can't walk.

Q: Can you tell us a little more about your injury and the treatment you received?

A: Sure. My right hip was broken just under the ball of my femur, the long bone at the top of your leg. It required immediate surgery and a metal plate and a bunch of screws to be screwed into both halves of my legbone so that the two parts of the bone would grow back together again. That surgery left me in the hospital for about a week, and I was under pretty heavy sedation for the pain, and then I had to stay in the rehabilitation center for another week before I was able to go home. I was in the wheelchair for another month before I was able to use a walker or crutches, and then it was another six weeks before I was able to walk short distances again. That whole time I was going to rehab three times a week and doing exercises every day at home too.

Q: And how are you doing today, a little over two years since the accident occurred?

A: I'm doing alright. Like I mentioned earlier, I can walk pretty much without a limp, but I still can't do anything really active or athletic that requires running around or anything like that. I'm able to sleep though the night okay now, and the pain doesn't bother me all that often except when the weather is about to change, which, unfortunately for me, seems to happen all too often here in central Florida. The doctors tell me that's never going to go away.

Q: Well, I guess at least you'll always know whether to bring an umbrella with you when you leave the house in the mornings. Now let's talk a little bit about the accident itself. 
A: Okay.

Q: When you first saw the truck, where was it in relation to the car you were in?

A: It was directly in front of us, a little to the right of the center of our car.

Q: Was the truck over the double yellow lines in the road that separate the two directions of travel?

A: Yes.

Q: In your estimation, how far over the double yellow lines?

A: It was about three-quarters of the way over into our lanes of traffic, so far that I didn't think we'd be able to swerve around it.

Q: And did the truck appear to swerve or deviate from its course at any time before the accident?

A: Yes, it did, it swerved to our right and ended up going off to our right, to the east.

Q: About how fast would you guess that you guys were going?

A: Not that fast. Probably 25 miles an hour or so.

Q: And at what speed would you estimate that the truck was going?

A: It had to have been over 40 miles per hour. We had slowed down, but it still came towards us very quickly and all we could see was the giant Shell tanker trailer swerving towards us.

Q: And then what happened?

A: Brock sort of hesitated for a quick second to figure out which direction was the safest way to get out of the way of the truck since we didn't really know where it was going to end up, and after he quickly turned to the left, to go west down Windsor, we saw the tail end of the trailer go sliding past us and we thought we had managed to escape an accident.

Q: But that wasn't the case, was it?

A: No, even though we barely missed colliding with the tanker, we were then suddenly hit from behind by Mr. McGraw, who had himself been hit on his passenger's side by the trailer and knocked into us, and that's when I went flying forward, 
hitting my head on the windshield and smashing into the dashboard and breaking my right hip, and then we hit the street lamp post which caused us to finally come to a stop.

Q: So you were fine up until the point when Mr. McGraw crashed into you, is that right?

A: Yeah, if he didn't hit us, I wouldn't have been hurt.

Q: Thank you, Ms. Johnson.

Plaintiff's Witness Amanda Johnson, Cross Examination by Howard Kurtz, Attorney for the Defendant

Howard Kurtz, Attorney for the Defendant (Questioner): Ms. Johnson, I'd like to ask you a few more questions about the events leading up to the accident and the details of the accident itself, if that's okay.

Amanda Johnson (Answerer): Of course.

Q: Ms. Johnson, could you please tell us exactly when you first noticed that the truck was on the wrong side of the road?

A: Before we got to the intersection, I could see that it was on the wrong side of the road. I could see the paint of the Shell logo and the chrome tank shining in the sun.

Q: And you are sure you could tell that the truck was on the wrong side of the road?

A: Yes, I'm sure.

Q: Ms. Johnson, if you saw the truck was on the wrong side of the road, why didn't you warn Mr. Collins?

A: I don't know. It happened very quickly. It was almost three quarters of the way over onto our side of the road, swerving to our right.

Q: Hmm. I see. Thank you, Ms. Johnson. No further questions.

Seth Blum, Attorney for the Plaintiff: Your Honor, we have no further witnesses to introduce, so the plaintiff rests.

Judge Robert C. Underwood: Thank you Mr. Blum. Mr. Kurtz, you may now present the defense's case. 
Howard Kurtz, Attorney for the Defendant: Thank you, Your Honor.

Defense Witness Tom Gorham, Direct Examination by Howard Kurtz, Attorney for the Defendant

Howard Kurtz, Attorney for the Defendant (Questioner): Hello, could you please state your name, city of residence, and occupation for the record?

Tom Gorham (Answerer): My name is Tom Gorham, I live in Orlando, and my wife and I own and operate a three-dimensional film and photography studio.

Q: How interesting! That must be a really exciting business for you two to be in. Now, could you please tell us where you were on the afternoon of Tuesday, July $8^{\text {th }}$, 2008 ?

A: I was stopped at the light at the corner of Windsor and Foothill, waiting to cross Foothill to the east, and I saw this whole big accident unfold.

Q: Can you tell us exactly what you saw?

A: Well, I first knew that something was wrong when I saw Mr. Collins's blue Toyota Camry flashing the lights and heard him laying on the horn as he came up to the intersection from the south. Very shortly after that, I saw the Shell tanker truck come skidding and fishtailing into the intersection from the north. He was pretty much swerving to his left, away from me, towards the opposite side of the intersection from where I was. I was on the west side, waiting to cross Foothill and head east, and he was swerving over that way with the trailer kind of swinging out behind him.

Q: Okay, so after you saw the two vehicles both entering the intersection, what happened next?

A: I saw Mr. Collins's car skirt around the trailer pretty much right in front of my car, when the trailer kind of swung around to its right, as the cab of the truck was making a hard left turn, and the trailer sort of swiped the passenger side of the white van. Right after that, the van rear-ended the Camry, and then the Camry went and ran into the streetlamp.

Q: And how good of a look did you get at this whole sequence of events?

A: I had a perfect view, pretty much a front-row seat to the whole thing.

Q: So did you see either Mr. Collins's or Ms. Johnson's heads strike the windshield of their car? 
A: Definitely.

Q: And when did you see that happen?

A: Right after their car crashed into the lamp post.

Q: Right. Now what happened next?

A: Well, I saw the guy with the black hair, McGraw, get out of the van right away and go over to the driver's side of Collins's car and it looked like he was checking to see if everyone was okay or to see how he could help. Collins got out of the car shortly after that, and while his head was bleeding a little bit, and he seemed a little dazed, he didn't really seem too badly hurt.

Q: Alright. Anything else?

A: Yeah, I stayed around to see if there was anything I could do, but the cops showed up and pretty much got it all under control and then the ambulance got there and I watched them take Ms. Johnson out of the car. It looked like her right leg was all messed up, and they loaded her and Mr. Collins into the ambulance and drove off.

Q: Thank you, Mr. Gorham. I have no further questions.

Defense Witness Tom Gorham, Cross Examination by Seth Blum, Attorney for the Plaintiff

Seth Blum, Attorney for the Plaintiff (Questioner): Mr. Gorham, you stated that you saw Mr. Collins's car flashing its lights and heard it honking its horn as it entered the intersection from the south, and that after that, you saw the Shell truck come skidding and screeching into the intersection. Now if Mr. Collins was honking and flashing his lights and everything, obviously in response to something, doesn't it seem strange to you that you didn't notice what he was reacting to before you noticed him?

Tom Gorham (Answerer): Well, maybe, I guess. I think I probably just noticed Mr. Collins's car first because he was the one that was right in front of me, and since the truck was all the way on the other side of the intersection, further away, maybe I didn't see it first for that reason. Or maybe the angle of everything made the Zales store sort of block my line of sight. I don't know really know why I saw things when I saw them, I just know what I saw.

Q: If you say so. Nothing more, Your Honor. 
Defense Witness John Kindt, Direct Examination by Howard Kurtz, Attorney for the Defendant

Howard Kurtz, Attorney for the Defendant (Questioner): Hello there, could you please state your name and occupation for the record?

John Kindt (Answerer): My name is John Kindt, and I am the security guard for the Zales jewelry store located on the northwest corner of the intersection of Foothill and Windsor Road in Clermont.

Q: Can you tell us where you were on the afternoon of Tuesday, July $8^{\text {th }}, 2008$ ?

A: Yeah, I was at work.

Q: Tell me, did you happen to witness the events at issue in this case?

A: I saw the tail end of it. I saw Mr. Collins's car almost run over the lady out walking her German Shepherd. She was walking on the sidewalk and nearly got creamed.

Q: Lucky thing for her that she didn't. So what else did you see then?

A: I saw the Shell trailer kind of smack the passenger side of Mr. McGraw's van and knock it into Mr. Collins's car.

Q: And did you see the consequences of the collision between the two vehicles?

A: Well, yeah. I saw Mr. Collins's car come crashing towards the store, and that lady and her dog, and hit the street lamp in front of our store.

Q: And did you see anybody get hurt?

A: Yeah, I saw Mr. Collins's head and Ms. Johnson's head smash into their windshield when they hit the lamp post. I was kind of surprised that the airbags didn't go off, but I guess they weren't going fast enough or something.

Q: And what else did you see?

A: Not much. I got a call about security issues for an upcoming delivery so I had to go look at some paperwork, and by the time I was through with that, the show was over.

Q: Thank you, Mr. Kindt. I have no more questions. 
Defense Witness John Kindt, Cross Examination by Seth Blum, Attorney for the Plaintiff

Seth Blum, Attorney for the Plaintiff: I have no questions for this witness, Your Honor.

Defense Witness Dr. Erik Dubberke, Direct Examination by Howard Kurtz, Attorney for the Defendant

Howard Kurtz, Attorney for the Defendant (Questioner): Hello there, could you please tell us your name and occupation?

Dr. Erik Dubberke (Answerer): My name is Erik Dubberke, and I am a trauma surgeon at South Lake Hospital here in Clermont.

Q: That's an emergency room doctor, right?

A: Yes, I'm one of the several surgeons on staff that are trained specifically for traumarelated procedures. Injuries, accident victims, things like that. Immediate and sometimes life-or-death repairs, as opposed to planned, preventative, or purely cosmetic procedures.

Q: Ahh. I see. Tell me, Dr. Dubberke, were you on duty on Tuesday, July $8^{\text {th }}, 2008$ ?

A: Yes, I was on call from noon until midnight that day.

Q: So, Dr. Dubberke, did you treat Ms. Amanda Johnson on that afternoon?

A: Yes I did. She was brought in by ambulance, and the paramedics had stabilized her condition, but it was pretty clear to them by the time that they arrived that she had suffered some sort of localized trauma to her right leg or hip area.

Q: And was that consistent with your observations?

A: Absolutely. We took some X-rays to see exactly what we were dealing with right after we cleaned up and stitched up her forehead. Luckily, her facial lacerations were superficial enough that I was able to use dissolvable sutures which tend to leave little or no scarring, but they don't work as well on really deep or difficult wounds.

Q: Thank heaven for small miracles. Now what did the X-rays reveal?

A: Ms. Johnson suffered a broken hip up near the ball in the joint where the right leg meets the pelvis. It's a very nasty sort of injury. 
Q: So how did you treat Ms. Johnson?

A: I inserted a metal plate into her right leg that is held in place by five metal screws that are screwed directly into her bones. By holding everything in place like that, it allows for the bone to gradually fuse back together at the site of the break.

Q: Sounds reasonable. Tell me, Dr. Dubberke, in your experience with trauma victims, are you at all able to judge the severity of accidents or make any determination about what caused or maybe even what could have prevented an injury?

A: To an extent. If what you're asking me is whether having worn her seat belt would have prevented Ms. Johnson's injury, then yes. I'm almost certain that her broken hip resulted from being thrown forward in a frontal crash.

Q: Not from having been rear-ended?

A: No, that type of impact typically results in whiplash and soft-tissue injuries, not being launched forward into the dashboard.

Q: Thank you, Dr. Dubberke. I don’t have any more questions.

Defense Witness Dr. Erik Dubberke, Cross Examination by Seth Blum, Attorney for the Plaintiff

Seth Blum, Attorney for the Plaintiff (Questioner): Dr. Dubberke, how long have you been working in the emergency room?

Dr. Erik Dubberke (Answerer): In my current capacity? Almost eight years. Counting assisting, residency, interning, volunteering, and all that? Probably another four, five years total on top of that.

Q: That's quite a long time. You must have seen some pretty remarkable, unusual, and awful things in that time.

A: Yeah, but you get used to it. If you can't, you don't last long.

Q: I bet. Tell me, have you ever seen anything that just defied explanation, or didn't make any sense, or just plain freak accidents or weird flukes?

A: Sure. The human body is a very complicated, complex organism, with an almost limitless capacity for things to go wrong. And the world can be a pretty strange place too. Just when you start to think you've seen it all, something you could have never envisioned in a hundred years comes through the doors. 
Q: I can only imagine. Thank you for all the good work you do, Doctor. Nothing further.

Defense Witness Officer Christopher Eaton, Direct Examination by Howard Kurtz, Attorney for the Defendant

Howard Kurtz, Attorney for the Defendant (Questioner): Good afternoon, Officer. Could you please give us your name, rank, department, and division?

Officer Christopher Eaton (Answerer): My name is Christopher Eaton, I'm a deputy with the Clermont Police Department, and I'm primarily assigned to the Patrol Section.

Q: Okay. And is the intersection of Foothill and Windsor in Clermont part of your assigned beat?

A: Yes, sir. It's right smack dab in the middle of it, in fact.

Q: And is that where you were on the afternoon of Tuesday, July $8^{\text {th }}, 2008$ ?

A: I was patrolling not too far away when I heard the call on the radio about an automobile accident which also potentially involved a gasoline truck. Mr. McGraw had called 911 pretty quickly, and it only took me a minute or two to get there, and I was the first responder on the scene.

Q: And what did you see when you arrived?

A: I observed Mr. Whitaker's Shell gasoline tanker truck stopped in the rightmost lane, on the south side of Windsor Road, in the eastbound direction. To the west of the intersection, on the north side of Windsor Road, in the westbound direction, I observed Mr. Collins's blue 2006 Toyota Camry at rest up with the front up against the lamp post, and Mr. McGraw's white 2000 Ford Econoline work van smashed up into the rear of Mr. Collins's car. Mr. McGraw's van had sustained additional damage to the rear passenger quarter panel area, and Mr. Collins's car had a broken windshield in the typical pattern found when the windshield is struck by the head of the occupants of the front seat. In this case, that would be Mr. Collins and Ms. Johnson.

Q: And you've seen a lot of car accidents during your time on the force, I imagine?

A: Yeah, that's probably one of the most frequent calls we get. Hardly a day goes by that I don't deal with at least one wreck. 
Q: I bet. And in your experience, when you've seen patients with head trauma and broken bones, injured in cars that end up with cracked windshields like Mr. Collins's car, do those accidents typically involve a car that has been rear-ended?

A: Well, I've never actually kept a count of something like that, but off the top of my head, I'd say that very few of them do, except for the sorts of multi-impact incidents like we had here.

Q: How do you mean?

A: Usually, passengers in cars who have cuts to their head and broken bones suffer their injuries as a result of having been forcefully thrown forward from the violence of a frontal impact. You know, running into something, not being run into from behind.

Q: Okay, well thank you very much for your assistance, Officer. I have no additional questions.

A: My pleasure, sir.

Defense Witness Officer Christopher Eaton, Cross Examination by Seth Blum, Attorney for the Plaintiff

Seth Blum, Attorney for the Plaintiff (Questioner): Officer Eaton, you've undoubtedly seen plenty of car accidents in your time patrolling, haven't you? Accidents of all kinds?

Officer Christopher Eaton (Answerer): Yes, sir. I couldn't even offer a guess as to the total number.

Q: And in your eyes, was this your typical rear-ending?

A: No, sir. Most rear-ending accidents do not involve an impact from a third vehicle like this incident.

Q: So this was a little out of the ordinary for how most rear-impact collisions occur?

A: Yes, sir. I'm not sure whether I can recall anything quite like this in all my years on the force where a semi trailer smacks into one vehicle which then crashes into another in a chain reaction like that. This was the result of some pretty unlikely combinations of forces and events.

Q: I'll say. Thank you, Officer. 
A: My pleasure, sir. 


\section{APPENDIX AD}

\section{Liability Jury Instructions and Verdict Form}

\section{$\underline{\text { Liability }}$}

Negligence is the failure to use reasonable care. Reasonable care is that degree of care which a reasonably careful person would use under like circumstances. Negligence may consist either in doing something that a reasonably careful person would not do under like circumstances, or in failing to do something that a reasonably careful person would do under like circumstances. To determine whether the defendant is liable for automobile negligence, you must determine whether the following conditions have been met:

a. On or about Tuesday, July $8^{\text {th }}, 2008$, the defendant, Michael McGraw, owned and operated a vehicle in Lake County, Florida.

b. At that time and place, defendant Michael McGraw negligently operated or maintained the motor vehicle so that it collided with the motor vehicle in which plaintiff Amanda Johnson was a passenger.

c. As a result, plaintiff Amanda Johnson suffered physical injuries described during the trial.

d. When determining liability, decide whether the defendant Michael McGraw was negligent using the burden of proof preponderance of the evidence: that is, based on the "greater weight of the evidence"

e. An additional question for your determination on the defense is whether some or all of Amanda Johnson's damages were caused by her failure to use a seat belt.

f. The issues for your determination on this question are whether the greater weight of the evidence shows that the automobile occupied by Amanda Johnson was equipped with an available and fully operational seat belt, that Amanda Johnson did not use the seat belt, that a reasonably careful person would have done so under the circumstances, and that Amanda Johnson's failure to use the seat belt produced or contributed substantially to producing the damages sustained by her.

g. If the greater weight of evidence does not support defendant Michael McGraw on each of these issues, then your verdict on this question should be for plaintiff Amanda Johnson.

h. If the greater weight of the evidence supports defendant on these issues, you should determine what percentage of Amanda Johnson's total damages were caused by her failure to use the seat belt. 
1. Based on the above legal definition of liability, do you find the defendant, Michael McGraw, liable for the plaintiff's injuries? (Check one)

YES, Michael McGraw is liable

NO, Michael McGraw is not liable

2. Using the scale below, how liable is Michael McGraw in this case?

\begin{tabular}{|c|c|c|c|c|c|c|c|c|}
\hline 1 & 2 & 3 & 4 & 5 & 6 & 7 & 8 & 9 \\
\hline \\
No - He is not liable \\
Yes - He is liable \\
\hline
\end{tabular}

3. How confident are you in your liability decision?

\begin{tabular}{|l|l|l|l|l|l|l|l|l|}
\hline 1 & 2 & 3 & 4 & 5 & 6 & 7 & 8 & 9 \\
\hline \multicolumn{4}{|c|}{ Not at all confident } \\
\hline
\end{tabular}

If your answer to question 1 is NO, your verdict is for defendant, and you should not proceed further. If your answer to question 1 is YES, please answer question 4.

4. Was there negligence on the part of Amanda Johnson which was a legal cause of her injury?

$$
\text { YES _ NO }
$$

5. Was there negligence on the part of Brock Collins which was a contributing legal cause of injury to Amanda Johnson?

YES _ NO N

6. State the percentage of any negligence, which was a legal cause of injury to Amanda Johnson that you charge to:

Michael McGraw $\%$

Brock Collins

Amanda Johnson

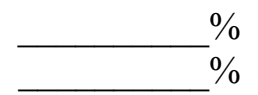


Total must be $100 \%$

(Note: For any response of "NO" to question 1, 2, or 3, place a zero as to that person in answering question 6.) 


\section{APPENDIX AE}

Damage Award Form

\section{Damage Award Determinations}

\section{Compensatory Damage Award:}

Below, determine the compensatory damages (if any) that Amanda Johnson should be awarded as a result of automobile negligence. Compensatory damages are based on a preponderance of the evidence. This means that if the weight of the evidence is in the plaintiff Amanda Johnson's favor, then compensatory damages should be awarded. In determining the amount of compensatory damages, consider the following:

a. Any economic losses or injuries incurred at the present time, or which will probably be incurred in the future, including: loss of earnings or wages, lost impairment of earning capacity, medical expenses, etc.

b. Any non-economic losses or injuries incurred at the present time, or which will probably be incurred in the future, including: pain and suffering, impairment in the quality of life, mental anguish, inconvenience, emotional stress, etc.

1. Dollar Award for economic damages

$\$$

2. Dollar Award for non-economic damages

$\$$

3. Total Compensatory Damage Award (Please add economic and non-economic damages)

$\$$

4. In a few sentences, explain why you awarded this total dollar amount.

\section{Punitive Damage Awards}

If you find for plaintiff Amanda Johnson and against defendant Michael McGraw, you should consider whether, in addition to compensatory damages, punitive damages are warranted in the circumstances of this case as punishment and as a deterrent to others.

Punitive damages are warranted if you find by clear and convincing evidence that Michael McGraw engaged in intentional misconduct or gross negligence.

5. Punitive damage award $\$$

6. In a few sentences, explain why you awarded this dollar amount. 


\section{APPENDIX AF}

Study Four Scoring of Inconsistency Detection Rates

\section{Verification of Inter-rater Reliability}

Skewness and kurtosis values for the distribution of "Yes" and "Partial" detection scores on the open ended questions exceeded twice the absolute value of their standard errors for all eight inconsistencies calculated for two research assistants individually using their data sets from their individual ratings done before discussion with each other, after discussion with each other, and collapsing the "Yes" and "Partial" scores into a single total of scored detections for both research assistants individually before discussion and again after discussion for at least one value on thirteen of the 17 response classifications with a distribution consisting of more than one score. The short answer questions were similarly non-normally distributed based on skewness and kurtosis values exceeding twice the absolute value of their standard errors for the same treatments and tests of data on thirteen of the 16 response classifications with a distribution of more than one score. Because of this non-normal distribution, I relied on Kendall's tau-b correlation to verify inter-rater reliability rather than Pearson's or Spearman's correlations.

\section{Open Ended and Short Answer Questions}

Although the research assistants used different thresholds of scoring for the open ended and short answer codings, the nature of the data set and the logistics of interpretation was common to both, so they will be discussed jointly for the purposes of this description. 
The way I quantified the open ended data is based on the way both research assistants scored the set of data, and it takes into account the fact that they were recording the total number of mentions of a detection for each inconsistency (this turned out to be something I later recoded into a binary "present/absent" variable, since certain inconsistencies lended themselves to being described in response to more than one question — especially among the open ended questions — while others would only be a natural response to one question) so the number of mentions, which is reflected in all Yes and Partial values, is not a reliable means of distinguishing detection rates. However, they remained useful as an index of agreement between research assistants, particularly when comparing the Before values (each assistant coded the data independently) to the After values (after meeting to compare the way they coded everything and to discuss and justify why they coded a particular response in a particular way with the goal of coming to a consensus if/when possible.)

For a full description of coding procedures and instructions, see Appendices $\mathrm{K}$ and L, but the primary difference between open ended and short answer coding instructions related to the degree of flexibility allowed in defining a "Yes" or a "Partial" detection. Since open-ended questions lack specificity by nature, the degree of flexibility was greater for the open ended responses. However, since the short answer questions were far more specific in their wording, the research assistants were instructed to be much more exclusive and strict in their scoring and interpretations.

Because of the room for interpretation inherent in these two subjective types of scores, I used the same two overall rates of detection as I did in Studies One and Two, with the strict criterion requiring that both research assistants each assign a "Yes" score 
for a participant to be given credit for the detection, while the loose criterion simply required that both research assistants assign some sort of credit (either a "Yes" or a "Partial" detection) to the response in order for it to counted as a detection.

\section{Multiple Choice Questions}

I did not split the multiple choice detection data into two separate loose and strict criterion levels as I had done in Studies One and Two (calculating the rates of detection for each inconsistency from the total number of participants as well as the percentage of detection among only those participants who correctly indicated having detected an inconsistency on at least one multiple choice question), as it was no longer my intention to identify which inconsistencies to retain and which to eliminate from the transcript. Instead, detection rates were simply the overall percentage of detections for each experimental condition.

\section{True or False Questions}

Interpreting the true or false inconsistency detection rates was straightforward. Four of the eight non-dummy questions were phrased in a manner such that an inconsistency detection corresponded with a "True" response, and four of eight were phrased in a manner where a "False" response indicated an inconsistency detection. Detection rates for each inconsistency were simply the percentage of participants who answered the non-dummy questions in a manner indicating a detection.

\section{Point Blank Questions}

Just as with the other objective forms of inconsistency detection questions, I did not employ separate methods of calculating a liberal, upper boundary of detection rates as 
well as a stricter, lower boundary of detection rates, but rather only counted the percentage of participants who correctly identified each inconsistency.

\section{Scales to Summarize Relative Ease of Detection for Each Inconsistency}

I did not use the same composite measures (strict: CombiHighYes and loose:

CombiMed) from Studies One and Two to compare the overall detection rates of each inconsistency across question types in Study Four because those measures were used for the specific purpose of evaluating rates of detection for each inconsistency individually and relative to all other inconsistencies in order to identify which inconsistencies to retain in the transcript (those detected by between approximately $33 \%$ and $66 \%$ of all participants) and which inconsistencies to eliminate (those detected by percentages of participants greater or less than that middle range). As mentioned in each section above, with the slight exception for the subjectively scored questions, I simply used the percentages of detection within each experimental condition for each inconsistency according to each question type without any additional adjustment or calculation. See Table AF1 for detection rate percentages for each of the eight inconsistencies according to emotion induced and question type. 
Table AF1

Study Four: Comparison of Mean Detection Ratings by Transcript Version

\begin{tabular}{|c|c|c|c|c|c|c|c|c|c|c|}
\hline & \multicolumn{4}{|c|}{ Detections } & \multirow[b]{3}{*}{$t$} & \multirow[b]{3}{*}{$\mathrm{df}^{*}$} & \multirow[b]{3}{*}{$p$} & & & \multirow[b]{3}{*}{$\begin{array}{c}\text { Cohen's } \\
d\end{array}$} \\
\hline & \multicolumn{2}{|c|}{$\begin{array}{c}\text { Inconsistent } \\
\text { Transcript }\end{array}$} & \multicolumn{2}{|c|}{$\begin{array}{l}\text { Consistent } \\
\text { Transcript }\end{array}$} & & & & \multicolumn{2}{|c|}{$95 \% \mathrm{CI}$} & \\
\hline & $M$ & $S D$ & $M$ & $S D$ & & & & $L L$ & $U L$ & \\
\hline PI4-Oil Co. & 1.61 & 1.60 & 0.16 & 0.37 & 8.77 & 109 & $<.001$ & 1.12 & 1.77 & 1.47 \\
\hline PI5-Car Model & 1.87 & 1.41 & 0.18 & 0.46 & 11.31 & 119 & $<.001$ & 1.39 & 1.98 & 1.79 \\
\hline PI7-Hair & 2.23 & 1.65 & 0.24 & 0.57 & 11.40 & 118 & $<.001$ & 1.64 & 2.34 & 1.83 \\
\hline PI9-Year & 1.39 & 1.39 & 0.22 & 0.44 & 7.99 & 118 & $<.001$ & 0.88 & 1.46 & 1.27 \\
\hline PI10-Color & 1.59 & 1.53 & 0.29 & 0.50 & 8.01 & 119 & $<.001$ & 0.97 & 1.61 & 1.28 \\
\hline PI1 1-Jared & 1.30 & 1.51 & 0.12 & 0.33 & 7.62 & 107 & $<.001$ & 0.87 & 1.49 & 1.28 \\
\hline PI12-Tree & 1.44 & 1.41 & 0.34 & 0.50 & 7.33 & 122 & $<.001$ & 0.80 & 1.40 & 1.16 \\
\hline PI13-Dog & 1.63 & 1.35 & 0.19 & 0.44 & 10.03 & 119 & $<.001$ & 1.15 & 1.72 & 1.59 \\
\hline $\begin{array}{l}\text { Open Ended } \\
\text { CombiHighYes }\end{array}$ & 2.15 & 1.90 & 0.04 & 0.24 & 10.95 & 101 & $<.001$ & 1.73 & 2.49 & 1.97 \\
\hline $\begin{array}{l}\text { Open Ended } \\
\text { CombiMed }\end{array}$ & 2.24 & 1.88 & 0.06 & 0.31 & 11.39 & 103 & $<.001$ & 1.80 & 2.56 & 1.98 \\
\hline Multiple Choice & 2.80 & 2.46 & 0.01 & 0.10 & 11.26 & 98 & $<.001$ & 2.30 & 3.28 & 2.18 \\
\hline True False & 4.08 & 2.35 & 0.48 & 0.83 & 14.39 & 122 & $<.001$ & 3.10 & 4.09 & 2.26 \\
\hline Point Blank & 4.03 & 2.21 & 1.22 & 1.48 & 10.51 & 171 & $<.001$ & 2.28 & 3.34 & 1.53 \\
\hline Total Detections & 13.06 & 7.91 & 1.76 & 1.83 & 13.86 & 108 & $<.001$ & 9.69 & 12.92 & 2.32 \\
\hline
\end{tabular}

Note. *Levene's test indicated unequal variances for all measures, so degrees of freedom have been adjusted from 196 to the values shown. $\mathrm{CI}=$ confidence interval; $L L=$ lower limit; $U L=$ upper limit. Total Detections represents the sum of detection rates for Open Ended CombiHighYes, Multiple Choice, True False, and Point Blank question types only, with possible total scores ranging from 0 to 32 detections. 
VITA

STEPHEN W. JOY

Born, Champaign, Illinois

1994-1998

B.A., Psychology

Augustana College

Rock Island, Illinois

1999-2002

J.D.

University of Illinois College of Law

Champaign, Illinois

2005-2009

M.S., Psychology

Florida International University

Miami, Florida

\section{PUBLICATIONS AND PRESENTATIONS}

Charman, S.D., Wells, G.L., \& Joy, S.W. (2011). The dud effect: Adding highly dissimilar fillers increases confidence in lineup identifications. Law and Human Behavior, 35, 479-500.

Joy, S.W. The effect of testimony mistranslations on bilingual and monolingual jurors. Poster session at the American Psychology-Law Society Conference. Vancouver, BC, March 2010.

Joy, S.W. Did he just say what I think he said? Effect of testimony mistranslations on bilingual and monolingual jurors. American Psychology-Law Society Conference. San Antonio, TX, March 2009.

Joy, S.W. Lost in translation, or simply mistranslated? Effect of testimony mistranslations on bilingual and monolingual jurors. Poster session at the American Psychology-Law Society Conference. Jacksonville, FL, March 2008.

Joy, S.W. Impact of task complexity on jury sentencing in noncapital felony cases. American Psychology-Law Society Conference. St. Petersburg, FL, March 2006.

Kindt, J.W., \& Joy, S.W. (2002). Internet gambling and the destabilization of national and international economies: Time for a comprehensive ban on gambling over the World Wide Web. Denver University Law Review, 80(1), 111-153. Reprinted in The Program in Arms Control, Disarmament, and International Security's Occasional Paper, Champaign, IL: University of Illinois at Urbana-Champaign, (September, 2007), 1-35; and Kindt, J.W. (Ed.), United States International Gambling Report Series: Gambling 
with crime, destabilized economies, and financial systems (Doc. No. 2.20 \& Doc. No. 2.30). Buffalo: William S. Hein \& Co., Inc. (2009).

Winter, R.J., \& Joy, S.W. (2012). Is Florida's self-defense law defensible? Monitor on Psychology, 43(8), 29.

Winter, R.J., \& Joy, S.W. (2011). 'Thanks for the recommendation, but I'd rather kill him' Monitor on Psychology, 42(8), 24.

Winter, R.J., \& Joy, S.W. (2010). Must the jury be unanimous? Monitor on Psychology, 41(3), 20. 